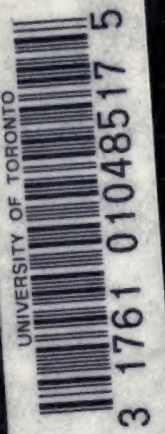

Handle with

EXTREME CARE

This volume is damaged or brittle and CANNOT be repaired!

- photocopy only if necessary

- return to staff

- do not put in bookdrop

Gerstein Science Information Centre

Umistot

Domerro

LIDFAPY 


\section{A COMPARISON OF METHODS FOR DETERMINING THE RESPIRATORY EXCHANGE OF MAN}

THORNE M. CARPENTER
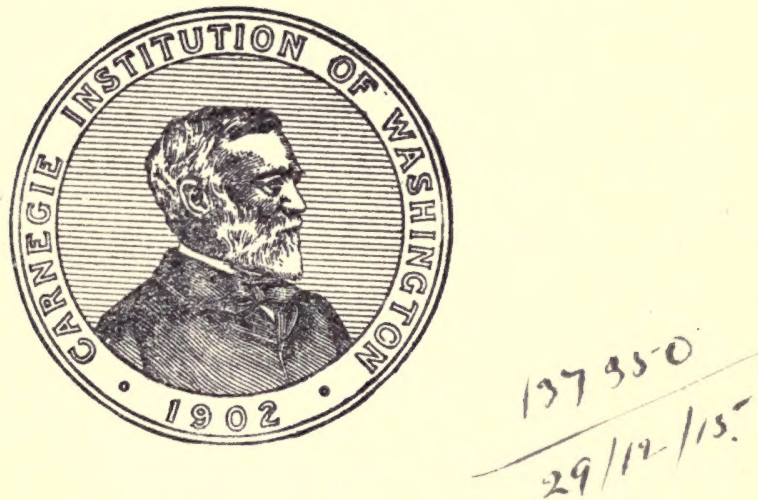

WASHINGTON, D. C.

Published by the Carnegie Institution of Washington 


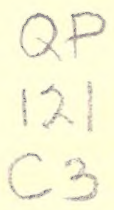

CARNEGIE INSTITUTION OF WASHINGTON

Publication No. 216

PRGSS OF GIBSON BROTHERS, INC.

WAsHINGTON, D. C. 

Digitized by the Internet Archive in 2008 with funding from Microsoft Corporation 


\section{CONTENTS.}

\section{PART I.}

PAGE.

Introduction . . . . . . .

Earlier comparisons of respiration apparatus . . . . . . . . . . . . . . . 10

Apparatus and technique used in the present study . . . . . . . . . . . . 12

Bed respiration calorimeter. . . . . . . . . . . . . . . . . . . . . 14

Benedict universal respiration apparatus. . . . . . . . . . . . . . . 21

Tension-equalizer unit. . . . . . . . . . . . . . . . . . . . 21

General plan of apparatus. . . . . . . . . . . . . . 21

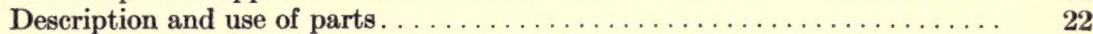

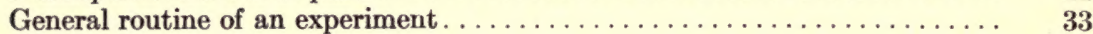

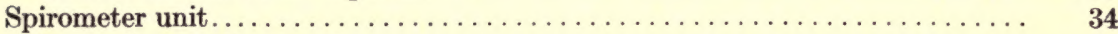

General plan of apparatus. . . . . . . . . . . . . . . $\ldots \ldots \ldots$

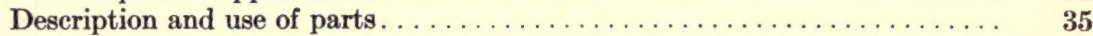

General routine of an experiment. . . . . . . . . $\ldots \ldots \ldots \ldots \ldots$

Oxygen supply for the universal respiration apparatus. . . . . . . . . . 46

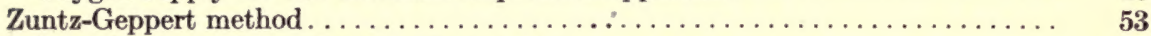

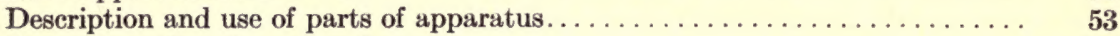

General routine of an experiment. . . . . . . . . . . . . . . . . 60

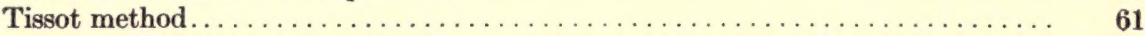

Description and use of parts of apparatus. . . . . . . 61

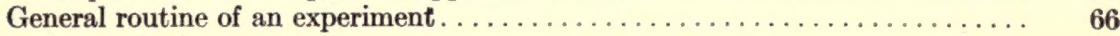

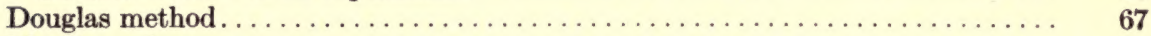

Mueller valves. . . . . . . . . . . . . . . . . . . . . . . . 70

Haldane gas-analysis apparatus. . . . . . . . . . . . . . . . . . 70

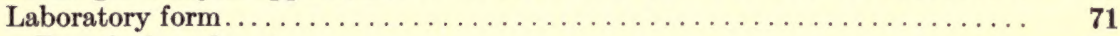

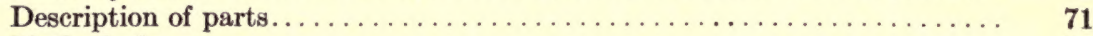

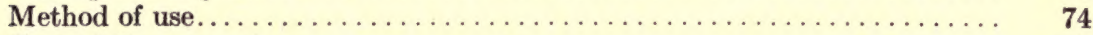

Care of the apparatus. . . . . . . . . . . . .

Testing the apparatus. . . . . . . . . . .

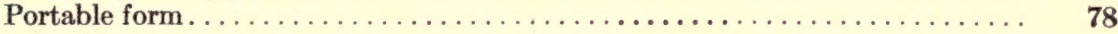

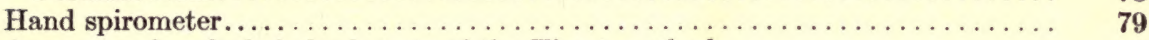

Apparatus for alcohol check-tests of the Tissot method . . . . . . . . . . . 80

\section{Part II.}

Comparisons of respiratory exchange as measured by different types of apparatus. .

Bed respiration calorimeter and Benedict respiration apparatus (tension-equalizer

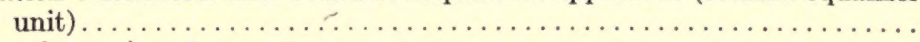

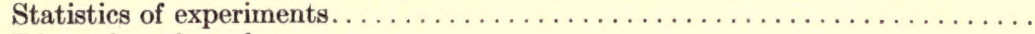

Discussion of results. . . . . . . . . . . . . . . . . . . . . . .

Sources of error in experiments with the bed calorimeter. . . . . . . . . . .

Sources of error in experiments with the Benedict respiration apparatus....

Differences in the individual comparisons. . . . . . . . . . . . . . . . .

The two types of the Benedict respiration apparatus (the tension-equalizer unit

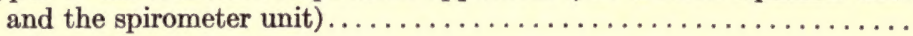

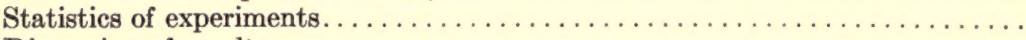

Discussion of results. ... . . . . . . . . . . . . . . . . . . . .

Zuntz-Geppert respiration apparatus and Benedict respiration apparatus (tension-

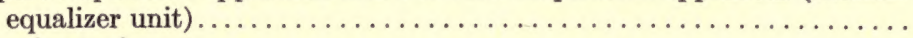

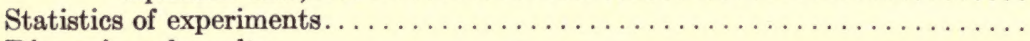

Discussion of results. . . . . . . . . . . . . . . . . . . . . . . . .

Zuntz-Geppert respiration apparatus and Benedict respiration apparatus (spi-

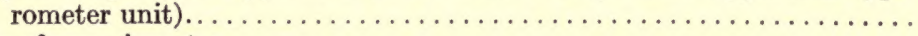

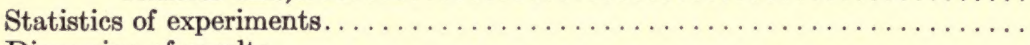

Discussion of results. . . . . . . . . . . . . . . . . . . . . . . . .

Tissot apparatus and Benedict respiration apparatus (tension-equalizer unit)....

Statistics of experiments.

Discussion of results 
Comparison of respiratory exchange-Continued.

Tissot apparatus and Benedict respiration apparatus (spirometer unit)....... 150

Statistics of experiments............................. 152

Discussion of results . . . . . . . . . . . . . . . . . . . . . . . . . . . 154

Douglas respiration apparatus and Benedict respiration apparatus (spirometer

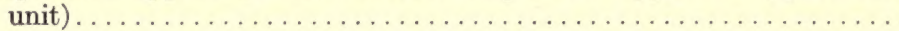

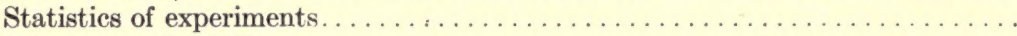

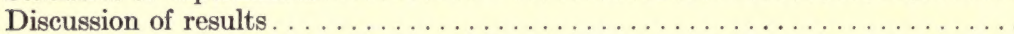

Mouth- and nose-breathing with the Benedict respiration apparatus (tension-

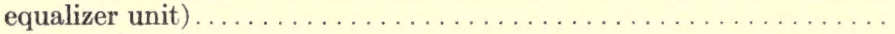

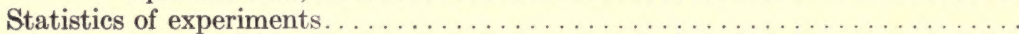

Discussion of results. . . . . . . . . . . . . . . . . . . . . . . . . .

Mouth- and nose-breathing with the Benedict respiration apparatus (spirometer unit) . . . . . . . . . . . . . . . . . . . . . . . . . .

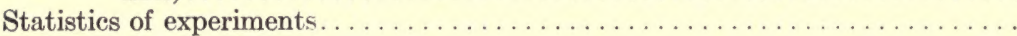

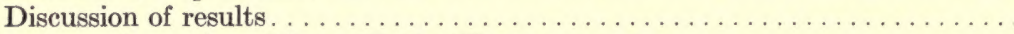

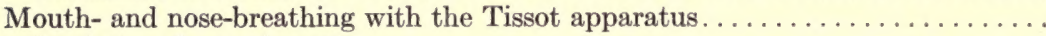

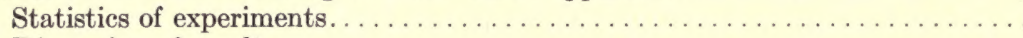

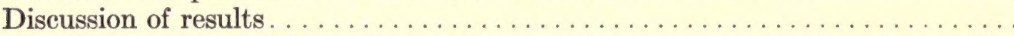

Mask and nosepieces with the Benedict respiration apparatus (spirometer unit). .

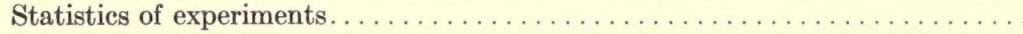

Discussion of results.

Glass and pneumatic nosepieces with the Benedict respiration apparatus (spirom-

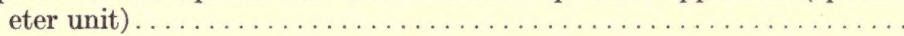

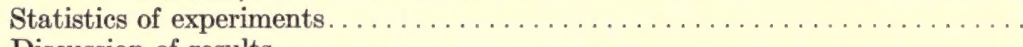

Discussion of results .

Mueller valves and Tissot spirometer and the Benedict respiration apparatus (spirometer unit) . . . . . . . . . . . . . . . . . . . . .

Statistics of experiments. . . . . . . . . . . . . . . . . . . . .

Discussion of results . . . . . . . . . . . . . . . . . . . . . . . .

Mueller valves and Tissot valves. . . . . . . . . . . . . . . . . . . .

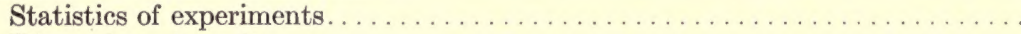

Discussion of results .

Benedict respiration apparatus (spirometer unit) with and without additional dead

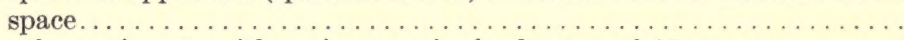

Statistics of experiments with an increase in dead space of 45 c.c...........

Statistics of experiments with an increase in dead space of 90 c.c..........

Statistics of experiments with an increase in dead space of 135 c.c..........

Statistics of experiments with an increase in dead space of 224 c.c..........

Discussion of results . . . . . . . . . . . . . . . . . . . . . . . . . . . . . . . . . . . .
Tissot apparatus with and without automatic counterpoise on the spirometer bell.

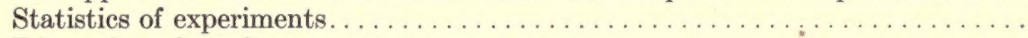

Discussion of results.

\section{PART III.}

Critical discussion of respiration apparatus and their technique........... 227

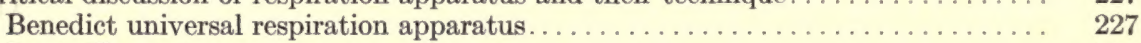

Zuntz-Geppert apparatus.................................... 234

Tissot apparatus..................................... 240

Douglas method ...................................... 248

Valves................................................ 250

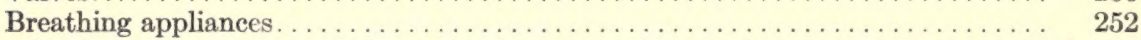

Pneumatic nosepieces................................ 253

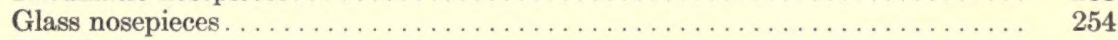

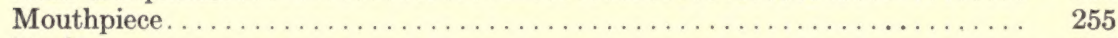

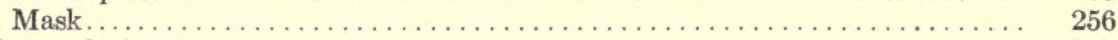

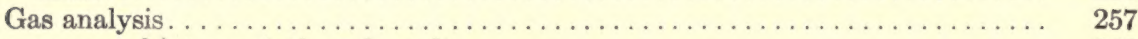

Accuracy and interpretation of results......................... 260 


\section{ILLUSTRATIONS.}

PAGE.

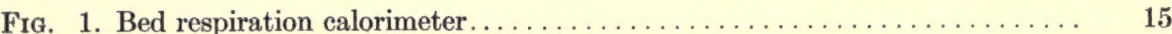

2. Air-circuit and purifying arrangements of tension-equalizer unit. . . . . 22

3. Arrangement of Benedict respiration apparatus (tension-equalizer unit). . 22

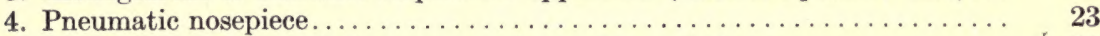

5. Tension-equalizer with three-way valve and mouthpiece.......... 25

6. Carbon-dioxide absorber and accompanying water-absorber........ 27

7. Moistener. . . . . . . . . . . . . . . . . . . . . . . . . . . . . 29

8. Apparatus used for tests of respiration apparatus with burning ether. . . . 31

9. Schematic outline of ventilation system of spirometer unit.......... 34

10. Detailed plan of ventilation system in spirometer unit . . . . . . . . . 35

11. Cross-section of the three-way valve, ventilating pipe, and connection for mouthpiece and moistener. . . . . . . . . . . . . . . . . 36

12. Details of moistener and connection for nosepieces . . . . . . . . . . . . 37

13. Details of spirometer, with recording attachments. . . . . . . . . . . . 38

14. Specimen graphic record of respiration . . . . . . . . . . . . . . 40

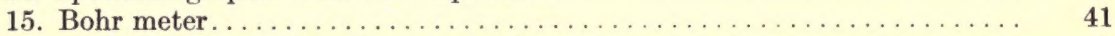

16. General view of the spirometer unit. . . . . . . . . . . . . . . . . 41

17. Specimen kymograph records in the calibration of the ventilation adder.. . 45

18. Mouthpiece and valves used in the Zuntz-Geppert apparatus . . . . . . . 54

19. Most recent form of the Zuntz valves. . . . . . . . . . . . . . . . . 54

20. Zuntz-Geppert apparatus, showing Elster meter, automatic sampling device,

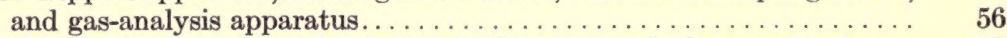

21. Caustic potash pipette used in the Zuntz-Geppert analysis apparatus.... $\quad 58$

22. Absorption pipette used in the Zuntz-Geppert analysis apparatus. . . . . . 58

23. Nosepieces and valves used with the Tissot method . . . . . . . . . . . 62

24. Modified glass nosepieces. . . . . . . . . . . . . . . . . . . . . . . . . 62

25. Apparatus for registering the respiration-rate used with the Tissot method. 63

26. Tissot spirometer with capacity of 50 liters. . . . . . . . . . . . . . . . 64

27. Tissot spirometer with capacity of 200 liters. . . . . . . . . . . . . . . 64

28. Apparatus for registering the volume of air in the Tissot spirometer. . . . 65

29. Mica-flap valve used with the Douglas method. . . . . . . . . . . . . 68

30. Rubber-flap valve used with the Douglas method. . . . . . . . . . . . . . 69

31. Mueller valve . . . . . . . . . . . . . . . . . . . . . . . . . . . . . 70

32. Haldane gas-analysis apparatus (laboratory form) . . . . . . . . . . . 72

33. Hand spirometer. . . . . . . . . . . . . . . . . . . . . . . . . 79

34. Apparatus used for alcohol check-tests of the Tissot method. . . . . . . . . 80

35. Type of respiration of subject H. F. T. as shown by chest pneumograph in the first period with the bed calorimeter on August $29,1911 \ldots \ldots \ldots$.

36. Type of respiration of subject H. F. T. in the first period with the bed

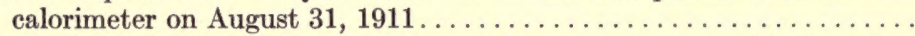

37. Probability curves for the series of comparison experiments with the spiro-

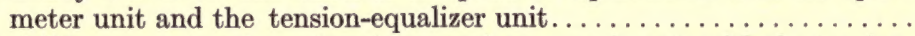

38. Probability curves for the series of comparison experiments with the tensionequalizer unit and the Zuntz-Geppert apparatus. . . . . . . . . . .

39. Types of respiration of subject $H$. F. T. in third and sixth periods with the spirometer unit on January $18,1912 \ldots \ldots \ldots \ldots \ldots \ldots \ldots \ldots \ldots \ldots \ldots \ldots \ldots \ldots \ldots \ldots \ldots$

40. Types of respiration of subject H. F. T. in first and second periods with the

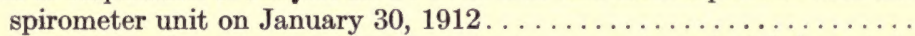

41. Types of respiration of subject P. F. J. in the seventh and eighth periods with the spirometer unit on February $7,1912 \ldots \ldots \ldots \ldots \ldots \ldots$

42. Probability curves for the series of comparison experiments with the spirometer unit and the Zuntz-Geppert apparatus...............

43. Probability curves for the series of comparison experiments with the tension-

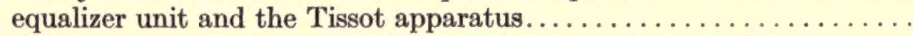

44. Probability curves for the series of comparison experiments with the spiro-

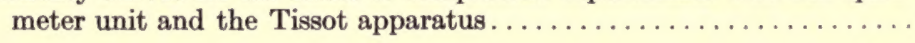


Fig. 45. Types of respiration of subject M. J. S. at end of second and fourth periods with the spirometer unit on July $19,1912 \ldots \ldots \ldots \ldots \ldots \ldots \ldots \ldots$

46. Probability curves for the series of comparison experiments with the spiro-

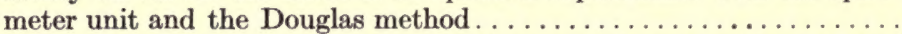

47. Probability curves for the series of comparison experiments with nose- and

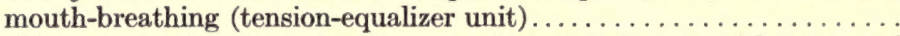

48. Probability curves for the series of comparison experiments with nose- and mouth-breathing (spirometer unit) $\ldots \ldots \ldots \ldots \ldots \ldots \ldots \ldots \ldots \ldots \ldots \ldots \ldots \ldots \ldots$

49. Probability curves for the series of comparison experiments with nose- and

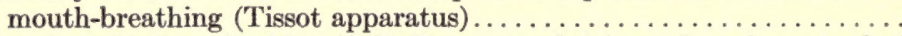

50. Types of respiration of subject L. E. E. as recorded from the spirometer bell in the second period on November $18,1912 \ldots \ldots \ldots \ldots \ldots \ldots \ldots$.

51. Probability curves for the series of comparison experiments with nosepieces and mask (spirometer unit).

52. Types of respiration of subject W. J. T. as shown by the pneumograph in the first two periods with the Mueller valves and Tissot spirometer on

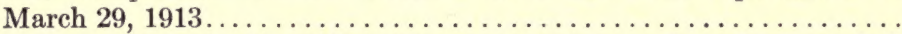

53. Type of respiration of subject W. J. T. as recorded from the spirometer bell in the second period with the spirometer unit on March 29, 1913...

54. Probability curves for the series of comparison experiments with the spi-

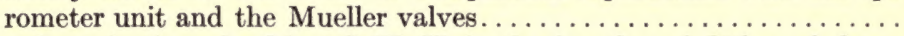

55. Type of respiration of subject J. H. H. in the fourth and fifth periods on

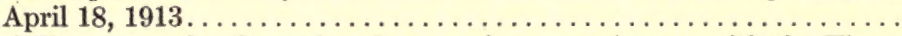

56. Probability curves for the series of comparison experiments with the Tissot valves and the Mueller valves.

PAGE.

165

172

179

183

188

190

193

196

196

199

201

205

57. Type of respiration of subject J. K. M. without additional dead space on September 20, 1912

58. Type of respiration of subject J. K. M. with 45 c.c. additional dead space

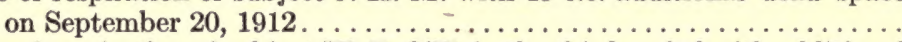

59. Type of respiration of subject W. F. O'H. in the third period with additional dead space on October $27,1912 \ldots \ldots \ldots \ldots \ldots \ldots \ldots \ldots \ldots \ldots$

60. Type of respiration of subject W.F. O'H. at the end of the third period without additional dead space on October $27,1912 \ldots \ldots \ldots \ldots \ldots \ldots$.

61. Type of respiration of subject W. F. O'H. in the early part of the second period without additional dead space on October 27, 1912........

62. Type of respiration of subject W. F. O'H. at the beginning of the fourth period without additional dead space on October $27,1912 \ldots \ldots \ldots \ldots$.

63. Type of respiration of subject J. W. P. in the second period with additional

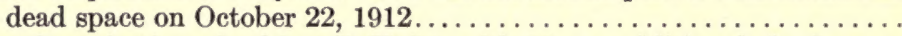

64. Type of respiration of subject J. K. M. with 90 e.c. additional dead space

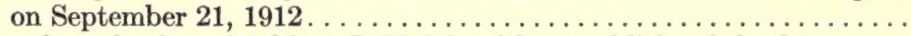

65. Type of respiration of subject J. K. M. without additional dead space on

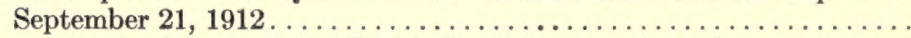

66. Type of respiration of subject T. M. C. without additional dead space on November 8, 1912.

67. Type of respiration of subject T. M. C. with 135 c.c. additional dead space

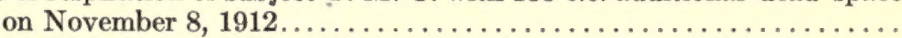

68. Type of respiration of subject P. F. J. without additional dead space on November 7, 1912.

69. Type of respiration of subject P. F. J. with 135 c.c. additional dead space

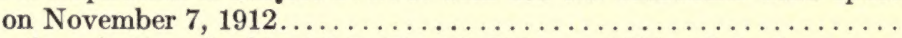

70. Type of respiration of subject J. B. T. with 224 c.c. additional dead space on

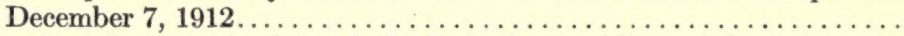

71. Type of respiration of subject J. B. T. without additional dead space on

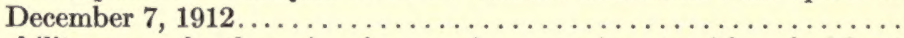

72. Probability curves for the series of comparison experiments with and without additional dead space (spirometer unit) ......................

73. Types of respiration of subject W. J. T. in second and third periods on

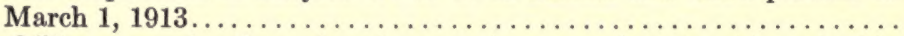

74. Probability curves for the series of comparison experiments with and without the counterpoise on the Tissot spirometer. . . . . . . . . . . . . 


\title{
A COMPARISON OF METHODS FOR DETERMINING THE RESPIRAT0RY EXCHANGE OF MAN.
}

\author{
By Thorne M. Carpenter.
}





\section{PART I.}

\section{INTRODUCTION.}

The development of apparatus for measuring the respiratory exchange of man has proceeded along two lines. In one type of apparatus the subject is completely inclosed in a chamber; in the other, the subject is attached to the respiration apparatus by means of some breathing appliance. The chamber type includes the respiration apparatus of Pettenkofer and Voit, ${ }^{1}$ Sondén and Tigerstedt, ${ }^{2}$ Jaquet, ${ }^{3}$ and Grafe, ${ }^{4}$ the Atwater-Benedict respiration calorimeter, ${ }^{5}$ and the respiration calorimeters of the Nutrition Laboratory. ${ }^{6}$ This type of apparatus is generally used for periods of not less than an hour and may be either a closed or open circuit. The apparatus without chambers are used for periods of about 15 minutes and may also be either closed or open circuit. In the latter case, the inspired and expired air are separated by valves. A mouthpiece, nosepiece, or mask is used for the breathing appliance. The open-circuit apparatus are represented by the apparatus of Speck, ${ }^{7}$ Zuntz-Geppert, ${ }^{8}$ Tissot, ${ }^{9}$ and Douglas. ${ }^{10}$ The closedcircuit apparatus include the two types of the Benedict apparatus, ${ }^{11}$ Rolly's ${ }^{12}$ modified Benedict apparatus, and that of Krogh. ${ }^{13}$

When the large amount of work on respiratory exchange carried out with these apparatus is considered, it will be seen that the importance of knowing whether the results obtained are reliable and physiologically comparable can hardly be overestimated. Recognizing the need of a comparative investigation into the reliability of the principal respiration apparatusin use to-day, the Director of the Nutrition Laboratory, in a trip to Europe in 1907, secured various apparatus for measuring the respiratory exchange, including particularly the Zuntz-Geppert and the Tissot respiration apparatus, with a view to comparing them with apparatus already being developed in this laboratory. Subsequently he arranged on two occasions for the writer to visit the laboratories in Berlin and Paris, where these methods were developed, and thus to become personally acquainted with the technique involved. The

\footnotetext{
${ }^{1}$ Pettenkofer and Voit, Ann. d. Chemie u. Pharm., II Supp. Bd., 1882, p. 52.

${ }^{2}$ Sondén and Tigerstedt, Skand. Archiv f. Physiol., 1895, 6, p. 1.

${ }^{3}$ Jaquet, Verhandl. d. Naturf. Gesellsch. in Basel, 1903, 15, p. 252.

${ }^{4}$ Grafe, Zeitschr. f. physiol. Chemie, 65, 1910, p. 1.

${ }^{5}$ Atwater and Benedict, Carnegie Inst. Wash. Pub. 42, 1905.

'Benedict and Carpenter, Carnegie Inst. Wash. Pub. 123, 1910.

'Speck, Physiologie des menschlichen Athmens nach eigenen Untersuchungen, Leipsic, 1892.

${ }^{8}$ Magnus-Levy, Archiv f. d. ges. Physiol., 1894, 55, p. 1.

Tissot, Journ. de physiol. et de pathol. gén., 1904, 6, p. 688.

${ }^{10}$ Douglas, Journ. Physiol., 1911, 42, Proc. Physiol. Soc. p. xvii.

${ }^{11}$ Benedict, Am. Journ. Physiol., 1909, 24, p. 345; Deutsch. Archiv f. klin. Med., 1912, 107 , p. 156.

${ }^{12}$ Rolly and Rosiewicz, Deutsch. Archiv f. klin. Med., 1911, 103, p. 58.

${ }^{13}$ Krogh, Skand. Archiv f. Physiol., 1913, 30, p. 375.
} 
following is a report of an extended comparative investigation of the different respiration apparatus used alone or in combination. While not all possible modifications have been studied, it is believed that the investigation covers enough lines for the results to be applied to respiration apparatus in general.

\section{EARLIER COMPARISONS OF RESPIRATION APPARATUS.}

A number of comparisons of the respiratory exchange obtained with various respiration apparatus have been made by different authors. These are all more or less in the nature of compilations and not direct determinations of the respiratory exchange by two or more methods on the same individual under identical conditions of food, body-weight, and time.

In 1897 Johansson $^{1}$ gave the results obtained on Zuntz with the respiration chamber at Stockholm and the Zuntz-Geppert apparatus in Berlin. The carbon-dioxide output per kilogram per hour as the result of two 2-hour periods September 21, 1897, in the chamber at Stockholm, was $0.304 \mathrm{gm}$., with a body-weight of $69.5 \mathrm{~kg}$. On October 1,1897 , at Berlin, the carbon-dioxide output was $0.285 \mathrm{gm}$. per kilogram per hour. Both values are designated by Johansson as having been obtained during complete muscular rest, although the protocols state that Zuntz was decidedly quieter in the experiment at Berlin than at Stockholm.

Durig, ${ }^{2}$ in his discussion on the results obtainable with the ZuntzGeppert method, gives a compilation of the determinations of the respiratory exchange for a number of subjects with the Zuntz-Geppert apparatus, the respiration chamber of Johansson, and the respiration calorimeter of Wesleyan University. The average results are given in table 1 .

TABLE 1.-Comparative compilation made by Durig of respiratory exchange determined by different methods.

\begin{tabular}{|c|c|c|c|c|c|}
\hline \multirow[b]{2}{*}{ Apparatus. } & \multirow[b]{2}{*}{$\begin{array}{c}\text { No. of } \\
\text { subjects. }\end{array}$} & \multicolumn{2}{|c|}{ Carbon-dioxide elimination. } & \multicolumn{2}{|c|}{ Oxygen absorption. } \\
\hline & & $\begin{array}{l}\text { Per kilogram } \\
\text { per minute. }\end{array}$ & $\begin{array}{l}\text { Per square } \\
\text { meter body- } \\
\text { surface per } \\
\text { minute. }\end{array}$ & $\begin{array}{l}\text { Per kilogram } \\
\text { per minute. }\end{array}$ & $\begin{array}{l}\text { Per square } \\
\text { meter body- } \\
\text { surface per } \\
\text { minute. }\end{array}$ \\
\hline $\begin{array}{l}\text { Zuntz-Geppert.......... } \\
\text { Johansson respiration } \\
\text { chamber.............. } \\
\text { Respiration calorimeter } \\
\text { (Wesleyan University) }\end{array}$ & 12 & $\begin{array}{l}\text { c.c. } \\
2.83 \\
2.75\end{array}$ & $\begin{array}{l}\text { c.c. } \\
93 \\
92\end{array}$ & $\begin{array}{c}c . c . \\
3.53\end{array}$ & $\begin{array}{l}c . c . \\
116\end{array}$ \\
\hline
\end{tabular}

1Johansson, Skand. Archiv f. Physiol., 1898, 8, p. 112.

${ }^{2}$ Durig, Denkschriften der mathematisch-naturwissenschaftlichen Klasse der kaiserlichen Akademie der Wissenschaften, Vienna, 1909, 86, pp. 120-121. 
Durig points out that the results agree very well, but calls attention to the fact that part of the experiments with the respiration calorimeter were made after food had been taken and a part with the subject fasting, and that all were during sleep. He also makes note of the fact that with the Zuntz-Geppert apparatus the skin respiration is not measured, but that this can scarcely be 1 per cent. The subjects with each apparatus were different, so that variations in body-weight and nationality may come into play as well as difference in respiration apparatus.

Benedict and Joslin ${ }^{1}$ have compared the results of the respiratory exchange of 5 normal subjects obtained with the bed calorimeter and the Benedict respiration apparatus ${ }^{2}$ in a reclining position and in the post-absorptive state, $i . e ., 12$ hours after the last meal. ${ }^{3}$ The results are given in table 2 . The figures were obtained by averaging all of the data for these five subjects which were available at the Nutrition Laboratory when the comparison was made. The experiments were not carried out expressly for the purpose of comparison, but were

TABLE 2.-Comparison of the metabolism of normal individuals as determined by the bed calorimeter and the respiration apparatus (Benedict and Joslin).

\begin{tabular}{|c|c|c|c|}
\hline $\begin{array}{c}\text { No. of } \\
\text { subjects. }\end{array}$ & Apparatus. & $\begin{array}{c}\text { Carbon-dioxide } \\
\text { per kilogram } \\
\text { per minute. }\end{array}$ & $\begin{array}{c}\text { Oxygen absorp- } \\
\text { tion per kilogram } \\
\text { per minute. }\end{array}$ \\
\hline & Bed calorimeter................ & 2.95 & c.c. \\
5 & Respiration apparatus..... & 2.90 & 3.51 \\
5 & & 3.52 \\
\hline
\end{tabular}

on different days and under different conditions of nourishment. It will be noted that the above figures agree fairly well with those calculated by Durig from experiments with the respiration calorimeter and the Zuntz-Geppert apparatus.

A similar comparison was made by Benedict and Joslin of the respiratory exchange of diabetics. The average results for 14 cases with different degrees of severity of the disease were as follows: With the bed calorimeter, 3.11 c.c. carbon dioxide produced per kilogram of body-weight per minute and 4.13 c.c. oxygen consumed; with the Benedict respiration apparatus, 3.13 c.c. carbon dioxide produced and 4.15 c.c. oxygen consumed. The results with both diabetic and normal subjects agree on the average remarkably well.

Loeffler ${ }^{4}$ gives measurements of the respiratory exchange of Gigon obtained with different apparatus at different times. The apparatus used were the Sondén-Tigerstedt chamber, the Jaquet chamber at

\footnotetext{
${ }^{1}$ Benedict and Joslin, Carnegie Inst. Wash. Pub. 136, 1910, p. 173.

${ }^{2}$ Benedict, Am. Journ. Physiol., 1909, 24, p. 345.

${ }^{3}$ Benedict and Catheart, Carnegie Inst. Wash. Pub. 187, 1913, p. 31.

'Loeffler, Archiv 1.d. ges. Physiol., 1912, 147, p. 203.
} 
Basel, and a spirometer constructed by Jaquet. Mueller valves were used with the spirometer. The results per hour in grams are given in table 3. The author says that the lower results obtained with the spirometer can be explained by the fact that the cutaneous respiration was not taken into account.

TABLE 3.-Comparison of the respiratory exchange of one subject with different respiration apparatus (Loeffer).

\begin{tabular}{|c|c|c|c|c|}
\hline Date. & $\begin{array}{c}\text { Carbon dioxide } \\
\text { produced. }\end{array}$ & $\begin{array}{c}\text { Oxygen } \\
\text { consumed. }\end{array}$ & $\begin{array}{l}\text { Respiratory } \\
\text { quotient. }\end{array}$ & Apparatus. \\
\hline $\begin{array}{l}\text { September } 1907 \ldots \\
\text { October } 1908 \ldots \\
\text { November } 1908 \ldots \\
\text { April } 1910 \ldots \\
\text { October } 1910 \ldots \\
\text { September } 1910 \ldots\end{array}$ & $\begin{array}{l}22.5 \\
23.8 \\
23.8 \\
22.7 \\
21.6 \\
20.8\end{array}$ & $\begin{array}{l}\cdots \\
21.6 \\
20.4 \\
19.5 \\
20.5\end{array}$ & $\begin{array}{l}\ldots \\
0.799 \\
0.811 \\
0.796 \\
0.740\end{array}$ & $\begin{array}{l}\text { Sondén-Tigerstedt. } \\
\text { Do. } \\
\text { Jaquet chamber. } \\
\text { Do. } \\
\text { Jaquet spirometer. } \\
\text { Do. }\end{array}$ \\
\hline
\end{tabular}

It must be noted that none of these comparisons are ideal. The experiments from which the data are drawn were carried out by different observers in different places; in one instance the comparison was made of experiments with wholly different groups of subjects. Furthermore, as the observations were not carried out on the same day, the differences in daily metabolism may have played a rôle, for the variations from day to day may be as high as 30 per cent. ${ }^{1}$

The measurements of the carbon-dioxide elimination may have been affected by two entirely different factors. One, which is purely physiological, is due to differences in the storage of glycogen. An individual with a large store of carbohydrate in the body will give a high respiratory quotient because of the preponderance of carbohydrate taking part in the daily metabolism and consequently a higher amount of carbon dioxide will be eliminated by such a subject than by one whose metabolism consists largely of the oxidation of fat. The other factor is the mechanics of respiration. If a respiration apparatus offers a hindrance to normal respiration, the ventilation of the lungs will be disturbed, with a consequent disturbance of the elimination of carbon dioxide. It is therefore very desirable to conduct the experiments with the various forms of respiration apparatus in such a manner that the only possible difference in the measurement of the respiratory exchange is due to the difference in the apparatus themselves.

\section{APPARATUS AND TECHNIQUE USED IN THE PRESENT STUDY.}

As has already been pointed out in the preceding discussion, for a fair comparison of the various methods for determining the respiratory exchange, the experiments with the apparatus compared should be made under conditions as nearly identical as possible. Accordingly 
it was made a fundamental principle of this investigation that the experiments with the two forms of apparatus selected for comparison should be carried out with the same subject and as nearly simultaneously as possible. While of course it was impossible to determine the respiratory exchange on the same subject with two apparatus at the same time, it was believed that by using the method of alternation on the same day the influence of sequence could be eliminated; furthermore, if a large number of comparisons were made with any two respiration apparatus, the multiplicity of results would eliminate any differences due to the individuality of the subject. Unfortunately the number of subjects used for many of the comparisons is not so large as would have been desirable, and also the same subjects were not used for all of the comparisons. This was due to the period (several years) over which the investigation was continued and the difficulty of being able to keep the subjects available for any great length of time.

Granting all these conditions are met, there still remains the question of a suitable base-line or standard. Given two sets of results with two forms of respiration apparatus, unless we know which is correct we have no way of assigning a value to the comparison. Unfortunately, we have no simple and accurate method of measuring normal respiration. The only apparatus which is at present available is the body plethysmograph used by Haldane and Priestley. ${ }^{1}$ The difficulties of getting an air-tight closure around the neck and of maintaining suitable temperature conditions must be very great with this apparatus, and it seems hardly practicable to attempt the measurement of the respiration volume under these conditions with any large number of subjects.

Investigations extending over several years have led us to believe that the respiration of a man inclosed in a respiration calorimeter, but free to move, is perfectly normal, for in such a chamber a subject may place himself in a perfectly comfortable position. The bed calorimeter ${ }^{2}$ of the Nutrition Laboratory permits measuring, with a high degree of accuracy and in periods of 3 hours or more, the respiratory exchange of a man in a reclining position. On the basis that the respiratory exchange is normal in the bed calorimeter, the results obtained with it have been compared with those obtained with the Benedict universal respiration apparatus; this apparatus has, in turn, been compared with others and modifications of the apparatus and conditions compared with each other.

Still another element in the whole question of comparable conditions has to be carefully considered, $i$. e., the elimination of external muscular activity. In several publications from this laboratory ${ }^{3}$ the impor-

\footnotetext{
${ }^{1}$ Haldane and Priestly, Journ. Physiol., 1905, 32, p. 242.

${ }^{2}$ Benedict and Carpenter, Carnegie Inst. Wash. Pub. 123, 1910.

${ }^{3}$ Benedict and Talbot, Am. Journ. Diseases of Children, 1912, 4, p. 130; Benedict, Deutsch Archiv f. klin. Med., 1912, 107, p. 158.
} 
tance of a graphic record of the degree of muscular rest on the part of the subject has been very thoroughly emphasized. Various methods of obtaining such a record have been employed in this research, which are subsequently described. Most of the men in these comparison tests were trained subjects and accustomed to keeping quiet during such experiments; the untrained subjects were also particularly instructed to refrain from all movements of body and limbs during the time of the experiment.

The apparatus used were the bed respiration calorimeter, the two types of the Benedict universal respiration apparatus, the ZuntzGeppert valves, meter, and gas-analysis apparatus, the Tissot nosepieces, valves, and spirometer, the Douglas bag and mica-flap valves, the Mueller valves, two forms of the Haldane gas-analysis apparatus, and a small hand spirometer. A detailed description of these apparatus follows.

\section{BED RESPIRATION CALORIMETER.}

The bed respiration calorimeter used in this research is in principle like the chair calorimeter which has been described in detail elsewhere. ${ }^{1}$ It has all the features of that apparatus, but the form of the chamber is particularly adapted to experiments with subjects in a reclining position.

The general principle of the apparatus is that of a closed-circuit system, consisting of a chamber with a ventilating apparatus attached. The ventilating apparatus removes the air continually from the chamber and provision is made for absorbing the water-vapor and the carbon dioxide from the air-current and for admitting oxygen to replace that used by the subject.

The general arrangement of the chamber and ventilating apparatus is shown in figure 1. The interior portion of the chamber consists of a copper shell, which is rigidly attached to a steel framework. ${ }^{2}$ In horizontal cross-section it is rectangular in shape and in vertical cross-section it is trapezoidal. The length is $220 \mathrm{~cm}$., the width $76 \mathrm{~cm}$., and the height $71 \mathrm{~cm}$. in front and $41 \mathrm{~cm}$. at the back. Its volume is about 950 liters. A rectangular opening at the front, $70 \mathrm{~cm}$. wide and $47 \mathrm{~cm}$. high, permits placing inside a subject lying upon a mattress. This opening is closed by a pane of plate glass, which is held in place and sealed air-tight by means of a soft wax of special composition seared over with a soldering iron.

The ventilation of the chamber is maintained by means of a rotary blower, ${ }^{3} F$, which draws the air from the chamber and forces it through

\footnotetext{
${ }^{1}$ Benedict and Carpenter, Carnegie Inst. Wash. Pub. 123, 1910.

${ }^{2}$ Since this was written, the bed calorimeter has been rcconstructed, using wood for the framework and "compo" board and cork for the outside insulating walls.

${ }^{3}$ For full description, see Benedict and Carpenter, Carnegie Inst. Wash. Pub. 123, 1910, p. 57. Recently the Crowell blower has been adopted with success.
} 
a pipe, $C$, extending to the rear, and passes it through purifiers, $1, K$, and 2 , in which the water and carbon dioxide are removed. The air then continues through a can containing sodium bicarbonate, which retains any traces of acid fumes that arise from the rapid passage of the air through the sulphuric acid. It finally returns to the chamber, oxygen being admitted from a weighed cylinder at some point between the

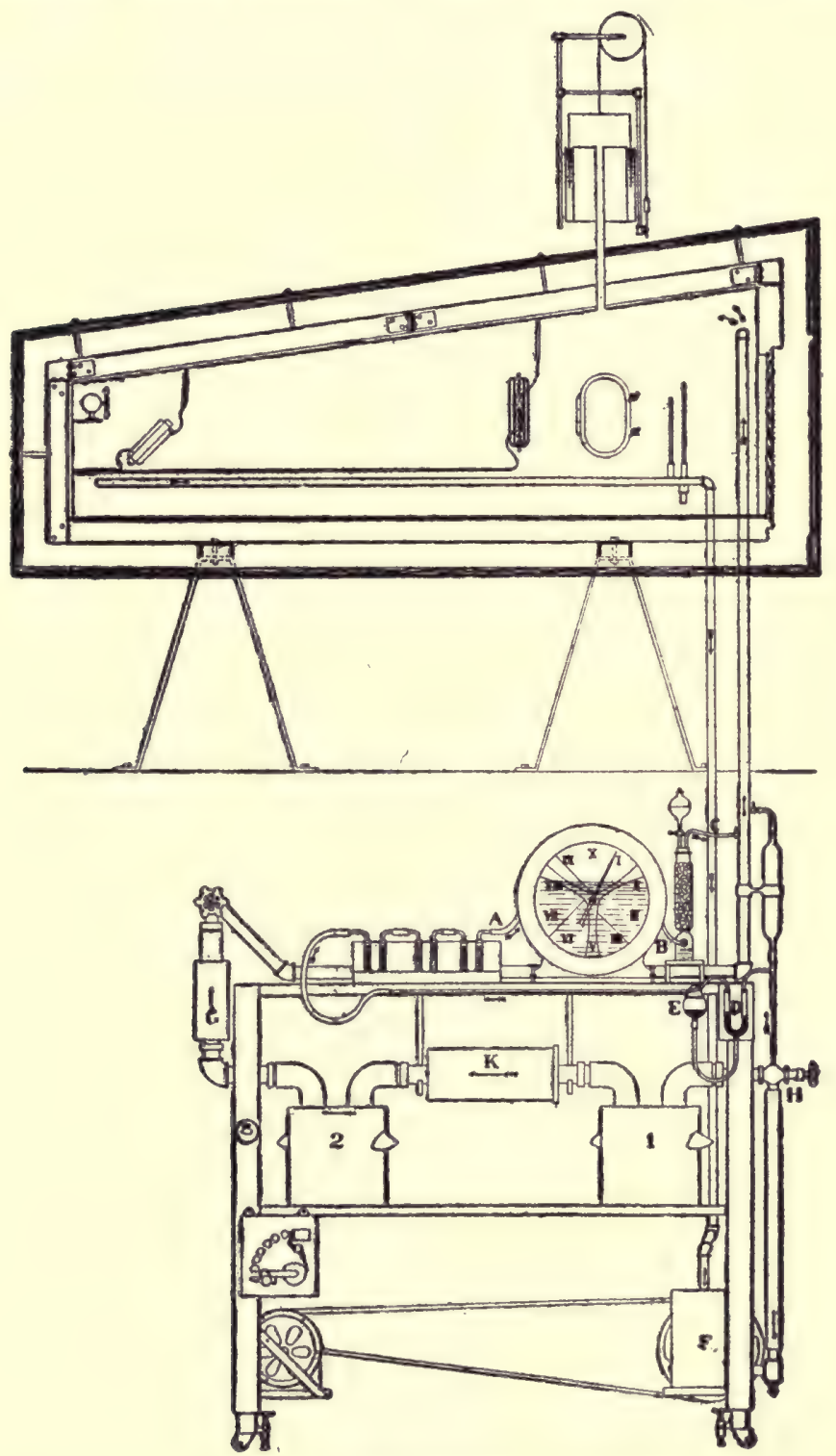

FiG. 1.-Bed respiration calorimeter.

$A$, tube leading to 10 -liter Bohr meter; $B$, tube leading from meter to drier; $C$, outgoing air pipe; $D$, mercury trap; $E$, leveling bulb for $D ; G$, sodium bicarbonate container; $F$, rotary blower; $\boldsymbol{H}$, valve; $\boldsymbol{K}$, soda-lime containgr; 1 and 8 , sulphuric-acid containers. 
sodium-bicarbonate can and the chamber. The air enters the chamber at the top, at a point near the front end. The average rate of ventilation is about 40 liters per minute; thus there is a wind movement in the chamber of about $1.6 \mathrm{~mm}$. per second. A thorough mixture of the air in the chamber is brought about by the use of an electric fan situated at the rear upper portion of the apparatus.

The water-vapor given off by the subject is removed by passing the circulating air-current through sulphuric acid contained in a porcelain vessel, the general shape and construction of which are shown in figure 1 (see 1 and 2). The air enters at the top of the vessel and is broken up in its passage through the acid by means of three concentric circles of openings; it then leaves at the top. Three liters of the strongest commercial sulphuric acid are used, the container and acid weighing about $18 \mathrm{~kg}$.

The carbon dioxide is removed by passing the air through slightly moist soda-lime. This is packed loosely in silver-plated brass cylindrical cans. (See $K$, fig. 1.) As the dry air in passing through the moist soda-lime absorbs water, another sulphuric-acid container, 2, is attached to the exit end of the carbon-dioxide absorber to absorb the water-vapor coming from the soda-lime.

All three pieces of apparatus are provided with couplings so that they may be detached and weighed, the weighings being made on a Sauter balance with an accuracy of $0.1 \mathrm{gm}$. A duplicate set of absorbers is provided and valves are placed at the ends of each series. By closing the valves attached to one set and opening those attached to the duplicate absorbers, the ventilating current may be deflected from one set to the other. This permits the division of the experiment into periods.

The supply of oxygen is maintained by automatic admission from a weighed cylinder. This cylinder contains when full about 100 cubic feet (2,800 liters) and weighs about $50 \mathrm{~kg}$. It is hung on one arm of a large Sauter balance and can be weighed with an accuracy of $0.1 \mathrm{gm}$. The admission of oxygen is regulated by the change in volume of the air in the apparatus. An opening in the side of the chamber is connected with a spirometer, ${ }^{1}$ this spirometer being simply a light copper cylinder which is counterpoised and suspended in water. As the watervapor and carbon dioxide are removed, the volume of air in the apparatus diminishes and the bell gradually sinks; oxygen is admitted from time to time to keep the bell at a convenient height. In actual practice with the apparatus, the admission is accomplished automatically by an electrical arrangement. When the bell drops to a certain point, an electric circuit is closed. In this electric circuit is an electro-

\footnotetext{
${ }^{1}$ Formerly another type of tension equalizer was used in which a rubber bathing-cap was attached to the upper end of a tin can. The details of its construction and use are given in Benedict and Carpenter, Carnegie Inst. Wash. Pub. No. 123, 1910, p. 71.
} 
magnet and the movement of its armature opens or closes the tube leading from the reduction valve on the cylinder to the chamber. The spirometer not only regulates the admission of the oxygen, but also provides for sudden changes in the air volume due to changes in the temperature of the air in the chamber or to changes in barometric pressure.

When a subject is breathing in the apparatus, it is not sufficient simply to weigh the absorbers and the oxygen cylinder in order to determine the amounts of carbon dioxide and water-vapor exhaled and the oxygen consumption in any given length of time, for the actual carbon-dioxide and water-vapor content of the air in the chamber may vary from time to time; the actual oxygen content may also vary because of variations in temperature and pressure as well as variations in amounts of carbon dioxide and water-vapor. Accordingly, the amounts of carbon dioxide and water-vapor in the air residual in the chamber should also be determined at the beginning and the end of the experimental period. At the same time a measurement of the temperature of the air in the apparatus should be made and the barometer read.

The water-vapor and carbon dioxide in the air-current were formerly determined in the following manner: A portion of the outcoming air was diverted at a point just before its entrance into the first sulphuricacid container. A mercury trap, $D$, shown in figure 1, served for opening and closing the branch tube. When the leveling bulb, $E$, was lowered, the mercury flowed away from the U-tube $D$ and allowed the air to pass through it. A small tube led from $D$ to a set of three U-tubes, $A$, containing sulphuric acid and pumice stone, soda-lime, and sulphuric acid and pumice stone, respectively. The exit tube of the last U-tube was connected with a 10-liter Bohr meter. ${ }^{1}$ From the Bohr meter a tube led to a drying-tower and then to the ingoing airpipe. The carbon dioxide and water-vapor of the outcoming air were determined by lowering the mercury level and passing 10 or 20 liters of air through the weighed $U$-tubes and meter, then raising the bulb again. The increases in weight of the U-tubes gave the amounts absorbed from the volume of air as indicated by the readings of the meter. The determination took place during the last 10 or 15 minutes of the experimental period.

In the winter season of 1911-12 another method of determining the carbon-dioxide and water-vapor content of the outcoming air was devised by Professor Benedict and used thereafter. According to this method, the water-vapor content is determined by calculation from the readings of a psychrometer ${ }^{2}$ installed inside the respiration chamber in

'See $A-B$, fig. 1, p. 15.

'This psychrometer is described by Benedict and Talbot in Carnegie Inst. Wash. Pub. 201, 1914 , p. 37. 
the outgoing air-pipe at a point near its exit from the chamber. The psychrometer consists of a dry thermometer and wet thermometer arranged with their bulbs inserted air-tight in the outgoing pipe. The wet bulb is kept moist by means of a thin layer of fine linen wrapped around the bulb, with its lower end dipping in a reservoir of water situated in a depression in the air-pipe. Both thermometers are placed near the front opening, so that they may be read with a lens to $0.01^{\circ} \mathrm{C}$. from outside of the chamber. Readings are taken during the last 5 minutes of the period. The carbon-dioxide content is determined by the analysis of a sample obtained by diverting a small current of the outgoing air through a glass sampler. This glass sampler is connected by rubber tubing between the mercury trap and the pipe leading to the ingoing air-current. Air runs through the sampler during the whole period. At the conclusion of the period the ends of the tube are closed, the sampler taken off, and another put in its place. The sample of air is then analyzed for carbon dioxide by means of the Sondén ${ }^{1}$ gas-analysis apparatus.

The temperature of the apparatus is obtained from the measurement of the changes in resistance of a set of thermometers placed at five different points in the chamber, approximately 2 or $3 \mathrm{~cm}$. from the wall. Their general construction is described in detail in a former publication. ${ }^{2}$ The barometer readings were obtained from a brass-scale mercury barometer equipped with a vernier reading to $0.05 \mathrm{~mm}$.

The carbon-dioxide production of a subject for an experimental period is obtained from the increase in weight of the soda-lime container and the sulphuric-acid container following it, plus or minus the changes in carbon-dioxide content of the air of the apparatus.

The oxygen consumption of the subject for an experimental period is obtained from the loss in weight of the cylinder corrected for the changes in oxygen content of the apparatus and the admission of nitrogen and argon with the oxygen. The change in the residual content of oxygen is calculated from the total volume of the air corrected to $0^{\circ} \mathrm{C}$. and $760 \mathrm{~mm}$. by subtracting from it the volume of carbon dioxide and water-vapor in the chamber at the end of the period. The volume of the nitrogen in the apparatus remains constant, except for the small amount present in the oxygen admitted from the cylinder, a correction being made for this on the loss in weight of the cylinder. ${ }^{3}$

The general routine of an experiment with the apparatus is as follows: When the subject is ready for the experiment a stethoscope is attached to the chest, and, in some instances, an electrical thermometer is inserted in the rectum. A pneumograph is also sometimes placed

\footnotetext{
${ }^{1} \mathrm{~A}$ detailed description of the most recent form of this apparatus is given by Benedict in Carnegie Inst. Wash. Pub. 166, 1912, p. 76.

'Benedict and Carpenter, Carnegie Inst. Wash. Pub. 123, 1910, pp. 28-29. ' 'Ibid., pp. 84 and 88.
} 
around the chest of the subject to record his respiration and activity, but more recently the latter has been recorded by means of a spring-bed arrangement. ${ }^{1}$ The subject lies upon an air-mattress placed upon a metal framework which can be readily slid into the chamber. When everything is in readiness, the subject is put into the chamber, the front opening is closed by the glass panel and sealed with wax; the heat-adjusting arrangements are put in order and the preliminary period is begun. During this preliminary period the assistant in charge of the calorimetric measurements brings the apparatus into equilibrium, so that there is no radiation through the walls and the absorption of heat by the water-current flowing through the apparatus is constant. ${ }^{2}$ When the equilibrium has been obtained, a determination is made of the residual content of the water-vapor and the carbon dioxide. After the determination of these two gases, the experiment is begun, the air-current being deflected from one side of the absorption system to the other and continued for a fixed period. At the end of the period the temperature is obtained by readings from a series of electrical resistance thermometers inside the respiration chamber, distributed at various points. The barometer is also read at the exact end of each period and the height of the spirometer taken in order to find the apparent volume of air inside the chamber. The oxygen cylinder and sulphuric-acid and soda-lime containers are then weighed. The experiment may be stopped at this point or another period begun. The usual length of periods is $\mathbf{4 5}$ minutes or an hour, and an experiment usually continues at least $1 \frac{1}{2}$ hours.

The accuracy of the measurement of the carbon-dioxide elimination and oxygen consumption has been carefully controlled theoretically by burning alcohol. ${ }^{3}$ The alcohol was introduced into the chamber through a copper tube, at the end of which a small enlargement was made in which was placed an asbestos wick. By means of this arrangement, small amounts of alcohol were burned in successive periods, these periods being each an hour or more in length. The alcohol was usually burned at the rate of about $14 \mathrm{gm}$. per hour, and as the amount burned could be determined to $0.01 \mathrm{gm}$., the error in weighing the alcohol was about 0.1 per cent. Considerable difficulty was experienced in the actual measurements of the alcohol on account of the changes in level of the alcohol in the lamp. This was finally overcome by means of a small manometer outside of the calorimeter; this manometer was arbitrarily filled to the same height at the end of each period. The results of two typical alcohol check experiments are given in tables 4 and 5 .

\footnotetext{
${ }^{1}$ Benedict, Carnegie Inst. Wash. Pub. 203, 1915, p. 311.

${ }^{2}$ As this publication does not deal with the calorimetric features of the apparatus, these are not described here. A full description is given by Benedict and Carpenter in Carnegie Inst. Wash. Pub. 123, 1910, pp. 10-53.

'Benedict, Riche, and Emmes, Am. Journ. Physiol., 1910, 26, p. 1.
} 
TABLE 4.-Alcohol check experiment. Bed calorimeter, January 7, 1910. (1-hour periods.)

\begin{tabular}{|c|c|c|c|c|c|c|c|}
\hline \multirow{2}{*}{ Period. } & \multirow{2}{*}{$\begin{array}{l}\text { Alcohol } \\
\text { burned. }\end{array}$} & \multicolumn{3}{|c|}{ Carbon dioxide. } & \multicolumn{3}{|c|}{ Oxygen. } \\
\hline & & Theory. & Found. & $\begin{array}{l}\text { Ratio of } \\
\text { found to } \\
\text { theory. }\end{array}$ & Theory. & Found. & $\begin{array}{l}\text { Ratio of } \\
\text { found to } \\
\text { theory. }\end{array}$ \\
\hline 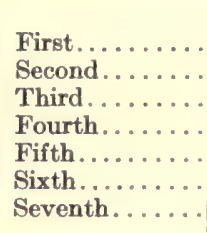 & $\begin{array}{l}g m . \\
13.2 \\
13.4 \\
12.6 \\
13.8 \\
13.1 \\
13.6 \\
13.6\end{array}$ & $\begin{array}{l}g m . \\
23.4 \\
23.8 \\
22.2 \\
24.4 \\
23.2 \\
24.0 \\
24.0\end{array}$ & $\begin{array}{l}g m . \\
23.5 \\
23.4 \\
22.3 \\
23.9 \\
24.0 \\
23.2 \\
23.9\end{array}$ & $\begin{array}{r}\text { p. ct. } \\
100.4 \\
98.3 \\
100.5 \\
98.0 \\
103.5 \\
96.7 \\
99.6\end{array}$ & $\begin{array}{l}g m . \\
25.5 \\
26.0 \\
24.3 \\
26.6 \\
25.3 \\
26.2 \\
26.2\end{array}$ & $\begin{array}{l}g m . \\
25.4 \\
26.5 \\
23.5 \\
26.5 \\
25.9 \\
25.5 \\
26.7\end{array}$ & $\begin{array}{r}\text { p. ct. } \\
99.6 \\
101.9 \\
96.7 \\
99.6 \\
102.4 \\
97.3 \\
101.9\end{array}$ \\
\hline Total... & 93.3 & 165.0 & 164.2 & 99.5 & 180.1 & 180.0 & 99.9 \\
\hline \multirow[b]{2}{*}{ Period. } & \multirow[b]{2}{*}{$\begin{array}{l}\text { Alcohol } \\
\text { burned. }\end{array}$} & \multicolumn{3}{|c|}{ Water-vapor. } & \multicolumn{3}{|c|}{ Heat. } \\
\hline & & Theory. & Found. & $\begin{array}{l}\text { Ratio of } \\
\text { found to } \\
\text { theory. }\end{array}$ & Theory. & Found. & $\begin{array}{l}\text { Ratio of } \\
\text { found to } \\
\text { theory. }\end{array}$ \\
\hline 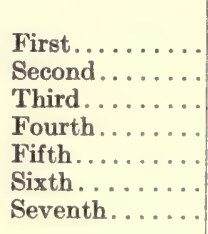 & $\begin{array}{l}g m . \\
13.2 \\
13.4 \\
12.6 \\
13.8 \\
13.1 \\
13.6 \\
13.6\end{array}$ & $\begin{array}{l}g m . \\
15.3 \\
15.6 \\
14.6 \\
16.0 \\
15.2 \\
15.8 \\
15.8\end{array}$ & $\begin{array}{l}g m . \\
16.1 \\
16.3 \\
14.9 \\
16.2 \\
15.7 \\
15.8 \\
15.8\end{array}$ & $\begin{array}{l}p . c t . \\
105.2 \\
104.5 \\
102.1 \\
101.3 \\
103.3 \\
100.0 \\
100.0\end{array}$ & $\begin{array}{c}c a l . \\
77.5 \\
79.0 \\
73.7 \\
81.0 \\
76.8 \\
79.6 \\
79.7\end{array}$ & $\begin{array}{c}\text { cal. } \\
76.4 \\
78.7 \\
71.3 \\
80.3 \\
75.9 \\
79.0 \\
79.5\end{array}$ & $\begin{array}{l}\text { p. ct. } \\
98.6 \\
99.6 \\
96.8 \\
99.1 \\
98.8 \\
99.2 \\
99.8\end{array}$ \\
\hline Total... & 93.3 & 177.4 & ${ }^{1} 78.4$ & 101.3 & 547.3 & 541.1 & 98.9 \\
\hline
\end{tabular}

This amount does not include the water-vapor for the first two periods, in which obviously moisture equilibrium was not established. The walls of this calorimeter are painted.

TABLE 5.-Alcohol check experiment. Bed calorimeter, February 15, 1912.

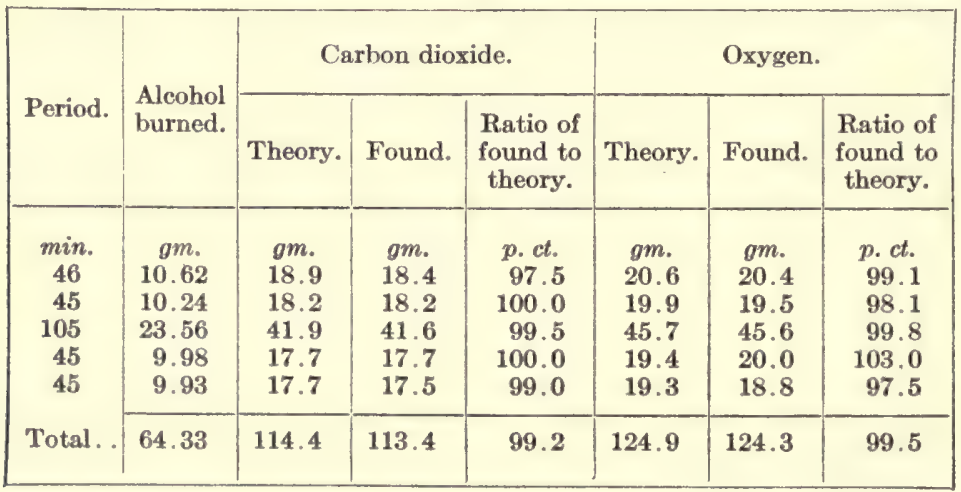




\section{BENEDICT UNIVERSAL RESPIRATION APPARATUS.}

Two types of the Benedict universal respiration apparatus have been used in this investigation: one, the tension-equalizer type and the other the spirometer type. The tension-equalizer apparatus was the first one to be developed and its use extended from about 1908 to 1912 ; the spirometer type was developed in 1911-12 and has been in use since that time. Both forms may be designated by the German word "Universalrespirationsapparat." It has been the common practice in this laboratory to call them units and this term will be used in this publication, i. e., tension-equalizer unit and spirometer unit, respectively.

\section{TENSION-EQUALIZER UNIT.'}

This apparatus is essentially the same as the respiration portion of the respiration calorimeters of this laboratory, except that it is constructed on a smaller scale and modified so that a subject can breathe by means of a suitable connection into and out of a moving current of air. The respiration may take place through the nose or mouth or through both. The water-vapor is removed from the air-current by sulphuric acid and the carbon dioxide is retained by soda-lime in weighable containers. The oxygen content of the apparatus is maintained at a constant volume by admission of oxygen into the moving current from a weighed cylinder or through a meter. The volume of the air in the apparatus and also in the respiratory tract of the subject must be the same at the end of the experimental period as at the beginning.

\section{General Plan of Apparatug.}

The general principle of the apparatus and the course of the aircurrent are shown diagrammatically in figure 2. The air expired by the subject passes into the moving current of air and is carried into the tension equalizer, then through the rotary blower, which keeps the air of the apparatus in circulation. After leaving the rotary blower it passes into the water absorber, where all the water in the air-current is retained, and then goes through the carbon-dioxide absorber. In the absorption of carbon dioxide, water-vapor is set free from the moist absorbent and this water is removed in a second water-absorber. To make the air respirable water-vapor is added to the air-current by passing it through a water-container. The circulating air then passes to the opening connected with the respiratory tract of the subject. Oxygen is admitted into the air-current at a point near the tension equalizer.

The general construction of the apparatus and arrangement of the several parts are shown in figure 3. The whole apparatus is mounted on a movable table. On a shelf at the bottom are the rotary blower

\footnotetext{
${ }^{1}$ A complete description of this apparatus has been given elsewhere. See Benedict, Am. Journ. Physiol., 1909, 24, p. 345.
} 
and the motor for driving it. A pipe connects the rotary blower to a Wolff bottle on the shelf above, containing sulphuric acid and pumice stone. To the exit end of this bottle a second Wolff bottle, also containing sulphuric acid and pumice stone, is attached, which is in turn connected with the carbon-dioxide absorber on the top shelf. Next in series is a third water-absorber, its lower portion containing sulphuric acid and the upper portion pumice stone. From this water-absorber a pipe leads to a moistener containing a dilute aqueous solution of sodium bicarbonate. This is connected to the three-way valve by a pipe and rubber tubing. The three-way valve opens to a connection for the nosepieces or other devices through which the subject breathes. The tension equalizer is inserted between the three-way valve and the
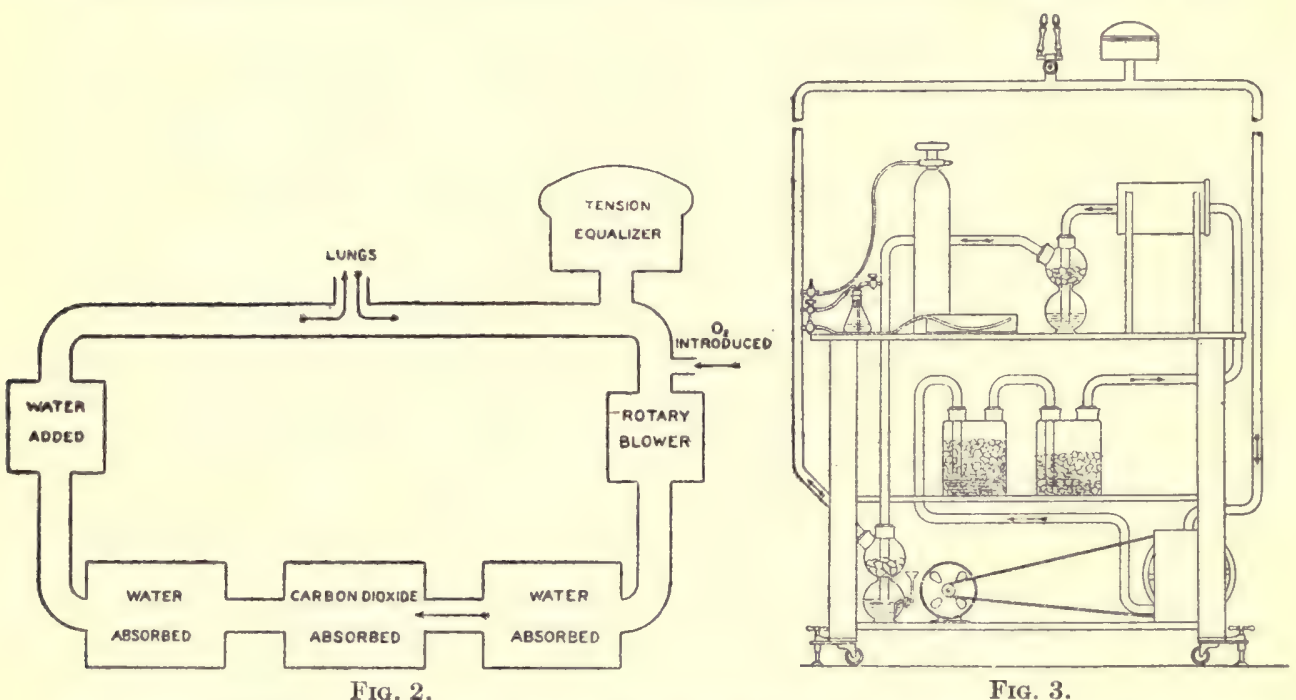

Fig. 3.

FIG. 2.-Diagrammatic scheme of air-circuit and purifying arrangements of tension-equalizer unit. FIG. 3.-Diagram showing arrangement of Benedict respiration apparatus (tension-equalizer unit. This shows nosepieces for breathing, tension equalizer, air-purifying apparatus, oxygen cylinder, and testing device for carbon dioxide.

rotary blower. Piping and rubber tubing lead from the tension equalizer to the rotary blower. Two petcocks are inserted in the pipe between the moistener and the three-way valve. One is attached to a delicate manometer; the other is for the admission of oxygen. At a point just beyond the third water-absorber is an arrangement for testing the completeness of the absorption of the carbon dioxide. Its exit is connected with the pipe leading from the air-moistener.

Description and Use of Parts.

Nosepieces.-In the development of the apparatus special nosepieces were devised, one of which is shown in figure 4. To conduct the air into and out of the nose, a piece of glass tubing, $a$, is used, which has a length of $6 \mathrm{~cm}$., an internal diameter of $7 \mathrm{~mm}$., and a wall thickness 
of $1.5 \mathrm{~mm}$., this tube being fire-polished at both ends. A small hole is cut in the end of a pure-gum finger-cot $b$, which is then slipped over the glass tube and tied carefully with silk thread. At the other end of the tube a one-hole rubber stopper, $c$, is attached. The finger-cot is then turned inside out and pulled back on itself in such a way as to be drawn over the rubber stopper, to which it is tied with silk thread. A hole is next made through the rubber stopper, in which a piece of small-bore glass tubing $d$ can be inserted, to which a short piece of rubber tubing is attached. By blowing air from a hand bulb through the rubber tubing, the finger-cot is inflated; the closing of the pinchcock $e$ serves to keep the air inclosed in the finger-cot. When the appliance is to be used, the deflated nosepieces are inserted into the nostrils and air is forced into each nosepiece in turn until they are sufficiently inflated to fit into the inequalities of the nostrils. The nosepieces should be tested for tightness by inflating them while they are entirely under water. If any part of the nosepieces leaks, bubbles will rise. The tightness of the fit in the nostril should also be tested by having the subject exhale against pressure. The subject first inhales deeply; the palm of the hand or a piece of cardboard is then placed against the opening of the three-way valve, and the subject attempts to exhale. If a leak occurs, it is detected by the sound of air escaping

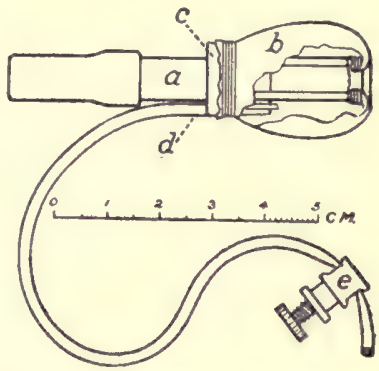

FIG. 4.-Pneumatic nosepiece. $a$, glass tube to which is fastened a rubber finger-cot, $b$, which is drawn over a rubber stopper, $c$. A capillary rubber tube, $d$, serves for dilating the $\cot b$; the clamp $e$ closes $d$ after $b$ is inflated.

between the nostril and rubber membrane of the nosepiece. The best test is made by covering the edges of the nostrils with soapsuds and applying pressure. Bubbles appear when there is a leak. The nosepieces are attached to the three-way valve by a piece of rubber tubing and a tube, to which are attached two metal tubes of approximately $6 \mathrm{~mm}$. internal diameter.

When the nosepieces are used, a tight closure of the mouth is sometimes obtained by placing two strips of surgeon's plaster over the mouth, from above the upper lip to below the lower lip. The subject draws in his lips and the surgeon's plaster is placed on them before they relax. This method can be used only when the subject is smooth shaven.

Mouthpiece.-The mouthpiece used, which is of the Denayrouse ${ }^{1}$ type, will be described in connection with the description of the ZuntzGeppert apparatus, the method of attachment being shown in the description of the later form of the Benedict respiration apparatus. ${ }^{2}$

\footnotetext{
${ }^{1}$ Regnard, Recherches expérimentales sur les variations pathologiques des combustions respiratoires, Paris, 1879 , p. 286.

${ }^{2}$ See pp. 25,36 , and 54 .
} 
Three-way valve.-With this apparatus the subject breathes into the open air up to the beginning of the experiment, but is at the same time attached to the apparatus by either the nosepiece or the mouthpiece. To provide for the instant deflection of the expired air into the closed circuit of the apparatus at the beginning of an experiment, a three-way valve is connected to the piping just before the tension equalizer. This three-way valve is an ordinary three-way plug-cock which is very carefully ground. To diminish the dead space a portion of one opening is cut off and the valve is soldered directly to the tee on the ventilating air-pipe. When it is in position, the side outlet opens directly to the air of the room, and connection is made with the ventilating air-system by turning the valve. In the early development of the apparatus the operator turned this valve by simply placing the fingers on the top of the plug and shifting it when necessary. Later a handle was added so that the valve could be turned without the subject's knowledge.

Piping, tubing, and couplings.-Standard $\frac{1}{2}$-inch piping is used, with an actual internal diameter of $15 \mathrm{~mm}$. The rubber tubing, which is common garden hose, with an internal diameter of $19 \mathrm{~mm}$., is fastened to the piping by wire or by special clamps. The total length of hose used in the apparatus is approximately 2 meters. The fittings are such as are commonly used for brass piping and are all of the same size as the piping. The couplings for connecting the different removable portions of the apparatus are ordinary $\frac{1}{2}$-inch garden-hose couplings. Between the different couplings rubber washers of suitable size are used, care being taken to have them of the best rubber.

Tension equalizer. - The tension equalizer consists of a rubber diaphragm fitted to a copper can $16 \mathrm{~cm}$. in diameter and $9 \mathrm{~cm}$. high. In the first form of apparatus ordinary hose-couplings were soldered on to the can at opposite sides near the bottom. Later well-ground unions were attached. A woman's pure-rubber bathing cap, such as can readily be purchased in local stores, is used for the rubber diaphragm. A cap of medium size permits fluctuation in the volume of respiration and consequently it is necessary to admit oxygen into the apparatus only occasionally. In using the apparatus, care should be taken that the diaphragm does not sink so low as to touch the sides of the metal can and thus produce a suction. The air coming into this tension equalizer contains carbon dioxide, and in order to make sure that it is completely swept out at the end of the experiment, a semicylindrical piece of sheet copper is soldered to the bottom and sides of the can near the entrance coupling in such a manner that when the air comes against this sheet it is deflected upward against the rubber diaphragm. This insures a circulatory movement of the air inside the tension equalizer. The tension equalizer with the three-way valve and mouthpiece are shown in figure 5.

Rotary blower.- The blower first used in the tension-equalizer unit was the so-called positive type, and has previously been described in 
detail. ${ }^{1}$ In this blower, a solid cylinder with two movable vanes attached is placed eccentrically inside a hollow cylindrical chamber. The rotary movements of the shaft and the compression and expansion of springs acting upon the vanes force the air through the blower. Later it was found that the blower manufactured by the J. Gilmer Crowell Company of Brooklyn, New York, was more satisfactory. This is mounted inside of a metal box, and may therefore be entirely immersed in oil with the exception of the portion of the shaft extending through the box to the driving pulley. Leaks around the shaft or in any portion of the blower may thus be readily detected. It is necessary of course to have the blower absolutely tight, as there is a difference of pressure between the inside and the atmosphere of at least $50 \mathrm{~cm}$. of water. $^{2}$ The large wheel on the shaft of the blower is belted directly

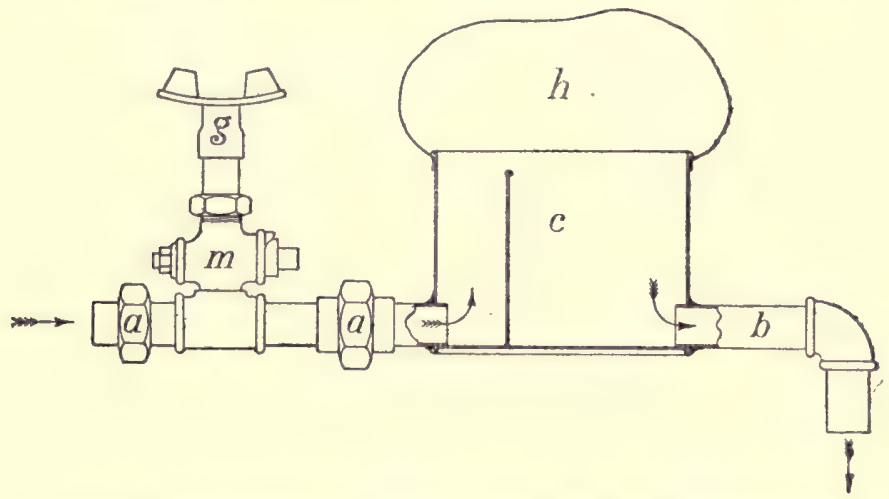

Fig. 5.-Tension-equalizer with three-way valve and mouthpiece.

$g$, rubber mouthpiece; $m$, three-way valve; $a$, union; $c$, tension-equalizer; $h$, rubber bathing cap; $b$, tube leading to rotary blower.

to $a \frac{1}{8} \mathrm{~h}$. p. electric motor. The driving-wheel is $26 \mathrm{~cm}$. in diameter, and by adjusting the size of the pulley on the motor, varying limits of speed may be obtained. The speed is also regulated by a resistance in series. Recently a bank of lamps in parallel and of varying candlepower has been placed in series with the motor; by varying the number of lamps used and their candle-power it is possible to get rates of speed ranging from 295 to 480 revolutions per minute. The rate of ventilation is usually adjusted to about 35 liters per minute. On the exit pipe leading from the blower a metal pipe and petcock are attached for trapping and drawing off any oil which may be mechanically carried forward. Having once determined the rate of flow and knowing the revolutions per minute of the blower shaft, the rate per minute can be taken as an index of the actual ventilation. Under ordinary conditions these blowers deliver about 120 c. c. of free air per revolution.

\footnotetext{
${ }^{1}$ Atwater and Benedict, Carnegie Inst. Wash. Pub. No. 42, 1905, p. 18.

${ }^{2}$ With none of the blowers which have been used in this laboratory and which have been properly taken care of has there been any leak. The blowers are remarkably satisfactory and efficient.
} 
Air-drier.-The air-current coming from the blower brings with it the water-vapor from the air-moistener and a certain amount of watervapor from the lungs of the subject. The method used in this apparatus of determining the carbon-dioxide production by weight necessitates the removal of water-vapor from the air-current before it reaches the carbon-dioxide absorbers, as any water-vapor reaching the soda-lime would be absorbed in the latter. The water-absorbers or air-driers used in this apparatus are two 4-liter Wolff bottles connected in series and containing sulphuric acid. These bottles are fitted with glass tubes of about the same diameter as the piping of the apparatus, the entrance tubes dipping about $2 \mathrm{~cm}$. into the acid. The usual method of use is to fill the first bottle to a certain level, and when sufficient water has been absorbed to increase the level of the liquid to a point determined by experience, the absorber is removed. If this routine is strictly followed, the second bottle never has to be replaced, these two absorbers being sufficient to remove all of the water-vapor from the air-current. In the earlier experimenting with this apparatus, the first bottle was filled with pumice stone and sulphuric acid added to half the height of the vessel. The second bottle was half filled with pumice stone, and acid then added to a one-third level. Later, instead of using pumice stone in the bottles, they were simply filled about two-thirds full with sulphuric acid, the entrance tubes dipping into the acid.

The glass tubes leading into and out of the Wolff bottles were made especially high for two reasons: First, if there were a slight back suction the acid would rise in the inlet tube so that considerable pressure would have to be overcome with this length of tube before the acid could travel back into the blower; second, the length of the exit tube enabled any sulphuric acid mechanically carried forward to drain back into the Wolff bottle. This mechanical carrying forward has more recently been prevented by the use of a special bulb with a perforated trap inside, which serves to catch the acid more efficiently and allows it to drain back into the bottle.

Carbon-dioxide absorbers. - The carbon-dioxide absorbers employed during the first two years after the apparatus was developed were constructed on the same principle as those used for the respiration calorimeter. ${ }^{1}$ They were made of brass tubing, which was silver-plated to resist the action of alkali. Their length was $26 \mathrm{~cm}$. and their diameter $12 \mathrm{~cm}$. A hose-coupling of standard size was soldered at each end for connecting with the rest of the apparatus. As the head of the can was removable, it could be easily filled. When the can was filled with granulated soda-lime of the size of half a pea, $60 \mathrm{gm}$. of carbon dioxide could be absorbed without allowing any to pass, with the circulating air moving at the rate of 35 liters per minute.

${ }^{1}$ See K, fig. 1, p. 15. 
At times this type of absorber proved difficult to make air-tight and another device was substituted in the spring of 1911, which is more efficient. This is shown in detail in figure 6 . It consists essentially of an ordinary 2-liter wide-mouth chemical bottle, with a two-holed rubber stopper in which iron pipes are inserted, one pipe, $b$, extending to the bottom of the container, the other being considerably shorter. These pipes are of standard size, with an internal diameter of $13 \mathrm{~mm}$. and an external diameter of $18 \mathrm{~mm}$. At the top a short pipe is fitted with a metal tee, $a$, which is used in filling the bottle. The long pipe, $b$, is fitted with an elbow, and hose-couplings ( $c$ and $c$ ) are attached to both pipes by rubber connections. To make sure that no particles of soda-lime enter the piping, the open end of the longer iron pipe is protected by a wire-gauze cap, $d, 9 \mathrm{~cm}$. long and $2 \mathrm{~cm}$. in diameter.

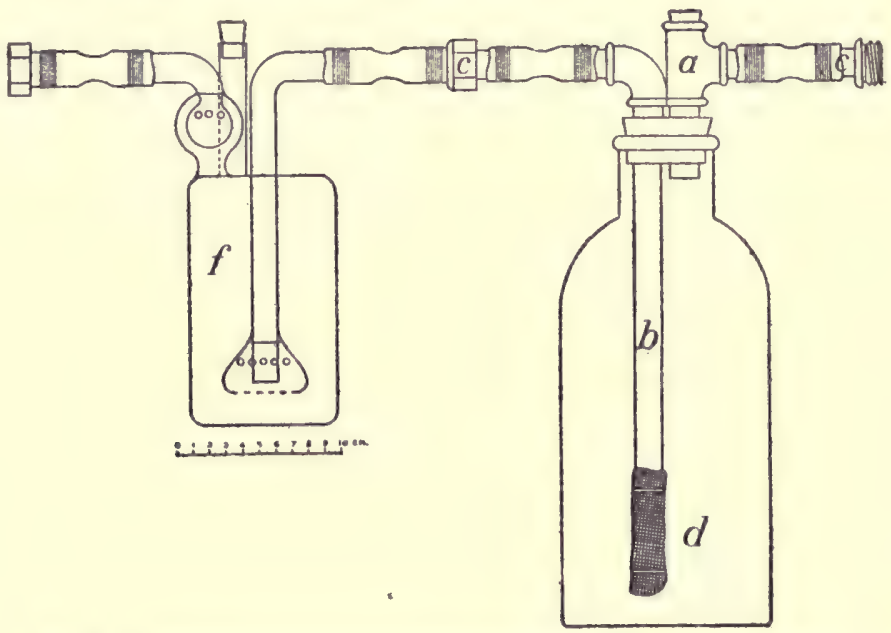

Fig. 6.-Carbon-dioxide absorber and accompanying water-absorber.

$a$, tee for filling absorber; $d$, wire gauze on the end of outgoing tube $b ; c, c$, entrance and outlet of air; $f$, water-absorber.

To prepare the absorber for use, the stopper is first removed from the opening in the tee, and the bottle is filled two-thirds full of soda-lime. It is then laid on its side and the tubes and wire-gauze protector are inserted. The bottle is again stood upright and the stopper pressed down firmly; it is then filled through a funnel inserted in the metal tee. After the opening in the tee has been closed by a rubber stopper, the pipes and connections are tested for tightness by a water manometer and then air is blown through them with the mouth to make sure that they are not clogged in any way. These bottles, when properly charged with $2,200 \mathrm{gm}$. of soda-lime sufficiently fine to pass through a sieve with a mesh of $3 \mathrm{~mm}$., should absorb about $100 \mathrm{gm}$. of carbon dioxide. This suffices for 15 to 25 experiments with a resting man, each experiment being 15 minutes long. 
The advantages of the glass bottle over the silver-plated can are the decreased expense of construction, the rapidity with which the bottle can be filled and closed, the readiness with which it may be made airtight, and the fact that, as the carbon dioxide is absorbed, the change in color of the soda-lime to a chalky white is easily seen. When this discoloration extends to the bottom, it is evident that the bottle should be refilled. Experimenting has shown that when air enters the bottle at the top and is withdrawn from the bottom through the tube, the results are more satisfactory than when the passage of air is reversed.

The method of making the soda-lime has been fully described in a previous publication. ${ }^{1}$ In order that the soda-lime may be efficient, it is always prepared in such a way that the finished product is slightly moist. Much of the difficulty found in the use of soda-lime as an absorbent for carbon dioxide has been due to the fact it was too dry.

Water-absorber.-In the passage of absolutely dry air through moist soda-lime, moisture is taken up by the air. As the carbon dioxide is determined by weight, it is necessary to know the amount of moisture leaving the carbon-dioxide absorber. In the first two or three years of experimenting with this apparatus, a form of absorbing vessel was used which was adapted from the bottom part of a 500 c.c. Kipp generator. The lower bulb was filled about half full of strong sulphuric acid. The upper bulb was filled with broken pumice stone and drenched with sulphuric acid. A bent glass tube led from the top of the bottle, through a rubber stopper, into the acid to a depth of 5 to $10 \mathrm{~mm}$. The side outlet in the upper bulb was used as the exit of the absorber. This form was employed for several years and proved satisfactory, but was subsequently replaced by the absorber devised by Dr. H. B. Williams, of the Department of Physiology at Columbia University, New York. The Williams absorber, which is shown in detail in figure 6 , is $9.5 \mathrm{~cm}$. in diameter and $15 \mathrm{~cm}$. high. It is so constructed that the air entering the apparatus is broken up into a number of bubbles during its passage into the acid by means of two concentric rows of openings. When charged with 450 c.c. of sulphuric acid, it can be relied upon to absorb completely at least $10 \mathrm{gm}$. of water-vapor from an air-current of 35 liters per minute without allowing any weighable amount to pass. The bottle is closed with a rubber stopper and fitted with hose-couplings at the ends; the outside is protected with a wire basket which has a handle for carrying. The Williams bottle and soda-lime container can be weighed together on a Sauter balance, their combined weight being about $5,000 \mathrm{gm}$.

Apparatus for testing completeness of carbon-dioxide absorption.A 100 c.c. Erlenmeyer flask with a two-hole rubber stopper is partly

\footnotetext{
${ }^{1}$ Atwater and Benedict, Carnegie Inst. Wash. Pub. 42, 1905, p. 29; also Benedict, Deutsch. Archiv f. klin. Med., 1912, 107, p. 166.
} 
filled with dilute barium-hydroxide solution. Through one hole of the rubber stopper is inserted a glass tube which extends below the surface of the liquid; in the other hole is a short piece of glass tubing. The end of the long glass tube is connected to a point in the piping just beyond the third water-absorber, while the short glass tube is connected to the piping going directly to the intake of the rotary blower. The method of testing is explained in detail on page 32 .

Moistener.-The air leaving the last water-absorber is absolutely dry and also has a slight odor of acid which, if not removed, would be extremely irritating to the respiratory tract of the subject. To moisten the air sufficiently for comfortable respiration and to remove the acid fumes, a part of a Kipp generator is used containing a solution of sodium bicarbonate. (See fig. 7.) The vessel is closed with a rubber stopper in which is inserted a brass tube with a number of perforations in its lower end, this tube dipping sufficiently into the liquid in the vessel for the perforations tobecovered. More recently sodium carbonate has been substituted for the sodium bicarbonate, as the latter gives off traces of carbon dioxide which may vitiate the results of the experiment. At the rate of 35 liters per minute, the air is saturated to about 65 per cent with this arrangement and the acid fumes are very efficiently removed.

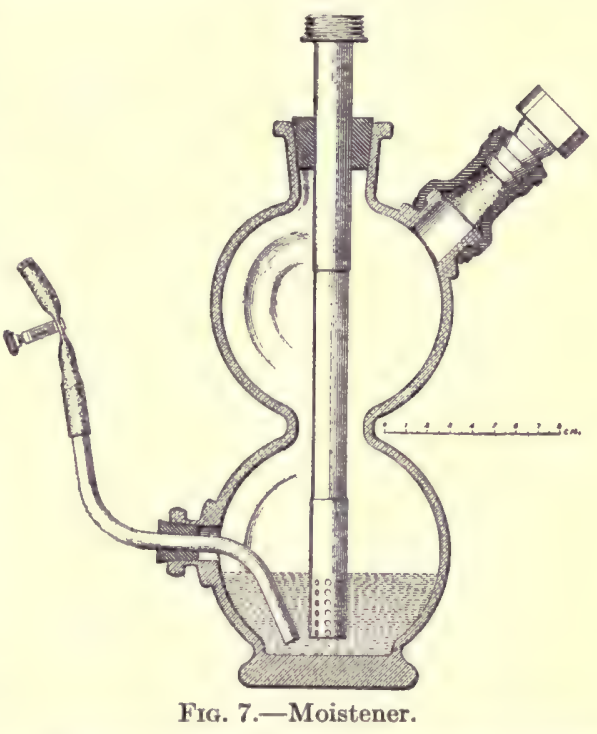

Air enters at top of upright tube, passes through holes into the water, and out of the side outlet in upper bulb.

Oxygen supply.-The oxygen for this apparatus has been supplied mainly by admission from a weighed cylinder. In the spring of 1911 the method of admission through a 1-liter Bohr meter was substituted, and this has since been used entirely. A more detailed description of both of these methods and a discussion of their merits will be given in the description of the spirometer unit of this apparatus.

Manometer.-In order to be absolutely certain that the volume of the air in the apparatus is the same at the end as at the beginning, it is necessary to have some method of measuring it. Instead of using the volume of the tension equalizer for this purpose, it is measured by determining the pressure on the tension equalizer with a very delicate Töpler ${ }^{1}$ manometer. This manometer has a glass tube bent in the arc

'A. Töpler, Wiedemann's Ann. d. Physik u. Chem., 1895, 56, p. 609. 
of a circle which contains a column or drop of petroleum. The movement of the petroleum along the arc of the circle for a few degrees is a very delicate measure of pressure. At the beginning of an experiment the tension equalizer is filled until a slight pressure is shown on the manometer; at the close of the experiment the tension equalizer may readily be brought to the same pressure.

Method of determining the carbon-dioxide excretion.-The general method for determining the carbon-dioxide excretion is by weight. As has already been pointed out, during an experiment the carbon dioxide is absorbed completely from the air-current as soon as it reaches the soda-lime container. At the close of the experiment, however, there is carbon dioxide in the air between the mouthpiece or connection to the subject and the carbon-dioxide absorber and it is necessary to sweep this out by continuing the ventilation for about half a minute after an experiment is over in order to absorb completely all of this carbon dioxide. The soda-lime container and its accompanying water-absorber are weighed together before the experiment and again after the experiment, the increase in weight representing the amount of carbon dioxide exhaled by the subject. The weight can then be converted to volume by the factor representing the relation between the weight of carbon dioxide and the volume. The volume per minute may be calculated from the length of the experimental period and the volume exhaled.

Method of determining the oxygen consumption.--The principle of the determination of the oxygen consumption by means of this apparatus has been briefly pointed out in an earlier part of the description. It involves several factors. In the first place, the volume of the apparatus must be the same at the beginning as at the end, and this is obtained by admitting air or oxygen into the apparatus before the experiment until a slight pressure is reached, as shown by the petroleum manometer. Then at the end of the experiment the same process is repeated, care being taken to have the pressure exactly the same as at the beginning. The other requirement is that the volume of the respiratory tract of the subject be the same at the beginning as at the end. In order to have this true, the experiment is begun at a point in the respiratory cycle which is apparently a constant one, the end of a normal expiration being taken. Numerous observations made in this laboratory with the pneumograph around the chest or abdomen seem to indicate that when the subject is breathing quietly, at rest, the subject empties the respiratory tract to about the same point each time. In practice with the respiration apparatus, therefore, it has been customary to begin the experimental period by turning the threeway valve exactly at the end of a normal expiration and to end the period in the same manner. Having made certain of these two conditions, the amount of oxygen admitted into the apparatus from the 
time the experiment begins until it is completed, is considered to be the actual amount used by the subject, provided there have been no changes in temperature or barometric pressure. A discussion of the whole question of the determination of the oxygen consumption with the unit respiration apparatus will be included in the discussion of the results obtained with it. ${ }^{1}$

Check tests of the respiration apparatus.-In the development of the respiration apparatus, it was thoroughly tested by experiments in which small quantities of ethyl ether were burned. For this purpose a combustion chamber of special construction was inserted in the ventilating air-pipe at the point where the three-way valve is ordinarily attached. This apparatus, which is shown in figure 8, consists of a large metal tee, $A$, of the standard 2-inch size (5 $\mathrm{cm}$. internal diameter). Into this is fastened an upright piece of pipe which is surrounded by a tin water-jacket, $J$. On the top an elbow is attached, into which a pipe, $C$, is screwed. To the bottom of the tee, $A$, is attached a short piece of pipe closed with a rubber stopper. Through this is passed, first, a brass tube connecting with the rubber tube, $B$, through which the ventilating current of air passes; second, a small brass pipe to which is attached a burner; and finally, two electric wires, $F$ and $F^{\prime \prime}$. Ether is supplied from a glass vessel, $G$, which is, as a matter of fact, an ordinary so-called sulphur-dioxide condensing tube. A current of air entering the ether tube at $H$ passes over the ether and becomes saturated with ether-vapor. It enters the combustion chamber, and issues from

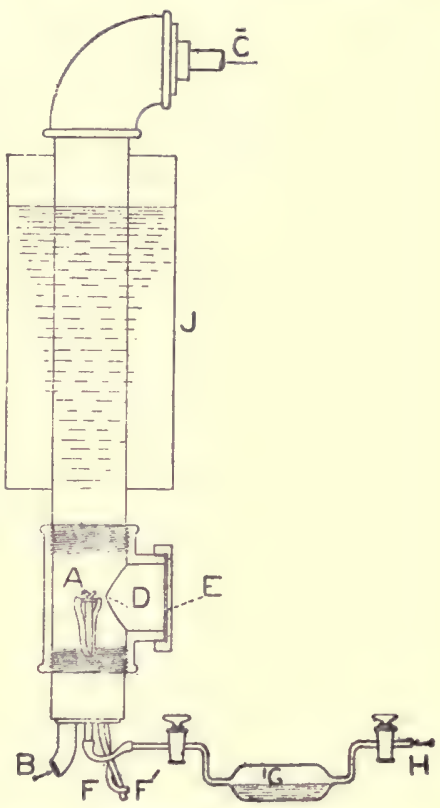

Fig. 8.-Apparatus used for tests of respiration apparatus with burning ether.

$A$, combustion chamber; $B$, ingoing ventilating air-current; $C$, outgoing air-current; $D$, burner; $E$, glass window; $F, F^{\prime}$, high-tension sparking-current lead wires; $G$, container for ether; $\boldsymbol{H}$, supply of air under pressure; $J$, water-cooler. the jet on the acetylene gas-burner, $D$. The vapor is ignited by causing a high-tension spark to jump across the wires $F, F^{\prime}$, by means of a spark coil. The heat developed from the combustion is absorbed readily by the water in the water-jacket. In order to have a constant flame, a steady air pressure must be maintained. This was secured by inserting a tee tube between the rotary blower and the first Wolff bottle. A small supply of air taken from this point carries the ether-vapor into the combustion chamber. 
In the experiment the ether vessel, $G$, is weighed before and after the period, and the amount of ether vaporized is thus accurately known. At the end of the experiment the ether-vapor is shut off and the ventilating air-current is allowed to circulate for several minutes to sweep out the carbon dioxide already formed and bring the whole apparatus to room temperature. The oxygen supply is continued until the apparatus has reached the same tension at the end as at the beginning. The loss in weight of the oxygen cylinder, the increase in weight of the carbon-dioxide absorbers, and the loss in weight of the ether container give the necessary data for calculating the theoretical amounts of carbon dioxide given off and oxygen consumed, and the amounts found by actual experimenting. The results of a typical 15-minute test are given in table $5 \mathrm{~A}$.

\begin{tabular}{|c|c|c|}
\hline & Found. & Required. \\
\hline Carbon dioxide, grams........ & 11.62 & 11.71 \\
\hline $\begin{array}{l}\text { Oxygen, grams } \ldots \ldots \ldots \\
\text { Respiratory quotient } \frac{\mathrm{CO}_{2}}{\mathrm{O}_{2}} \cdots \cdots\end{array}$ & $\begin{array}{c}12.78 \\
0.662\end{array}$ & $\begin{array}{r}12.78 \\
0.666\end{array}$ \\
\hline
\end{tabular}

Test for leaks in the apparatus.-Obviously, with this apparatus, based as it is upon the closed-circuit principle, there must be absolutely no leakage of air during experiments. In order to demonstrate this, tests for leaks are frequently made. The general method used is to admit oxygen or air into the apparatus until a slight tension is reached, as shown by the petroleum manometer, then to ventilate the apparatus for a moment or two in order to equalize the pressure throughout. The tension equalizer diminishes in volume slightly, this being due to air trapped between the acid-containers. The ventilation is stopped and oxygen or air admitted to bring the tension to the desired point. The apparatus is then again ventilated for $\mathbf{1 5}$ minutes and when the ventilation is stopped the tension is noted. Change in pressure is evidence of a leak, as otherwise the manometer would remain constant.

Tests for completeness of carbon-dioxide absorption.-In order to be sure that the soda-lime is absorbing the carbon dioxide completely from the air-current, a portion of the circulating air is diverted through the apparatus containing barium-hydroxide solution (see p. 28) for about one minute. This test is usually made during the latter half of the period. If carbon dioxide is present, a turbidity will be seen in the solution.

Test for completeness of water-vapor absorption.- Since the carbondioxide excretion is determined by weight, the air entering the sodalime container must be dry; furthermore, the last water-absorber must remove completely the water-vapor given off in the soda-lime container. 
To determine the completeness of absorption, so-called efficiency tests are made, as follows: The weights of the soda-lime container and the accompanying water-absorber are each taken separately. The two absorbers are then connected with the rest of the apparatus and the ventilation is continued for 15 or 20 minutes. If the water-absorber is efficient, the loss in weight of the carbon-dioxide absorber and the gain in weight of the water-absorber are equal. In general practice they agree within $0.02 \mathrm{gm}$., which is the limit of weighing. Occasionally the absorption has been incomplete, and this of course is indicated by the fact that the increase in weight of the sulphuric-acid container is less than the decrease in weight of the soda-lime container. It also sometimes happens that the Wolff bottles, $i$. e., the first two waterabsorbers or air-driers, are deficient. This is shown by the fact that the increase in weight of the acid-container accompanying the sodalime container is greater than the decrease in weight of the latter. In the later experimenting with this apparatus, the common practice has been to test the efficiency of the Wolff bottles by weighing the carbondioxide absorber and the third water-absorber together; if no change in weight is found during the 15 or 20 minute test, it is assumed that all parts of the apparatus are efficient. There is, of course, the slight possibility that the actual loss through the two Wolff bottles may be equivalent to an actual loss in the third water-absorber. Even if this occurs, however, it will not in any way affect the carbon-dioxide determination, as the net result will be the same.

General Routine of an Experiment.

The general method of determining the respiratory exchange of a subject with this apparatus is as follows: The subject assumes the position which he is to maintain during the experiment, lying or sitting, as the case may be, and should maintain that position for at least half an hour previous to the experiment. After the preliminary test for tightness, the nosepieces or mouthpiece is inserted, and the subject breathes into the open air until the experimental period begins. The carbon-dioxide absorbers are weighed; the oxygen cylinder, if used, is also weighed, or if the meter is used a reading is made before the experiment begins. After a few minutes of quiet and regular respiration, the three-way valve is turned by an assistant, who does this in so far as possible exactly at the end of a normal expiration. The subject then breathes into the apparatus, and the experiment is continued the determined length of time. At the conclusion of the experiment, the valve is again turned at the end of a normal expiration. During the experiment oxygen is admitted occasionally or continuously at such a rate as to prevent the rubber diaphragm touching the bottom of the tension equalizer. Toward the latter part of the experimental period a test is made for the completeness of the absorption of carbon 
dioxide by passing air 2 or 3 minutes through the barium-hydroxide container, as previously described. After the experimental period is over, the ventilation is stopped and oxygen is admitted until the pressure is the same as at the beginning of the experiment. The carbon-dioxide absorbers are then disconnected and weighed, and the oxygen cylinder is also weighed. The loss in weight of the oxygen cylinder and the gain in weight of the carbon-dioxide absorber and accompanying water-absorber give respectively the quantities of oxygen consumed and carbon dioxide exhaled.

\section{SPIROMETER UNIT.}

The spirometer unit was developed in the winter of 1911-12, and a description of it was published at that time. ${ }^{1}$ Subsequently a number of modifications were made in the apparatus; it is accordingly desirable to give a complete description in English of the apparatus in its present form.

\section{General Plan of Apparatus.}

The general principle of the spirometer type of the universal respiration apparatus is the same as that of the tension-equalizer type. The subject breathes into a closed volume of air which is kept in motion by a rotary blower. The water-vapor and carbon dioxide of the expired air are removed by suitable absorbers and oxygen is admitted to the apparatus. The volume of the system must be the same at the beginning and end or its changes known. A spirometer bell, suspended in oil or water, is substituted for the tension equalizer, the vertical movements of the bell giving quantitatively the volume alterations of the respiratory tract. A device is included for adding the inspiratory volumes and some mechanical changes to assist in manipulation and opera-

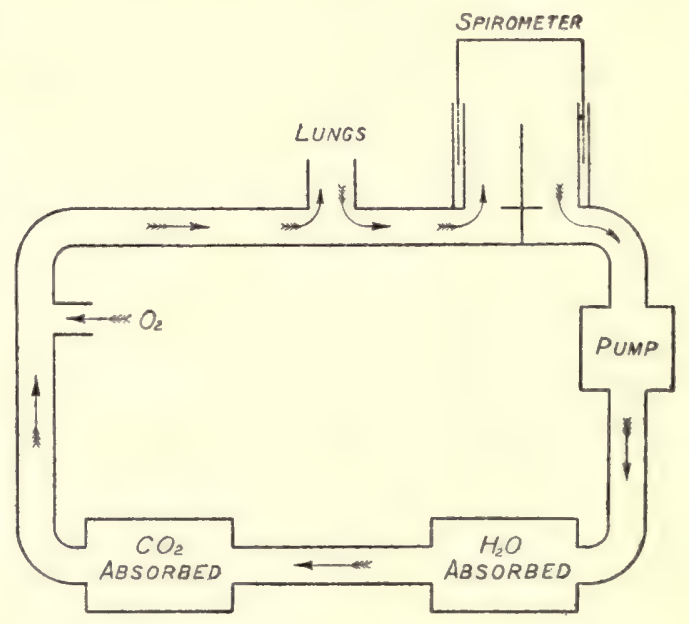

Frg. 9.-Schematic outline of ventilation system of spirometer unit. tion have also been made.

The general scheme of the apparatus may be seen in figure 9 . After the air leaves the rotary blower, it passes first through a waterabsorber, next through a carbon-dioxide absorber, and then through the spirometer, returning from there to the pump or rotary blower. 
Oxygen is admitted after the air leaves the carbon-dioxide absorber and between this point and the spirometer connection is made with the respiratory tract of the subject. The general plan of the apparatus with its different parts is shown in figure 10. From the rotary pump the air passes in turn into a trap, two Williams bottles containing sulphuric acid, a soda-lime container, a sulphuric-acid container or Williams bottle, a can containing dry sodium bicarbonate to remove acid fumes, and finally reaches the respiratory tract of the subject. From there it passes into the spirometer and returns to the rotary pump or blower.

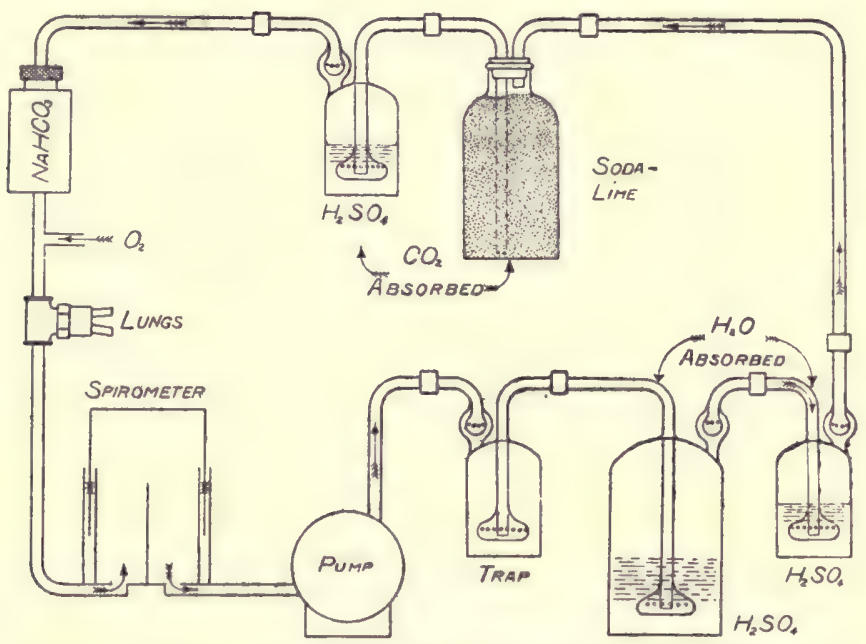

Frg. 10.-Detailed plan of ventilation system in spirometer unit.

Degchiption and Use of Parto.

Rotary blower.- The rotary blower in this apparatus is the same as that used in the tension-equalizer unit, and described in that connection (see p. 24).

Trap.-In using the apparatus a stoppage occasionally occurs which is due either to improperly packed soda-lime containers or to improper manipulation. If pressure is developed in the air-circuit beyond the blower, which can not be released when the ventilation is stopped, the acid from the two Williams bottles will be forced back into the blower. In order to avoid the delay in experimenting required to remove this acid, it has been found advisable to insert a trap for catching the acid when such pressure occurs. For this purpose an empty Williams bottle, reversed, has been inserted in the air-circuit, and thus, when pressure occurs, the acid will run up into the tube which extends to the bottom of the bottle. This empty bottle is sufficient to retain all acid which may come into it due to back pressure.

Water-absorbers.-For absorbing the water-vapor from the expired air and from the air of the apparatus, two Williams bottles in series are used, each filled with 450 c.c. of strong commercial sulphuric acid. 
Carbon-dioxide absorbers.-The carbon dioxide is absorbed by sodalime, which is placed in containers of the same type as those employed for the tension-equalizer unit, of which a detailed description is given on page 27. A Williams bottle containing sulphuric acid is placed after the soda-lime container in order to remove the water which is given off in the absorption of carbon dioxide by the soda-lime.

Retention of acid fumes.-In this apparatus, instead of the air being passed through water containing sodium bicarbonate, it is carried through dry sodium bicarbonate in a brass container, $10.5 \mathrm{~cm}$. in diameter and $11 \mathrm{~cm}$. in height. This container is connected to the apparatus in a vertical position and is packed with alternate layers of cotton and sodium bicarbonate in such a manner that when placed in the ventilating system the layers are in a horizontal position. The bicarbonate and cotton may without renewal be used for several months of experimenting.

Three-way valve, mouthpiece, nosepieces, and moistener.-The threeway valve used for the passage of air from the respiratory tract of the subject to the circulating air-current is the same as that in the older form of apparatus. A cross-section of the valve is shown in figure 11. In the same figure a cross-section is given of the ventilating pipe, the connection for attaching the mouthpiece, and the newer form of air moistener.

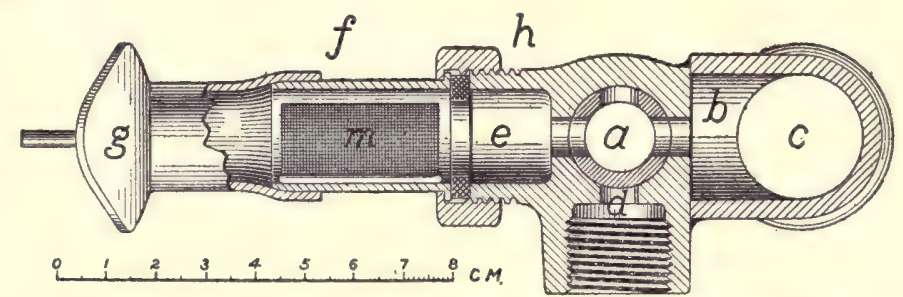

FIG. 11.-Cross-section of the three-way valve, ventilating pipe, and connection for mouthpiece and moistener.

The three-way valve, $a$, is connected to the main air pipe, $c$, by means of a tee, $b$. The mouthpiece, $g$, is fastened to the metal tube, $f$, which is connected to the three-way valve, $a$, by means of the collar, $h ; d$, opening to outside air; $e$, opening between mouthpiece and three-way valve; $m$, metal gauze of moistening apparatus.

An ordinary three-way plug cock, $a$, is used for the three-way valve. This is ground very carefully and sufficient metal taken from it so that it may be soldered directly to the tee, $b$, on the ventilating pipe, $c$. The manipulation and use of the valve are the same as with the tensionequalizer unit (see page 33 ).

The mouthpiece is attached by means of a cylindrical piece of brass tubing, $f$, which is $3 \mathrm{~cm}$. in length and $20 \mathrm{~mm}$. in diameter. This is connected to the three-way valve by a collar, $h$, which screws to the threaded part of the three-way valve. A rubber washer makes a tight closure. The mouthpiece, which is shown as $g$ in figure 11, is the same as that used with the other types of respiration apparatus. A detailed description is given in connection with the Zuntz-Geppert apparatus. 
The nosepieces are of the same form as those described on page 23 (fig. 4), and are attached to the three-way valve by means of an arrangement similar to that used for the mouthpiece. It has two brass tubes ( $d, d$, fig. 12), to which the nosepieces are fastened by means of rubber tubing.

A special device for moistening the inspired air is also shown in figure 12. It is commonly assumed that the expired air is saturated with moisture at $37^{\circ} \mathrm{C}$., so that when the air breathed into the apparatus by the subject strikes a tube which is colder than $37^{\circ} \mathrm{C}$., a deposit or condensation takes place. The moistener in the spirometer unit is constructed to take advantage of this fact. A piece of copper gauze ( $m$ in fig. 11 and $a$ in fig. 12) is rolled into a cylinder and inserted in the tube connecting the nosepieces with the three-way valve. This is done in such a manner that the air entering the nose or mouth passes on both sides of the copper gauze. To facilitate the removal of this
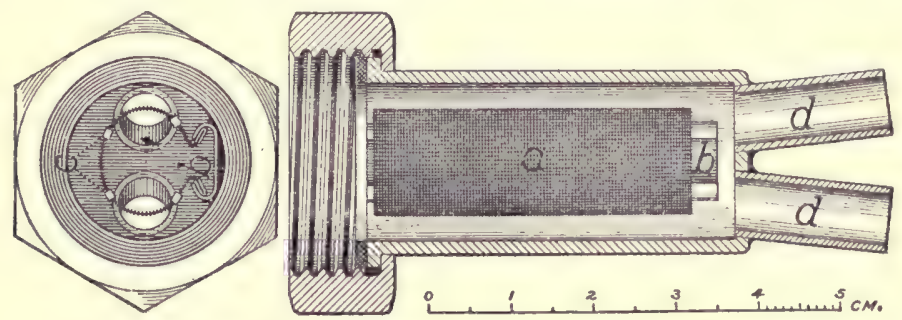

FIG. 12.-Details of moistener and connection for nosepieces.

The air-moistener, $a$, is inside of a brass tube, to the end of which are connected the tubes $d$ and $d$ for holding the nosepieces. At the left is shown a lateral cross-section. The metal ridge, $b$, holds the moistener $a$. Rubber bands for holding the linen on the moistener are shown at $c$.

moistener, the edges of the gauze fit into a small strip of metal, $b$, soldered to the inside of the tube. Fine cambric is wrapped about the gauze and kept in place longitudinally by a rubber band, $c$, or by sewing it on. In actual use this cambric is saturated with water, so that the dry air, before entering the nose or mouth, becomes partially saturated as it passes over the moistening device. As some moisture from the expired air is unquestionably deposited, the original amount of water is but slowly evaporated. When once thoroughly drenched, this moistener gives satisfactory service for several experimental periods of 15 minutes and can be readily removed and re-moistened or sterilized whenever necessary.

Spirometer.-The essential modification in this type of respiration apparatus is the insertion of a spirometer in the ventilating air-current, consisting of a cylinder suspended free in a bath of water or oil and counterpoised. Air enters and leaves the bell through tubes connected with the apparatus, the bell rising and falling as the pressure increases or decreases. Devices are attached to record the movements of the bell, which show the quantitative changes in the volume of the respira- 


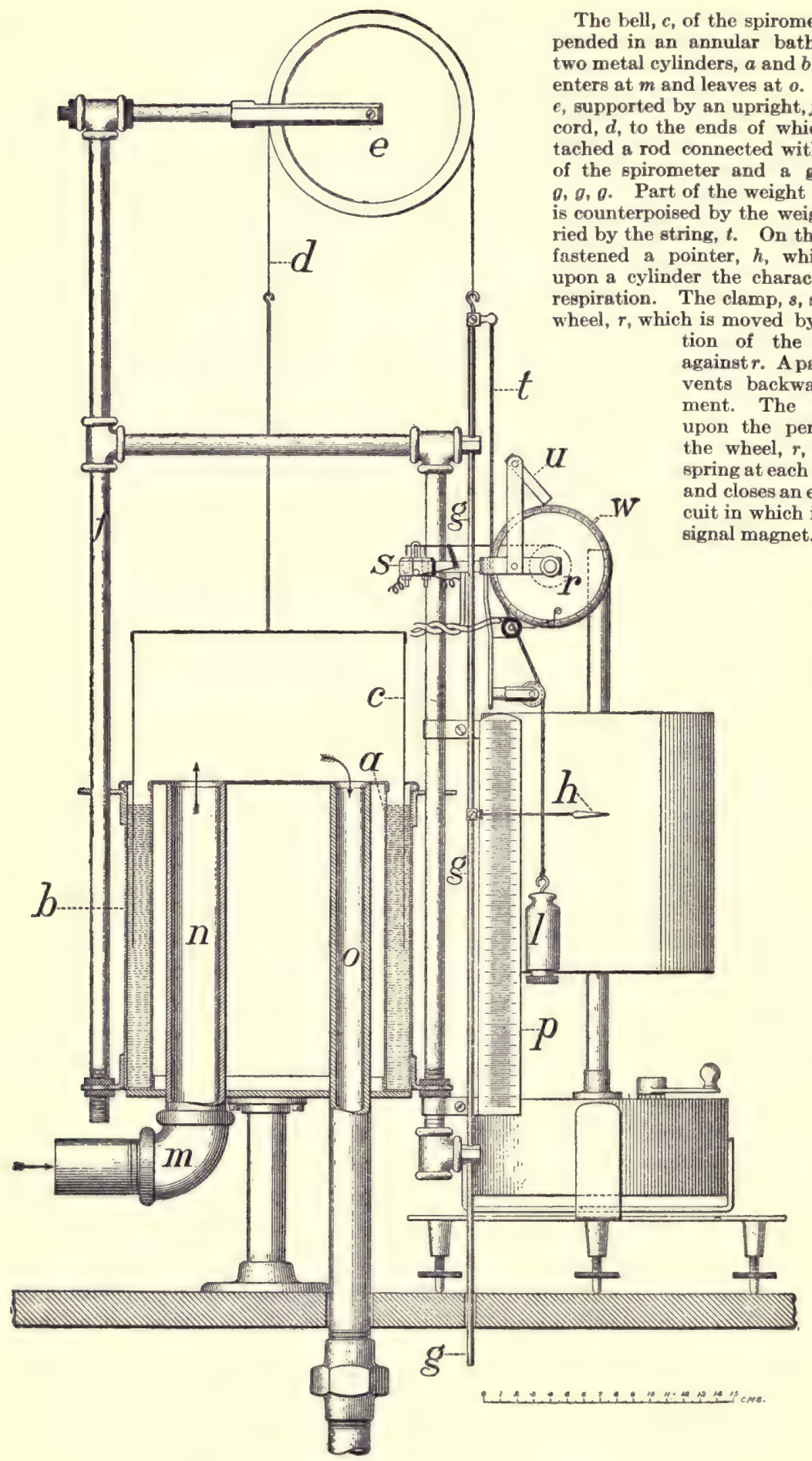

FIG. 13.-Details of spirometer, with recording attachments. 
tory system of the subject. The readings of the spirometer at the beginning and the end of the experiment are also used in determining the oxygen consumption. This spirometer is shown in figure 10, and in more detail in figure 13.

The spirometer bell is cylindrical in form and constructed of the lightest-weight sheet-copper, with the seams shellacked instead of soldered, as the heat required for soldering would tend to distort the shape of the bell. The internal diameter is about $166 \mathrm{~mm}$. and the maximum vertical excursion is $135 \mathrm{~mm}$.; the fluctuating volume is therefore 2 to 3 liters. The total weight of the bell is not far from $100 \mathrm{gm}$. It is suspended in an annular bath of water or oil between two copper cylinders, $a$ and $b$, the inner cylinder being covered at the top except for the openings of the air-pipes, $n$ and $o$. The bell is suspended by a silk cord, $d$, running over a grooved aluminum wheel, $e$, fastened to an upright, $f$. The weight is accurately counterpoised by a guide-rod, $g$, a pointer, $h$, and a weight, $l$. When properly adjusted, the bell is in equilibrium at nearly any point. The most perfect equilibrium is arbitrarily adjusted at about the midway position of the bell. The spirometer is connected with the ventilating circuit by means of a tube leading from the three-way valve to a short piece of rubber tubing attached to the elbow, $m$, at the bottom of the spirometer. The metal tube, $n$, through which the air enters, is continued to the top of the inner cylinder. The air leaves the spirometer bell through a smaller pipe, $o$, which can be connected directly to the intake end of the blower. A millimeter scale, $p$, fastened to the frame of the spirometer and a pointer, $h$, attached to the guide-rod permit readings of the height of the bell.

Device for obtaining a graphic record of the respiration.-The spirometer bell rises and falls with each respiration; this movement is recorded graphically. To the guide-rod, $g$, is attached a horizontal piece of steel wire; to the free end of this wire is fastened a small pointer, $h$, of parchment paper or celluloid. When the bell rises or falls, the movement is recorded upon the moving drum of a kymograph, the record showing not only the amount of air inspired or expired, but also the length and depth of the respiration. A specimen record is given in figure 14. On this respiratory curve the beginning of the experimental period is shown at 1 . No oxygen was admitted into the apparatus until the point 2 , when an attempt was made to add the oxygen as rapidly as the subject consumed it. At $S$ the valve was again turned so that the subject breathed into the open air. At 4 A he began breathing into the apparatus, but, as will be seen, the valve was turned too soon in the respiratory cycle. No oxygen was admitted into the apparatus and at 5 , the valve was turned. The subject then breathed into the open air until the valve was again turned at $6 \mathrm{~B}$, the record showing that this was done too late in the respiratory cycle. At 7 the valve was opened to the outside air and the record was ended. The time in minutes is recorded on the lowest line. 
Device for measuring the total inspiratory ventilation.--The line directly below the respiration curve in figure 14 was made by the device for recording the total inspiratory ventilation, or the so-called "ventilation adder." An aluminum wheel, ${ }^{1} r$ (fig. 13), is attached to the support, $s$, in such a manner that at each movement of the spirometer bell in a downward direction, that is, at each inhalation, the wheel is mechanically moved by the upward motion of the cord, $t$. A pawl, $u$, prevents any perceptible backward motion as the cord is drawn down by the counterpoise, $l$. By means of a platinum wire, against which a projecting point, $w$, touches, and a signal magnet not shown in the figure, the total number of revolutions of the wheel can be recorded upon the kymograph drum. The fractional revolution is noted from the reading of a series of numbers on the periphery of the wheel. Each revolution of the wheel corresponds to a movement of the bell through $228 \mathrm{~mm}$., and consequently to a volume of about 4,900 c.c. From the total

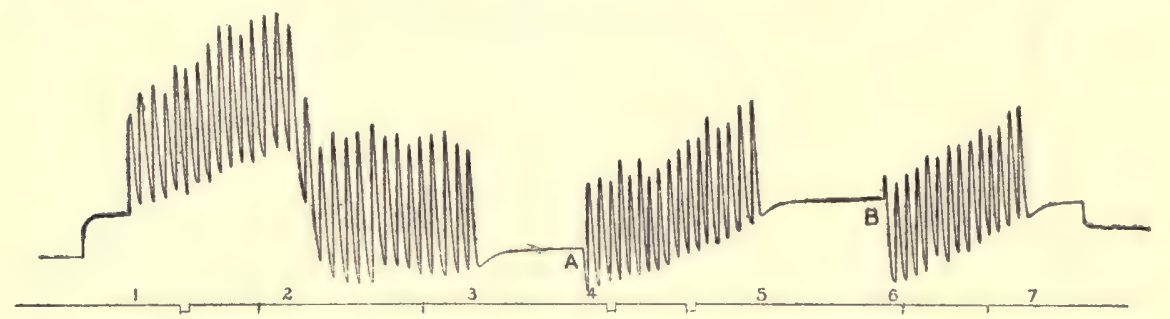

FIG. 14.-Specimen graphic record of respiration.

Lowest line, time; middle line, revolutions of the wheel, $r$ (fig. 13). Between 1 and 2 , 4 and 5 , and 6 and 7 , no oxygen was admitted; between 2 and 3 oxygen was admitted at approximately the rate that it was used. At $A$ the three-way valve was turned too early and at $B$ too late.

number of revolutions and the value per revolution, a calculation may be made of the total amount of air inspired during the time the subject is breathing into the apparatus. ${ }^{2}$

Device for registering number of respirations. - The number of respirations in an experiment can be counted from the record made by the excursions of the pointer attached to the counterpoise of the spirometer bell. Since the counting requires considerable time, it is planned to do this automatically by an electrical counting device. The contact portion of this arrangement, which has already been installed on one of the respiration apparatus, is shown in detail in figure 13. One end of a platinum wire is fastened loosely around the axis of the aluminum

\footnotetext{
${ }^{1}$ An aluminum wheel, devised by Professor W. T. Porter in connection with his work adder and manufactured by the Harvard Apparatus Co., was used for this purpose.

${ }^{2}$ During the period of an inspiration, the influence of the absorption of carbon dioxide on the one hand and the admission of oxygen on the other involve two more or less compensatory corrections when a high degree of extreme accuracy is desired.
} 



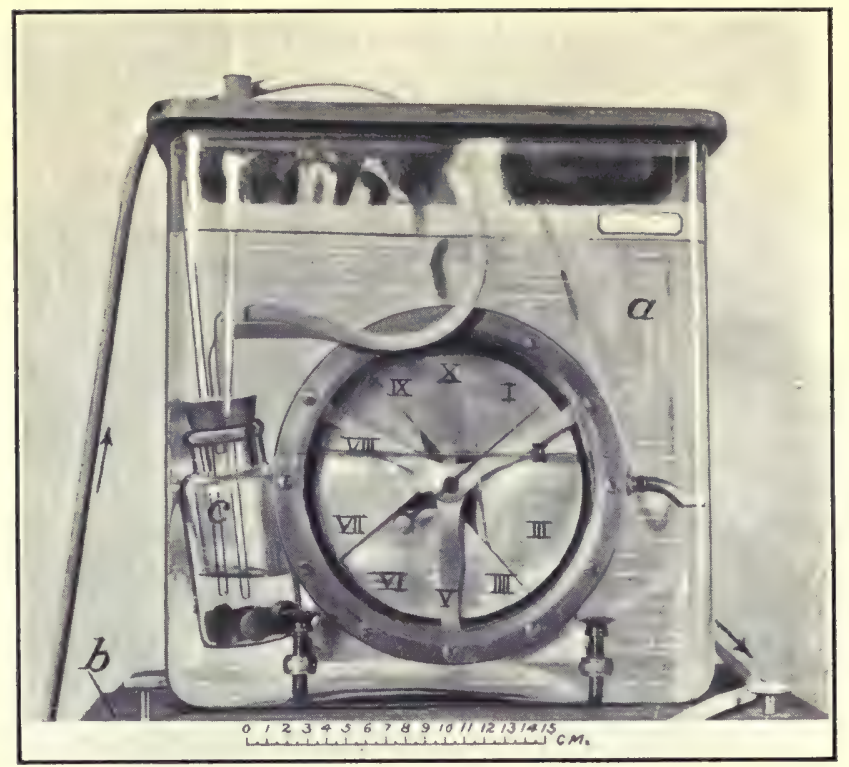

FIG. 15.-Bohr meter. $a$, water bath; $b$, leveling board; $c$, moistener. (For description, see page 47.)

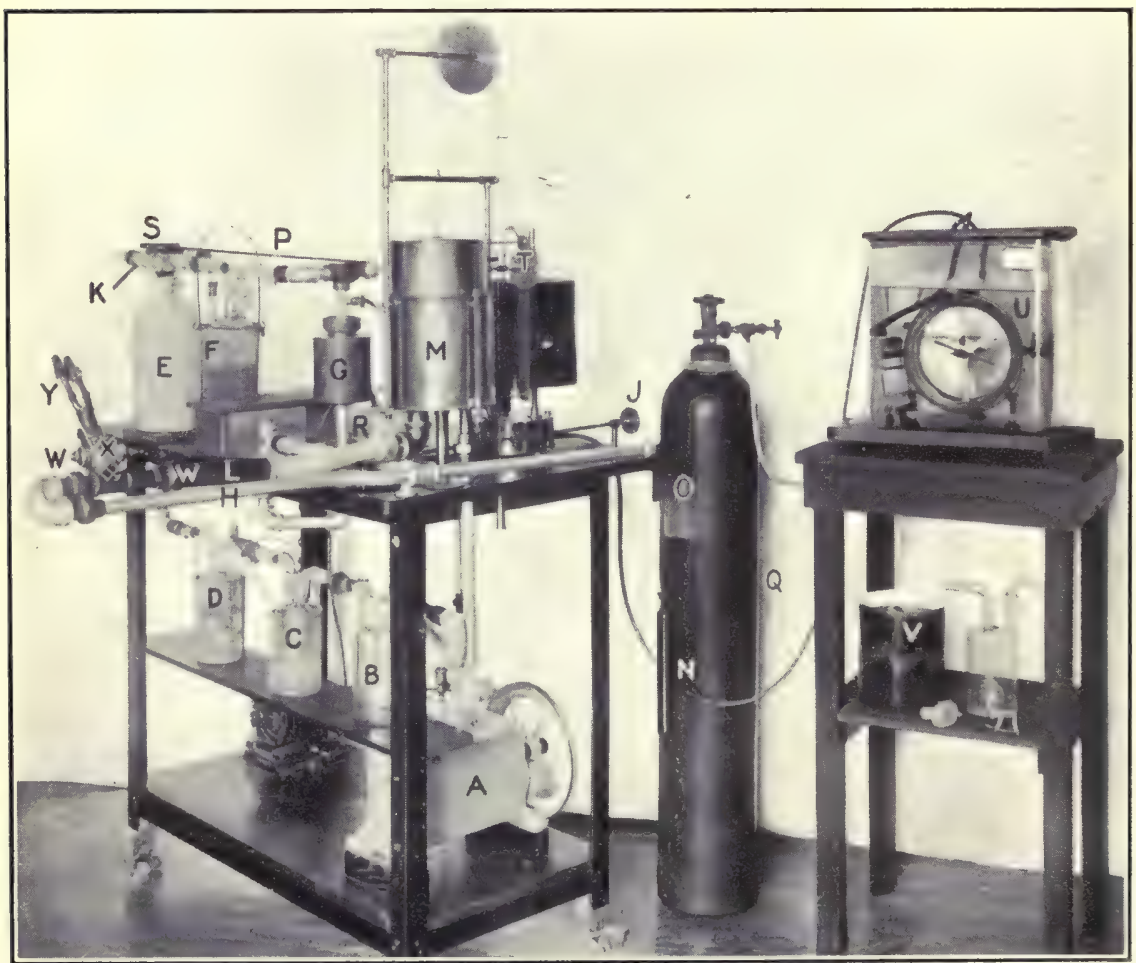

FIG. 16.-General view of the spirometer unit.

$A$, rotary blower; $B$, trap; $C$ and $D$, Williams bottles containing sulphuric acid for absorbing water-vapor; $\boldsymbol{E}$, soda-lime container for absorbing carbon dioxide; $\vec{F}$, Williams bottle containing sulphuric acid; $G$, sodium-bicarbonate can; $H$, air-pipe leading from $G$ to three-way valve, $X$; $J$, handle for turning three-way valve; $L$, large tube leading from three-way valve to spirometer $M ; N$, weight counterbalancing movable arm, $L H W W ; O$, clutch for holding movable arm in place; $K P$, handle and rod for turning two three-way valves at either end of carbon-dioxide absorption system; $Q$, large oxygen cylinder; $R$, Erlenmeyer flask partly filled with bariumhydroxide solution; $S$, three-way valve; $T$, ventilation adder wheel; $U$, Bohr meter immersed in water; $V$, typical graphic record; $X$, three-way valve; $Y$, pneumatic nosepieces; $W W$, connections for changing angle of three-way valve. 
wheel, $r$. The middle portion of this wire is kept in contact with the guide-rod, $g, g$, by means of a spring. The outer or free end of the wire has two platinum points which dip into two mercury cups. When the spirometer moves downward and the rod upward, these two points are lifted out of the mercury cups, thus breaking the circuit in which the two cups are installed. When the rod moves downward, the two platinum points dip deep into the mercury and the circuit is closed. The constant make and break of this circuit can be made to actuate a small magnet. Ultimately a mechanical counter of some type will be installed in the circuit which can be read at the beginning and end of an experiment, the difference between the two readings giving the number of respirations for the whole experiment.

Mechanical arrangement of the apparatus.-A general view of the spirometer unit is shown in figure 16. Standard $\frac{1}{2}$-inch piping is used throughout the apparatus, except for the tube leading from the threeway valve to the spirometer. Half-inch garden-hose couplings connect the several parts. For assistance in manipulating the apparatus with subjects at varying levels, the portion of piping which runs either side of the three-way valve is arranged so that it forms a part of a flexible arm with a movable joint at the point where it is attached to the table. This is counterpoised by the weight, $N$, and may be fixed in any position by the clamp, $O$. Loosening the couplings either side of the three-way valve permits the raising or lowering of the three-way valve and the nosepieces. The air, on leaving the three-way valve, passes through the tube, $L$, and the supplementary rubber tube into the spirometer, $M$. From the spirometer it descends to the pipe below the table and into the rotary blower, $A$. It then passes through the trap, $B$, and into the two Williams bottles, $C$ and $D$. The air from this point passes upward to the three-way valve, $S$, and then into the carbondioxide absorber, $E$, and subsequently into the Williams bottle, $F$. The sodium-bicarbonate can for removing the acid fumes is shown as $G$. The air then returns along the table to the pipe $H$ and back to the three-way valve. The handle of the three-way valve is shown at $J$. The device containing barium hydroxide is shown at $R$.

Care of the apparatus. - In the manipulation and running of the apparatus for routine work a number of points should be observed for keeping the apparatus in good mechanical condition. The blower should occasionally be oiled internally through the oil-cup situated just above the blower. The shaft should also be oiled at times by unscrewing the two rods which close the openings around the shaft.

The Williams bottles on the lower section of the table in which the water is absorbed from the circulating air-current should be refilled occasionally. The usual routine is to renew the first bottle each day when a series of experiments is being carried on. A record is also kept 
of the changes in weight of the second bottle; when it has gained $10 \mathrm{gm}$. of water-vapor it is rejected and another substituted. The method of insuring the efficiency of the soda-lime containers has already been given in the description of the tension-equalizer unit, and applies to this apparatus. The efficiency of the Williams bottle following the carbon-dioxide absorber is also safeguarded as described on p. 32 .

Before each experiment the three-way valve should be taken out, thoroughly sterilized, and lubricated with vaseline in such a manner that it will turn easily without danger of a leak. The mouthpiece, moistener, and nosepieces should also be sterilized before each experiment and again immediately after the experiment.

The bell of the spirometer should be examined occasionally to make sure that it does not touch the copper walls of the bath. It should hang perfectly vertical and move up and down midway between the two cylinders. The ventilation adder contact should likewise be inspected before the experiment is begun to find if it works properly when the wheel is placed at zero.

Calibration of the bell of the spirometer.-The records of the movement of the spirometer bell up or down are used in the measurement of the oxygen consumption and also in the measurement of the volume of respiration, each millimeter representing a certain quantitative relation of volume (usually 21 to 23 c.c.). This value may be ascertained in several different ways. It may be calculated from the height and the diameter of the spirometer bell by the usual method of calculating the volume of a cylinder. This assumes that the bell is a perfect cylinder, with no irregularities in any part. Another method is to invert the bell of the spirometer, fill it with water at a definite temperature, and compare the weights obtained before and after filling it. In using this method the bottom of the cylinder must be well supported to prevent bulging; the cylinder must also be absolutely level, otherwise it is impossible to fill the cylinder to its full capacity. A third method of calibrating the bell, and the most common in this laboratory, is by the admission of a definite quantity of air or oxygen through a Bohr meter. A description of this meter is given in connection with the description of the method of admitting oxygen to the apparatus (see page 47). The spirometer bell is pushed down to the lowest possible limit and a reading on a millimeter scale is taken. Air or oxygen is then passed through the meter into the bell of the spirometer; when the bell has risen to its full height, the oxygen or air is shut off. From the reading of the meter, the factor of the meter, and the number of millimeters to which the bell has risen, the value per millimeter may be calculated. A correction should be made for temperature if the temperatures of the meter and the spirometer are markedly different. 
A specimen calibration of the bell of a spirometer follows:

Height of bell at start, $42 \mathrm{~mm}$.; at end, $175 \mathrm{~mm}$.

Oxygen admitted, 2.935 liters; factor of meter, 0.9623 ; temperature of meter, $18.8^{\circ} \mathrm{C}$; ; temperature of water in spirometer, $19.2^{\circ} \mathrm{C}$. $(2.935 \times(273.0+19.2) \times 0.9623) \div(175-42) \times(273.0+18.8)=$ 21.28 c.c. per $\mathrm{mm}$.

The volume represented by each millimeter rise of the bell is therefore 21.28 c.c.

Calibration of the ventilation adder.- The periphery of the wheel of the ventilation adder is milled. The pawl above the wheel is triangular at the end and engages in this milling as the bell moves in an upward direction. Notwithstanding this arrangement, however, there is some slight backward movement. Theoretically the value in c.c. of one revolution of the ventilation adder wheel should be equivalent to the circumference of the wheel in millimeters multiplied by the value in c.c. of a millimeter of the bell of the spirometer. The calibration can be carried out in a number of different ways. The bell of the spirometer may be filled with air or oxygen and readings taken of the level of the spirometer and of the ventilation adder wheel; the bell may then be pushed down until it is empty and a second reading taken of the level of the spirometer bell and of the ventilation adder wheel. As this method does not take into account any backward movement, the calibration should be carried out under as nearly the same conditions as possible as those which are present when the subject is breathing into and out of the apparatus. This may be accomplished by connecting a bulb to the opening of the three-way valve, this bulb being connected to another bulb filled with water, the upper and lower portions of the bulb being marked. The first bulb may be alternately filled and emptied to the upper and lower marks by raising and lowering the second bulb. An up and down motion of the spirometer bell is thus produced, simulating respiration. If the exact volume between the two marks on the first bulb is known, also the number of movements or strokes and the number of revolutions of the ventilation adder, the value per revolution may then be calculated.

This method was used in the development of the apparatus, but recently the ventilation adder has been calibrated by a more convenient method in which the small hand spirometer, described in detail on page 79, has been used. This hand spirometer consists of an inverted cylinder which moves in a bath between two concentric cylinders on the same principle as the spirometer of the respiration apparatus. A handle is fastened to this inverted cylinder by which it can be moved up and down in a rigid framework, the length of the stroke (vertical movement) being adjusted by a set-screw. The general method of calibrating the ventilation adder with this apparatus is as follows: The small spirometer is connected to the three-way valve, the ventilation adder wheel is set at zero, and the kymograph drum is brought near the writing-point of the spirometer on the respiration apparatus. 
The kymograph is next set in motion and the three-way valve opened between the small hand spirometer and the large spirometer. Regular movements up and down are then made with the small spirometer, care being taken that the beginning of the movement at the bottom and the end of the movement at the top are made slowly so as not to jar the ventilation adder wheel. This is continued until the wheel has revolved a number of times. The kymograph record is then coated with a fixative and when it is dry a number of measurements of the records of the strokes are made, using a pair of dividers and a millimeter scale, and estimating to about $0.1 \mathrm{~mm}$. The average of ten measurements is then multiplied by the value per millimeter of the bell of the spirometer ( 21.33 c.c. in the example given) and the total number of strokes. This gives the total volume required to move the ventilation adder wheel the number of revolutions which has taken place. Dividing this volume by the number of revolutions gives the "apparent" volume per revolution. A sample calculation and calibration is given:

Length of movement of the bell of spirometer, $26.1 \mathrm{~mm}$.

Number of movements, 82. Number of revolutions of the aluminum wheel, 9.10.

Calculation: $(82 \times 26.1 \times 21.33) \div 9.10=5.02$ liters, volume per revolution.

Back-lash.-In the actual use of the ventilation adder wheel there is a certain amount of backward movement each time that the spirometer bell moves in an upward direction. This is due to the fact that the edge of the wheel is milled and the transverse grooves are wide enough to permit some backward motion before the pawl fits firmly into the groove. In order to determine the amount of this backward movement, calibrations of the ventilation adder may be made with two different lengths of stroke. If the same number of complete revolutions are obtained, the value per stroke for the back-lash may then be calculated from the difference in number of strokes and the difference in total volume for the complete number of revolutions. This has been done in a number of calibrations and the results are as follows:

Calibration with $7.04 \mathrm{~mm}$. movement of the bell of the spirometer gave, as a result, 5.49 liters per revolution of the ventilation adder wheel.

Calibration with $26.06 \mathrm{~mm}$. movement of the bell of the spirometer gave, as a result, 5.05 liters per revolution of the ventilation adder wheel.

The number of movements of the bell for 9 revolutions of the ventilation adder wheel, with $7.04 \mathrm{~mm}$. per movement, was 248 greater than that with $26.06 \mathrm{~mm}$. movement.

The difference in volume for 9 revolutions amounted to 3.96 liters. Therefore, the amount of backward movement of wheel at each stroke was $3.96 \div 248=0.016$ liter per movement. 
The record on the kymograph drum made in the two calibrations is shown in figure 17. When a rubber band is placed around the pawl, the back-lash is increased, but the use of this rubber band is found desirable, as the sound of the metal pawl striking against the corrugations of the wheel attracts the attention of the subject and makes him conscious of his respiration.

Kymograph records.-It is the general custom in this laboratory to smoke the kymograph records heavily, so as to give a sharp contrast and to enable us to reproduce them in whole or in part by using the original record as a negative. The greatest care is taken to keep the curves from accidental abrasion, and to arrange the recording devices so that there need be little alteration in reproducing the record for publication. An effort is made to adjust the speed of the kymo-
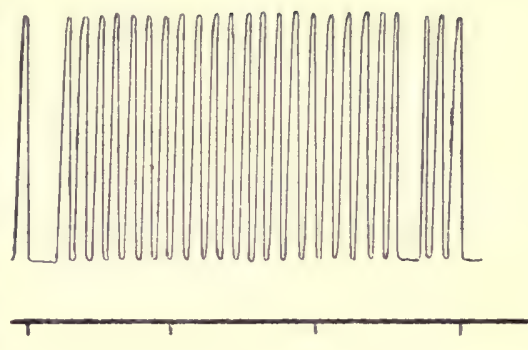
graph to a uniform rate, so that the experimental records may all be comparable.

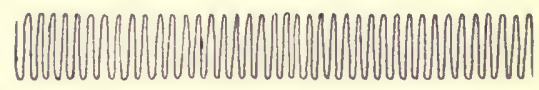

FIG. 17.- Specimen kymograph records made in the calibration of the ventilation adder.

The upper portion shows the record made with a stroke of 556 c.c. and the lower with a stroke of 150 c.c. The complete revolutions of the ventilation adder wheel are recorded by the signal magnet in the horizontal line below each kymograph record.

\section{General Routine of an Expertment.}

The general routine of a respiration experiment with this apparatus is practically the same as with the tension-equalizer unit. There are, however, some additional manipulations required, owing to the increase in number of observations. The subject, after securing a comfortable position, is attached to the apparatus by means of either the mouthpiece or nosepieces. Before the experiment is actually begun, the carbon-dioxide absorbers are weighed and the meter reading or the weight of the oxygen cylinder is obtained. The spirometer level is set at such a height (as indicated by a millimeter scale) that there will be no danger of all the air being drawn out of the spirometer bell by the subject in a deep inspiration. The contact of the ventilation adder is set at zero and the kymograph is adjusted so that the time marker, the revolution counter, and the pointer of the spirometer bell will write freely. The height of the spirometer bell may be read either while the apparatus is running or before the ventilation has been started. Of course it is necessary to use the same method of reading at the end of the experiment as at the beginning. Everything being in readiness, and the air of the apparatus circulating, the three-way valve is then 
turned at the end of a normal expiration and the subject begins inspiring from the apparatus. Oxygen is admitted either continuously or intermittently. If the meter is used, the movement of the pointer is recorded each time it passes the zero-point of the meter. At the end of the experiment the valve is turned as with the older type of apparatus. After running a few minutes, oxygen is admitted into the apparatus until the spirometer is at the same level as at the beginning of the experiment. For convenience this admission of oxygen may be omitted in actual practice, care being taken to read the height of the spirometer and then to correct for the actual difference in level between the beginning and end of the experiment. A reading of the ventilation adder is also taken at the end of the experiment and noted on the record sheet. During the latter half of the experimental period the completeness of absorption of the carbon dioxide is tested, as with the older apparatus, by deflecting a portion of the air-current through a solution of barium hydroxide.

\section{OXYGEN SUPPLY FOR THE UNIVERSAL RESPIRATION APPARATUS.}

In connection with the direct determination of the oxygen consumption of the subject it is necessary to admit the oxygen in such a manner that it can be easily and accurately weighed or measured. It is also necessary to have the supply free from carbon dioxide and water-vapor, or to make some provision for removing these gases. In the earlier experimenting, oxygen was admitted from a small cylinder containing about 150 liters of the gas. As the kind of oxygen first purchased contained both carbon dioxide and water, the cylinder was provided with tubes for the removal of these impurities. A rubber bag attached to a tee which was connected with the valve prevented any sudden escape of the gas through the tubes when the valve was opened.

These small cylinders were used for some time, but there were a number of disadvantages in connection with their use. It was necessary to make sure that the bag was absolutely deflated each time that the cylinder was used, and that the connections on the carbon-dioxide and water-vapor absorbers were absolutely tight. These latter parts, being fragile, were easily broken, and whenever such a break occurred the determination of the oxygen was lost for that particular experiment. The fitting of the purifying apparatus to the cylinders also required considerable time.

For a brief period in the early development of the apparatus an oxygen generator was used which furnished oxygen by the generation of the gas from the action of water on sodium peroxide. A tin can containing fused sodium peroxide was held in the bottom of a container by means of springs. Holes were punched in the top and bottom of the can to allow the admission of water. The can of sodium peroxide 
was covered with a bell having an exit and a valve at the top. When this valve was opened, the water entered the can of sodium peroxide and gas was generated. The gas thus formed was remarkably pure, containing only moisture. It was, therefore, still necessary to have a drier. One objection to this apparatus was the fact that during generation intense heat was formed which interfered with accurate weighing.

This method of supplying oxygen did not prove so practical as the use of cylinders, and when it was found that the oxygen from the Linde Air Products Company of Buffalo, New York, contained very little nitrogen and practically no weighable amount of carbon dioxide and water, their product was substituted. Small cylinders were obtained, containing about 150 liters of the gas, with approximately 3 per cent of nitrogen. ${ }^{1} \quad$ A reduction valve was attached by means of which the flow of oxygen into the apparatus could readily be regulated. While the quality of the oxygen and the method of admission were both satisfactory, provided the reduction valve was in perfect condition, it was frequently found that the reduction valve did not work properly or that it was leaking. A Bohr experimental gas-meter of 1-liter capacity was therefore tested in the spring of 1911 and adopted; at the present time there are at least five of these meters in use in the Nutrition Laboratory.

The Bohr meter as set up and used is shown in figure 15 (page 41). Each scale division corresponds to 5 c.c., while the numerals correspond to 0.1 liter. The whole meter is immersed in an aquarium jar filled with water. This insures uniformity of temperature throughout the meter and surrounding medium, and precludes measurable temperature change in a 15-minute experiment. A moistener is placed in front of the meter so as to provide for the complete saturation of the air passing through it, thus preventing the evaporation of the water in the meter. This moistener consists of a wide-mouth bottle, $c$, in which a three-holed rubber stopper provided with tubes is inserted. One tube dips below the level of the water and the other provides for the exit of the gas. A third tube, which extends from below the surface of the meter to above the water in the aquarium jar, serves as a safety valve in case there is back pressure. The use of this is referred to later. The bottle is weighted down with shot. The thermometer inserted through the cover of the aquarium jar indicates the temperature of the water.

The requirements for accuracy in the use of the meter are accurate measurements of the barometric pressure and the temperature, complete saturation of the air with water-vapor, and a knowledge of the mechanical factor of the apparatus. The first three conditions can

\footnotetext{
${ }^{1}$ Formerly the impurity was considered to be nitrogen, but it has recently been found that this impurity is nearly all argon and our calculations are made upon this basis.
} 
easily be met. The mechanical correction factor can be obtained by calibration tests, ${ }^{1}$ in which a cylinder of oxygen is used, the amount of gas passing through the meter being computed from the loss in weight and from the known chemical composition of the gas. Before the meter is calibrated it should be accurately leveled by means of the leveling screws on the meter and on the board upon which it rests. It should also be filled to the level at which it is to be used, the best level being that indicated by the manufacturers by the lines marked upon the rim. The meter and aquarium jar with the surrounding water should stand long enough before calibration for the whole mass to come into temperature equilibrium, otherwise the temperature of

TABLE 6.-Results of independent calibrations of a 1-liter Bohr meter by two operators.

\begin{tabular}{|c|c|c|c|}
\hline \multicolumn{2}{|l|}{ P. F. J. } & \multicolumn{2}{|l|}{ T. M. C. } \\
\hline Date. & Per cent. & Date. & Per cent. \\
\hline 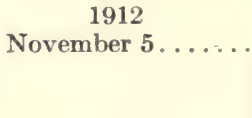 & $\begin{array}{l}99.39 \\
99.24 \\
99.96\end{array}$ & $\begin{array}{c}1912 \\
\text { November } 6 . . . . .\end{array}$ & $\begin{array}{l}98.97 \\
99.96 \\
98.59\end{array}$ \\
\hline Average ... . . . . & 99.53 & Average.. . & 99.17 \\
\hline November $6 . . . .$. & $\begin{array}{l}98.90 \\
99.10\end{array}$ & November $15 . . .$. & $\begin{array}{l}98.70 \\
98.71\end{array}$ \\
\hline Average........ & 99.00 & Average.... & 98.71 \\
\hline November $14 . .$. . & $\begin{array}{r}100.10 \\
99.94 \\
99.77\end{array}$ & & \\
\hline Average........ & 99.94 & & \\
\hline Average of all.... & 99.50 & Average of all.... & 98.94 \\
\hline
\end{tabular}

the bath may not indicate the temperature of the meter. It is also necessary that the cylinder connections be absolutely air-tight. This may be tested by weighing the cylinder at intervals of 15 to 20 minutes; if no change in weight takes place, the connections are tight. The cylinder is then connected to the entrance tube of the moistening apparatus and the gas is passed through at approximately the rate to be used during an experiment. Usually this has been about 4 liters in 10 to 15 minutes. The two or three calibrations made in this manner should agree within 0.5 per cent, and the limits of error between two sets of calibrations made by two people on separate days should agree on the average within at least 1 per cent. 
Accuracy in filling the meter for the several calibrations is also an important consideration. The meter should always be filled to within at least $1 \mathrm{~mm}$. of the same level each time and, if the other observations are made with sufficient accuracy and uniformity, the only cause for variation in the mechanical factor should be the level. Calibrations independently made by two observers after emptying and refilling the meter each day are given in table 6 .

That the difference in level of the water inside the meter makes a difference in the factor of the meter is shown by some experiments which were carried out by Dr. E. P. Cathcart, ${ }^{1}$ of the London Hospital Medical College. In these tests approximately 4 liters of oxygen were passed through the meter in from 2 to 3 minutes. The volume at $0^{\circ}$ and $760 \mathrm{~mm}$. as measured by the meter was computed from the meter readings and the records of the temperature and barometer; the true volume was computed from the loss in weight of the oxygen cylinder. The correction factors, which are given in table 7 , were calculated by

TABLE 7.-Results of Cathcart's experiments on the effect

of varying levels of water in the meter.

\begin{tabular}{|c|c|c|}
\hline $\begin{array}{l}\text { No. of experiments } \\
\text { averaged. }\end{array}$ & Level. & $\begin{array}{l}\text { Correction } \\
\text { factor. }\end{array}$ \\
\hline $\begin{array}{r}11 \\
3 \\
4 \\
3\end{array}$ & $\begin{array}{r}\text { Line mark...... } \\
3 \mathrm{~mm} \text {. above... } \\
8 \mathrm{~mm} \text {. above... } \\
13 \mathrm{~mm} \text {. above... }\end{array}$ & $\begin{array}{c}\text { p. ct. } \\
103.3 \pm 0.8 \\
98.7 \pm 0.3 \\
92.9 \pm 0.6 \\
84.7 \pm 0.3\end{array}$ \\
\hline
\end{tabular}

dividing the true volume of gas leaving the cylinder by the amount computed from the meter readings. It will be seen from table 7 that there was a marked change in the correction factor of the meter when the water-level was increased in height. It is also quite possible to have the level of the water so low that the meter will not record at all.

It has been pointed out that the meter must be calibrated under exactly the same conditions as used in the experiment. One of these conditions is rapidity of admission. If the oxygen is admitted at the rate of 1 liter in 3 or 4 minutes, it should be calibrated at that rate; if more rapidly, it should be calibrated at the higher rate. The effect of the rate of admission upon the correction factor is clearly shown in the series of results which were obtained by Dr. Cathcart in connection with an experiment on muscular work. (See table 8.) The time varied from the rate of 4 liters in 21 seconds to the rate of 4 liters in 9 minutes 30 seconds. It will be seen that up to the rate of 4 liters in 2 minutes 6 seconds, the correction factor varies with the rate that the gas passed through the meter. During the calibrations particular

\footnotetext{
'Research Associate of the Nutrition Laboratory in 1911-12. The results of these tests have been previously published in a description of the spirometer unit. (Benedict, Deutsch. Archiv klin. Med., 1912, 107, p. 183.)
} 
care is of course taken to insure that all of the observations are made as uniformly as possible.

It is of interest to note the average accuracy of meters in actual experimenting. An opportunity was given for observing this in connection with a study on the effect of a carbohydrate-free diet upon four young men during the winter season of 1912-13. Both meters and oxygen cylinders were used in these experiments. The type of oxygen cylinder and valve employed will be subsequently described. The cylinders were weighed to approximately $0.01 \mathrm{gm}$. on the balance regularly used in connection with the respiration apparatus; the meters were read as usual, and the barometer and temperature observed during each period of admission. Each of the four meters was in charge of each of four observers at various times, so that the series of results probably represents as nearly as can be the actual range of accuracy with

TABLE 8.-Results of Cathcart's experiments on the effect of the rate at which oxygen is passed through the meter.

\begin{tabular}{|c|c|c|c|c|c|}
\hline $\begin{array}{l}\text { Time for } \\
\text { record of } \\
4 \text { liters. }\end{array}$ & $\begin{array}{c}\text { Correction } \\
\text { factor. }\end{array}$ & $\begin{array}{l}\text { Time for } \\
\text { record of } \\
4 \text { liters. }\end{array}$ & $\begin{array}{l}\text { Correction } \\
\text { factor. }\end{array}$ & $\begin{array}{l}\text { Time for } \\
\text { record of } \\
4 \text { liters. }\end{array}$ & $\begin{array}{c}\text { Correction } \\
\text { factor. }\end{array}$ \\
\hline $\begin{array}{cl}\min . & \text { sec. } \\
0 & 21.2\end{array}$ & $\begin{array}{l}p . c t . \\
106.5\end{array}$ & $\begin{array}{cl}\min . & \text { sec. } \\
0 & 36\end{array}$ & $\begin{array}{l}\text { p. ct. } \\
104.4\end{array}$ & $\begin{array}{cl}\min . & \text { sec. } \\
3 & 37\end{array}$ & $\begin{array}{l}\text { p.ct. } \\
102.8\end{array}$ \\
\hline $\begin{array}{ll}0 & 26\end{array}$ & 106.9 & $0 \quad 57$ & 103.6 & $\begin{array}{ll}6 & 31\end{array}$ & 103.2 \\
\hline $0 \quad 32$ & 105.8 & 112 & 103.6 & 800 & 101.9 \\
\hline $0 \quad 35$ & 105.0 & 26 & 102.5 & $9 \quad 30$ & 102.7 \\
\hline
\end{tabular}

these meters in use. Table 9 shows the correction factors obtained, assuming that the loss in weight of the oxygen cylinder was accurately measured and that there was no leak of oxygen during the experiments. From an examination of the results it would appear that the range in percentage accuracy is \pm 2 per cent, that the average deviation for the four series was from \pm 0.37 to \pm 0.75 per cent, and that the majority of the figures are within this variation. Three of the observations with meter No. 2 do not appear to have been made with sufficient care, i.e., the first one on December 27 (100.4 per cent) and the first two on December 28. On the latter date there was evidently a compensation error which brought the first value well above the average and the other considerably below. In general, however, the figures for meter No. 2 are reliable. Similarly, it is believed that the percentages for the other three meters are representative of the accuracy with which one can use the meter.

Mention has been made of the various types of valves and connections which have been used with the oxygen cylinders. As has been stated, the reduction valves supplied with the oxygen cylinders or which were purchased in Europe were at times so inefficient that the substitution of the meter proved of much advantage. Subsequently it was found that a needle-valve, sold by the Charles E. Beseler Co., of 
New York City, and the Lunkenheimer angle needle-valve were tight to the pressure obtained in oxygen cylinders when filled to 100 atmospheres or more. Threaded collars and fittings were obtained from the manufacturers of the cylinders and substituted for the fittings on the needle-valve; the needle-valves were then attached to the small

TABLE 9.-Correction factors of the Bohr meters, as shown by results obtained in actual use.

\begin{tabular}{|c|c|c|c|c|c|c|c|}
\hline \multicolumn{2}{|c|}{ Meter No. 2.} & \multicolumn{2}{|c|}{ Meter No. 5.} & \multicolumn{2}{|c|}{ Meter No. 7.} & \multicolumn{2}{|c|}{ Meter No. 8.} \\
\hline Date. & P. ct. & Date. & P. ct. & Date. & P. ct. & Date. & P. ct. \\
\hline \multirow{2}{*}{$\begin{array}{c}1912 \\
\text { December } 27\end{array}$} & 100.4 & $\begin{array}{c}1912 \\
\text { December } 27\end{array}$ & & 1912 & & 1912 & \\
\hline & $\begin{array}{r}95.4 \\
96.7\end{array}$ & December 28 & $\begin{array}{l}91.5 \\
98.2 \\
98.5\end{array}$ & December 27 & $\begin{array}{l}103.3 \\
102.8 \\
102.9\end{array}$ & December 27 & $\begin{array}{l}100.9 \\
100.7 \\
101.0\end{array}$ \\
\hline \multirow[t]{5}{*}{ December 28} & $\begin{array}{l}98.2 \\
93.4\end{array}$ & December 28 & 97.9 & & 103.6 & & 100.8 \\
\hline & $\begin{array}{l}95.4 \\
96.1\end{array}$ & & $\begin{array}{l}97.8 \\
98.8\end{array}$ & & $\begin{array}{l}103.4 \\
102.7\end{array}$ & & $\begin{array}{l}101.6 \\
100.5\end{array}$ \\
\hline & 95.9 & & 98.6 & & 103.0 & & 102.0 \\
\hline & 95.9 & & 99.7 & December 28 & 103.0 & December 28 & 101.4 \\
\hline & 96.5 & & 98.5 & & 103.2 & & 101.5 \\
\hline \multirow{9}{*}{ December 29} & 95.4 & & 98.8 & & 102.6 & & 101.1 \\
\hline & 96.2 & December 29 & 98.3 & & 103.4 & & 101.3 \\
\hline & 95.7 & & 98.4 & & 104.3 & & 102.4 \\
\hline & $\begin{array}{l}98.3 \\
97.2\end{array}$ & & $\begin{array}{l}98.2 \\
99.2\end{array}$ & . & 102.9 & & 101.1 \\
\hline & 96.3 & & 99.2 & & $\begin{array}{l}102.9 \\
103.9\end{array}$ & & 101.6 \\
\hline & 96.9 & & 98.9 & & 102.3 & & 101.9 \\
\hline & 95.8 & & 98.1 & & 103.2 & December 29 & 102.0 \\
\hline & 95.6 & & 98.6 & December 29 & 103.0 & & 100.9 \\
\hline & 95.9 & & 98.0 & & 102.6 & & 101.4 \\
\hline \multirow{5}{*}{ December 30} & 95.9 & December 30 & 97.6 & & 103.3 & December 30 & 101.2 \\
\hline & 96.0 & & 98.8 & December 30 & 102.5 & - & 101.2 \\
\hline & 96.2 & & 97.7 & & 103.4 & & 101.0 \\
\hline & 96.2 & & 98.0 & & 102.2 & & 101.7 \\
\hline & $\begin{array}{l}95.5 \\
96.7\end{array}$ & & $\begin{array}{l}98.3 \\
97.8\end{array}$ & & $\begin{array}{l}103.3 \\
102.5\end{array}$ & Average. & 1013 \\
\hline \multirow{3}{*}{ December 31} & 95.9 & December 31 & 98.2 & & 102.4 & Av. devia- & \\
\hline & $\begin{array}{l}96.3 \\
95.8\end{array}$ & & $\begin{array}{l}98.4 \\
98.2\end{array}$ & & 104.1 & tion..... & \pm .37 \\
\hline & & & 98.4 & & 102.6 & & \\
\hline \multirow{3}{*}{$\begin{array}{l}\text { Average... } \\
\text { Av. devia- } \\
\text { tion.... }\end{array}$} & 96.3 & & 98.1 & & & & \\
\hline & \pm .75 & & $\begin{array}{l}98.8 \\
98.0 \\
98.4 \\
97.9 \\
98.4\end{array}$ & $\begin{array}{c}\text { Average... } \\
\text { Av. devia- } \\
\text { tion..... }\end{array}$ & $\begin{array}{l}103.0 \\
+\quad .41\end{array}$ & & \\
\hline & & $\begin{array}{c}\text { Average... } \\
\text { Av. devia- } \\
\text { tion.... }\end{array}$ & $\begin{array}{l}98.3 \\
\pm .37\end{array}$ & & & & \\
\hline
\end{tabular}

oxygen cylinders. The cylinders, thus fitted, have been more or less used since that time.

It is somewhat difficult to state which method of measurement is preferable, as both the cylinder method and the meter method have their disadvantages. The use of the oxygen cylinder and valves 
requires an additional weighing; furthermore, if the valve is not absolutely tight, the whole apparatus for determining the oxygen is useless. The valves also vary in their closeness of fit; occasionally one is found which leaks slightly and again another will remain tight for a number of months. It is also sometimes difficult to obtain a collar which fits closely against the valve opening of the cylinder.

The meter method has an advantage in that the rate of admission can be noted and a leak detected while the experiment is in progress. Furthermore with the meter a large cylinder of oxygen, $i$. e., with a capacity of 100 cubic feet, may be used, this supply being sufficient for a period of several months without renewal. Among the disadvantages is the fact that occasionally the noting of the number of liters used is inadvertently omitted. The operator, in looking over the other factors of the experiment, may discover this omission, but the results may be of such a character that the addition of 1 liter may or may not correct the evident error. Several attempts have been made to avoid this error by providing an automatic recording attachment. This has been in most instances electrical. The pointer attached to the moving drum of the meter is provided with a short rod at right angles to it, so that when passing a contact at the top of the meter a circuit is closed. Several different kinds of contact have been inserted in the top of the meter, but none of them has as yet proved absolutely reliable and they can not be recommended. With an electrical recording device, the full amount of oxygen to be supplied must be admitted during the experimental period, as otherwise the record will not give the true value. Another method for preventing this error of omission has been instituted by Mr. H. L. Higgins, of the Laboratory staff. Instead of admitting the oxygen at such a rate as to equal the consumption of the gas by the subject, he allows the volume of the apparatus to diminish gradually for the first 3 or 4 minutes, and then admits quite rapidly 1 liter of oxygen. At the end of the seventh or eighth minute the process is repeated and again at the end of the tenth or twelfth minute. If this routine is adhered to, there is no danger of omitting the recording of a liter. The only disadvantage is that during the time of admitting the gas rapidly there is liable to be a distortion of the respiration record. Occasionally, through oversight, oxygen has been admitted to the meter when the exit pipe to the apparatus was closed. This caused such a pressure inside the meter that the glass face was blown out. Recently, at the suggestion of Mr. L. E. Emmes, of this laboratory, a device has been used which prevents such an accident. ${ }^{1}$ A third glass tube is inserted in the moistener with the lower end below the level of the water in the moistener and the upper end above the level of the water in the water-bath. When pressure accumulates, this acts as a safety valve and allows the release of the gas before sufficient pressure can be accumulated to cause damage. 
The choice of the two methods of admitting oxygen, $i$. e., from a weighed eylinder or through a meter, depends upon the facilities of the laboratory and the limits of its finances. If a weighed cylinder is used it is necessary to have at least two small cylinders which can be alternated or else one small and one large cylinder from which the small one can be refilled occasionally. The equipment necessary for the use of a meter comprises a good barometer, a 1-liter Bohr meter, a glass jar large enough to immerse the meter, a small oxygen cylinder for calibration purposes, and a large cylinder for general supply. Most experimental laboratories where respiration work is carried on are equipped with barometers, so that the additional equipment actually required would ordinarily be the Bohr meter and glass tank and a large supply of oxygen. After a meter is once installed and properly calibrated it should remain in good condition indefinitely, although occasional calibrations should be made. One meter has been in use in this laboratory for 6 months without calibration and when it was recalibrated by an operator who had had no experience with it, the results agreed to within 1 per cent of the correction factor which had been in use previously. It should be stated that in this case the meter was taken out of the bath and the water in it removed; the meter was then refilled, put back into the tank, and re-leveled before calibration.

The use of a meter involves more calculation in obtaining the results of experiments than the use of a weighed cylinder, but a cylinder requires the additional time of weighing which practically offsets the increase in calculations. Accordingly, so far as time is concerned, there is no advantage in either case. In general, it would appear from the experience in this laboratory with cylinder and meters that the use of the latter is preferable because there is less likelihood of the loss of the determination of oxygen with the use of the meter if the proper method of admission is used and ordinary precautions are taken.

\section{ZUNTZ-GEPPERT METHOD. ${ }^{1}$}

The successful use of the Zuntz-Geppert method in this investigation is largely due to the courtesy of Professor Zuntz. During a stay of several weeks in the Institute of Animal Physiology at Berlin, I had the privilege of acquiring the technique of this method under the immediate supervision of Professor Zuntz, and wish here to express my thanks for the assistance rendered me at that time and for the many helpful points obtained pertaining to the study of the respiratory exchange.

DESCRIPTION AND USE OF PARTS OF APPARATUS.

A detailed description of the mouthpiece and nose-clip, the valves, and the various parts of the sampling and gas-analysis apparatus is given in the following pages. The general principle employed in the Zuntz-Geppert method of determining the respiratory exchange is 
as follows: The subject of the experiment breathes through a mouthpiece attached to a tee connecting two glass valves which separate the inspired and expired air. The expired air is measured by means of a moist gas-meter. A sample of the air is taken over water by an automatic apparatus and is then analyzed in a special gas-analysis apparatus in which the carbon dioxide is absorbed by potassium hydroxide and the oxygen absorbed by phosphorus.

Mouthpiece.-The mouthpiece used, which is shown at $C$ in figures 18 and 19, is the original Denayrouse type. ${ }^{1}$ It is constructed of soft, pure-gum rubber and consists of an elliptical piece of rubber or flange, having an opening in the center, $2 \mathrm{~cm}$. in diameter, to which a rubber tube is attached. This flange is placed between the lips and gums.

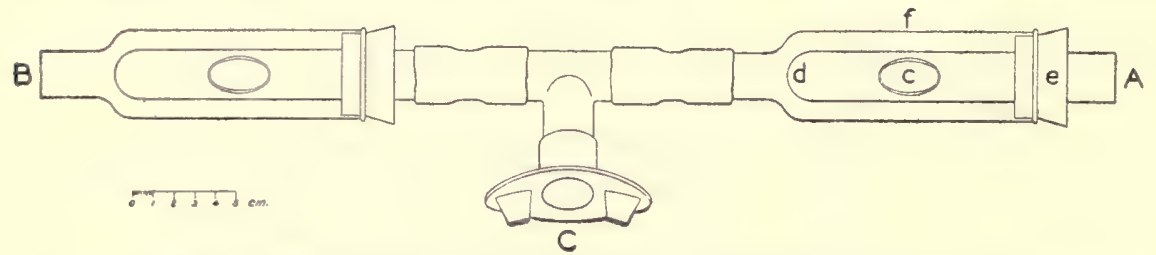

Fig. 18.-Mouthpiece and valves used in the Zuntz-Geppert apparatus.

Air enters at $A$, is drawn into the mouth through the mouthpiece $C$, and is exhaled at $B, c$, opening which is covered by a membrane; $d$, inside tube of valve; $e$, rubber stopper; $f$, outside cylinder of valve.

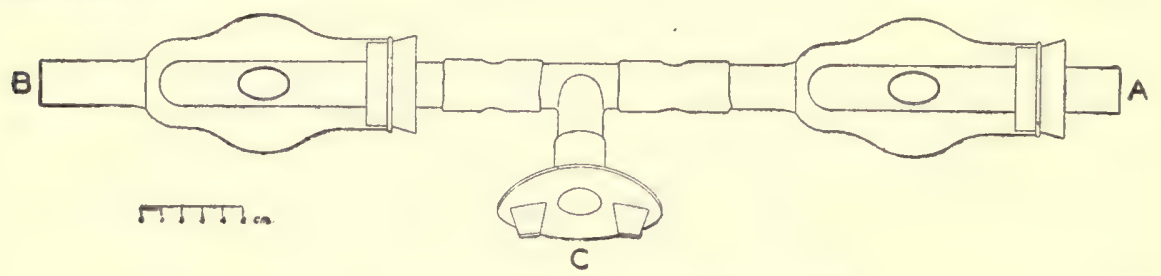

FIG. 19.-Most recent form of the Zuntz valves.

The enlargement in the outside cylinder permits a very free play of the membrane around the inside cylinder, and also serves to hold water for moistening the inspired air and the membrane; air enters at $A$ and leaves at $B ; C$, mouthpiece.

Two small flanges attached at right angles to the larger flange enable the subject to grasp it with the teeth and thus keep it in place. This type of mouthpiece is the most generally used when mouth-breathing is employed.

Nose-clip.-The nose-clip is also of the type most commonly used, $i$. e., a flat steel spring consisting of a band of metal about $15 \mathrm{~mm}$. wide, on the inside of which are flat pads which fit against the sides of the nose. The tight closure of the nostrils depends upon the proper placing of the nose-clip and upon the tension of the spring.

Valves.-The valves used are shown in figure 18. A glass tube, with an internal diameter of $22 \mathrm{~mm}$. and a length of $25 \mathrm{~cm}$., is rounded over

'P. Regnard, Recherches expérimentales sur les variations pathologiques des combustions respiratoires, Paris, 1879, p. 286. 
at one end, $d$, and closed. In the side of the tube, and about one-third of the length from the closed end, is an elliptical opening, $c$, which has a smooth edge. A thin membrane is tied around this tube in such a way that it fits loosely; a slit is made in the membrane on the side opposite to the opening, $c$. Zuntz and his co-workers have most commonly used calves' intestine for this purpose, but Durig ${ }^{1}$ has substituted fish membrane. We have also employed a very thin tambour rubber. The glass tube is inserted in a rubber stopper, $e$, which fits into the end of a cylinder, $f, 45 \mathrm{~mm}$. in diameter and $19 \mathrm{~cm}$. in length. The other end of the cylinder is constricted to about the same size as the smaller tube. When air is pushed in at $e$ or drawn through the opposite end it distends the membrane, which opens and allows the air to pass through at $c$. When the pressure in $f$ is slight, the membrane closes and fits against the smaller tube, $d$. In tying on the membrane there should be a play of several millimeters between the tube and the membrane. One of these valves is attached by rubber tubing to each end of the glass tee, connecting with the rubber mouthpiece. The whole arrangement, with the exception of the membrane covering, is shown in figure 18, the air entering at $A$ and leaving at $B$.

Another and more recent form of valve is shown in figure 19. Instead of the outside cylinder being of uniform diameter, an enlargement has been made so that the membrane, when distended, will not adhere to the outer tube. Water can also be placed in the enlarged portion, which assists in moistening the ingoing air and, of still more importance, moistens the membrane in the ingoing air-tube.

Elster meter.-The gas-meter used for measuring the expired air is shown in figure $20 .^{2}$ It has four dials, three of which give 10, 100, and 1,000 liters, while the fourth, which is the largest one, gives liters and parts of a liter to 0.02 liter. The meter is filled with water to a certain level, which is determined by opening the cap at $A$. When water flows out through this opening, the meter is sufficiently full for measuring purposes. As different levels require different correction factors and a difference in level is produced by the evaporation of the water, we have attached a side tube with a millimeter seale, $W$, in such a way as to show the actual level of the water at any time. This water-gage has proved of distinct advantage in working with the Elster meter. The need of some indication of the level of the water in the meter is very clearly shown in the calibration tests made by Cathcart with different levels in the Bohr meter. (See table 7, page 49.) For obtaining the temperature of the meter or of the air passing through it, thermometers may be inserted as shown in figure 20 at $V$ and $V$.

Meter thermo-barometer.-In order to obtain the amount of gas passing through the meter at $0^{\circ} \mathrm{C}$. and $760 \mathrm{~mm}$. mercury pressure, 


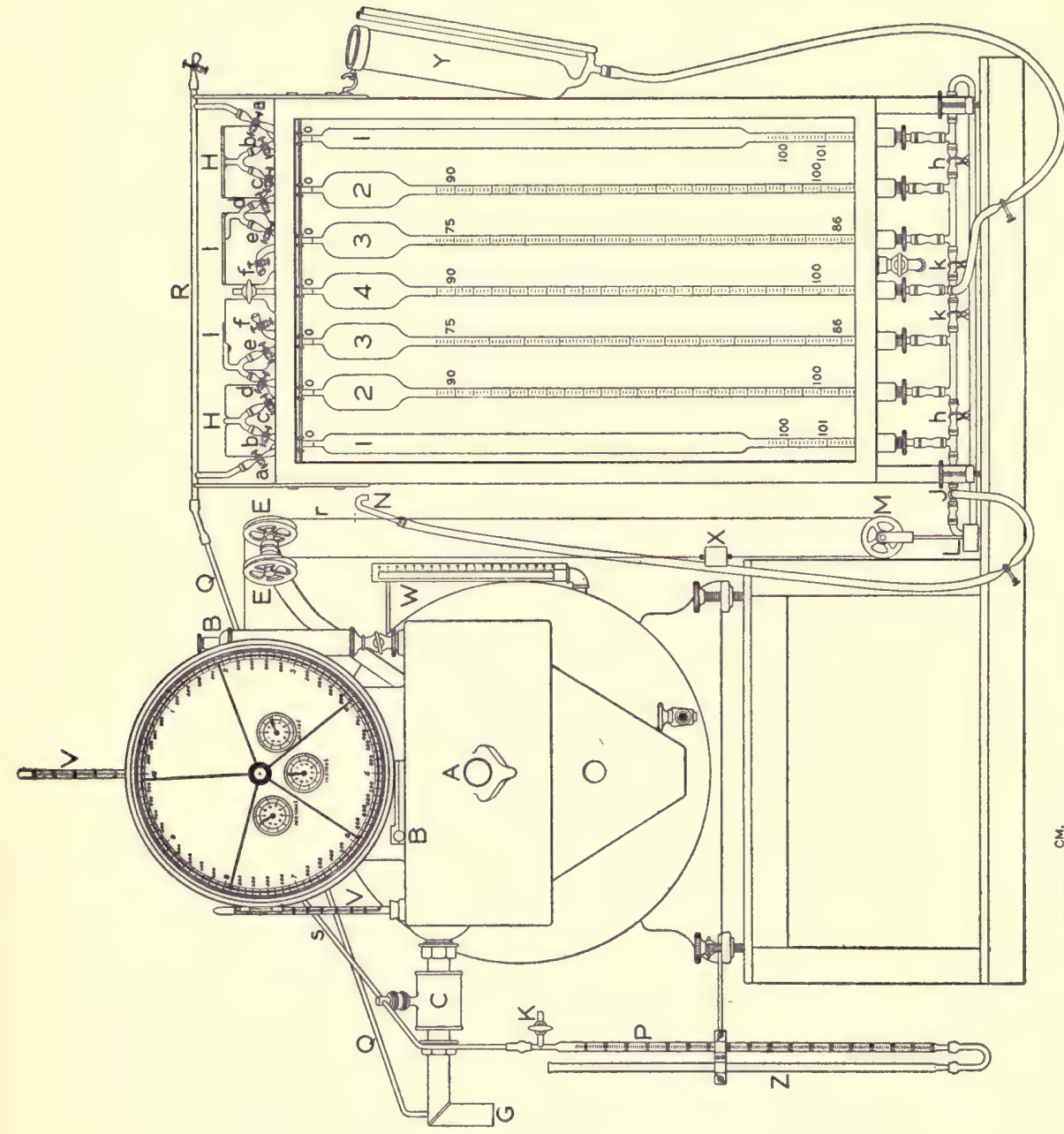

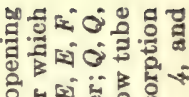
\% 于 का

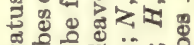

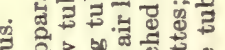

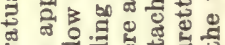

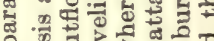
每

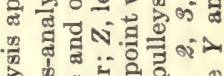

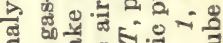
If F. 界

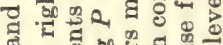
에 o

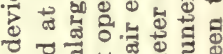

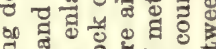
句

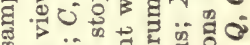

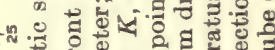

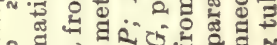
赵 狚击

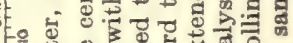
委 落

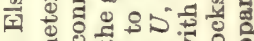
o

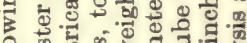

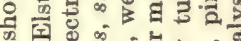
5 0 \%

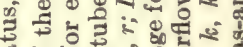

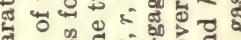

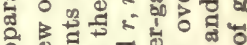

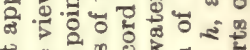

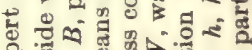

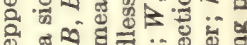
o. 0 . 5 o

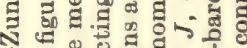

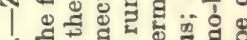

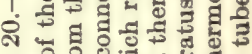

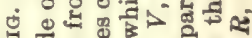

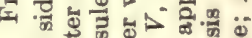
물

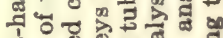

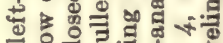
呐ㄹ 要

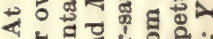

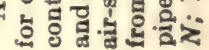


Zuntz has devised an automatic method for indicating a volume of 100 c.c. of air at the conditions under which the air passes through the meter. A thin-walled metal capsule containing a few drops of water is placed inside the air-tube $G$ entering the meter and another in the tube $T$ leading from the meter. The location of these capsules is shown at $C$ and $D$ in figure 20 . The two capsules are connected by a small metal tube $s, s$, which in turn is connected with the graduated glass tube, $P$, shown at the side of the meter. This graduated tube is partly filled with water and actuated by a leveling tube, $Z$. The method of use is as follows: The volume, 100 c.c. at $0^{\circ} \mathrm{C}$. and $760 \mathrm{~mm}$. pressure, is calculated to the volume at the average temperature of the meter and the barometric pressure, the latter being corrected for the tension of the aqueous vapor in the meter at the time of use. A stopcock, $K$, at the side of the graduated glass tube, $P$, is opened to the air and air is drawn into the graduated glass tube by means of the leveling tube, $Z$, to the point corresponding to the volume calculated. The glass stopcock, $K$, is then closed. The reading of the graduated tube gives the volume of 100 c.c. at the observed temperature and pressure.

Automatic sampling device.-Another arrangement connected with the meter provides for taking automatically a small sample from the air as it enters. To the central axis of the meter, which is extended at the back, are fastened 4 or 5 concentric pulleys of different sizes (see $U$ ). Around one of these pulleys passes an endless cord, $r, r, r$, which is carried over pulleys at the top of the meter and then forward to pulleys on the front of the meter. These are shown in figure 20 at $E, E$, and $F$. This endless cord then extends downward to a loose pulley, $M$, somewhat below the level of the meter. The cord is kept taut by the weight $L$. Upon the right-hand side of the cord as it is carried over the two pulleys $E$ and $E$, is attached a glass overflow tube, $N$, with an open end, which is connected by a rubber tube to the bottom of the analytical apparatus at $J$. The weight of the overflow tube, $N$, and of the rubber connections is counterpoised by means of the weight $X$. Theoretically the weight of the exit tube and connections should be greater than the weight used to counterbalance it, so that no pressure will be produced in the meter and thus hinder respiration.

The routine of sampling is asfollows: Before an experiment is begun, the measuring burettes, 1 and 1 , on the gas-analysis apparatus are filled with acidulated water. The overflow tube $N$ is then lifted to a height somewhat above the zero-mark on the burettes. As all of the connections are open, each movement of the meter lowers automatically the tube $N$ so that the water-levels in the sampling burettes, 1 and 1 , are at the same time gradually and automatically lowered. The rapidity with which this is done can be regulated by placing the cord on different pulleys at the back of the meter. The air is thus drawn through the sampling tube, $Q, Q$, which extends from the large ingoing air-pipe $G$ 
over the top of the meter to the capillary tube $R$ connected with burettes, 1 and 1 , of the gas-analysis apparatus.

Gas-analysis apparatus.-The general principle of the gas-analysis apparatus is as follows: The gases to be analyzed are automatically collected over acidulated water in two burettes of similar construction in the manner just described. After being measured by leveling at atmospheric pressure, the air is then passed into a 30 per cent solution of caustic-potash in pipettes of special construction containing glass tubes. One of the caustic-potash pipettes is shown in figure 21 . Another form of pipette is shown in figure 22. After absorption of the carbon dioxide has taken place, the residual gases are drawn back into two other burettes, where they are again measured at atmospheric

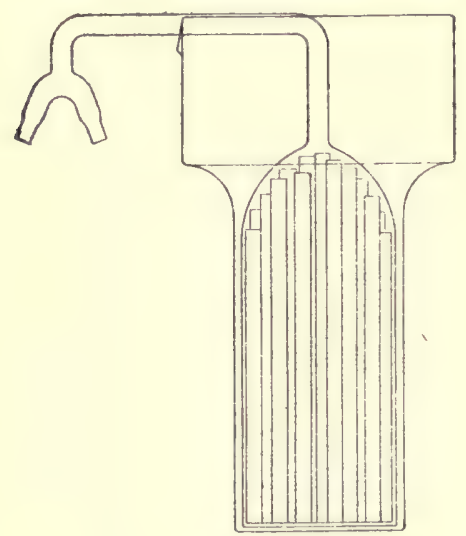

FIG. 21.

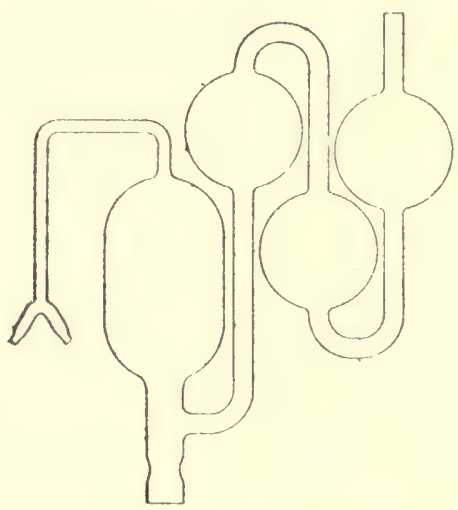

FIG. 22.

FIG. 21.-Caustic potash pipette used in the Zuntz-Geppert analysis apparatus.

The inside cylinder is filled with glass tubes which give a large surface for absorption of carbon dioxide. The pipette for the absorption of oxygen is of similar construction, but the glass tubes are replaced by stick yellow phosphorus.

FIG. 22.-Absorption pipette used in the Zuntz-Geppert analysis apparatus.

It may contain either caustic potash solution for absorption of carbon dioxide or sodium hydrosulphite for the absorption of oxygen.

pressure and the temperature of the bath. They are then driven into pipettes containing phosphorus, where the oxygen is absorbed; finally, the remaining gas, or nitrogen plus argon, is measured.

The general construction of the gas-analysis apparatus may be seen in figure 20. A glass tank filled with water contains 7 burettes. The two outside burettes, 1 and 1 , are designed to measure the collected gas and are therefore graduated in 0.02 c.c. only from -100 to +101 c.c. They are connected at the top by the $Y$ capillary connections, $a, a$, to the capillary tube $R$ above the apparatus for drawing in the sample, and by the connections, $b, b$, to the caustic-potash pipettes, $H$ and $H$. When the sample is drawn from the atmosphere or from the air going through the meter, the clamps at $a$ and $a$ are open, while the clamps at 
$b$ and $b$ are closed, thus furnishing connection between the overflow tube $N$ and the burettes. Next to the two sample-measuring burettes, 1 and 1 , are two more burettes, 2 and 2 , which are graduated from 90 to 100 c.c. and are used for measuring the gas after the carbon dioxide has been absorbed. These are connected by the $Y$ connections, $c$ and $c$, and $d$ and $d$, to the caustic-potash pipettes, $H$ and $H$, and to the phosphorus pipettes, $I$ and $I$, respectively. On the inside of these burettes are two additional burettes, 3 and 3 , graduated from 75 to 86 c.c., in which the gas is measured after the oxygen has been absorbed in the phosphorus pipettes, $I$ and $I . \quad Y$ connections at the top ( $e$ and $e$, and $f$ and $f$ ) lead to the phosphorus pipettes, $I$ and $I$, and to the open air, respectively. The connections between the pipettes and burettes are made by means of capillary rubber tubing, and closure is made of this rubber tubing by means of spring clamps, as shown in figure 20 . In the center of the seven burettes is the special burette, 4 , known as the "analysis thermo-barometer." Corrections for changes in barometric pressure and the temperature of the water-bath are made by means of the readings taken upon this burette. The burette 4 at the beginning of the experiment is filled with a definite amount of water; the stopcock is then closed at the top and the reading taken by means of the leveling bulb $Y$, which is at the right of the figure. When a reading is made, the water-levels in the arms of the leveling bulb and that in the burette are brought to the same horizontal plane.

Routine of gas analysis. - The analysis of the air is carried out as follows: After the sample has been drawn into burettes, 1 and 1, the pinchcock on the tube $J N$ is closed, and the pinchcocks $k, k$ and $h, h$ are opened; after a few minutes a reading is taken, using the leveling bulb, $Y$, at the right. A simultaneous reading is taken of burette $4-$ the so-called "analysis thermo-barometer." Several readings are taken at intervals of a minute or so until the changes in all three burettes are alike or give constant readings. The air in these two burettes is then driven over into the pipettes $H$ and $H$ by opening the pincheocks $b$ and $b$. When all of the gas has been driven into the pipettes, the pinchcocks are closed, and the gas is allowed to remain for at least 10 minutes to insure complete absorption of the carbon dioxide. The leveling bulb $Y$ is then lowered and hung on a hook at the right-hand side of the tank, the pinchcocks $c$ and $c$ being opened so that the gas will descend slowly into burettes 2 and 2 . The gas should be drawn into these burettes very slowly in order that they may drain properly. After the gas has been drawn in, the solutions in the two caustic-potash pipettes $H$ and $H$ are drawn to the same point that they were before the analysis was started. The pinchcocks $c$ and $c$ are then closed and readings are taken of burettes 2 and 2 , and of the analysis thermobarometer, 4, until they become constant. The gas is then driven into pipettes $I$ and $I$, which contain stick yellow phosphorus. Here the 
absorption of oxygen which requires about 10 minutes, takes place. The pinchcocks, $e$ and $e$, are now opened and the gas is drawn into burettes 3 and 3 by the routine carried out after the carbon dioxide had been absorbed. When the gas has all been drawn into burettes 3 and 3 , the water in the phosphorus pipettes, $I$ and $I$, is drawn to a definite point in the capillary tube and closure is made by shutting the pinchcocks $e$ and $e$. A reading is then taken of the gas in the burettes 3 and 3 and of the analysis thermo-barometer 4. The gas is finally expelled into the open air by opening the pinchcocks $f$ and $f$. The water-level in the burettes 3 and 3 is finally set at zero, and the apparatus is ready for another analysis.

\section{GENERAL ROUTINE OF AN EXPERIMENT.}

The general method of carrying out a respiration experiment with the Zuntz-Geppert apparatus is as follows: In rest experiments the subject usually lies on his back upon a couch for about half an hour before the experimental period begins. The valves are placed in a convenient position for the subject and so that he does not support them. The outgoing valve is connected to the moist gas-meter by a piece of rubber tubing 20 to $25 \mathrm{~mm}$. in diameter and of suitable length, usually from 1 to 2 meters. When the period for the experiment is determined, the subject inserts the mouthpiece, puts on the noseclip, and begins breathing through the valves. Usually outdoor air is supplied. The operator then takes readings of the Elster meter every minute. When these become constant, the actual experimental period is begun. The overflow tube, $N$, from the burettes in the gasanalysis apparatus is raised to such a height that when the pincheocks $a$ and $a$ are opened air will be drawn into burettes 1 and 1 . The time is noted and a reading of the Elster meter is taken at exactly the beginning of the period. A reading of the meter thermo-barometer is also taken. Pinchcocks $a$ and $a$ are then opened and the air drawn into the burettes 1 and 1 . Readings are made of the Elster meter every minute throughout the experimental period, which is usually of 15 to 20 minutes' duration. The time required for emptying the burettes must be so regulated that it will coincide with the duration of the period. This is done by the proper adjustment of the endless cord, $r, r, r$, upon the concentric pulleys, $U$, at the back of the meter. When burettes 1 and 1 are full of air, the pinchcocks are closed, the time is noted, and the readings are taken of the meter and the meter thermo-barometer. The experimental period is then ended.

Several experiments may be made in succession by drawing air into the sampling burettes as soon as the first two samples have been sent over into the potash pipette. A short interval should be allowed for the gases in the burette to reach constant temperature or constant readings. A new experiment may then be begun. 
The Zuntz-Geppert method has been the leading method for a number of years for determining the gaseous metabolism in short periods of both man and animals. The method has been and is now in use in a large number of clinics and laboratories, and we are indebted to it for a great advance in the modern knowledge of the respiratory exchange under normal and pathological conditions.

TISSOT METHOD.

The Tissot method of determining the respiratory exchange has found greatest use in the French laboratories. In Chauveau's laboratory a large amount of work on the mechanics of respiration as well as on the gaseous metabolism of man has been carried out with this method. More recently it has been quite extensively applied by Amar ${ }^{1}$ in the study of muscular work of various kinds.

During a European trip in 1908, I studied the technique of this method in Chauveau's laboratory in Paris, and am indebted to Professor Chauveau and Dr. Tissot for the privileges accorded me at that time and to Mr. Jules Mansion for much personal assistance.

The method as described by Tissot $^{2}$ is essentially the following: The subject breathes through glass nosepieces of special design attached to a pair of valves which separate the inspired and expired air. The expired air is conducted by means of rubber tubing into an automatically counterpoised spirometer. The gas collected in the spirometer is sampled after the experiment is finished and analyzed by means of a gas-analysis apparatus. ${ }^{3}$

DESCRIPTION AND USE OF PARTS OF APPARATUS.

A description of the nosepieces, valves, and method of collecting the expired air is given here in detail. ${ }^{4}$

Nosepieces.-The nosepieces are made of glass tubing in one end of which a bulb is blown. These are shown in figure 23 ( $A$ and $A$ ) connected to the tee-piece $B$ by rubber tubing of suitable size, this tubing being of varying length to permit flexibility in use. Different sizes of glass tubing and bulbs may be used to adjust the nosepieces to the nostrils of the various subjects. They are inserted as deeply into the nose as is comfortable for the subject and are tested by putting the fingers over the open ends and attempting to exhale.

Modified glass nosepieces.-During this investigation an attempt has been made to modify the glass nosepieces so that they would fit more closely into the nostrils and be more comfortable. These modified nosepieces are shown in figure 24. They are made of ordinary glass tubing with a flat bulb blown at one end. The nosepiece is bent so

${ }^{1}$ Amar, Journ. de physiol, et de pathol. gén., 1913, 15, p. 62.

'Tissot, Journ. de physiol. et de pathol. gén., 1904, 6, p. 688.

${ }^{3}$ Tissot, Traite de Physique Biologie, Paris, 1901, 1, p. 717.

${ }^{4}$ For description of apparatus for alcohol check tests, see p. 80 . 
that when placed in the nostril the other end can be easily attached to the connecting piece $B$ (fig. 23) without stress being put upon the nostril. The view at $A$ (fig. 24) shows it as it appears from above when placed in the nostril and at $B$ from the side.

Valves.-The valves used in the Tissot method are the Thiry valves. ${ }^{1}$ Two of these are shown in figure $23(C$ and $C)$. A very thin brass flap, $D$, hinged on one edge, rests against a brass tube, $E, 15 \mathrm{~mm}$. in diameter. The edge of the tube is tapered where the flap $D$ rests against it, so that there is a minimum amount of surface in contact between $D$ and $E$. The brass tube $E$ is inserted in a collar $F$, which screws into the ring $G$. This ring encircles a glass tube, $H, 23 \mathrm{~mm}$. in diameter and $30 \mathrm{~mm}$. in length. A collar, $K$, with attached brass tube, $J$, fits over the end of the glass tube $H$. The glass tube is cemented into the parts $G$ and $K$ by sealing-wax. The tee-piece $B$ joins the two valves and the nosepieces. When the valve is in action, the air enters

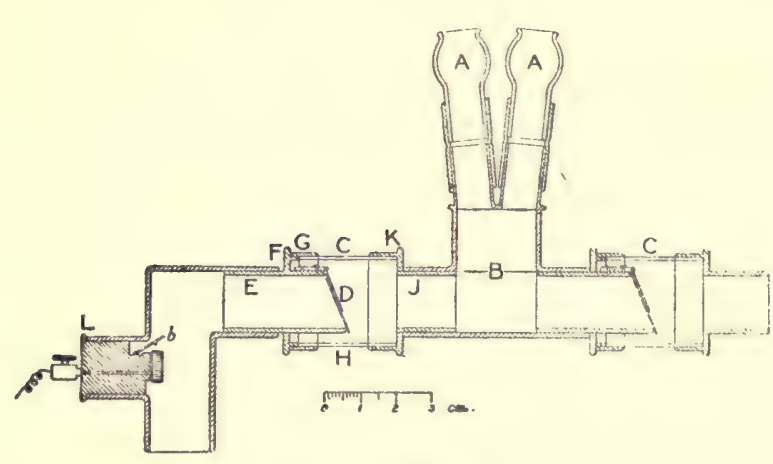

FIG. 23.

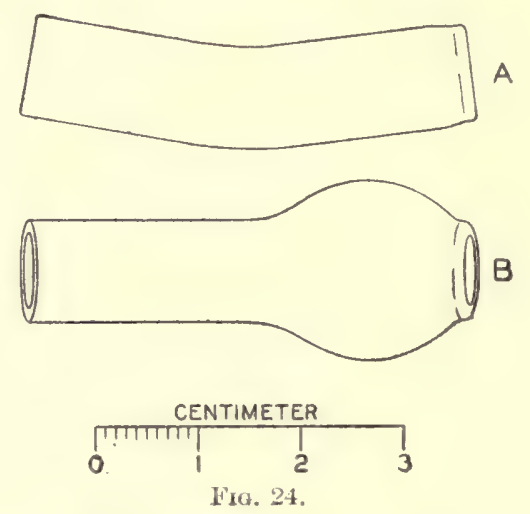

FIG. 23.-Nosepieces and valves used with the Tissot method.

$A, A$, nosepieces; $B$, tee piece connecting two valves $C, C ; D$, flap of valve; $E$, inlet of valve; $J$, outlet of valve; $H$, glass tube to which are sealed brass shoulder, $K$, and $\operatorname{ring}, G ; F$, threaded part fitting into $G ; L$, part of apparatus for registering respirations; $b$, thin copper flap to which are attached two electrical contacts.

FIG. 24.-Modified glass nosepieces.

$A$, view from above when placed in the nostril; $B$, view from side when placed in the nostril.

at $E$, raising the flap $D$, and leaves at $J$. The valves and nosepieces are supported upon the head of the subject by means of straps or strings connecting the valves with a small round cap which fits over the head. With this arrangement the nosepieces can be forced into the nose and it is possible for the subject to maintain any position.

Apparatus for registration of respiration-rate.-The number of respirations in a particular experiment can be obtained by attaching to the valves a fitting which contains a mercury contact of special design. This is shown at $L$ in figure 23. A perspective view is given in figure 25 . 
The very thin metal flap rises when the air is drawn in; when the air is blown out this metal flap drops back in place, making a contact in the two mercury cups $a$ and $a^{\prime}$. If wires are led from these mercury cups to a signal magnet and battery, the respiration can be recorded on a kymograph.

Spirometer.- The spirometers used with the Tissot method are also of special design, very well made, and the parts are easily adjusted. Figures 26 and 27 show the 50-liter and 200-liter types respectively. The bell of the spirometer, which is made of very thin copper, is cylindrical in form, with a conical top, and is suspended in a water-bath between the double walls of a hollow cylinder. The height of the 50-liter bell is $60 \mathrm{~cm}$. and the diameter $33 \mathrm{~cm}$., while the height of the $200-$ liter bell is $73 \mathrm{~cm}$. and the diameter $65 \mathrm{~cm}$. An opening at $Z$ permits the insertion of a rubber stopper with a thermometer and tube for sampling. This rubber stopper may be removed when the spirometer is emptied after an experiment. The air coming from the subject or from any other

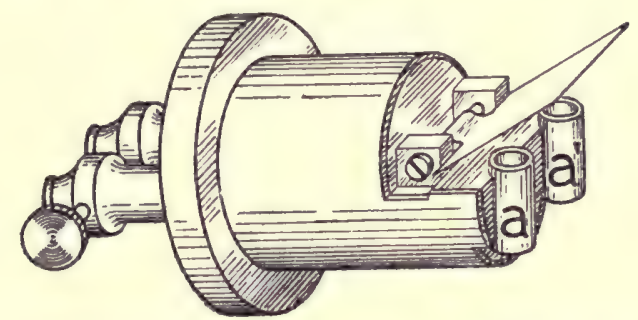

FIG. 25.-Apparatus for registering the respiration-rate used with the Tissot method.

The flap has attached to it two platinum points which dip into the mercury-containing cups $a, a^{\prime}$; the flap rises and falls at each respiration.

source enters the spirometer at the bottom through a three-way cock, $A$. This three-way cock may also be so turned that the air passes out into the room. The major portion of the weight of the spirometer bell is counterpoised by the weight $R$. The automatic adjustment of the counterpoise is, however, accomplished in the following manner: A glass cylinder, $C$, is made of such size that when filled to the level of the water in the spirometer, the weight of water in the cylinder exactly equals the increase in weight of the spirometer bell, due to its new position. When the bell rises or falls, water is added to or taken from the cylinder $C$ by means of the siphon tube $D$. Any increase or decrease in the weight of the bell due to the varying displacements of the volume of water by the mass of metal in the spirometer bell is thus exactly counterpoised by a like increase or decrease in the weight of the water in the cylinder. The bell and the cylinder $C$ are supported by means of a thin steel band, $E$, which is carried over the aluminum wheel $F$ (fig. 26) or aluminum wheels $F$ and $G$ (fig. 27), the band fitting into flat grooves in the wheels. The bearings of the 


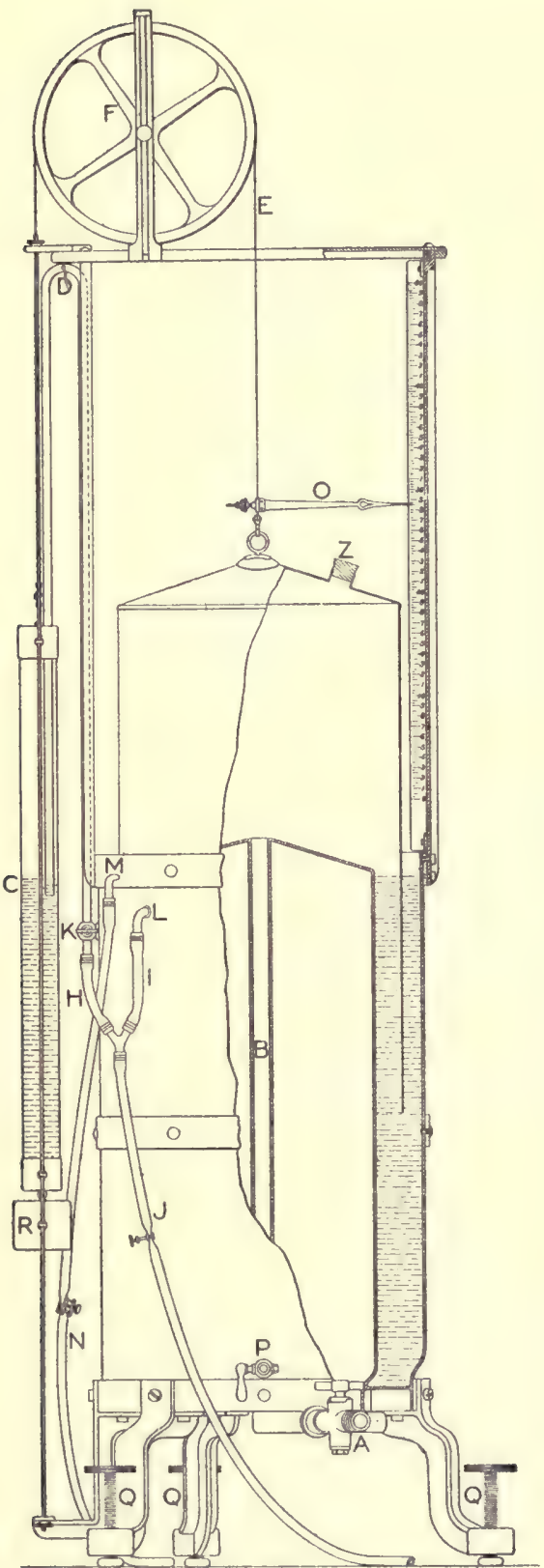

FIG. 26.

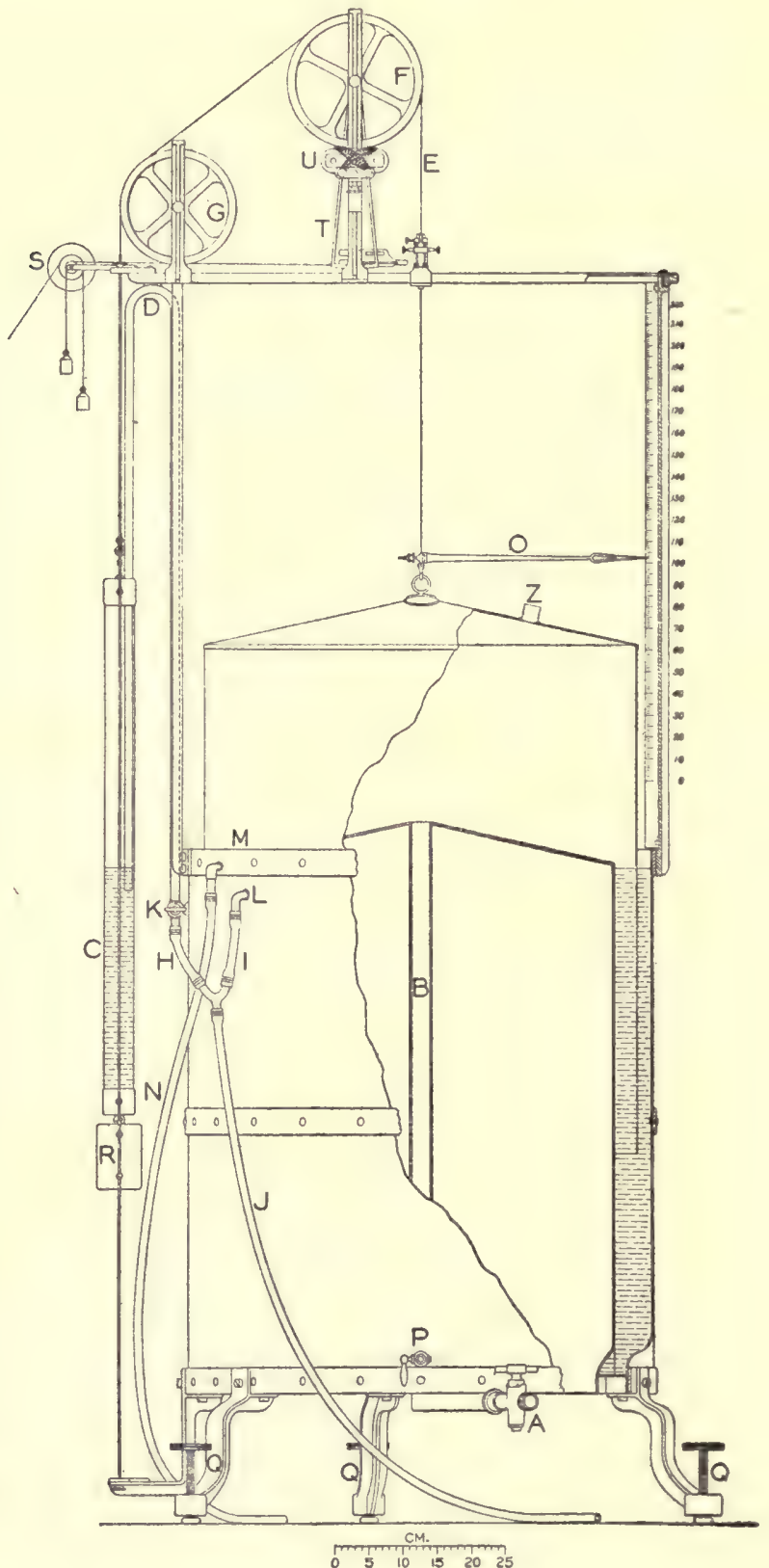

FIG. 27.

F1G. 26.-Tissot spirometer with capacity of 50 liters.

$A$, three-way valve connecting air in bell of spirometer with outside air; $B$, tube leading to inside of bell; $C$, counterpoise tube compensating for changes in weight of bell; $D$, siphon tube connecting $C$ with water in tank; $E$, flat steel band supporting spirometer; $F$, wheel over which runs $E ; H$, rubber tube connecting siphon tube with supply tube $J ; I$, branch of supply-water tube leading to tank at $L ; M, N$, overflow tube from tank; $O$, pointer; $P$, cock for emptying tank; $Q, Q$, leveling screws; $\boldsymbol{R}$, lead counterpoise; $\boldsymbol{Z}$, opening for gas sampling.

FrG. 27.-Tissot spirometer with capacity of 200 liters.

All letters appearing in figure 26 are on this drawing and refer to the same parts. $G$, additional aluminum wheel; $S$, multiplying pulley; $T$, movable arc for writing respiration volume; $U$, electromagnet. 
aluminum wheels are steel points, fitting into sockets. The upright position of the counterpoise cylinder $C$ is determined and maintained by means of two brass rods on which the cylinder travels. These are firmly fastened when the cylinder is placed in position, and, when properly adjusted, permit the rise and fall of the cylinder with a minimum amount of friction. The siphon tube $D$ is also so arranged that it does not touch the cylinder $C$ at any point. To send water into the cylinder $C$, the three-way cock $K$ is so turned that water flows through the rubber tubes $J$ and $H$ (the connection with the rubber tube $I$ being closed) and then through the siphon tube $D$ into the cylinder. When the cylinder is filled to the same level as that in the tank, the threeway cock $K$ is so turned that connection is made between the tank of the spirometer and the siphon. The level of the water in the tank of the spirometer is maintained by a constant flow of water through the tube $J I$, and into the opening $L$; the overflow passes out of the tank through the opening $M$ and the rubber tube $N$. A scale is shown at the right-hand side of the apparatus which, in the 50-liter spirometer, is divided into 0.25 liter, while in the 200-liter spirometer it is divided into 0.5 liter. The aluminum pointer $O$ fastened upon the metal band above the spirometer indicates the position of

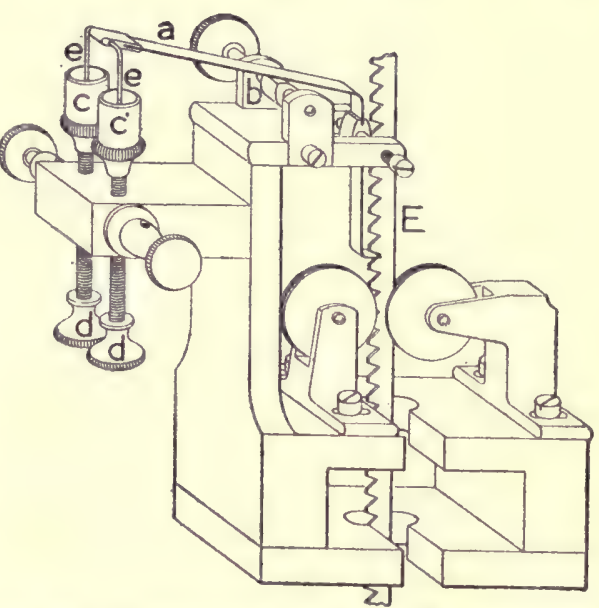

Fro. 28.-Apparatus for registering the volume of air in the Tissot spirometer.

$E$, portion of band supporting the bell of the spirometer; $a$, lever actuated by the saw-teeth on the band $E$ as the bell rises; $e, e$, points dipping into the mercury cups $c, c^{\prime}$, as each tooth of $E$ moves upward past $a ; d, d$, adjustment screws; $b$, eccentric for raising $a$ when latter is not in use. the bell. The 50-liter spirometer may be read to 0.05 liter, and the 200-liter apparatus to 0.1 liter. The movements of the bell of the spirometer when properly adjusted can be made sensitive to $0.1 \mathrm{~mm}$. water pressure. The cock $P$ at the bottom of the tank of the spirometer provides for emptying the tank when desired. The level of the whole apparatus can be adjusted by means of the leveling screws $Q, Q, Q$.

A pparatus for registering the volume of air in the spirometer.-A special attachment upon the bar supporting the aluminum wheels permits the automatic registration of each liter of gas as the spirometer is filled. On the metal band, $E$, between 0 and 50 or 0 and 200, are saw-teeth which are so cut that when the band moves upward it operates a thin metal lever which rises and falls with the movement of the metal band. This special attachment is shown in figure 28. A section of the metal 
band $E$ is shown, and two guiding pulleys which can be adjusted so as to keep the band in place with a minimum amount of friction. As the metal band rises, it pushes the lever $a$ outward, causing the ends $e$ and $e$ to rise out of the two mercury cups $c$ and $c^{\prime}$. The lever $a$ then drops back into the indentation between two teeth, and the two points $e$ and $e$ again dip in the mercury cups $c$ and $c^{\prime}$. Each time the points dip into the mercury cups, a contact is made which closes an electric circuit connected with a signal magnet, and thus each liter can be recorded as the spirometer is being filled. The mercury cups $c$ and $c^{\prime}$ can be adjusted by means of the screws $d$ and $d$. When not in use the lever $a$ may be raised out of the mercury cups by means of the eccentric $b$.

Device for recording the volume of inspiration or expiration.-An adjustment was designed by Tissot in connection with this spirometer, so that either the volume of inspiration or the volume of expiration may be recorded. The arrangement is shown on a small scale in figure 27 at $U, T$. A segment of a wheel, $T$, is suspended loosely on the shaft of the wheel $F$. A row of metal teeth is fastened at a point on the segment $T$ opposite the rim of the wheel $F$, and a rubber ring is cemented in a groove on this wheel opposite to the teeth. An electro-magnet, $U$, is fastened to the upright supporting the wheel $F$, the armature of the magnet being attached to the arc $T$. A thread runs from the are $T$ to the multiplying pulley $S$. The electro-magnet $U$ is connected in a circuit with the two mercury cups $a$ and $a^{\prime}$ in the apparatus shown in figure 25 .

The operation of the system when recording the volume of expiration is as follows: The apparatus shown in figure 27 is attached to the outgoing valve. When the subject inspires, the flap shown in figs. 23 and 25 rests against the cups $a, a^{\prime}$ (fig. 25) and the circuit thus closed actuates the electro-magnet $U$ (fig. 27). The arc $T$ is held motionless. During expiration the flap is raised and the circuit broken. The are $T$ moves in the same direction as the wheel $F$, as it $(T)$ is held against the wheel because of the friction of the metal teeth against the rubber ring on the wheel $F$. The motion of the arc $T$ is communicated to the pulley $S$ by a thread. At the end of an expiration, $T$ drops back to its original position, owing to the action of the electro-magnet $U$, its circuit being closed. If a moving pointer writing on a kymograph is connected to $S$, the movements of $T$ may be recorded.

\section{GENERAL ROUTINE OF AN EXPERIMENT.}

In making an experiment by this method, the valves are first tested for tightness. This may be done by inserting the nosepieces with the valves attached into the nose and putting pressure against the ends of the valves. Rubber tubing of about $20 \mathrm{~mm}$. internal diameter connects the valves with the spirometer. The valves and nosepieces may be supported by means of a special cap and strings or by means of 
the clamp upon a burette standard. The latter has been of common use in this laboratory, as all of the experiments made with this apparatus have been with the subject lying upon a couch. With the bell of the spirometer at zero, a reading is taken of the pointer, and the threeway valve $A$ is turned so that the expired air enters the spirometer bell. The subject then breathes for a definite length of time, during which period the air is collected in the spirometer. The valve is again turned at the end of the experiment, a reading of the position of the spirometer bell is made, records taken of the temperature and the barometric pressure, and finally a sample of air is drawn from the spirometer and analyzed.

For the air analyses Tissot has used a special gas-analysis apparatus, ${ }^{1}$ with a burette of about 100 c.c. capacity, in which he absorbs the carbon dioxide over potash and the oxygen over phosphorus, or determines the oxygen by explosion with hydrogen. Personal experience with this apparatus has shown that it is very complicated and difficult to operate, and that it possesses no distinct advantage over the other forms of gas-analysis apparatus used in this research. In connection with the work on the Tissot method in this laboratory the accompanying gas analyses were almost exclusively made with the Haldane gas-analysis apparatus subsequently described in this publication.

\section{DOUGLAS METHOD.}

The Douglas ${ }^{2}$ method of determining the respiratory exchange is of more recent origin than the other methods used in this investigation, but it promises to be widely utilized because of its simplicity and the portability of the apparatus required to make determinations of the gaseous metabolism. In the researches of the Nutrition Laboratory it has been employed by Mr. H. L. Higgins on a trip in the Alps. ${ }^{3}$ During my visit to Oxford, Dr. Douglas demonstrated to me the technique of the method and subsequently gave me further information regarding the details of the apparatus by correspondence and during a visit to the Nutrition Laboratory. For these courtesies I wish to express my thanks.

The Douglas method may be briefly described as follows: The subject breathes through a mouthpiece by means of valves into a rubber tube having an inside diameter of at least $20 \mathrm{~mm}$. At a suitable distance from the expiratory valve, a three-way valve of large bore is attached which is connected with a wedge-shaped reservoir bag made of rubber-lined cloth. The expired air collected in this bag is measured at the end of the experiment by passing it through a meter and a

\footnotetext{
${ }^{1}$ Tissot, Traité de Physique Biologique, Paris, 1901, 1, p. 717.

${ }^{2}$ Douglas, Journ. Physiol., 1911, 42; Proc. Physiol. Soc., p. xvii. Douglas, Haldane, Henderson, and Schneider, Phil. Trans., 1913, 203, p. 217.

${ }^{3}$ Galeotti, Barkan, Giuliani, Higgins, Signorelli, Viale, Gli effetti dell'alcool sulla fatica in montagna. Reale Accademia dei Lincei, Rome, 1914, and Arch. d. Fisiol., 1914, 12, p. 277.
} 
sample is analyzed. By supporting the tube and valves on a light framework placed upon the head and resting the bag upon a second frame on the back, the respiration apparatus may be carried quite easily a considerable distance. The accessory apparatus required for this method of determining the respiratory exchange are a meter for measuring the gas collected in the bag, samplers for collecting the samples of air, and a gas-analysis apparatus.

In experimenting the bag is placed in a suitable position and a support arranged for the valves and tubing. The subject then inserts the mouthpiece and commences respiration, with the three-way valve so turned that the air expired passes out into the surrounding atmosphere. After equilibrium of respiration has been established, the threeway valve is turned so that the expired air will enter the bag. The experiment is then continued the determined length of time, this being limited by the size of the bag used and the kind of experiment. After the experiment is ended, the gas in the bag, when thoroughly mixed, is forced through a meter, the barometric pressure and the temperature of the meter being recorded. A sample of the gas is also taken for analysis. The bag should be emptied completely, which can be done by rolling it up when nearly empty and allowing it to flatten naturally. This process for expelling the air should likewise be used before the experiment in order to insure the same residual volume as at the end of the experiment. The rate of diffusion through the wall of the bag must be determined by analysis, as a bag allowing any determinable escape of carbon dioxide during the carrying out of a respiration experiment

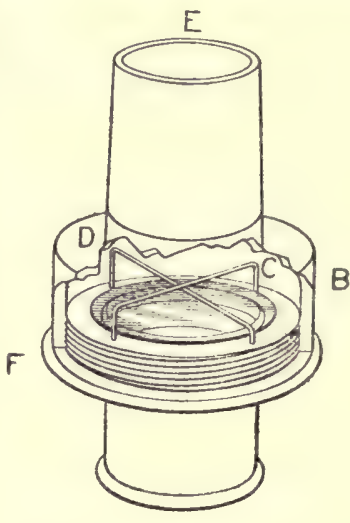

A

FIG. 29.-Mica-flap valve used with the Douglas method.

The valve is shown with a portion cut away so that the interior is seen. The direction of the air-current is from $A$ to $E$ and is determined by the movements of the mica flap $C$, the crosswires $D, D$, keeping the flap in place. can not be used. The tests can be made by filling the bag with expired air and taking samples for analysis at such intervals as will correspond with the length of time the expired air ordinarily remains in the bag.

In using the Douglas method in this research, two bags were employed. One of these - a gas bag of practically pure gum-was supposed to contain 100 liters, but without appreciable pressure would not hold more than 20 to 30 liters. The other bag was the largest used by Douglas and was capable of containing 100 liters. This was made to order of heavy rubber cloth according to measurements given by Douglas in a private communication. A 10-liter Bohr meter was used for measuring the gas in the bag. Samples of the air were collected over mercury 
in 100 c.c. gas samplers, the analyses being made with the laboratory form of the Haldane gas-analysis apparatus. ${ }^{1}$

In connection with this series of experiments two types of valves were used (figs. 29 and 30), both manufactured by Siebe, Gorman and Co., Ltd., of London, England, and used by them in their mine-rescue apparatus. The form shown in figure 29 consists of a metal tube, $20 \mathrm{~mm}$. in diameter, with an enlargement at $B$. Across the opening of this enlargement, a thin mica disk $(C)$ rests upon a very narrow metallic edge. When air enters at $A$, this disk is raised, the upward movement being limited by the cross-wires above the disk. When the air presses against the top of the disk, the mica flap falls again into place, so that no air can pass back through the opening $A$; the general direction of the air is thus from $A$ to $E$. The valve may be taken apart by unscrewing at $F$. A pair of these valves is used in separating inspired and expired air.
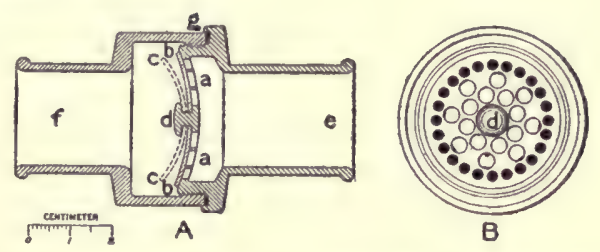

FIG. 30.-Rubber-flap valve used with the Douglas method.

The cross-section $A$ shows the general construction, and $B$ the openings of the valve. A rubber flap connected at $d$ opens and closes, the position when closed being indicated by $b b$, and when open by $c$. The direction of the air-current is from $e$ to $f$.

The other form of valve is shown in figure 30 , the cross-section being designated $A$ and the face of the opening through the valve $B$. This valve is essentially a metal tube, with a concave disk across its bore, in which there are a number of openings; a rubber flap covers the openings in the disk. When the valve is used as an inspiratory valve this flap opens and closes as the subject inspires and expires. The size and arrangement of the openings are shown in $B$, while $A$ shows the disk with the openings in cross-section at $a, a$. The position of the rubber flap when closed against the openings is indicated by $b, b$ in $A$, and when open by the dotted lines $c, c$. The rubber flap, which is circular in shape, is held in place by a knob, $d$, over which it is slipped. The direction of the air in passing through the valve is from $e$ to $f$. The parts of the valve may be separated by unscrewing it at $g$.

In the experiments carried out by the Douglas method, the pneumatic nosepieces shown in figure 4 and the Tissot valves shown in figure 23 were also used, but this did not produce any alterations in the general principle of the method. 


\section{MUELLER VALVES.}

The Mueller ${ }^{1}$ valves have long been used for studies of the respiration and respiratory exchange, and while many newer forms of valves have been developed and are in use, this form still finds application in a number of laboratories. Their continued use ${ }^{2}$ is doubtless due to the fact that they can be easily and inexpensively constructed from materials that are found in any well-equipped laboratory. The principle of the valve is simple, being that of an ordinary wash-bottle, the liquid in the bottle acting as a seal and preventing the air from going in more than one direction.

One of the valves constructed for this research is illustrated in figure 31 . It was made of a 1-liter wide-mouth bottle, in the neck of which was inserted a two-hole rubber stopper $(C)$. The inlet tube was an elbow of thin-walled brass tubing $(A)$, with an internal diameter of $25 \mathrm{~mm}$., of which the longer arm was inserted in one hole of the rubber stopper; the lower end of the tubing extended nearly to the bottom of the bottle. A shorter elbow $(B)$ of the same material was inserted in the other hole in the stopper and served as the exit tube. Two valves of this type were connected with a brass tee made of the same kind of tubing. Sufficient water was used in the valves to barely seal the lower end of the tube $D$. In use a valve was properly supported on each side of the subject, the intake tube being connected with the subject by a mouthpiece and the exit tube to the spirometer by means of rubber tubing.

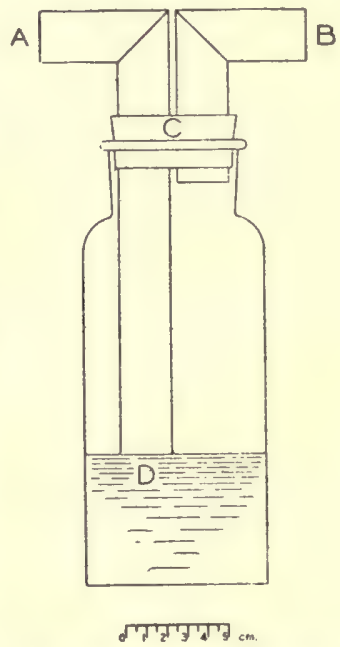

Fig. 31.-Mueller valve.

$A$, inlet tube; $B$, outlet tube; $C$, 2-holed rubber stopper; $D$, water seal. Air enters at $A$, passes through $D$, and leaves at $B$.

\section{HALDANE GAS-ANALYSIS APPARATUS.}

Several forms of apparatus for the analysis of various mixtures of gases have been devised by Haldane. Two of the forms, the laboratory and the portable gas-analysis apparatus, have found considerable application in the analysis of atmospheric air, mine air, and expired air. They differ mainly in their size and portability. The laboratory form is adapted for laboratory work only, as it requires considerable space and permanent installation. The portable form is constructed on the same principle, but is of a size suitable for carrying easily from room to room or into mines, ships, or any other of air are possible. 
LABORATORY FORM.

The laboratory form of the Haldane gas-analysis apparatus has been used considerably for analyses of atmospheric and expired air in connection with the respiration experiments conducted in this research. A detailed description of the apparatus, its method of use, and some of the modifications in technique made in this laboratory will therefore be given. Descriptions of this apparatus have previously been published by Haldane. ${ }^{1}$

The general principle of the apparatus is as follows: The gas to be analyzed is taken into a burette surrounded by a water-jacket, and is there saturated with water-vapor over mercury and measured. In the same water-jacket is a control tube, which is of about the same volume as the burette. The measuring burette and the control tube can be put into connection with one another through a manometer containing dilute potash solution. The control tube can be set at atmospheric pressure and compensates for the changes in temperature and pressure. The gas is first freed from carbon dioxide by means of potassium hydroxide, then from oxygen by absorption with potassium pyrogallate, measurements being made before and after each operation. From the differences of the three readings, the volumes of the carbon dioxide and of the oxygen can be calculated.

Description of Parts.

The apparatus in detail is shown in figure 32. A measuring burette, $A$, is placed in a cylindrical water-jacket, $B$. The total content of the burette is 21 c.c., 15 c.c. of this being included in the bulb at the upper part of the burette. From 15 c.c. to 21 c.c. it is graduated to 0.01 c.c.; the total length of the divided portion is $60 \mathrm{~cm}$.; the bore is $4 \mathrm{~mm}$. At the top of the burette is a stopcock, $C$, with two outlets arranged so that air can be drawn through one outlet from the sampler and air can be sent through the other outlet to the absorption pipettes. The lower part of the burette extends through a rubber stopper at the bottom of the water-jacket and is connected to the leveling bulb $D$ by means of rubber tubing.

The pipette $E$, for the absorption of carbon dioxide, consists of a cylindrical bulb, $13 \mathrm{~cm}$. in length and $30 \mathrm{~mm}$. in diameter. It can be put in communication with the burette $A$ by means of the two rightangle stopcocks $F$ and $G$. At the bottom of the potash pipette $E$ is a glass tee $H$, one branch of which is connected by rubber tubing to the leveling bulb $I$ containing potash. The other branch connects to a three-way stopcock, $J$, which in turn is connected to a compensation tube, $K$. The pipette for the absorption of oxygen is shown at $L$. This is connected to the burette $A$ by means of the two right-angle stopcocks $F$ and $G$, and is filled with potassium pyrogallate which can

'Haldane, Journ. Physiol., 1898, 22, p. 465; Methods of air analysis, London, 1912. 
be introduced through the leveling bulb and tube $M$ and rubber tubing $N$. Haldane recommends that the extra bulbs on the oxygen pipette be filled with potassium pyrogallate, as this protects the pyrogallate in the pipette. The stopcock $F$ can be turned so that the gas from the

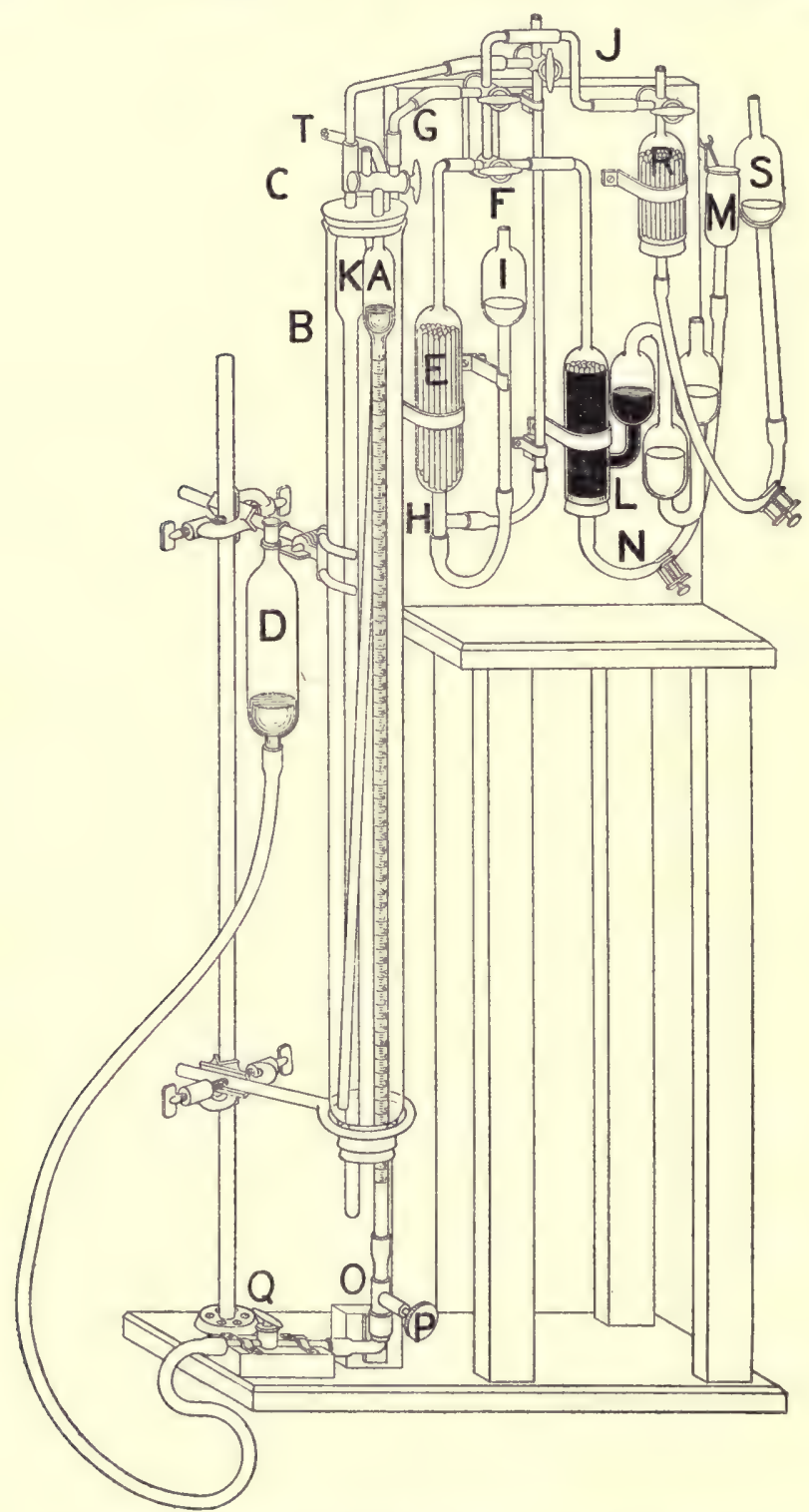

Frg. 32.-Haldane gas-analysis apparatus (laboratory form).

${ }^{A},{ }^{\prime}$ burette; $B$, water jacket; $C$, three-way stopcock; $D$, leveling bulb, connecting with burette $A ; E$, potash pipette; $F$ and $G$, right-angle stopcocks; $H$, tee connecting $E$ with leveling bulb $I$ and three-way stopcock, $J ; K$, compensating tube; $L$, potassium pyrogallate pipette; $M, N$, bulb and tubing for introducing potassium pyrogallate into $L ; O, P$, arrangement for fine adjustment of meroury level in $A ; Q$, stopcock; $R$, combustion pipette contsining stick yellow phosphorus; $S$, leveling bulb; $T$, tube for forcing air into water-bath. 
burette, $A$, can be introduced into the potash pipette $E$ or into the potassium pyrogallate pipette $L$, at will, but not simultaneously into both. Level marks on the two pipettes show the height to which the solutions are drawn.

The potassium pyrogallate is made by dissolving $10 \mathrm{gm}$. of pyrogallic acid in 100 c.c. of a nearly saturated solution of caustic potash. The specific gravity of the caustic potash should be 1.55. The potassium pyrogallate is kept in a closed bottle and should be prepared some time before it is to be used.

To compensate for changes in temperature and pressure, another tube, $K$, of the same size and construction as the burette $A$, and containing a few cubic centimeters of water is inserted in the waterjacket $B$, parallel with the burette, and is connected to the potash pipette $E$ through a three-way stopcock $J$. When the three-way stopcock $J$ is opened to the outside air, the level on the tube below the stopcock and the level on the potash pipette $E$ may be set at atmospheric pressure by raising or lowering the bulb $I$, the potash solution acting as a manometer. After the level has been set, the stopcock $J$ is closed to the outside air. The air on each side of the potash solution is then at the same pressure.

In the original Haldane apparatus the mercury in the burette $A$ is raised or lowered by means of the long cylindrical leveling bulb, constructed of tubing similar to that used for the burette. In this laboratory it was found somewhat difficult to use this type of leveling bulb, owing to the fact that occasionally the clamp which held it did not grip the tube firmly enough to prevent its slipping. When this occurred the potash or the potassium pyrogallate solution would be drawn over into the burette, causing considerable inconvenience. The leveling bulb has, therefore, been so modified that the manipulation is much easier. At the bottom of the burette $A$ is placed a piece of rubber tubing with a metal tube, $O$, surrounding it. Inside the latter is a flat metal piece which presses against the rubber tubing and can be moved by means of a fine adjusting screw, $P$. A common glass stopcock, $Q$, is placed between $O$ and the leveling bulb $D$ and connected to the latter by means of rubber tubing. In manipulation, the glass leveling bulb $D$ is raised or lowered until the mercury is nearly at the point desired. The stopcock $Q$ is then closed and the final adjustment of the mercury level in the burette, $A$, is made by the fine adjustment screw $P$, which alters the pressure on the rubber tube. No accidents of the character described above have occurred since this was adopted.

The original Haldane apparatus contains a combustion pipette for the oxidation of carbon monoxide or methane. In this laboratory there has been no occasion for using this pipette for the purpose designed. It has therefore been utilized to advantage in experimenting with phosphorus as an absorbent for oxygen. The upper of the two right-angle stopcocks, $G$, leads to the combustion pipette $R$ on the 
upper right-hand portion of the apparatus. . This combustion pipette is provided with a three-way stopcock. The ignition tubes inside the pipette have been removed and it has been filled with stick yellow phosphorus of suitable length and amount, so that 21 c.c. of air can be introduced into the combustion pipette. A leveling bulb, $S$, containing water, is attached by means of rubber tubing to the lower portion of the combustion pipette. It has been possible with this arrangement to compare directly on the same apparatus the absorption of oxygen by means of potassium pyrogallate and the absorption of oxygen by means of phosphorus.

Method of Use.

An analysis of atmospheric air or expired air is carried out in the following manner: The air in the apparatus is first freed from carbon dioxide and oxygen, in order that all of the capillaries may be filled with nitrogen. A small portion of air is then drawn into the apparatus through the stopcock, $C$, at the top of the burette, $A$, passed into the potash in $E$, and then into the potassium pyrogallate in $L$ until constant readings are obtained. Before any readings are made the levels on the potash pipette are set. This is done by lowering the mercury and shutting the stopcock, $Q$, when the mercury has come to the proper point, making the final adjustment by means of the adjustment screw, $P$, at the bottom. The angle stopcock, $F$, situated between the potash pipette, $E$, and the potassium pyrogallate pipette, $L$, is then turned so that communication exists between the burette, $A$, and the potash pipette, $E$. The stopcock, $J$, situated between the potash pipette and the compensating tube, is then opened to the air, and the levels in the tube leading from the potash pipette, $E$, and in the tube connecting the compensating tube, $K$, and the potash pipette, $E$, with the three-way stopcock, $J$, are set. It is advisable to place leveling marks on these two tubes when the apparatus is first put into use by taking out the three-way stopcock, $J$, and the angle stopcock, $F$, and allowing the liquid to settle to its own level. The two levels will then obviously be at atmospheric pressure. After these two levels have been set, the three-way stopcock, $J$, connecting the potash pipette and the compensating tube, is closed and there is no need of opening it again during any immediately succeeding analysis or series of analyses. It must be pointed out, however, that this setting of levels should be done on the residual sample of gas, $i$. e., nitrogen, rather than on the sample of gas to be analyzed. If this is not done, the first measurement of the sample to be analyzed will be incorrect. After all of the connecting tubes have been filled with nitrogen, the nitrogen is expelled from the burette into the open air.

The drawing of the sample may take place either by the washing method or by forcing mercury out through the connections to the 
sampler and then drawing air from the sampler through the connections. The drawing of the sample by the washing method is carried out as follows: An additional three-way stopcock is attached to the stopcock, $C$, above the burette. One of the branches is attached to the sampler. Air is then drawn through the tube from the sampler into the burette, $A$, in portions of about 15 c.c., and rejected through the free opening of the extra three-way stopcock. The amount of washing depends in part upon the amount of gas available, but the process should be carried out two or three times at least. When the amount of gas is small, it is necessary to use the other method, that is, by filling with mercury the space between the stopcock, $C$, attached to the burette and the sampler and then drawing the mercury up through the tube into the burette, $A$. The former method has ordinarily been used in this laboratory, as in practically all cases the sample to be analyzed was of such size that a considerable amount could be rejected in the washing method. In all washing and sampling arrangements the gas must always be under pressure, so that if any of the connections are not tight, the leak would be outward rather than inward, as a leak inward would produce a change in the composition of the gas.

After the final washing is completed, the amount required is drawn into the burette, $A$. The stopcock, $C$, is then reversed and the leveling performed by means of the leveling bulb, $D$, and the device at the bottom of the burette. The burette should contain sufficient water to saturate the gas thoroughly before the setting is made and the actual reading is taken. The water in the water-jacket, $B$, should be stirred by forcing in a little air through the tube, $T$. The two right-angle stopcocks, $F$ and $G$, should then be turned in such a way that the gas is in connection with the pipette, $E$. A reading is then taken. The gas is passed back and forth several times, care being taken not to force the mercury up into the stopcock, $C$, at the top of the burette. A reading is then taken, the levels being set again as before. The difference between the two readings gives the amount of carbon dioxide absorbed from the sample. In order to make sure that all of the carbon dioxide is absorbed, it may be passed again into the pipette and a reading taken.

After the carbon dioxide is absorbed, the air is then passed into the potassium pyrogallate pipette to absorb the oxygen. The routine which has been carried out in this laboratory is as follows: After the air is sent back and forth into the pyrogallate five times, it is left in the pyrogallate pipette for a few minutes, then drawn out and passed back and forth in the potash pipette five times. It is next drawn from the potash pipette and forced back and forth in the potassium pyrogallate pipette five times, and again sent into the potash pipette, when the first reading is taken. After the air has been sent back and forth into the potash once and into the pyrogallate five times readings are again taken. This routine is repeated until the last two readings are constant 
within 0.001 c.c. The final readings are then taken and from the difference between the reading after the carbon dioxide is absorbed and the reading after the oxygen is absorbed, the amount of oxygen in the sample is calculated.

Absorption of oxygen by phosphorus.-In many analyses of expired air and atmospheric air made in this laboratory, phosphorus instead of potassium pyrogallate has been used for the absorption of oxygen. The general routine is as follows: After the carbon dioxide has been absorbed in the usual way, the air is sent through the upper of the two right-angle stopcocks, $G$, into the pipette, $R$, which contains sticks of phosphorus, and is allowed to remain there for 3 minutes. It is next drawn over into the burette, $A$, once, then put back into the phosphorus again for 1 minute, sent into the potash pipette, $E$, five times, and finally into the phosphorus pipette, $R$, for 1 minute, when a reading is taken. After the first reading the air is sent into the phosphorus pipette for one minute and into the potash once and the second reading taken. This process is repeated until the readings are constant. The air is then sent over into the phosphorus pipette and at the end of 5 minutes the final reading is taken. The additional 5 minutes is allowed to insure complete absorption, as Durig ${ }^{1}$ has pointed out that even when apparently all the oxygen has been absorbed there may still be minute traces which require a longer time.

The use of phosphorus as an absorbent has proved extremely satisfactory. It has the advantage over the potassium pyrogallate that it does not have to be renewed so frequently, that the meniscus of water is much easier to set in the capillary connecting tube, and that the absorption can be carried out without the continuous raising and lowering of the bulb $D$. In order to obtain the quickest absorption with the potassium pyrogallate, it is necessary to drive the gas back and forth many times, and this constant raising and lowering of the mercury bulb is very tiring. There is also the advantage that should the liquid over the phosphorus pipette be drawn up into the connections no serious harm is done, while with the potassium pyrogallate it is necessary to take out all of the stopcocks and thoroughly clean them with acid before the apparatus can be used again. The phosphorus pipette is kept covered from the light by means of a metal shield which is taken off only during analysis. In one apparatus stick phosphorus has been in use for 8 months and shows no signs of deterioration.

Comparison of potassium pyrogallate and phosphorus as absorbents for oxygen.-To make sure that the results obtained by phosphorus were comparable with those obtained by the absorption with potassium pyrogallate, a number of comparisons on both atmospheric air and expired air were carried out in this laboratory. It will be seen by reference to table 10 that the results of the two series of analyses are

${ }^{1}$ Durig, Denkschriften der mathematisch-naturwissenschaftlichen Klasse der kaiserlichen Akademie der Wissenschaften, 1909, 86, p. 119. 
comparable. I am much indebted to Miss Alice Johnson and Miss Grace A. Dunning for assistance in the alterations in the apparatus and for very painstaking work in making the analyses. As an illustration of the adaptability of the apparatus, it may be mentioned that the latter analyst had had no experience with it previous to June 1912.

TABLE 10.-Comparison of potassium pyrogallate and phosphorus as absorbents for oxygen with Haldane gas-analysis apparatus (laboratory form).

\begin{tabular}{|c|c|c|c|c|}
\hline \multirow[b]{2}{*}{ Date. } & \multirow[b]{2}{*}{ Analyst. } & \multirow[b]{2}{*}{ Kind of air. } & \multicolumn{2}{|c|}{ Oxygen absorbed by- } \\
\hline & & & $\begin{array}{l}\text { Potassium } \\
\text { pyrogallate. }\end{array}$ & Phosphorus. ${ }^{1}$ \\
\hline 1912 & \multirow{6}{*}{ A. J..... } & & & \\
\hline May 29 & & Room air... & 20.94 & 20.91 \\
\hline & & .... do..... & 20.96 & 20.92 \\
\hline June 28 & & Outdoor... & 20.96 & $\ldots \ldots$ \\
\hline June 29 & & .....do.... & $\ldots \ldots$ & 20.95 \\
\hline & & ..... do..... & & 20.96 \\
\hline \multirow[t]{2}{*}{ July 6} & \multirow[b]{5}{*}{ G. A. D. } & .... do..... & 20.94 & 20.97 \\
\hline & & .... do..... & 20.95 & 20.95 \\
\hline July 8 & & .....do..... & 20.95 & 20.95 \\
\hline \multirow{2}{*}{ July 10} & & Expired air. & 15.40 & 15.41 \\
\hline & & .... do..... & 16.18 & 16.20 \\
\hline July 11 & A. J.... & .... do..... & 16.84 & 16.88 \\
\hline \multirow[t]{3}{*}{ July 12} & \multirow{3}{*}{ G. A. D. . } & ..... do..... & $(18.06$ & $(18.05$ \\
\hline & & ..... do..... & 18.04 & 18.09 \\
\hline & & ..... do.... & & 18.10 \\
\hline July 13 & \multirow[t]{4}{*}{ A. $\mathbf{J} . .}$. & $\ldots$. . do.... & 16.64 & 16.67 \\
\hline July 15 & & .... do. .... & 16.90 & 16.95 \\
\hline \multirow[t]{5}{*}{ July 16} & & .... do.... & $(17.15$ & 17.11 \\
\hline & & .... do.... & 17.19 & 17.15 \\
\hline & G. A. D. & ... do do... & 17.19 & 17.21 \\
\hline & A. J. & .... do.... & $\{16.89$ & $\int 16.87$ \\
\hline & & .....do..... & 16.88 & 16.84 \\
\hline July 17 & & ..... do.... & 15.71 & 15.70 \\
\hline \multirow[t]{2}{*}{ July 18} & G. A. D. & $\ldots$... do..... & 17.11 & 17.09 \\
\hline & A. J.... & $\ldots$...do.... & 17.39 & 17.40 \\
\hline \multirow[t]{3}{*}{ July 19} & G. A. D. . & .... do..... & 16.89 & 16.90 \\
\hline & & $\ldots$. . do.... & 16.73 & 16.75 \\
\hline & & $\ldots$ do..... & 16.54 & 16.58 \\
\hline
\end{tabular}

${ }^{1}$ Results inclosed in braces were obtained from one sample of the gas.

Care of the Apparatus.

The burette should always be kept thoroughly clean to insure correct results. If a poor grade of rubber tubing is used for the connections this may cause trouble in several ways. The mercury may become dirty from the sulphur and other material in the rubber tubing and thus require frequent cleaning. Also, if tubing containing much free sulphur is used on the connections of the potash pipette it may cause error in the determination of carbon dioxide. The best grade of puregum rubber tubing should be employed for practically all of the connections, and for the connection between the leveling bulb and burette a heavy-walled tubing must be used. The joints of the apparatus should fit as closely as possible, $i$. e., glass to glass, thus minimizing the 
dead space. All of the stopcocks should be absolutely tight. They may be tested by drawing the air into the burette and then, after connecting with the stopcock which is to be tested, putting the air in the burette under pressure. If there is a leak the volume will gradually decrease. A leak may also be shown by putting the air in the burette under diminished pressure and the liquid in the potash pipette or the potassium pyrogallate pipette will gradually rise, owing to the suction, if the stopcocks connecting these parts leak. In manipulating the apparatus care should be taken as far as possible to have the parts under pressure when the setting of the potash levels is begun, otherwise if there is suction the potash will rise into the connections, thus requiring cleaning with acid and the lubrication of the stopcocks.

Testing thie Apparatug.

The apparatus is regularly tested in this laboratory by analyses of outdoor air. The outdoor air remains uniform in composition and the standard for carbon dioxide and oxygen has been taken as 0.03 per cent for the former and 20.94 per cent ${ }^{1}$ for the latter. The limits of accuracy commonly allowed have been 0.03 to 0.04 per cent in parallels, this being a plus or minus error of 0.02 per cent. If the figures obtained are not within the limits of accuracy, the analysis is continued or a search is made for the cause. Generally, however, with the laboratory form of the apparatus, it is not difficult to obtain duplicates within 0.01 or 0.02 per cent for both carbon dioxide and oxygen.

The burette should be calibrated by some standard method of calibration.

PORTABLE FORM.

The portable form of the Haldane apparatus has likewise been used in this laboratory for the analysis of outdoor air and expired air. The results of a series of analyses of outdoor air made with this apparatus by G. A. D. are given in table 11. Phosphorus was used for the absorption of oxygen.

TABLE 11.-Results of analyses of atmospheric air with the portable Haldane gas-analysis apparatus.

\begin{tabular}{|c|c|c|c|c|c|}
\hline Date. & $\begin{array}{l}\text { Carbon } \\
\text { dioxide. }\end{array}$ & Oxygen. & Date. & $\begin{array}{l}\text { Carbon } \\
\text { dioxide. }\end{array}$ & Oxygen. \\
\hline 1913 & p. ct. & p. ct. & 1913 & p. ct. & p. ct. \\
\hline May 5 & 0.04 & 20.96 & May 8 & 0.03 & 20.98 \\
\hline \multirow{2}{*}{ May 6} & .04 & 20.98 & May 14 & .04 & 20.94 \\
\hline & .04 & 20.95 & May 17 & .04 & 20.95 \\
\hline \multirow[t]{4}{*}{ May 7} & .04 & 20.92 & May 23 & .04 & 20.93 \\
\hline & .04 & 20.93 & July 14 & .04 & 20.97 \\
\hline & .04 & 20.95 & & .04 & 20.93 \\
\hline & .04 & 20.95 & & & \\
\hline
\end{tabular}

\footnotetext{
${ }^{1}$ The value for oxygen used in this investigation is 20.94. Haldane gives 20.93 and Benedict 20.95. It is immaterial which value is used when the limits of error allowed are those given above.
} 
This form of apparatus is exactly the same in principle as that of the laboratory type, but it has a wider range of application because of its portability. It has recently been more generally used in this laboratory than the larger form, particularly in the analyses of samples of alveolar air. ${ }^{1}$ Both forms of apparatus are recommended because of the accuracy with which gas analyses can be made.

\section{HAND SPIROMETER.}

In connection with many tests of respiration apparatus, some method for imitating the respiration of man was found necessary. A small leather bellows, with the intake valve sealed up, was first employed. This was attached to the opening of either a pair of valves or one of the forms of respiration apparatus, and an attempt made to simulate respiration, but air-tight closure could not be obtained. The success of the spirometer of the Benedict respiration apparatus ${ }^{2}$ suggested the construction of a smaller form for the purpose, which could be operated by hand. A diagram of the hand spirometer is shown in figure 33 .

In this apparatus a heavy copper cylinder, $A$, is inverted in a double-walled annular bath of water or oil. From an opening, $B$, in the top of the cylinder forming the inner wall of the bath a tube leads down through the bottom of the spirometer, then makes a rightangle joint, the lower end of the tube being open to the air at $C$. For raising and lowering the bell of the spirometer, a long handle is provided which runs through an opening in the top crosspiece of the frame attached to the spirometer. The height to which the bell may be raised is regulated by means of a set-screw, $E$, which is placed upon the rod of the handle, thus determining the amount of air put into or out of the spirometer. The total content of the spirometer is about 1 liter. The height of the bell is $20.5 \mathrm{~cm}$. and the diameter of the cross-section $8 \mathrm{~cm}$. The whole apparatus is mounted on a small block and thus can be set up on any flat surface wherever needed.

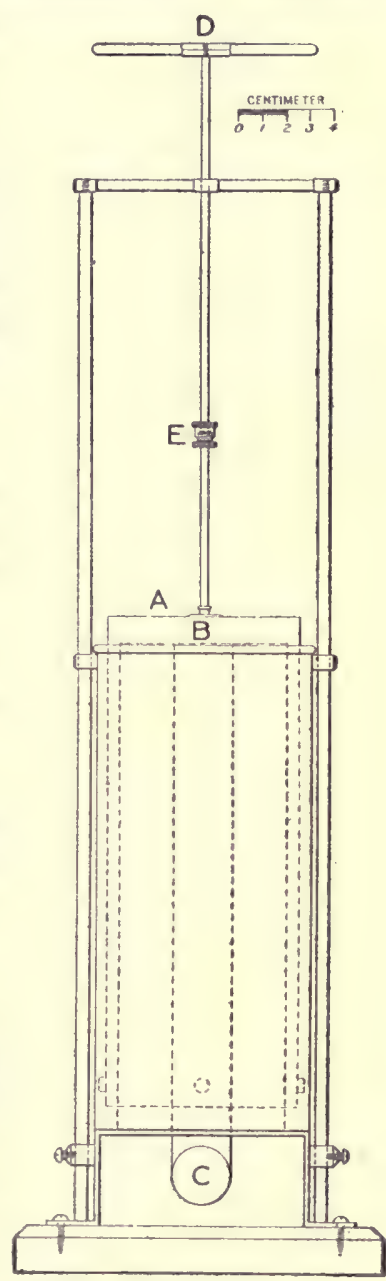

FIG. 33.--Hand spirometer.

The apparatus consists of a copper cylinder, $A$, immersed in a double-walled annular bath. An opening, $B$, in the top of the inner cylinder of the bath, connects with the outside air through the tube, $C$, which makes a right-angle bend at the bottom. The bell, $A$, is raised and lowered by the handle, $D$, the height to which it is raised being controlled by the set screw, $\boldsymbol{E}$. 
In use the hand spirometer is attached to the tee piece between a pair of valves or connected with the three-way valve of the unit respiration apparatus; then, by raising or lowering the bell, the valves may be opened or closed as in ordinary respiration or the tension equalizer or the spirometer of the unit respiration apparatus may be made to rise and fall. By the use of this apparatus it is possible to simulate respiration closely so far as volume and time are concerned. With the bath filled with water the spirometer is also used for the efficiency tests on the unit-respiration apparatus and for the calibration of the ventilation adder. ${ }^{1}$ In some experiments with a pair of valves carbon dioxide has been introduced between the valves at such a rate as would simulate the production of this gas by man. The apparatus has proved extremely useful in testing respiration apparatus.

\section{APPARATUS FOR ALCOHOL CHECK-TESTS OF THE TISSOT METHOD.}

To test the accuracy of the Tissot method $^{2}$ for the measurement of the respiratory exchange, an apparatus was devised for making experiments with burning alcohol. The general arrangement is shown in figure 34 . A burette, $A$, divided into 0.01 c.c. and with a capacity of a little over 5 c.c., was connected to a lamp, $B$, by means of rubber tubing and capillary copper tubing. A screw pinchcock, $O$, on the rubber tubing, controlled the flow of alcohol from the burette. The lamp was a brass cup with an opening about $0.5 \mathrm{~cm}$. diameter and was inclosed in a glass chamber, $D$, made of the outside part of a Zuntz valve (see fig. 19, page 54). The upper end of this chamber was connected by a tee piece, $E$, to the hand spirometer, $M N$, and by a second tee piece, $G$, to a Tissot valve, $J$. The open end in the tee piece, $G$, was closed by a rubber stopper, $K$. The lower end of the chamber, $D$, was connected with a second Tissot valve by a tee piece, $F$, the open end of which was closed by a rubber stopper, $L$. The whole apparatus was mounted by means of clamps and rings upon a large ring stand.

A test with this apparatus was carried out as follows: The burette $A$ was filled with alcohol, which was allowed to pass out through the tubing and lamp, and when they were free from air-bubbles the screw pinchcock $O$ was

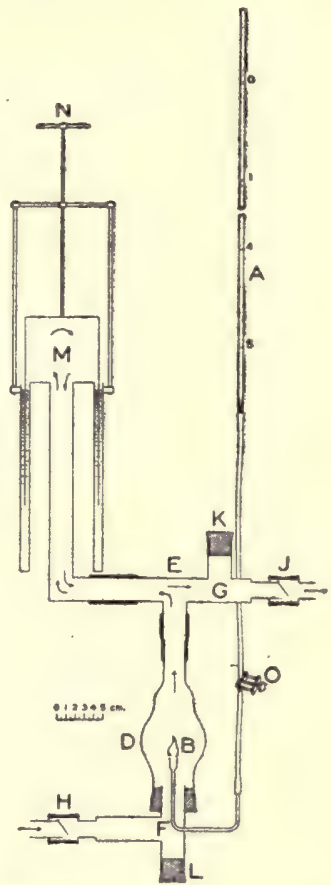

Frg. 34.-Apparatus used for alcohol check-tests of the Tissot method.

$A$, burette; $B$, alcohol lamp; $D$, glass chamber; $E$, $F$, and $G$, brass tee pieces; $H$ and $J$, Tissot valves; $K$ and $L$, rubber stoppers; $M$, $N$, hand spirometer; $O$, pinchcock. 
closed. The burette was then filled again, and the flow of alcohol was regulated by manipulation of the pinchcock $O$. The stoppers $L$ and $K$ were next taken out and the alcohol flowing from $B$ was lighted by means of a wax taper. When the flame was burning regularly, the stoppers $L$ and $K$ were put in place and the bell, $M$, of the hand spirometer immediately raised by means of the handle $N$. Regular up-and-down movements of the bell were then made, causing the air to enter the apparatus at $H$ with each upward movement and to leave at $J$ with each downward movement. After a few minutes, a reading was taken of the burette $A$ and the three-way valve (see $A$, figs. 26 and 27) on the Tissot spirometer was turned so that the air leaving $J$ entered the spirometer. After a suitable length of time had elapsed another reading of the burette was made and the threeway valve on the Tissot spirometer was turned to its original position. A sample of air was then taken from the spirometer and analyzed as for a respiration experiment. From the volume and composition of the air in the spirometer a calculation was made of the carbon dioxide produced and the oxygen consumed by the burning alcohol and the results were compared with the theoretical amounts computed from the amounts of alcohol burned. 



\section{PART II.}

\section{COMPARISONS OF RESPIRATORY EXCHANGE AS MEASURED BY DIFFERENT TYPES OF APPARATUS.}

The following comparisons of respiration apparatus and methods were made in this study:

Bed respiration calorimeter and Benedict universal respiration apparatus (tension-equalizer unit).

The two types of the Benedict universal respiration apparatus, $i$. e., tension-equalizer unit and spirometer unit.

Zuntz-Geppert apparatus and tension-equalizer unit.

Zuntz-Geppert apparatus and spirometer unit.

Tissot apparatus and tension-equalizer unit.

Tissot apparatus and spirometer unit.

Douglas respiration apparatus and spirometer unit.

Mouth- and nose-breathing with tension-equalizer unit.

Mouth- and nose-breathing with spirometer unit.

Mouth- and nose-breathing with Tissot apparatus.

Mask and nosepieces with spirometer unit.

Glass and pneumatic nosepieces with spirometer unit.

Mueller valves and Tissot spirometer with spirometer unit.

Mueller valves and Tissot spirometer with Tissot valves and spirometer.

Spirometer unit with and without additional dead space.

Tissot apparatus with and without automatic counterpoise on the spirometer bell.

The statistics and detailed results for all of the experiments in the various series are given in the following pages. Except in a few instances, the experiments were made in the morning and with the subject in the post-absorptive state, ${ }^{1} i$.e e. 12 hours after the last meal. The two forms of apparatus were ordinarily placed side by side, so that either could be used with but little delay, thus minimizing the time between the periods with the two apparatus and securing a uniform environment. The subject usually lay upon a husk mattress or upon an air mattress especially made for the purpose. As a rule he wore his ordinary clothing and was lightly covered with blankets. His head rested comfortably upon a pillow. In the bed calorimeter he lay nearly always upon his side, but in the experiments with the other forms of respiration apparatus he lay upon his back. In a few experiments he sat up in a chair. Even in the longer calorimeter experiments he was requested to keep as nearly as possible absolutely quiet, especially in the later experimenting, and was also required to keep awake.

The two apparatus were used either alternately or in series, the periods following each other as rapidly as technique would permit. Ordinarily the subject lay down upon the couch or entered the apparatus at least half an hour before the experiment began. In the statistics of the experiments the length of this preliminary period will be given 
whenever a record is available. In all but the calorimeter experiments the experimental periods approximated 15 minutes in length, varying not more than 5 minutes from this.

The pulse-rate in a few of the calorimeter experiments was counted by the subject, but generally the count was made by the observer by means of a Bowles stethoscope placed over the heart of the subject. From 3 to 5 counts for each period were taken by the observer, every count being a full minute in duration.

In the earlier experiments the respiration-rate was counted by the observer three times in each period for ten respirations and the rate per minute calculated. Subsequently information regarding the rate and general character of the respiration was obtained by means of a tambour, pointer, and kymograph drum attached either to a pneumograph fastened about the lower chest of the subject halfway between the nipples and the umbilicus or by the recording apparatus on the spirometer of the spirometer-unit respiration apparatus. ${ }^{1}$

In the experiments first carried out, the muscular activity was noted by the observer, although an incomplete record of the degree of muscular repose was obtained in many of the experiments by means of the pneumograph used for recording the respiration; in some instances a second pneumograph was placed about the hips, as suggested by Mr. H. L. Higgins, of the Laboratory staff. In later experimenting a special form of bed-rest was used which gave an exact record of the muscular movements of the subject. ${ }^{2}$

A considerable number of the subjects used in this research were members of the Laboratory staff; many of these had previously been subjects of similar experiments or had assisted in carrying out the experimental routine and were therefore familiar with the apparatus developed in this laboratory; they were not, however, so familiar with the other forms of apparatus. The subjects not members of the Laboratory staff were mostly medical students. The ages of the men experimented upon ranged between 18 and 35 years. ${ }^{3}$

To avoid repetition in presenting the statistics for the comparisons of the various apparatus and varying conditions of use, a preliminary statement is made of the general features peculiar to the series under consideration. The details are then given of the individual experiments, any exceptions to the general routine or changes in the apparatus being noted. The results of the experiments are presented in a general table accompanying the statistics for each series. In these tables the data for the two forms of apparatus compared are grouped separately, those for the periods with the apparatus first used preceding. The tables show the time of beginning each period and in some series the duration of the periods, the carbon dioxide eliminated and the oxygen

\footnotetext{
1See p. 39 .

${ }^{2}$ Benedict, Carnegie Inst. Wash. Pub. 203, 1915, p. 311.

${ }^{3}$ The basal metabolism for these subjects has previously been reported together with age, height, and weight. See Benedict, Emmes, Roth, and Smith, Journ. Biol. Chem., 1914, 18, p. 139.
} 
absorbed per minute in cubic centimeters, the respiratory quotient calculated to the nearest $0.005,{ }^{1}$ and the average pulse-rate calculated usually to the nearest 0.5 beat per minute. The average number of respirations per minute is also given; if the latter is obtained by counting only three times during a period, this is given to the nearest whole number; with a graphic record it is given to 0.1 respiration per minute. In certain comparisons the total ventilation of the lungs per minute, reduced to $0^{\circ} \mathrm{C}$. and $760 \mathrm{~mm}$. pressure, the volume per respiration calculated to $37^{\circ} \mathrm{C}$. moist and prevailing pressure, and the composition of the expired air are also included in the tables. The experimental data are arranged in all cases in chronological order by subjects. The detailed results are followed by a summary and discussion.

\section{BED RESPIRATION CALORIMETER AND BENEDICT RESPIRATION APPARATUS (TENSION-EQUALIZER UNIT).}

The development of the bed respiration calorimeter ${ }^{2}$ and the Benedict universal respiration apparatus (tension-equalizer unit ${ }^{3}$ ) was carried on simultaneously and extended over several years. During this time many opportunities were given for comparative respiration experiments. In these comparisons the periods with each apparatus were generally in series, the apparatus first used varying. Occasionally the experiment consisted of alternating series of periods with the two apparatus. Usually the bed calorimeter and respiration apparatus were in the same room, and as, in this comparison, the mattress was placed upon a framework or metal plate, the subject could be readily transferred from one apparatus to the other without muscular activity on his part. As it was necessary to delay the beginning of an experiment with the calorimeter until temperature equilibrium had been obtained, the time intervening between the series with the two apparatus varied in length according to which apparatus was first used. When changing from the respiration apparatus to the bed calorimeter, a considerable intermission - never more than one hour-was required before experimenting could again begin, while in changing from the bed calorimeter to the respiration apparatus, the succeeding period could usually be begun in about 15 minutes.

The pulse-rate was measured by means of the Bowles stethoscope, except that in a number of the calorimeter experiments the records were made by the subject himself. With the stethoscope the pulserate was counted by the observer as frequently as once in 5 minutes in the calorimeter periods and as frequently as every 2 or 3 minutes in the periods with the tension-equalizer unit.

The respiration-rate was counted from observation by an assistant or a graphic record was obtained with the chest pneumograph. The

\footnotetext{
${ }^{1}$ The average respiratory quotient is calculated from the average carbon dioxide and the average oxygen figures.

'See p. 14.

See p. 21.
} 
latter method was used unless otherwise stated. In the later calorimeter experiments no record was obtained of the respiration-rate.

Graphic records of the muscular activity were secured with the chest pneumograph, with the occasional addition of the hip pneumograph, or by the special form of bed-rest previously referred to. ${ }^{1}$ In the longer calorimeter experiments absolute muscular repose for the whole time was difficult to obtain and the subjects were inclined to fall asleep. They were not required to keep awake in the earlier experiments with this apparatus, but later were asked to do so, if possible, and requested not to change the position more than once in a period.

Both laboratory assistants and medical students were used as subjects, the latter being employed more particularly in the later comparisons. While the medical students were unfamiliar with the two apparatus, this proved to be no detriment to the experiments, as they quickly became accustomed to the apparatus and the routine. The statistics of the 36 experiments are given in the following pages. The majority of the experiments with the bed calorimeter were carried out by Mr. L. E. Emmes.

\section{STATISTICS OF EXPERIMENTS.}

F. G. B., March 1, 1909.-Bed calorimeter, two 1-hour periods; tensionequalizer unit, three 8- to 10-minute periods; preliminary period, 1 hour 21 minutes. Mouthpiece used with tension-equalizer unit. Pulse-rate taken by subject in calorimeter, by observer (three counts in each period) with tension-equalizer unit. Respiration-rate with calorimeter recorded by chest pneumograph; with tension-equalizer unit counted by observer. Subject urinated in calorimeter at $8^{\mathrm{h}} 50^{\mathrm{m}}$ a. m., turned over on his side twice during each period. Awake and fairly quiet with both apparatus, except as noted.

F. G. B., March 2, 1909.-Bed calorimeter, five 30-minute periods; tensionequalizer unit, three 10-minute periods; preliminary period, 1 hour 1 minute. Mouthpiece used with tension-equalizer unit. Pulse-rate counted few times by subject in calorimeter; by observer with tension-equalizer unit. Respiration-rate recorded by chest pneumograph with calorimeter; observed by assistant with tension-equalizer unit; two counts with each apparatus. Subject turned over twice in calorimeter, urinating immediately after the beginning of the first period; otherwise quiet and awake with both apparatus.

F. G. B., November 15, 1910.-Bed calorimeter, two 1-hour periods; tensionequalizer unit, three 15-minute periods; preliminary period, 1 hour 4 minutes. Mouthpiece used with tension-equalizer unit. Pulse-rate taken by subject in calorimeter. Considerable activity during first period with calorimeter and subject turned over on side once during second period; quiet in periods with tension-equalizer unit. Both pulse- and respiration-rates regular.

$J$. A. R., March 20, 1909.-Tension-equalizer unit, three 10-minute periods; bed calorimeter, two 1-hour periods. Pneumatic nosepieces used with tensionequalizer unit. Pulse-rate counted by observer from stethoscope with both apparatus, several counts in each period; respiration-rate recorded by chest pneumograph. Subject quiet and awake with both apparatus.

T.M.C., March 23, 1909.- Subject had a small cup of clear coffee at 6 a. m.; experiment began at $8^{\mathrm{h}} 5^{\mathrm{m}}$ a. $\mathrm{m}$. Three series: Tension-equalizer unit, four 10-minute periods; bed calorimeter, two 1-hour periods; finally, tension- 
equalizer unit, three 10-minute periods. Pneumatic nosepieces used with tension-equalizer unit. Pulse-rate counted from stethoscope by observer through whole experiment, but only two or three records made in each period with respiration apparatus; respiration-rate obtained from pneumograph with bed calorimeter and from observation by assistant with tension-equalizer unit; two counts made in each period with latter apparatus. Subject asleep much of first period in calorimeter, but awake in second period, and also in periods with the tension-equalizer unit. He stated that the breathing was perfectly free throughout.

T. M. C., July 12, 1910.-Tension-equalizer unit, four 15-minute periods; bed calorimeter, two 1-hour periods. Pneumatic nosepieces used with tensionequalizer unit. Subject asleep nearly all of first period in calorimeter. Pulserate with tension-equalizer unit for most part uniform; six counts in each period. A number of records of pulse-rate obtained in calorimeter, with variations first period, 72 to 56 ; second period, 73 to 59 . Respiration rate with tensionequalizer unit uniform in depth and character; also uniform in calorimeter.

T. M. C., November 16, 1910.-Tension-equalizer unit, five 10-to 15-minute periods; bed calorimeter, two 1-hour periods. Pneumatic nosepieces used with tension-equalizer unit. Subject comfortable in periods with the tensionequalizer unit. In bed calorimeter, he read a magazine for first half of first period and was asleep most of the second half; complained that pneumograph made him uncomfortable in calorimeter. Pulse-rate uniform in periods with both apparatus; only two counts obtained in second period with bed calorimeter. Respiration-rate also uniform in periods with both apparatus.

J. J. C., November 3, 1910.-Bed calorimeter, two 1-hour periods; tensionequalizer unit, three 15-minute periods; pneumatic nosepieces used with tension-equalizer unit. Subject asleep most of time in calorimeter. Errors in oxygen determinations in first and third periods with tension-equalizer unit. Pulse- and respiration-rates uniform with both apparatus.

J. J. C., November 8, 1910.-Bed calorimeter, one period $1 \frac{1}{2}$ hours, one period 1 hour; tension-equalizer unit, three 15-minute periods; preliminary period, 1 hour 26 minutes. Pneumatic nosepieces with tension-equalizer unit. Subject asleep most of time in bed calorimeter. Pulse- and respiration-rates uniform for most part with both forms of apparatus.

J. J. C., November 10, 1910.-Three series: Tension-equalizer unit, six 15-minute periods, bed calorimeter, two 1-hour periods; finally tensionequalizer unit, three 12- to 15 -minute periods; preliminary period, 32 minutes. An attempt was made to begin the experiment at $7^{\mathrm{h}} 55^{\mathrm{m}} \mathrm{a} . \mathrm{m}$. but the results of this period have been omitted, as the metabolism of the subject had not then reached its rest level. Subject rather drowsy in some of the periods with tension-equalizer unit and asleep most of the time in bed calorimeter. In the first series with tension-equalizer unit pulse-rate variable but respiration-rate uniform; in bed calorimeter, pulse- and respiration-rates fairly uniform; in last series with tension-equalizer unit, both pulse- and respiration-rates uniform.

J. J. C., November 15, 1910.-Tension-equalizer unit, three 10-minute periods, bed calorimeter, three 1-hour periods. Pneumatic nosepieces used with tension-equalizer unit. Subject slept greater part of time in calorimeter, waking occasionally. Only a few counts of pulse-rate obtained with each apparatus; uniform in character. Respiration-rate also uniform.

V. G., November 4, 1910.-Bed calorimeter, one period $1 \frac{1}{2}$ hours, one period 1 hour; tension-equalizer unit, four 15-minute periods; preliminary period, 1 hour 16 minutes. Pneumatic nosepieces used with tension-equalizer unit. Subject very quiet with both apparatus; asleep most of time in calorimeter. Errors in oxygen determination in two periods due to leaks in tension-equalizer 
unit. Pulse-rate variable in bed calorimeter; pulse- and respiration-rates fairly uniform with tension-equalizer unit.

V. G., November 7, 1910.-Bed calorimeter, two 1-hour periods; tensionequalizer unit, three 15 -minute periods; preliminary period, 1 hour 6 minutes. Pneumatic nosepieces used with tension-equalizer apparatus. Subject asleep greater part of time in calorimeter; quiet with tension-equalizer apparatus. Pulse- and respiration-rates fairly uniform throughout experiment.

L. E. E., December 9, 1910.-Tension-equalizer unit, two 15-minute periods; bed calorimeter, three 45-minute periods. Pneumatic nosepieces used with tension-equalizer apparatus. Subject very quiet throughout experiment. Pulse-rate uniform with tension-equalizer unit; with calorimeter, variable, rate in first period being 55 to 62 , in second period 50 to 58 , and in third period 55 to 62 . Respiration-rate uniform throughout experiment as counted from pneumograph.

R. J.C., June 16, 1911.-Tension-equalizer unit, three 15-minute periods; bed calorimeter, three 45-minute periods. Pneumatic nosepieces used with tension-equalizer unit. Subject quiet in calorimeter in first period; somewhat restless in second and third periods in this apparatus. Pulse- and respirationrates uniform with tension-equalizer unit. Pulse-rate variable in last two calorimeter periods; no respiration-rate recorded with this apparatus.

H.F.T., A ugust 17, 1911.-Bed calorimeter, one 99-minute period, tensionequalizer unit, four 15-minute periods; preliminary period, approximately 2 hours 18 minutes. Pneumatic nosepieces used with tension-equalizer unit. Subject was quiet with tension-equalizer unit. Pulse- and respiration-rates uniform throughout experiment, except that in last part of bed-calorimeter period there was a tendency toward apnœa.

H. F. T., August 24, 1911.-Bed calorimeter, two 45-minute periods; tension-equalizer unit, four 15-minute periods. Pneumatic nosepieces with tension-equalizer unit. Subject quiet throughout experiment; pulse- and respiration-rates uniform in each period.

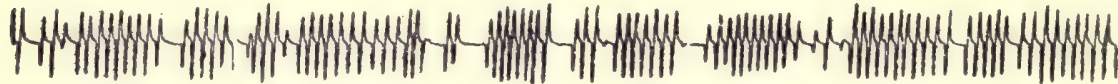

\footnotetext{
FIG. 35.-Type of respiration of subject H. F. T. as shown by chest pneumograph in the first period with the bed calorimeter on August 29, 1911.
}

H. F. T., August 29, 1911.-Five series: First, tension-equalizer unit, three 15-minute periods; second, bed calorimeter, two 45-minute periods; third, tension-equalizer unit, three 15-minute periods; fourth, bed calorimeter, two 45-minute periods, fifth, tension-equalizer unit, two 15-minute periods. Pneumatic nosepieces with tension-equalizer unit. Subject quiet throughout experiment. In the fifth period of the experiment, $i . e$. , the second period in the first series with the bed calorimeter, the determination of the oxygen consumption was defective. The pulse-rate was uniform throughout. With the exception of some apnœea shown in the first series with the tension-equalizer unit and in the first period of the first series with the bed calorimeter, the respiration-rate was also uniform, particularly in the second series with the tensionequalizer unit. The type of respiration obtained with this subject is shown in figure 35 .

H.F.T., August 31, 1911.-Four series: First, tension-equalizer unit, four 15-minute periods; second, bed calorimeter, two 45-minute periods; third, tension-equalizer unit, three 15-minute periods; fourth, bed calorimeter, two 45-minute periods. Pneumatic nosepieces used with tension-equalizer unit. 
Subject quiet. Pulse-rate uniform throughout experiment, especially in first two series. During the first series with the bed calorimeter the records obtained with the chest pneumograph showed the respiration to be decidedly irregular, but no quantitative determinations could be secured. In the period beginning at $10^{\mathrm{h}} 19^{\mathrm{m}}$ a. m., there was considerable apnoea, approaching Cheyne-Stokes respiration. During the second period in this series the respiration was much more uniform in character. In the other periods of the experiment the respiration-rate was uniform with both apparatus. Figure 36 gives curve obtained for the respiration of this subject.

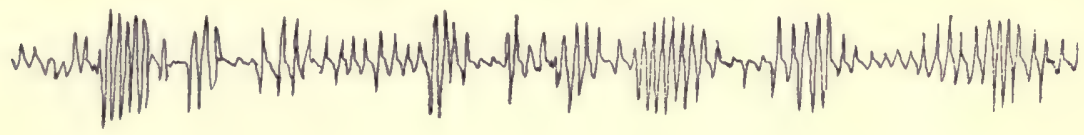

FIG. 36.-Type of respiration of subject H. F. T. in the first period with the bed calorimeter on August 31, 1911. Note the frequency of apncea.

Dr. P. R., October 24, 1911.-Bed calorimeter, three 45-minute periods; tension-equalizer unit, four 15-minute periods; preliminary period, 1 hour 3 minutes. Pneumatic nosepieces with tension-equalizer unit. Subject very quiet throughout experiment; slept part of time in bed calorimeter. Pulseand respiration-rates uniform.

K. H. A., February 26, 1912.-Bed calorimeter, four 45-minute periods; tension-equalizer unit, four 15-minute periods; preliminary period, 1 hour 5 minutes. Pneumatic nosepieces used with tension-equalizer unit. Subject quiet, though awake, in calorimeter; with tension-equalizer unit, slight movement in first period and drowsy in third period. Pulse-rate uniform throughout experiment. No respiration records in calorimeter periods; with tensionequalizer unit, respiration-rate uniform.

K. H. A., March 14, 1912.-Bed calorimeter, four 45-minute periods; tension-equalizer unit, four 15-minute periods; preliminary period, 50 minutes. Pneumatic nosepieces used with tension-equalizer unit. Subject quiet and awake throughout experiment. Pulse-rate uniform. No records of respiration in bed calorimeter periods, but those for the tension-equalizer unit were uniform.

I. A. F., March 19, 1912.--Bed calorimeter, three 45-minute periods; tension-equalizer apparatus, three 12- to 15-minute periods; preliminary period, 1 hour 9 minutes. Subject quiet for the most part in calorimeter except for slight movement after beginning of each period; more active in first period than in last two periods. With tension-equalizer unit, subject quiet and awake. Pulse-rate uniform throughout experiment. No respiration records taken in calorimeter periods; respiration-rate uniform in periods with tension-equalizer unit.

S. A.R., March 16, 1912.- Subject had very light breakfast at $8^{\mathrm{h}} 30^{\mathrm{m}}$ a. m.; experiment began at $1^{\mathrm{h}} 25^{\mathrm{m}}$ p. m. Bed calorimeter, three 45-minute periods; tension-equalizer unit, three 15-minute periods; preliminary period, 45 minutes. Results of two additional periods with tension-equalizer unit not included in table, as subject was disturbed, causing leakage of air. Subject quiet throughout experiment. Pulse-rate fairly uniform in calorimeter and uniform with tension-equalizer unit. No respiration records made with calorimeter; rate uniform with tension-equalizer unit.

S. A. R., March 20, 1912. - Subject had very light breakfast early in the morning; experiment began at $2^{\mathrm{h}} 19^{\mathrm{m}}$ p. m. Bed calorimeter, two 1-hour periods; tension-equalizer unit, four 11- to 15-minute periods; preliminary 
period, 1 hour 25 minutes. Pneumatic nosepieces used in periods with tensionequalizer unit. Subject fairly quiet in calorimeter, except for a part of the last period; quiet with tension-equalizer unit. Pulse-rate fairly uniform in calorimeter and also for the most part with the tension-equalizer unit. Respiration records not made in calorimeter; rate uniform with tension-equalizer unit.

S. A. R., A pril 2, 1912.-Bed calorimeter, three 45-minute periods; tensionequalizer unit, four 14-minute periods; preliminary period approximately 1 hour 15 minutes. In calorimeter subject breathed through nosepieces and the three-way valve, which had been detached from the respiration apparatus and placed in the calorimeter chamber. The openings of the valve had been adjusted in such a way that the changes in resistance were the same as when he breathed through it on the tension-equalizer unit; he also lay on his back as in experiments with the tension-equalizer unit. Pneumatic nosepieces were used in the tension-equalizer unit periods. When taken out of calorimeter subject said he was somewhat tired. Was quiet in the tension-equalizer periods. Pulse-rate uniform throughout whole experiment, especially with bed calorimeter. No respiration records obtained with calorimeter; with tension-equalizer unit respiration-rate uniform.

S. A. R., A pril 5, 1912.-Tension-equalizer unit, three 13- to 15-minute periods; bed calorimeter, three 45-minute periods. Pneumatic nosepieces used with tension-equalizer unit. Nosepieces and three-way valve used in calorimeter as in the preceding experiment; surgeon's plaster was also used over the lips so that subject could not breathe through mouth. Subject sleepy during periods with tension-equalizer unit, particularly in the first period. Stated at end of calorimeter periods that the inability to change his position due to the use of nosepieces and three-way valve was tiring, also that the nosepieces were somewhat too closely fitted. He slept part of the time in calorimeter. Pulse-rate uniform with tension-equalizer unit except in first period; with calorimeter but few records of pulse-rate obtained, but these were fairly uniform. Respiration-rate with tension-equalizer unit uniform; no records taken with calorimeter.

P. F. J., March 15, 1912.-Bed calorimeter, three 45-minute periods; tension-equalizer unit, four 15-minute periods; preliminary period, 48 minutes. Pneumatic nosepieces used with tension-equalizer unit. Subject quiet and awake in calorimeter. Pulse-rate fairly uniform throughout experiment. No respiration records with calorimeter; with tension-equalizer unit fairly uniform.

P.F.J., March 18, 1912.-Bed calorimeter, three 45-minute periods; tensionequalizer unit, four 15-minute periods; preliminary period, approximately 1 hour 38 minutes. Pneumatic nosepieces used with tension-equalizer unit. Subject quiet and awake throughout the experiment. Pulse-rate uniform. Respiration-rate also uniform in tension-equalizer unit periods, but no records taken in calorimeter periods.

P. F. J., March 29, 1912.-Tension-equalizer unit, four 15-minute periods; bed calorimeter, three 45-minute periods. Subject quiet with the tensionequalizer unit; complained of acid fumes in one period, but said that in the third period he could tell no difference between breathing room air and the air in the apparatus. In the calorimeter periods he was more restless. The pulse-rate with the tension-equalizer unit was uniform, but was somewhat more variable in the calorimeter periods. Respiration-rate with respiration apparatus uniform; no records obtained with the calorimeter.

P. F. J., A pril 8, 1912.-Bed calorimeter, two 1-hour periods; tensionequalizer unit, three 12- to 14-minute periods; preliminary period, 55 minutes. Subject fairly quiet in calorimeter; uneasy and restless in last two periods with tension-equalizer unit, and said he had great difficulty to keep from coughing. 
Pulse-rate varied in first calorimeter period from 68 to 74 and in second period from 73 to 85 . Only a few records of pulse-rate in experiments with tensionequalizer unit. No records of respiration in calorimeter periods; respiration with tension-equalizer unit uniform.

M. A. M., March 20, 1912.-Bed calorimeter, three 45-minute periods, one 1-hour period; tension-equalizer unit, four 11- to 15-minute periods; preliminary period, 1 hour 20 minutes. Mouthpiece used with tension-equalizer unit. Subject awake in the calorimeter; quiet with tension-equalizer unit. Pulse-rate uniform in calorimeter periods; variable with tension-equalizer unit. Respiration-rate not taken in calorimeter; exceptionally uniform with tension-equalizer unit.

M. A. M., March 22, 1912.-Tension-equalizer unit, five 15-minute periods; bed calorimeter, three 1 -hour periods; preliminary period, 37 minutes. Mouthpiece used with tension-equalizer unit. Subject quiet and awake throughout the experiment. At $9^{\mathrm{h}} 30^{\mathrm{m}}$ a. $\mathrm{m}$. he arose and urinated, lying down again at $9^{\mathrm{h}} 32^{\mathrm{m}}$ a. m. Pulse- and respiration-rates very uniform in periods with the tension-equalizer unit. Pulse-rate fairly uniform in bed calorimeter, but no respiration records taken.

E. P. C., April 6, 1912.-Tension-equalizer unit, four 13- to 15-minute periods; bed calorimeter, two 1-hour periods. Mouthpiece used with tensionequalizer unit. Subject somewhat drowsy at times with tension-equalizer unit; quiet in first period with calorimeter, but slightly restless in second period. Pulse-rate for most part regular. Depth of respiration irregular with tension-equalizer unit; no records of respiration made with bed calorimeter.

J. E. F., April 6, 1912.-Bed calorimeter, two 1-hour periods; tensionequalizer unit, four 15-minute periods; preliminary period, 1 hour 7 minutes. Subject very quiet in calorimeter and quiet with tension-equalizer unit. Pulse-rate uniform in bed calorimeter and fairly uniform in periods with tension-equalizer unit. No respiration records taken with bed calorimeter; rate very regular with tension-equalizer unit.

J.E.F., April 8, 1912.-Bed calorimeter, two 1-hour periods; tensionequalizer unit, three 13-minute periods; preliminary period, 1 hour 7 minutes. Experiments carried out in afternoon. Subject very quiet in calorimeter. Pulse-rate for the most part uniform in calorimeter; somewhat variable with tension-equalizer unit. No respiration records taken with calorimeter; rate uniform with tension-equalizer unit.

\section{DISCUSSION OF RESULTS.}

The results of all of the comparisons made with the bed calorimeter and the tension-equalizer unit are given in table 12. The general averages for the two apparatus show a close agreement in the respiratory exchange. The carbon dioxide with the bed calorimeter is 190 c.c. per minute and with the tension-equalizer unit 185 c.c. per minute. The figures for the oxygen consumption show a similar agreement, being 223 c.c. with the bed calorimeter and 227 c.c. with the respiration apparatus. The respiratory quotient is slightly higher with the bed calorimeter than with the respiration apparatus, $i$. e., 0.850 against 0.815. The pulse-rate is practically the same with both apparatus, or 58.5 and 59.5 respectively; the respiration-rate is slightly higher with the bed calorimeter, the average result for that apparatus being $\mathbf{1 5 . 0}$ as compared with 13.2 for the tension-equalizer unit. 
TABLE 12.-Respiratory exchange in comparison experiments with the bed calorimeter and the Benedict respiration apparatus (tension-equalizer unit). (Without food.)

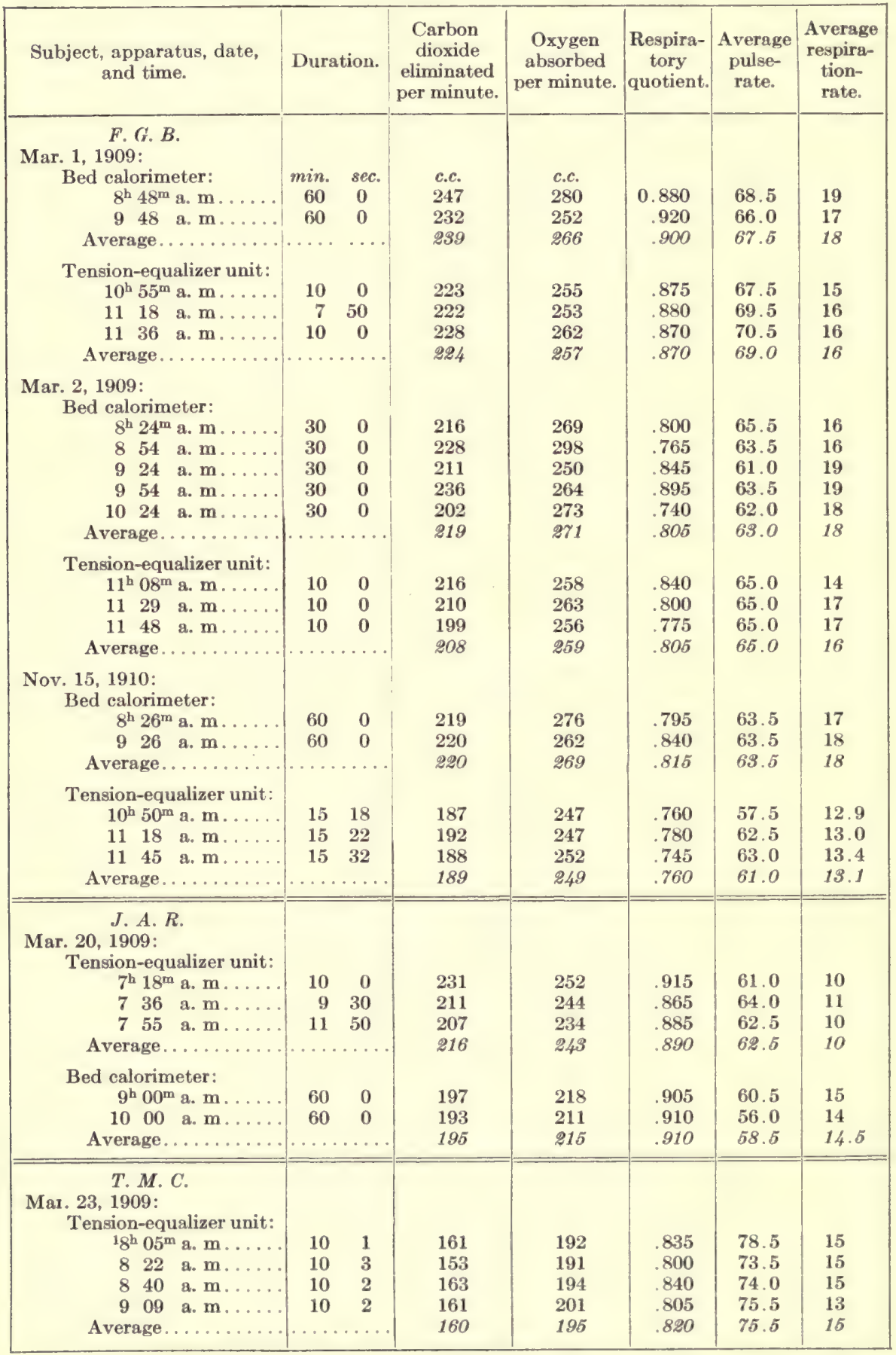

1Subject had a small cup of elear coffee at 6 a.m. 
TABLE 12.-Respiratory exchange in comparison experiments with the bed calorimeter and the Benedict respiration apparatus (tension-equalizer unit). (Without food.)-Continued.

\begin{tabular}{|c|c|c|c|c|c|c|}
\hline $\begin{array}{l}\text { Subject, apparatus, date, } \\
\text { and time. }\end{array}$ & Duration. & $\begin{array}{c}\text { Carbon } \\
\text { dioxide } \\
\text { eliminated } \\
\text { per minute. }\end{array}$ & $\begin{array}{c}\text { Oxygen } \\
\text { absorbed } \\
\text { per minute. }\end{array}$ & $\begin{array}{c}\text { Respira- } \\
\text { tory } \\
\text { quotient. }\end{array}$ & $\begin{array}{c}\text { Average } \\
\text { pulse- } \\
\text { rate. }\end{array}$ & $\begin{array}{l}\text { Average } \\
\text { respira- } \\
\text { tion- } \\
\text { rate. }\end{array}$ \\
\hline 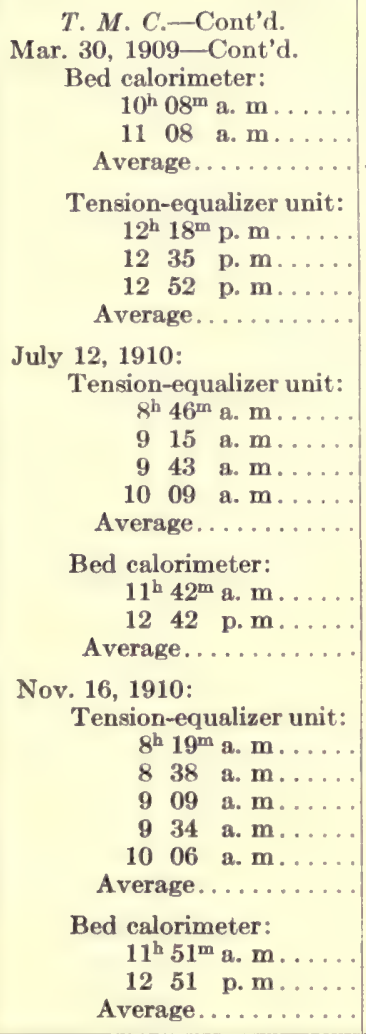 & \begin{tabular}{|rr}
10 & 4 \\
15 & 3 \\
14 & 59 \\
15 & 56 \\
15 & 1 \\
$\ldots \ldots$ & $\ldots$ \\
& \\
60 & 0 \\
60 & 0 \\
$\ldots \ldots$ & $\ldots .$.
\end{tabular} & $\begin{array}{l}168 \\
150 \\
148 \\
152 \\
156 \\
155\end{array}$ & $\begin{array}{l}191 \\
177 \\
183 \\
190 \\
192 \\
187\end{array}$ & $\begin{array}{l}.880 \\
.845 \\
.810 \\
.800 \\
.810 \\
.830\end{array}$ & $\begin{array}{l}72.0 \\
71.5 \\
69.5 \\
66.0 \\
68.0 \\
69.5\end{array}$ & $\begin{array}{l}15.2 \\
12.4 \\
12.1 \\
12.0 \\
11.7 \\
12.7\end{array}$ \\
\hline 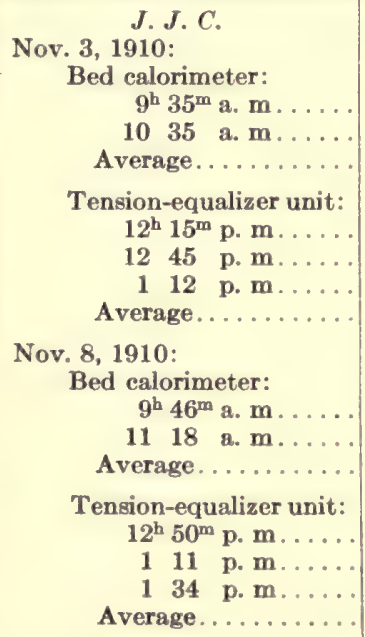 & $\begin{array}{cc}14 & 30 \\
14 & 46 \\
14 & 15 \\
\ldots & \ldots\end{array}$ & $\begin{array}{l}192 \\
183 \\
193 \\
189\end{array}$ & $\begin{array}{c}(263) \\
232 \\
\cdots \\
282\end{array}$ & $\begin{array}{c}(.730) \\
.790 \\
. . \\
.815\end{array}$ & $\begin{array}{l}56.0 \\
55.5 \\
58.0 \\
56.5\end{array}$ & $\begin{array}{l}18.7 \\
18.6 \\
18.5 \\
18.6\end{array}$ \\
\hline
\end{tabular}


TABLE 12.-Respiratory exchange in comparison experiments with the bed calorimeter and the Benedict respiration apparatus (tension-equalizer unit). (Without food.)-Continued.

\begin{tabular}{|c|c|c|c|c|c|c|c|}
\hline $\begin{array}{l}\text { Subject, apparatus, date, } \\
\text { and time. }\end{array}$ & Dura & tion. & $\begin{array}{c}\text { Carbon } \\
\text { dioxide } \\
\text { eliminated } \\
\text { per minute. }\end{array}$ & $\begin{array}{c}\text { Oxygen } \\
\text { absorbed } \\
\text { per minute. }\end{array}$ & $\begin{array}{c}\text { Respira- } \\
\text { tory } \\
\text { quotient. }\end{array}$ & $\begin{array}{l}\text { Average } \\
\text { pulse- } \\
\text { rate. }\end{array}$ & $\begin{array}{l}\text { Average } \\
\text { respira- } \\
\text { tion- } \\
\text { rate. }\end{array}$ \\
\hline \multicolumn{8}{|l|}{$\begin{array}{l}\text { J.J.C.C-Cont'd. } \\
\text { Nov. 10, 1910: }\end{array}$} \\
\hline $\begin{array}{l}\text { 1ension-equanzer unit: } \\
8^{\mathrm{h}} 16^{\mathrm{m}} \text { a. } \mathrm{m} . . .\end{array}$ & 15 & 12 & $\begin{array}{l}c . c . \\
188\end{array}$ & 217 & .865 & 65.5 & 16.5 \\
\hline 844 a. m...... & & 34 & 170 & 219 & .780 & 55.0 & 14.5 \\
\hline 910 a. $\mathrm{m} \ldots .$. & 14 & 59 & 183 & 221 & .825 & 58.5 & 13.6 \\
\hline 955 a. $\mathrm{m} \ldots \ldots$ & 15 & 10 & 185 & 223 & .830 & 55.5 & 14.3 \\
\hline 1037 a. $\mathrm{m} \ldots$... & 15 & 5 & 174 & & & 57.5 & 15.1 \\
\hline 1105 a. m..... & 15 & 8 & 184 & 218 & .840 & 61.5 & 17.0 \\
\hline Average............ & & & 181 & 220 & .825 & 59.0 & 15.2 \\
\hline 153 p. m......... & & 0 & 195 & 221 & .0885 & $\begin{array}{l}55.5 \\
60.5\end{array}$ & $\begin{array}{l}18 \\
17\end{array}$ \\
\hline Average.......... & $\cdots$ & & 192 & 221 & .870 & 58.0 & 18 \\
\hline \multirow{2}{*}{\multicolumn{8}{|c|}{$\begin{array}{l}\text { Tension-equalizer unit: } \\
3^{\mathrm{h}} 05^{\mathrm{m}} \text { p. } \mathrm{m} \ldots \ldots\end{array}$}} \\
\hline & & 26 & 198 & 233 & .850 & 59.5 & 18.8 \\
\hline 328 p. m..... & 14 & 58 & 194 & 245 & .795 & 60.0 & 19.3 \\
\hline 346 p. $m \ldots$. & 12 & 9 & 194 & 247 & .785 & 59.5 & 20.3 \\
\hline Average........... & & & 195 & 242 & .805 & 59.5 & 19.5 \\
\hline \multicolumn{8}{|l|}{ Nov. 15, 1910: } \\
\hline \multicolumn{8}{|l|}{$\begin{array}{r}\text { Tension-equalizer unit: } \\
8^{\mathrm{h}} 57^{\mathrm{m}} \text { a. m ...... }\end{array}$} \\
\hline 915 a. m...... & 10 & 7 & 182 & 230 & .790 & 59.0 & 14.4 \\
\hline 952 a. $\mathrm{m} . .$. & 10 & 14 & 186 & 233 & .800 & 56.5 & 14.8 \\
\hline Average........ & & & 186 & 289 & .810 & 58.0 & 14.4 \\
\hline \multicolumn{8}{|l|}{$\begin{array}{l}\text { Bed calorimeter: } \\
12^{\mathrm{h}} 34^{\mathrm{m}} \text { p. } \mathrm{m} .\end{array}$} \\
\hline $\begin{array}{c}12^{\mathrm{h}} 34^{\mathrm{m}} \text { p. } \mathrm{m} . \\
142 \text { p. } \mathrm{m} .\end{array}$ & $\begin{array}{l}68 \\
60\end{array}$ & $\begin{array}{l}0 \\
0\end{array}$ & $\begin{array}{l}193 \\
189\end{array}$ & & & $\begin{array}{l}58.0 \\
60.0\end{array}$ & 15 \\
\hline $\begin{array}{lll}1 & 42 & \text { p. } m \ldots \\
2 & 42 & \text { p. } m \ldots\end{array}$ & 68 & $\begin{array}{l}0 \\
0\end{array}$ & $\begin{array}{l}189 \\
187\end{array}$ & $\begin{array}{l}230 \\
224\end{array}$ & .820 & $\begin{array}{l}60.0 \\
59.0\end{array}$ & $\begin{array}{l}15 \\
15\end{array}$ \\
\hline Average............ & 0 & $\cdots$ & 190 & 227 & .835 & 59.0 & 15 \\
\hline \multicolumn{7}{|l|}{$\begin{array}{l}V . G . \\
\text { Nov. 4, 1910: }\end{array}$} & \\
\hline $\begin{array}{c}9^{\mathrm{h}} 26^{\mathrm{m}} \text { a. } \mathrm{m} . \ldots \\
1056 \text { a. m..... }\end{array}$ & $\begin{array}{l}90 \\
60\end{array}$ & $\begin{array}{l}0 \\
0\end{array}$ & $\begin{array}{l}201 \\
204\end{array}$ & $\begin{array}{l}238 \\
240\end{array}$ & $\begin{array}{l}.845 \\
.850\end{array}$ & $\begin{array}{l}61.0 \\
59.0\end{array}$ & $\begin{array}{l}20 \\
20\end{array}$ \\
\hline Average.............. & & & 202 & 289 & .850 & 60.0 & 20 \\
\hline \multicolumn{8}{|l|}{ Tension-equalizer unit: } \\
\hline $12^{\mathrm{h}} 45^{\mathrm{m}}$ p. m...... & 14 & 50 & 193 & $\cdots$ & … & 55.5 & 15.8 \\
\hline 106 p. m..... & 15 & 30 & 200 & & & 54.0 & 16.9 \\
\hline 140 p. m...... & 15 & 2 & 196 & 251 & .785 & 57.0 & 17.6 \\
\hline 214 p. m..... & 15 & 27 & 184 & 243 & .755 & 56.0 & 17.6 \\
\hline Average........... & & & 198 & 247 & .780 & 55.5 & 17.0 \\
\hline \multirow{2}{*}{\multicolumn{8}{|c|}{$\begin{array}{l}\text { Nov, 7, 1910: } \\
\text { Bed calorimeter: }\end{array}$}} \\
\hline & & & 205 & 224 & .920 & 61.5 & 19 \\
\hline 1006 a. m..... & 60 & 0 & 211 & 221 & .955 & 56.5 & 19 \\
\hline Average............. & & & 208 & 228 & .935 & 59.0 & 19 \\
\hline Tension-equalizer unit: & & & & & & & \\
\hline $11^{\mathrm{h}} 30^{\mathrm{m}}$ a. $\mathrm{m} . \ldots$ & 15 & 7 & 207 & 222 & .930 & 54.5 & 18.1 \\
\hline 1154 a. m...... & 15 & 1 & 190 & 232 & .820 & 54.5 & 17.2 \\
\hline 1218 p. m...... & 15 & 5 & 201 & 231 & .870 & 57.0 & 18.0 \\
\hline Average............. & & & 199 & 288 & .875 & 65.5 & 17.8 \\
\hline
\end{tabular}


TABLE 12.-Respiratory exchange in comparison experiments with the bed calorimeter and the Benedict respiration apparatus (tension-egualizer unit). (Without food.)-Continued.

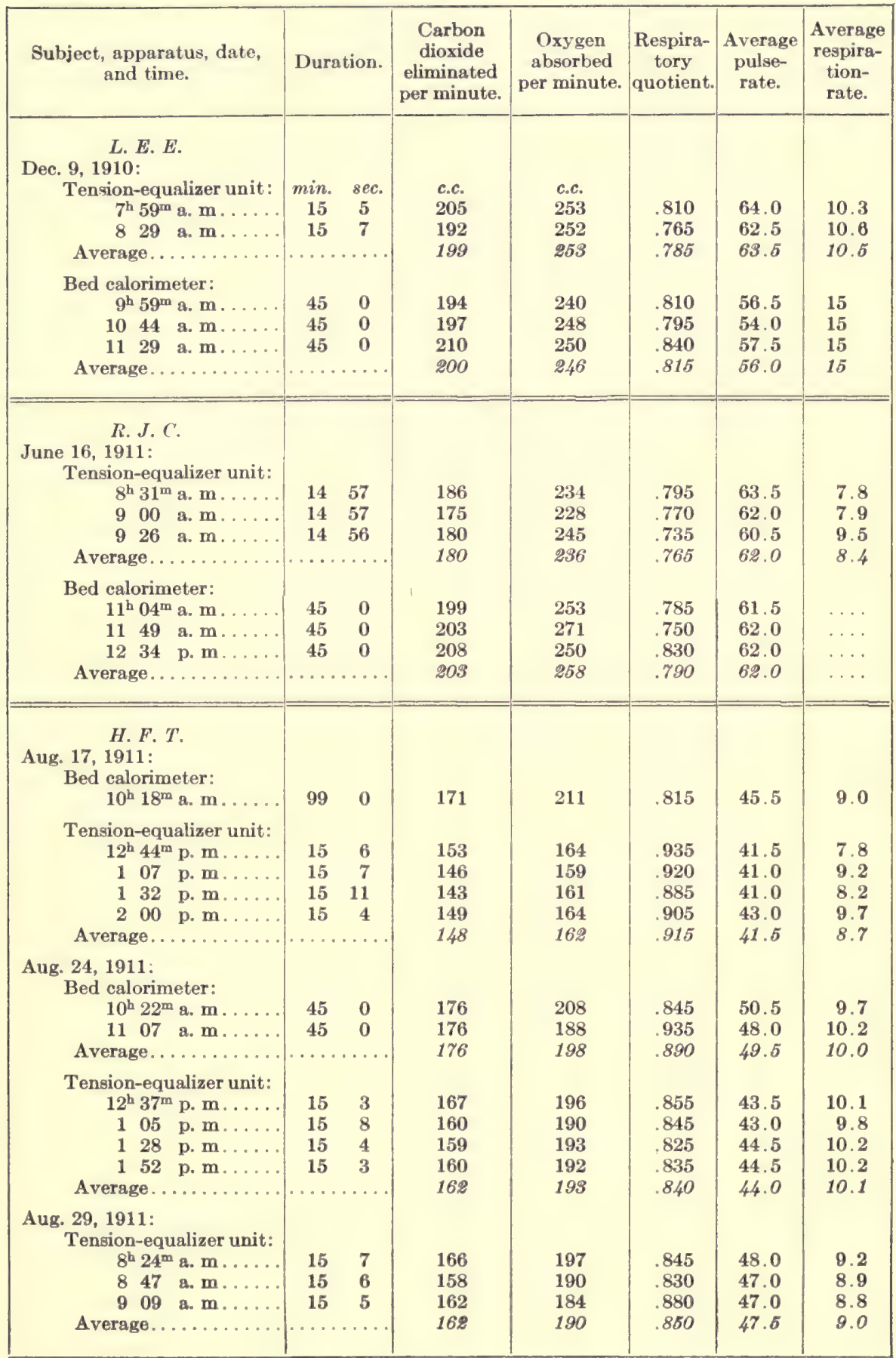


TABLE 12.-Respiratory exchange in comparison experiments with the bed calorimeter and the Benedict respiration apparatus (tension-equalizer unit). (Without food.)-Continued.

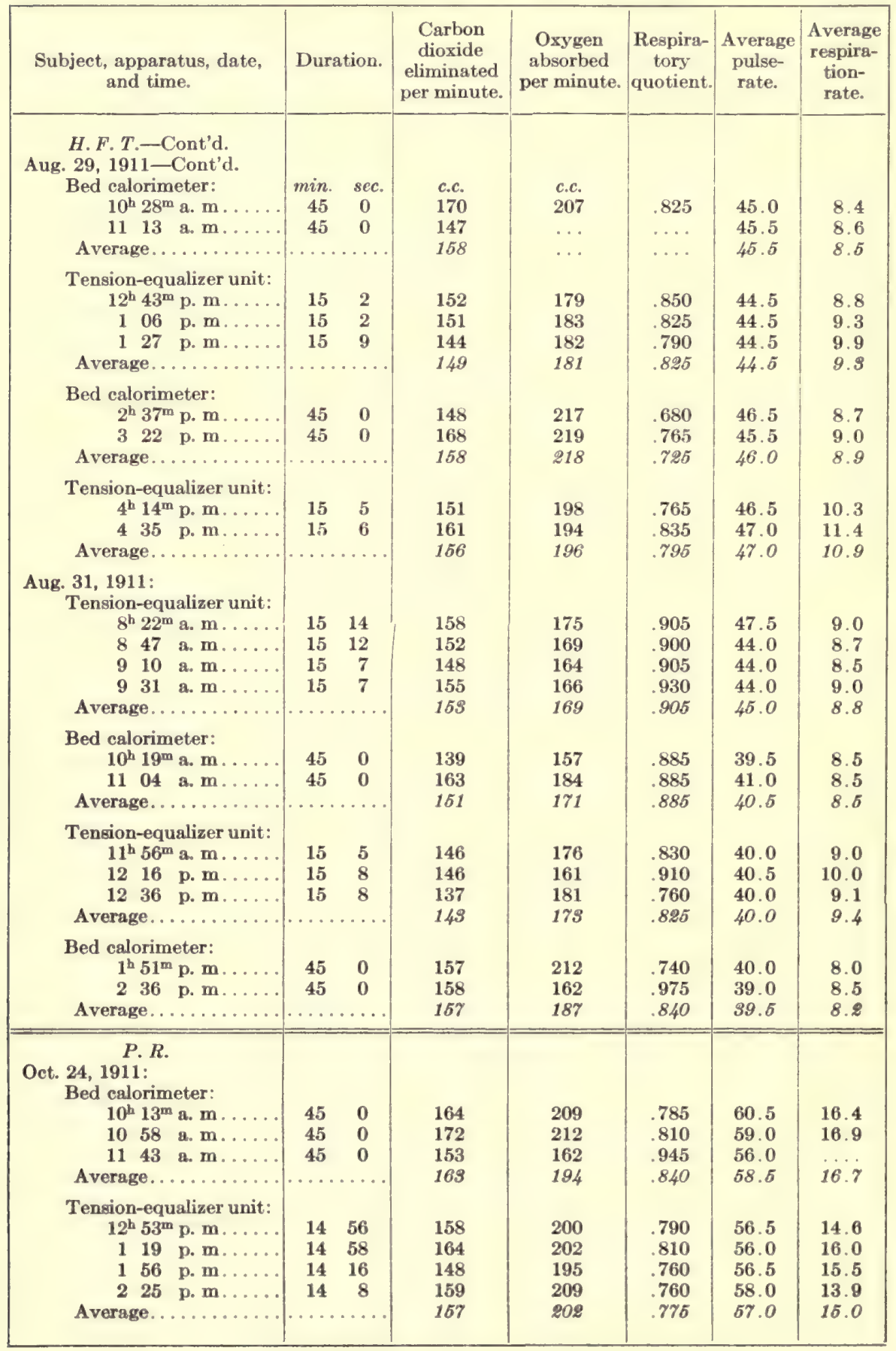


TABLE 12.-Respiratory exchange in comparison experiments with the bed calorimeter and the Benedict respiration apparatus (tension-equalizer unit). (Without food.) - Continued.

\begin{tabular}{|c|c|c|c|c|c|c|}
\hline $\begin{array}{l}\text { Subject, apparatus, date, } \\
\text { and time. }\end{array}$ & Duration. & $\begin{array}{c}\text { Carbon } \\
\text { dioxide } \\
\text { eliminated } \\
\text { per minute. }\end{array}$ & $\begin{array}{c}\text { Oxygen } \\
\text { absorbed } \\
\text { per minute. }\end{array}$ & $\begin{array}{c}\text { Respira- } \\
\text { tory } \\
\text { quotient. }\end{array}$ & $\begin{array}{l}\text { Average } \\
\text { pulse- } \\
\text { rate. }\end{array}$ & $\begin{array}{l}\text { Average } \\
\text { respira- } \\
\text { tion- } \\
\text { rate. }\end{array}$ \\
\hline 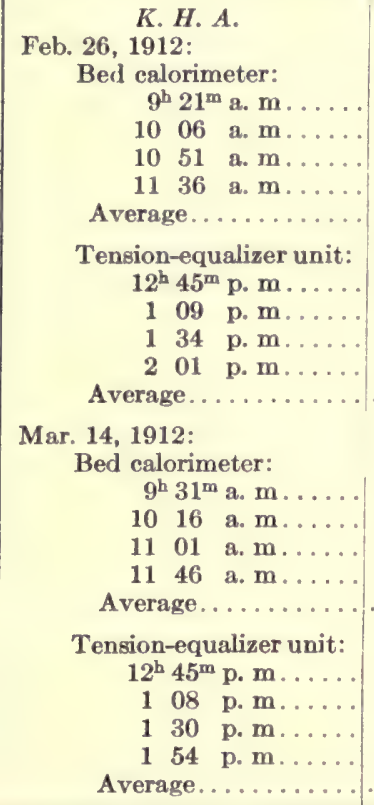 & $\mid \begin{array}{cc}\min . & \text { sec. } \\
45 & 0 \\
45 & 0 \\
45 & 0 \\
45 & 0 \\
\ldots \ldots & \ldots \\
15 & 10 \\
15 & 4 \\
15 & 5 \\
15 & 7 \\
\ldots \ldots\end{array}$ & $\begin{array}{l}c . c . \\
204 \\
199 \\
200 \\
196 \\
200\end{array}$ & $\begin{array}{l}\text { c.c. } \\
234 \\
232 \\
240 \\
229 \\
234\end{array}$ & $\begin{array}{l}.860 \\
.815 \\
.815 \\
.830 \\
.825\end{array}$ & $\begin{array}{l}47.5 \\
52.5 \\
47.5 \\
51.0 \\
49.5\end{array}$ & $\begin{array}{l} \\
\ldots \\
\ldots \\
\cdots \\
\cdots \\
\cdots\end{array}$ \\
\hline 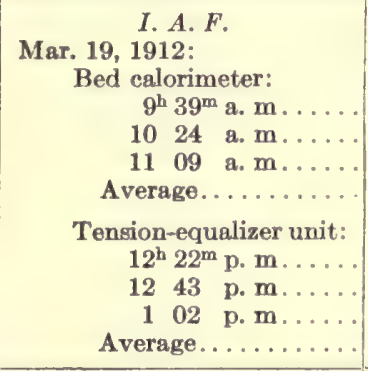 & $\begin{array}{cc}13 & 41 \\
14 & 30 \\
12 & 23 \\
\ldots & \ldots\end{array}$ & $\begin{array}{l}200 \\
174 \\
200 \\
191\end{array}$ & $\begin{array}{l}250 \\
247 \\
237 \\
245\end{array}$ & $\begin{array}{l}.800 \\
.705 \\
.845 \\
.780\end{array}$ & $\begin{array}{l}69.5 \\
69.0 \\
69.0 \\
69.0\end{array}$ & $\begin{array}{l}12.9 \\
12.4 \\
11.5 \\
12.9\end{array}$ \\
\hline 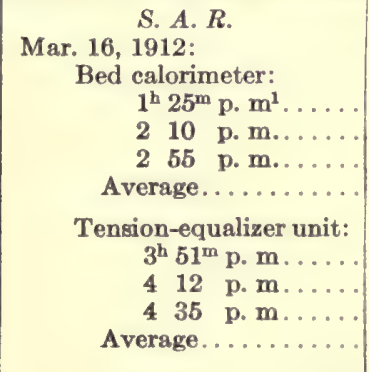 & $\begin{array}{cc}14 & 44 \\
14 & 48 \\
14 & 52 \\
\ldots & \ldots\end{array}$ & $\begin{array}{l}194 \\
181 \\
180 \\
185\end{array}$ & $\begin{array}{l}262 \\
241 \\
247 \\
250\end{array}$ & $\begin{array}{l}.740 \\
.750 \\
.730 \\
.740\end{array}$ & $\begin{array}{l}69.5 \\
67.0 \\
66.5 \\
67.5\end{array}$ & $\begin{array}{l}11.1 \\
11.0 \\
11.0 \\
11.0\end{array}$ \\
\hline
\end{tabular}

${ }^{1}$ Subject had light breakfast at $8^{\text {h }} 30^{\text {m }}$ a. $m$. 
TABLE 12.-Respiratory exchange in comparison experiments with the bed calorimeter and the Benedict respiration apparatus (tension-equalizer unit). (Without food.)-Continued.

\begin{tabular}{|c|c|c|c|c|c|c|}
\hline $\begin{array}{l}\text { Subject, apparatus, date, } \\
\text { and time. }\end{array}$ & Duration. & $\begin{array}{c}\text { Carbon } \\
\text { dioxide } \\
\text { eliminated } \\
\text { per minute. }\end{array}$ & $\begin{array}{c}\text { Oxygen } \\
\text { absorbed } \\
\text { per minute. }\end{array}$ & $\begin{array}{c}\text { Respira- } \\
\text { tory } \\
\text { quotient. }\end{array}$ & $\begin{array}{c}\text { Average } \\
\text { pulse- } \\
\text { rate. }\end{array}$ & $\begin{array}{l}\text { Average } \\
\text { respira- } \\
\text { tion- } \\
\text { rate. }\end{array}$ \\
\hline $\begin{array}{l}\text { S. A. R.-Cont'd. } \\
\text { Mar. } 20,1912 \text { : } \\
\text { Bed calorimeter: } \\
2^{\mathrm{b}} 19^{\mathrm{m}} \text { p. } \mathrm{m}^{2} \ldots \ldots \\
319 \quad \text { p. m. } \ldots \ldots \\
\text { Average......... }\end{array}$ & $\begin{array}{cc}\min . & \text { sec. } \\
60 & 0 \\
60 & 0 \\
\ldots \ldots\end{array}$ & $\begin{array}{l}\text { c.c. } \\
173 \\
184 \\
178\end{array}$ & $\begin{array}{l}c . c . \\
217 \\
215 \\
216\end{array}$ & $\begin{array}{l}.795 \\
.855 \\
.825\end{array}$ & $\begin{array}{l}55.5 \\
55.0 \\
55.5\end{array}$ & $\begin{array}{l}\ldots \\
\ldots \\
\ldots\end{array}$ \\
\hline 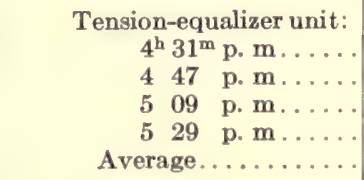 & \begin{tabular}{cc|}
11 & 13 \\
13 & 11 \\
14 & 36 \\
14 & 35 \\
$\cdots$ & $\ldots$
\end{tabular} & $\begin{array}{l}183 \\
176 \\
166 \\
167 \\
173\end{array}$ & $\begin{array}{l}231 \\
230 \\
223 \\
235 \\
230\end{array}$ & $\begin{array}{l}.790 \\
.765 \\
.745 \\
.710 \\
.750\end{array}$ & $\begin{array}{l}54.5 \\
55.5 \\
58.0 \\
58.5 \\
56.5\end{array}$ & $\begin{array}{l}11.7 \\
12.1 \\
12.0 \\
12.5 \\
12.1\end{array}$ \\
\hline $\begin{array}{l}\text { Apr. 2, 1912: } \\
\text { Bed calorimeter: } \\
9^{\mathrm{h}} 15^{\mathrm{m}} \text { a. } \mathrm{m} \ldots \ldots \\
10 \text {. } \ldots 0 \text { a. } \mathrm{m} \ldots \ldots \\
10 \quad 45 \quad \text { a. } \mathrm{m} \ldots \ldots \\
\text { Average.......... }\end{array}$ & $\begin{array}{cc}45 & 0 \\
45 & 0 \\
45 & 0 \\
\ldots & \ldots\end{array}$ & $\begin{array}{l}177 \\
186 \\
174 \\
179\end{array}$ & $\begin{array}{l}199 \\
199 \\
190 \\
196\end{array}$ & $\begin{array}{l}.890 \\
.935 \\
.920 \\
.915\end{array}$ & $\begin{array}{l}50.5 \\
54.0 \\
48.5 \\
51.0\end{array}$ & $\begin{array}{l}\cdots \\
\cdots \\
\cdots \\
\cdots\end{array}$ \\
\hline 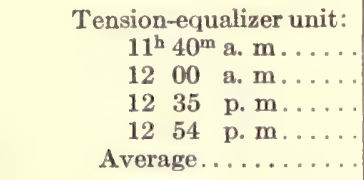 & $\begin{array}{rr}14 & 8 \\
14 & 12 \\
14 & 3 \\
14 & 5 \\
& \end{array}$ & $\begin{array}{c}2(222) \\
188 \\
192 \\
188 \\
189\end{array}$ & $\begin{array}{c}219 \\
2(206) \\
222 \\
221 \\
221\end{array}$ & $\begin{array}{c}2(1.010) \\
2(0.910) \\
.865 \\
.850 \\
.855\end{array}$ & $\begin{array}{l}63.5 \\
53.5 \\
55.0 \\
57.5 \\
57.5\end{array}$ & $\begin{array}{l}12.6 \\
11.5 \\
12.2 \\
12.4 \\
18.2\end{array}$ \\
\hline 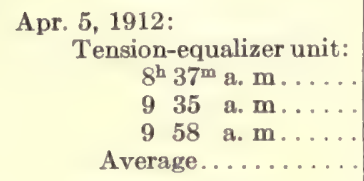 & $\begin{array}{cc}15 & 1 \\
13 & 12 \\
15 & 10 \\
\ldots \ldots & \ldots\end{array}$ & $\begin{array}{l}178 \\
168 \\
178 \\
175\end{array}$ & $\begin{array}{l}214 \\
209 \\
213 \\
212\end{array}$ & $\begin{array}{l}.835 \\
.805 \\
.830 \\
.825\end{array}$ & $\begin{array}{l}51.5 \\
52.5 \\
51.5 \\
52.0\end{array}$ & $\begin{array}{l}12.4 \\
11.5 \\
11.7 \\
11.9\end{array}$ \\
\hline 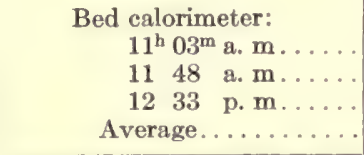 & $\begin{array}{cc}45 & 0 \\
45 & 0 \\
45 & 0 \\
\ldots \ldots\end{array}$ & $\begin{array}{l}169 \\
172 \\
183 \\
175\end{array}$ & $\begin{array}{l}198 \\
207 \\
209 \\
204\end{array}$ & $\begin{array}{l}.855 \\
.835 \\
.880 \\
.855\end{array}$ & $\begin{array}{l}49.0 \\
52.5 \\
51.0 \\
51.0\end{array}$ & $\begin{array}{l}\cdots \\
\ldots \\
\ldots \\
\ldots\end{array}$ \\
\hline 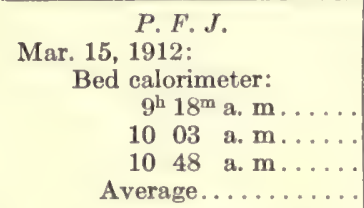 & $\begin{array}{cc}45 & 0 \\
45 & 0 \\
45 & 0 \\
\ldots & \ldots\end{array}$ & $\begin{array}{l}197 \\
190 \\
199 \\
195\end{array}$ & $\begin{array}{l}223 \\
218 \\
244 \\
928\end{array}$ & $\begin{array}{l}.880 \\
.870 \\
.815 \\
.855\end{array}$ & $\begin{array}{l}75.0 \\
72.5 \\
70.5 \\
72.5\end{array}$ & $\begin{array}{l}\cdots \\
\cdots \\
\cdots \\
\cdots\end{array}$ \\
\hline 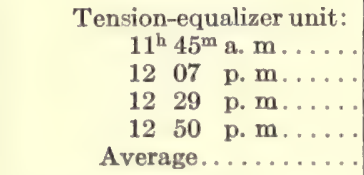 & $\begin{array}{ll}14 & 41 \\
14 & 45 \\
14 & 52 \\
14 & 54 \\
& \end{array}$ & $\begin{array}{l}187 \\
178 \\
187 \\
189 \\
185\end{array}$ & $\begin{array}{l}256 \\
242 \\
249 \\
243 \\
248\end{array}$ & $\begin{array}{l}.735 \\
.735 \\
.750 \\
.780 \\
.745\end{array}$ & $\begin{array}{l}70.5 \\
71.5 \\
70.0 \\
72.0 \\
71.0\end{array}$ & $\begin{array}{l}8.1 \\
7.4 \\
7.5 \\
9.7 \\
8.2\end{array}$ \\
\hline $\begin{array}{l}\text { Mar. } 18,1912 \\
\text { Bed calorimeter: } \\
9^{\mathrm{h}} 38^{\mathrm{m}} \text { a. } \mathbf{m} \ldots \ldots \\
1023 \text { a. } \mathbf{m} \ldots \ldots \\
1108 \text { a. } \ldots \ldots \ldots \\
\text { Average........... }\end{array}$ & $\begin{array}{cc}45 & 0 \\
45 & 0 \\
45 & 0 \\
\ldots \ldots \ldots\end{array}$ & $\begin{array}{l}210 \\
180 \\
209 \\
200\end{array}$ & $\begin{array}{l}211 \\
220 \\
224 \\
219\end{array}$ & $\begin{array}{l}.995 \\
.820 \\
.930 \\
.915\end{array}$ & $\begin{array}{l}69.0 \\
61.0 \\
69.0 \\
66.5\end{array}$ & $\begin{array}{l}\ldots \\
\cdots \\
\ldots \\
\ldots\end{array}$ \\
\hline
\end{tabular}


TABLE 12.-Respiratory exchange in comparison experiments with the bed calorimeter and the Benedict respiration apparatus (tension-equalizer unit). (Without food.)-Continued.

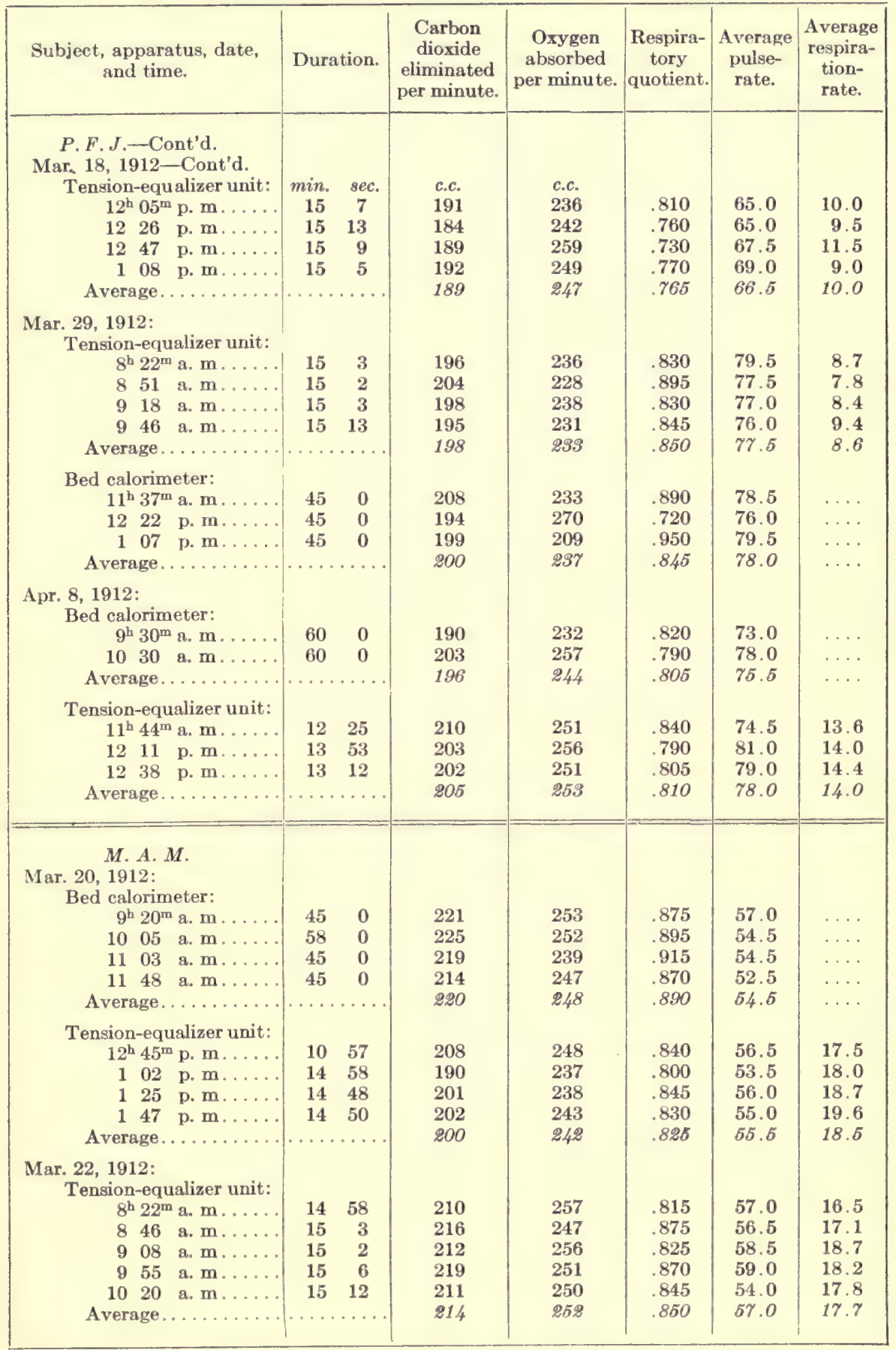


TABLE 12.--Respiratory exchange in comparison experiments with the bed calorimeter and the Benedict respiration apparatus (tension-equalizer unit). (Without food.)-Continued.

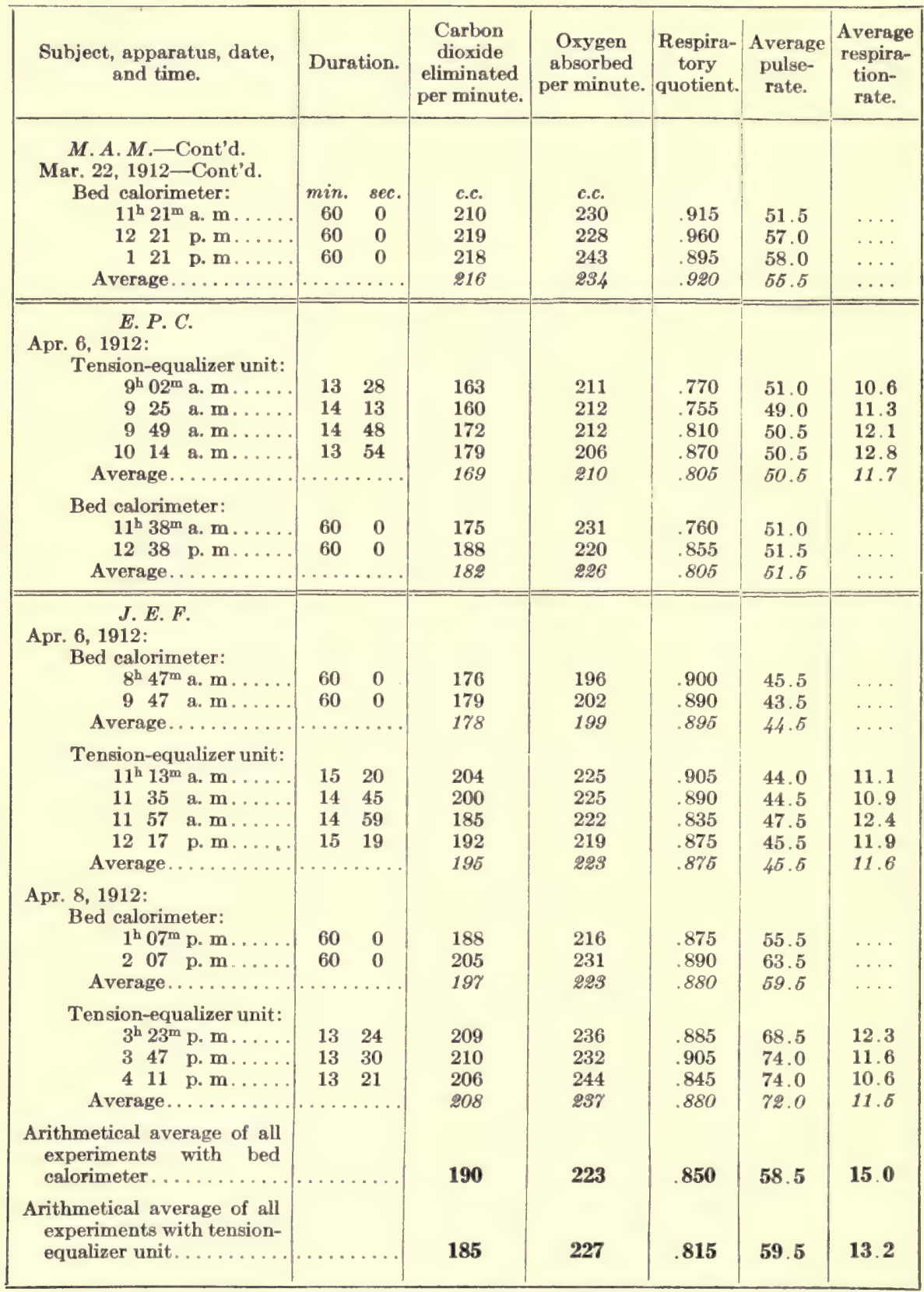

A critical examination of the table shows that in many of the experiments the respiratory quotient is noticeably higher with the bed calorimeter, irrespective of the order in which the experiments were 
made. Before discussing this apparent discrepancy, the general question of conditions under which the experiments were carried out and the various sources of error may be considered.

The chamber of the bed ealorimeter is not large enough to permit much movement by the subject, but is sufficiently large for a man of medium size to turn from one side to the other. The subjects were requested to remain quiet throughout the experimental period, and although they usually made an attempt to do this, it was very difficult for them to remain in one position for two or three hours. With the opportunity for freedom of movement afforded by the bed calorimeter there was more or less movement by the subject, and as he could be seen only through the glass window at the end of the calorimeter and a small glass porthole on the side, it was not possible to observe accurately the degree of muscular repose. Some of the subjects found themselves so comfortable in the calorimeter that it was impossible for them to keep awake. This was especially true of J. J. C. and V. G. The other subjects were awake for the greater part of the time.

During the experiments with the tension-equalizer unit, the subject lay flat upon his back, with nosepieces inserted in the nose, or a mouthpiece in the mouth. The connections with the ventilating apparatus were so short that movements of the head were not possible, and any other major movements, such as movements of the arms or legs, could easily be recorded by the observer. Accordingly, the subject lay either in a comfortable relaxed position, which would be conducive to a low metabolism, or in a condition of tenseness, which would tend to increase the metabolism.

It will be seen, therefore, that it was somewhat difficult to compare the metabolism as measured by these two apparatus, for to determine the accuracy of measurement it is necessary to assume that the metabolism to be measured is the same. With the same degree of muscular repose in both cases, other conditions being alike, the results of the measurement would agree, provided the two apparatus were equally accurate. If, on the other hand, the two forms of apparatus gave theoretically the same results, any differences in the metabolism would be due to differences in the degree of muscular repose.

In addition to this possible difference in the metabolism due to difference in the muscular repose, there are various sources of error with both the bed calorimeter and the tension-equalizer unit, which may affect the measurement of the metabolism. Those for the bed calorimeter will first be considered.

Sources of Error in Experiments with the Bed Calorimeter.

Errors in measuring the carbon-dioxide elimination.-The errors in the measurement of the carbon-dioxide elimination have to do mainly with the weighing of the absorption vessels. The carbon-dioxide absorbers 
used were extremely large for the amounts of carbon dioxide to be absorbed. The water-absorber following the carbon-dioxide absorbers weighs 18 kilograms, and it is possible to weigh it to $\pm 0.1 \mathrm{gm}$.; an error of $0.1 \mathrm{gm}$. in weighing would be equivalent to about 0.5 to 1 per cent for a period in which the carbon-dioxide elimination was $25 \mathrm{gm}$. When the results of the whole experiment are considered, the percentage error becomes very much smaller, as the experiment is usually two hours in length.

Errors in measuring the residual carbon dioxide.-In the former method of determining the residual carbon dioxide, it was possible to have a larger error in the measurement of this factor than with the later method, i. e., that by which the sample of air is taken from the outgoing air-current and analysis made by means of the Sondén-Pettersson gas-analysis apparatus. With the later method it is possible to determine the percentage of carbon dioxide in the residual air to within 0.002 per cent. With a volume of 950 liters, which is approximately the volume of the bed calorimeter, this would be equal to about 0.02 liter or $0.04 \mathrm{gm}$. This is an error far too small for consideration in this connection. That the actual composition of the outgoing air undergoes mathematically the same fluctuations in percentage composition as does the entire air of the chamber has been demonstrated by analyzing samples taken at different points in the chamber, the results showing no variations due to the difference in location.

Error in determining the water-vapor in the chamber. - The method formerly used in determining the amount of moisture in the chamber was that of diverting a small stream of air from the outgoing air-current through a set of U-tubes, one of which contained sulphuric acid and pumice stone. Twenty liters of air were passed through these U-tubes and the increase in weight of the tubes was noted; from this increase in weight and the volume of the sample, the amount of moisture inside of the chamber was calculated. The errors in this method have to do mainly with the errors in weighing. The U-tubes weighed about 60 $\mathrm{gm}$., and the possible error was 1 to $5 \mathrm{mg}$. The amount of moisture absorbed in the 20-liter sample, although varying, was usually about $100 \mathrm{mg}$. It will be seen, therefore, that the error might be \pm 5 per cent. This error chiefly affects the determination of the oxygen consumption, as the calculation of the amount of oxygen present in the apparatus at the end of an experimental period necessitates the deduction of the volume occupied by the water-vapor in the chamber air; consequently any error in determining the latter volume results in a similar error in the calculated volume of oxygen.

The more recent method of determining the residual water-vapor, that is, the use of the wet- and dry-bulb thermometer inside of the chamber, gives determinations with a high degree of accuracy. The determinations obtained by this method have been carefully checked by duplicate determinations made with the Sondén hygrometer and the results agreed very closely. 
Errors in the determination of the residual oxygen.-The principal error in experiments with the bed calorimeter is incidental to the measurement of the oxygen consumption. The oxygen consumption of the subject is determined from the amount admitted to the chamber during the experimental period and from the changes in the oxygen content of the air in the chamber. The chief difficulty met with is in the measurement of the residual oxygen, especially as this measurement is affected by the combined errors in the determination of the residual water-vapor and the residual carbon dioxide.

The measurement of the residual oxygen is also affected by errors in the determination of the average temperature of the air in the apparatus. The average temperature changes of the apparatus are obtained from readings of the changes in resistance of a set of copper-wire resistance thermometers. These are placed at five different points adjacent to the wall of the chamber and consequently give only the changes in the temperature of the layer of air next to the walls. When the subject is inside the chamber there is a thermal gradient of approximately $37^{\circ} \mathrm{C}$. (the temperature of the man's body) to about $12^{\circ} \mathrm{C}$. for the air next to the cooling pipes. If the man moves he may produce a slight heating of the air about him, but this heating effect will not be shown by a variation in the thermometer readings. It will, however, affect the volume of the air in the chamber, causing it to expand slightly, with a consequent rise in the rubber diaphragm of the tensionequalizer or the spirometer bell. If this increase in volume occurs just prior to the end of a period, and this record is used in calculating the volume to $0^{\circ} \mathrm{C}$. and $760 \mathrm{~mm}$., the average temperature reading will be too low, so that the results supposedly correct will indicate an oxygen content of the chamber which is actually too large. This difficulty in the accurate measurement of the average temperature of the air in the chamber has always been recognized in observations made with the respiration calorimeter and has been thoroughly discussed in another publication. ${ }^{1}$ It has, however, become even more apparent as experience with the apparatus has accumulated.

To prevent such errors in the measurement of the residual oxygen, the subjects have been cautioned to remain perfectly quiet during the last 15 minutes of an experimental period. Various interpretations of this request have been made by the different subjects and undoubtedly some have made major movements which resulted in erroneous determinations of the oxygen consumption. The importance of this bodymovement control at the end of an experimental period became so marked that in a series of night experiments with a subject who fasted 31 days a procedure was adopted to register the change in volume during the last 5 minutes. ${ }^{2}$ A recording device was attached to the spirom- 
eter which would give a graphic record of the movements of the spirometer bell during the last 5 minutes of the period. An assistant, by pressing a key, connected this recording device in circuit with a signal magnet when the kymograph drum was started; the key was then pressed each minute until the end of the period. If the subject was absolutely quiet and there was no marked change in the temperature or the barometric pressure, the decrease in volume would be relatively constant. If, however, the pointer showed an upward movement of the spirometer bell, the experimental period was continued until the record showed that the subject was perfectly quiet. In this way the expansion of the air due to any movement of the subject was controlled and the volume of the apparatus as shown by the spirometer at the end of the period was the actual volume.

The measurement of the average temperature of the air may also be affected by the fact that the subject, when he first enters the apparatus, warms the clothing with which he is covered and then warms the air next to the clothing; accordingly, during the preliminary period and possibly during the first period of the experiment, there may be a gradual warming of the air in the chamber which is not indicated by the thermometers. The record of the temperature at the beginning of the experimental period would therefore be lower than the actual average temperature of the apparatus, since the thermal gradient between the man and the air next to the wall had not been established. The resulting measurement of the oxygen content of the air in the chamber would therefore be too large, the calculated oxygen consumption too small, and the respiratory quotient too high. The chamber itself is actually a large air thermometer, the changes of which are shown by the movements of the diaphragm of the tension equalizer or the bell of the spirometer.

The difficulties met with in securing the average temperature and the fact that the errors in the determination of the residual carbon dioxide and water-vapor all affect the calculation of the oxygen content of the air make the determinations of the oxygen consumption extremely difficult and the experimental period should be of such length that these errors would play a very small percentage rôle. Many of the experiments in this comparison are relatively short and it would undoubtedly have been desirable to have had them longer. It was impracticable, however, to keep the subject inside the chamber for a longer period and still have him sufficiently quiet for comparison purposes.

Sources of Error in Experiments with the Benedict Regpiration Apparatus.

As has already been shown in the description of the Benedict respiration apparatus, ${ }^{1}$ the principle is exactly the same as that of the bed calorimeter, except that it is applied on a much smaller scale. There is 
no chamber and the man breathes into the apparatus by means of a mouthpiece or nosepieces. The same errors apply to the measurements of the various factors, except that with this apparatus the effect of muscular activity on the temperature of the air in the apparatus plays no rôle. The measurements are affected, however, by changes in the barometric pressure or in the temperature of the air in the apparatus.

Errors due to variations in barometric pressure and temperature of the apparatus.-The total volume of the spirometer unit is about 10 liters; the volume of the tension-equalizer unit is somewhat less. Assuming the volume of the apparatus to be 10 liters, a variation of $1 \mathrm{~mm}$. in the barometric pressure would change the total absolute volume 0.013 liter, but a change of $1 \mathrm{~mm}$. in 15 minutes, which is the ordinary length of an experimental period with this apparatus as used in the Nutrition Laboratory, is an enormous barometric change under ordinary conditions. Should there be such a change in the barometric pressure, however, the error in the determination of the oxygen consumption would equal but 1 c.c. per minute.

A change of $1^{\circ} \mathrm{C}$. in the temperature of the apparatus results in a change in the volume of 37 c.c., or a little over 2 c.c. per minute in a 15-minute period. The total change in the temperature of the apparatus is rarely over $1^{\circ} \mathrm{C}$., so that omitting both the reading of the barometer at the beginning and end of an experiment and the record of the change in temperature of the apparatus would produce an error too small to be ordinarily considered.

Errors in determining the carbon-dioxide elimination and oxygen consumption.-The errors in weighing may be $\pm 0.01 \mathrm{gm}$. If the meter is used for measuring the oxygen, the error may be larger than this, i. e., about \pm 1 per cent, which would be equivalent to 2 c.c. per minute with an oxygen consumption of 225 c.c.

There is also a possibility of error due to the incomplete absorption of carbon dioxide if the apparatus is not properly controlled. If the soda-lime used in the carbon-dioxide absorber is not completely efficient, the carbon dioxide may accumulate in the system instead of being absorbed. This source of error has, however, been eliminated during the past two or three years by the test for the presence of carbon dioxide in the air after it leaves the soda-lime container.

Occasionally, through carelessness, it may happen that the water absorbers in the lower part of the apparatus are deficient, and some of the water-vapor in the air from the lungs of the man escapes absorption. In this case, it will be absorbed by the sulphuric acid in the waterabsorber following the soda-lime container. Since this water-absorber should only absorb the water taken up by the air from the moist sodalime, and the amount of carbon dioxide produced is determined by noting the increase in the combined weights of the soda-lime container and the following sulphuric-acid container, the record of the carbon 
dioxide will be larger than that actually produced. By means of a large number of experiments, a system of controls has been introduced which makes it practically impossible for such inefficiency to occur. In these experiments the amount of water which it is possible for these absorbers to retain has been carefully determined and it has been made a part of the experimental routine to change the absorbers before they reach this point. So long as this routine is followed, there will be no danger of an incomplete absorption of the water-vapor. It must be remembered that the rate of ventilation must be the same as that when the efficiency tests were carried out. It is obvious that in all experiments the air entering the soda-lime container must be dried to the same degree of humidity as the air leaving its accompanying waterabsorber. Usually this means absolute desiccation, but with extremely high rates of ventilation and with large quantities of water in the air, slight traces of water-vapor may escape from the air-drier preceding the carbon-dioxide absorber and an equivalent amount may escape absorption in the water-absorber following the soda-lime container. With two air-driers used in series, the second one, even under the most exacting conditions with muscular work, rarely gains over $0.1 \mathrm{gm}$.

Physiological influences upon the measurement of the respiratory exchange.-Aside from the sources of error in the actual manipulation of the apparatus, there is also the possibility of physiological influences. When the subject is inside the chamber, all of the carbon dioxide eliminated and all of the oxygen consumed are measured; with the subject on the respiration apparatus, the assumption must be made that the content of the respiratory tract of the man is the same at the end of the experiment as at the beginning - that is, the experiment must be begun and ended in such a manner that the subject has in his lungs exactly the same amount of air at both periods. The end of a normal expiration has always been used for the beginning of the experiment. This assumes that the man breathes always to the same depth and that the air left in the lungs is the same in volume at the end of each expiration. If the subject begins the experiment with deep breathing and ends the experiment with shallow breathing, it is quite possible that there may be a difference in the total volume of the lungs; in that case, the determination of the oxygen consumption will be in error. Later experiments with the more recent type of this apparatus have shown that in the majority of experiments the subjects breathed so regularly that the assumption that there was the same amount of air in the lungs at the beginning and end of the experiments is justifiable. There are exceptions to this, however, and it is quite possible that many irregularities which have been obtained with the earlier form of the respiration apparatus may be due to this fact. As a control on the regularity of the breathing, a pneumograph was usually placed around the chest and a graphic record secured. It is very difficult, however, 
to obtain an adequate idea of minute differences in the volumes of respiration by means of the pneumograph and an error of 40 c.c. would be equivalent to an error of 2 to 3 c.c. per minute in the measurement of the oxygen consumption.

If only one 15-minute experiment were considered, no definite conclusions could be drawn. If, however, three 15-minute experiments are made consecutively and the results obtained agree closely, it may be concluded that the error due to the variations in volume of air in the lungs is extremely small or else it is a constant one, but from all of our experience with respiration experiments, changes in the volume of air in the lungs have rarely to be considered.

If the particular form of respiration apparatus used caused any change in the respiration, this would also lead to differences in the determination of the respiratory exchange. For example, if the conditions obtaining with this apparatus tended to make the subject breathe deeper and more rapidly than under ordinary conditions, it may be seen that the results would be abnormal because of the abnormal character of the breathing. When the subject is in the bed calorimeter, there is no mechanical reason for his breathing abnormally. The carbon-dioxide content of the air in the chamber may, however, be higher than normal and, indeed, may be as high as 1 per cent. While this would lead to an increase in the volume of respiration, the increase would not be so great as to alter the metabolism. With the respiration apparatus, air free from carbon dioxide is supplied to the subject, but there is a possibility that the mechanics of the apparatus may produce abnormal ventilation of the lungs. During this series of comparison experiments a test was made of this point in several of the bed-calorimeter experiments ${ }^{1}$ by having subjects breathe through the nosepieces and three-way valve of the respiration apparatus, which had been detached for the purpose and placed inside the bed calorimeter. The results indicated that the use of the nosepieces and valve had practically no influence upon the respiratory exchange. The only effect noted was due to the fact that the subject was obliged to lie upon his back during the whole period without changing his position; he was therefore more weary at the end of the experiment than when he had more freedom of movement.

\section{Diffenences in the Indvidual Comparisons.}

Considering all of these possible sources of error in conducting experiments with the two apparatus, it may be said that those with the bed calorimeter are mainly of physical or technical origin and with the respiration apparatus are principally of physiological origin. With these possibilities in mind, the differences in the individual comparisons may be considered. These differences are shown in table 13, in 
which the values obtained with the bed calorimeter have been used as a basis of calculation and the difference between these results and those obtained with the respiration apparatus is expressed as plus or minus, according to whether the latter are higher or lower than those obtained with the bed calorimeter.

TABLE 13.-Variations of average results obtained with the Benedict respiration apparatus (tension-equalizer unit) from those obtained with the bed calorimeter.

\begin{tabular}{|c|c|c|c|c|c|c|}
\hline Subject. & Date. & $\begin{array}{c}\text { Carbon } \\
\text { dioxide } \\
\text { eliminated } \\
\text { per minute. }\end{array}$ & $\begin{array}{c}\text { Oxygen } \\
\text { absorbed } \\
\text { per minute. }\end{array}$ & $\begin{array}{c}\text { Respiratory } \\
\text { quotient. }\end{array}$ & $\begin{array}{c}\text { Average } \\
\text { pulse-rate. }\end{array}$ & $\begin{array}{l}\text { Average } \\
\text { respira- } \\
\text { tion-rate. }\end{array}$ \\
\hline \multirow{3}{*}{ F. G. B... } & 1909 & c.c. & c.c. & & & \\
\hline & $\int \mathrm{Mar}, 1$ & -15 & -9 & -0.03 & +1.5 & -2 \\
\hline & $\operatorname{Mar}_{1910} 2$ & -11 & -12 & 0 & +2 & -2 \\
\hline F. G. B.......... & $\begin{array}{l}\text { Nov. } 15 \\
1909\end{array}$ & -31 & -20 & -.055 & -2.5 & -4.9 \\
\hline J. A. R........... & Mar. 20 & +21 & +28 & -.02 & +4.0 & -4.5 \\
\hline \multirow[t]{4}{*}{ 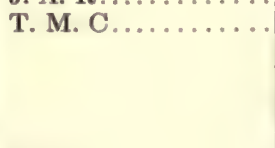 } & Mar. 23 & +1 & -4 & +.02 & +7.0 & 0 \\
\hline & 1910 & -1 & -3 & +.005 & +6.0 & 0 \\
\hline & (July 12 & -1 & +6 & -.04 & +3.5 & -0.4 \\
\hline & Nov. 16 & -1 & +6 & -.025 & +7.0 & -2.8 \\
\hline \multirow{5}{*}{ J. J. C. . . . . . . } & Nov. 3 & +1 & +10 & -.035 & +1.5 & +2.6 \\
\hline & Nov. 8 & +6 & +22 & -.06 & +1.0 & +2.5 \\
\hline & Nov. 10 & -11 & -1 & -.045 & +1.0 & -2.8 \\
\hline & & +3 & +21 & -.065 & +1.5 & +1.5 \\
\hline & Nov, 15 & -4 & +2 & -.025 & -1.0 & -0.6 \\
\hline \multirow{3}{*}{$\begin{array}{l}\text { V. G......... } \\
\text { L. E. E. . } \ldots \ldots \ldots\end{array}$} & Nov. 4 & -9 & +8 & -.07 & -4.5 & -3.0 \\
\hline & Nov. 7 & -9 & +6 & -.04 & -3.5 & -1.2 \\
\hline & ${ }_{1911}^{\text {Dee. }} 9$ & -1 & -7 & -.03 & +7.5 & -4.5 \\
\hline \multirow[t]{2}{*}{ 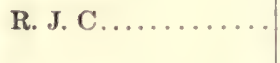 } & June 16 & -23 & -22 & -.025 & 0 & \\
\hline & (Aug. 17 & -23 & -49 & +.10 & -4.0 & -0.3 \\
\hline \multirow{4}{*}{ H. F. T. . } & Aug. 24 & -14 & -5 & -.05 & -5.5 & +0.1 \\
\hline & Aug. 29 & & -24 & +.055 & $\begin{array}{r}0.5 \\
+\quad 0.5\end{array}$ & $\begin{array}{r}1.0 \\
+1.0\end{array}$ \\
\hline & Aug. 31 & +2 & -2 & +.02 & $\begin{array}{r}+4.5 \\
\end{array}$ & +0.3 \\
\hline & & -14 & -14 & -.015 & +0.5 & +1.2 \\
\hline P. R... & $\begin{array}{l}\text { Oct. } 24 \\
1912\end{array}$ & -6 & +8 & -.065 & -1.5 & -1.6 \\
\hline \multirow{2}{*}{ K. H. A. . } & Feb. 26 & -12 & +2 & -.060 & -4.5 & $\ldots \ldots$ \\
\hline & Mar. 14 & -3 & +10 & -.05 & -2.5 & $\ldots$ \\
\hline \multirow[t]{2}{*}{ I. A. F.......... } & Mar. 19 & -7 & +2 & -.035 & +4.5 & $\ldots \ldots$ \\
\hline & Mar. 16 & -9 & +10 & -.07 & -1.5 & $\ldots$ \\
\hline \multirow{3}{*}{ S. A. R.......... } & Mar. 20 & -5 & +14 & -.075 & +1.0 & $\ldots$ \\
\hline & Apr. 2 & +10 & +15 & -.06 & +6.5 & $\ldots$ \\
\hline & Apr. 5 & 0 & +8 & -.03 & +1.0 & $\ldots$. \\
\hline \multirow{4}{*}{ 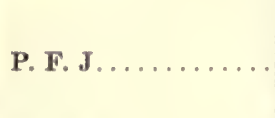 } & Mar. 15 & -10 & +20 & -.11 & -1.5 & $\ldots$ \\
\hline & Mar. 18 & -11 & +28 & -.15 & 0 & $\ldots .$. \\
\hline & Mar. 29 & -2 & -4 & +.005 & -0.5 & $\ldots$ \\
\hline & Apr. 8 & $+\overline{9}$ & +9 & +.005 & +2.5 & $\ldots$. \\
\hline \multirow{5}{*}{ 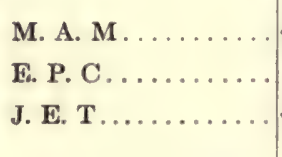 } & $\int$ Mar. 20 & -20 & -6 & -.065 & $\begin{array}{r}1.0 \\
\end{array}$ & $\ldots$ \\
\hline & Mar. 22 & -2 & +18 & -.07 & +1.5 & $\ldots$. \\
\hline & Apr. 6 & -13 & -16 & 0 & -1.0 & .... \\
\hline & $\{$ Apr. 6 & +17 & +24 & -.02 & +1.0 & $\ldots \ldots$ \\
\hline & Apr. 8 & +11 & +14 & 0 & +12.5 & $\ldots$ \\
\hline \multicolumn{2}{|l|}{ Average } & 9 & 13 & 0.045 & 3.0 & 1.8 \\
\hline
\end{tabular}


An inspection of the data given in table 13 shows that in many of the comparisons the differences between the results obtained with the two forms of apparatus are considerable. With the values for the carbon-dioxide production, it will be found that in 24 out of 39 comparisons $^{1}$ the average values with the respiration apparatus varied more than plus or minus 5 c.c. per minute; in only 6 of these experiments was the carbondioxide production higher with the respiration apparatus.

Adopting the same limits of difference with values for the oxygen consumption, we find that in 30 experiments this factor varied more than plus or minus 5 c.c. per minute; in 20 of these experiments the values are greater with the respiration apparatus.

The respiratory quotient shows differences greater than plus or minus 0.04 in 18 experiments; in only 2 of these experiments was this value higher with the respiration apparatus.

The differences in the values for the pulse-rate varied somewhat widely, but not much stress can be laid upon them, owing to the difficulty in making records of the pulse-rate in the bed calorimeter. It must be pointed out that unless the records of the pulse-rate are sufficiently frequent to represent a true average, the averages can not be used as possible indications of differences in the respiratory exchange.

If each comparison is considered as a whole, the following may be accepted as giving comparable results with the two apparatus: T. M. C., all experiments; J. J. C., November 3 and 15; V. G., November 4; the one experiment with L. E. E.; H. F. T., August 31, first experiment; the one experiment with I. A. F.; S. A. R., April 5; and P. F. J., March 29. In addition to these there are nine other experiments in which the respiratory quotient shows good agreement. As pointed out previously, the primary cause in the differences in the values for the carbondioxide production and oxygen consumption is the difference in the muscular activity, while it is believed that the primary cause of the differences in the respiratory quotient is the difficulty of measuring correctly the oxygen consumption in the bed calorimeter.

An extraordinarily good opportunity for comparing respiratory quotients obtained with the bed calorimeter and the Benedict respiration apparatus presented itself in the measurement of the respiratory exchange of a man who fasted for 31 days. Such a comparison is made in table 14, which shows the respiratory quotients obtained each night while the subject was in the bed calorimeter and the respiratory quotients obtained with him immediately after he was removed from the chamber. ${ }^{2}$ The quotients given for the bed calorimeter are values for the entire night period, i. e., from about $10 \mathrm{p}$. m. to $7 \mathrm{a} . \mathrm{m}$., while those given for the respiration apparatus are the averages of three

\footnotetext{
'While there were only 36 experimental days, it is considered that on three of these days two individual comparisons were made.

The experiments in the morning were with the spirometer unit.
} 
15-minute experiments each morning. This man was an unusually good subject, as in the morning experiments he was absolutely quiet and his respiration was remarkably uniform. The period in the bed calorimeter was so long that the possibilities of error previously noted played scarcely any rôle. The values obtained show a high degree of uniformity, indicating that the respiratory exchange was practically the same with both apparatus so far as the relation between the carbon dioxide and the oxygen values is concerned. The amount of the gaseous exchange can not be considered in the experiments with this particular subject, as in the bed calorimeter he was asleep more or less of the time and with the respiration apparatus he was wide awake.

TABLE 14.-Respiratory quotients for a fasting man in experiments with the bed calorimeter and the Benedict respiration apparatus (spirometer unit).

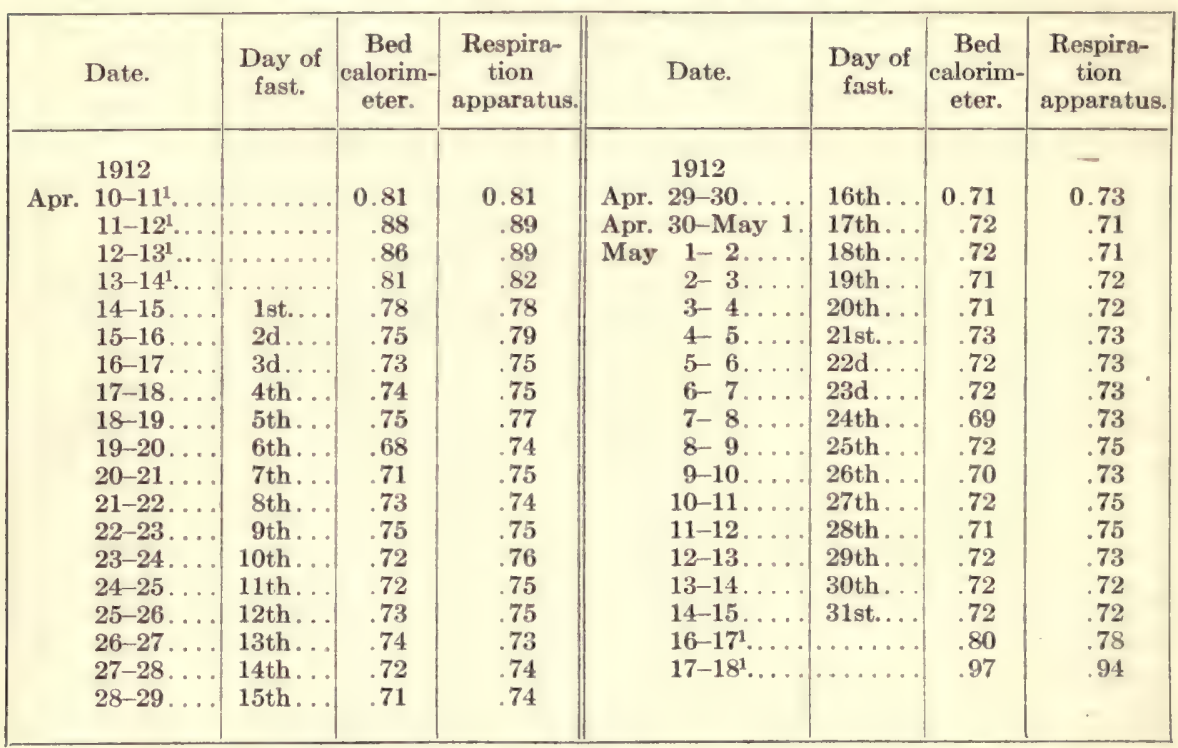

${ }^{1}$ On the days preceding and following the fast the night experiments were made after the ingestion of food. The subject was without breakfast during the morning respiration experiments.

In conclusion it can be stated that agreement between the respiratory quotients obtained with the bed calorimeter and the respiration apparatus is possible and that comparable values for both carbon dioxide and oxygen can be secured with both apparatus. It is difficult, however, to secure such agreement, as it is not easy to make sure of the same degree of muscular activity in experiments with both apparatus or to determine correctly the oxygen consumption of a subject while inside the bed calorimeter.

At the moment of writing, experiments are in progress with a new chamber designed by Professor Benedict primarily for the determination of the respiratory quotient. The results of these experiments will 
show the possible differences which may exist between the respiratory quotient for a man in a chamber perfectly free to breathe naturally and that for a man connected with a respiration apparatus.

\section{THE TWO TYPES OF THE BENEDICT RESPIRATION APPARATUS (THE TENSION-} EQUALIZER UNIT AND THE SPIROMETER UNIT).

During the later comparison of the bed calorimeter and the tensionequalizer type of the Benedict universal respiration apparatus, a modified form - the spirometer unit ${ }^{1}$-was developed, perfected, and put into use. While the spirometer unit was not employed in the comparison with the bed calorimeter, it was used in some of the comparisons with other respiration apparatus, and it was therefore considered advisable to compare the respiratory exchange as determined by both the tension-equalizer unit and the spirometer unit. If the two types of apparatus gave comparable results, it could logically be concluded that results found comparable with either the tension-equalizer unit or the spirometer unit would also be comparable with those obtained with the bed calorimeter.

Several comparison experiments were accordingly carried out with the two types of this respiration apparatus. The general routine and accessory apparatus were practically the same as in the comparisons of the tension-equalizer unit and the bed calorimeter. The subjects were in the post-absorptive condition and always lay upon a couch; the preliminary period was approximately 30 minutes long, the experimental periods usually being 15 minutes in length. As the two types of apparatus were placed side by side, the subject, with couch, could be readily moved from one apparatus to the other without muscular activity on his part. The tension-equalizer unit and the spirometer unit were alternated or used in series, $i . e$., several periods carried out with one apparatus followed by several periods with the other. Pneumatic nosepieces were used throughout the comparison.

The pulse-rate was recorded by means of a Bowles stethoscope, and the respiration-rate with a tambour, pointer, and kymograph attached to a chest pneumograph. Graphic records of the activity were obtained with a pneumograph fastened about the hips of the subject and connected with a tambour and kymograph. All of the young men had previously acted as subjects in the comparison experiments with the bed calorimeter and the tension-equalizer unit. Three of these, K. H. A., J. B. T., and J. K. M., were assistants in the Nutrition Laboratory; the others were medical students. The statistics of the 9 comparisons follow. 
STATISTICS OF EXPERIMENTS.

H. B. L., March 5, 1912.-Tension-equalizer unit, 4 periods; spirometer unit, 4 periods; the two forms of apparatus alternated. Subject frequently complained that he was getting tired and said that while he noticed no particular difference between the two types of apparatus, he found it somewhat difficult to remain quiet during the whole experiment. Respiration-rate fairly regular.

S. A.R., March 2S, 1912.-A light breakfast; experiment began $1^{\text {h }} 40^{\mathrm{m}}$ p. m. Tension-equalizer unit, 5 periods; spirometer unit, 2 periods; first 3 periods with tension-equalizer unit, then apparatus alternated for 4 periods. In the first and second periods the subject complained that the air supplied seemed somewhat dry; water was added to the moistener ${ }^{1}$ after the second period; the subject then stated that he found breathing much easier. Pulserate fairly uniform. Respiration-rate for the most part uniform, but character varied slightly at times. In last period of experiment (with tension-equalizer unit), the depth of respiration varied somewhat, being wave-like and approximating Cheyne-Stokes respiration, but the pneumograph gave no idea of the quantitative variations.

S. A. R., A pril 1, 1912.-Tension-equalizer unit, 5 periods; spirometer unit, 4 periods; first two periods with tension-equalizer unit in series, then apparatus alternated. Respiration regular in rate and depth.

J. A. F., March 26, 1912.- Spirometer unit, 3 periods; tension-equalizer unit, 3 periods; apparatus alternated throughout experiment. Subject not mcuh accustomed to apparatus, as he had been experimented upon but once before. Pulse-rate uniform in the individual periods. Respiration regular throughout experiment.

K.H.A., May 21, 1912.- Spirometer unit, 3 periods; tension-equalizer unit, 3 periods; apparatus alternating throughout experiment. Subject stated that he noted no difference between the two apparatus. He also said that at the beginning of the first period with each apparatus he noticed a slight odor, but that this soon passed away. Pulse-rate in individual periods uniform. Respiration uniform in character and depth, except that in the second period with the spirometer unit there was a tendency to irregularity in depth.

K. H. A., May 25, 1912.- Spirometer unit, 3 periods; tension-equalizer unit, 3 periods; apparatus alternating throughout experiment; preliminary period, 39 minutes. Subject stated that the nosepieces in the first period were inflated too much and therefore fitted too closely. Pulse-rate in individual periods uniform; respiration exceptionally uniform throughout the experiment.

J. B. T., May 27, 1912.- Spirometer unit, 2 periods; tension-equalizer unit, 3 periods; first and fourth periods, spirometer unit; remaining periods, tensionequalizer unit. Pulse-rate uniform in individual periods; respiration uniform in rate and depth.

J. B. T., May 29, 1912.-Spirometer unit, 2 periods; tension-equalizer unit, 3 periods; first and second periods with spirometer unit; remaining periods with tension-equalizer unit. In the fourth period (tension-equalizer unit) the barium-hydroxide test ${ }^{2}$ showed that the carbon dioxide had not been wholly absorbed from the air and the results obtained for the carbon-dioxide production are therefore in error. In the last period (same apparatus) the valve was not turned soon enough at the beginning of the period and the result for the oxygen consumption is therefore too low. The respiration in the entire experiment was very uniform in character. 
J. K. M., May 28, 1912.-Spirometer unit, 2 periods; tension-equalizer unit, 2 periods; apparatus alternated. Subject drowsy toward the end of the experiment and it was difficult to keep him awake. Pulse-rate uniform in most of the periods except in the last period with the spirometer unit, when the pulse-rate was higher in the first part of the period than later. Respiration uniform in character and depth throughout experiment.

\section{DISCUSSION OF RESULTS.}

In table 15 the results are given not only for each period of the experiment but also an average for each apparatus in the individual experiments and for all of the periods in the 9 comparisons. The data in the table include the time of beginning the periods, the averages for the carbon-dioxide elimination and oxygen consumption, the respiratory quotient, and the average pulse- and respiration-rates. The average values obtained with the two methods are as follows: Carbon-dioxide elimination, 197 c.c. for the tension-equalizer unit and 198 c.c. for the spirometer unit; oxygen consumption, 231 c.c. and 233 c.c. respectively; respiratory quotient, 0.855 and 0.850 ; pulse-rate, 58.5 and 59.5 ; and the respiration-rate, 12.8 and 14.1. These grand averages show an extraordinarily good agreement.

TABLE 15.-Respiratory exchange in comparison experiments with the two types of the Benedict respiration apparatus. (Without food.)

\begin{tabular}{|c|c|c|c|c|c|}
\hline $\begin{array}{l}\text { Subject, apparatus, date, } \\
\text { and time. }\end{array}$ & $\begin{array}{c}\text { Carbon } \\
\text { dioxide } \\
\text { eliminated } \\
\text { per minute. }\end{array}$ & $\begin{array}{c}\text { Oxygen } \\
\text { absorbed } \\
\text { per minute. }\end{array}$ & $\begin{array}{c}\text { Respira- } \\
\text { tory } \\
\text { quotient. }\end{array}$ & $\begin{array}{c}\text { Average } \\
\text { pulse- } \\
\text { rate. }\end{array}$ & $\begin{array}{l}\text { Average } \\
\text { respira- } \\
\text { tion } \\
\text { rate. }\end{array}$ \\
\hline 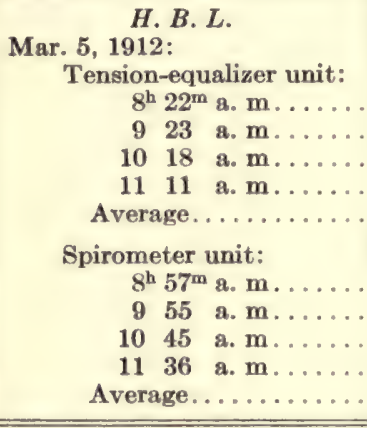 & $\begin{array}{l}c . c . \\
218 \\
203 \\
229 \\
207 \\
214\end{array}$ & $\begin{array}{l}c . c . \\
241 \\
234 \\
268 \\
262 \\
251\end{array}$ & $\begin{array}{r}0.905 \\
.870 \\
.855 \\
.790 \\
.855\end{array}$ & $\begin{array}{l}63.0 \\
66.0 \\
69.0 \\
72.5 \\
67.5 \\
\end{array}$ & $\begin{array}{l}14.5 \\
14.7 \\
14.0 \\
14.4 \\
14.4\end{array}$ \\
\hline 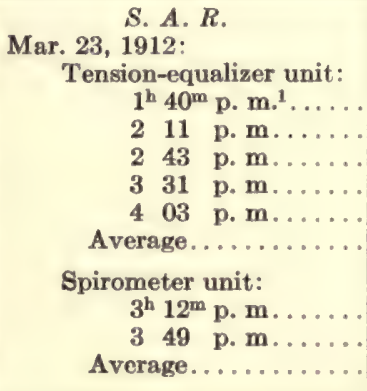 & $\begin{array}{l}167 \\
161 \\
159 \\
156 \\
147 \\
158\end{array}$ & $\begin{array}{l}225 \\
212 \\
194 \\
\ldots 01 \\
201 \\
208\end{array}$ & $\begin{array}{l}.740 \\
.760 \\
.820 \\
.735 \\
.760\end{array}$ & $\begin{array}{l}30.0 \\
50.5 \\
48.5 \\
49.0 \\
49.5\end{array}$ & $\begin{array}{l}12.8 \\
13.3 \\
12.9 \\
12.5 \\
11.6 \\
12.6\end{array}$ \\
\hline
\end{tabular}


TABLE 15.-Respiratory exchange in comparison experiments with the two types of the Benedict respiration apparatus. (Without food.)-Continued.

\begin{tabular}{|c|c|c|c|c|c|}
\hline $\begin{array}{l}\text { Subject, apparatus, date, } \\
\text { and time. }\end{array}$ & $\begin{array}{c}\text { Carbon } \\
\text { dioxide } \\
\text { eliminated } \\
\text { per minute. }\end{array}$ & $\begin{array}{c}\text { Oxygen } \\
\text { absorbed } \\
\text { per minute. }\end{array}$ & $\begin{array}{l}\text { Respira- } \\
\text { tory } \\
\text { quotient. }\end{array}$ & $\begin{array}{l}\text { Average } \\
\text { pulse- } \\
\text { rate. }\end{array}$ & $\begin{array}{c}\text { Average } \\
\text { respira- } \\
\text { tion- } \\
\text { rate. }\end{array}$ \\
\hline \multirow{2}{*}{\multicolumn{6}{|c|}{$\begin{array}{l}\text { S.A.R.-Cont'd. } \\
\text { Apr. 1, 1912: } \\
\text { Tension-equalizer unit: }\end{array}$}} \\
\hline & c.c. & $\begin{array}{l}\text { c.c. } \\
212\end{array}$ & & & \\
\hline 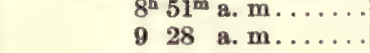 & $\begin{array}{l}194 \\
188\end{array}$ & $\begin{array}{l}212 \\
203\end{array}$ & $\begin{array}{l}.915 \\
.925\end{array}$ & $\begin{array}{l}54.0 \\
51.5\end{array}$ & $\begin{array}{l}12.0 \\
11.6\end{array}$ \\
\hline 1021 a. m....... & 190 & 196 & .970 & 50.0 & 11.4 \\
\hline 1114 a. m.. & 207 & 212 & .975 & 58.5 & 12.2 \\
\hline 1215 p. m........ & 190 & 201 & .945 & 54.0 & 11.4 \\
\hline Average............ & 194 & 805 & .945 & 53.5 & 11.7 \\
\hline \multicolumn{6}{|l|}{$\begin{array}{l}\text { Spirometer unit: } \\
g^{\mathrm{h}} 57^{\mathrm{m}} \mathrm{a} . \mathrm{m} .\end{array}$} \\
\hline 1049 a. m... & 182 & 200 & .905 & 49.5 & $\begin{array}{l}12.8 \\
11.9\end{array}$ \\
\hline 1145 a. m..... & 184 & 210 & .875 & 49.0 & 12.0 \\
\hline 1241 p. m......... & 189 & 193 & .980 & 53.0 & 12.6 \\
\hline Average............ & 189 & 204 & .985 & 50.5 & 18.8 \\
\hline \multicolumn{6}{|l|}{$\begin{array}{l}\text { J.A. F. } \\
\text { Mar. 26, 1912: }\end{array}$} \\
\hline \multirow{2}{*}{\multicolumn{6}{|c|}{$\begin{array}{l}\text { Mar. 26, 1912: } \\
\text { Spirometer unit: } \\
g^{\mathrm{h}} 13^{\mathrm{m}} \text { a. m. }\end{array}$}} \\
\hline & 186 & 224 & .830 & 69.0 & 12.4 \\
\hline 958 a. m.. & 187 & & & 71.5 & 13.6 \\
\hline 1041 a. m............. & 186 & 217 & .860 & 70.5 & 14.1 \\
\hline Average $\ldots \ldots \ldots \ldots$ & 186 & 221 & .840 & 70.5 & 13.4 \\
\hline \multicolumn{6}{|l|}{ Tension-equalizer unit: } \\
\hline 1020 a.m...... & 182 & 219 & .830 & 70.5 & 13.5 \\
\hline 1101 a. m...... & 182 & 220 & .825 & 70.0 & 14.6 \\
\hline Average....... & 183 & 228 & .825 & 69.5 & 13.6 \\
\hline \multirow{3}{*}{\multicolumn{6}{|c|}{$\begin{array}{c}K . H . A . \\
\text { May } 21,1912: \\
\text { Spirometer unit: } \\
8^{\text {th } 52^{\mathrm{m}} \text { a. m. }} .\end{array}$}} \\
\hline & & & & & \\
\hline & 184 & 219 & .840 & 46.5 & 12.5 \\
\hline 1013 a. m. & 189 & 232 & .815 & 44.5 & 13.6 \\
\hline 1129 a. m. . & 200 & 229 & .870 & 49.0 & 13.2 \\
\hline Average............ & 191 & 287 & .840 & 46.5 & 13.1 \\
\hline \multirow{2}{*}{\multicolumn{6}{|c|}{$\begin{array}{c}\text { Tension-equalizer unit: } \\
9^{\mathrm{h}} 38^{\mathrm{m}} \text { a. } \mathrm{m} . . .\end{array}$}} \\
\hline & 194 & 235 & .825 & 50.5 & 12.7 \\
\hline 1049 a. m....... & 193 & 230 & .840 & 47.5 & 14.1 \\
\hline 1203 p. m...... & 189 & 240 & .785 & 49.0 & 14.1 \\
\hline Average......... & 192 & 235 & .815 & 49.0 & 13.6 \\
\hline \multirow{2}{*}{\multicolumn{6}{|c|}{$\begin{array}{l}\text { May 25, 1912: } \\
\text { Spirometer unit: }\end{array}$}} \\
\hline & & & & & \\
\hline 956 a. m..... & 198 & 232 & .855 & 55.5 & 14.4 \\
\hline 1053 a. m.... & 201 & 238 & .845 & 56.0 & 13.8 \\
\hline Average............. & 193 & 281 & .835 & 55.5 & 13.9 \\
\hline \multicolumn{6}{|l|}{ Tension-equalizer unit: } \\
\hline $9^{\text {h }} 24^{\mathrm{m}}$ a. m..... & 212 & 249 & .850 & 54.0 & 14.2 \\
\hline 1024 a. m... & 193 & $\begin{array}{l}229 \\
235\end{array}$ & .840 & 51.5 & 14.4 \\
\hline 1119 a. m....... & 200 & 235 & .850 & 55.0 & 15.1 \\
\hline Average.............. & 202 & 238 & .850 & 53.5 & 14.6 \\
\hline \multicolumn{6}{|l|}{$\begin{array}{c}J . B . T . \\
\text { May } 27,1912:\end{array}$} \\
\hline $9^{\mathrm{h}} 12^{\mathrm{m}}$ a. m. . & $\begin{array}{l}207 \\
226\end{array}$ & $\begin{array}{l}263 \\
256\end{array}$ & $\begin{array}{l}.790 \\
880\end{array}$ & $\begin{array}{l}70.5 \\
68.5\end{array}$ & 14.0 \\
\hline 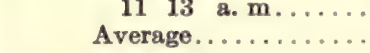 & 217 & $\begin{array}{l}200 \\
860\end{array}$ & .835 & 69.5 & 13.9 \\
\hline
\end{tabular}


TABLs 15.-Respiratory exchange in comparison experiments with the two types of the Benedict respiration apparatus. (Without food.)-Continued.

\begin{tabular}{|c|c|c|c|c|c|}
\hline $\begin{array}{l}\text { Subject, apparatus, date, } \\
\text { and time. }\end{array}$ & $\begin{array}{c}\text { Carbon } \\
\text { dioxide } \\
\text { eliminated } \\
\text { per minute. }\end{array}$ & $\begin{array}{c}\text { Oxygen } \\
\text { absorbed } \\
\text { per minute. }\end{array}$ & $\begin{array}{c}\text { Respira- } \\
\text { tory } \\
\text { quotient. }\end{array}$ & $\begin{array}{c}\text { Average } \\
\text { pulse- } \\
\text { rate. }\end{array}$ & $\begin{array}{l}\text { Average } \\
\text { respira- } \\
\text { tion- } \\
\text { rate. }\end{array}$ \\
\hline 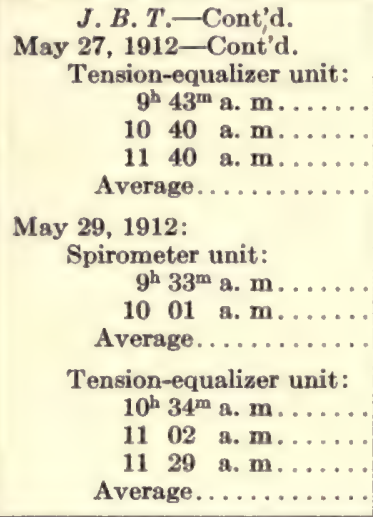 & $\begin{array}{c}224 \\
1(197) \\
224 \\
284\end{array}$ & $\begin{array}{c}246 \\
245 \\
2(228) \\
246\end{array}$ & $\begin{array}{c}.910 \\
3(.805) \\
3(.985) \\
.910\end{array}$ & $\begin{array}{l}64.0 \\
63.0 \\
67.0 \\
64.5\end{array}$ & $\begin{array}{l}10.4 \\
11.8 \\
11.7 \\
11.8\end{array}$ \\
\hline 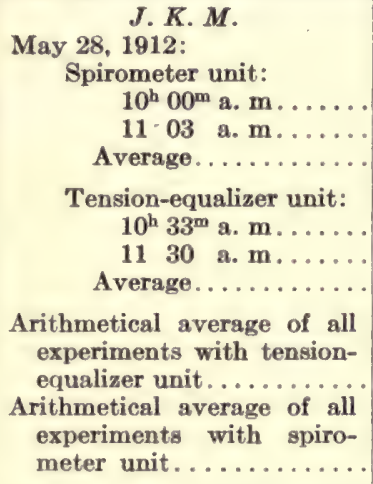 & $\begin{array}{l}187 \\
184 \\
186\end{array}$ & $\begin{array}{l}214 \\
234 \\
224\end{array}$ & $\begin{array}{l}.820 \\
.900 \\
.855 \\
\\
.875 \\
.790 \\
.830\end{array}$ & $\begin{array}{l}57.5 \\
63.5 \\
60.5 \\
53.5 \\
51.5 \\
52.5\end{array}$ & $\begin{array}{l}16.4 \\
17.3 \\
16.9 \\
12.1 \\
14.0 \\
13.1\end{array}$ \\
\hline
\end{tabular}

${ }^{1}$ Carbon dioxide present in system; omitted from average.

${ }^{2}$ Valve turned too late at beginning of period; omitted from average.

${ }^{3}$ Omitted in calculating the average for the experiment.

To find whether this agreement is actual, the differences in each experiment between the averages for the two apparatus are brought together in table 16, the values obtained for the spirometer unit being used as the base-line. In some cases the difference found is somewhat larger than is indicated by the grand averages in table 15, the greatest difference being with S. A. R. on March 23, 1912. In the other experiments the differences found are no larger than would be expected under the conditions of experimenting.

In this summing up of results, not only the averages should be considered, but also the general picture of the respiratory exchange as measured by the two types of apparatus. A careful examination of the 
results from this point of view shows that the measured respiratory exchange was practically the same with both forms of the respiration apparatus.

TABLE 16.-Variations of average results obtained with the tension-equalizer unit from those obtained with the spirometer unit.

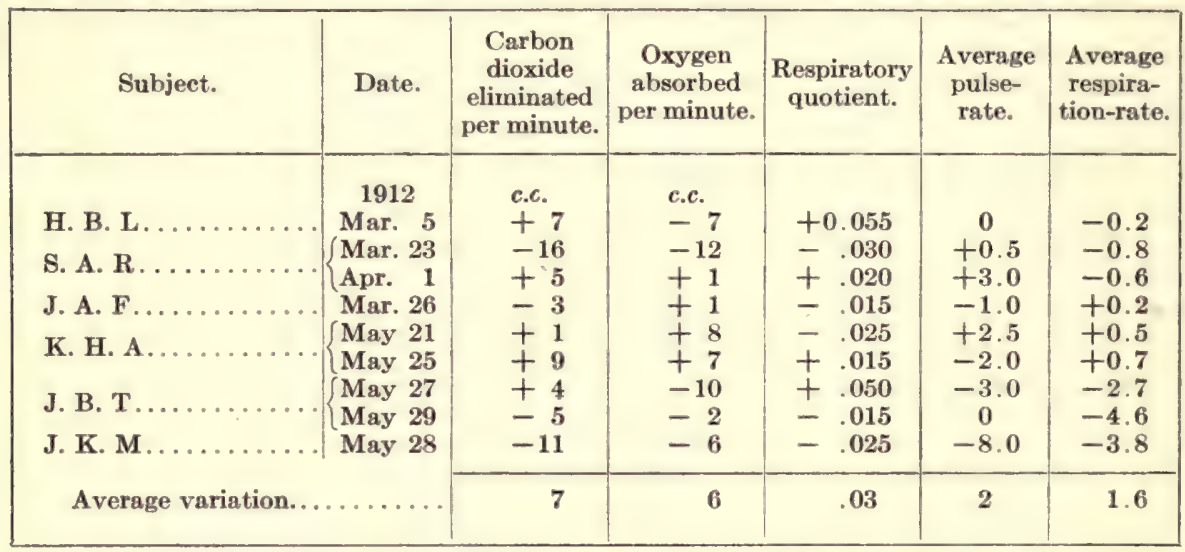

In considering the statistics of the individual periods, it is of interest to calculate the percentage uniformity of results obtained with the two apparatus. The results of such a calculation are best shown by probability curves which have been plotted from data obtained in the following manner:

The difference between the results for an individual period and the average for the apparatus on that day was first found; this difference when divided by the average result obtained with the apparatus in that experiment gave the percentage variation for the period. The percentage number of periods varying more than 0.5 per cent from the average results was then found by determining the number of periods showing this variation and dividing this number by the total number of periods.

For example, in the experiment with H. B. L. on March 5, the difference between the carbon-dioxide elimination for the first period with the tension-equalizer unit (218 c.c.) and the average carbon-dioxide elimination with that apparatus for the day (214 c.c.) was 4 c.c.; this divided by the average carbon-dioxide elimination ( 214 c.c.) gives, as the percentage variation for that period, 1.87 per cent. With the tension-equalizer unit there were 26 periods in which the carbon-dioxide elimination varied more than 0.5 per cent from the average of the carbon-dioxide elimination with this apparatus. This number of periods divided by the total number of periods (30) gives 87 per cent as the percentage number of periods with the tension-equalizer unit varying more than 0.5 per cent from the grand average of the carbondioxide elimination. 
This calculation has been made for all the five factors observed, not only for a variation of 0.5 per cent but also for variations of 1 per cent, 1.5 per cent, 2 per cent, and so on. The results of these calculations with both forms of apparatus are given in the probability curves shown in figure 37 , the ordinates representing the percentage of the total number of periods, the abscissæ representing the percentage variation of the number of periods indicated. The percentage of the total number of

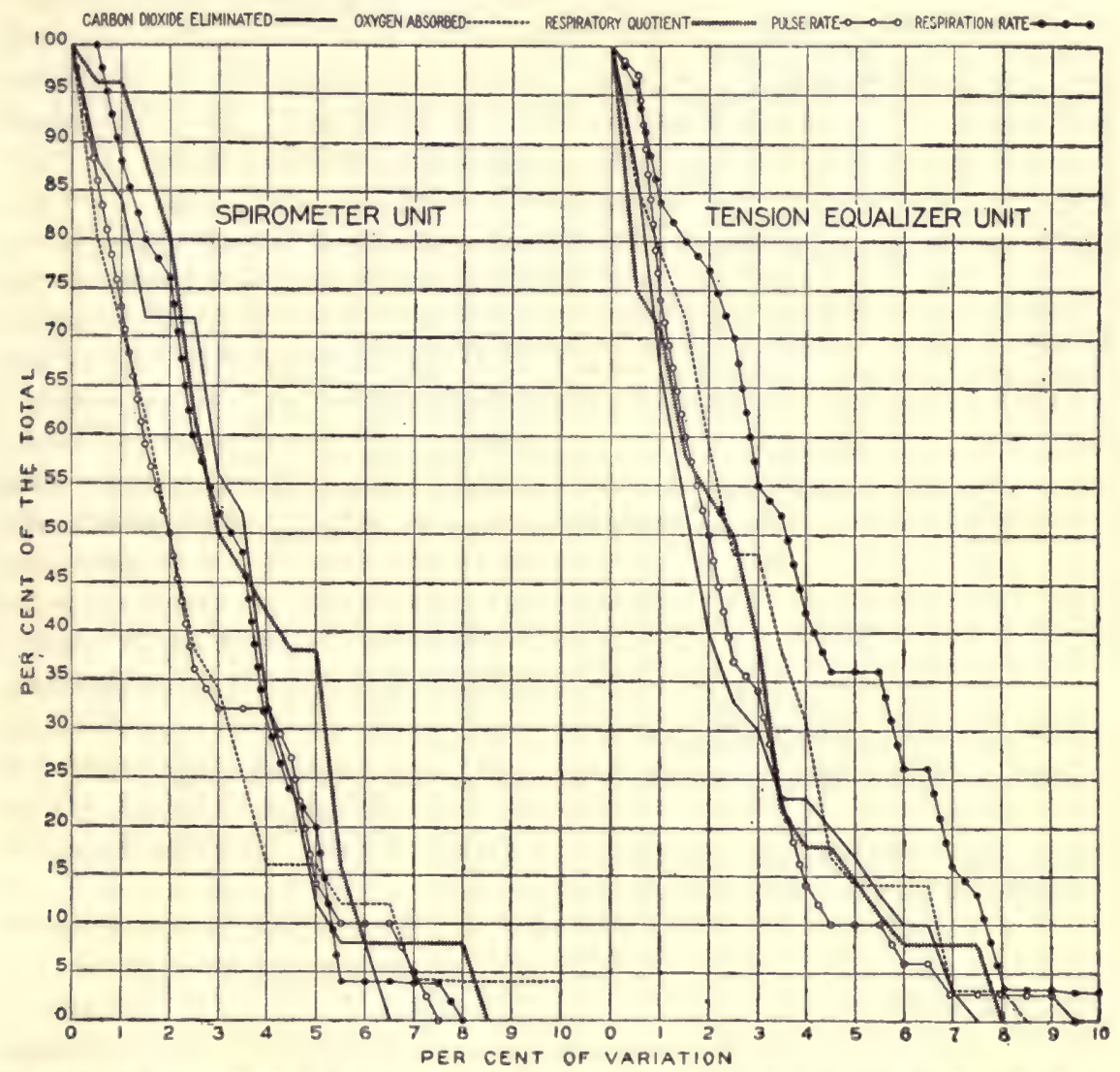

FIG. 37.-Probability curves for the series of comparison experiments with the spirometer unit and the tension-equalizer unit.

The ordinates indicate the percentage of the total number of periods; the abscissæ indicate the percentage of variation from the average.

periods is plotted in intervals of 5 per cent and the percentage variation in intervals of 0.5 per cent.

In this laboratory a series of three periods in a respiration experiment is considered perfectly satisfactory if the range in figures for the carbon-dioxide elimination and the oxygen consumption does not exceed 10 c.c. This would be approximately equal to an average deviation of 2.5 per cent for the carbon-dioxide elimination and 2.15 
per cent for the oxygen consumption. If the ordinates in figure 37 are examined, it will be noted that of the two apparatus the spirometer unit shows the larger number of periods having a variation from the average carbon-dioxide elimination greater than 2.5 per cent, the number of periods showing such excess variation being some 40 per cent larger than with the tension-equalizer unit. The curves for the oxygen consumption, however, show a greater uniformity in the results obtained with the two forms of apparatus. This greater difference in variation for the carbon-dioxide elimination with the spirometer unit and the parallelism in the oxygen consumption is shown at all points in these curves for the two apparatus. The curves for the respiratory quotient show a difference similar to that in the carbon-dioxide curves.

The pulse-rate curves are remarkably parallel, indicating that the conditions of the experiments were, in general, about the same so far as activity and metabolic intensity were concerned. Not much stress can be laid upon this parallelism, however, as the measurement of the pulse-rate was the least accurate of the data obtained. All of the other observations were made for the entire period and the average is therefore a true average, but the pulse-rate was taken only at intervals, the entire time occupied in taking the records amounting to only onethird of the experimental period. From our general experience with pulse-rates, it is evident that no assumption can be made that five counts of one minute each at intervals during the 15-minute experimental period will give an average as accurate as the averages obtained for the other measurements. It is believed, however, that the lack of refinement in measuring the pulse-rate applies in equal degree to the results obtained for both apparatus and the average pulse-rates for the two apparatus are therefore comparable. The figures would therefore indicate that the variations in the pulse-rate are nearly the same in both series of experiments.

The cause for the lesser uniformity of results for the carbon-dioxide measurement with the spirometer type of apparatus lies, probably, in the differences in ventilation of the lungs with this apparatus. Since the ventilation was not measured with either type of apparatus, these variations are not known. The difference, however, can not be ascribed to greater irregularities in the respiration-rate when the spirometer unit was used, as the percentage variations in the respiration-rate for the two apparatus are nearly parallel.

In summarizing, it may be stated that on the average the two forms of apparatus give the same results in the measurement of the respiratory exchange under like conditions and that the tension-equalizer unit gives somewhat more uniform results in the determination of the carbondioxide elimination and the respiratory quotients. 
ZUNTZ-CEPPERT RESPIRATION APPARATUS AND BENEDICT RESPIRATION APPARATUS (TENSION-EQUALIZER UNIT).

In the first series of experiments comparing the respiratory exchange as measured by the Benedict respiration apparatus and the ZuntzGeppert apparatus, ${ }^{1}$ the tension-equalizer unit was used and, in all but one experiment, the pneumatic nosepieces. With the Zuntz-Geppert apparatus, the ordinary form of rubber mouthpiece was employed, also the common form of valve (see fig. 18, page 54) with fish-membrane or thin rubber covering. The samples of expired air in the experiments with this apparatus were collected in the burettes of the Zuntz-Geppert gas-analysis apparatus and analyzed immediately after the experimental period. The volume of expired air was converted to $0^{\circ} \mathrm{C}$. and $760 \mathrm{~mm}$. by means of the readings of the thermo-barometer. The expired air was conducted from the subject to the Elster meter through a rubber tube with an internal diameter of $20 \mathrm{~mm}$. and a length of 1 to 2 meters.

The regular routine was followed in carrying out the experiments, any exceptions being noted in the statistics. ${ }^{2}$ While the apparatus first used varied in the different experiments, in all cases they were alternated with each period. The total number of periods varied from 6 to 8 , following each other as rapidly as technique would permit. They were usually 15 minutes in length, but in some cases varied from this by 5 minutes, either more or less. Prior to the periods with the Zuntz-Geppert apparatus, a preliminary determination was made of the rate of ventilation of the lungs by noting with a stopwatch the time required for the expiration of 20 liters of air. When it was found that the rates for two successive periods were uniform, the experimental period with the Zuntz-Geppert apparatus was begun.

The pulse-rate in all of the experiments was obtained by means of the Bowles stethoscope; usually three separate counts were made in each period. The respiration-rate was secured during the first few experiments by noting the time for 10 respirations and then calculating the rate per minute; three counts were obtained in this way. Subsequently a pneumograph around the lower part of the chest was used, by means of which a graphic record was made of the respiration for the whole period. The muscular activity was noted by the observer, although in the experiments in which the respiration was obtained with the chest pneumograph incomplete graphic records of the activity were also secured. The methods used in later experimenting for securing a graphic record of the muscular activity were not developed at the time when this series of experiments was carried out.

The subjects were members of the Laboratory staff, and while all of them were more or less familiar with the tension-equalizer unit, they were not all accustomed to the Zuntz-Geppert apparatus. 
The statistics of the 11 comparisons follow. Mr. J. A. Riche carried out the experiments with the Zuntz-Geppert apparatus and made all the air analyses.

In addition to the data which have been given in the previous comparisons, the figures are also given for the total ventilation of the lungs per minute, reduced to $0^{\circ} \mathrm{C}$. and $760 \mathrm{~mm}$. pressure, and the volume per respiration calculated to $37^{\circ} \mathrm{C}$. and atmospheric pressure, corrected for the tension of aqueous vapor in the lungs. The composition of the expired air, as obtained from the Zuntz-Geppert gas-analysis apparatus, is also included in the table for the periods in which the Zuntz-Geppert respiration apparatus was used. These figures represent the average of two analyses, agreeing usually to within 0.04 per cent for both the carbon dioxide and the oxygen.

\section{STATISTICS OF EXPERIMENTS.}

T. M. C., June 24, 1910.-Tension-equalizer unit, 4 periods; Zuntz-Geppert apparatus, 3 periods; preliminary period, 4 minutes; apparatus alternated. But few counts of the pulse-rate in each period. Respiration-rate recorded by pneumograph; uniform in character. Rate of preliminary ventilation for 20 liters with Zuntz-Geppert apparatus:

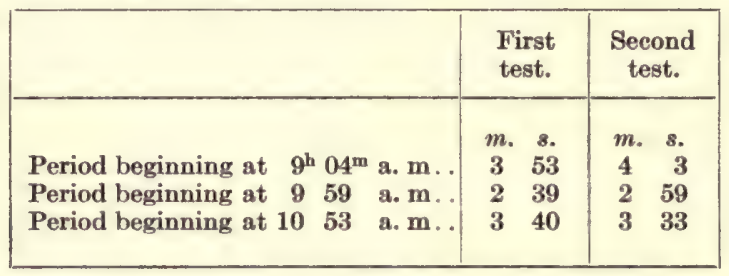

T. M. C., June 29, 1910.-Tension-equalizer unit, 3 periods; Zuntz-Geppert apparatus, 3 periods; preliminary period, 18 minutes; apparatus alternated. Subject stated that during first period he felt as if he were breathing against pressure and that there was so much air in the tension equalizer that his breathing was necessarily shallow for a short time. No difficulty was experienced in the following periods with this apparatus. Only a few counts of pulse-rate in each period; uniform in character. Respiration-rate obtained with pneumograph; uniform for individual periods. Rate of preliminary ventilation for 20 liters with Zuntz-Geppert apparatus:

\begin{tabular}{|c|c|c|}
\hline & $\begin{array}{l}\text { First } \\
\text { test. }\end{array}$ & $\begin{array}{c}\text { Second } \\
\text { test. }\end{array}$ \\
\hline $\begin{array}{l}\text { Period beginning at } 9^{\mathrm{h}} 19^{\mathrm{m}} \text { a. } \mathrm{m} \text {. } \\
\text { Period beginning at } 1022 \text { a. } \mathrm{m} \text {. } \\
\text { Period beginning at } 11 \quad 15 \text { a. } \mathrm{m} \text {. }\end{array}$ & $\begin{array}{cc}m . & 8 . \\
3 & 41 \\
3 & 21 \\
2 & 16\end{array}$ & $\begin{array}{cc}m . & s . \\
3 & 50 \\
3 & 16 \\
2 & 25\end{array}$ \\
\hline
\end{tabular}

J. J. C., June 8, 1910.-Tension-equalizer unit, 4 periods; Zuntz-Geppert apparatus, 4 periods; preliminary period, 35 minutes; apparatus alternated. In third period with tension-equalizer unit, subject very sleepy. Respirationrate counted by observer; in all periods but one very uniform, but in third period with Zuntz-Geppert apparatus it showed a tendency toward irregularity. 
J. J. C., June 13, 1910.-Zuntz-Geppert apparatus, 4 periods; tensionequalizer unit, 4 periods; preliminary period, 28 minutes; apparatus alternated. Subject asleep in last period. No respiration-rates taken by pneumograph and only a few counts made of pulse- and respiration-rates in each period. Rate of preliminary ventilation for 20 liters with Zuntz-Geppert apparatus:

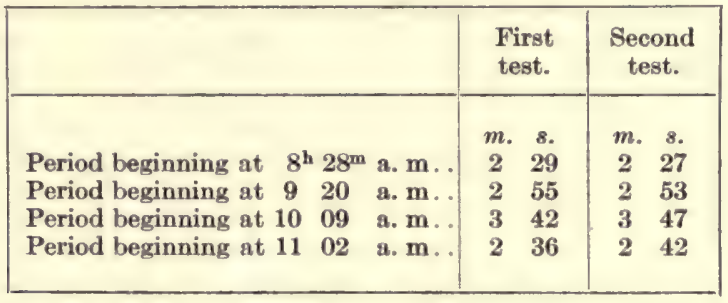

J. J. C., June 25, 1910.-Zuntz-Geppert apparatus, 3 periods; tensionequalizer unit, 3 periods; preliminary period, 49 minutes; apparatus alternated. Pulse-rate counted at three or four different times during each period and, so far as the individual periods were concerned, was quite regular. Respirationrate taken with pneumograph; rates comparatively uniform in each period. Rate of preliminary ventilation for 20 liters with Zuntz-Geppert apparatus:

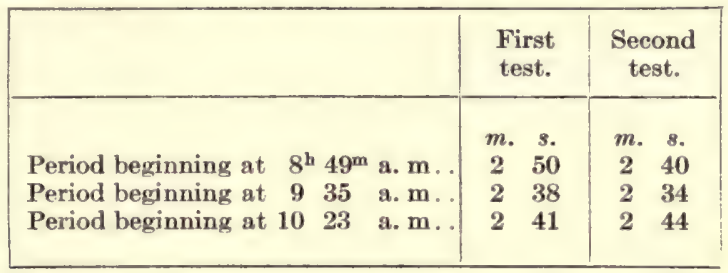

A. G. E., July 18, 1910.-Zuntz-Geppert apparatus, 3 periods; tensionequalizer unit, 3 periods; apparatus alternated. Pulse-rate counted only few times in each period; approximately uniform. Respiration-rate obtained with pneumograph; rates uniform, except in second period with Zuntz-Geppert apparatus, when there was considerable fluctuation in type and depth. Rate of preliminary ventilation for 20 liters with Zuntz-Geppert apparatus:

\begin{tabular}{|c|c|c|c|c|}
\hline & $\begin{array}{l}\text { First } \\
\text { test. }\end{array}$ & $\begin{array}{c}\text { Second } \\
\text { test. }\end{array}$ & $\begin{array}{l}\text { Third } \\
\text { test. }\end{array}$ & $\begin{array}{c}\text { Fourth } \\
\text { test. }\end{array}$ \\
\hline Period beginning at $9^{\mathrm{h}} 28^{\mathrm{m}}$ a. $\mathrm{m}$. & $\begin{array}{cc}m . & 8 . \\
2 & 37\end{array}$ & $\begin{array}{cc}m . & 8 . \\
3 & 10\end{array}$ & $\begin{array}{cc}m . & s . \\
3 & 45\end{array}$ & $\begin{array}{cc}m . & s . \\
3 & 30\end{array}$ \\
\hline Period beginning at 1029 a. m. . & 340 & 350 & . & . \\
\hline Period beginning at 1131 & 327 & 348 & . & . \\
\hline
\end{tabular}

L. E. E., July 6, 1910.-Zuntz-Geppert apparatus, 3 periods; tensionequalizer unit, 3 periods; apparatus alternated. Subject somewhat restless during experiment; stated in first period with Zuntz-Geppert apparatus that the noseclip troubled him considerably, and complained of noseclip in all periods in which it was used. Pulse-rate only 3 to 4 counts in each period; uniform as to individual periods. Respiration-rate recorded with pneumograph. With Zuntz-Geppert apparatus respiration seemed to be more labored in first period but respiration-rate approximately uniform with this apparatus. With tension-equalizer unit a number of delayed respirations in latter half of 
first period, suggesting apnœea; in second period, the type persisted but less apparent than in first period; in third period, but little, if any, of this type of respiration; respiration uniform otherwise throughout periods with this apparatus. Rate of preliminary ventilation for 30 liters first period and 20 liters second and third periods with Zuntz-Geppert apparatus:

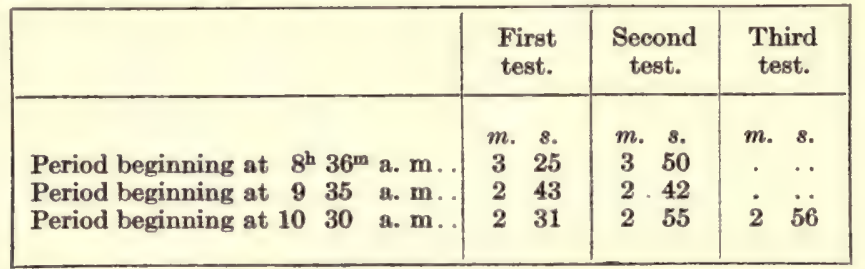

L. E. E., July 14, 1910.-Tension-equalizer unit, 3 periods; Zuntz-Geppert apparatus, 3 periods; apparatus alternated. Pulse-rate counted three times in each period; uniform in most of the periods, except in the first with the Zuntz-Geppert apparatus, when the range was from 47 to 54 in the three counts. Respiration-rate obtained with pneumograph. In first period with each apparatus, respiration-rate comparatively uniform. In second period with tensionequalizer apparatus, there was a tendency toward irregularity and a wave-like respiration, $i . e$., at intervals subject took a deep breath and the depth of respiration would then gradually decrease; in second period with Zuntz-Geppert apparatus, there was a very decided irregularity, approaching Cheyne-Stokes respiration. In third period with each apparatus, respiration-rate comparatively uniform. Rate of preliminary ventilation for 20 liters with ZuntzGeppert apparatus:

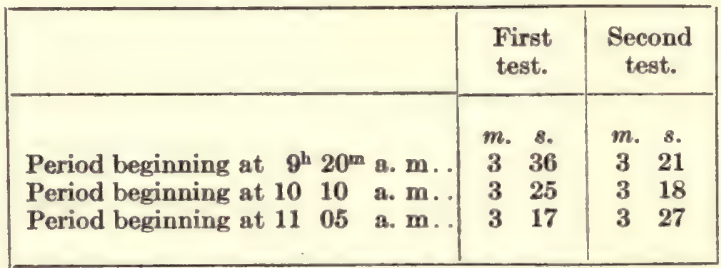

H. L. H., July 16, 1910.-Zuntz-Geppert apparatus, 3 periods; tensionequalizer unit, 3 periods; apparatus alternated. Pulse-rate counted three times in each period; respiration-rate obtained with pneumograph uniform in character. Rate of preliminary ventilation for 20 liters with ZuntzGeppert apparatus:

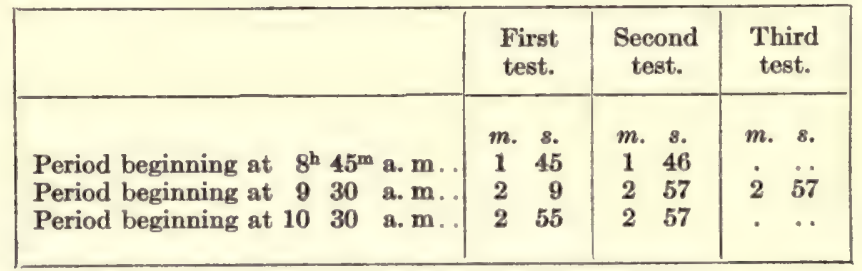

H. L. H., July 25, 1910.-Zuntz-Geppert apparatus, 3 periods; tensionequalizer unit, 3 periods; preliminary period, 30 minutes; apparatus alternated. Subject said there was only a slight resistance to respiration in periods with Zuntz-Geppert apparatus. Pulse-rate obtained three times in each period; 
uniform. Respiration-rate obtained with pneumograph; rate remarkably uniform in all periods. Rate of preliminary ventilation for 20 liters with Zuntz-Geppert apparatus:

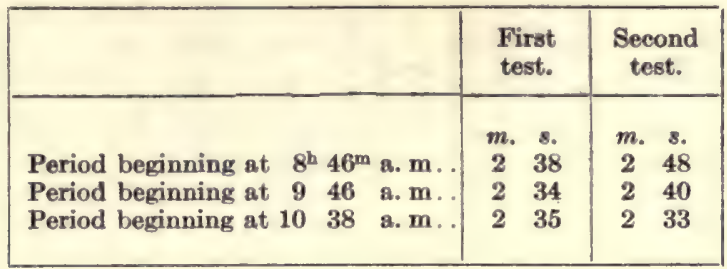

D. J. M., July 1, 1910.-Tension-equalizer unit, 3 periods; Zuntz-Geppert apparatus, 3 periods; preliminary period, 15 minutes; apparatus alternated. Mouthpiece used with tension-equalizer unit, as subject said nosepieces irritated his nose. Subject more or less restless during experiment, as flies troubled him somewhat; also moved lower part of body; was asleep during second period with each apparatus. Pulse-rate counted three times in every period. Respiration-rate obtained with pneumograph, but little could be determined as to character, as apparatus was not well placed; rate in individual periods fairly uniform. Rate of preliminary ventilation for 20 liters with Zuntz-Geppert apparatus:

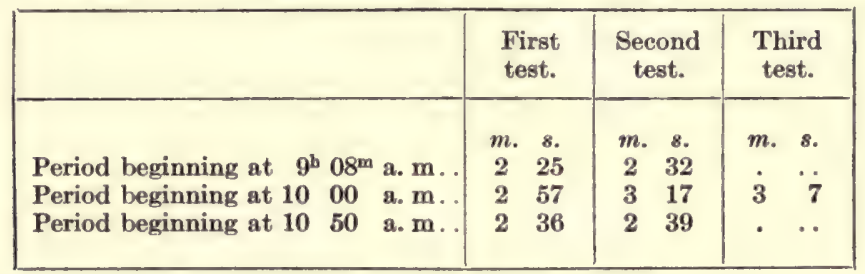

\section{DISCUSSION OF RESULTS.}

The data for the individual periods and the averages for each apparatus, both for each experiment and for all the periods in the series, are given in table 17. The grand averages for the carbon-dioxide elimination for the two apparatus show a difference of only 4 c.c. per minute, being 190 c.c. for the tension-equalizer unit and 186 c.c. per minute for the Zuntz-Geppert apparatus. The averages for the oxygen consumption differ only 3 c.c., these being 224 c.c. per minute and 227 c.c. per minute respectively. The average respiratory quotients, pulse-rate, and respiration-rate show a similar good agreement. The values for the tension-equalizer unit are: Respiratory quotient, 0.850 ; pulse-rate, 63.0; respiration-rate 15.9; for the Zuntz-Geppert apparatus, respiratory quotient, 0.820 ; pulse-rate 64.5 ; respiration-rate 17.0 . 
TABLE 17.-Respiratory exchange in comparison experiments with the $Z$ untz-Geppert apparatus ${ }^{1}$ and the Benedict respiration apparatus (tension-equalizer unit). (Without food.)

\begin{tabular}{|c|c|c|c|c|c|c|c|c|c|}
\hline \multirow{2}{*}{$\begin{array}{l}\text { Subject, date, method, } \\
\text { and time. }\end{array}$} & \multirow{2}{*}{ 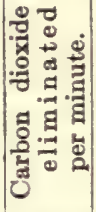 } & \multirow{2}{*}{ 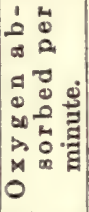 } & \multirow{2}{*}{ 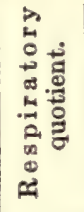 } & \multirow{2}{*}{ 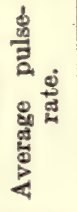 } & \multirow{2}{*}{ 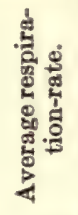 } & \multirow{2}{*}{ 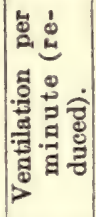 } & \multirow{2}{*}{ 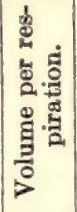 } & \multicolumn{2}{|c|}{$\begin{array}{l}\text { Composition of } \\
\text { expired air. }\end{array}$} \\
\hline & & & & & & & & $\begin{array}{l}\text { Carbon } \\
\text { dioxide. }\end{array}$ & Oxygen. \\
\hline \multicolumn{10}{|l|}{$\begin{array}{c}T . M . C . \\
\text { June 24, 1910: } \\
\text { Tension-equalizer unit: }\end{array}$} \\
\hline $\begin{array}{r}\text { Tension-equalizer unit: } \\
8^{\mathrm{h}} 34^{\mathrm{m}} \text { a. } \mathrm{m} . . .\end{array}$ & $\begin{array}{l}\text { c.c. } \\
149\end{array}$ & c.c. & & 67.0 & 14.0 & $\begin{array}{l}\text { liters. } \\
\ldots\end{array}$ & c.c. & $\begin{array}{l}\text { p. ct. } \\
\text {. . . }\end{array}$ & p.ct. \\
\hline 929 a. m..... & 151 & 178 & 0.850 & 66.5 & 14.3 & $\ldots \ldots$ & $\ldots$. & & $\ldots \ldots$ \\
\hline 1026 a. m..... & 153 & 194 & .790 & 67.0 & 15.2 & $\ldots \ldots$ & $\ldots$. & $\ldots \ldots$ & $\ldots \ldots$ \\
\hline 1117 a. m..... & 153 & 187 & .820 & $\cdots \cdots$ & 14.1 & $\cdots \cdots$ & $\ldots$. & $\ldots \ldots$ & $\ldots \ldots$ \\
\hline Average........... & 152 & 186 & .815 & 67.0 & 14.4 & & $\cdots \cdots$ & & $\cdots \cdots$ \\
\hline $\begin{array}{l}\text { Zuntz-Geppert: } \\
\quad 9^{\mathrm{h}} 04^{\mathrm{m}} \text { a. } \mathbf{m} \ldots \ldots\end{array}$ & 146 & 178 & .820 & 72.5 & 15.3 & 5.09 & 403 & 2.95 & 17.50 \\
\hline 959 a. m....... & 153 & 191 & .800 & 71.0 & 15.7 & 5.22 & 401 & 2.99 & 17.37 \\
\hline 1053 a. m...... & 150 & 188 & .795 & 72.0 & 15.9 & 5.09 & 388 & 3.01 & 17.34 \\
\hline Average........... & 150 & 186 & .805 & 72.0 & 15.6 & 5.13 & 397 & 2.98 & 17.40 \\
\hline \multicolumn{10}{|l|}{$\begin{array}{l}\text { June } 29,1910 \text { : } \\
\text { Tension-equalizer unit: } \\
\qquad 8^{\mathrm{h}} 48^{\mathrm{m}} \text { a. m. . . . . . }\end{array}$} \\
\hline $\begin{array}{l}8^{\mathrm{h}} 48^{\mathrm{m}} \text { a. } \mathrm{m} \ldots \ldots \\
\mathbf{9} \quad 55 \text { a. } \mathrm{m} \ldots \ldots\end{array}$ & $\begin{array}{l}149 \\
126\end{array}$ & 159 & .010 & 62.0 & 13.4 & & & & $\ldots \ldots$ \\
\hline 1050 a. $\mathrm{m} \ldots \ldots$ & 146 & 185 & .790 & 65.5 & 13.2 & & & & $\ldots \ldots \ldots$ \\
\hline & 140 & 176 & .795 & 65.5 & 13.4 & & & & $\ldots \ldots$ \\
\hline \multicolumn{10}{|l|}{ Zuntz-Geppert: } \\
\hline 1022 a. $\mathrm{m} \ldots .$. & 138 & 179 & .775 & 66.5 & 19.5 & 5.11 & 316 & 2.78 & 17.54 \\
\hline 1115 a. m...... & 133 & 182 & .730 & 68.5 & 21.5 & 5.38 & 304 & 2.55 & 17.68 \\
\hline Average........... & 134 & 179 & .750 & 67.5 & 20.1 & 5.16 & 311 & 2.68 & 17.59 \\
\hline \\
\hline $\begin{array}{r}\text { Tension-equalizer unit: } \\
8^{\mathrm{h}} 35^{\mathrm{m}} \text { a. } \mathrm{m}\end{array}$ & 226 & 252 & .895 & 75.0 & 19 & & & & \\
\hline 919 a. m.... & 209 & 239 & .875 & 64.5 & 20 & & & $\ldots$ & \\
\hline 1010 a. m..... & 201 & 226 & .890 & 57.0 & 18 & & & $\cdots$ & \\
\hline 1055 a. m..... & 189 & 223 & .850 & 58.0 & 20 & & & ... & \\
\hline Average............ & 206 & 235 & .875 & 63.5 & 19 & & & & \\
\hline $\begin{array}{l}\text { Zuntz-Geppert: } \\
\qquad 8^{\mathrm{h}} 56^{\mathrm{m}} \text { a. } \mathrm{m} .\end{array}$ & 197 & 230 & .860 & 70.0 & 19 & 6.39 & 408 & 3.12 & 17.38 \\
\hline 945 a. $\mathrm{m}^{2} \ldots \ldots$ & 184 & 233 & .790 & 52.0 & (13) & $(5.45)$ & $(512)$ & $(3.41)$ & $(16.79)$ \\
\hline 1036 a. m..... & 177 & 206 & .860 & 59.5 & 19 & 6.13 & 388 & 2.93 & 17.61 \\
\hline 1118 a. m...... & 184 & 224 & .825 & 60.5 & 19 & 6.55 & 420 & 2.85 & 17.58 \\
\hline Average........... & 186 & 223 & .830 & 61.5 & 19 & 6.36 & 405 & 2.97 & 17.52 \\
\hline \multicolumn{10}{|l|}{$\begin{array}{l}\text { June 13, 1910: } \\
\text { Zuntz-Geppert: }\end{array}$} \\
\hline $8^{\mathrm{h}} 28^{\mathrm{m}}$ a. m... & 197 & 221 & .890 & 73.5 & 23 & 7.00 & 368 & 2.85 & 17.78 \\
\hline 920 a. m..... & 184 & 233 & .790 & 60.5 & 19 & 6.41 & 407 & 2.91 & 17.40 \\
\hline 1009 a. m..... & 170 & 192 & .880 & 60.5 & 20 & 5.99 & 363 & 2.87 & 17.73 \\
\hline 1102 a. m....... & 167 & 200 & .835 & 58.5 & 17 & 5.00 & 356 & 3.37 & 17.00 \\
\hline Average.......... & 180 & 212 & .850 & 63.5 & 20 & 6.10 & 374 & 3.00 & 17.48 \\
\hline $\begin{array}{c}\text { Tension-equalizer unit: } \\
8^{\mathrm{h}} 50^{\mathrm{m}} \text { a. } \mathrm{m}\end{array}$ & 198 & 218 & 910 & 61.5 & 16 & & & & \\
\hline 941 a. m..... & 197 & 217 & .910 & 59.0 & 18 & & & & \\
\hline 1029 a. m..... & 198 & 203 & .975 & 60.0 & 18 & & & & \\
\hline 1128 a. m..... & 206 & 217 & .950 & 63.5 & 20 & & & & \\
\hline Average.......... & 200 & 214 & .935 & 61.0 & 18 & & & & \\
\hline
\end{tabular}

${ }^{1}$ The samples were collected and analyzed in the Zuntz gas-analysis apparatus. 'Figures in parentheses were omitted in calculating the average. 
TABLE 17.-Respiratory exchange in comparison experiments with the Zuntz-Geppert apparatus and the Benedict respiration apparatus (tension-equalizer unit). (Without food.)-Continued.

\begin{tabular}{|c|c|c|c|c|c|c|c|c|c|}
\hline \multirow{2}{*}{$\begin{array}{c}\text { Subject, date, method, } \\
\text { and time. }\end{array}$} & \multirow{2}{*}{ 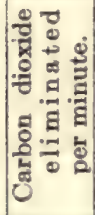 } & \multirow{2}{*}{ 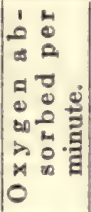 } & \multirow{2}{*}{ 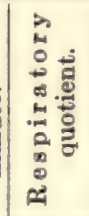 } & \multirow{2}{*}{ 兽 } & \multirow{2}{*}{ 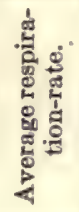 } & \multirow{2}{*}{ 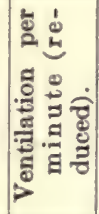 } & \multirow{2}{*}{ 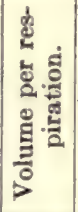 } & \multicolumn{2}{|c|}{$\begin{array}{l}\text { Composition of } \\
\text { expired air. }\end{array}$} \\
\hline & & & & & & & & $\begin{array}{l}\text { Carbon } \\
\text { dioxide. }\end{array}$ & Oxygen. \\
\hline 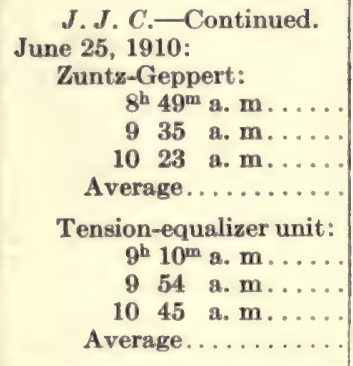 & $\begin{array}{l}\text { c.c. } \\
198 \\
202 \\
199 \\
200\end{array}$ & $\begin{array}{l}c . c . \\
245 \\
241 \\
240 \\
242\end{array}$ & $\begin{array}{r}0.810 \\
.835 \\
.830 \\
.885\end{array}$ & $\begin{array}{l}68.0 \\
62.5 \\
58.5 \\
63.0\end{array}$ & $\begin{array}{l}18.4 \\
21.1 \\
18.0 \\
19.9\end{array}$ & $\begin{array}{l}\text { liters. } \\
6.82 \\
7.23 \\
7.26 \\
7.10\end{array}$ & $\left|\begin{array}{l}\ldots \\
\ldots \\
\ldots \\
\ldots\end{array}\right|$ & $\mid \begin{array}{c}\ldots \\
\ldots \\
\ldots \\
\ldots \\
\ldots\end{array}$ & $\begin{array}{c}p . c t . \\
17.42 \\
17.66 \\
17.69 \\
17.69\end{array}$ \\
\hline 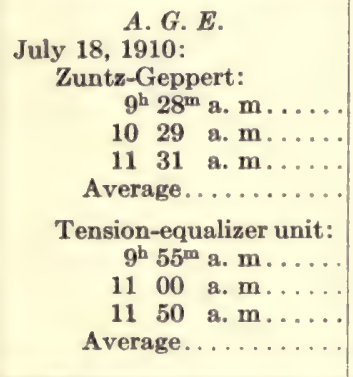 & $\begin{array}{l}196 \\
194 \\
190 \\
193\end{array}$ & $\begin{array}{l}227 \\
215 \\
214 \\
219\end{array}$ & $\begin{array}{l}.865 \\
.900 \\
.890 \\
.880\end{array}$ & $\begin{array}{l}60.5 \\
63.0 \\
68.0\end{array}$ & $\begin{array}{r}12.7 \\
12.7 \\
14.9 \\
13.4 \\
13.9 \\
13.7 \\
13.7 \\
13.8\end{array}$ & $\mid \begin{array}{c}\ldots \\
\ldots \\
\ldots \\
\cdots \\
\cdots\end{array}$ & $\mid \begin{array}{c}\cdots \\
\cdots \\
\cdots \\
\cdots \\
\cdots\end{array}$ & $\mid \begin{array}{c}\ldots \\
\ldots \\
\ldots \\
\ldots \\
\ldots\end{array}$ & $\mid \begin{array}{l}\ldots \ldots \\
\ldots \ldots \\
\ldots \ldots \\
\ldots \ldots \\
\cdots\end{array}$ \\
\hline 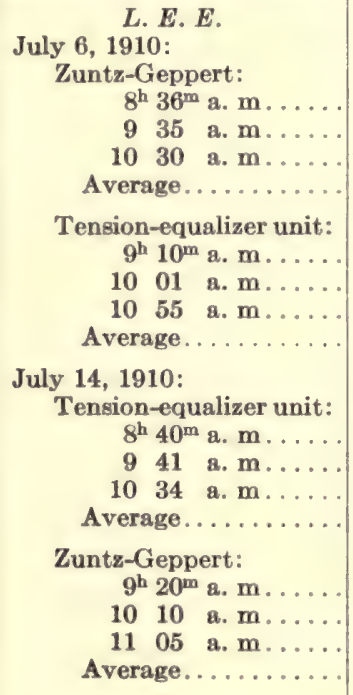 & $\begin{array}{l}202 \\
215 \\
222 \\
213\end{array}$ & $\begin{array}{l}231 \\
232 \\
244 \\
836\end{array}$ & $\begin{array}{l}.840 \\
.820 \\
.780 \\
.815\end{array}$ & $\begin{array}{l}60.5 \\
59.5 \\
58.5 \\
59.5\end{array}$ & $\begin{array}{l}13.6 \\
13.4 \\
13.3 \\
13.4\end{array}$ & $\mid \begin{array}{c}\cdots \\
\cdots \\
\cdots \\
\cdots \\
\cdots\end{array}$ & $\begin{array}{l}\cdots \cdots \\
\cdots \\
\cdots \\
\cdots\end{array}$ & $\left.\mid \begin{array}{c}\ldots \\
\ldots \\
\ldots \\
\ldots \\
\ldots\end{array}\right]$ & $\mid \begin{array}{l}\ldots \ldots \\
\ldots \ldots \\
\cdots \ldots \\
\cdots\end{array}$ \\
\hline
\end{tabular}


TABLE 17.-Respiratory exchange in comparison experiments with the Zuntz-Geppert apparatus and the Benedict respiration apparatus (tension-equalizer unit). (Without food.)-Continued.

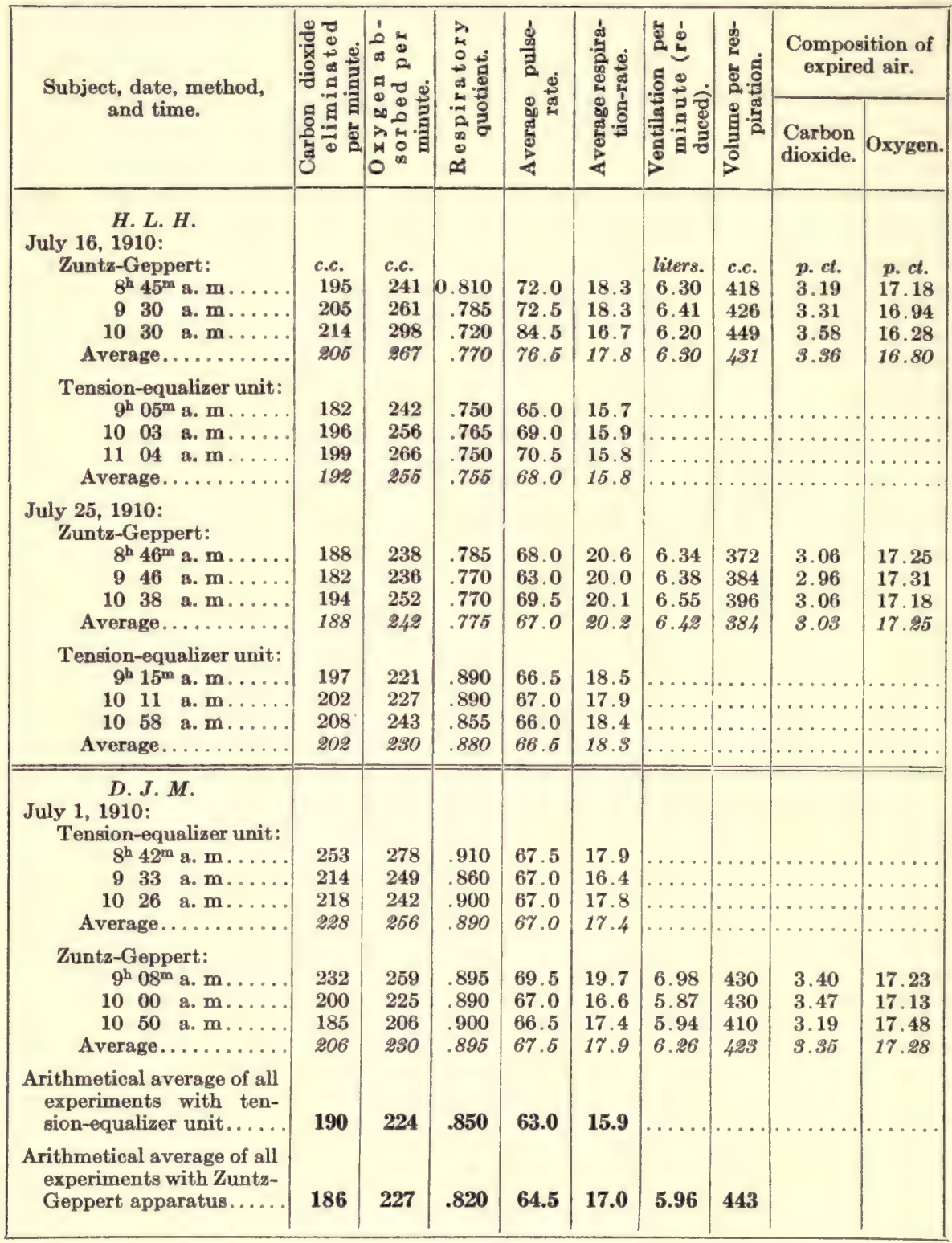

As in the previous comparisons, the differences between the averages for the two apparatus have been calculated for each experiment, using the values for the tension-equalizer unit as a base-line, and are given in table 18. The results show that this difference is sometimes plus and sometimes minus, and somewhat large in several of the compari- 
sons. The average variation is 12 c.c. for the carbon-dioxide production, 10 c.c. for the oxygen consumption, and 0.045 for the respiratory quotient.

An examination of the statistics shows that there was more or less variation in the conditions during experimenting. A few comparisons, however, show results for each apparatus which are, on the whole, entirely comparable, as, for example, the experiments with T. M. C., A. G. E., L. E. E. (July 14), and D. J. M. The averages for D. J. M. are not in close agreement, but if the periods are arranged in the order in which they were carried out it will be seen that the results give slowly descending values independent of the apparatus. This subject had been somewhat active previous to the experiment in running on errands and was accordingly not in the best of condition for such observation. The largest differences between the two apparatus are shown by the subject J. J. C., these being both plus and minus. This

TABLE 18. -Variations of average results obtained with the Zuntz-Geppert apparatus from those obtained with the Benedict respiration apparatus (tension-equalizer unit).

\begin{tabular}{|c|c|c|c|c|c|c|}
\hline Subject. & Date. & \begin{tabular}{|c|} 
Carbon \\
dioxide \\
eliminated \\
per minute.
\end{tabular} & $\begin{array}{c}\text { Oxygen } \\
\text { absorbed } \\
\text { per minute. }\end{array}$ & $\begin{array}{c}\text { Respira- } \\
\text { tory } \\
\text { quotient. }\end{array}$ & $\begin{array}{l}\text { Average } \\
\text { pulse- } \\
\text { rate. }\end{array}$ & $\begin{array}{c}\text { Average } \\
\text { respira- } \\
\text { tion- } \\
\text { rate. }\end{array}$ \\
\hline 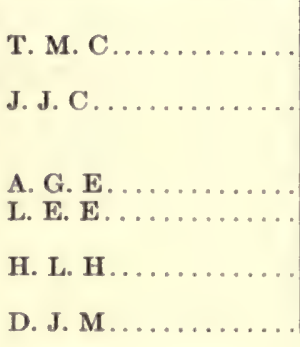 & \begin{tabular}{lr}
\multicolumn{2}{c}{1910} \\
June & 24 \\
June & 29 \\
June & 8 \\
June & 13 \\
June & 25 \\
July & 18 \\
July & 6 \\
July & 14 \\
July & 16 \\
July & 25 \\
July & 1
\end{tabular} & $\begin{array}{l}\text { c.c. } \\
-2 \\
-6 \\
-20 \\
-20 \\
+15 \\
-2 \\
+15 \\
+3 \\
+13 \\
-14 \\
-22\end{array}$ & $\begin{array}{l}\text { c.c. } \\
\pm 0 \\
+3 \\
+12 \\
-2 \\
+24 \\
+15 \\
+1 \\
+3 \\
+12 \\
+12 \\
-26\end{array}$ & $\begin{array}{l}-0.010 \\
-\quad .045 \\
-.045 \\
-.085 \\
-.025 \\
-.065 \\
+.065 \\
+.005 \\
+.015 \\
+.105 \\
+.005\end{array}$ & $\begin{array}{l}+5.0 \\
+2.0 \\
-2.0 \\
+2.5 \\
+6.5 \\
-4.0 \\
-3.5 \\
-1.0 \\
+8.5 \\
+1.5 \\
+0.5\end{array}$ & $\begin{array}{l}+1.2 \\
+6.7 \\
\pm 0 \\
+2.0 \\
+0.9 \\
-0.4 \\
-1.6 \\
-1.2 \\
+2.0 \\
+1.9 \\
+0.5\end{array}$ \\
\hline Average variation. & & 12 & 10 & .045 & 3.0 & 1.7 \\
\hline
\end{tabular}

subject was most difficult to control because of his inability to keep awake; in all probability the variations are due more to differences in wakefulness rather than to actual differences in the method of determining the respiratory exchange. An examination of the pulse-rate tends to confirm this, as the records show somewhat wide variations for the individual periods. The pulse-rate in the comparisons with other subjects also shows somewhat wide variations. As the differences in this factor are both plus and minus, there is no evidence that the pulserate is higher with one apparatus than with the other.

The percentage of uniformity in the results with the two apparatus has also been calculated for this comparison and used as a basis for plotting probability curves. (See fig. 38.) These curves show that the general uniformity is practically the same with both apparatus, 
with a tendency for the results with the tension-equalizer unit to be the more nearly uniform. The differences in the uniformity of the pulserate are somewhat marked; this again tends to confirm the belief that the cause for the differences in the respiratory exchange is due to the differences in muscular repose. It must be noted in this connection that, at the time this comparison was made, the necessity for absolute muscular repose and a uniform degree of wakefulness was not so well known as it was in the comparison of the Zuntz-Geppert apparatus with

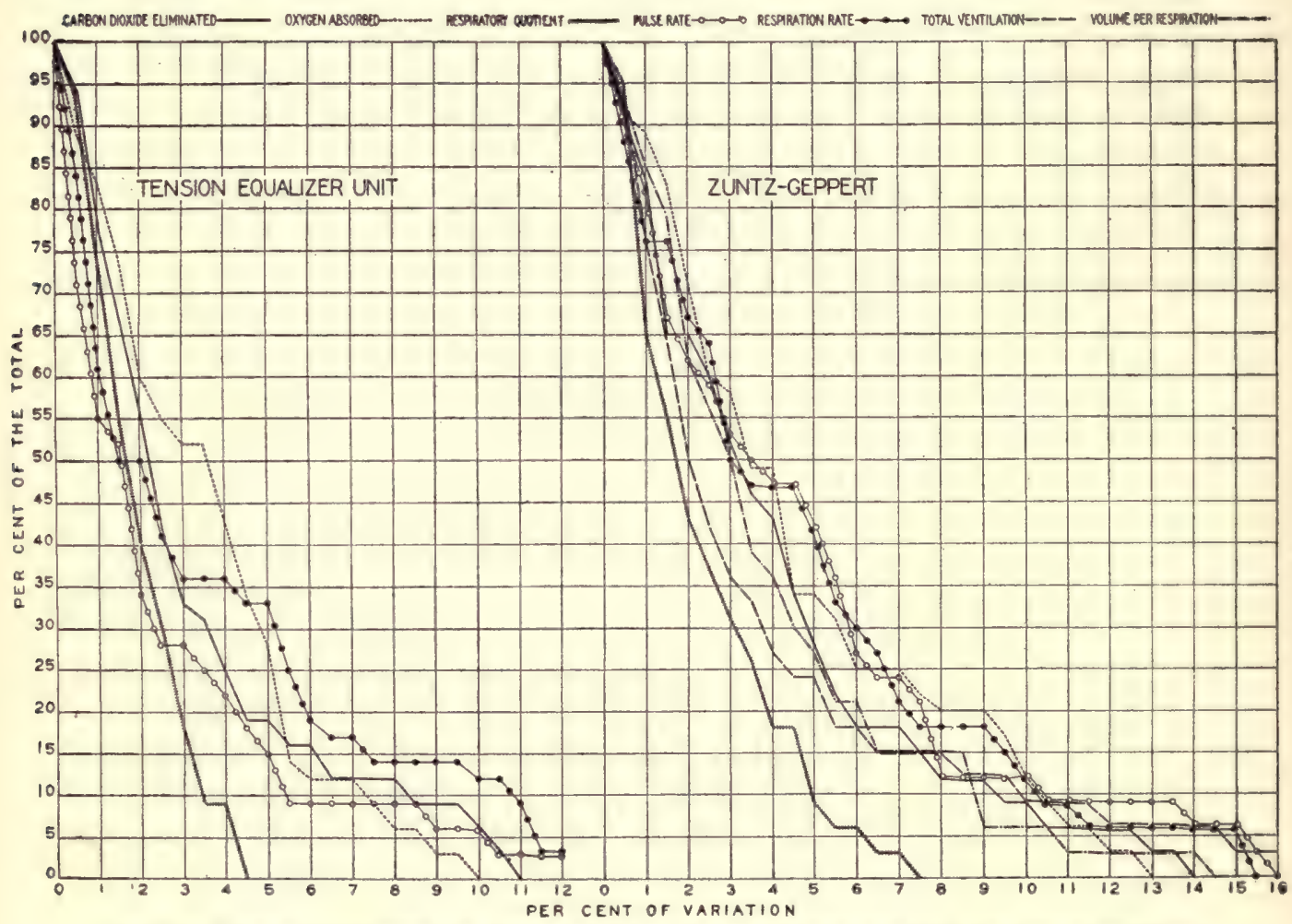

FIG. 38.-Probability curves for the series of comparison experiments with the tension-equalizer unit and the Zuntz-Geppert apparatus.

The ordinates indicate the percentage of the total number of periods and the abscissa the percentage of variation from the average.

the spirometer unit, and that no graphic method of recording the degree of muscular repose was used.

The general conclusion from the results obtained in the comparison of the tension-equalizer unit and the Zuntz-Geppert apparatus is that the two forms of apparatus give practically the same results in the measurement of the respiratory exchange. 
ZUNTZ-GEPPERT RESPIRATION APPARATUS AND BENEDICT RESPIRATION APPARATUS (SPIROMETER UNIT).

In addition to the foregoing series of experiments, in which the ZuntzGeppert respiration apparatus was compared with the tension-equalizer type of the Benedict respiration apparatus, a second series of experiments was conducted in which the same apparatus was compared with the spirometer type of the Benedict apparatus. The Zuntz gasanalysis apparatus was not used in this series of experiments, but the samples of air were collected over mercury in a tourniquet apparatus or in gas-samplers of about 300 c.c. capacity, and the analyses were made later with the laboratory form of the Haldane gas-analysis apparatus. As this procedure is not strictly according to the ZuntzGeppert method, the second series of experiments can not be considered as an actual comparison of the Zuntz-Geppert apparatus and the spirometer unit. The essential principle of the Zuntz-Geppert method of the measurement of the expired air and the method of aliquot sampling for analysis was, however, adhered to in this comparison.

The preliminary ventilation in the experiments with the ZuntzGeppert apparatus was usually obtained for several minutes preceding the experimental period, and observations are given for the preceding 5 minutes when they were secured. As a rule, the pneumatic nosepieces were used with the spirometer unit and the ordinary form of rubber mouthpiece with the Zuntz-Geppert apparatus. The pulserate was, as in previous comparisons, obtained with the Bowles stethoscope, in nearly all cases 5 counts being made in a 15 -minute period. The chest pneumograph was ordinarily used for obtaining the respiration-rate, especially in the experiments with the Zuntz-Geppert apparatus. With the spirometer unit it was obtained by means of the recording device attached to the drum of the spirometer, but in some cases the pneumograph was also used. In practically all of the experiments a record of the activity was secured from a pneumograph placed about the hips of the subject, so that slight movements of the body or of the legs would be recorded. The subjects used in this comparison series differ somewhat from those employed in the earlier comparisons, the majority being untrained men. They were mostly medical students who were obtainable in the early morning before attending lectures. The statistics and results of the 22 experiments are given in the following pages. In addition to the data given in the earlier comparison, the average barometric pressure and the average temperature of the air in the apparatus are recorded.

\section{STATISTICS OF EXPERIMENTS.}

H. F. T., January 18, 1912.-Spirometer unit, 4 periods; Zuntz-Geppert apparatus, 2 periods; first three and last periods, spirometer unit; fourth and fifth periods, Zuntz-Geppert apparatus. Pneumatic nosepieces used with both apparatus. No preliminary ventilation records were taken with the 
Zuntz-Geppert apparatus, but the subject began breathing into the apparatus as soon as the attachments were made and a sample of air was taken 1 or 2 minutes afterwards.

Pulse-rate for the most part regular. Respiration irregular. Sections of curves obtained in this experiment are reproduced in figure 39 to show the types of respiration exhibited by this subject at different times, and also to show their relation to the results. In the first period with the spirometer unit, the respiration was frequently delayed. This was wholly unconscious. The subject had frequently been used for experiments and was therefore accustomed to this apparatus. In the second period, on the contrary, there was a decided increase in the ventilation of the lungs and the effect upon the results is clearly shown. Again in the third period with the same apparatus, the respiration was apnœic. In the first period with the Zuntz-Geppert apparatus, the respiration was not distinctively apnoic and the cause for the low carbondioxide production is not so apparent as with the other apparatus. Unfortu-
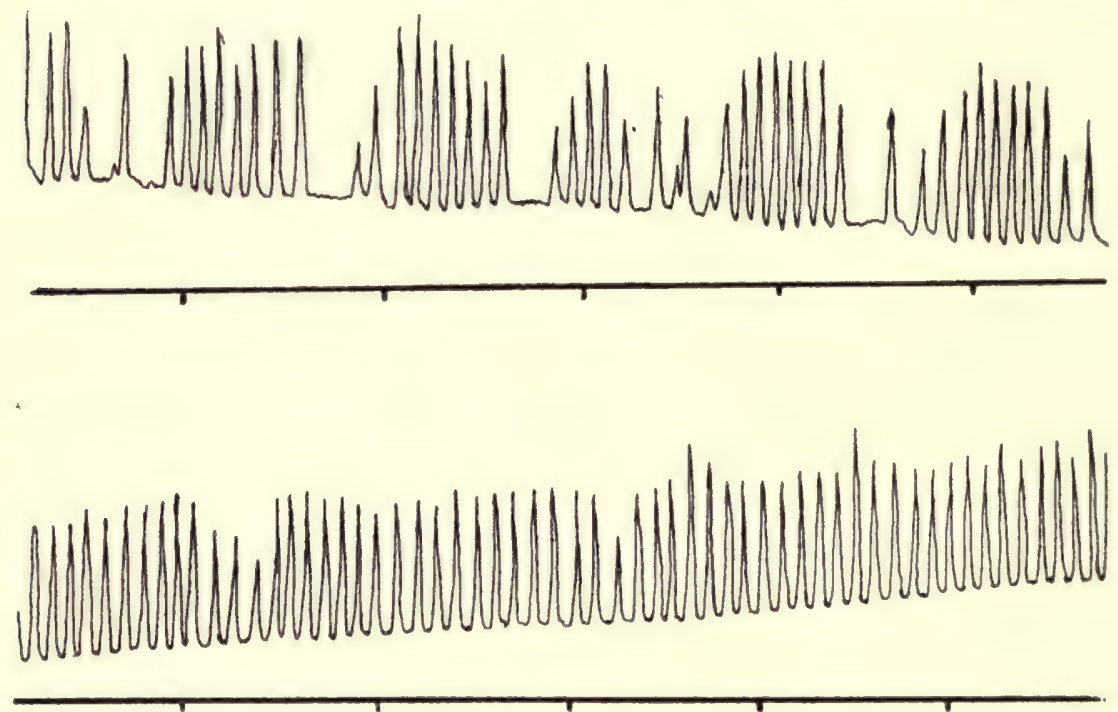

Fig. 39.-Types of respiration of subject H. F. T. with the spirometer unit on January 18, 1912. Three-fifths original size.

Upper curve, third period; lower curve, sixth period; time lines, minutes.

nately the recording apparatus was not adjusted to show the differentiation between the types very clearly. In the last period of the experiment (with the spirometer unit), the respiration was very regular. Average barometric pressure, $766.0 \mathrm{~mm}$.; average temperature of air with the spirometer unit, $22.3^{\circ} \mathrm{C}$.; with the Zuntz-Geppert apparatus, $20.7^{\circ} \mathrm{C}$.

H. F. T., January 19, 1912.-Spirometer unit, 4 periods; Zuntz-Geppert apparatus, 3 periods; apparatus alternated. No preliminary ventilation was recorded with the Zuntz-Geppert apparatus. Respiration again varying in character; with spirometer unit, more or less apnoic in first period of experiment, markedly apnœic in third period, and for the most part uniform in fifth and seventh periods; with Zuntz-Geppert apparatus, apnœic throughout second period of experiment, with slow rate and total ventilation of lungs slow; long pauses between respirations in fourth period; also many long pauses 
in sixth period. Average barometric pressure, $753.1 \mathrm{~mm}$.; average temperature of air with spirometer unit, $24.3^{\circ} \mathrm{C}$.; with Zuntz-Geppert apparatus, $22.0^{\circ} \mathrm{C}$.

H. F. T., January 27, 1912.- Spirometer unit, 4 periods; Zuntz-Geppert apparatus, 2 periods; periods with each apparatus in series. Subject lay on side instead of on back as usual. He said that, in second period with ZuntzGeppert apparatus, inhalation seemed difficult and on examination it was found that the membrane on the ingoing valve was dry. Pulse-rate for the most part uniform. Respiration-rate uniform in all periods. Average barometric pressure, $755.7 \mathrm{~mm}$; average temperature of the air in the spirometer unit, $20.8^{\circ} \mathrm{C}$.; in the Zuntz-Geppert apparatus, $17.8^{\circ} \mathrm{C}$.

H. F. T., January 29, 1912.-Zuntz-Geppert apparatus, 3 periods; spirometer unit, 2 periods; periods with each apparatus in series. With the ZuntzGeppert apparatus the more recent form of Zuntz valves (see fig. 19, page 54) and covering of fish membrane were used. With spirometer unit, the newer form of moistener (see fig. 12, page 37) was employed. Mouthpiece used with both apparatus. Subject lay on right side throughout experiment. In second period with spirometer unit, subject said that his throat became somewhat dry; the moistener was therefore moistened and in the second period with this apparatus the subject said that the air seemed more agreeable. Pulse-rate in individual periods for the most part uniform. In the first period of the experiment the pneumograph was not properly adjusted, so that a good record of the respiration was not obtained. In the second period there were a number of apnceic respirations, this being even more marked in the third period. In the last two periods of the experiment (with the spirometer unit), respiration-rate uniform. Average barometric pressure, $766.1 \mathrm{~mm}$. ; average temperature of air in spirometer unit, $21.8^{\circ} \mathrm{C}$.; in ZuntzGeppert apparatus, $19.5^{\circ} \mathrm{C}$.

H. F. T., January 30, 1912.-Spirometer unit, 3 periods; Zuntz-Geppert apparatus, 3 periods; periods with each apparatus in series. Subject lay on
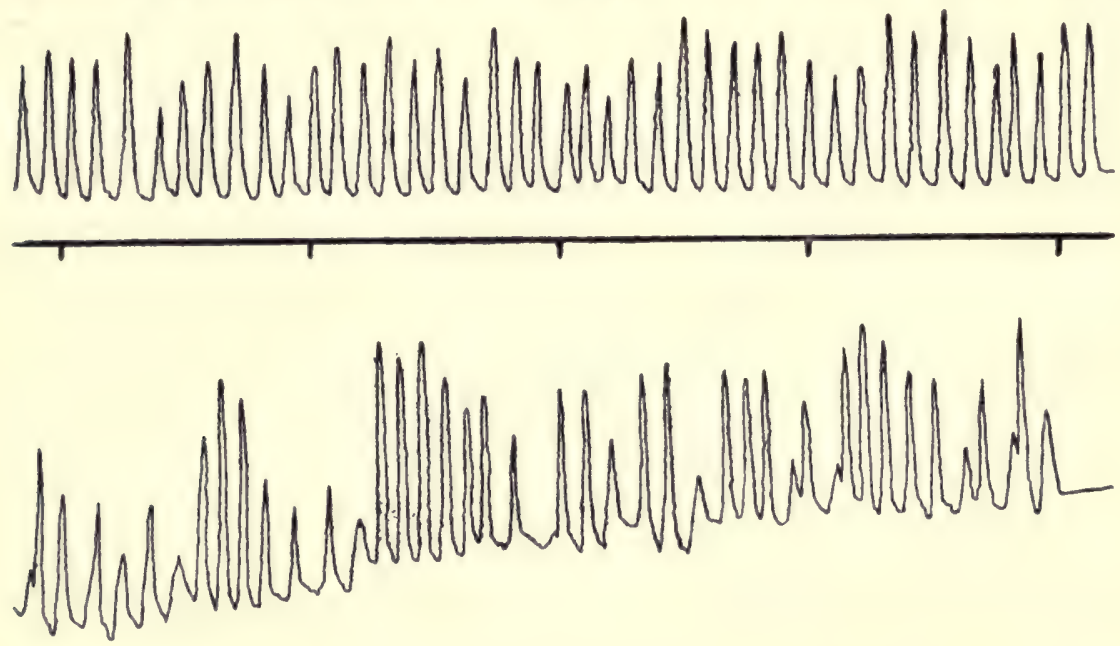

FIG. 40.-Types of respiration of subject H. F. T. with the spirometer unit on January 30, 1912. Three-fifths original size.

Upper curve, first period; lower curve, second period; time lines, minutes. 
side during whole experiment; stated that in second period with spirometer unit he was quite drowsy. Pulse-rate uniform in all experiments. Respiration-rate with spirometer unit very uniform in first period, but in the last twothirds of second period and in third period somewhat irregular. With Zuntz-Geppert apparatus, respiration-rate in practically all three periods very uniform. Sections of curves obtained with the spirometer unit are given in figure 40. Average barometric pressure, $753.3 \mathrm{~mm}$; ; average temperature of the air in the spirometer unit, $21.0^{\circ} \mathrm{C}$.; in the Zuntz-Geppert apparatus, $18.4^{\circ} \mathrm{C}$.

K. H. A., February 2, 1912.-Spirometer unit, 4 periods; Zuntz-Geppert apparatus, 2 periods; periods with each apparatus in series. Pneumatic nosepieces with spirometer unit, mouthpiece with Zuntz-Geppert apparatus. Pulse-rate fairly uniform in most of the periods. Respiration-rate regular in all periods; in third period with spirometer unit there was a tendency for the depth of expiration to vary. Average barometric pressure, $750.4 \mathrm{~mm}$.; average temperature of air with spirometer unit, $23.0^{\circ} \mathrm{C}$.; with Zuntz-Geppert apparatus, $20.6^{\circ} \mathrm{C}$.

K. H. A., February 19, 1912.-Spirometer unit, 5 periods; Zuntz-Geppert apparatus, 2 periods; periods with each apparatus in series. In second period subject opened his mouth twice, allowing air to escape; data for oxygen consumption not given in table, therefore, although the figures for carbondioxide elimination are given. In third, fourth, and fifth periods, respiration regular in rate and fairly regular in amount. In fourth period, tendency shown for air in respiratory tract at end of respiration to be irregular. Subject said that in this period the nosepieces had been inserted too deeply, which interfered somewhat with breathing. Average barometric pressure, $760.8 \mathrm{~mm}$; ; average temperature of

\begin{tabular}{|c|c|}
\hline $\begin{array}{c}\text { Period } \\
\text { beginning } \\
9^{\mathrm{h}} 59^{\mathrm{m}} \text { a. } \mathrm{m} .\end{array}$ & $\begin{array}{c}\text { Period } \\
\text { beginning } \\
10^{\mathrm{h}} 31^{\mathrm{m}} \mathrm{a} . \mathrm{m} .\end{array}$ \\
\hline liters. & liters. \\
5.60 & 4.00 \\
4.00 & 5.75 \\
4.05 & 4.95 \\
4.25 & 4.90 \\
4.15 & 4.65 \\
\hline
\end{tabular}
air in spirometer unit, 21.6 $6^{\circ} \mathrm{C}$; in Zuntz-Geppert apparatus, $15.6^{\circ} \mathrm{C}$. The preliminary ventilation by minutes preceding the two periods with the ZuntzGeppert apparatus is shown herewith.

H.H.A., February 3,1912.-Zuntz-Geppert apparatus, 2 periods; spirometer unit, 3 periods; periods with each apparatus in series. Subject drowsy at times. Pulse-rate for the most part regular in individual periods. Respiration-rate regular in all periods. Average barometric pressure, $754.3 \mathrm{~mm}$; average temperature of air in spirometer unit, $20.1^{\circ} \mathrm{C}$.; in Zuntz-Geppert apparatus, $18.8^{\circ} \mathrm{C}$.

H. H. A., February 6, 1912.-Spirometer unit, 4 periods; Zuntz-Geppert apparatus, 3 periods; periods with each apparatus in series. Pulse-rate in individual periods for the most part uniform. Respiration-rate in all periods uniform. Average barometric pressure, $757.3 \mathrm{~mm}$; average temperature of air in spirometer unit, $20.9^{\circ} \mathrm{C}$.; in Zuntz-Geppert apparatus, $18.7^{\circ} \mathrm{C}$.

H. H. A., February 8, 1912.-Zuntz-Geppert apparatus, 3 periods; spirometer unit, 2 periods; periods with each apparatus in series. Nosepieces with Zuntz-Geppert apparatus, mouthpiece with spirometer unit. Subject said in general he preferred the mouthpiece, but when used with the spirometer unit there was a tendency for the mouth to become dry. His preference was therefore to use the nosepieces for the spirometer unit and the mouthpiece with the Zuntz-Geppert apparatus instead of the reverse, as in the experiment. Both pulse-rate and respiration-rate uniform in all of the periods. Average barometric pressure, $754.0 \mathrm{~mm}$; average temperature of air in spirometer unit, $19.0^{\circ} \mathrm{C}$; in Zuntz-Geppert apparatus, $17.3^{\circ} \mathrm{C}$. 
H. H. A., February 10, 1912.-Spirometer unit, 3 periods; Zuntz-Geppert apparatus, 2 periods; periods with each apparatus in series. Pulse-rate uniform. Respiration uniform in all periods, both in rate and in amount. Average barometric pressure, $758.7 \mathrm{~mm}$; average temperature of air in spirometer unit, $19.0^{\circ} \mathrm{C}$.; in Zuntz-Geppert apparatus, $17.5^{\circ} \mathrm{C}$.

P. F. J., February 5, 1912.-Spirometer unit, 4 periods; Zuntz-Geppert apparatus, 3 periods; periods with each apparatus in series. Subject stated that in third period with spirometer unit he found it difficult to breathe, especially in inhaling. Respiration-rate uniform in all the periods. Average barometric pressure, $753.5 \mathrm{~mm}$; average temperature of air in spirometer unit, $20.3^{\circ} \mathrm{C}$.; in Zuntz-Geppert apparatus, $18.3^{\circ} \mathrm{C}$.

P.F.J., February 7, 1912.-Zuntz-Geppert apparatus, 4 periods; spirometer unit, 4 periods; periods with each apparatus in series. Subject said it was difficult to state which of the two apparatus was the easier, the difference being with the nosepieces and mouthpiece rather than with the apparatus. So far as resistance was concerned, he noted no difference between the two. Respiration uniform, except that in third period with spirometer unit there was a slight tendency toward the end for it to be shallow and more rapid. Sections of the kymograph records, showing the types of respiration, are given in figure 41. Average barometric pressure, $760.0 \mathrm{~mm}$.; average temperature of air in spirometer unit, $22.7^{\circ} \mathrm{C}$.; in Zuntz-Geppert apparatus, $19.4^{\circ} \mathrm{C}$.
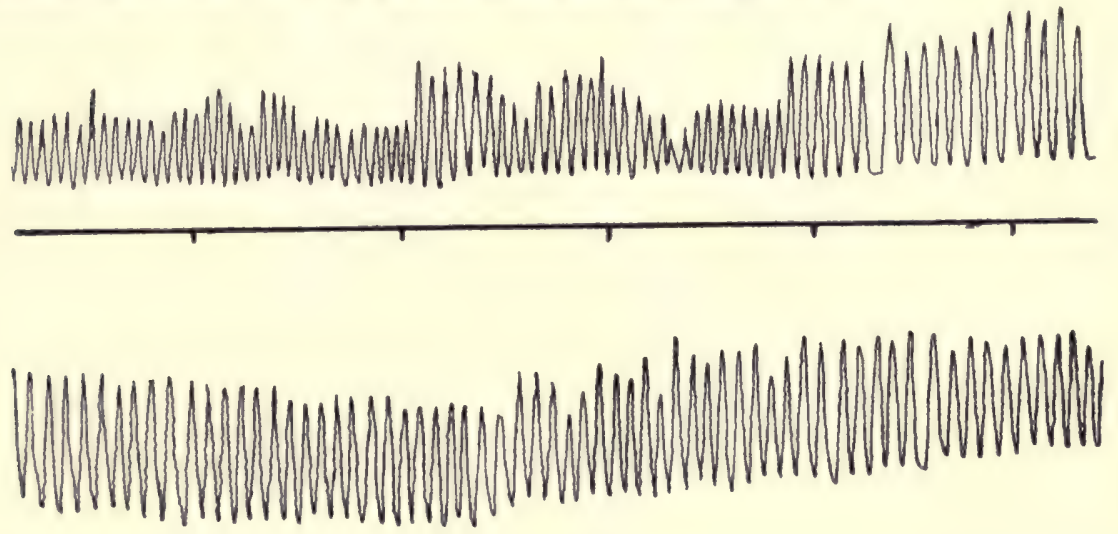

FIG. 41.-Types of respiration of subject P. F. J. with the spirometer unit on February $7,1912$. Three-fifths original size.

Upper curve, seventh period; lower curve, eighth period; time lines, minutes.

J.E.F., February 12, 1912.-Spirometer unit, 4 periods; Zuntz-Geppert apparatus, 2 periods; periods with each apparatus in series. Pulse-rate varied somewhat in the different periods. Respiration-rate uniform so far as the individual periods were concerned. Average barometric pressure, $762.8 \mathrm{~mm}$; ; average temperature of air in spirometer unit, $19.4^{\circ} \mathrm{C}$.; in Zuntz-Geppert apparatus, $18.9^{\circ} \mathrm{C}$. The preliminary ventilation by minutes preceding the two periods with the Zuntz-Geppert apparatus is shown herewith.

H. W. E., February 14, 1912.-Spirometer unit, 3

\begin{tabular}{|c|c|}
\hline $\begin{array}{c}\text { Period } \\
\text { beginning } \\
11^{\mathrm{h}} 07^{\mathrm{m}} \mathrm{a} . \mathrm{m} .\end{array}$ & $\begin{array}{c}\text { Period } \\
\text { beginning } \\
11^{\mathrm{h}} 40^{\mathrm{m}} \mathrm{a} . \mathrm{m} .\end{array}$ \\
\hline liters. & liters. \\
6.0 & 4.55 \\
6.4 & 4.40 \\
7.0 & 5.15 \\
8.35 & 4.25 \\
7.2 & 3.70 \\
\hline
\end{tabular}
periods; Zuntz-Geppert apparatus, 2 periods; periods with each apparatus in series. Pulse-rate in individual periods uniform. With spirometer unit, res- 
piration in first two periods uniform and regular. In third period somewhat irregular, with a number of pauses and shallow respirations; in this period he was drowsy and seemed to be nearly asleep. With the Zuntz-Geppert apparatus respiration somewhat irregular, as shown by the pneumograph record, the position of the chest varying at different times. There were also a number of pauses in the respiration. Average barometric pressure, $770 \mathrm{~mm}$.; average temperature of air in the spirometer unit, 21.1 ${ }^{\circ} \mathrm{C}$; ; in Zuntz-Geppert apparatus, $19.9^{\circ} \mathrm{C}$. The preliminary ventilation by minutes preceding the two periods with the Zuntz-Geppert apparatus is shown herewith.

H. B. L., February 20, 1912.-Spirometer unit, 4

\begin{tabular}{|c|c|}
\hline $\begin{array}{c}\text { Period } \\
\text { beginning } \\
8^{\mathrm{h}} 00^{\mathrm{m}} \text { a. m. }\end{array}$ & $\begin{array}{c}\text { Period } \\
\text { beginning } \\
8^{\mathrm{h}} 38^{\mathrm{m}} \mathrm{a.m} .\end{array}$ \\
\hline liters. & liters. \\
$\mathbf{5 . 2 5}$ & $\mathbf{4 . 4 5}$ \\
$\mathbf{4 . 9 0}$ & $\mathbf{5 . 9 0}$ \\
$\mathbf{4 . 9 5}$ & 3.95 \\
$\mathbf{4 . 5 5}$ & 4.50 \\
$\mathbf{5 . 1 5}$ & $\cdots$ \\
\hline
\end{tabular}
periods; Zuntz-Geppert apparatus, 3 periods; periods with each apparatus in series. Nosepieces used with both forms of apparatus. In the periods with the Zuntz-Geppert apparatus subject was drowsy and in one of them he was asleep. Of the two forms of apparatus he preferred the spirometer unit, as he found it easier to breathe with this apparatus. He was unable to tell when the three-way valve was thrown, as he detected no difference between the room air and the air in the ventilating circuit. Pulse-rate had wide range in all periods, varying as much as

\begin{tabular}{|c|c|c|}
\hline $\begin{array}{c}\text { Period } \\
\text { beginning } \\
10^{\mathrm{h}} 14^{\mathrm{m}} \mathrm{a} . \mathrm{m} .\end{array}$ & $\begin{array}{c}\text { Period } \\
\text { beginning } \\
10^{\mathrm{h}} \mathbf{4 9} \mathrm{m} \mathrm{a} . \mathrm{m} .\end{array}$ & $\begin{array}{c}\text { Period } \\
\text { beginning } \\
11^{\mathrm{h}} 28^{\mathrm{m}} \mathrm{a.m} .\end{array}$ \\
\hline liters. & liters. & liters. \\
5.7 & 5.6 & 6.1 \\
6.6 & 6.1 & 6.55 \\
4.7 & 5.55 & 4.50 \\
4.1 & 6.25 & 4.05 \\
5.0 & 6.60 & 3.85 \\
\hline
\end{tabular}
10 beats per minute. Respiration with both apparatus very uniform. Average barometric pressure, $757.5 \mathrm{~mm}$.; average temperature of air in spirometer unit, $20.2^{\circ} \mathrm{C}$; in Zuntz-Geppert apparatus, $18.6^{\circ} \mathrm{C}$. The preliminary ventilation by minutes preceding the three periods with the Zuntz-Geppert apparatus is shown herewith.

H. B. L., February 21, 1912. - Subject had light breakfast; experiment began $12^{\text {h }} 49^{\mathrm{m}}$ p. m. Zuntz-Geppert apparatus, 4 periods; spirometer unit, 4 periods; apparatus alternating for the most part. Pulse-rate varied somewhat in individual periods. Respiration uniform in each period. Average barometric pressure, 757.3 $\mathrm{mm}$. ; average temperature of air in spirometer unit, $20.1^{\circ} \mathrm{C}$;

\begin{tabular}{|c|c|c|c|}
\hline $\begin{array}{c}\text { Period } \\
\text { beginning } \\
12^{\mathrm{h}} 49^{\mathrm{m}} \text { p. m. }\end{array}$ & $\begin{array}{c}\text { Period } \\
\text { beginning } \\
2^{\mathrm{h}} 08^{\mathrm{m}} \mathrm{p} . \mathrm{m} .\end{array}$ & $\begin{array}{c}\text { Period } \\
\text { beginning } \\
3^{\mathrm{h}} 29^{\mathrm{m}} \mathrm{p} . \mathrm{m} .\end{array}$ & $\begin{array}{c}\text { Period } \\
\text { beginning } \\
4^{\mathrm{h}} 23^{\mathrm{m}} \mathrm{p.} \mathrm{m} .\end{array}$ \\
\hline liters. & liters. & liters. & liters. \\
$\mathbf{5 . 6}$ & $\mathbf{6 . 9}$ & $\mathbf{7 . 9 5}$ & $\mathbf{6 . 7}$ \\
$\mathbf{6 . 2}$ & $\mathbf{5 . 3}$ & $\mathbf{5 . 1}$ & $\mathbf{5 . 2}$ \\
$\mathbf{5 . 5}$ & $\mathbf{4 . 6}$ & 6.25 & $\mathbf{4 . 9}$ \\
$\mathbf{5 . 0}$ & $\mathbf{4 . 2 5}$ & $\mathbf{5 . 7}$ & $\mathbf{6 . 0}$ \\
$\mathbf{5 . 7}$ & $\mathbf{5 . 1}$ & $\mathbf{6 . 6 5}$ & $\mathbf{5 . 9}$ \\
\hline
\end{tabular}
in Zuntz-Geppert apparatus, $16.9^{\circ} \mathrm{C}$. The preliminary ventilation by minutes preceding the four periods with the Zuntz-Geppert apparatus is shown herewith.

H. B. L., February 28, 1912. - Subject had light breakfast at about 7 a.m.; experiment began $2^{\mathrm{h}} 10^{\mathrm{m}}$

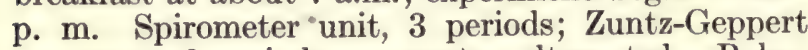
apparatus, 2 periods; apparatus alternated. Pulserate varied in most of the periods. With spirometer unit, respiration varied, being regular in the first half of period but apnœic in character in the last part of the period. This was especially apparent in the first and second periods. With the Zuntz-Geppert appa-

\begin{tabular}{|c|c|}
\hline $\begin{array}{c}\text { Period } \\
\text { beginning } \\
3^{\mathrm{h}} 13^{\mathrm{m}} \mathrm{p} . \mathrm{m} .\end{array}$ & $\begin{array}{c}\text { Period } \\
\text { beginning } \\
4^{\mathrm{h}} 17^{\mathrm{m}} \mathrm{p} . \mathrm{m} .\end{array}$ \\
\hline liters. & liters. \\
5.95 & 5.70 \\
5.75 & 5.90 \\
6.80 & 7.10 \\
5.40 & 5.45 \\
7.10 & 5.75 \\
\hline
\end{tabular}


ratus, respiration was regular in rate but varying in depth, the position of the chest as indicated by the pneumograph being different at different times. Average barometric pressure, $754.5 \mathrm{~mm}$.; average temperature of air in both apparatus, $20.5^{\circ} \mathrm{C}$. The preliminary ventilation by minutes preceding the two periods with the Zuntz-Geppert apparatus is shown herewith.

M. B., February 22, 1912.- Spirometer unit, 2 periods; Zuntz-Geppert apparatus, 2 periods; apparatus alternated. Pulse-rate fairly uniform in the different periods. Pneumograph with Zuntz-Geppert apparatus did not work properly and accordingly the curves do not show the character of the respiration plainly. Average barometric pressure, $735.3 \mathrm{~mm}$.; average temperature of air in spirometer unit, $19.3^{\circ} \mathrm{C}$.; in Zuntz-Geppert apparatus, $15.4^{\circ} \mathrm{C}$. The preliminary ventilation by minutes preceding

\begin{tabular}{|c|c|}
\hline $\begin{array}{c}\text { Period } \\
\text { beginning } \\
9^{\mathrm{h}} 02^{\mathrm{m}} \mathrm{a.} \mathrm{m} .\end{array}$ & $\begin{array}{c}\text { Period } \\
\text { beginning } \\
\mathbf{9}^{\mathrm{h}} \mathbf{5 5 ^ { \mathrm { m } } \mathrm { a } . \mathrm { m } .}\end{array}$ \\
\hline liters. & liters. \\
$\mathbf{4 . 5}$ & 6.55 \\
$\mathbf{5 . 2 5}$ & $\mathbf{4 . 1 5}$ \\
$\mathbf{4 . 8 5}$ & $\mathbf{5 . 1 0}$ \\
$\mathbf{5 . 1 0}$ & $\mathbf{3 . 5 0}$ \\
$\mathbf{5 . 0 0}$ & $\mathbf{4 . 4 5}$ \\
\hline
\end{tabular}
the three periods with the Zuntz-Geppert apparatus is shown herewith.

M. B., February 27, 1912.-Zuntz-Geppert apparatus, 3 periods; spirometer unit, 2 periods; apparatus alternated for the most part. Pulse-rate for the most part uniform. Respiration regular in the individual periods but varying from one period to another. Average barometric pressure, $740.3 \mathrm{~mm}$.; average temperature of air in spirometer unit, $21.0^{\circ} \mathrm{C}$.; in Zuntz-Geppert apparatus, $18.7^{\circ} \mathrm{C}$. The preliminary ventilation by minutes preceding the three periods with the Zuntz-Geppert apparatus is shown herewith.

M. B., March 2, 1912.- Subject had light breakfast at about 7 a. m.; experiment began $1^{\mathrm{h}} 12^{\mathrm{m}}$ p. m. Spirometer unit, 3 periods; Zuntz-Geppert apparatus, 3 periods; apparatus alternated. Mouthpiece used for both apparatus. Pulse-rate regular. Respiration uniform in individual periods. Average barometric pressure, $766.9 \mathrm{~mm}$.; average temperature of air in spirometer unit, $20.6^{\circ} \mathrm{C}$; in Zuntz-Gep-

\begin{tabular}{|c|c|c|}
\hline $\begin{array}{c}\text { Period } \\
\text { beginning } \\
8^{\mathrm{h}} 28^{\mathrm{m}} \text { a. m. }\end{array}$ & $\begin{array}{c}\text { Period } \\
\text { beginning } \\
9^{\mathrm{h}} 50^{\mathrm{m}} \text { a. m. }\end{array}$ & $\begin{array}{c}\text { Period } \\
\text { beginning } \\
10^{\mathrm{h}} 41^{\mathrm{m}} \text { a. } \mathrm{m} .\end{array}$ \\
\hline $\begin{array}{l}\text { liters. } \\
5.1 \\
5.6 \\
4.9 \\
5.35 \\
\ldots \ldots\end{array}$ & $\begin{array}{l}\text { liters. } \\
5.8 \\
\mathbf{4 . 9} \\
\mathbf{5 . 8 5} \\
\mathbf{5 . 5 5} \\
\mathbf{5 . 6 0}\end{array}$ & $\begin{array}{l}\text { liters. } \\
\mathbf{5 . 1} \\
\mathbf{4 . 9} \\
\mathbf{4 . 8 5} \\
\mathbf{5 . 1} \\
\mathbf{5 . 3 5}\end{array}$ \\
\hline
\end{tabular}

\begin{tabular}{|c|c|c|}
\hline $\begin{array}{c}\text { Period } \\
\text { beginning } \\
\mathbf{1}^{\mathrm{h}} 50^{\mathrm{m}} \mathrm{p.} \mathrm{m} .\end{array}$ & $\begin{array}{c}\text { Period } \\
\text { beginning } \\
2^{\mathrm{h}} \mathbf{5} 3^{\mathrm{m}} \mathrm{p} . \mathrm{m} .\end{array}$ & $\begin{array}{c}\text { Period } \\
\text { beginning } \\
4^{\mathrm{h}} 01^{\mathrm{m}} \mathrm{p} . \mathrm{m} .\end{array}$ \\
\hline liters. & liters. & liters. \\
4.65 & 4.2 & 4.4 \\
5.05 & 4.8 & 5.9 \\
5.00 & 4.85 & 5.2 \\
4.05 & 4.75 & 5.0 \\
3.85 & 5.4 & 6.1 \\
\hline
\end{tabular}
pert apparatus, $19.1^{\circ} \mathrm{C}$. 'The preliminary ventilation by minutes preceding the three periods with the Zuntz-Geppert apparatus is shown herewith.

Ma. B., February 29, 1912.-Spirometer unit, 3 periods; Zuntz-Geppert apparatus, 2 periods; first, second, and last periods with spirometer unit, third and fourth periods with Zuntz-Geppert apparatus. Pneumatic nosepieces used with both apparatus. Subject active in first period with Zuntz-Geppert apparatus, but in others fairly quiet. Pulse-rate regular; also respiration. Average barometric pressure, $762.7 \mathrm{~mm}$.; average temperature of air in spirometer unit, 21.1 ${ }^{\circ} \mathrm{C}$.; in Zuntz-Geppert apparatus,

\begin{tabular}{|c|c|}
\hline $\begin{array}{c}\text { Period } \\
\text { beginning } \\
\mathbf{\theta}^{\mathrm{h}} \mathbf{4 6 ^ { \mathrm { m } } \mathrm { a. } \mathrm { m } .}\end{array}$ & $\begin{array}{c}\text { Period } \\
\text { beginning } \\
10^{\mathrm{h}} \mathbf{4} 8^{\mathrm{m}} \mathrm{a.} \mathrm{m} .\end{array}$ \\
\hline liters. & liters. \\
$\mathbf{6 . 3 5}$ & $\mathbf{5 . 5}$ \\
$\mathbf{6 . 3 5}$ & 5.9 \\
$\mathbf{6 . 4 5}$ & $\mathbf{5 . 4 5}$ \\
6.4 & 5.95 \\
6.9 & 5.2 \\
\hline
\end{tabular}
$20.6^{\circ} \mathrm{C}$. The preliminary ventilation by minutes preceding the two periods with the Zuntz-Geppert apparatus is shown herewith. 


\section{DISCUSSION OF RESULTS.}

The results of the experiments are given in table 19, with an average for each apparatus for every experiment and a general average for each apparatus for the whole series of comparisons. The greatest average difference in the respiratory exchange with the two methods is shown in the results for the carbon-dioxide elimination, that with the ZuntzGeppert apparatus being 176 c.c. and for the spirometer unit 182 c.c. The average oxygen consumption with the two apparatus is nearly identical, i.e., 220 c.c. for the Zuntz-Geppert apparatus and 219 c.c. for the spirometer unit; the respiratory quotient is 0.80 for the ZuntzGeppert apparatus and 0.83 for the spirometer unit. The average pulse-rate is $\mathbf{5 8 . 5}$ for both apparatus. There is only a slight difference in the average respiration-rate, which is $\mathbf{1 2 . 3}$ for the Zuntz-Geppert apparatus as compared with 12.5 for the spirometer unit. The average ventilation per minute and volume per respiration are slightly lower with the Zuntz-Geppert apparatus (4.45 liters and 448 c.c., respectively) than with the spirometer unit (4.76 liters and 480 c.c., respectively).

TABLE 19.-Respiratory exchange in comparison experiments with the Zuntz-Geppert apparatus and the Benedict respiration apparatus (spirometer unit).

(Without food.)

\begin{tabular}{|c|c|c|c|c|c|c|c|c|c|}
\hline \multirow{2}{*}{$\begin{array}{l}\text { Subject, date, method, } \\
\text { and time. }\end{array}$} & \multirow{2}{*}{ 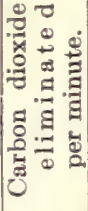 } & \multirow{2}{*}{ 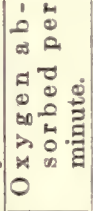 } & \multirow{2}{*}{ 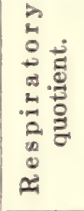 } & \multirow{2}{*}{ 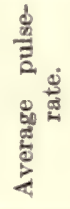 } & \multirow{2}{*}{ 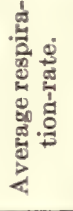 } & \multirow{2}{*}{ 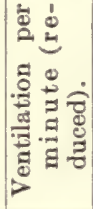 } & \multirow{2}{*}{ 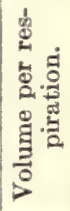 } & \multicolumn{2}{|c|}{$\begin{array}{l}\text { Composition of } \\
\text { expired air. }\end{array}$} \\
\hline & & & & & & & & $\begin{array}{l}\text { Carbon } \\
\text { dioxide. }\end{array}$ & Oxygen \\
\hline $\begin{array}{c}H . F . T . \\
\text { Jan. 18, } 1912:\end{array}$ & & & & & & & & & \\
\hline $\begin{array}{l}\text { Spirometer unit: } \\
6^{\mathrm{h}} 38^{\mathrm{m}} \text { a. } \mathrm{m}\end{array}$ & $\begin{array}{l}c . c . \\
166\end{array}$ & $\begin{array}{l}c . c \\
195\end{array}$ & 0.850 & 51.5 & 9.5 & $\begin{array}{r}\text { liters. } \\
4.23\end{array}$ & $\begin{array}{l}\text { c.c. } \\
534\end{array}$ & p. ct. & p. ct. \\
\hline 702 a. 1 & 188 & 185 & 1.015 & 51.5 & 10.4 & 5.19 & 599 & & \\
\hline 728 a. 1 & 177 & 191 & 0.925 & 51.5 & 9.0 & 4.40 & 587 & & \\
\hline 840 a. $n$ & 172 & 212 & .810 & 53.5 & 10.4 & 4.97 & 573 & & \\
\hline Average. & 176 & 196 & .900 & 52.0 & 9.8 & 4.70 & 573 & & \\
\hline Zuntz-Geppert: & & & & & & & & & \\
\hline $\begin{array}{l}7^{\mathrm{h}} 56^{\mathrm{m}} \text { a. } \mathrm{n} \\
8 \quad 20 \text { a. } \mathrm{r}\end{array}$ & $\begin{array}{l}152 \\
171\end{array}$ & $\begin{array}{l}189 \\
199\end{array}$ & 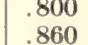 & $\begin{array}{l}53.0 \\
53.0\end{array}$ & $\begin{array}{l}11.4 \\
10.4\end{array}$ & $\begin{array}{l}4.20 \\
4.57\end{array}$ & $\begin{array}{l}433 \\
517\end{array}$ & $\begin{array}{l}3.71 \\
3.83\end{array}$ & $\begin{array}{l}16.55 \\
16.65\end{array}$ \\
\hline $\begin{array}{c}820 \text { a. m... } \\
\text { Average........ }\end{array}$ & $\begin{array}{l}171 \\
162\end{array}$ & 194 & .830 & 53.0 & 10.9 & 4.39 & 475 & 3.77 & 16.60 \\
\hline $\begin{array}{l}\text { Jan. 19, 1912: } \\
\text { Spirometer unit: }\end{array}$ & & & & & & & & & \\
\hline $6^{\mathrm{h}} 19^{\mathrm{m}}$ a. m & 195 & 213 & .915 & 52.0 & 9.1 & 4.76 & 638 & & \\
\hline 714 a. $\mathrm{n}$ & 157 & 189 & .830 & 49.5 & 9.3 & 4.42 & 580 & & \\
\hline 806 a. $m$ & 184 & 198 & .93 & 54 & 9.9 & 5.07 & 627 & & \\
\hline 851 a. m. & 166 & 202 & .820 & 54.5 & 11.1 & 5.28 & 582 & & \\
\hline Average ........ & 176 & 201 & .875 & 52.5 & 9.9 & 4.88 & 607 & & \\
\hline Zuntz-Geppert: & & & & & & & & & \\
\hline $\begin{array}{l}6^{\mathrm{h}} 50^{\mathrm{m}} \text { a. } \mathrm{n} \\
740 \text { a. } \mathrm{n}\end{array}$ & $\begin{array}{l}134 \\
147\end{array}$ & $\begin{array}{l}182 \\
195\end{array}$ & .735 & $\begin{array}{l}48.5 \\
49.0\end{array}$ & $\begin{array}{r}8.4 \\
10.4\end{array}$ & $\begin{array}{l}3.04 \\
4.14\end{array}$ & $\begin{array}{l}320 \\
483\end{array}$ & $\begin{array}{l}3.85 \\
3.70\end{array}$ & $\begin{array}{l}10.11 \\
16.36\end{array}$ \\
\hline 831 a. m. & 158 & 223 & .710 & 52.5 & 11.2 & 4.83 & 516 & 3.42 & 16.50 \\
\hline Average........ & 146 & 200 & .730 & 50.0 & 10.0 & 4.20 & 506 & 3.65 & 16.32 \\
\hline $\begin{array}{l}\text { Jan. 27, 1912: } \\
\text { Spirometer unit: }\end{array}$ & & & & & & & & & \\
\hline & 185 & 213 & .870 & 56.5 & 11.2 & 5. 1 & 563 & & \\
\hline 654 a. $\mathrm{m}$. & 15 & 198 & .785 & 56.5 & 9.9 & 4. & 510 & & \\
\hline 716 a. $\mathrm{m}$. & 159 & 198 & .805 & 53 & 9.8 & 4.3 & 537 & & \\
\hline 735 a. $\mathrm{m}$. & 171 & 212 & .805 & 53.0 & 10.6 & 4.64 & 533 & & \\
\hline 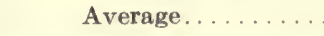 & 168 & 205 & .820 & 55.0 & 10.4 & 4.57 & 536 & & \\
\hline
\end{tabular}


TABLE 19.-Respiratory exchange in comparison experiments with the Zuntz-Geppert apparatus and the Benedict respiration apparatus (spirometer unit). (Without food.)-Continued.

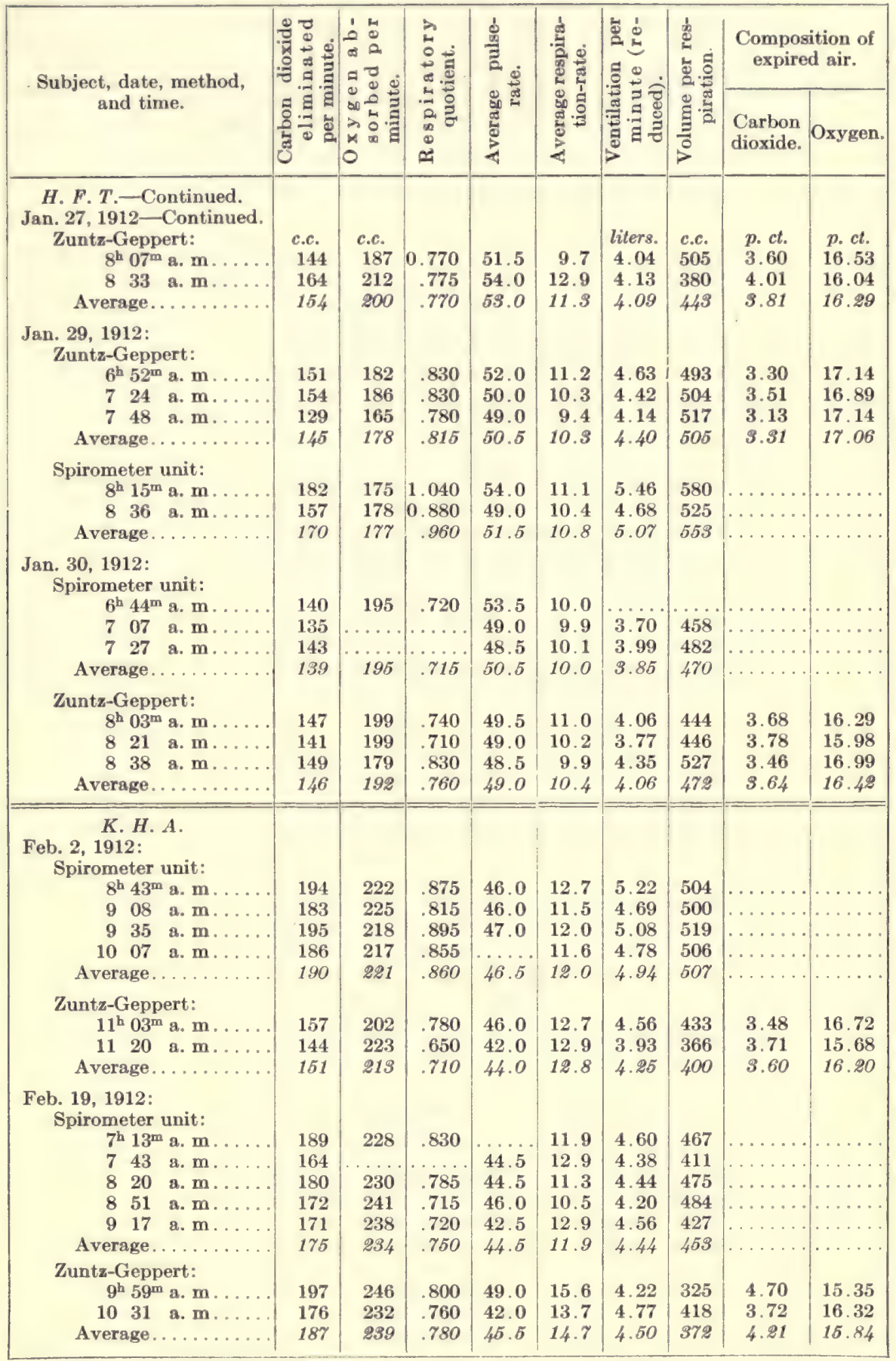


TABLE 19.-Respiratory exchange in comparison experiments with the Zuntz-Geppert apparatus and the Benedict respiration apparatus (spirometer unit). (Without food.)-Continued.

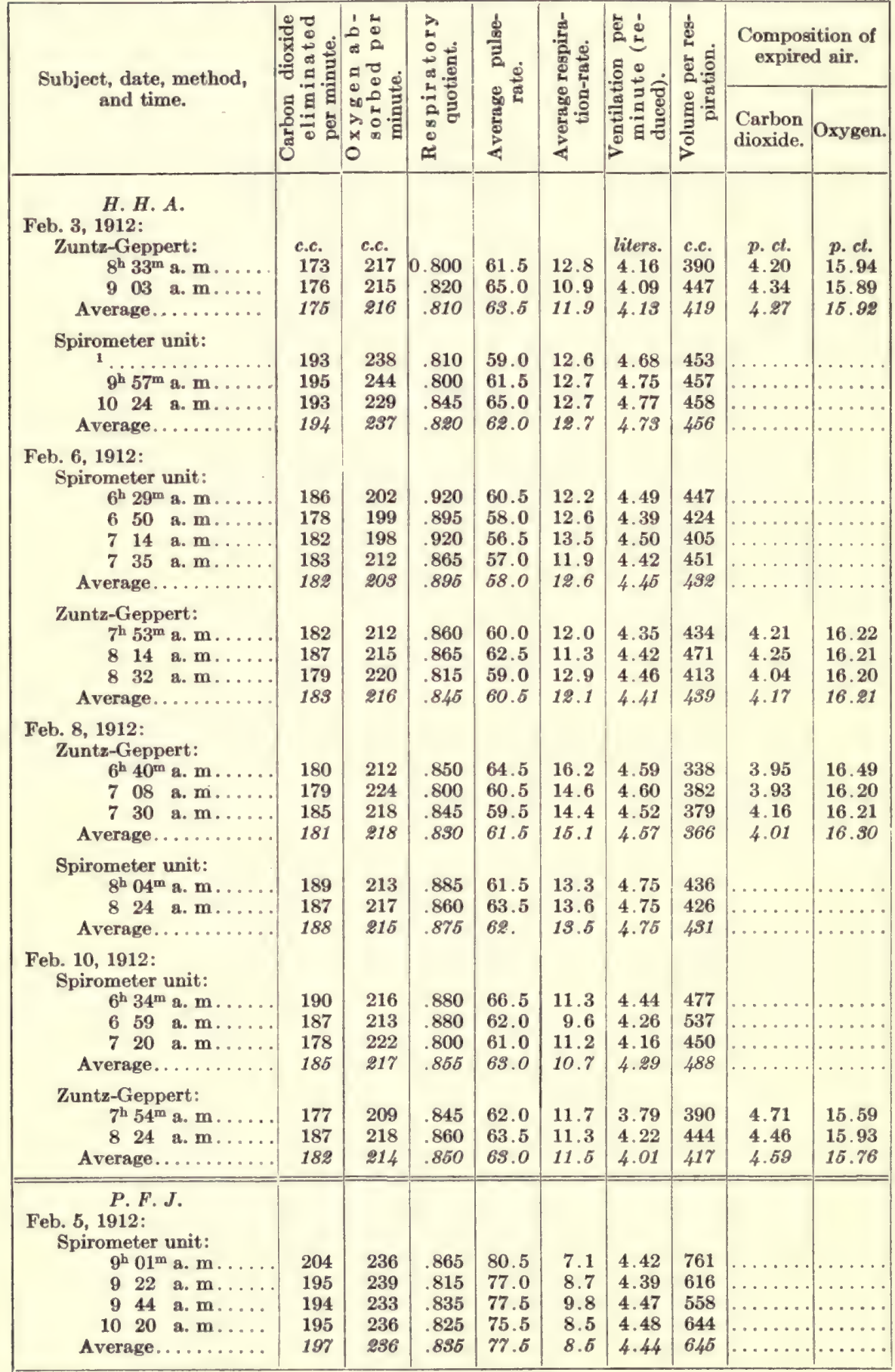


TABLE 19.-Respiratory exchange in comparison experiments with the Zuntz-Geppert apparatus and the Benedict respiration apparatus (spirometer unit). (Without food.) - Continued.

\begin{tabular}{|c|c|c|c|c|c|c|c|c|c|}
\hline \multirow{2}{*}{$\begin{array}{l}\text { Subject, date, method, } \\
\text { and time. }\end{array}$} & \multirow{2}{*}{ 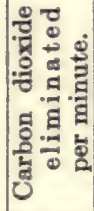 } & \multirow{2}{*}{ 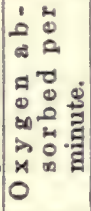 } & \multirow{2}{*}{ 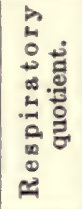 } & \multirow{2}{*}{ 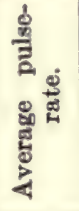 } & \multirow{2}{*}{ 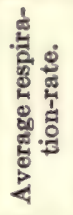 } & \multirow{2}{*}{ 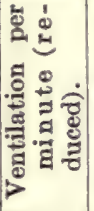 } & \multirow{2}{*}{ 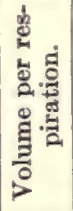 } & \multicolumn{2}{|c|}{$\begin{array}{l}\text { Composition of } \\
\text { expired air. }\end{array}$} \\
\hline & & & & & & & & $\begin{array}{l}\text { Carbon } \\
\text { dioxide. }\end{array}$ & Oxygen \\
\hline $\begin{array}{l}\text { P. F.J. - Continued. } \\
\text { Feb. 5, 1912-Continued. }\end{array}$ & & & & & & & & & \\
\hline $\begin{array}{l}\text { Zuntz-Geppert: } \\
10^{\mathrm{h}} 48^{\mathrm{m}} \text { a. m. . . . . }\end{array}$ & $\begin{array}{l}\text { c.e. } \\
177\end{array}$ & $\begin{array}{c}\text { c.c. } \\
236\end{array}$ & 0.750 & 71.5 & 8.0 & $\begin{array}{c}\text { liters. } \\
\mathbf{3 . 6 9}\end{array}$ & $\begin{array}{l}\text { c.c. } \\
556\end{array}$ & $\begin{array}{l}\text { p. ct. } \\
4.83\end{array}$ & $\begin{array}{l}\text { p. } c t . \\
14.89\end{array}$ \\
\hline 1123 a. m. & 191 & 248 & .770 & 71.0 & 7.8 & 4.17 & 641 & 4.62 & 15.27 \\
\hline 1144 a. m. & 200 & 270 & .740 & 72.5 & 8.0 & 4.31 & 653 & 4.67 & 15.02 \\
\hline Average........... & 189 & 251 & .755 & 71.5 & 7.9 & 4.06 & 617 & 4.71 & 15.06 \\
\hline \multicolumn{10}{|l|}{$\begin{array}{l}\text { Feb. 7, 1912: } \\
\text { Zuntz-Geppert: }\end{array}$} \\
\hline $8^{\mathrm{h}} 45^{\mathrm{m}}$ a. m & 207 & 259 & .800 & 80.5 & 6.3 & 4.20 & 789 & 4.96 & 15.03 \\
\hline 912 a. $\mathrm{m}$. & 208 & 257 & .810 & 77.5 & 7.5 & 4.30 & 677 & 4.87 & 15.19 \\
\hline 936 a. m. & 190 & 251 & .755 & 75.0 & 8.5 & 3.85 & 546 & 4.96 & 14.75 \\
\hline 1006 a.m. & 194 & 250 & .775 & 70.5 & 8.6 & 4.24 & 591 & 4.61 & 15.32 \\
\hline Average........ & 800 & 254 & .785 & 76.0 & 7.7 & 4.15 & 651 & 4.85 & 15.07 \\
\hline \multicolumn{10}{|l|}{ Spirometer unit: } \\
\hline $10^{\mathrm{h}} 35^{\mathrm{m}}$ a. $\mathrm{m}$ & 188 & 244 & .770 & 64.0 & 10.8 & 4.58 & 514 & & \\
\hline 1100 a. $\mathrm{m}$. & 188 & 229 & .820 & 67.5 & 10.8 & 4.57 & 513 & & \\
\hline 1120 a. $\mathrm{m}$. & 172 & 232 & .740 & 61.5 & 14.0 & 4.38 & 379 & & \\
\hline 1141 a. $\mathrm{m}$. & 188 & 247 & .760 & 69.0 & 11.2 & 4.58 & 496 & & \\
\hline Average....... & 184 & 238 & .775 & 65.5 & 11.7 & 4.53 & 476 & & \\
\hline \multirow{2}{*}{\multicolumn{10}{|c|}{$\begin{array}{l}\text { J.E. F. } \\
\text { Feb. 12, 1912: } \\
\text { Spirometer unit: }\end{array}$}} \\
\hline & & & & & & & & & \\
\hline 920 a. m. & 201 & 241 & .835 & 57.5 & 8.9 & 4.92 & 666 & & \\
\hline 944 a. m. & 183 & 227 & .805 & 56.0 & 11.2 & 4.88 & 525 & & \\
\hline 1011 a. m. & 194 & 227 & .855 & 54.5 & 9.7 & 4.99 & 620 & & \\
\hline Average....... & 195 & 248 & .805 & 57.0 & 9.7 & 4.99 & 616 & & \\
\hline \multicolumn{10}{|l|}{ Zuntz-Geppert: } \\
\hline $11^{\text {h }} 07^{m}$ a. $n$ & 194 & 254 & .765 & 50.5 & 11.3 & 5.29 & 559 & 3.70 & 16.38 \\
\hline 1140 a. $\mathrm{m}$ & 184 & 245 & .750 & 54.0 & 11.8 & 4.04 & 404 & 4.59 & 15.19 \\
\hline Average..... & 189 & 250 & .760 & 52.5 & 11.6 & 4.67 & 482 & 4.15 & 15.79 \\
\hline \multicolumn{10}{|l|}{$\begin{array}{l}H \cdot W \cdot \boldsymbol{E} . \\
.\end{array}$} \\
\hline $\begin{array}{l}\text { Spirometer unit: } \\
6^{\mathrm{h}} 31^{\mathrm{m}} \text { a. } \mathrm{m}\end{array}$ & 18 & & & & & & & & \\
\hline 655 a. $\mathrm{m}$. & 186 & 214 & .870 & 48.0 & 11.0 & 4.53 & 492 & & \\
\hline 721 a.m & 201 & 204 & .985 & 51.0 & 10.9 & 4.98 & 546 & & \\
\hline Average........ & 191 & 210 & .910 & 49.0 & 11.1 & 4.69 & 504 & & \\
\hline \multicolumn{10}{|l|}{ Zuntz-Geppe } \\
\hline $8^{\text {h }} 00^{m}$ a. m & 201 & 221 & .910 & 50.0 & 10.9 & 4.60 & 495 & 4.39 & 16.24 \\
\hline 838 a. m. & 194 & 237 & .820 & 47.5 & 10.2 & 4.59 & 530 & 4.26 & 15.97 \\
\hline Average....... & 198 & 229 & .865 & 49.0 & 10.6 & 4.60 & 513 & 4.33 & 16.11 \\
\hline \multicolumn{10}{|l|}{$\begin{array}{l}\text { H. B. L. } \\
\text { Feb. 20, 1912: }\end{array}$} \\
\hline \multicolumn{10}{|l|}{ Spirometer unit: } \\
\hline 833 a. $\mathrm{m}$. & 177 & 229 & .775 & 63.0 & 13.4 & 4.7 & 428 & & \\
\hline 900 a. $\mathrm{m}$. & 151 & 206 & .735 & 60.5 & 13.2 & 4.2 & 376 & & \\
\hline 929 a. m..... & 179 & 218 & .820 & 61.5 & 13.8 & 4.7 & 446 & & \\
\hline Average......... & 177 & 223 & .795 & 64.0 & 13.7 & 4.7 & 419 & & \\
\hline
\end{tabular}


TABLE 19.-Respiratory exchange in comparison experiments with the $Z$ untz-Geppert apparatus and the Benedict respiration apparatus (spirometer unit). (Without food.)-Continued.

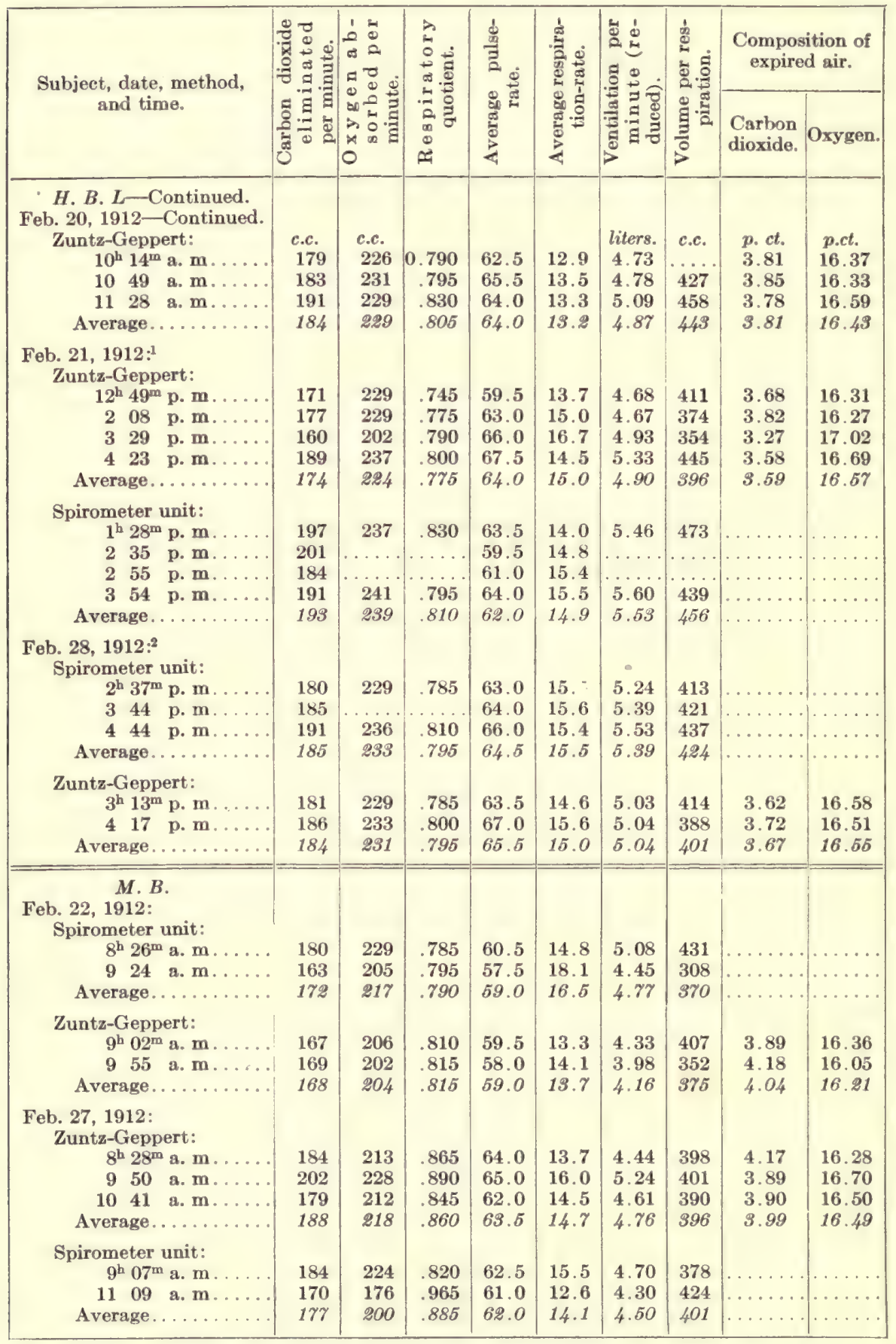

${ }^{1}$ Subject had light breakfast.

2Subject had light breakfast about 7 a. m. 
TABLE 19.-Respiratory exchange in comparison experiments with the Zuntz-Gepperl apparatus and the Benedict respiration apparatus (spirometer unit). (Without food.)-Continued.

\begin{tabular}{|c|c|c|c|c|c|c|c|c|c|}
\hline \multirow{2}{*}{$\begin{array}{l}\text { Subject, date, method, } \\
\text { and time. }\end{array}$} & \multirow{2}{*}{ 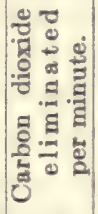 } & \multirow{2}{*}{ 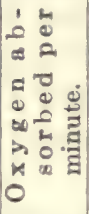 } & \multirow{2}{*}{ 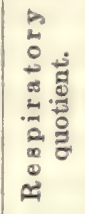 } & \multirow{2}{*}{ 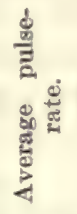 } & \multirow{2}{*}{ 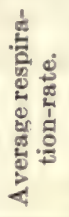 } & \multirow{2}{*}{ 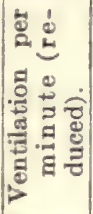 } & \multirow{2}{*}{ 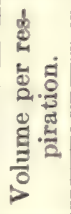 } & \multicolumn{2}{|c|}{$\begin{array}{l}\text { Composition of } \\
\text { expired air. }\end{array}$} \\
\hline & & & & & & & & $\begin{array}{l}\text { Carbon } \\
\text { dioxide. }\end{array}$ & Oxygen. \\
\hline 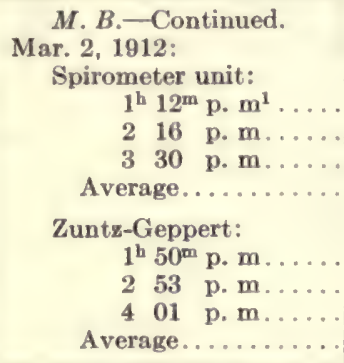 & $\begin{array}{l}c . c . \\
171 \\
166 \\
179 \\
172\end{array}$ & $\begin{array}{l}c . c . \\
225 \\
216 \\
218 \\
220\end{array}$ & $\begin{array}{r}0.760 \\
.770 \\
.820 \\
.780\end{array}$ & $\begin{array}{l}65.5 \\
64.0 \\
65.5 \\
65.0\end{array}$ & $\begin{array}{l}10.8 \\
11.3 \\
14.7 \\
12.3\end{array}$ & $\begin{array}{l}\text { liters. } \\
4.49 \\
4.38 \\
5.37 \\
4.75\end{array}$ & $\begin{array}{l}\text { c.c. } \\
379 \\
416 \\
364 \\
386\end{array}$ & $\begin{array}{c}\quad p . c t . \\
\cdots \cdots \\
\cdots \cdots \\
\cdots \cdots \\
\cdots \cdots \\
\cdots\end{array}$ & $\begin{array}{l}\text { p. ct. } \\
\cdots \cdots \\
\cdots \cdots \\
\cdots \cdots \\
\cdots \cdots\end{array}$ \\
\hline 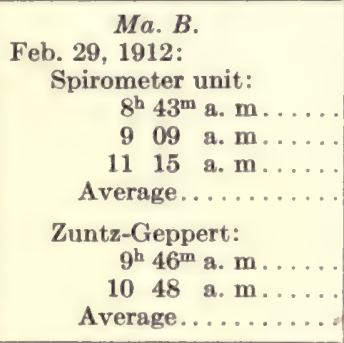 & $\begin{array}{l}217 \\
226 \\
220 \\
221\end{array}$ & $\begin{array}{l}257 \\
265 \\
266 \\
263\end{array}$ & $\begin{array}{l}.845 \\
.855 \\
.825 \\
.840\end{array}$ & $\begin{array}{l}68.5 \\
63.5 \\
55.5 \\
62.5\end{array}$ & $\begin{array}{l}19.9 \\
19.8 \\
21.4 \\
20.4\end{array}$ & $\begin{array}{l}5.85 \\
6.05 \\
6.02 \\
5.97\end{array}$ & $\begin{array}{l}355 \\
368 \\
339 \\
354\end{array}$ & $\begin{array}{l}3.94 \\
4.28 \\
4.11\end{array}$ & $\begin{array}{l}16.52 \\
15.97 \\
16.25\end{array}$ \\
\hline $\begin{array}{l}\text { Arithmetical average of all } \\
\text { experiments with spi- } \\
\text { rometer unit. . . . . . . . } \\
\text { Arithmetical average of all } \\
\text { experiments with } \mathrm{Zuntz-} \\
\text { Geppert apparatus..... }\end{array}$ & 182 & 219 & .830 & 58.5 & 12.5 & 4.76 & 480 & & \\
\hline
\end{tabular}

${ }^{1}$ Subject had light breakfast at about 7 a. m.

The differences in the individual experiments are given in table 20, the values for the spirometer unit being used for the base-line. If these differences are considered, it will be found that on the whole the variations between the two apparatus are not very large. The greatest differences are found with the subject $\mathrm{H}$. F. T., who was an extremely difficult subject to work with, as, without consciousness on his part, his respiration showed frequent periods of apnœea. It will be seen that in nearly all of the experiments with this subject, the carbondioxide production with the Zuntz-Geppert apparatus is lower than with the spirometer unit and that there is a somewhat marked difference in the respiratory quotient. There is likewise a large difference in the volume per respiration, this volume being much smaller with the Zuntz-Geppert apparatus than with the spirometer unit. The subject K. H. A. shows a somewhat wide variation on February 2. In the experiment on this date, all of the periods with the spirometer unit 
preceded those with the Zuntz-Geppert apparatus, which may in part account for the difference in the results. The value of 144 c.c. per minute for the carbon-dioxide production for the period beginning at $11^{\mathrm{h}} 20^{\mathrm{m}}$ a. $\mathrm{m}$. is probably incorrect, although there are no indications that there was an error in the technique. The results with H. H. A. show good agreement, with the exception of those obtained in the first experiment, in which the values for the spirometer unit show a markedly higher metabolism than those for the Zuntz-Geppert apparatus. With P. F. J. the differences are both plus and minus. The high average obtained for the oxygen consumption on February 5 with

TABLE 20.-Variations of average results obtained with the Zuntz-Geppert apparatus from those obtained with the Benedict respiration apparatus (spirometer unit).

\begin{tabular}{|c|c|c|c|c|c|c|c|c|}
\hline Subject. & Date. & $\begin{array}{c}\text { Carbon } \\
\text { dioxide } \\
\text { elimi- } \\
\text { nated per } \\
\text { minute. }\end{array}$ & $\begin{array}{c}\text { Oxygen } \\
\text { absorbed } \\
\text { per } \\
\text { minute. }\end{array}$ & $\begin{array}{l}\text { Respira- } \\
\text { tory } \\
\text { quotient. }\end{array}$ & $\begin{array}{c}\text { Average } \\
\text { pulse- } \\
\text { rate. }\end{array}$ & $\begin{array}{c}\text { Average } \\
\text { respira- } \\
\text { tion- } \\
\text { rate. }\end{array}$ & $\left\{\begin{array}{l}\text { Ventila- } \\
\text { tion per } \\
\text { minute } \\
\text { (reduced). }\end{array}\right.$ & $\begin{array}{l}\text { Volume } \\
\text { per } \\
\text { respira- } \\
\text { tion. }\end{array}$ \\
\hline \multirow{6}{*}{ H. F. T.... } & 1912 & c.c. & c.e. & & & & & \\
\hline & Jan. 18 & -14 & -2 & -0.07 & +1.0 & +1.1 & -0.31 & -98 \\
\hline & Jan. 19 & -30 & -1 & -.145 & -2.5 & +.1 & -.68 & -101 \\
\hline & Jan. 27 & -14 & -5 & -.050 & -2.0 & +.9 & -.48 & -93 \\
\hline & Jan. 29 & -25 & +1 & -.155 & -1.0 & -.5 & -.67 & -48 \\
\hline & Jan. 30 & +7 & -3 & +.045 & -1.5 & +.4 & +.21 & +2 \\
\hline \multirow[t]{2}{*}{ K. H. A.... } & Feb. 2 & -39 & -8 & -.150 & -2.5 & +.8 & -.69 & -107 \\
\hline & Feb. 19 & +12 & +5 & +.03 & +1.0 & +2.8 & +.06 & -81 \\
\hline \multirow[t]{4}{*}{ H. H. А.... } & Feb. 3 & -19 & -21 & -.01 & +1.5 & -.8 & -.60 & -37 \\
\hline & Feb. 6 & +1 & +13 & -.05 & +2.5 & -.5 & -.04 & +7 \\
\hline & Feb. 8 & -7 & +3 & -.045 & -1.0 & +1.6 & -.18 & -65 \\
\hline & Feb. 10 & -3 & -3 & -.005 & 0 & +.8 & -.28 & -71 \\
\hline \multirow[t]{2}{*}{ P. F. J..... } & Feb. 5 & -8 & +15 & -.08 & -6.0 & -.6 & -.38 & -28 \\
\hline & Feb. 7 & +16 & +16 & +.01 & +10.5 & -4.0 & -.38 & +75 \\
\hline J. E. F. & Feb. 12 & -6 & +8 & -.045 & -4.5 & +1.9 & -.26 & -134 \\
\hline H. W. E.... & Feb. 14 & +7 & +19 & -.045 & 0 & -.5 & -.09 & +9 \\
\hline \multirow[t]{3}{*}{ H. B. L.... } & Feb. 20 & +7 & +6 & +.01 & 0 & -.5 & +.17 & +24 \\
\hline & Feb. 21 & -19 & -15 & -.035 & +2.0 & +.1 & -.63 & -60 \\
\hline & Feb. 28 & -1 & -2 & 0 & +1.0 & -.5 & -.35 & -23 \\
\hline \multirow[t]{3}{*}{ M. B..... } & Feb. 22 & -4 & -13 & +.025 & 0 & -2.8 & -.61 & +5 \\
\hline & Feb. 27 & +11 & +18 & -.025 & +1.5 & +.6 & +.26 & -5 \\
\hline & Mar. 2 & -3 & -9 & +.02 & -.5 & -2.5 & -.39 & +37 \\
\hline Ma. B. & Feb. 29 & -4 & -5 & 0 & -2.5 & -2.2 & -.64 & -14 \\
\hline \multicolumn{2}{|c|}{ Average variation.... } & 12 & 9 & .05 & 2.0 & 1.2 & .38 & 51 \\
\hline
\end{tabular}

the Zuntz-Geppert apparatus is due, in part at least, to the high value obtained in the last period. If this figure were excluded the results obtained for the oxygen consumption with the Zuntz-Geppert apparatus would be similar to those secured with the spirometer unit. The other subjects also show both plus and minus variations, so that the results obtained with both forms of apparatus are, on the average, comparable.

If the probability curves for the carbon-dioxide results are examined (see fig. 42), it will be found that the total number of periods varying 1,2 , and 3 per cent from the average is practically the same with both forms of apparatus. For instance, in 43 per cent of the periods, the 
results with the spirometer unit vary more than 3 per cent, while with the Zuntz-Geppert apparatus 50 per cent of the periods show this degree of variation. The same is true of the curves for the oxygen consumption, these two being even more nearly parallel than those for carbon-dioxide production. On the other hand, when the curves for the respiratory quotient are plotted, it is seen that there is a very marked difference, the

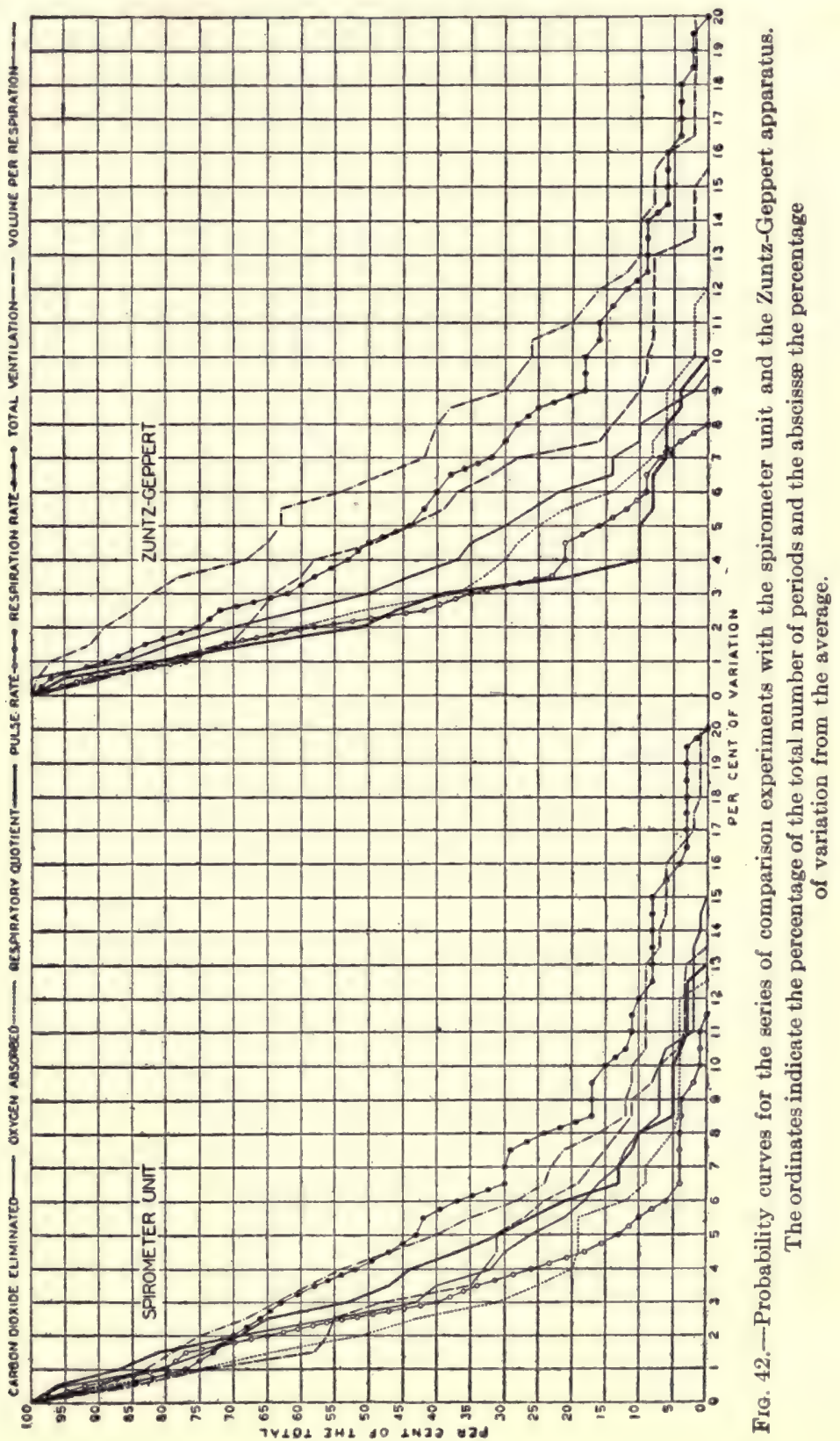


respiratory quotients with the Zuntz-Geppert apparatus being much more uniform than those with the spirometer unit. For example, in 53 per cent of the periods with the spirometer unit the respiratory quotient varies 3 per cent or more from the average for the experiment, while with the Zuntz-Geppert apparatus only 39 per cent of the periods vary more than 3 per cent. Both pulse-rate and respiration-rate have approximately the same degree of uniformity with both apparatus, while the total ventilation and the volume per respiration are more nearly uniform with the spirometer unit than with the Zuntz-Geppert apparatus.

As will be seen from table 20, the averages of the differences between the experiments are somewhat large, showing that, in general, the agreement in the results with the two forms of apparatus is not particularly good. This lack of agreement is probably due in part to the fact that the subjects were not familiar with the apparatus. The difference between the averages of all the results with both types of apparatus is small, however, (see table 19) and in general the two apparatus give essentially the same results in the measurement of the respiratory exchange.

\section{TISSOT APPARATUS AND BENEDICT RESPIRATION APPARATUS (TENSION-EQUALIZER UNIT).}

In the first series of experiments in which the Benedict respiration apparatus and the Tissot apparatus were compared, the tensionequalizer unit was used and the study was carried out in the same manner as in previous comparisons. In five of the experiments the 50-liter Tissot spirometer was employed, the remaining comparisons being made with the 200-liter Tissot spirometer. The pneumatic nosepieces were used in all of the experiments but one.

The expired air collected in the Tissot spirometer was sampled by drawing portions through a glass tube inserted in a rubber stopper placed in the opening at the top of the copper bell. (See $Z$ in figs. 26 and 27, p. 64.) This tube was attached to a glass sampler, with a capacity of 150 c.c. or 300 c.c., which was filled with mercury and connected with a leveling-bulb. The sampler was provided with three-way glass stopcocks. A sample of air was drawn by opening the stopcocks and lowering the leveling-bulb; when the sampler was full of air, the leveling-bulb was raised and the upper stopcock turned so that the air was expelled into the room. When the sampler was again full of mercury, the leveling-bulb was lowered and the upper stopcock turned so that a second portion of air was drawn from the spirometer; this sample was also rejected. Finally, a third portion was drawn and reserved for analysis. The analysis was made with the laboratory form of the Haldane gas-analysis apparatus, in which the carbon dioxide was absorbed by caustic potash and the oxygen by potassium pyrogallate. Duplicate analyses of the sample usually agreed to within less than 0.04 per cent for both carbon dioxide and oxygen. In some cases two samples were drawn and one portion from each analyzed. 
Usually the apparatus were alternated in each experiment, the apparatus first used varying. In two experiments the periods with each apparatus were in series. The duration of the periods was, as a rule, approximately 15 minutes, except when the 50-liter spirometer was used, when they were only 10 minutes in length. No special preliminary ventilation was obtained with either apparatus, the periods beginning about 5 minutes after everything was in readiness.

The pulse-rate was obtained by means of a Bowles stethoscope placed over the heart of the subject. Usually five separate counts, each a full minute in duration, were made during a 15-minute period. A graphic record of the respiration was secured with a pneumograph placed about the lower chest of the subject and connected with a tambour and kymograph. The external muscular activity of the subject was controlled with a pneumograph placed about the hips. Only two subjects were used for this series of experiments, H. F. T. and K. H. A., all but one of the experiments being made with $\mathrm{H}$. F. T. Both were young men who were accustomed to respiration experiments of this type. The statistics of the 10 experiments are given in the following pages. In addition to the data usually recorded, the average barometric pressure and the average temperature of the air in the apparatus are given.

\section{STATISTICS OF EXPERIMENTS.}

H. F. T., August 8, 1911.-Tension-equalizer unit, 4 periods; Tissot spirometer, 3 periods; first two periods with tension-equalizer unit; apparatus alternated thereafter. Pneumatic nosepieces; 50-liter spirometer. Preliminary ventilation for 10 to 12 minutes in the periods with the Tissot apparatus. Pulse-rate for the most part uniform in all of the periods. Respiration in the various periods similar and fairly regular. This subject had a tendency to irregularity in length of individual respirations and some respirations were longer than others, with pauses at the end of an expiration. Average barometric pressure $760.4 \mathrm{~mm}$. ; average temperature of air in apparatus $24.4^{\circ} \mathrm{C}$.

H. F. T., August 9, 1911.-Tissot apparatus, 4 periods; tension-equalizer unit, 3 periods; periods with each apparatus in series; preliminary period, 7 minutes. 50-liter spirometer. The nosepieces were not removed during the entire series with the Tissot apparatus. Pulse-rate very uniform. Respiration in the first three periods with the Tissot apparatus somewhat irregular, with many periodic pauses; with the tension-equalizer unit, somewhat more regular. Average barometric pressure, $759.2 \mathrm{~mm}$.; average temperature of air in apparatus, $23.9^{\circ} \mathrm{C}$.

H. F.T., August 23, 1911.-Tension-equalizer unit, 4 periods; Tissot apparatus, 3 periods; first two and last two periods with tension-equalizer unit. Pneumatic nosepieces, both types of apparatus; with Tissot apparatus, 50liter spirometer; nosepieces inserted about 10 minutes before each period began. Pulse- and respiration-rates fairly regular. Average barometric pressure, $758.9 \mathrm{~mm}$.; average temperature of air in apparatus, $19.2^{\circ} \mathrm{C}$.

H.F.T., August 26, 1911.-Tissot apparatus, 5 periods; tension-equalizer apparatus, 4 periods; apparatus alternated. With Tissot apparatus, 50-liter spirometer; nosepieces inserted 10 minutes before each period began. Pulserate uniform. Respiration-rate essentially uniform in all periods. Average 
barometric pressure, $763.3 \mathrm{~mm}$; average temperature of air in apparatus, $19.7^{\circ} \mathrm{C}$.

H. F. T., September 6, 1911.-Tension-equalizer unit, 4 periods; Tissot apparatus, 3 periods; periods with tension equalizer and Tissot apparatus in series. With Tissot apparatus, 200-liter spirometer. Range in pulse-rate about 4 beats per minute in each period. Respiration-rate uniform in entire series. Average barometric pressure, $752.3 \mathrm{~mm}$; average temperature of air in apparatus, $24^{\circ} \mathrm{C}$.

H.F.T., September 13, 1911.-Tissot apparatus, 5 periods; tension-equalizer unit, 6 periods; apparatus usually alternated. Pneumatic nosepieces with both apparatus; 200-liter spirometer with Tissot apparatus. Pulse-rate and respiration-rate fairly uniform in the individual periods. Average barometric pressure, $760.6 \mathrm{~mm}$.; average temperature of air in apparatus, $20.5^{\circ} \mathrm{C}$.

H. F. T., September 15, 1911.- Tension-equalizer unit, 6 periods; Tissot apparatus, 6 periods; preliminary period, 13 minutes; apparatus alternated. With Tissot apparatus, 200-liter spirometer. Nosepieces inserted approximately 5 minutes before periods with Tissot apparatus began. Both pulserate and respiration-rate comparatively uniform. In third period with tension-equalizer unit, too much oxygen was admitted and the subject accordingly exhaled against some pressure; the respiration was in consequence slightly increased in volume. Average barometric pressure, $766.1 \mathrm{~mm}$.; average temperature of air in apparatus, $20.0^{\circ} \mathrm{C}$.

H.F.T., September 18, 1911.-Tissot apparatus, 4 periods; tension-equalizer unit, 4 periods; apparatus alternated. With Tissot apparatus, 200-liter spirometer. Pulse-rate uniform, except that the range in the last period with each apparatus was about 4 beats per minute. Respiration-rate fairly uniform, with occasional pauses at end of expiration. Average barometric pressure, $762.9 \mathrm{~mm}$. ; average temperature of air in apparatus, $20.9^{\circ} \mathrm{C}$.

H. F. T., September 22, 1911.-Tension-equalizer unit, 6 periods; Tissot apparatus, 6 periods; apparatus alternated. With Tissot apparatus, 200-liter spirometer. Both pulse-rate and respiration-rate regular. Average barometric pressure, $763.6 \mathrm{~mm}$.; average temperature of air in apparatus, $21.9^{\circ} \mathrm{C}$.

K. H. A., August 5, 1911.-Tension-equalizer unit, 6 periods; Tissot apparatus, 2 periods; all but fourth and seventh periods with tension-equalizer unit. Pneumatic nosepieces with tension-equalizer unit; mouthpiece and 50liter spirometer with Tissot apparatus. Both pulse-rate and respiration-rate uniform. Average barometric pressure, $762.0 \mathrm{~mm}$., average temperature of air in apparatus, $23.1^{\circ} \mathrm{C}$.

\section{DISCUSSION OF RESULTS.}

The results for the periods in all of the experiments and the averages for each apparatus, both for the individual experiments and for all of the periods in the study, are given in table 21. It will be seen that the grand averages of the values obtained with both apparatus are practically identical. These are as follows, the values for the tensionequalizer unit preceding: Carbon-dioxide elimination, 165 c.c. and 167 c.c. ; oxygen absorption, 193 c.c., and 194 c.c.; respiratory quotient, 0.855 and 0.860 ; pulse-rate, 47.0 and 48.0 ; respiration-rate, 10.1 and 10.2 . The average values obtained with the Tissot apparatus for the ventilation of the lungs is 4.26 liters; volume per respiration, 503 c.c. 
TABLE 21. - Respiratory exchange in comparison experiments with the Tissot apparatus and the Benedict respiration apparatus (tension-equalizer unit). (Without food.)

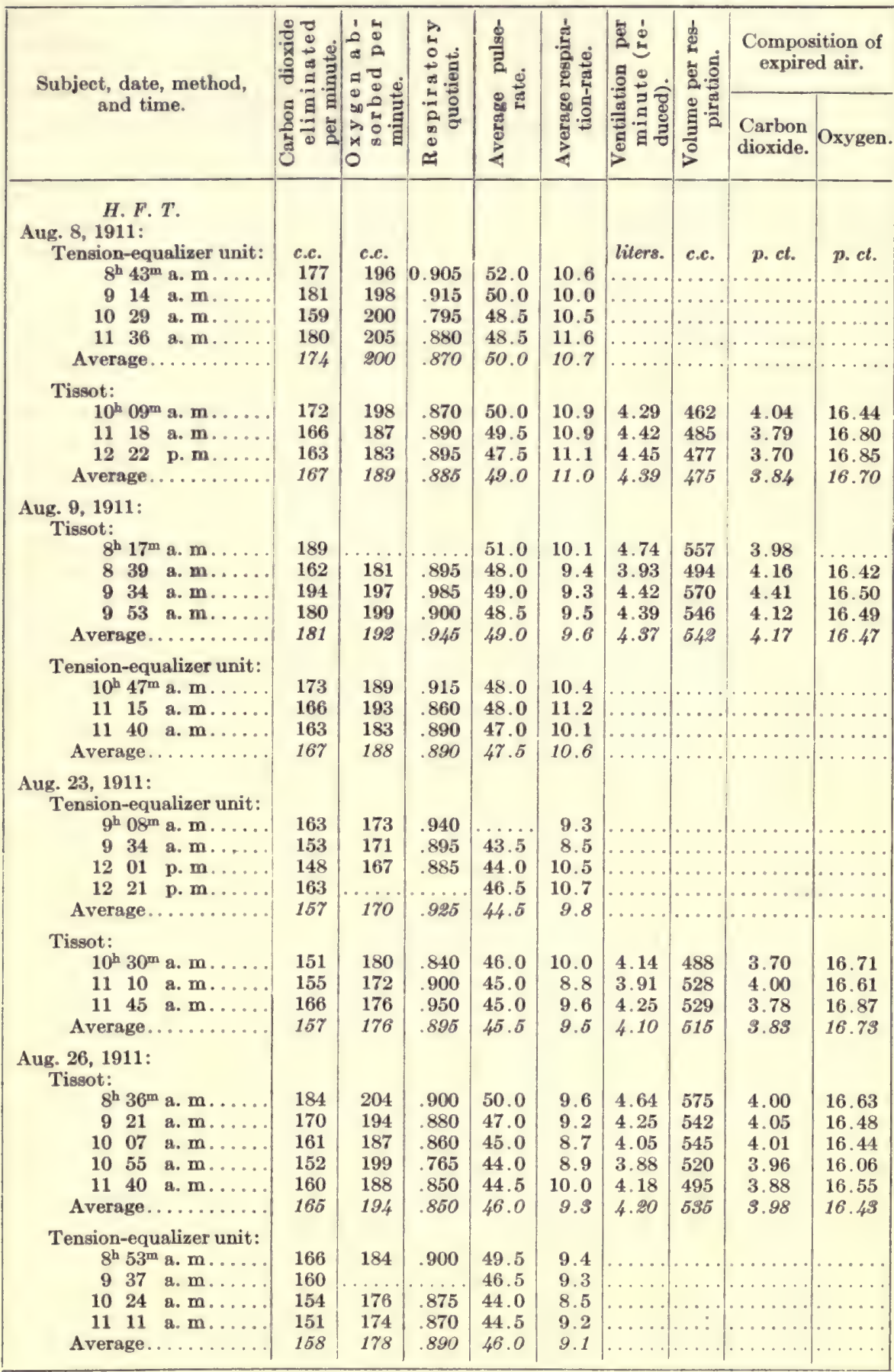


TABLE 21.-Respiratory exchange in comparison experiments with the Tissot apparatus and the Benedict respiration apparatus (tension-equalizer unit). (Without food.)-Continued.

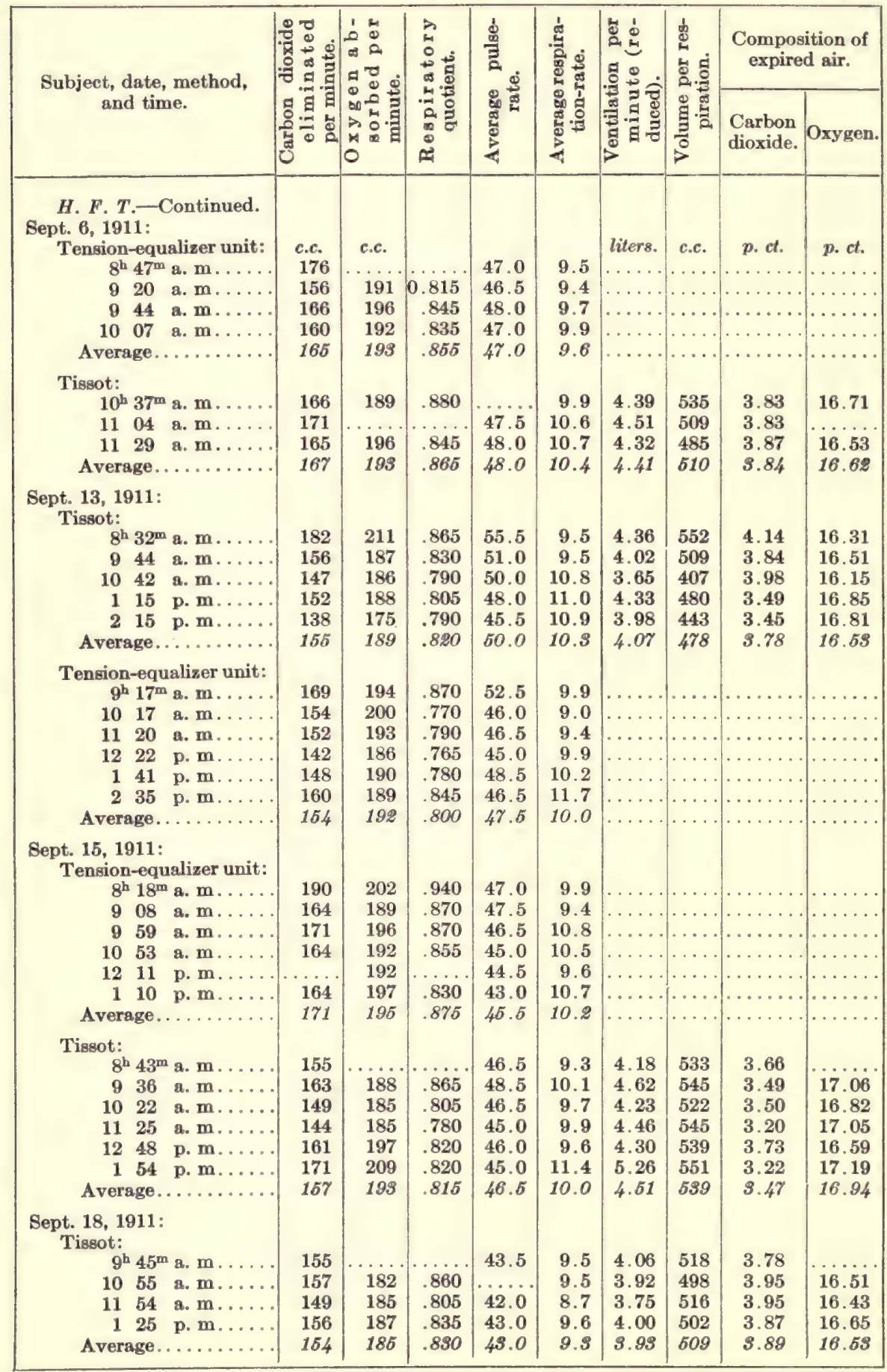


TISSOT AND BENEDICT METHODS.

TABLE 21.-Respiratory exchange in comparison experiments with the Tissot apparatus and the Benedict respiration apparatus (tension-equalizer unit). (Without food.)-Continued.

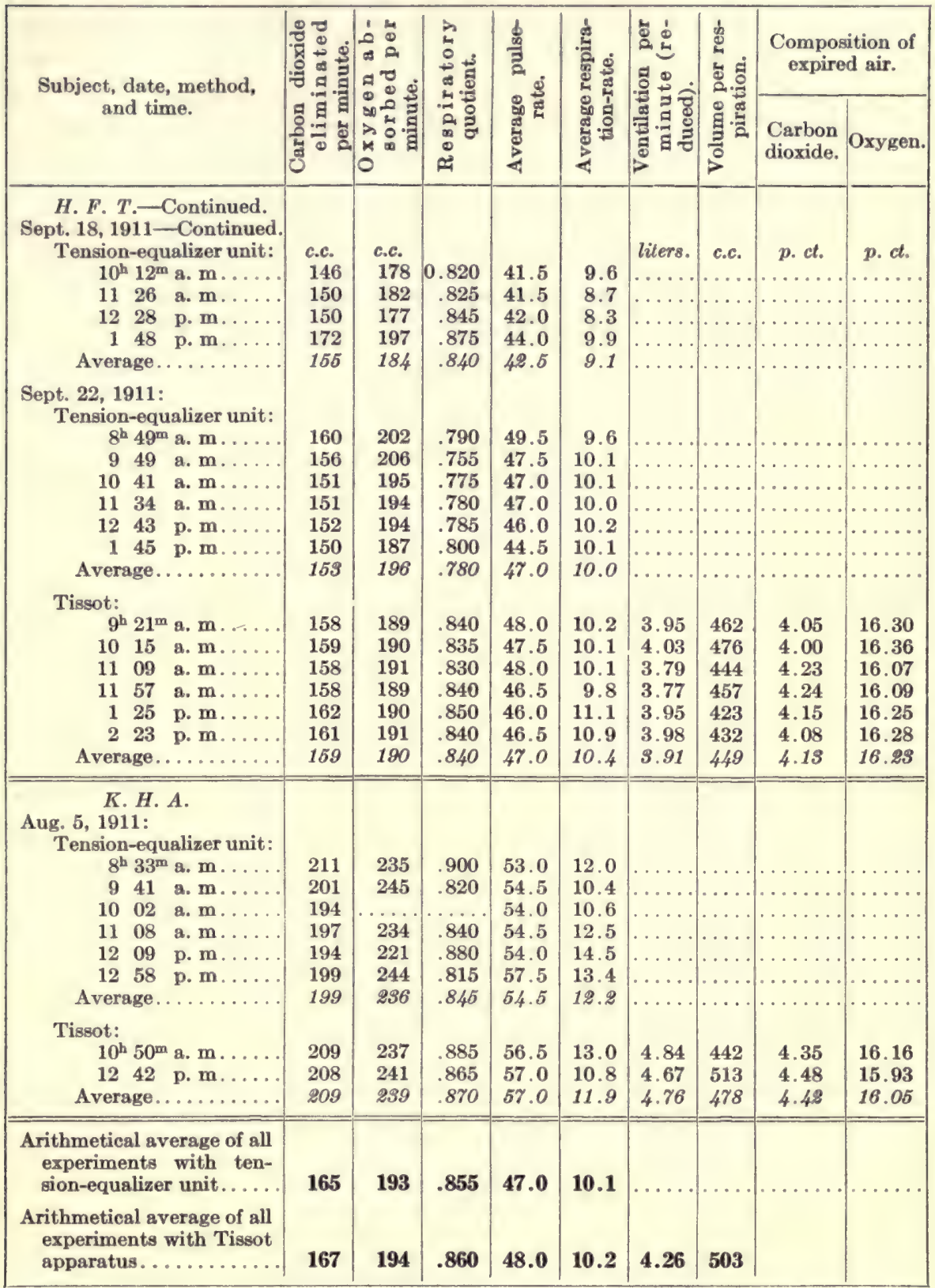


The variations in the averages for both apparatus in each experiment are given in table 22, the values for the tension-equalizer unit being used for the base-line. These variations ranged for the carbondioxide elimination from +14 to -14 , with an average of \pm 6 ; for the oxygen consumption from +16 to -11 , with an average of \pm 5 ; for the respiratory quotient from +0.06 to -0.06 , with an average of \pm 0.035 . The average pulse-rate and respiration-rate do not show much variation.

The data for the probability curves have also been calculated, and the curves are given in figure 43. The uniformity for all of the factors measured is practically the same with both types of apparatus. As has been stated, all of the comparisons but one were made with the same

TABLE 22.-Variations of average results obtained with the Tissot apparatus from those obtained with the tension-equalizer unit.

\begin{tabular}{|c|c|c|c|c|c|c|}
\hline Subject. & Date. & $\begin{array}{c}\text { Carbon } \\
\text { dioxide } \\
\text { eliminated } \\
\text { per minute. }\end{array}$ & $\begin{array}{c}\text { Oxygen } \\
\text { absorbed } \\
\text { per minute. }\end{array}$ & $\begin{array}{l}\text { Respira- } \\
\text { tory } \\
\text { quotient. }\end{array}$ & $\begin{array}{c}\text { Average } \\
\text { pulse- } \\
\text { rate. }\end{array}$ & $\begin{array}{l}\text { Average } \\
\text { respira- } \\
\text { tion- } \\
\text { rate. }\end{array}$ \\
\hline $\begin{array}{l}\text { H. F. T. . . . } \\
\text { к. Н. А...... }\end{array}$ & $\begin{array}{l}1911 \\
\text { Aug. } 8 \\
\text { Aug. } 9 \\
\text { Aug. } 23 \\
\text { Aug. } 26 \\
\text { Sept. } 6 \\
\text { Sept. } 13 \\
\text { Sept. } 15 \\
\text { Sept. } 18 \\
\text { Sept. } 22 \\
\text { Aug. } 5\end{array}$ & $\begin{array}{l}c . c . \\
-7 \\
+14 \\
0 \\
+7 \\
+2 \\
+1 \\
-14 \\
-1 \\
+6 \\
+10\end{array}$ & $\begin{array}{l}c . c . \\
-11 \\
+4 \\
+6 \\
+16 \\
0 \\
-3 \\
-2 \\
+1 \\
-6 \\
+\quad 3\end{array}$ & $\begin{array}{l}+0.015 \\
+\quad .055 \\
-\quad .030 \\
-\quad .040 \\
+\quad .010 \\
+\quad .020 \\
-\quad .060 \\
-\quad .010 \\
+\quad .060 \\
+\quad .025\end{array}$ & $\begin{array}{l}-1.0 \\
+1.5 \\
+1.0 \\
0 \\
+1.0 \\
+2.5 \\
+1.0 \\
+.5 \\
0 \\
+2.5\end{array}$ & $\begin{array}{l}+0.3 \\
-1.0 \\
-\quad .3 \\
+.2 \\
+.8 \\
+.3 \\
+.2 \\
+.2 \\
+.4 \\
-.3\end{array}$ \\
\hline Average variation... & & 6 & 5 & 0.035 & 1.0 & 0.4 \\
\hline
\end{tabular}

subject. The results obtained with this subject are more likely to be variable than with many other subjects because of frequent apnœa, but a study of the variations will show that these are as likely to be in one direction as in the other, while the average difference is small. In the experiment which contained the greatest number of periods-that on September 13 - the averages are almost identical. The results as a whole indicate that the respiratory exchange as measured by the Tissot apparatus and the tension-equalizer unit is essentially the same.

\section{TISSOT APPARATUS AND BENEDICT RESPIRATION APPARATUS (SPIROMETER UNIT).}

The second series of experiments comparing the respiratory exchange as measured by the Benedict respiration apparatus and the Tissot apparatus was made with the spirometer unit, the pneumatic nosepieces being used unless otherwise stated. With the Tissot apparatus, the 200-liter spirometer was used and also the glass nosepieces, except as noted in the statistics. The samples of air for the Tissot apparatus 
were collected and analyzed in the same manner as in the previous comparison with the tension-equalizer unit. The periods with the two forms of apparatus were either alternated or in series, and usually of about 15 minutes duration, with a preliminary ventilation of approximately 5 minutes for each period.

The pulse-rate was obtained as usual by means of a Bowles stethoscope, with ordinarily 5 counts in each 15 -minute period. In the periods with the spirometer unit the respiration was recorded from the

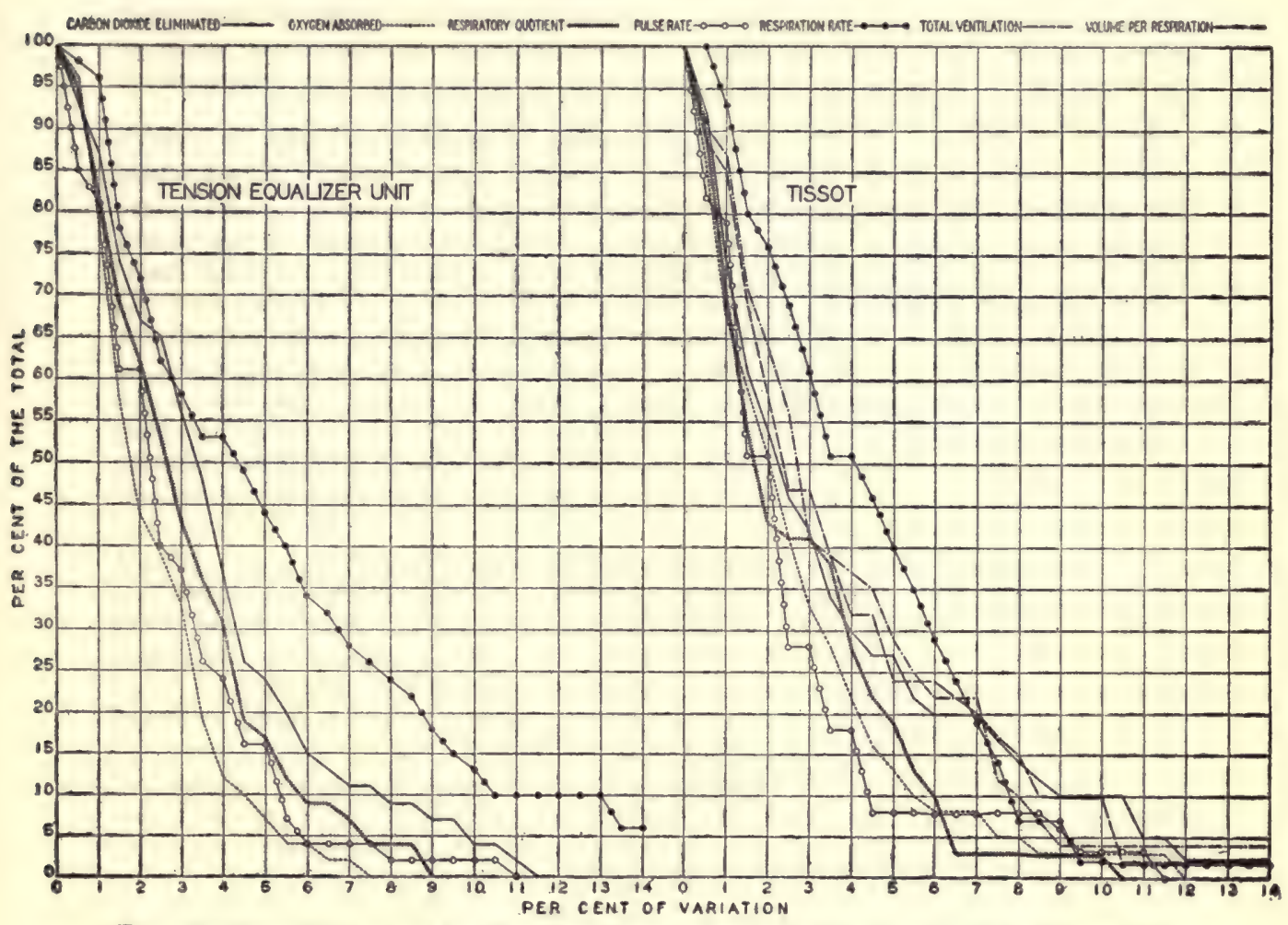

FIG. 43.-Probability curves for the series of comparison experiments with the tension-equalizer unit and the Tissot apparatus.

The ordinates indicate the percentage of the total number of periods and the abscissæ the percentage of variation from the average.

spirometer bell; with the Tissot apparatus, the records were made from the chest pneumograph as in the previous comparison. Except in the experiments with E. W. H., the muscular activity was recorded from a pneumograph placed about the hips of the subject, as in the first series of comparisons with the Tissot apparatus. The subjects were all assistants in the Laboratory, with the exception of J.H. H., and had acted as subjects in previous respiration experiments with the spirometer unit. J. H. H. was familiar with both of the apparatus compared, although he had never been used before in a comparison experiment. 
The statistics of the 17 experiments are given in the following pages. As in the previous comparison, the average barometric pressure and the average temperature of the air in the apparatus are added to the data usually presented.

\section{STATISTICS OF EXPERIMENTS.}

K. H. A., June 4, 1912.-Spirometer unit, 5 periods; Tissot apparatus, 3 periods; preliminary period, 1 hour 2 minutes; first two periods, spirometer unit; apparatus alternated thereafter. Glass nosepieces tested with soapsuds for periods with Tissot apparatus. Pulse-rate varied in range in individual periods from 3 to 9 beats per minute. Respiration-rate remarkably uniform in all periods. Average barometric pressure, $755.7 \mathrm{~mm}$.; average temperature of air in apparatus, $27.2^{\circ} \mathrm{C}$.

K. H. A., June 7, 1912.-Tissot apparatus, 4 periods; spirometer unit, 4 periods; preliminary period, 49 minutes; apparatus alternated. Glass nosepieces tested with soapsuds for each period with Tissot apparatus and found without leak. Subject drowsy at times. Pulse-rate uniform. Respirationrate very uniform, but depth of respiration varied somewhat. Average barometric pressure, $758.2 \mathrm{~mm}$.; average temperature of air in apparatus, $21.7^{\circ} \mathrm{C}$.

P. F. J., June 5, 1912.- Spirometer unit, 5 periods; Tissot apparatus, 2 periods; first three periods with spirometer unit, apparatus alternating thereafter. Glass nosepieces for Tissot apparatus tested with soapsuds. Respiration-rate in third and last periods of experiment (with spirometer unit) somewhat irregular toward end of periods, probably due to drowsiness of subject. Average barometric pressure, $758.8 \mathrm{~mm}$.; average temperature of air in apparatus, $24.5^{\circ} \mathrm{C}$.

P. F. J., June 8, 1912.--Tissot apparatus, 3 periods; spirometer unit, 4 periods; periods with each apparatus in series. Range in pulse-rate approximately 4 beats per minute in the individual periods. Respiration with spirometer unit in first two periods regular; in third period very rapid and shallow; in fourth period regular. Respiration with Tissot apparatus regular throughout all periods. Average barometric pressure, $764.6 \mathrm{~mm}$.; average temperature of air in apparatus, $20.8^{\circ} \mathrm{C}$.

J. B. T., June 10, 1912.-Spirometer unit, 4 periods; Tissot apparatus, 3 periods; first two periods with spirometer unit, apparatus alternating thereafter. Respiration-rate in all periods regular. Average barometric pressure, $765.2 \mathrm{~mm}$.; average temperature of air in apparatus, $20.2^{\circ} \mathrm{C}$.

J. B. T., June 12, 1912.-Tissot apparatus, 3 periods; spirometer unit, 4 periods; periods with each apparatus in series. Tests made with soapsuds before each period for leaks around nosepieces. Both pulse-rate and respiration-rate regular in all periods. Average barometric pressure, $754.8 \mathrm{~mm}$.; average temperature of air in apparatus, $24.1^{\circ} \mathrm{C}$.

J. B. T., June 21, 1912.-Spirometer unit, 4 periods; Tissot apparatus, 3 periods; preliminary period, 39 minutes; first two periods with spirometer unit, apparatus alternating thereafter. Pulse-rate uniform, except in last period of experiment (with Tissot apparatus) when there was a range of 5 beats per minute. Respiration-rate in all periods regular. Average barometric pressure, $760.1 \mathrm{~mm}$; ; average temperature of air in apparatus, $25.7^{\circ} \mathrm{C}$.

J. W. P., June 14, 1912.-Tissot apparatus, 3 periods; spirometer unit, 4 periods; periods with each apparatus in series. Pneumatic nosepieces with both apparatus. Pulse-rate in first period with Tissot apparatus irregular; in other periods fairly regular, range being approximately 3 to 4 beats per minute. Respiration regular in rate, but somewhat irregular in depth in all 
of the periods; particularly in last two periods of experiment (with spirometer unit). Average barometric pressure, $760.2 \mathrm{~mm}$.; average temperature of air in apparatus, $21.2^{\circ} \mathrm{C}$.

J.W. P., June 27, 1912.-Spirometer unit, 4 periods; Tissot apparatus, 2 periods; first two periods with spirometer unit, apparatus alternating thereafter. Pneumatic nosepieces used with both forms of apparatus and tested for tightness with soapsuds. Pulse-rate very regular in all periods. Respiration-rate regular in each period. Average barometric pressure, $766.2 \mathrm{~mm}$.; average temperature of air in apparatus, $21.8^{\circ} \mathrm{C}$.

J. K. M., June 20, 1912.-Tissot apparatus, 4 periods; spirometer unit, 4 periods; first two periods with Tissot apparatus, then apparatus alternating, and last two periods with spirometer unit. Subject somewhat drowsy in second period of experiment (with Tissot apparatus). Range of pulse-rate from 4 to 5 beats per minute in all periods. Respiration-rate in all periods regular. Average barometric pressure, $754.9 \mathrm{~mm}$; average temperature of air in apparatus, $24.0^{\circ} \mathrm{C}$.

J. K. M., June 26, 1912.-Spirometer unit, 4 periods; Tissot apparatus, 3 periods; preliminary period, 46 minutes; first two periods with spirometer unit, apparatus alternating thereafter. Subject drowsy in second period of experiment (with spirometer unit), also in fifth period (with Tissot apparatus). He said he preferred the Tissot apparatus because of absence of vibration. Pulse-rate varied in different periods in range up to 5 beats per minute. Respiration-rate regular in all of the periods; in last period with spirometer unit somewhat irregular in depth. Average barometric pressure, $755.5 \mathrm{~mm}$. ; average temperature of air in apparatus, $28.6^{\circ} \mathrm{C}$.

J.K. M., June 29, 1912.- Spirometer unit, 4 periods; Tissot apparatus, 3 periods; first two periods with spirometer unit, then apparatus alternating. Nosepieces tested for tightness with soapsuds. Pulse-rate varying in range in individual periods from 3 to 9 beats per minute. Respiration-rate regular in all periods with both apparatus. Average barometric pressure, $755.6 \mathrm{~mm}$.; average temperature of air in apparatus, $27.4^{\circ} \mathrm{C}$.

E. W. H. June 24, 1912.-Spirometer unit, 3 periods; Tissot apparatus, 3 periods; first two periods with spirometer unit, then apparatus alternating, last two periods with Tissot apparatus. Subject sat in a Morris chair, as his respiration while lying on his back was so irregular and deep at times that it was found impracticable to experiment with him in the latter position. Subject somewhat uneasy in several of the periods, especially in the fifth period (with spirometer unit), when he moved considerably. This uneasiness affected the results. Pulse-rate irregular and wide in range. Respiration irregular and uneven in depth. Average barometric pressure, $762.1 \mathrm{~mm}$; average temperature of air in apparatus, $27.9^{\circ} \mathrm{C}$.

E. W. H., June 28, 1912.-Spirometer unit, 4 periods; Tissot apparatus, 3 periods; first three periods and fifth period with spirometer unit; remaining periods with Tissot apparatus. Subject sitting in chair. Nosepieces tested with soapsuds. Subject said he liked the spirometer unit better than the Tissot apparatus. Pulse-rate irregular, varying widely in the individual periods. Respiration somewhat irregular in depth and rate. Average barometric pressure, $761.9 \mathrm{~mm}$.; average temperature of air in apparatus, $24.6^{\circ} \mathrm{C}$.

J. H. H., A pril 14, 1913.-Spirometer unit, 3 periods; Tissot apparatus, 3 periods; preliminary period, 1 hour 14 minutes; periods with each apparatus in series. Mouthpiece used. Pulse-rate fairly regular. Normal respirationrate, 19 per minute; ${ }^{1}$ respiration during experiment uniform in character in

${ }^{1}$ In the later experiments it was made a part of the routine to record the normal respiration-rate before the experiment began. 
both series of periods. Average barometric pressure, $755.2 \mathrm{~mm}$; average temperature of air in apparatus, $16.5^{\circ} \mathrm{C}$.

J. H. H., A pril 16, 1913.- - Tissot apparatus, 3 periods; spirometer unit, 3 periods; preliminary period, 1 hour 6 minutes; periods with each apparatus in series. Mouthpiece used. Subject said he noted but little difference between the apparatus. Pulse-rate very uniform. Normal respiration-rate 19 to 20 per minute; respiration very uniform in character throughout experiment. Average barometric pressure, $751.8 \mathrm{~mm}$.; average temperature of air with Tissot apparatus, $17.6^{\circ} \mathrm{C}$.; with spirometer unit, $20^{\circ} \mathrm{C}$.

J. H. H., A pril 17, 1913.-Tissot apparatus, 4 periods; spirometer unit, 4 periods; preliminary period, 1 hour 12 minutes; apparatus alternated. Mouthpiece used. Pulse-rate very uniform. Normal respiration-rate 22 per minute; respiration in experiment uniform in all periods. Average barometric pressure, $756 \mathrm{~mm}$.; average temperature of air in apparatus, $18.4^{\circ} \mathrm{C}$.

\section{DISCUSSION OF RESULTS.}

The results of the comparisons of the respiratory exchange as measured with the Tissot apparatus and the spirometer unit are given in table 23. The grand averages for the two methods show a difference of 2 c.c. for the carbon-dioxide production, that for the Tissot apparatus being 192 c.c. and for the spirometer unit 190 c.c. The values for the oxygen consumption vary 9 c.c., being 242 c.c. for the Tissot apparatus and 233 c.c. for the spirometer unit. The average respiratory quotients are within $0.02, i . e ., 0.795$ for the Tissot apparatus and 0.815 for the spirometer unit. The other factors agree very fairly, the pulse-rate and respiration-rate for the Tissot apparatus being 60.5 and 13.9 respectively and for the spirometer unit 60.5 and 12.4 respectively. The ventilation of the lungs is almost identical with the two forms of apparatus, 5 liters and 4.96 liters, but the volume per respiration is somewhat smaller with the Tissot apparatus, this being 445 c.c. as compared with 509 c.c. 
TABLE 23.-Respiratory exchange in comparison experiments with the Tissot apparatus and the Benedict respiration apparatus (spirometer unit). (Without food.)

\begin{tabular}{|c|c|c|c|c|c|c|c|c|c|}
\hline \multirow{2}{*}{$\begin{array}{l}\text { Subject, date, method, } \\
\text { and time. }\end{array}$} & \multirow{2}{*}{ 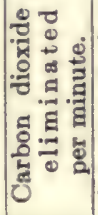 } & \multirow{2}{*}{ 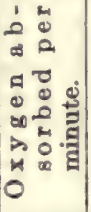 } & \multirow{2}{*}{ 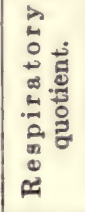 } & \multirow{2}{*}{ 递 } & \multirow{2}{*}{ 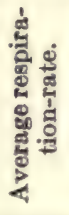 } & \multirow{2}{*}{ 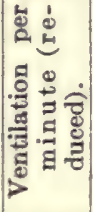 } & \multirow{2}{*}{ 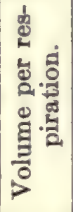 } & \multicolumn{2}{|c|}{$\begin{array}{l}\text { Composition of } \\
\text { expired air. }\end{array}$} \\
\hline & & & & & & & & $\begin{array}{l}\text { Carbon } \\
\text { dioxide. }\end{array}$ & Oxygen \\
\hline \multicolumn{10}{|l|}{$\begin{array}{r}\text { K. H. A. } \\
\text { June 4, 1912: }\end{array}$} \\
\hline $\begin{array}{l}\text { Spirometer unit: } \\
\qquad 9^{\mathrm{h}} 10^{\mathrm{m}} \mathrm{a} \cdot \mathrm{m} . . .\end{array}$ & $\begin{array}{l}\text { c.c. } \\
187\end{array}$ & $\begin{array}{l}\text { c.c. } \\
235\end{array}$ & 0.795 & 55.5 & 12.6 & $\begin{array}{l}\text { liters. } \\
4.77\end{array}$ & $\begin{array}{l}\text { c.c. } \\
460\end{array}$ & 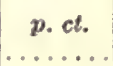 & p.ct. \\
\hline 930 a. m..... & 183 & 233 & .785 & 52.5 & 14.9 & 4.96 & 405 & & \\
\hline 1021 a. $m \ldots$. & 195 & 248 & .785 & 55.5 & 13.6 & 5.12 & 459 & & \\
\hline 1105 a. $\mathbf{m} \ldots$ & 183 & 231 & .790 & 53.5 & 13.8 & 4.92 & 435 & & \\
\hline 1146 a. m.... & 202 & 252 & .800 & 56.0 & 12.7 & 5.18 & 497 & & \\
\hline Average........... & 190 & 240 & .790 & 54.5 & 13.5 & 4.99 & 464 & & . \\
\hline $\begin{array}{l}\text { Tissot: } \\
9^{\mathrm{h}} 55^{\mathrm{m}} \text { a. m..... }\end{array}$ & 195 & 244 & .800 & 56.0 & 13.5 & 4.96 & 440 & 3.95 & 16.23 \\
\hline 1042 a. m.......... & 195 & 247 & .790 & 55.0 & 14.2 & 4.99 & 419 & 3.94 & 16.22 \\
\hline 1125 a. m..... & 189 & 237 & .800 & 52.0 & 15.7 & 5.11 & 392 & 3.72 & 16.50 \\
\hline Average........... & 193 & 243 & .795 & 54.5 & 14.5 & 5.02 & 417 & 3.87 & 16.32 \\
\hline \multicolumn{10}{|l|}{$\begin{array}{l}\text { June } 7,1912: \\
\text { Tissot: }\end{array}$} \\
\hline $9^{\mathrm{h}} 01^{\mathrm{m}}$ a. m. . . . . & 179 & 232 & .770 & 44.0 & 14.4 & 4.74 & 395 & 3.80 & 16.28 \\
\hline 942 a. m..... & 169 & 211 & .800 & 46.0 & 14.9 & 4.36 & 352 & 3.91 & 16.30 \\
\hline 1037 a. m..... & 197 & 237 & .830 & 51.0 & 15.0 & 5.26 & 423 & 3.76 & 16.59 \\
\hline 1115 a. $\mathrm{m} \ldots$ & 180 & 231 & .780 & 45.0 & 14.9 & 4.89 & 389 & 3.71 & 16.45 \\
\hline Average........... & 181 & 288 & .795 & 46.5 & 14.8 & 4.81 & 390 & 3.80 & 16.41 \\
\hline $\begin{array}{l}\text { Spirometer unit: } \\
\qquad 9^{\mathrm{h}} 22^{\mathrm{m}} \text { a. m } \ldots \ldots\end{array}$ & 181 & 216 & .840 & 44.5 & 14.6 & 5.09 & 423 & & \\
\hline 1004 a. m...... & 179 & 221 & .810 & 44.5 & 15.1 & 5.09 & 409 & & \\
\hline 1057 a. m..... & 180 & 208 & .865 & 45.0 & 15.1 & 5.21 & 419 & & \\
\hline 1135 a. $\mathrm{m} \ldots$ & 181 & 220 & .825 & 45.0 & 14.4 & 5.08 & 428 & & $\ldots \ldots$ \\
\hline Average............. & 180 & 216 & .835 & 45.0 & 14.8 & 5.12 & 420 & $\ldots \ldots$ & $\ldots \ldots$ \\
\hline \multirow{2}{*}{\multicolumn{10}{|c|}{$\begin{array}{c}P . F . J . \\
\text { June 5, 1912: } \\
\text { Spirometer unit: }\end{array}$}} \\
\hline & & & & & & & & & \\
\hline 915 a. m..... & 183 & 225 & .815 & 69.0 & 7.1 & 4.26 & 728 & & \\
\hline 1009 a. m.... & 181 & 218 & .830 & 66.5 & 9.4 & 4.38 & 566 & & \\
\hline 1100 a. $\mathrm{m} \ldots \ldots$ & 182 & 230 & .790 & 67.0 & 10.1 & 4.66 & 559 & & . . \\
\hline 1141 a. m.... & 188 & 239 & .785 & 69.0 & 9.2 & 4.54 & 598 & & \\
\hline Average............ & 186 & 227 & .820 & 68.5 & 9.0 & 4.52 & 617 & & \\
\hline Tissot: & & & & & & & & & \\
\hline $10^{\mathrm{h}} 36^{\mathrm{m}}$ a. m. & 188 & 235 & .800 & 65.5 & 8.7 & 4.51 & 620 & 4.19 & 15.96 \\
\hline 1120 a. m..... & 185 & 237 & .780 & 66.0 & 11.3 & 4.61 & 487 & 4.04 & 16.03 \\
\hline Average........... & 186 & 236 & .790 & 66.0 & 10.0 & 4.56 & 554 & 4.12 & 16.00 \\
\hline \multicolumn{10}{|l|}{$\begin{array}{c}\text { June } 8,1912: \\
\text { Tissot: }\end{array}$} \\
\hline $8^{\mathrm{h}} 50^{\mathrm{m}}$ a. $\mathrm{m} \ldots$ & 216 & 242 & .895 & 71.5 & 10.4 & 5.35 & 597 & 4.06 & 16.52 \\
\hline 913 a. $\mathrm{m} \ldots .$. & 208 & 239 & .870 & 70.5 & 10.5 & 5.17 & 585 & 4.05 & 16.44 \\
\hline 937 a. $m \ldots$ & 206 & 232 & .890 & 67.0 & 11.0 & 5.21 & 558 & 3.98 & 16.59 \\
\hline Average........... & 210 & 238 & .880 & 69.5 & 10.6 & 5.24 & 580 & 4.03 & 16.62 \\
\hline Spirometer unit: & 108 & & & & & 510 & & & \\
\hline 1027 a. $\mathrm{m} \ldots$ & $\begin{array}{l}198 \\
196\end{array}$ & $\begin{array}{l}229 \\
223\end{array}$ & $\begin{array}{l}.865 \\
.880\end{array}$ & $\begin{array}{l}69.5 \\
69.0\end{array}$ & $\begin{array}{l}11.3 \\
12.7\end{array}$ & $\begin{array}{l}5.19 \\
5.31\end{array}$ & $\begin{array}{l}502 \\
502\end{array}$ & & \\
\hline 1100 a. m..... & 183 & 213 & .860 & 64.5 & 14.4 & 5.04 & 421 & & $\ldots \ldots$ \\
\hline 1138 a. $\mathrm{m} \ldots \ldots$ & 203 & 226 & .900 & 72.0 & 12.9 & 5.43 & 506 & & $\ldots \ldots$ \\
\hline Average............ & 195 & 283 & .875 & 69.0 & 12.8 & 5.24 & 495 & & \\
\hline
\end{tabular}


TABUE 23.-Respiratory exchange in comparison experiments with the Tissot apparatus and the Benedict respiration apparatus (spirometer unit). (Without food.)-Continued.

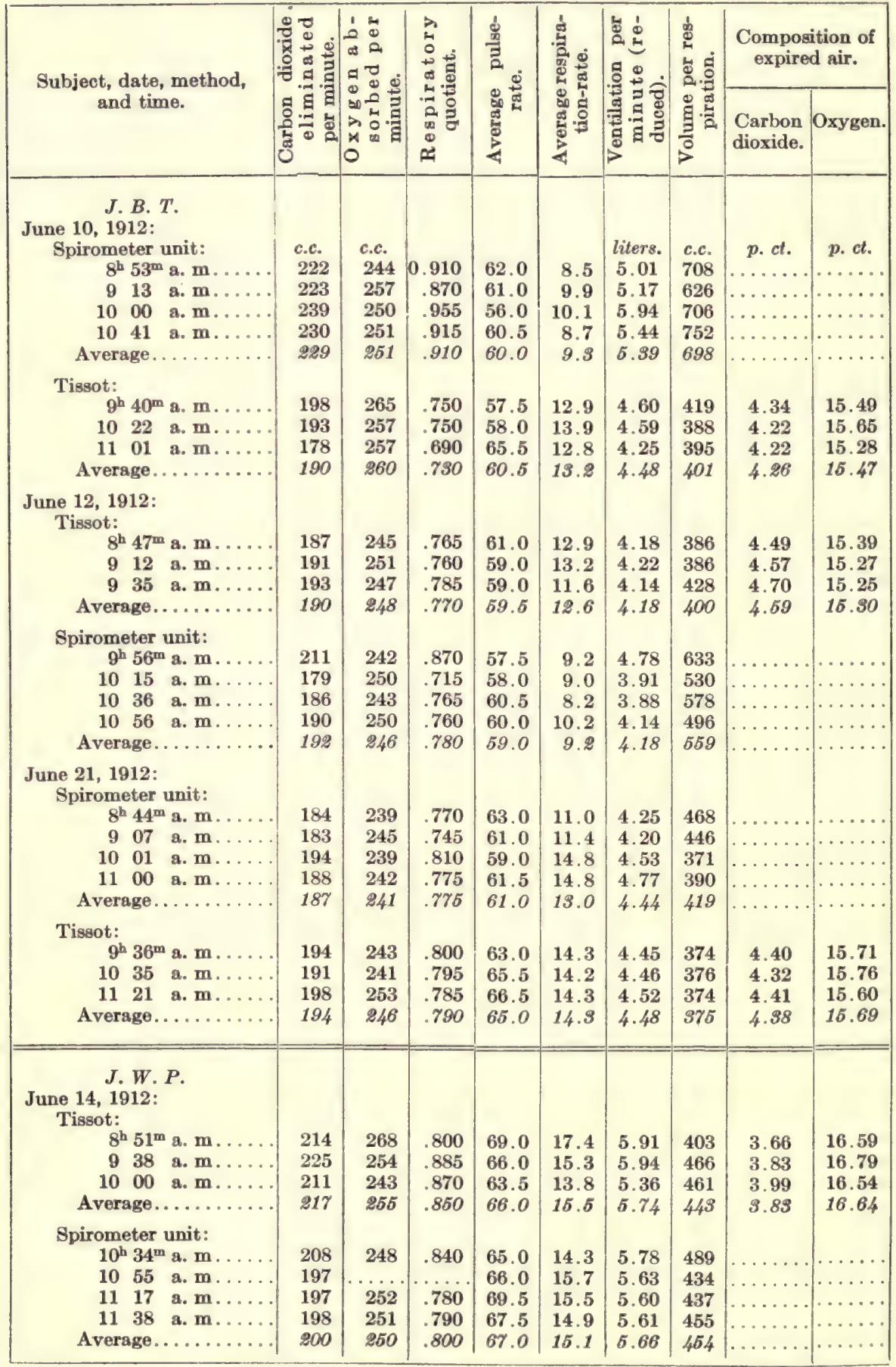


TABLE 23.-Respiratory exchange in comparison experiments with the Tissot apparatus and the Benedict respiration apparatus (spirometer unit). (Without food.) - Continued.

\begin{tabular}{|c|c|c|c|c|c|c|c|c|c|}
\hline \multirow{2}{*}{$\begin{array}{l}\text { Subject, date, method, } \\
\text { and time. }\end{array}$} & \multirow{2}{*}{ 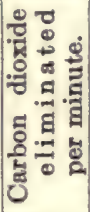 } & \multirow{2}{*}{ 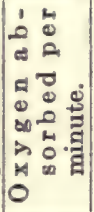 } & \multirow{2}{*}{ 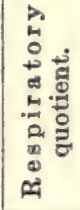 } & \multirow{2}{*}{ 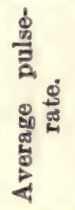 } & \multirow{2}{*}{ 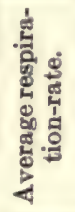 } & \multirow{2}{*}{ 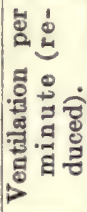 } & \multirow{2}{*}{ 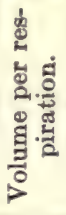 } & \multicolumn{2}{|c|}{$\begin{array}{l}\text { Composition of } \\
\text { expired air. }\end{array}$} \\
\hline & & & & & & & & $\begin{array}{l}\text { Carbon } \\
\text { dioxide. }\end{array}$ & Oxygen. \\
\hline \multicolumn{10}{|l|}{$\begin{array}{l}J . W . P .- \text { Continued. } \\
\text { June } 27,1912 \text { : }\end{array}$} \\
\hline $\begin{array}{l}\text { Spirometer unit: } \\
\qquad 8^{\mathrm{h}} 54^{\mathrm{m}} \text { a. m...... }\end{array}$ & $\begin{array}{l}c . c . \\
200\end{array}$ & $\begin{array}{l}\text { c.c. } \\
220\end{array}$ & 0.910 & 60.5 & 10.6 & $\begin{array}{l}\text { liters. } \\
5.27\end{array}$ & $\begin{array}{l}\text { c.c. } \\
597\end{array}$ & & p. ct. \\
\hline 921 a. m....... & 199 & 225 & .885 & 59.5 & 12.8 & 5.51 & 517 & & \\
\hline 1011 a. m..... & 197 & 241 & .815 & 59.0 & 13.6 & 5.53 & 488 & & \\
\hline 1058 a. m..... & 204 & 241 & .845 & 60.5 & 12.0 & 5.54 & 554 & & \\
\hline Average........... & 200 & 282 & .860 & 60.0 & $1 \% .3$ & 5.46 & 539 & & \\
\hline \multicolumn{10}{|l|}{ Tissot: } \\
\hline $10 \quad 33$ a. m..... & 199 & 247 & .805 & 60.5 & 18.9 & 5.99 & $\begin{array}{l}453 \\
381\end{array}$ & $\begin{array}{l}5.08 \\
3.31\end{array}$ & $\begin{array}{l}16.91 \\
17.04\end{array}$ \\
\hline Average........... & 807 & 250 & .830 & 61.0 & 17.7 & 5.98 & 408 & 3.45 & 16.98 \\
\hline $\begin{array}{l}\text { J.K. } M . \\
\text { June 20. }\end{array}$ & \multicolumn{9}{|c|}{$\begin{array}{l}\text { June 20, 1912: } \\
\text { Tissot: }\end{array}$} \\
\hline $8^{\mathrm{h}} 30^{\mathrm{m}}$ a. m. . . . . & 175 & 214 & .815 & 57.0 & 16.0 & 4.34 & 328 & 4.05 & 16.21 \\
\hline 859 a. m.... & 166 & 200 & .830 & 54.5 & 15.3 & 4.13 & 326 & 4.05 & 16.27 \\
\hline $9 \begin{array}{lll}9 & 50 & \text { a. }\end{array} \ldots$ & 186 & 232 & .800 & 57.0 & 16.9 & 4.72 & 333 & 3.97 & 16.23 \\
\hline 1036 a. m....... & 179 & 231 & .775 & 54.5 & 14.9 & 4.42 & 354 & 4.08 & 15.96 \\
\hline Average........... & 177 & 219 & .810 & 56.0 & 15.8 & 4.40 & 335 & 4.04 & 16.17 \\
\hline \multicolumn{10}{|l|}{ Spirometer unit: } \\
\hline 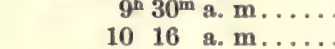 & $\begin{array}{l}177 \\
178\end{array}$ & $\begin{array}{l}213 \\
214\end{array}$ & $\begin{array}{l}.830 \\
.830\end{array}$ & $\begin{array}{l}52.0 \\
53.5\end{array}$ & $\begin{array}{l}13.6 \\
15.2\end{array}$ & $\begin{array}{l}4.35 \\
4.61\end{array}$ & $\begin{array}{l}390 \\
370\end{array}$ & & \\
\hline 1103 a.m.... & 195 & 225 & .865 & 56.5 & 15.0 & 5.02 & 408 & & \\
\hline 1126 a. $\mathrm{m} \ldots$ & 181 & 224 & .810 & 55.0 & 13.9 & 4.53 & 398 & & \\
\hline Average........... & 183 & 219 & .835 & 54.5 & 14.4 & 4.63 & 392 & $\ldots$. & \\
\hline \multicolumn{10}{|l|}{$\begin{array}{l}\text { June 26, 1912: } \\
\text { Spirometer unit: }\end{array}$} \\
\hline $\begin{array}{l}\text { Spirometer unit: } \\
\qquad 8^{\mathrm{b}} 51^{\mathrm{m}} \text { a. } \mathrm{m} . \ldots\end{array}$ & 178 & 222 & .800 & 57.0 & 14.9 & 4.85 & 396 & & \\
\hline 915 a. $\mathrm{m} \ldots$ & 172 & 215 & .800 & 53.0 & 14.8 & 4.73 & 386 & & \\
\hline 1002 a. m.... & 181 & 219 & .825 & 56.5 & 16.9 & 5.10 & 368 & & \\
\hline 1054 a. m..... & 174 & 207 & .840 & 54.5 & 15.7 & 4.82 & 377 & $\ldots \ldots \ldots$ & $\ldots \ldots$ \\
\hline Average........... & 176 & 216 & .815 & 55.5 & 15.6 & 4.88 & 382 & $\ldots \ldots \ldots$ & $\ldots \ldots$ \\
\hline \multicolumn{10}{|l|}{ Tissot: } \\
\hline $9^{\mathrm{h}} 38^{\mathrm{m}}$ a. $\mathrm{m}$....... & 165 & 217 & .755 & 56.5 & 14.9 & 4.35 & 352 & 3.81 & 16.20 \\
\hline 1026 a. m.... & 162 & 216 & .750 & 52.5 & 13.7 & 4.05 & 353 & 4.02 & 15.89 \\
\hline 1117 a. m.... & 169 & 223 & .760 & 55.0 & 14.5 & 4.31 & 355 & 3.95 & 16.04 \\
\hline Average.......... & 165 & 219 & .755 & 54.5 & 14.4 & 4.24 & 358 & 3.93 & 16.04 \\
\hline \multicolumn{10}{|l|}{$\begin{array}{l}\text { June 29, 1912: } \\
\text { Spirometer unit: }\end{array}$} \\
\hline $\begin{array}{l}\text { Spirometer unit: } \\
8^{\mathrm{b}} 47^{\mathrm{m}} \text { a. } \mathrm{m} .\end{array}$ & 164 & 224 & .730 & 58.5 & 15.6 & 4.67 & 364 & & \\
\hline 910 a. m..... & 163 & 209 & .780 & 56.0 & 15.0 & 4.66 & 378 & & $\ldots$ \\
\hline 954 a. m.... & 163 & 208 & .785 & 54.0 & 14.8 & 4.53 & 373 & & $\ldots \ldots$ \\
\hline 1041 a. m..... & 173 & 206 & .840 & 54.0 & 14.7 & 4.82 & 399 & $\ldots \ldots$ & $\ldots \ldots$ \\
\hline Average.......... & 166 & 212 & .785 & 55.5 & 15.0 & 4.67 & $\$ 79$ & & $\ldots \ldots$ \\
\hline Tissot: & & & & & & & & & \\
\hline $9^{\mathrm{h}} 31^{\mathrm{m}}$ a. $\mathrm{m}$ & 176 & 229 & .765 & 61.5 & 15.9 & 4.65 & 353 & 3.81 & 16.24 \\
\hline 1015 a. m.... & 152 & 204 & .750 & 54.5 & 14.3 & 4.15 & 347 & 3.71 & 16.29 \\
\hline 1106 a. m.... & 164 & 220 & .750 & 53.0 & 14.6 & 4.47 & 369 & 3.72 & 16.28 \\
\hline Average........... & 164 & 218 & .750 & 56.5 & 14.9 & 4.42 & 356 & 3.75 & 16.27 \\
\hline
\end{tabular}


TABLE 23.-Respiratory exchange in comparison experiments with the Tissot apparatus and the Benedict respiration apparatus (spirometer unit). (Without food.)-Continued.

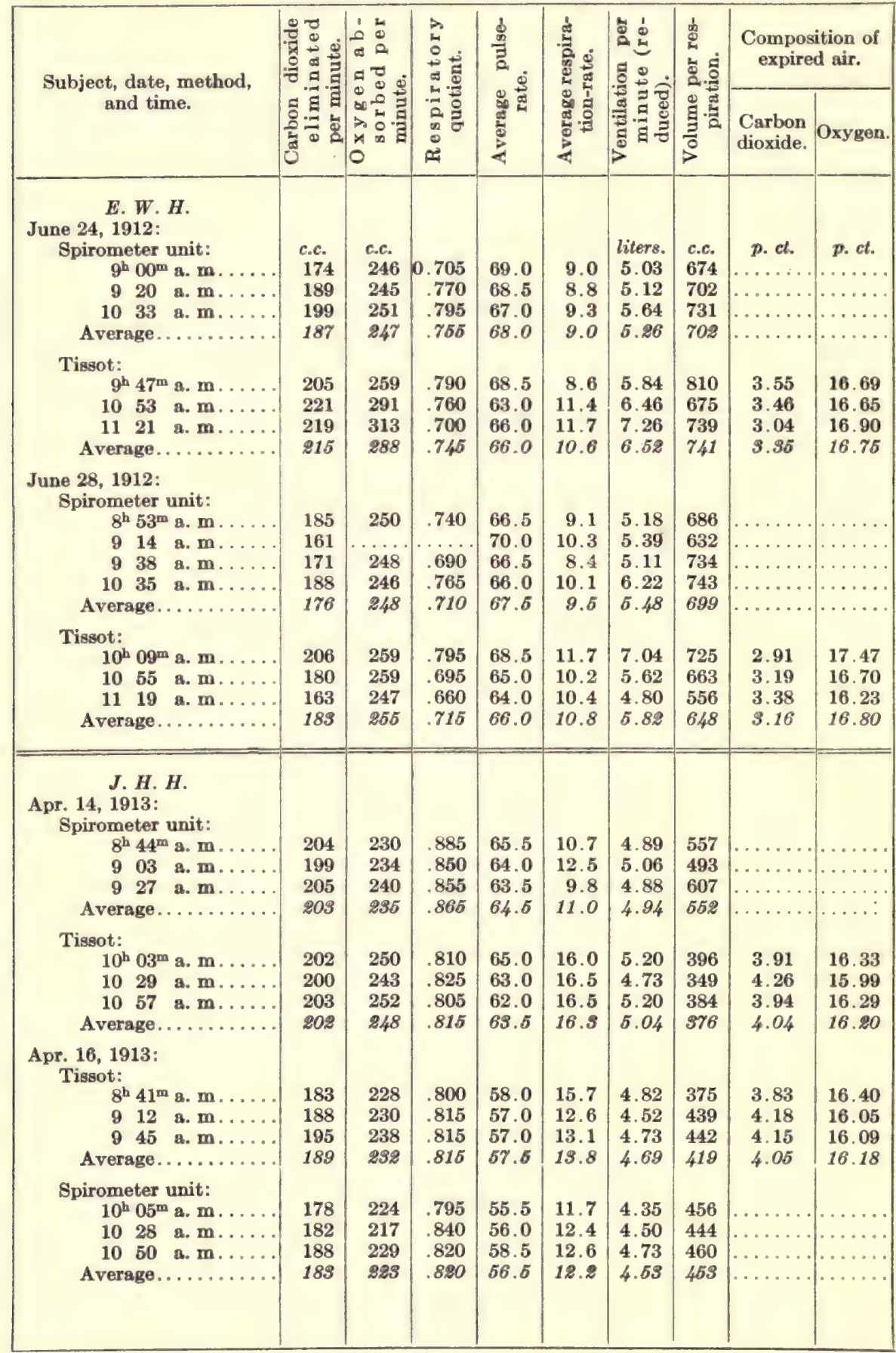


TABLE 23.-Respiratory exchange in comparison experiments with the Tissot apparalus and the Benedict respiration apparatus (spirometer unit). (Without food.)-Continued.

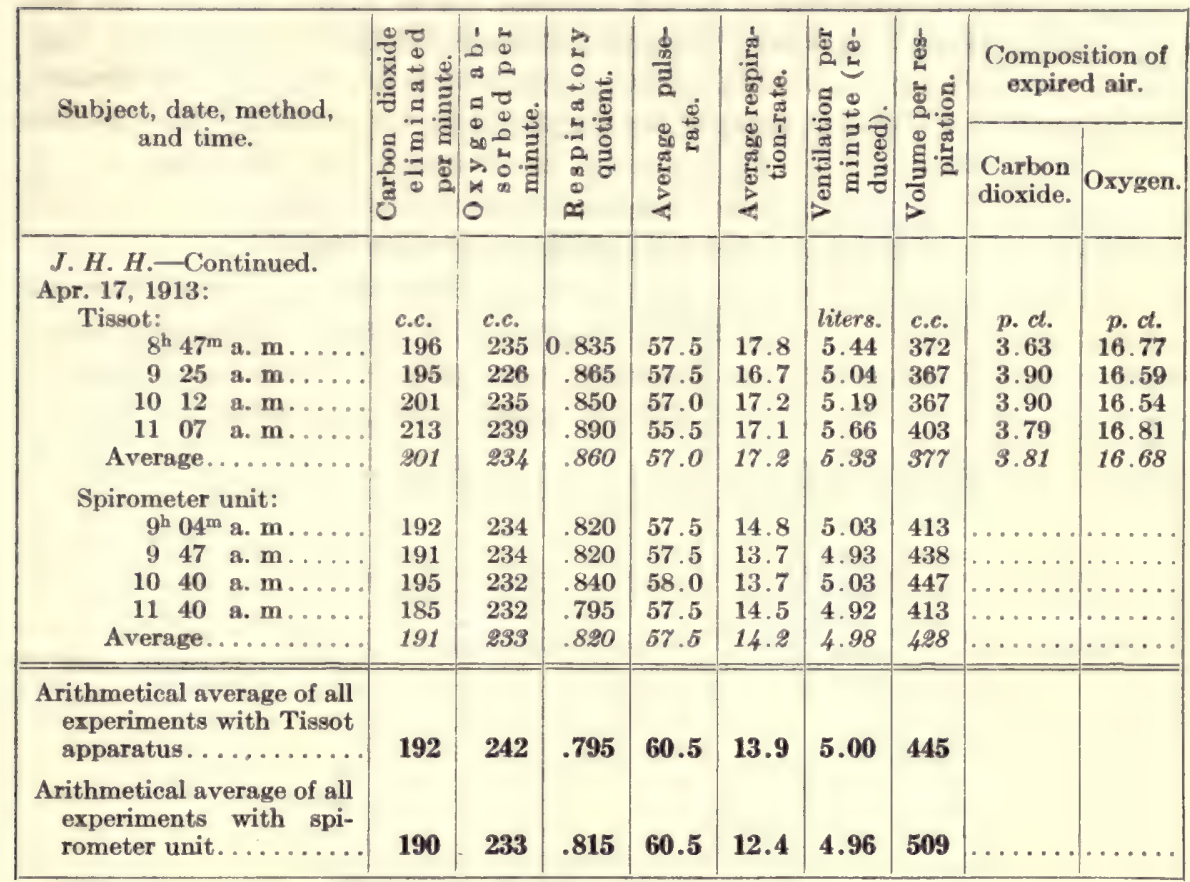

If the individual comparisons are considered, it will be seen from table 24, in which the values for the spirometer unit are used as a baseline, that the averages are not truly representative of the individual experiments, since some of the values show large variations. With K. H. A. the comparisons give, on the whole, fairly good results. With P. F. J. the second experiment shows a higher metabolism with the Tissot apparatus, but it will be noted that the periods with that apparatus were in the early part of the morning, while the periods with the spirometer unit were in the last part, so that the differences may be partly accounted for by the difference in the time of day. With J. B. T. the first experiment shows a marked difference in the carbondioxide production; from a comparison of the figures obtained in this experiment for the total ventilation of the lungs and the volume of respiration, it is apparent that the subject over-ventilated the lungs in the periods with the spirometer unit. An average value of 698 c.c. per respiration is distinctly abnormal for most subjects. The other two comparisons with the same subject gave on the whole very good results. With J. W. P. the values for the Tissot apparatus are usually higher than those for the spirometer unit; in one of the experiments with this subject, the periods with the Tissot apparatus preceded those with the spirometer unit. With J. K. M. the differences are not large and on the whole the comparisons gave very fair results. In the second 
experiment with this subject he was drowsy at times, this having an influence on the uniformity of the results. The subject $\mathrm{E}$. W. H. was distinctly difficult to work with because of his restlessness; the high values for the carbon dioxide and oxygen shown in the first experiment, which tend to raise the general average value, were wholly due to this fact. The subject J. H. H. gave on the average very fair values. It may be noted in this connection that this subject earlier in the year was used with the spirometer unit with very poor results.

The average variations for all of the subjects were: Carbon-dioxide production, \pm 9 c.c.; oxygen consumption, +9 c.c.; respiratory quotient, \pm 0.035 ; ventilation of the lungs per minute, \pm 0.31 liter; volume per respiration, \pm 78 c.c. It will be noted, however, that the volume

TABLE 24.-Variations of average results obtained with the Tissot apparatus from those obtained with the spirometer unit.

\begin{tabular}{|c|c|c|c|c|c|c|c|c|}
\hline Subject. & Date. & $\begin{array}{c}\text { Carbon } \\
\text { dioxide } \\
\text { elimi- } \\
\text { nated per } \\
\text { minute. }\end{array}$ & $\begin{array}{c}\text { Oxygen } \\
\text { absorbed } \\
\text { per } \\
\text { minute. }\end{array}$ & $\begin{array}{l}\text { Respira- } \\
\text { tory } \\
\text { quotient. }\end{array}$ & $\begin{array}{c}\text { Average } \\
\text { pulse- } \\
\text { rate. }\end{array}$ & $\begin{array}{c}\text { Average } \\
\text { respira- } \\
\text { tion- } \\
\text { rate. }\end{array}$ & $\begin{array}{c}\text { Ventila- } \\
\text { tion per } \\
\text { minute } \\
\text { (reduced). }\end{array}$ & $\begin{array}{l}\text { Volume } \\
\text { per } \\
\text { respira- } \\
\text { tion. }\end{array}$ \\
\hline \multirow{4}{*}{$\begin{array}{l}\text { K. H. A... } \\
\text { P. F. J.... }\end{array}$} & 1912 & & c.c. & & & & liters. & \\
\hline & June 4 & +3 & +3 & +0.005 & 0 & +1.0 & +0.03 & -47 \\
\hline & June 7 & +1 & +12 & -.040 & +1.5 & 正 & -.31 & -30 \\
\hline & June 5 & 0 & +9 & -.030 & -1.5 & +1.0 & +.04 & -63 \\
\hline \multirow{4}{*}{ J. B. T. } & June 8 & +15 & +15 & +.005 & +0.5 & -2.2 & 0 & +85 \\
\hline & June 10 & -39 & +9 & -.180 & +0.5 & +3.9 & -.91 & -297 \\
\hline & June 12 & -2 & +2 & -.010 & +0 & +3 & 0 & -159 \\
\hline & June 21 & +7 & +5 & +.015 & +4 & +1.3 & +0.04 & -44 \\
\hline \multirow{2}{*}{ J. W. P... } & June 14 & +17 & +5 & +.050 & -1.0 & +.4 & +.08 & -11 \\
\hline & June 27 & +7 & +18 & -.030 & +1 . & +5.4 & +.52 & -131 \\
\hline \multirow{3}{*}{ J. K. M... } & June 20 & -6 & 0 & -.025 & +1.5 & +1.4 & -.23 & -57 \\
\hline & June 26 & -11 & +3 & -.06 & -1 & -1.2 & -.64 & -29 \\
\hline & June 29 & -2 & +6 & -.035 & +1 . & -.1 & -.25 & -23 \\
\hline \multirow[t]{2}{*}{ E. W. H. . } & June 24 & +28 & +41 & -.010 & -2.0 & +1.6 & +1.26 & +39 \\
\hline & $\begin{array}{c}\text { June } 28 \\
1913\end{array}$ & +7 & +7 & +.005 & -1.5 & +1.3 & +0.34 & -51 \\
\hline \multirow[t]{3}{*}{ J. H. H... } & Apr. 14 & -1 & +13 & -.050 & -1.0 & +5.3 & +.10 & -176 \\
\hline & Apr. 16 & +6 & +9 & -.005 & +1.0 & +1.6 & +.16 & -34 \\
\hline & Apr. 17 & +10 & +1 & +.040 & -.5 & +3.0 & +.35 & -51 \\
\hline \multicolumn{2}{|c|}{ Average variation.... } & 9 & 9 & 0.035 & 1.0 & 2.0 & 0.31 & 78 \\
\hline
\end{tabular}

per respiration in all but two series is decidedly lower with the Tissot apparatus than with the spirometer unit. This is due, in some cases at least and particularly with J. H. H., to the fact that the respirationrate is higher with the Tissot apparatus and more nearly approaches the normal. The fact that all the variations for the oxygen consumption are plus indicates that the metabolism with the Tissot apparatus was slightly higher than with the spirometer unit.

The degree of uniformity in the results has been calculated and the percentage of the total variation from the average is given in the form of curves in figure 44. The several factors are comparatively uniform 
in the measurements. The respiratory quotient shows a slightly better uniformity with the Tissot apparatus than with the spirometer unit. On the whole, the results indicate that with good subjects it is possible to obtain comparable results in the measurement of the respiratory exchange with both types of apparatus.

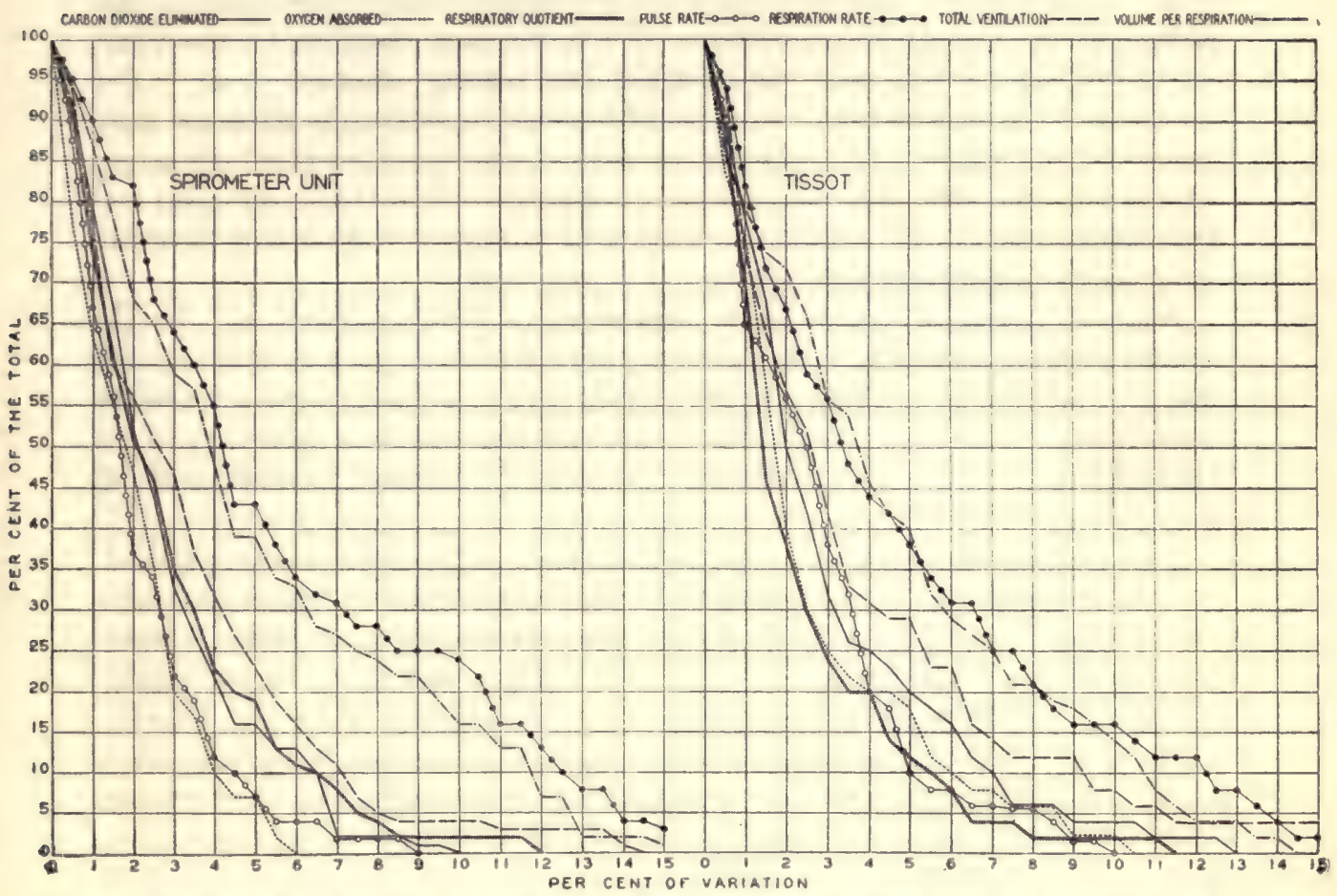

Fıg. 44.-Probability curves for the series of comparison experiments with the spirometer unit and the Tissot apparatus.

The ordinates indicate the percentage of the total number of periods and the abscissæ the percentage of variation from the average.

\section{DOUGLAS RESPIRATION APPARATUS AND BENEDICT RESPIRATION APPARATUS (SPIROMETER UNIT).}

Although the Douglas respiration apparatus had not been used in this laboratory for regular respiration experiments, it was deemed advisable to compare the gaseous metabolism as measured by the Douglas method with that measured by the spirometer unit. A description of the Douglas method has been given in a previous section of this report. ${ }^{1}$

For the earlier experiments in the series a bag was purchased which was made from a fairly good grade of rubber and was supplied with a tube leading into it through which air could be introduced. This bag was supposed to have a capacity of 100 liters, but it was found that it 
would hold only about 25 to 30 liters without noticeable pressure. The periods in which the bag was used were only about 5 minutes in length, with a preliminary period of 10 minutes.

This bag was used up to and including July 3, 1912, when another bag was secured of rubber-covered cloth which was of the same dimensions as the larger bag described by Douglas. The possibility of the diffusion of carbon dioxide through the rubber cloth was tested by partly filling the bag with expired air and taking samples from time to time. No appreciable change in the carbon-dioxide content was found in the length of time which would elapse between the beginning of a period and the time of taking the sample. This bag was used for the remainder of the series, the duration of the periods being $10 \mathrm{~min}$ utes, with a preliminary period of 5 minutes.

Several types of valves were employed in this comparison. In all of the experiments with the first bag and also in a part of the experiments with the larger bag, the rubber-flap valves described on page 69 were used. In some of the later experiments use was also made of both the mica-flap valves ordinarily employed with the Douglas method and the Tissot valves.

The routine was the same as in the other comparisons, except that in a number of the experiments the subject occupied a reclining chair, this position being more convenient with the Douglas method. Both the mouthpiece and the nosepieces were used as noted in the statistics. As in other comparisons, the pulse-rate was determined by the Bowles stethoscope. The respiration was recorded from the chest pneumograph in the periods with the Douglas apparatus and from the movements of the spirometer bell in the periods with the spirometer unit. The degree of muscular repose was determined in nearly all of the experiments with the spirometer unit by means of the lever bed-spring arrangement, ${ }^{1}$ the only exception being the first experiment with E.W.H. This device was also used in many of the experiments with the Douglas method, but in some of the experiments the only indications of the quietness of the subject were obtained from the records of the chest pneumograph. None of the subjects were familiar with the Douglas apparatus, but, with the exception of M. J. S., they had all had previous experience with the spirometer unit. The statistics of the 16 experiments in this comparison are given in the following pages. My thanks are due to Mr. L. E. Emmes for assistance in carrying out a considerable number of the experiments.

\section{STATISTICS OF EXPERIMENTS.}

E. W. H., June 21, 1912.-Spirometer unit, 3 periods; Douglas apparatus, 2 periods; apparatus alternated. Pneumatic nosepieces with both apparatus; rubber-flap valves and small bag with Douglas apparatus. Subject sat in reclining chair; was very difficult to work with, owing to his restlessness and 
irregularity in respiration. Complained of pressure of the chest pneumograph in first period of experiment (with spirometer unit), saying that it caused a desire to breathe through the mouth. In second period (with Douglas apparatus) he found it fatiguing to breathe; in fourth period (with same apparatus) he moved his head and said that it ached slightly; he found it difficult to breathe toward end of period. Pressure in bag at end of period, $11 \mathrm{~mm}$. of water. In third period with spirometer unit (last period of experiment), he was very restless and tired, saying that he felt like getting up and jumping. Pulse-rate uniform. Respiration fairly uniform, except in third period with spirometer unit. Average barometric pressure and average temperature of air in apparatus were: Spirometer unit, $761.6 \mathrm{~mm}$. and $21.3^{\circ} \mathrm{C}$., respectively; Douglas apparatus, $761.3 \mathrm{~mm}$. and $20.3^{\circ} \mathrm{C}$., respectively.

K. H. A., June 24, 1912.-Spirometer unit, 3 periods; Douglas apparatus, 3 periods; preliminary period, 44 minutes; apparatus alternated. Subject lying on couch; pneumatic nosepieces with both apparatus; rubber-flap valves and small bag with Douglas apparatus. Subject stated that at the end of first period with Douglas apparatus it was very much more difficult to breathe than with spirometer unit. Pressure in bag at end of periods 7 to $8 \mathrm{~mm}$. of water. In second and third periods with Douglas apparatus he found it easier to breathe. Pulse-rate in all periods except first and respiration in all periods approximately uniform. Average barometric pressure and temperature of air in apparatus were: Spirometer unit, $763.5 \mathrm{~mm}$. and $22.1^{\circ} \mathrm{C}$., respectively; Douglas apparatus, $763.4 \mathrm{~mm}$. and $22.9^{\circ} \mathrm{C}$., respectively.

K. H. A., June 26, 1912.-Spirometer unit, 3 periods; Douglas apparatus, 3 periods; preliminary period, 39 minutes; apparatus alternated. Subject lying on couch; pneumatic nosepieces with both apparatus, rubber-flap valves and small bag with Douglas apparatus. Pressure in bag at end of experiment approximately 8 to $9 \mathrm{~mm}$. of water. Pulse-rate uniform in all periods, also respiration-rate. Average barometric pressure and average temperature of air in apparatus were: Spirometer unit, $757.0 \mathrm{~mm}$. and $22.8^{\circ} \mathrm{C}$., respectively; Douglas apparatus, $756.8 \mathrm{~mm}$. and $23.2^{\circ} \mathrm{C}$., respectively.

P.F. J., June 25, 1912.-Spirometer unit, 3 periods; Douglas apparatus, 3 periods; preliminary period, 34 minutes; apparatus alternated. Subject lying on couch; nosepieces with both apparatus, and rubber-flap valves and small bag with Douglas apparatus. In first two periods with spirometer unit subject complained of acid fumes. In first period with Douglas apparatus he noted but little difference between the two apparatus. In second period with this apparatus he thought there was some difficulty in breathing toward the end. Pressure in bag about $8 \mathrm{~mm}$. of water. Pulse-rate uniform in all periods but first. Respiration-rate uniform. Average barometric pressure and temperature of air in apparatus were: Spirometer unit, $763.1 \mathrm{~mm}$. and $23.2^{\circ} \mathrm{C}$., respectively; Douglas apparatus, $763.0 \mathrm{~mm}$. and $23.3^{\circ} \mathrm{C}$., respectively.

P.F. J., July 2, 1912.-Spirometer unit, 3 periods; Douglas apparatus, 3 periods; preliminary period, 53 minutes; apparatus alternated. Subject lying on couch; nosepieces with both apparatus; rubber-flap valves and small bag with Douglas apparatus. In first period with Douglas apparatus subject found it difficult to inhale but not to exhale, and said that he preferred the spirometer unit, as breathing with latter was easier. Pulse-rate fairly uniform. Respiration-rate in each period uniform; in second period with Douglas apparatus, respiration-rate markedly faster, but with no apparent cause. Average barometric pressure and average temperature of air in apparatus were: Spirometer unit, $768.8 \mathrm{~mm}$. and $21.0^{\circ} \mathrm{C}$., respectively; Douglas apparatus, $768.7 \mathrm{~mm}$. and $20.1^{\circ} \mathrm{C}$., respectively. 
J. B. T., June 27, 1912.-Spirometer unit, 3 periods; Douglas apparatus, 3 periods; preliminary period, 34 minutes; apparatus alternated. Subject lying on couch; pneumatic nosepieces with both apparatus and rubber-flap valves and small bag with Douglas apparatus. Subject said with Douglas method it was difficult to exhale. Pressure in bag at end of experiment 7 to $8 \mathrm{~mm}$. of water. Both pulse-rate and respiration-rate uniform in all periods. Average barometric pressure and temperature of air in apparatus were: Spirometer unit, $767.9 \mathrm{~mm}$. and $22.8^{\circ} \mathrm{C}$., respectively; Douglas apparatus, $767.8 \mathrm{~mm}$. and $22.8^{\circ} \mathrm{C}$., respectively.

J.K. M., July 1, 1912.-Spirometer unit, 3 periods; Douglas apparatus, 2 periods; preliminary period, 57 minutes; first two periods with spirometer unit, then apparatus alternated. Subject lying on couch; pneumatic nosepieces with both apparatus, and rubber-flap valves and small bag with Douglas apparatus. Subject stated that he noted no difference between methods. Pressure on bag at end of experiment $6 \mathrm{~mm}$. Both pulse-rate and respirationrate fairly uniform. Average barometric pressure and temperature of air in apparatus were: Spirometer unit, $767.0 \mathrm{~mm}$. and $21.3^{\circ} \mathrm{C}$, respectively; Douglas apparatus, $767.1 \mathrm{~mm}$. and $22.4^{\circ} \mathrm{C}$., respectively.

J.K. M., July 3, 1912.-Spirometer unit, 3 periods; Douglas apparatus, 3 periods; preliminary period, 57 minutes; apparatus alternated. Subject lying on couch; nosepieces used with both apparatus, and rubber-flap valves and small bag with Douglas apparatus. Subject said that there was a slight resistance to exhaling. Pressure on bag at end of experiment $6 \mathrm{~mm}$. of water. Subject drowsy in first two periods with spirometer unit; wide awake in last period. In last period with Douglas apparatus he had a great desire to get through with the experiment. Pulse-rate and respiration-rate both uniform. Average barometric pressure and temperature of air in apparatus were: Spirometer unit, $765.8 \mathrm{~mm}$. and $21.6^{\circ} \mathrm{C}$., respectively; Douglas apparatus, $765.9 \mathrm{~mm}$. and $22.3^{\circ} \mathrm{C}$. respectively.

S. A. R., July 20, 1912.-Douglas apparatus, 3 periods; spirometer unit, 3 periods; apparatus alternated. Subject lying on couch; pneumatic nosepieces with spirometer unit; mouthpiece, rubber-flap valves and large bag with Douglas apparatus. Subject thought Douglas method easier than spirometer unit. Pressure in bag at end of experiment 4 to $5 \mathrm{~mm}$. of water. Pulse-rate comparatively uniform. In all periods there was a tendency to apnœe in respiration, more particularly with Douglas method. Average barometric pressure and temperature of air in apparatus were: Spirometer unit, 767.4 $\mathrm{mm}$. and $21.1^{\circ} \mathrm{C}$., respectively; Douglas apparatus, $767.6 \mathrm{~mm}$. and $19.8^{\circ} \mathrm{C}$., respectively.

M.J. S., July 19, 1912.-Spirometer unit, 2 periods; Douglas apparatus, 2 periods; preliminary period, 2 hours; apparatus alternated. Subject lying on couch; glass nosepieces with spirometer unit and mouthpiece with special moistener with Douglas apparatus; rubber-flap valves and large bag with Douglas apparatus. Pulse-rate varied somewhat in first period with each apparatus. Respiration irregular in periods with spirometer unit, particularly in the last few minutes. The type of respiration is shown in figure 45 . Average barometric pressure and average temperature of air in apparatus were: Spirometer unit, $757.9 \mathrm{~mm}$. and $20.8^{\circ} \mathrm{C}$., respectively; Douglas apparatus, $758.4 \mathrm{~mm}$. and $21.7^{\circ} \mathrm{C}$., respectively.

M. J. S., July 22, 1912.-Douglas apparatus, 4 periods; spirometer unit, 4 periods; preliminary period, 52 minutes; apparatus alternated. Subject lying on couch; pneumatic nosepieces with spirometer unit, mouthpiece with Douglas apparatus; mica-flap valves and large bag with Douglas apparatus. Intake valve arranged so that flap was horizontal, in order to be sure that it 
would close properly during expiration. The expiration valve was nearly horizontal. During first two or three minutes with Douglas method the intake valve did not appear to close properly, as the bag fell slightly at the beginning of each inspiration; the subject also stated that the air did not seem pure, except when he inspired deeply. In third period with Douglas apparatus the intake valve was placed at the end of a long rubber tube, so that it hung below the couch and was vertical. The subject stated that it was very easy to breathe with this arrangement of the valve. Pulse-rate very uniform. Tendency toward deep respiration at end of second period with Douglas apparatus; deeper respirations than normal in other periods
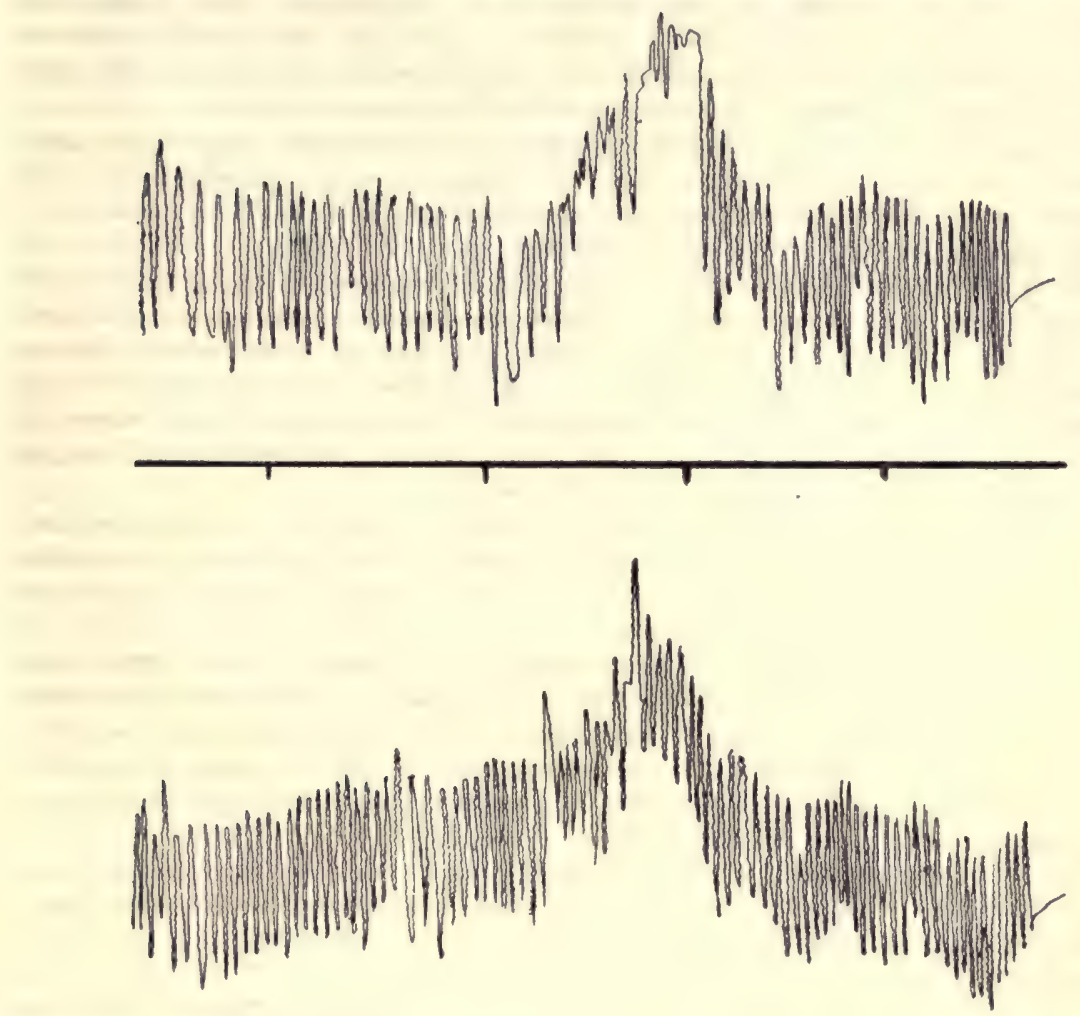

FIG. 45.-Types of respiration of subject M. J. S. at end of second and fourth periods with the spirometer unit on July 19, 1912. Time line, minutes. Original size.

with this apparatus. Respirations perfectly normal with spirometer unit. Average barometric pressure and average temperature of air in apparatus were: Spirometer unit, $756.3 \mathrm{~mm}$. and $22.5^{\circ} \mathrm{C}$., respectively; Douglas apparatus, $756.2 \mathrm{~mm}$. and $22.7^{\circ} \mathrm{C}$., respectively.

M. J. S., July 24, 1912.-Douglas apparatus, 3 periods; spirometer unit, 3 periods; preliminary period, 52 minutes; apparatus alternated. Subject lying on couch; with Douglas method. Tissot valves, glass nosepieces, and large bag; with spirometer unit, pneumatic nosepieces. Subject preferred Douglas apparatus, as glass nosepieces easier to breathe through. Subject 
tired in last period. Pressure in bag at end of experiment about $4 \mathrm{~mm}$. of water. Pulse-rate comparatively regular. Respiration for the most part uniform, except in last period, when subject occasionally took a deep breath. Average barometric pressure and temperature of air in apparatus were: Spirometer unit, $754.3 \mathrm{~mm}$. and $20.4^{\circ} \mathrm{C}$., respectively; Douglas apparatus, $754.8 \mathrm{~mm}$. and $20.9^{\circ} \mathrm{C}$., respectively.

M. J. S., July 25, 1912.-Douglas apparatus, 3 periods; spirometer unit, 3 periods; apparatus alternated. Subject lying on couch. With Douglas method, Tissot valves, glass nosepieces, and large bag; with spirometer unit, pneumatic nosepieces which were tested with soapsuds for leaks. Subject preferred Douglas method, as less resistance to breathing. Both pulse-rate and respiration-rate comparatively uniform. Average barometric pressure and temperature of air in apparatus were: Spirometer unit, $751.1 \mathrm{~mm}$. and $20.5^{\circ} \mathrm{C}$.; Douglas method, $751.3 \mathrm{~mm}$. and $21.6^{\circ} \mathrm{C}$., respectively.

M. J. S., July 26, 1912.-Douglas apparatus, 3 periods; spirometer unit, 3 periods; apparatus alternated. Subject lying on couch; mouthpiece with both apparatus; rubber-flap valves and large bag used with Douglas apparatus; Douglas bag supported vertically. Subject said he found it more difficult to inhale with rubber-flap valves than with the Tissot valves and preferred the spirometer unit in this experiment. Pressure in bag at end of experiment about $5 \mathrm{~mm}$. of water. Pulse-rate uniform throughout experiment. Respiration comparatively uniform, except in last period, when there was considerable irregularity in last half. Average barometric pressure and average temperature were: Spirometer unit, $751.0 \mathrm{~mm}$. and $19.8^{\circ} \mathrm{C}$., respectively; Douglas apparatus, $751.0 \mathrm{~mm}$. and $19.0^{\circ} \mathrm{C}$., respectively.

J. B. T., November 15, 1912. - Spirometer unit, 3 periods; Douglas apparatus 3 periods; apparatus alternated. Subject sitting in reclining chair; pneumatic nosepieces, with surgeon's plaster over lips and soapsuds around nosepieces with both apparatus; mica-flap valves and large bag with Douglas apparatus. Subject found no difference in breathing with either of the apparatus. Pulse-rate during experiment comparatively uniform. Normal respirationrate, 18 per minute; respiration during experiment very uniform in character. Average barometric pressure and temperature of air in apparatus were: Spirometer unit, $756.4 \mathrm{~mm}$. and $20.4^{\circ} \mathrm{C}$., respectively; Douglas apparatus, $756.2 \mathrm{~mm}$. and $19.4^{\circ} \mathrm{C}$., respectively.

T. M. C., November 16, 1912.-Spirometer unit, 3 periods; Douglas apparatus, 3 periods; apparatus alternated. Subject sitting in reclining chair; mouthpiece used with both apparatus; mica-flap valves and large bag with Douglas apparatus. Subject stated he found it a little more difficult to breathe into Douglas bag. Pulse-rate uniform. Average respiration-rate before experiment 14 per minute; respiration during experiment very uniform. Average barometric pressure and temperature of air in apparatus were: Spirometer unit, $764.3 \mathrm{~mm}$. and $18.5^{\circ} \mathrm{C}$., respectively; Douglas apparatus $764.3 \mathrm{~mm}$. and $18.0^{\circ} \mathrm{C}$., respectively.

\section{DISCUSSION OF RESULTS.}

The results of the several comparisons with the Douglas method and the spirometer unit are given in table 25, together with averages for each experiment and a general average for each apparatus for the whole series of comparisons. The general averages for the respiratory exchange with the Douglas apparatus are lower than those with the spirometer unit, being 178 c.c. for the carbon dioxide eliminated, 224 c.e. 
for the oxygen consumption, and $\mathbf{0 . 7 9 5}$ for the respiratory quotient as compared with 189 c.c., 231 c.c. and 0.820 respectively for the same factors with the spirometer unit. The average pulse-rate for the two methods is essentially identical, $i . e ., 62$ per minute for the Douglas apparatus and 61.5 for the spirometer unit. The other averages show slight variations, the values being for the Douglas apparatus 15.3 per minute for the respiration-rate, 5.15 liters for the ventilation of the lungs, and 431 c.c. for the volume per respiration, and for the spirometer unit, 14.3 per minute, 5.04 liters, and 445 c.c. respectively for the same factors. The variations in the individual comparisons are given in table 26 , the values for the spirometer unit being used for the basis of calculation. A study of tables 25 and 26 shows that the values fluctuate widely and that the differences between the two apparatus are noticeable.

TABLE 25.-Respiratory exchange in comparison experiments with the Douglas method and the Benedict respiration apparaius (spirometer unit). (Without food.)

\begin{tabular}{|c|c|c|c|c|c|c|c|c|c|}
\hline \multirow{2}{*}{$\begin{array}{l}\text { Subject, date, method, } \\
\text { and time. }\end{array}$} & \multirow{2}{*}{ 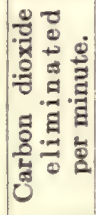 } & \multirow{2}{*}{ 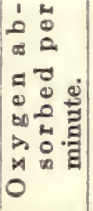 } & \multirow{2}{*}{ 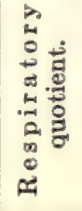 } & \multirow{2}{*}{ 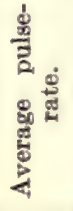 } & \multirow{2}{*}{ 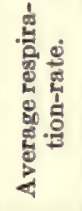 } & \multirow{2}{*}{ 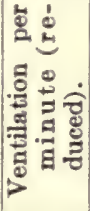 } & \multirow{2}{*}{ 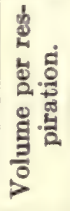 } & \multicolumn{2}{|c|}{$\begin{array}{l}\text { Composition of } \\
\text { expired air. }\end{array}$} \\
\hline & & & & & & & & $\begin{array}{l}\text { Carbon } \\
\text { dioxide. }\end{array}$ & Oxygen \\
\hline $\begin{array}{l}E \cdot W \cdot H . \\
\text { June } 21,1912: \\
\text { Spirometer unit: } \\
9^{\mathrm{h}} 13^{\mathrm{m}} \text { a. } \mathbf{m} .\end{array}$ & $\begin{array}{l}\text { c.c. } \\
198\end{array}$ & $\begin{array}{l}c . c . \\
243\end{array}$ & 0.815 & 70.0 & 9.6 & $\begin{array}{l}\text { liters. } \\
5.30\end{array}$ & $\begin{array}{l}c . c . \\
666\end{array}$ & p. ct. & p. ct. \\
\hline 1028 a. $m$. & 234 & 250 & .935 & 68.5 & 9.9 & 7.56 & 922 & & \\
\hline 1145 a. $\mathrm{m}$. & 233 & 255 & .915 & 70.0 & 10.4 & 7.63 & 886 & & \\
\hline Average....... & 222 & 249 & .890 & 69.5 & 10.0 & 6.83 & 825 & & \\
\hline Douglas: & & & & & & & & & \\
\hline $10^{\mathrm{h}} 00^{\mathrm{m}} \mathrm{a} \cdot \mathrm{m}$. & $\begin{array}{l}166 \\
203\end{array}$ & 236 & .705 & 69.5 & 10.0 & 5.06 & $\begin{array}{l}611 \\
719\end{array}$ & $\begin{array}{l}3.33 \\
3.11\end{array}$ & 16.56 \\
\hline $\begin{array}{r}11 \text { a. } 16 . \\
\text { Average......... }\end{array}$ & $\begin{array}{l}203 \\
185\end{array}$ & $\begin{array}{l}274 \\
255\end{array}$ & .740 & $\begin{array}{l}69.0 \\
69.5\end{array}$ & $\begin{array}{l}11.1 \\
10.6\end{array}$ & $\begin{array}{l}6.60 \\
5.89\end{array}$ & 665 & & $\begin{array}{l}17.02 \\
16.79\end{array}$ \\
\hline $\begin{array}{c}\text { K. H. A. } \\
\text { June 24, 1912: } \\
\text { Spirometer unit: } \\
9^{\mathrm{h}} 04^{\mathrm{m}} \mathrm{a} . \mathrm{m}\end{array}$ & 200 & 240 & .835 & 580 & 139 & & & & \\
\hline 1035 a. m. & 180 & 220 & .820 & 48.5 & 14.0 & & & & \\
\hline 1145 a. $\mathrm{m}$. & 192 & 224 & .855 & 48.0 & 14.5 & 5.24 & 436 & & \\
\hline Average......... & 191 & 228 & .840 & 51.5 & 14.1 & 5.24 & 486 & & \\
\hline $\begin{array}{l}\text { Douglas: } \\
\qquad 9^{\mathrm{h}} 50^{\mathrm{m}} \text { a. } \mathrm{m} .\end{array}$ & 188 & 232 & .805 & 50.0 & & 4.77 & & 3.9 & 16.25 \\
\hline 1115 a. m. & 171 & 214 & .800 & 45.5 & 13.8 & 4.85 & 423 & 3.55 & 16.72 \\
\hline 1220 p. m. & 166 & 216 & .770 & 45.0 & 13.9 & 4.84 & 420 & 3.49 & 16.68 \\
\hline Average....... & 175 & 221 & .790 & 47.0 & 18.9 & 4.82 & 422 & 3.68 & 16.65 \\
\hline $\begin{array}{l}\text { June 26, 1912: } \\
\text { Spirometer unit: } \\
8^{\mathrm{h}} 55^{\mathrm{m}} \mathrm{g}, \mathrm{m}\end{array}$ & & & & & & 4.83 & 422 & & \\
\hline $\begin{array}{lll}855^{m i n} & \text { a. } \mathrm{m} . \\
9 & 57 & \text { a. } \mathrm{m}\end{array}$ & $\begin{array}{l}177 \\
192\end{array}$ & $\begin{array}{l}245 \\
235\end{array}$ & .820 & $\begin{array}{l}53.5 \\
51.5\end{array}$ & $\begin{array}{l}13.9 \\
15.2\end{array}$ & $\begin{array}{l}4.83 \\
5.31\end{array}$ & 424 & & \\
\hline 1058 a. $\mathrm{m}$. & 195 & 233 & .835 & 50.0 & 15.6 & 5.21 & 406 & & \\
\hline Average........ & 188 & 288 & .790 & 61.5 & 14.9 & 5.12 & 417 & & \\
\hline
\end{tabular}


TABLE 25.-Respiratory exchange in comparison experiments with the Douglas method and the Benedict respiration apparatus (spirometer unit). (Without food.)-Continued.

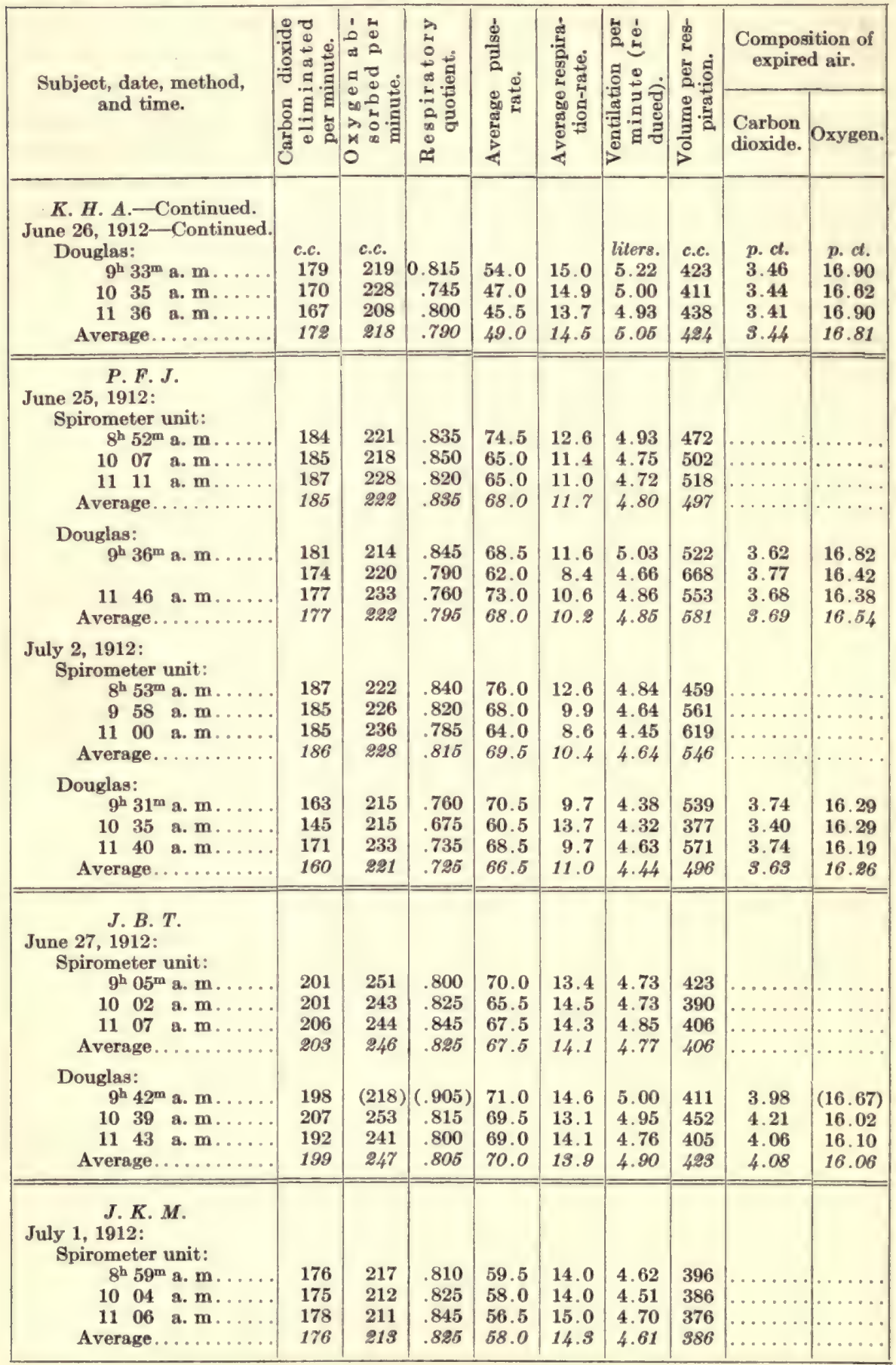


TABLE 25.-Respiratory exchange in comparison experiments with the Douglas method and the Benedict respiration apparatus (spirometer unit). (Without food.)-Continued.

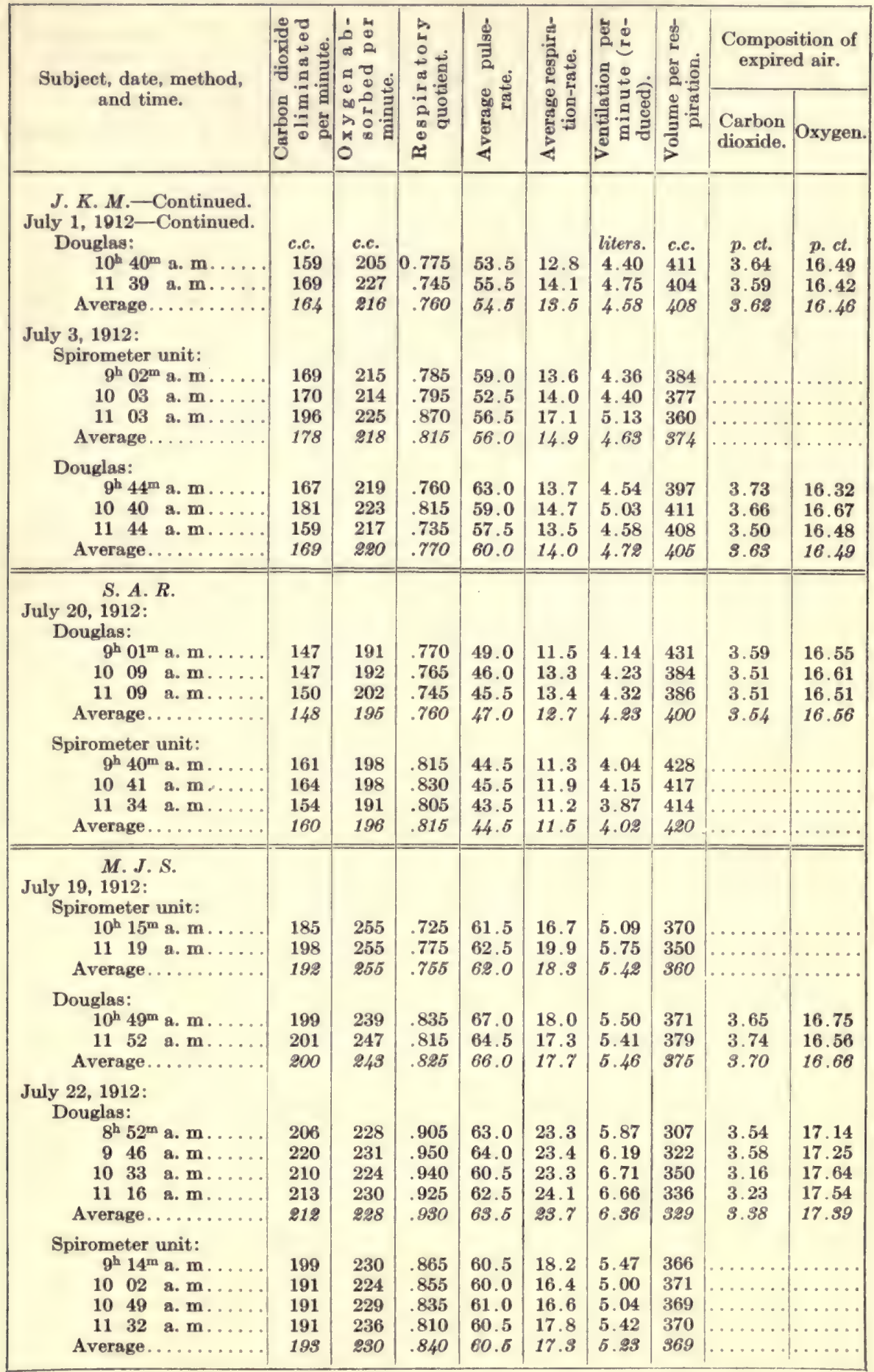


TABLE 25.-Respiratory exchange in comparison experiments with the Douglas method and the Benedict respiration apparatus (spirometer unit). (Without food.)-Continued.

\begin{tabular}{|c|c|c|c|c|c|c|c|c|c|}
\hline \multirow{2}{*}{$\begin{array}{l}\text { Subject, date, method, } \\
\text { and time. }\end{array}$} & \multirow{2}{*}{ 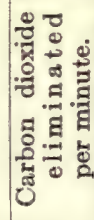 } & \multirow{2}{*}{ 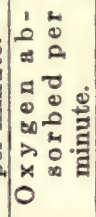 } & \multirow{2}{*}{ 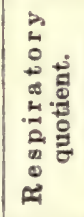 } & \multirow{2}{*}{ 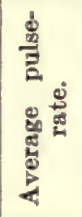 } & \multirow{2}{*}{ 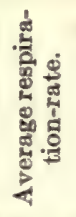 } & \multirow{2}{*}{ 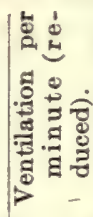 } & \multirow{2}{*}{ 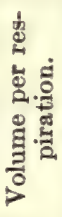 } & \multicolumn{2}{|c|}{$\begin{array}{l}\text { Composition of } \\
\text { expired air. }\end{array}$} \\
\hline & & & & & & & & $\begin{array}{l}\text { Carbon } \\
\text { dioxide. }\end{array}$ & Oxygen \\
\hline \multirow{2}{*}{\multicolumn{10}{|c|}{$\begin{array}{l}M . J . S .- \text { Continued. } \\
\text { July 24, 1912: } \\
\text { Douglas: }\end{array}$}} \\
\hline & $\begin{array}{l}\text { c.c. } \\
191\end{array}$ & $\begin{array}{l}\text { c.c. } \\
223\end{array}$ & 0.855 & 61.0 & 25.5 & $\begin{array}{l}\text { liters. } \\
6.05\end{array}$ & $\begin{array}{l}\text { c.c. } \\
289\end{array}$ & $\begin{array}{l}\text { p. ct. } \\
3.19\end{array}$ & $\begin{array}{l}p . c t . \\
17.36\end{array}$ \\
\hline 1007 a. m..... & 193 & 229 & .840 & 61.0 & 22.6 & 5.77 & 311 & 3.37 & 17.10 \\
\hline 1110 a. m. & 156 & 190 & .820 & 59.0 & 25.2 & 5.06 & 245 & 3.12 & 17.32 \\
\hline Average......... & 180 & 214 & .840 & 60.5 & 24.4 & 5.63 & 282 & 3.28 & 17.26 \\
\hline \multicolumn{10}{|l|}{ Spirometer unit: } \\
\hline 1040 a. m. & 197 & 227 & .870 & 61.5 & 17.3 & 5.32 & 375 & & \\
\hline 1145 a. $\mathrm{m}$. & 201 & 233 & .865 & 62.0 & 18.3 & 5.53 & 369 & & \\
\hline Average......... & 197 & 230 & .855 & 61.5 & 17.8 & 5.94 & 365 & & \\
\hline \multicolumn{10}{|l|}{$\begin{array}{l}\text { July 25, 1912: } \\
\text { Douglas: }\end{array}$} \\
\hline $\mathbf{9}^{\mathrm{h}} 08^{\mathrm{m}}$ a. m & 190 & 236 & .805 & 64.0 & 20.1 & 5.97 & 363 & 3.20 & 17.15 \\
\hline 1012 a. $\mathrm{m}$. & 214 & 280 & .765 & 64.0 & 19.4 & 6.29 & 397 & 3.43 & 16.70 \\
\hline 1128 a. $\mathrm{m}$. & 188 & 240 & .785 & 61.5 & 24.2 & 6.00 & 304 & 3.18 & 17.12 \\
\hline Average......... & 197 & 252 & .780 & 63.0 & 21.2 & 6.09 & 355 & 3.27 & 16.99 \\
\hline \multirow{2}{*}{\multicolumn{10}{|c|}{$\begin{array}{l}\text { Spirometer unit: } \\
9^{\mathrm{h}} \mathbf{4 0 ^ { \mathrm { m } }} \text { a. } \mathrm{m} .\end{array}$}} \\
\hline 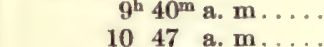 & & & & & & 5.23 & 408 & & \\
\hline $\begin{array}{l}1047 \text { a. m. } \\
11 \text { 58 a.m. }\end{array}$ & 185 & 231 & .800 & 63.5 & 17.3 & 5.12 & 363 & & \\
\hline 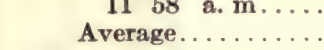 & $\begin{array}{l}191 \\
188\end{array}$ & 245 & .780 & 63.5 & 19.9 & 5.43 & 334 & & \\
\hline \multirow{2}{*}{\multicolumn{10}{|c|}{$\begin{array}{l}\text { July 26, 1912: } \\
\text { Douglas: }\end{array}$}} \\
\hline & & & & & & & & & \\
\hline 957 a. m. & $\begin{array}{l}180 \\
180\end{array}$ & 213 & .845 & $\begin{array}{l}64.0 \\
61.0\end{array}$ & $\begin{array}{l}20.0 \\
16.2\end{array}$ & 5.16 & $\begin{array}{l}353 \\
390\end{array}$ & $\begin{array}{l}3.30 \\
3.54\end{array}$ & \\
\hline 1048 a. $\mathrm{m}$. & 187 & 226 & .825 & 58.0 & 16.5 & 5.11 & 379 & 3.69 & 16.68 \\
\hline Average......... & 185 & 220 & .840 & 61.0 & 17.6 & 5.34 & 374 & 3.61 & 16.81 \\
\hline \multicolumn{10}{|l|}{ Spirometer unit: } \\
\hline & 199 & 239 & .835 & 60.5 & 17.1 & 5.03 & 360 & & \\
\hline 1024 a. m. & 190 & 235 & .810 & 59.5 & 18.3 & 5.04 & 337 & & \\
\hline 1118 a. $\mathrm{m}$. & 201 & 233 & .865 & 60.5 & 19.2 & 5.47 & 349 & & \\
\hline Average......... & 197 & 236 & .835 & 60.0 & 18.2 & 5.18 & 349 & & \\
\hline \\
\hline \multicolumn{9}{|l|}{$\begin{array}{l}\text { Nov. 15, 1912: } \\
\text { Spirometer unit: }\end{array}$} & \\
\hline 959 a. m. & 222 & 270 & .820 & 69.0 & $\begin{array}{r}9.0 \\
10.1\end{array}$ & $\begin{array}{l}4.02 \\
4.88\end{array}$ & 587 & & \\
\hline 1057 a. $\mathrm{m}$. & 204 & 265 & .770 & 67.0 & 10.1 & 4.78 & 576 & & \\
\hline Average........ & 212 & 263 & .805 & 69.6 & 9.8 & 4.73 & 585 & & \\
\hline \multicolumn{10}{|l|}{ Douglas: } \\
\hline $9^{\mathrm{h}} 33^{\mathrm{m}}$ a. m. & 174 & 222 & .780 & 78.5 & 13.3 & 5.02 & 459 & 3.49 & 16.71 \\
\hline 1031 a. $\mathrm{m}$. & 186 & 237 & .790 & 70.0 & 11.5 & 4.64 & 491 & 4.05 & 16.07 \\
\hline 1129 a. $\mathrm{m}$. . & 172 & 220 & .780 & 71.0 & 10.0 & 4.44 & 541 & 3.91 & 16.21 \\
\hline Average........... & 177 & 226 & .785 & 73.0 & 11.6 & 4.70 & 497 & 3.82 & 16.38 \\
\hline \multicolumn{10}{|l|}{$\begin{array}{l}\quad T . M . C . \\
\text { Nov. } 16,1912:\end{array}$} \\
\hline $\begin{array}{l}\text { Spirometer unit: } \\
8^{\mathrm{h}} 21^{\mathrm{m}} \text { a. m. }\end{array}$ & 160 & 189 & .845 & 73.0 & 12.6 & 4.64 & 443 & & \\
\hline 912 a. $\mathrm{m}$. & 157 & 192 & .820 & 70.5 & 14.9 & 4.99 & 403 & & \\
\hline 1007 a. m.... & 162 & 204 & .795 & 71.5 & 13.8 & 4.87 & 424 & & \\
\hline Average........... & 160 & 195 & .820 & 71.6 & 18.8 & 4.85 & 428 & & \\
\hline
\end{tabular}


TABLE 25.-Respiratory exchange in comparison experiments with the Douglas method and the Benedict respiration apparatus (spirometer unit). (Without food).-Continued.

\begin{tabular}{|c|c|c|c|c|c|c|c|c|c|}
\hline \multirow{2}{*}{$\begin{array}{l}\text { Subject, date, method, } \\
\text { and time. }\end{array}$} & \multirow{2}{*}{ 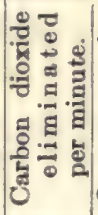 } & \multirow{2}{*}{ 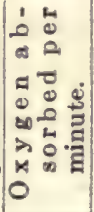 } & \multirow{2}{*}{ 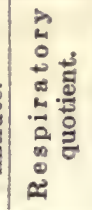 } & \multirow{2}{*}{ 递 } & \multirow{2}{*}{ 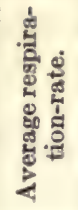 } & \multirow{2}{*}{ 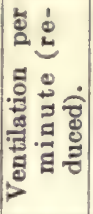 } & \multirow{2}{*}{ 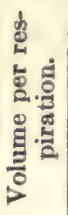 } & \multicolumn{2}{|c|}{$\begin{array}{l}\text { Composition of } \\
\text { expired air. }\end{array}$} \\
\hline & & & & & & & & $\begin{array}{l}\text { Carbon } \\
\text { dioxide. }\end{array}$ & Oxygen. \\
\hline 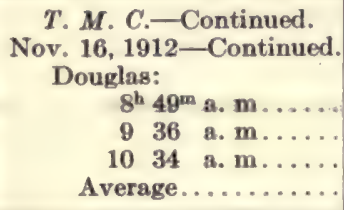 & $\begin{array}{l}\text { c.c. } \\
140 \\
156 \\
146 \\
147\end{array}$ & $\begin{array}{l}c . c . \\
174 \\
199 \\
188 \\
187\end{array}$ & $\begin{array}{r}0.805 \\
.785 \\
.775 \\
.785\end{array}$ & $\begin{array}{l}76.0 \\
73.5 \\
75.0 \\
75.0\end{array}$ & $\begin{array}{l}12.3 \\
14.5 \\
14.9 \\
13.9\end{array}$ & $\begin{array}{l}\text { liters. } \\
\mathbf{5} .13 \\
\mathbf{5 . 6 6} \\
\mathbf{5 . 4 2} \\
\mathbf{5 . 4 0}\end{array}$ & $\begin{array}{l}\text { c.c. } \\
502 \\
469 \\
437 \\
469\end{array}$ & $\begin{array}{l}\text { p. ct. } \\
2.76 \\
2.78 \\
2.73 \\
2.76\end{array}$ & $\begin{array}{l}\text { p. ct. } \\
17.69 \\
17.59 \\
17.63 \\
17.64\end{array}$ \\
\hline $\begin{array}{l}\text { Arithmetical average of all } \\
\text { experiments with spi- } \\
\text { rometer unit. . . . . . . . . } \\
\text { Arithmetical average of all } \\
\text { experiments with Doug- } \\
\text { las method.............. }\end{array}$ & 189 & 231 & .820 & 61.5 & 14.3 & 5.04 & 445 & & \\
\hline
\end{tabular}

The experiments with the smaller bag were carried out previous to July 4, 1912. The first experiment in the series, that with E. W. H. on June 21, can not be considered satisfactory, as the variations are so large in the individual periods. The other comparisons in which this bag was used show a fair uniformity in the results. In all of the experiments with the smaller bag the carbon-dioxide elimination is

TABLE 26.-Variations of average results obtained with the Douglas respiration apparatus from those obtained with the Benedict respiration apparatus (spirometer unit).

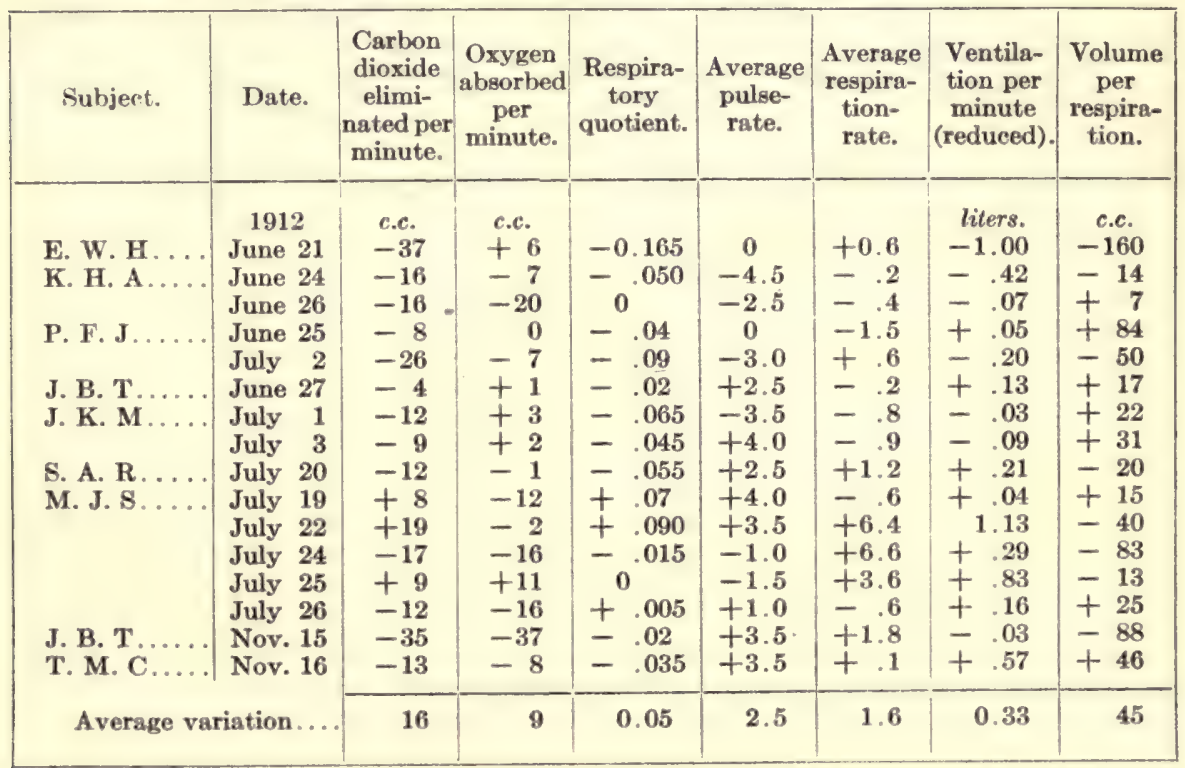


lower with the Douglas method than with the spirometer unit and some of the experiments also show lower values for the oxygen consumption. In the majority of the experiments the pulse-rate, the respiration-rate, and the ventilation per minute are likewise lower with the Douglas method.

In the experiments with the larger bag, $i$. $e$, those following July 4, 1912 , the fluctuations between the averages are both plus and minus. In general they are all comparatively consistent in their differencesthat is to say, when there is a smaller carbon-dioxide output with the

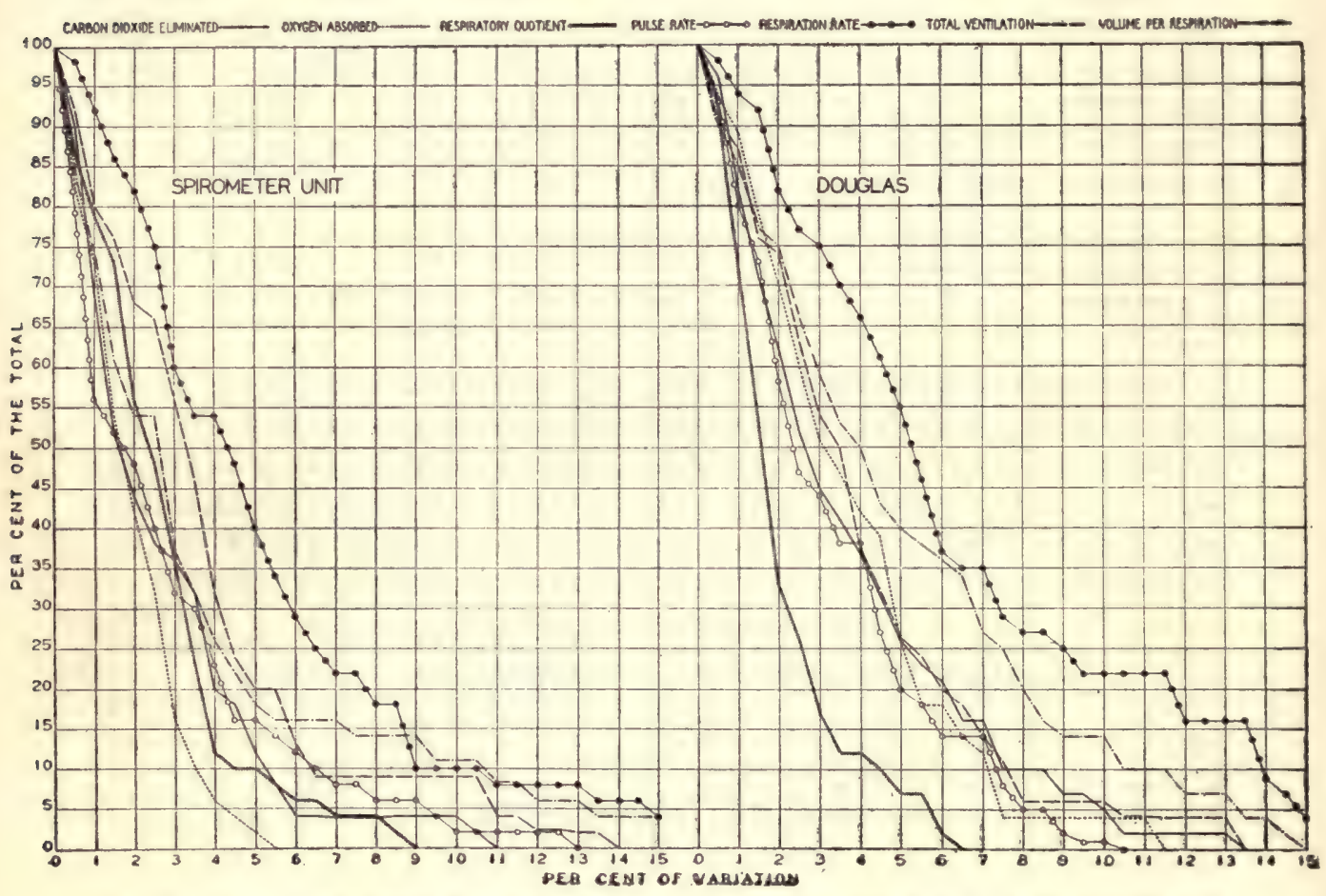

Fig. 46.-Probability curves for the series of comparison experiments with the spirometer unit and the Douglas method.

The ordinates indicate the percentage of the total number of periods and the abscissa the percentage of variation from the average.

Douglas apparatus, there is also a smaller oxygen intake, this being true in five cases out of seven. In general, the results are more satisfactory with the larger bag than with the smaller.

The probability curves are given in figure 46 , which show that as a whole the results with the spirometer unit are much more uniform so far as the carbon-dioxide and oxygen are concerned than are those with the Douglas method; on the contrary, the experiments with the Douglas method show much more uniform respiratory quotients. The other factors have about the same degree of uniformity. 
This comparison does not give such satisfactory results as have been obtained in the preceding comparisons. A general discussion of the use of the Douglas apparatus will be found in a subsequent section of this report.

\section{MOUTH- AND NOSE-BREATHING WITH THE BENEDICT RESPIRATION APPARATUS (TENSION-EQUALIZER UNIT).}

During the development of the tension-equalizer type of the Benedict respiration apparatus, the subject breathed through the rubber mouthpiece. After the pneumatic nosepieces were devised, either the mouthpiece or the nosepieces were used according to the preference of the subject, the majority of the experiments being carried out with the nosepieces. It was accordingly important to know whether the respiratory exchange when the subject breathed through the mouth differed from that when he breathed through the nose, $i$. e., when the mouthpiece was used rather than the nosepieces. Several experiments were therefore carried out at different times to study this particular point. They were distinctly comparison experiments in that the conditions were the same in all of the periods except for the change in the method of breathing.

The rubber mouthpiece and noseclip were those which are commonly employed with the Zuntz-Geppert apparatus; the nosepieces were the pneumatic nosepieces regularly used with the Benedict universal respiration apparatus. In nearly every experiment a series of periods was first carried out with one type of breathing, this series being followed by a second series of periods with the other type of breathing. The pulse-rate was determined with the Bowles stethoscope. The respiration-rate was secured from a pneumograph fastened around the chest of the subject, but in some of the experiments the graphic record was obtained by means of a side-tube connected with the three-way valve (see $m$ in fig. 5). If a manometer were connected to this tube, it would show oscillations in pressure corresponding to the inspirations and expirations of the subject. Instead of using a manometer for this purpose, a tambour and kymograph were connected, the movements of the pointer on the tambour giving a graphic record of the respiration. In the experiments in 1911, a graphic record of the muscular activity was obtained by means of a pneumograph placed about the hips of the subject. All of the subjects were members of the laboratory staff and were therefore more or less accustomed to respiration experiments of this kind. The statistics of the nine experiments are given in the following pages.

\section{STATISTICS OF EXPERIMENTS.}

J. J. C., November 5, 1910.-Mouthpiece, 3 periods; nosepieces, 3 periods; preliminary period, about 1 hour 55 minutes; mouthpiece and nosepieces alternated. Mouthpiece held in place by rubber bandage secured with an elastic strap passed around the head and fastened at the back with a buckle. 
This precaution was necessary, as this particular subject had a tendency to fall asleep during an experiment; the mouth would then relax, with consequent danger of leakage of air. In first period with mouthpiece, subject asleep at beginning and drowsy throughout period; similar conditions in second period with nosepieces; in third period with nosepieces, more awake and moved arms; as a rule somewhat more awake in periods with mouthpiece, owing to discomfort caused by mouthpiece and noseclip. Subject preferred nosepieces to mouthpiece. Respiration-rate fairly regular in all but second period with mouthpiece.

F. G. B., November 11, 1910.-Nosepieces, 4 periods; mouthpiece, 4 periods; preliminary period, about 1 hour 33 minutes; periods with nosepieces and mouthpiece in series. Respiration-rate secured by means of side outlet in three-way valve. Subject urinated after first period. At end of second period, subject stated that his neck was in a strained position but that rest of body was relaxed. Also said that air seemed dry; water was therefore added to moistener. With mouthpiece was troubled with saliva and found noseclip uncomfortable after first 5 minutes. Noticed a vibration of air with the mouthpiece at first but soon became accustomed to it. Pulse- and respirationrates uniform.

T. M. C., November 14, 1910.-Nosepieces, 7 periods; mouthpiece, 3 periods; preliminary period, 15 minutes; periods with nosepieces and mouthpiece in series. Respiration-rate secured by means of side outlet in three-way valve. Elastic bandage, about $5 \mathrm{~cm}$. wide, used over mouth in first, second, fourth, and fifth periods with nosepieces in the hope of finding some method of insuring a perfect closure of the mouth. Subject stated that bandage was somewhat uncomfortable, particularly in first part of period, and that probably most men, after once using the bandage, would have learned to keep the mouth closed without the necessity of resorting to such a method as this. The kymograph record of the respiration showed a tendency, during the mouthpiece periods, for slightly wider excursions; in the middle of the last period with the mouthpiece there were a number of very wide excursions, indicating a pressure on the tension equalizer. Pulse-rate was regular throughout the experiment.

H. F. T., June 27, 1911.-Nosepieces, 4 periods; mouthpiece, 3 periods; periods with nosepieces and mouthpiece in series. Subject stated that he experienced no discomfort in breathing by either method, but that there was a tendency for the saliva to increase with the mouthpiece. Pulse- and respiration-rates regular.

H. F. T., September 8, 1911.-Nosepieces, 3 periods; mouthpiece, 4 periods; preliminary period, 41 minutes; periods with nosepieces and mouthpiece in series. Pulse-rate regular.

H. F. T., September 9, 1911.-Mouthpiece, 4 periods; nosepieces, 3 periods; preliminary period, 35 minutes; periods with mouthpiece and nosepieces in series. During the last two periods with the nosepieces, the subject had a great desire to urinate but on the whole was quiet throughout the series. Pulseand respiration-rates uniform.

K. H. A., September 23, 1911.--Nosepieces, 4 periods; mouthpiece, 4 periods; periods with nosepieces and mouthpiece in series. Pulse-rate very even. In all of the periods there was a very distinct tendency, shown at the beginning, for the subject to breathe slowly and regularly. This was in all probability due to his anticipation of the turning of the three-way valve connecting him with the circulating air of the apparatus; respiration-rate otherwise regular.

K. H. A., September 28, 1911.-Nosepieces, 3 periods; mouthpiece, 3 periods; periods with nosepieces and mouthpiece in series. Pulse-rate fairly uniform in individual periods. Respiration-rate comparatively uniform throughout experiment. 
K. H. A., September 30, 1911.-Mouthpiece, 3 periods; nosepieces, 3 periods; periods with mouthpiece and nosepieces in series. In second period with nosepieces, subject asleep for a very short time. Pulse-rate somewhat variable. Respiration-rate regular throughout experiment.

\section{DISCUSSION OF RESULTS.}

The results of the experiments comparing nose- and mouth-breathing with the tension-equalizer unit are given in table 27. The average of the results shows that the respiratory exchange was practically the same with the two methods of breathing, being within such close limits that it is difficult to see any actual difference. The difference between the average results obtained for the carbon-dioxide elimination is 4 c.c. and for the oxygen consumption, 1 c.c. The pulse- and respiration-rates were essentially the same with both methods of breathing.

TABLE 27.-Respiratory exchange in comparison experiments with mouth-breathing and nosebreathing-Benedict respiration apparatus (tension-equalizer unit). (Without food.)

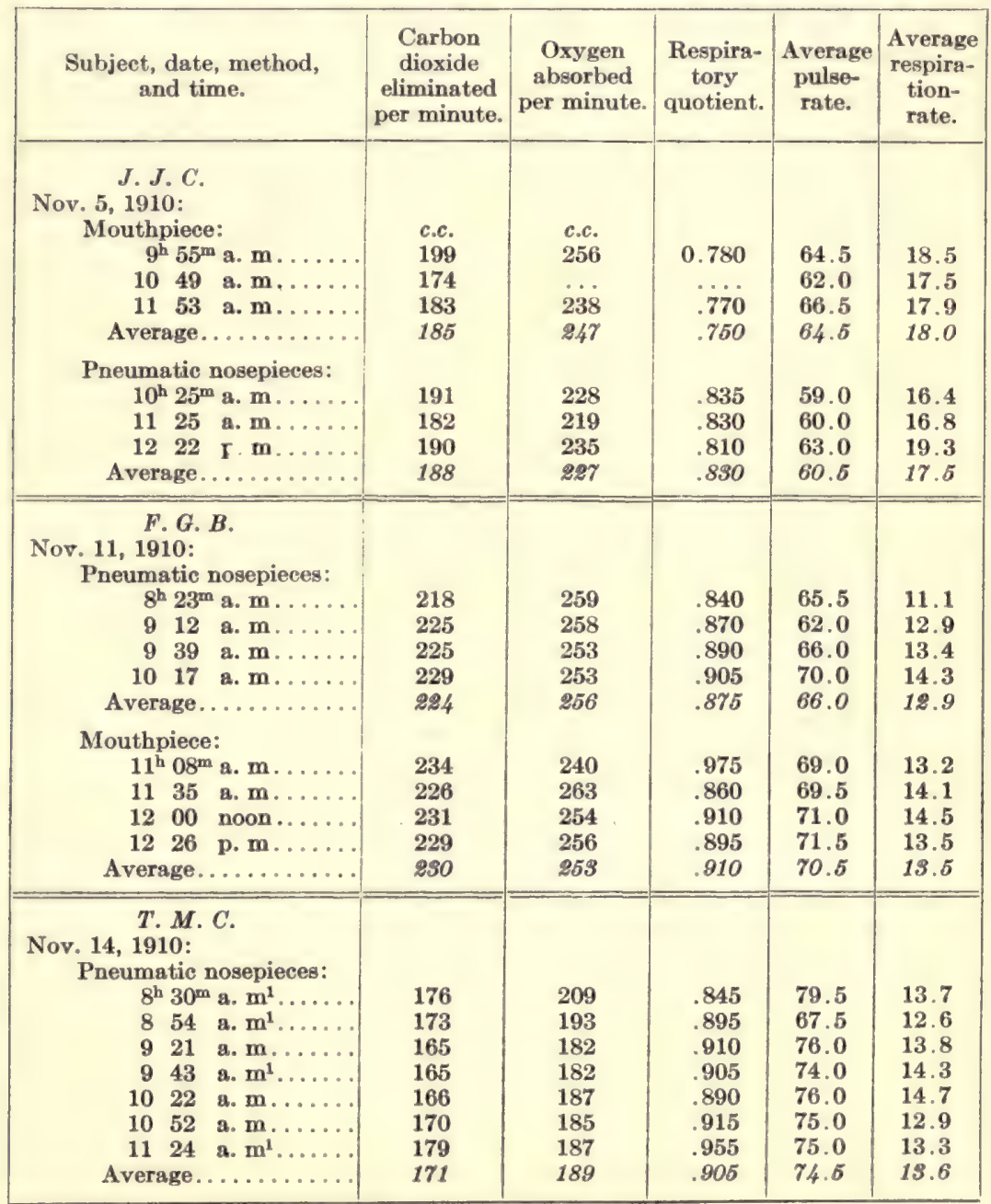


TABLE 27.-Respiratory exchange in comparison experiments with mouth-breathing and nose-breathing-Benedict respiration apparatus (tension-equalizer unit). (Without food.)-Continued.

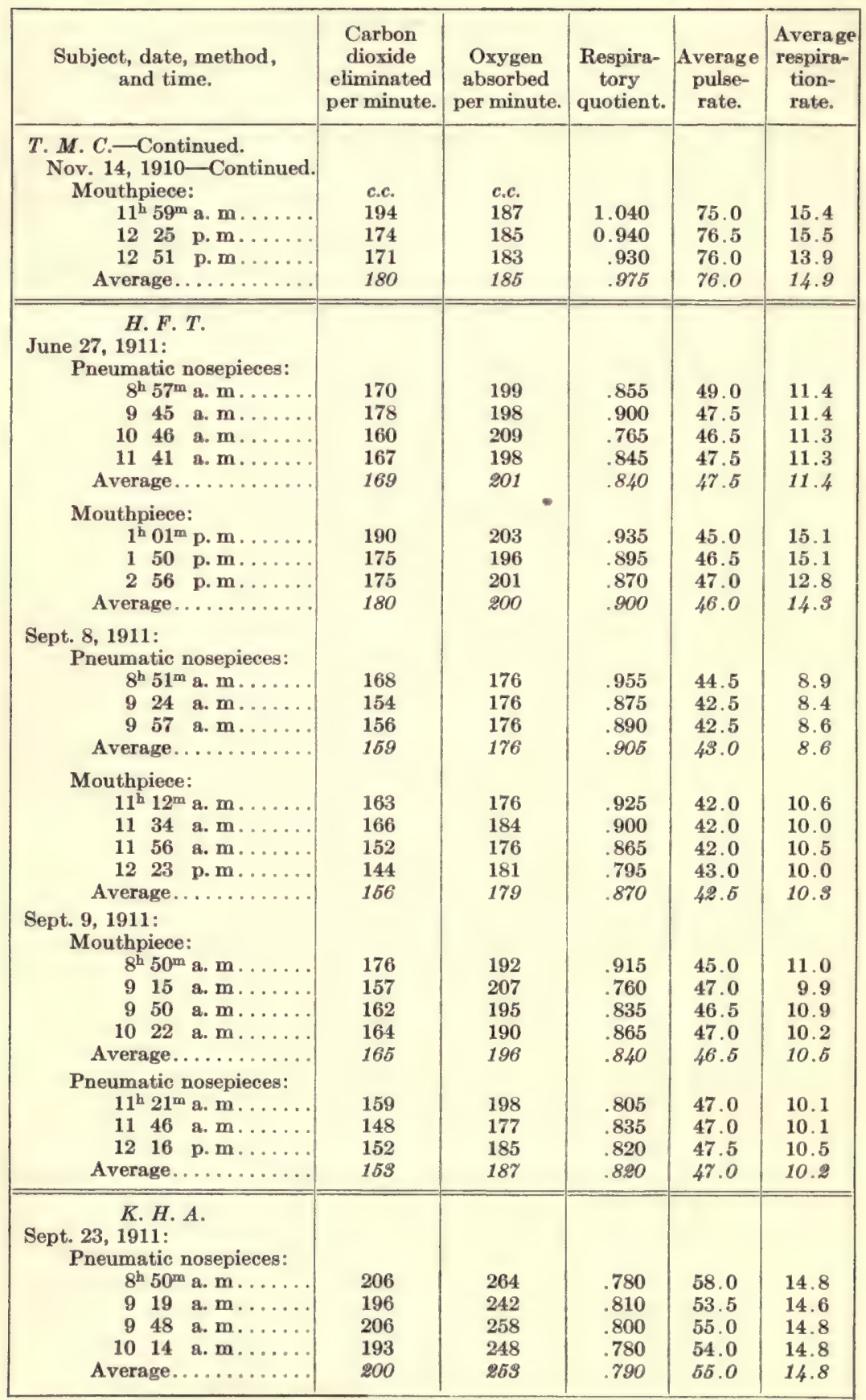


TABLE 27.-Respiratory exchange in comparison experiments with mouth-breathing and nose-breathing-Benedict respiration apparatus (tension-equalizer unit). (Without food.)-Continued.

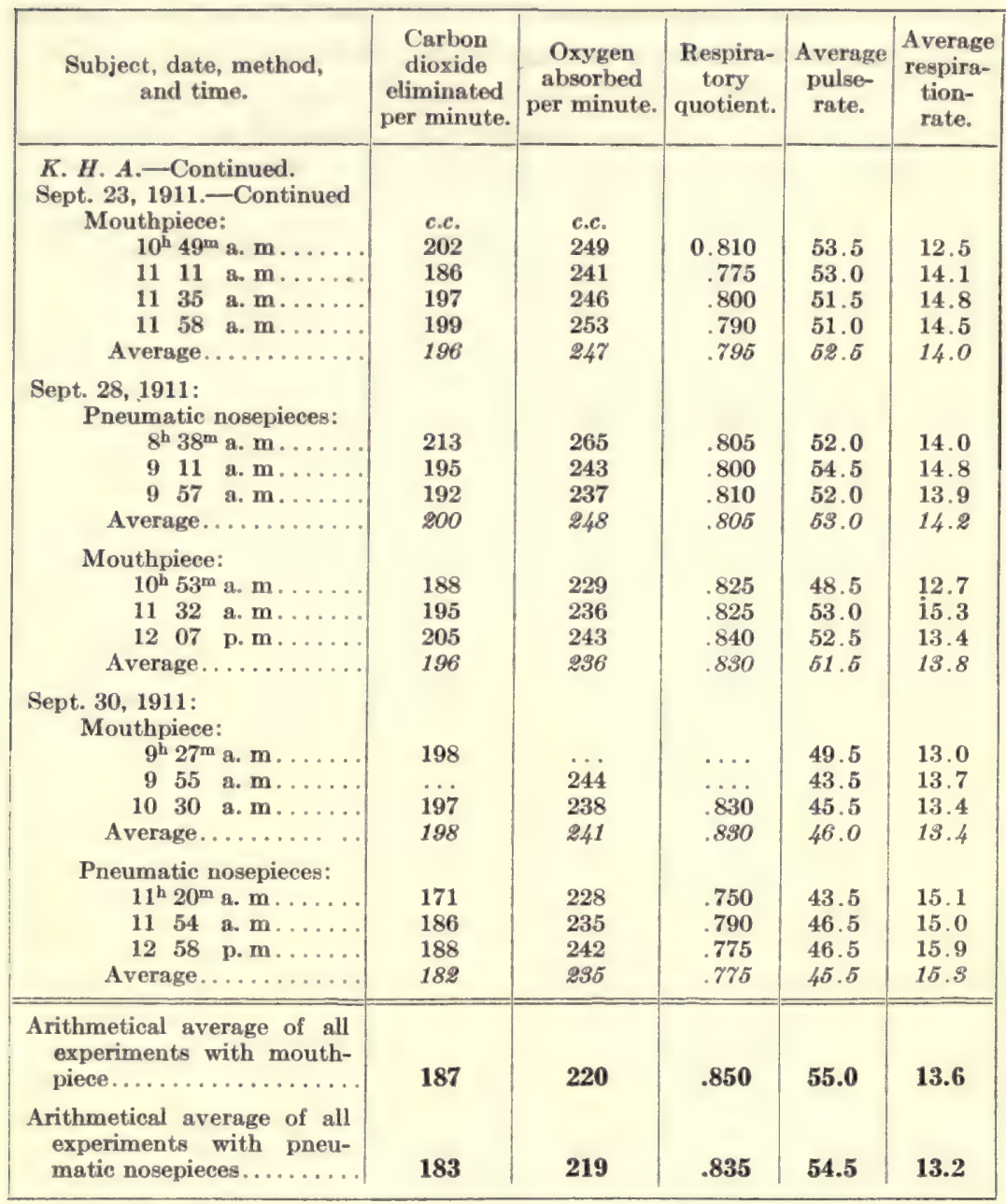

The differences between the average values obtained with the two methods are given in table 28 , the values with the nose-breathing being used as the base-line. The agreement between the results obtained with the two methods in the individual experiments is, as a whole, very fair. The greatest variations for the carbon-dioxide elimination are those for H. F. T. on June 27 and September 9, when the amounts obtained with the mouth-breathing exceeded those with the nosebreathing by 11 c.c. and 12 c.c. respectively, and for K. H. A. on September 30 , when the carbon-dioxide elimination with the mouthpiece was 16 c.c. higher. On the contrary, in two experiments with $\mathrm{K}$. H. A., the results obtained with the mouth-breathing were slightly 
lower. The first four subjects showed a tendency to a slightly higher respiration-rate with the mouthpiece than with the nosepieces while with K. H. A., the reverse was true. The differences in the respirationrate were not marked, however, in any of the experiments. With the first three subjects the pulse-rate was slightly higher in the mouthpiece periods than in those with the nosepieces. With H. F. T., the pulserate was slightly lower with the mouthpiece, while with K. H. A. it varied.

It must be noted that all of these subjects were fairly well-trained. The first, J. J. C., had been used in a great many experiments; as previously stated, on account of his tendency to fall asleep during an experiment, it was difficult to obtain the same degree of wakefulness

TABLE 28.-Variations of average results obtained with mouth-breathing from those obtained with nose-breathing (tension-equalizer unit)

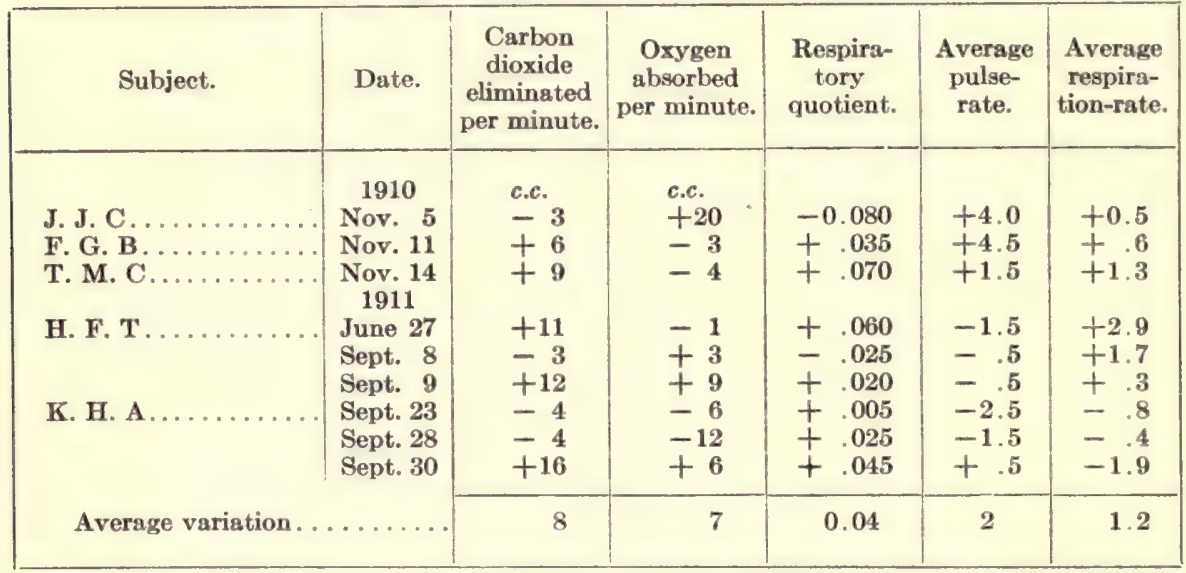

throughout a series of periods. F. G. B. and T. M. C. were both well trained in respiration experiments and accustomed to apparatus for nose- and mouth-breathing. As has already been noted, H. F. T. was a peculiar subject because of his occasional apnœic respiration. It is probable that with the mouthpiece he had a tendency to breathe more regularly than with the nosepieces. With this subject the carbondioxide elimination was usually higher with the mouthpiece than with the nosepieces. K. H. A. was also familiar with the apparatus; he had no peculiarities of respiration and was able to maintain nearly the same degree of quietness and wakefulness throughout the experiments.

In these comparisons the preliminary period of breathing through the particular appliance being tested was not very long, continuing usually less than 5 minutes. Consequently, if there were a tendency shown with the mouthpiece toward deeper breathing or toward an exaggerated respiration, it would have been apparent, as the period began so soon after the mouthpiece was inserted that there was no 
opportunity for compensation. The general indications are, however, that the respiration-rate and the respiratory quotient were practically the same with both methods of breathing.

The probability curves plotted from the variations of the individual periods from the average are given in figure 47. The pulse-rate and the oxygen consumption are slightly more uniform with mouth-breathing than with nose-breathing, but the respiratory quotient is more uniform when the nosepieces are used. In general, there appeared to be no difference in the respiratory exchange with the two methods. Consequently either mouthpiece or nosepieces may be used with the tension-equalizer unit without affecting the results.

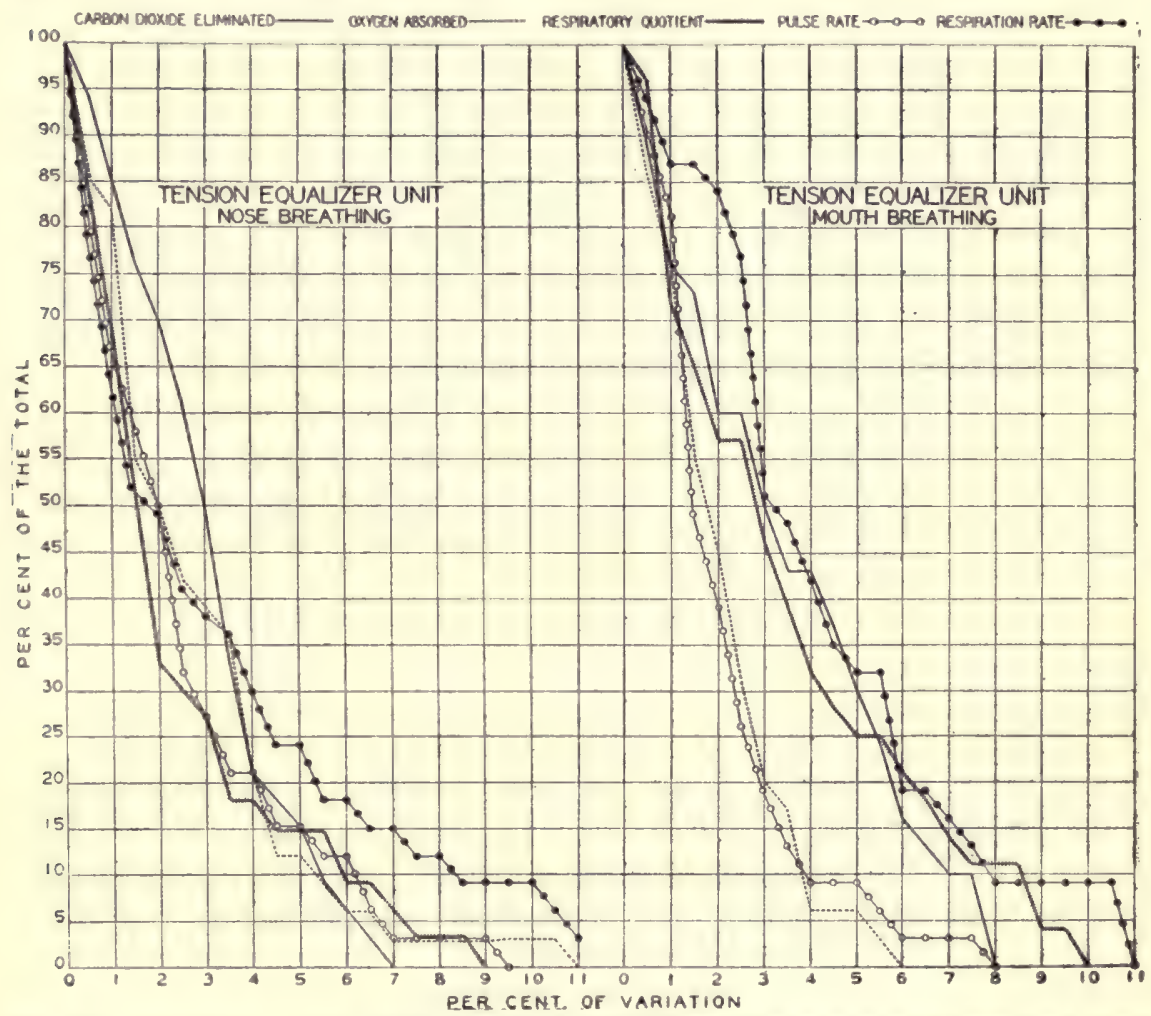

Fig. 47.-Probability curves for the series of comparison experiments with nose- and mouthbreathing (tension-equalizer unit).

The ordinates indicate the percentage of the total number of periods and the abscissm the percentage of variation from the average.

MOUTH- AND NOSE-BREATHING WITH THE BENEDICT RESPIRATION APPARATUS (SPIROMETER UNIT).

In the results previously given of a series of comparison experiments ${ }^{1}$ it was shown that the respiratory exchange with the two forms of the Benedict respiration apparatus - the tension-equalizer unit and the spirometer unit-was essentially the same, but in those experiments 
mouth- and nose-breathing were not compared. Accordingly, in addition to the series of comparisons made with the tension-equalizer unit on the effect of changing the method of breathing, a second series was made with the spirometer unit. Since this type of apparatus provides for a qualitative and quantitative graphic record of the ventilation, the differences in the character of the respiration can be studied.

The pneumatic nosepieces were used in all of the experiments but one. The respiration-rate was recorded from the bell of the spirometer and the pulse-rate was, as usual, obtained with the Bowles stethoscope. A record of the muscular activity was secured by means of a pneumograph placed about the hips or from the self-recording bed. With the exception of M.J. S., the subjects were all accustomed to the apparatus. The details of the five experiments in the series are here given.

\section{STATISTICS OF EXPERIMENTS.}

P. F. J., February 14, 1912. - Subject had breakfast about $7^{\text {h }} 30^{\mathrm{m}}$ a. m.; experiment began at $10^{\mathrm{h}} 40^{\mathrm{m}}$ a. m. Pneumatic nosepieces, 2 periods; mouthpiece, 2 periods; preliminary period, 15 minutes; periods with nosepieces and mouthpiece alternating. Subject stated that he was somewhat sleepy in the second period and that he liked the nosepieces better than the mouthpiece. No observations of the pulse-rate. Average barometric pressure, $769.5 \mathrm{~mm}$.; average temperature of air in apparatus, $22.1^{\circ} \mathrm{C}$.

P. F. J., July 10, 1912.-Pneumatic nosepieces, 3 periods; mouthpiece, 3 periods; periods with nosepieces and mouthpiece alternating. Subject uniformly quiet. Pulse-rate uniform except in fifth period, when it was low; respiration was rapid and shallow in this period, but otherwise fairly uniform. Average barometric pressure, $759.4 \mathrm{~mm}$.; average temperature of air in apparatus, $22.5^{\circ} \mathrm{C}$.

J. K. M., July 9, 1912.-Pneumatic nosepieces, 3 periods; mouthpiece, 3 periods; preliminary period, 57 minutes; periods with nosepieces and mouthpiece alternating. Subject preferred nosepieces, as in breathing through mouthpiece his mouth became dry. Pulse-rate uniform in first three periods; in fourth, a tendency to fall; in fifth and sixth, a tendency to rise. Respiration-rate fairly uniform in all except the second period for each type of breathing, when there was some apnœa. Average barometric pressure, $760.8 \mathrm{~mm}$. average temperature, $24.2^{\circ} \mathrm{C}$.

T. M. C., July 11, 1912.-Pneumatic nosepieces, 3 periods; mouthpiece, 3 periods; periods with nosepieces and mouthpiece alternating. Subject quiet throughout experiment; said it seemed easier to breathe with mouthpiece. During periods with mouthpiece he swallowed frequently. Slightly drowsy at the end of third period with nosepieces. Pulse-rate very uniform; respiration-rate uniform. Average barometric pressure, $755.7 \mathrm{~mm}$.; average temperature of air in apparatus, $22.3^{\circ} \mathrm{C}$.

M. J. S., July 27, 1912.-Mouthpiece, 3 periods; glass nosepieces, 2 periods; periods with mouthpiece and nosepieces alternating. Subject made a few slight movements during the second and third periods with mouthpiece. Pulse- and respiration-rates uniform. Average barometric pressure, 754.9 $\mathrm{mm}$.; average temperature of air in apparatus, $21.1^{\circ} \mathrm{C}$. 


\section{DISCUSSION OF RESULTS.}

The results of this series of comparisons are given in table 29, in which the general averages show that the respiratory exchange with the two methods of breathing was nearly the same. The difference for the average carbon-dioxide elimination with the mouthpiece and the nosepieces was 5 c.c. and for the oxygen consumption 5 c.c. The respiratory quotient was practically identical for the two types of breathing. The pulse-rate, respiration-rate, total ventilation of the lungs, and volume of respiration may likewise be said to be the same for both methods, the slight difference being within the limits of error of measurement.

TABLE 29.-Respiratory exchange in comparison experiments with mouth-breathing and nosebreathing-Benedict respiration apparatus (spirometer unit). (Without food.)

\begin{tabular}{|c|c|c|c|c|c|c|c|}
\hline $\begin{array}{l}\text { Subject, date, method, } \\
\text { and time. }\end{array}$ & $\begin{array}{l}\text { Carbon } \\
\text { dioxide } \\
\text { elimin- } \\
\text { ated per } \\
\text { minute. }\end{array}$ & $\begin{array}{c}\text { Oxygen } \\
\text { absorbed } \\
\text { per } \\
\text { minute. }\end{array}$ & $\begin{array}{l}\text { Respira- } \\
\text { tory } \\
\text { quotient. }\end{array}$ & $\begin{array}{l}\text { Average } \\
\text { pulse- } \\
\text { rate. }\end{array}$ & $\begin{array}{l}\text { Average } \\
\text { respira- } \\
\text { tion-rate. }\end{array}$ & $\begin{array}{l}\text { Ventila- } \\
\text { tion per } \\
\text { minute } \\
\text { (reduced). }\end{array}$ & $\begin{array}{l}\text { Volume } \\
\text { per } \\
\text { respira- } \\
\text { tion. }\end{array}$ \\
\hline \multicolumn{8}{|l|}{$\begin{array}{l}P . F . J \\
\text {. }\end{array}$} \\
\hline Pneumatic nosepieces: & c.c. & c.c. & 0000 & & 154 & liters. & c.c. \\
\hline 1130 a. $\mathrm{m} . .$. & $\begin{array}{l}231 \\
215\end{array}$ & $\begin{array}{l}254 \\
224\end{array}$ & $\begin{array}{r}.990 \\
.960\end{array}$ & & $\begin{array}{l}15.4 \\
11.3\end{array}$ & $\begin{array}{l}6.00 \\
5.15\end{array}$ & $\begin{array}{l}465 \\
544\end{array}$ \\
\hline Average.......... & 928 & 229 & .975 & & 13.4 & 5.58 & 505 \\
\hline Mouthpiece: & & & & & & & \\
\hline $11^{\mathrm{h}} 05^{\mathrm{m}}$ a. $\mathrm{m}$........ & 206 & 238 & .865 & & 13.6 & 5.15 & 452 \\
\hline 1156 a. m.... & 228 & 228 & 1.000 & & 10.4 & 5.33 & 612 \\
\hline Average.......... & 217 & 283 & 0.930 & & 12.0 & 5.24 & 538 \\
\hline \multirow{2}{*}{\multicolumn{8}{|c|}{$\begin{array}{l}\text { July 10, 1912: } \\
\text { Pneumatic nosepieces: } \\
9^{\mathrm{h}} 04^{\mathrm{m}} \mathrm{a} . \mathrm{m}\end{array}$}} \\
\hline & 176 & 209 & .845 & 66.5 & 12.4 & 4.34 & 424 \\
\hline 1015 a. m..... & 184 & 207 & .890 & 65.0 & 9.8 & 4.37 & 540 \\
\hline 1109 a. m... & 154 & 211 & .730 & 59.5 & 15.0 & 4.10 & 331 \\
\hline Average. . . . . . . . . . & 171 & 209 & .820 & 63.5 & 12.4 & 4.87 & 438 \\
\hline \multicolumn{8}{|l|}{ Mouthpiece: } \\
\hline 1041 a. m..... & 183 & 212 & .860 & 65.0 & 10.3 & 4.26 & 501 \\
\hline 1140 a. m.... & 182 & 234 & .780 & 67.0 & 7.7 & 4.28 & 674 \\
\hline Average.......... & 185 & 282 & .835 & 65.5 & 9.1 & 4.26 & 577 \\
\hline \multicolumn{8}{|l|}{$\begin{array}{l}J . K \cdot M \\
\text { July } 9,1912:\end{array}$} \\
\hline $\begin{array}{l}\text { July 9, 1912: } \\
\text { Pneumatic nosepieces: }\end{array}$ & & & & & & & \\
\hline $9^{\mathrm{h}} 07^{\mathrm{m}}$ a. m.... & 181 & 237 & .765 & 55.5 & 14.3 & 4.63 & 391 \\
\hline 1010 a. m.... & 167 & 228 & .735 & 54.0 & 14.3 & 4.38 & 370 \\
\hline 1120 a. m.... & 185 & 234 & .790 & 57.5 & 13.4 & 4.52 & 408 \\
\hline Average.......... & 178 & 233 & .765 & 55.5 & 14.0 & 4.51 & 390 \\
\hline Mouthpiece: & & & & & & & \\
\hline $9^{\mathrm{h}} 44^{\mathrm{m}}$ a. m . . . . . & 184 & 243 & .755 & 58.0 & 16.5 & 4.88 & 357 \\
\hline 1038 a. m.... & 174 & 228 & .765 & 55.0 & 17.5 & 4.79 & 331 \\
\hline 1146 a. m.... & 197 & 236 & .830 & 60.0 & 16.8 & 4.80 & 346 \\
\hline Average........... & 185 & 236 & .785 & 57.5 & 16.9 & 4.88 & 345 \\
\hline
\end{tabular}


TABLE 29.-Respiratory exchange in comparison experiments with mouth-breathing and nosebreathing-Benedict respiration apparatus (spirometer unit). (Without food.)-Continued.

\begin{tabular}{|c|c|c|c|c|c|c|c|}
\hline $\begin{array}{l}\text { Subject, date, method, } \\
\text { and time. }\end{array}$ & $\begin{array}{l}\text { Carbon } \\
\text { dioxide } \\
\text { elimin- } \\
\text { ated per } \\
\text { minute. }\end{array}$ & $\begin{array}{c}\text { Oxygen } \\
\text { absorbed } \\
\text { per } \\
\text { minute }\end{array}$ & $\begin{array}{c}\text { Respira- } \\
\text { tory } \\
\text { quotient. }\end{array}$ & $\begin{array}{c}\text { Average } \\
\text { pulse- } \\
\text { rate. }\end{array}$ & $\begin{array}{c}\text { Average } \\
\text { respira- } \\
\text { tion-rate. }\end{array}$ & $\begin{array}{l}\text { Ventila- } \\
\text { tion per } \\
\text { minute } \\
\text { (reduced). }\end{array}$ & $\begin{array}{l}\text { Volume } \\
\text { per } \\
\text { respira- } \\
\text { tion. }\end{array}$ \\
\hline 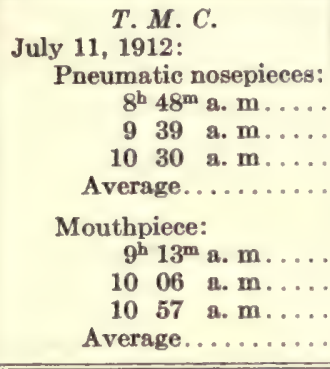 & $\begin{array}{l}c . c . \\
167 \\
160 \\
153 \\
160\end{array}$ & $\begin{array}{l}c . c . \\
186 \\
181 \\
201 \\
189\end{array}$ & $\begin{array}{r}0.895 \\
.885 \\
.760 \\
.845 \\
\\
.955 \\
.880 \\
.800 \\
.870\end{array}$ & $\begin{array}{l}72.0 \\
68.5 \\
69.5 \\
70.0\end{array}$ & $\begin{array}{l}14.0 \\
15.0 \\
15.2 \\
14.7\end{array}$ & $\begin{array}{r}\text { liters. } \\
4.69 \\
4.50 \\
4.60 \\
4.60\end{array}$ & $\begin{array}{l}c . c . \\
408 \\
365 \\
369 \\
381\end{array}$ \\
\hline 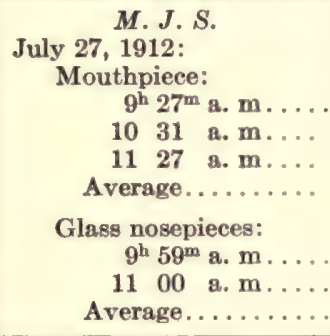 & $\begin{array}{l}200 \\
194 \\
208 \\
201\end{array}$ & $\begin{array}{l}246 \\
240 \\
251 \\
246 \\
\\
\\
239 \\
240 \\
240\end{array}$ & $\begin{array}{l}.815 \\
.810 \\
.830 \\
.815 \\
\\
.805 \\
.800 \\
.805\end{array}$ & $\begin{array}{l}67.0 \\
65.0 \\
64.5 \\
65.5 \\
\\
63.5 \\
62.0 \\
63.0\end{array}$ & $\begin{array}{l}18.4 \\
18.0 \\
18.1 \\
18.2 \\
\\
19.5 \\
18.4 \\
19.0\end{array}$ & $\begin{array}{l}5.58 \\
5.37 \\
5.76 \\
5.57 \\
\\
5.56 \\
5.33 \\
5.45\end{array}$ & $\begin{array}{l}370 \\
364 \\
388 \\
374 \\
\\
348 \\
353 \\
351\end{array}$ \\
\hline $\begin{array}{l}\text { Arithmetical average of } \\
\text { all experiments with } \\
\text { mouthpiece........... } \\
\text { Arithmetical average of } \\
\text { all experiments with } \\
\text { nosepieces........... }\end{array}$ & 190 & 225 & .845 & 64.5 & 14.5 & 4.96 & 439 \\
\hline
\end{tabular}

The variations between the averages for each day are given in table 30 , the results obtained with the nosepieces being taken as the baseline. Both the carbon-dioxide elimination and the oxygen consumption were higher with the mouthpiece in four out of five experiments, while the pulse-rate was higher in three out of the four experiments in which this factor was observed. It will be noted that the individual variations between the averages are not large, the greatest being with P. F. J. on July 10, when the difference between the averages for the oxygen consumption is 13 c.c. This greater difference is caused by the higher value obtained in the last period of the experiment, when the oxygen consumption was notably higher than in the other two periods with the mouthpiece. Two low values were obtained for the carbondioxide elimination, one with J. K. M. in the second period with the nosepieces on July 9, and one with P. F. J. in the third period with the nosepieces on July 10. In both of these instances the subject was somewhat drowsy and the ventilation of the lungs was consequently irregular. 
The probability curves are given in figure 48 . If these are examined it will be seen that the uniformity of the carbon-dioxide measurement is about the same for the two methods of breathing, with a variation within 2 per cent; when the variation is larger than this, the uniformity is greater with the subject breathing through the nosepieces. The values for the oxygen consumption also show greater uniformity with the nosepiece method even with a small variation, but with a variation

TABLE 30.-Variations of average results obtained with mouth-breathing from those obtained with nose-breathing (spirometer unit).

\begin{tabular}{|c|c|c|c|c|c|c|c|c|}
\hline Subject. & Date. & $\begin{array}{l}\text { Carbon } \\
\text { dioxide } \\
\text { elimin- } \\
\text { ated per } \\
\text { minute. }\end{array}$ & $\begin{array}{c}\text { Oxygen } \\
\text { absorbed } \\
\text { per } \\
\text { minute. }\end{array}$ & $\begin{array}{c}\text { Respira- } \\
\text { tory } \\
\text { quotient. }\end{array}$ & $\begin{array}{l}\text { Average } \\
\text { pulse- } \\
\text { rate. }\end{array}$ & $\begin{array}{l}\text { Average } \\
\text { respira- } \\
\text { tion-rate. }\end{array}$ & $\begin{array}{l}\text { Ventila- } \\
\text { tion per } \\
\text { minute } \\
\text { (reduced). }\end{array}$ & $\begin{array}{l}\text { Volume } \\
\text { per } \\
\text { respira- } \\
\text { tion. }\end{array}$ \\
\hline $\begin{array}{l}\text { P. F. J.. } \\
\text { J. K. M. } \\
\text { T. M. C.. } \\
\text { M. J. S. }\end{array}$ & \begin{tabular}{lr}
\multicolumn{2}{c}{1912} \\
Feb. & 14 \\
July & 10 \\
July & 9 \\
July & 11 \\
July & 27
\end{tabular} & $\begin{array}{l}\text { c.c. } \\
-4 \\
+14 \\
+7 \\
+4 \\
+8\end{array}$ & $\begin{array}{l}\text { c.e. } \\
+\quad 4 \\
+13 \\
+3 \\
+1 \\
+\quad 6\end{array}$ & $\begin{array}{l}-0.045 \\
+\quad .015 \\
+\quad .02 \\
+.025 \\
+.01\end{array}$ & $\begin{array}{l}+2.0 \\
+2.0 \\
-0.5 \\
+2.5\end{array}$ & $\begin{array}{l}-1.4 \\
-3.3 \\
+2.9 \\
+1.6 \\
-\quad .8\end{array}$ & $\begin{array}{l}\text { liters. } \\
-0.34 \\
-.01 \\
+.31 \\
+.30 \\
+.12\end{array}$ & $\begin{array}{r}c . c . \\
+\quad 27 \\
+147 \\
-45 \\
-15 \\
+\quad 23\end{array}$ \\
\hline \multicolumn{2}{|c|}{ Average variation... } & 7 & 5 & 0.025 & 1.5 & 2.0 & 0.22 & 51 \\
\hline
\end{tabular}

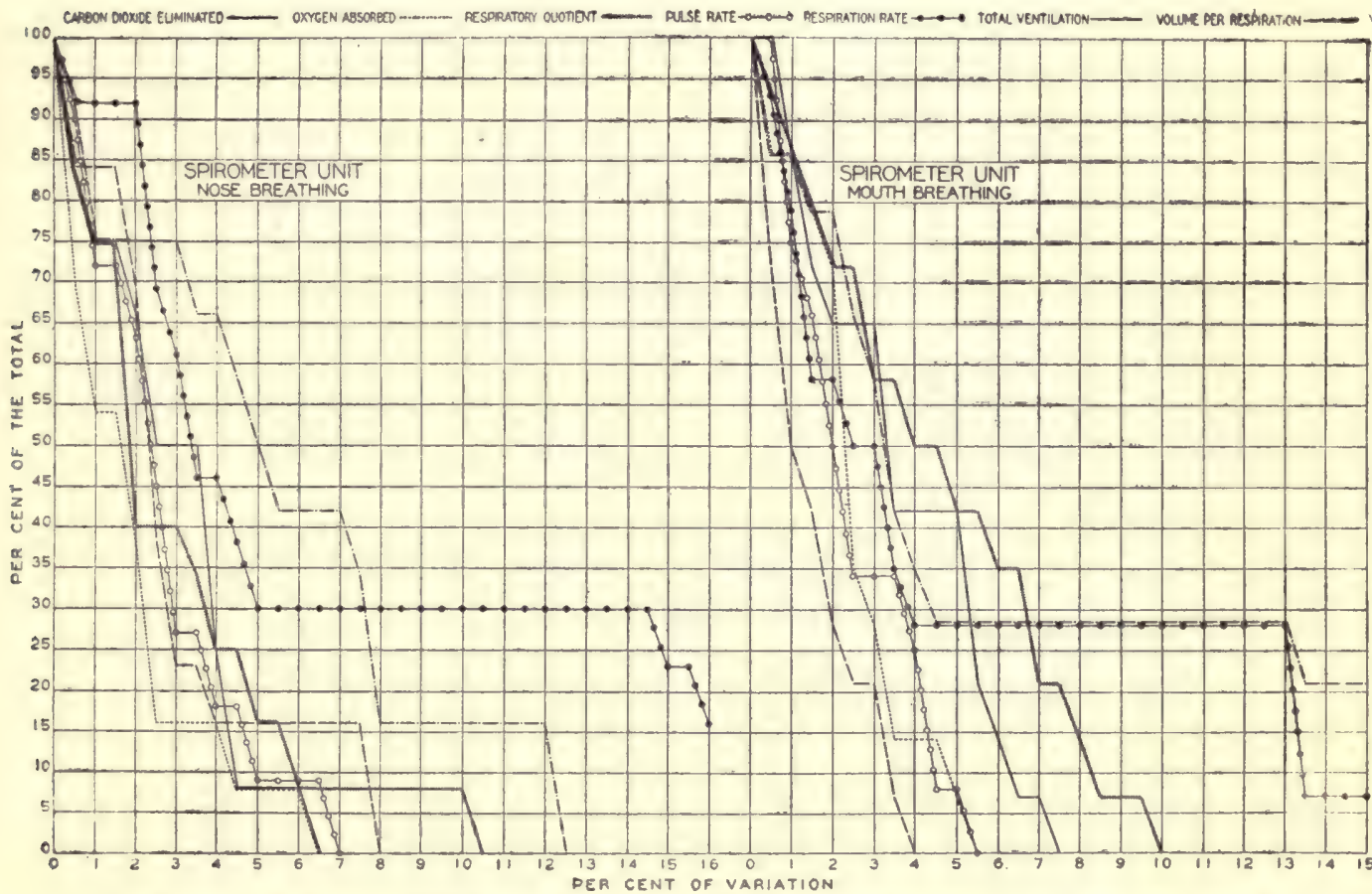

FIG. 48.-Probability curves for the series of comparison experiments with nose- and mouthbreathing (spirometer unit).

The ordinates indicate the percentage of the total number of periods and the abscissæ the percentage of variation from the average. 
of 3.5 per cent, the percentage of the total is nearly the same. The curves for the respiratory quotient show much greater uniformity with the nose-breathing, while the curves for the pulse-rate have approximately the same degree of uniformity with both types of breathing. The respiration-rate is somewhat more uniform with the mouth-breathing when the limits of variation are considered as 2.5 per cent, but beyond this there is approximately similar uniformity. The total ventilation of the lungs is much nearer uniformity with the mouthbreathing; this is shown to some extent in the volume per respiration.

The results of the comparisons would indicate that there is a slightly higher metabolism with mouth-breathing, but that this is due to the fact that the subjects are usually more awake with this type of breathing and that this produces a more regular and uniform ventilation. That the volume of air left in the lungs at the end of each expiration is, however, more uniform with nose-breathing is indicated by the greater uniformity of the oxygen consumption and the respiratory quotient with nose-breathing. The variations between the two methods shown by these comparisons with the spirometer unit are so small that either the mouthpiece or the nosepieces may be properly used in respiration experiments.

\section{MOUTH- AND NOSE-BREATHING WITH THE TISSOT APPARATUS.}

In rest experiments with the Tissot apparatus, nosepieces are ordinarily used, but in work experiments recently carried out by Amar ${ }^{1}$ a mouthpiece was employed. Both nosepieces and mouthpiece were used in the comparison study made with the Tissot apparatus and it was therefore of interest to determine the difference, if any, in the respiratory exchange in mouth- and nose-breathing with the Tissot apparatus. A series of experiments with three subjects was therefore made in which the rubber mouthpiece and the Siebe-Gorman noseclip were used in the mouth-breathing periods and the round glass nosepieces for the nose-breathing periods. Each experiment began with a nose-breathing period, the use of the nosepieces and mouthpiece alternating throughout the experiment. The samples of expired air were collected over mercury as in the earlier comparisons with this apparatus and the analyses were made with the Haldane apparatus. No preliminary ventilation was obtained, the periods usually beginning within 5 minutes of the adjustment of the mouthpiece or nosepieces. The pulse-rate was obtained by means of the Bowles stethoscope, the respiration-rate from a pneumograph fastened about the chest, and a record of the degree of muscular repose from a pneumograph placed about the hips. The subjects were all assistants in the Laboratory and were familiar with the apparatus. The statistics of the five experiments in this series follow.

\footnotetext{
${ }^{1}$ Amar, Le moteur humain. Paris, 1914. Journ. de Physiol. et de Pathol., 1913, 15, p. 62.
} 


\section{STATISTICS OF EXPERIMENTS.}

J. K. M., June 13, 1912.-Nosepieces, 3 periods; mouthpiece, 3 periods; periods with nosepieces and mouthpiece alternating. In first two periods, nosepieces tested for tightness with soapsuds. Respiration-rate fairly regular in all periods. Range of pulse-rate, 4 to 10 beats. Average barometric pressure, $752.1 \mathrm{~mm}$.; average temperature of air in apparatus, $22.5^{\circ} \mathrm{C}$.

J. K. M., June 18, 1912.-Nosepieces, 3 periods; mouthpiece, 3 periods; periods with nosepieces and mouthpiece alternating. Range of pulse-rate 5 to 8 beats. Respiration-rate regular in all periods. Average barometric pressure, $753 \mathrm{~mm}$.; average temperature of air in apparatus, $23^{\circ} \mathrm{C}$.

J. B. T., June 15, 1912.-Nosepieces, 3 periods; mouthpiece, 3 periods; periods with nosepieces and mouthpiece alternating. Subject preferred nosepieces. Pulse-rate ranged from 4 to 7 beats per minute. Respiration-rate regular in all periods. Average barometric pressure, $762.4 \mathrm{~mm}$.; average temperature of air in apparatus, $18.3^{\circ} \mathrm{C}$.

K. H. A., June 19, 1912.-Nosepieces, 3 periods; mouthpiece, 3 periods; periods with nosepieces and mouthpiece alternating. Subject preferred nosepieces. Range in pulse-rate 5 to 7 beats. Average barometric pressure, $756.1 \mathrm{~mm}$. ; average temperature of air in apparatus, $22.9^{\circ} \mathrm{C}$.

K. H. A., June 22, 1912.--Nosepieces, 3 periods; mouthpiece, 3 periods; periods with nosepieces and mouthpiece alternating. Subject said that his mouth became dry in periods with mouthpiece and that he preferred nosepieces to mouthpiece; in second period with nosepieces, was a little drowsy. Pulserate varied, with a range as high as 8 beats per minute in some periods. Respiration-rate very regular. Average barometric pressure, $763.8 \mathrm{~mm}$.; average temperature of air in apparatus, $25.6^{\circ} \mathrm{C}$.

\section{DISCUSSION OF RESULTS.}

The results of the five experiments comparing the respiratory exchange with mouth- and nose-breathing on the Tissot apparatus are given in table 31 . On the whole the averages show that the respiratory exchange with the two types of breathing does not differ markedly. The carbon-dioxide production is 6 c.c. higher and the respiratory quotient is 0.025 higher with the mouth-breathing than with the nosebreathing. The averages for the other factors are practically identical. 
TABLE 31.-Respiratory exchange in comparison experiments with mouth-breathing and nosebreathing-Tissot apparatus. (Without food.)

\begin{tabular}{|c|c|c|c|c|c|c|c|c|c|}
\hline \multirow{2}{*}{$\begin{array}{l}\text { Subject, date, method, } \\
\text { and time. }\end{array}$} & \multirow{2}{*}{ 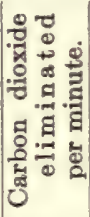 } & \multirow{2}{*}{ 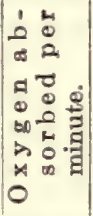 } & \multirow{2}{*}{ 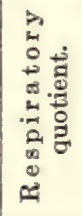 } & \multirow{2}{*}{ 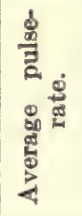 } & \multirow{2}{*}{ 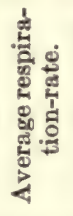 } & \multirow{2}{*}{ 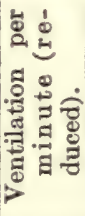 } & \multirow{2}{*}{ 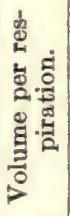 } & \multicolumn{2}{|c|}{$\begin{array}{l}\text { Composition of } \\
\text { expired air. }\end{array}$} \\
\hline & & & & & & & & $\begin{array}{l}\text { Carbon } \\
\text { dioxide. }\end{array}$ & Oxygen \\
\hline $\begin{array}{l}\text { J.K. } M . \\
\text { June } 13,1912:\end{array}$ & & & & & & & & & \\
\hline $\begin{array}{c}\text { Nosepieces: } \\
8^{\mathrm{h}} 39^{\mathrm{m}} \text { a. } 1\end{array}$ & $\begin{array}{l}\text { c.c. } \\
183 .\end{array}$ & $\begin{array}{l}\text { c.e. } \\
238\end{array}$ & 0.770 & 57.0 & 14.2 & $\begin{array}{l}\text { liters. } \\
4.52\end{array}$ & $\begin{array}{l}\text { c.c. } \\
389\end{array}$ & $\begin{array}{l}\text { p.ct. } \\
4.08\end{array}$ & $\begin{array}{l}\text { p. } c t . \\
15.93\end{array}$ \\
\hline 950 a. & 177 & 227 & .775 & 53.0 & 14.5 & 4.40 & 371 & 4.04 & 16.02 \\
\hline 1043 a. $\mathrm{m}$ & 172 & $\cdot 217$ & .790 & 53.0 & 14.4 & 4.38 & 372 & 3.95 & 16.21 \\
\hline Average........ & 177 & 227 & .780 & 54.5 & 14.4 & 4.48 & 377 & 4.02 & 16.05 \\
\hline $\begin{array}{l}\text { Mouthpiece: } \\
\qquad 9^{\mathrm{h}} 21^{\mathrm{m}} \text { a. m }\end{array}$ & 178 & 224 & .795 & 56.5 & 13.8 & 4.04 & 358 & 4.45 & 15.62 \\
\hline 1016 a. $n$ & 185 & 222 & .830 & 57.5 & 14.7 & 4.26 & 355 & 4.37 & 15.91 \\
\hline $11 \quad 10$ a. I & 185 & 226 & .820 & 54.5 & 13.7 & 4.19 & 374 & 4.46 & 15.74 \\
\hline Average...... & 183 & 224 & .815 & 56.0 & 14.1 & 4.16 & $\$ 62$ & 4.49 & 15.76 \\
\hline $\begin{array}{l}\text { June 18, 1912: } \\
\text { Nosepieces: }\end{array}$ & & & & & & & & & \\
\hline & 177 & 241 & .735 & 62.0 & 15.3 & 4.42 & 353 & 4.05 & 15.78 \\
\hline 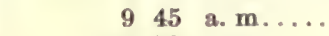 & 175 & 233 & .750 & 59.5 & 15.5 & 4.33 & 341 & & 15.84 \\
\hline 1058 a. $n$ & 169 & 231 & .730 & 59.5 & 15.5 & 4.24 & 334 & 4.03 & 15.79 \\
\hline Average..... & 174 & 235 & .740 & 60.5 & 15.4 & 4.38 & 343 & 4.05 & 15.80 \\
\hline Mouthpiece: & & & & & & & & & \\
\hline $9^{\overline{\mathrm{h}}} 22^{\mathrm{m}} \mathrm{a}$ & 178 & 236 & .755 & 61.5 & 16.6 & 4.55 & 335 & 3.94 & 16.02 \\
\hline 1017 a. 1 & 173 & 238 & .725 & 61.0 & 16.2 & 4.59 & 346 & 3. & 16.06 \\
\hline 1126 a. 1 & 189 & 247 & .765 & 60.5 & 16.7 & 4.83 & 354 & & 16.09 \\
\hline Average..... & 180 & 240 & .750 & 61.0 & 16.5 & 4.66 & 345 & 8.89 & 16.06 \\
\hline $\begin{array}{l}J . B . T . \\
1912:\end{array}$ & & & & & & & & & \\
\hline $8^{\mathrm{h}} 36^{\mathrm{m}}$ a. $n$ & 193 & 255 & .755 & 69.0 & 13.4 & 4.54 & 409 & 4.2 & \\
\hline 937 в. $\mathrm{x}$ & 203 & 260 & .780 & 67.5 & 13.9 & 4.62 & 401 & & 15.57 \\
\hline 1030 a. $m$ & 195 & 257 & .760 & 62.5 & 15.8 & 4.68 & 357 & 4. & 15. \\
\hline Average....... & 197 & 257 & .765 & 66.5 & 14.4 & 4.61 & 389 & 4.30 & 15.63 \\
\hline Mouthpiece: & & & & & & & & & \\
\hline 0 & $\begin{array}{l}203 \\
200\end{array}$ & $\begin{array}{l}255 \\
262\end{array}$ & .800 & $\begin{array}{l}65.5 \\
66.5\end{array}$ & $\begin{array}{l}13.3 \\
11.8\end{array}$ & $\begin{array}{l}4.41 \\
4.19\end{array}$ & $\begin{array}{l}400 \\
428\end{array}$ & & \\
\hline 1100 a. m & $\begin{array}{l}200 \\
204\end{array}$ & 275 & .745 & 70.0 & 15.9 & 4.68 & $\begin{array}{l}420 \\
355\end{array}$ & & 15.39 \\
\hline Average........ & 202 & 264 & .765 & 67.5 & 13.7 & 4.43 & 394 & 4.61 & 15.27 \\
\hline $\begin{aligned} & K . H . \\
& \text { Une } 19,1912:\end{aligned}$ & & & & & & & & & \\
\hline $9^{\mathrm{h}} 04^{\mathrm{m}} \mathrm{a}$. & & 271 & .745 & 52.0 & 15.6 & 5.60 & 437 & & \\
\hline $\begin{array}{llll}9 & 59 & \text { a. }\end{array}$ & & 249 & .780 & 45 . & 14.9 & 5.13 & 419 & & \\
\hline 1048 a.m. & & 266 & .775 & 51.0 & 15.3 & 5.50 & 437 & & 33 \\
\hline Average........ & 201 & 262 & .765 & 49.5 & 15.8 & 5.41 & 431 & 3.75 & 16.93 \\
\hline Mouthpiece: & & & & & & & & & \\
\hline & & 254 & .795 & 50.0 & 13.3 & 4.41 & 403 & 4.59 & \\
\hline $\begin{array}{lll}10 & 25 & \mathrm{a} . \\
11 & 12 & \mathrm{a} .\end{array}$ & & 253 & .810 & 50.0 & 12.9 & 5.11 & 482 & 4.05 & \\
\hline $\begin{array}{c}11 \quad 12 \quad \text { a. } \\
\text { Average.... }\end{array}$ & $\begin{array}{l}211 \\
206\end{array}$ & $\begin{array}{l}254 \\
254\end{array}$ & .830 & $\begin{array}{l}50.0 \\
50.0\end{array}$ & $\begin{array}{l}12.8 \\
13.0\end{array}$ & $\begin{array}{l}5.14 \\
4.89\end{array}$ & $\begin{array}{l}489 \\
458\end{array}$ & $\begin{array}{l}4.13 \\
4.26\end{array}$ & $\begin{array}{l}16.17 \\
15.98\end{array}$ \\
\hline $\begin{array}{l}\text { June 22, 1912: } \\
\text { Nosepieces: }\end{array}$ & & & & & & & & & \\
\hline $8^{\mathrm{h}} 51^{\mathrm{m}}$ a. m. & 206 & 262 & .785 & 59.0 & 15.0 & 5.61 & 450 & 3.70 & 16.48 \\
\hline 943 a. $n$ & 19 & 232 & .835 & 52.5 & 15.9 & 5.34 & 404 & 3.64 & 16.75 \\
\hline $\begin{array}{llll}10 & 39 & \text { a. } \mathrm{n}\end{array}$ & 213 & 248 & .860 & 55.5 & 15.9 & 5.75 & 435 & 3.74 & 16.75 \\
\hline Average...$\ldots \ldots$ & 204 & 247 & .825 & 55.5 & 15.6 & 5.57 & 430 & 3.69 & 16.66 \\
\hline
\end{tabular}


TABLE 31.-Respiratory exchange in comparison experiments with mouth-breathing and nosebreathing-Tissot apparatus. (Without food.) - Continued.

\begin{tabular}{|c|c|c|c|c|c|c|c|c|c|}
\hline \multirow{2}{*}{$\begin{array}{l}\text { Subject, date, method, } \\
\text { and time. }\end{array}$} & \multirow{2}{*}{ 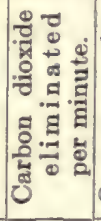 } & \multirow{2}{*}{ 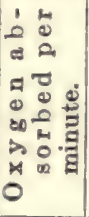 } & \multirow{2}{*}{ 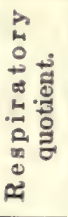 } & \multirow{2}{*}{ 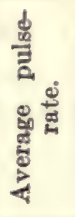 } & \multirow{2}{*}{ 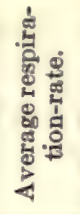 } & \multirow{2}{*}{ 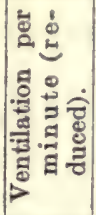 } & \multirow{2}{*}{ 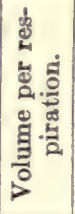 } & \multicolumn{2}{|c|}{$\begin{array}{l}\text { Composition of } \\
\text { expired air. }\end{array}$} \\
\hline & & & & & & & & $\begin{array}{l}\text { Carbon } \\
\text { dioxide. }\end{array}$ & Oxygen. \\
\hline 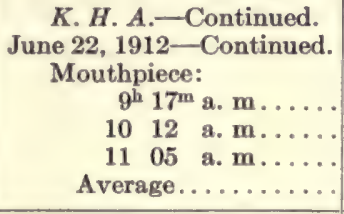 & $\begin{array}{l}c . c . \\
215 \\
217 \\
208 \\
213\end{array}$ & $\begin{array}{l}\text { c.c. } \\
257 \\
253 \\
245 \\
252\end{array}$ & $\begin{array}{l}.835 \\
.855 \\
.850 \\
.845\end{array}$ & $\begin{array}{l}55.0 \\
56.0 \\
52.0 \\
54.6\end{array}$ & $\begin{array}{l}14.0 \\
13.8 \\
13.8 \\
13.9\end{array}$ & $\begin{array}{l}\text { liters. } \\
5.25 \\
5.47 \\
5.11 \\
5.88\end{array}$ & $\begin{array}{l}c . c . \\
451 \\
477 \\
446 \\
458\end{array}$ & $\begin{array}{l}\text { p. ct. } \\
4.11 \\
3.99 \\
4.09 \\
4.06\end{array}$ & $\begin{array}{c}\text { p. } c t . \\
16.22 \\
16.45 \\
16.31 \\
16.39\end{array}$ \\
\hline 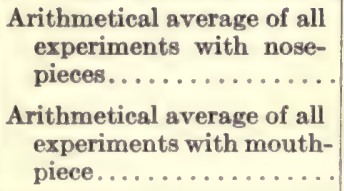 & 191 & 247 & .775 & 57.5 & 15.0 & 4.87 & 394 & & \\
\hline
\end{tabular}

The differences found between the results for the mouth-breathing and those for the nose-breathing for the individual experiments are given in table 32, those for the nose-breathing being taken as the base-line. It will be noted that in every instance the carbon-dioxide elimination was higher with the mouth-breathing than with the nosebreathing; the oxygen consumption was also higher in three of the

TABLE 32.-Variations of average results obtained with mouth-breathing from those obtained with nose-breathing (Tissot apparatus).

\begin{tabular}{|c|c|c|c|c|c|c|c|c|}
\hline Subject. & Date. & $\begin{array}{l}\text { Carbon } \\
\text { dioxide } \\
\text { elimin- } \\
\text { ated per } \\
\text { minute. }\end{array}$ & $\begin{array}{c}\text { Oxygen } \\
\text { absorbed } \\
\text { per } \\
\text { minute. }\end{array}$ & $\begin{array}{c}\text { Respira- } \\
\text { tory } \\
\text { quotient. }\end{array}$ & $\begin{array}{c}\text { Average } \\
\text { pulse- } \\
\text { rate. }\end{array}$ & $\begin{array}{c}\text { Average } \\
\text { respira- } \\
\text { tion- } \\
\text { rate. }\end{array}$ & $\begin{array}{l}\text { Ventila- } \\
\text { tion per } \\
\text { minute } \\
\text { (reduced). }\end{array}$ & $\begin{array}{l}\text { Volume } \\
\text { per } \\
\text { respira- } \\
\text { tion. }\end{array}$ \\
\hline $\begin{array}{l}\text { J. K. М... } \\
\text { J. B. T.... } \\
\text { K. H. А... }\end{array}$ & $\begin{array}{c}1912 \\
\text { June } 13 \\
\text { June } 18 \\
\text { June } 15 \\
\text { June } 19 \\
\text { June 22 }\end{array}$ & $\begin{array}{l}c . c . \\
+6 \\
+6 \\
+5 \\
+5 \\
+9\end{array}$ & $\begin{array}{l}\text { c.c. } \\
-3 \\
+5 \\
+7 \\
-8 \\
+5\end{array}$ & $\begin{array}{l}+0.035 \\
+\quad .01 \\
+\quad .0 \\
+\quad .045 \\
+\quad .02\end{array}$ & $\begin{array}{l}+1.5 \\
+\quad .5 \\
+1.0 \\
+\quad .5 \\
-1.0\end{array}$ & $\begin{array}{l}-0.3 \\
+1.1 \\
-.7 \\
-2.3 \\
-1.7\end{array}$ & $\begin{array}{r}\text { liters. } \\
-0.27 \\
+\quad .33 \\
-\quad .18 \\
-\quad .52 \\
-\quad .29\end{array}$ & $\begin{array}{l}c . c . \\
-15 \\
+\quad 2 \\
+5 \\
+27 \\
+28\end{array}$ \\
\hline \multicolumn{2}{|c|}{ Average variation.... } & 6 & 6 & 0.020 & 1.0 & 1.2 & 0.32 & 15 \\
\hline
\end{tabular}

five experiments, and both the respiratory quotient and pulse-rate were higher in four of the five experiments. On the contrary, in four of the five experiments both the respiration-rate and the ventilation of the lungs were lower with the mouth-breathing, but the difference was not large enough to be of significance. The results therefore tend to show that with this apparatus there was a slightly higher respiratory exchange with mouth-breathing than with nose-breathing. 
Since the increased carbon-dioxide elimination is not accompanied by an increase in the total ventilation, it is evident that there must have been a slightly more economical ventilation with mouth-breathing than with nose-breathing.

The probability curves for these comparison experiments are given in figure 49. The curves for the carbon-dioxide elimination do not show very much difference, but those for the oxygen consumption are slightly more uniform with the mouth-breathing. The pulse-rate is

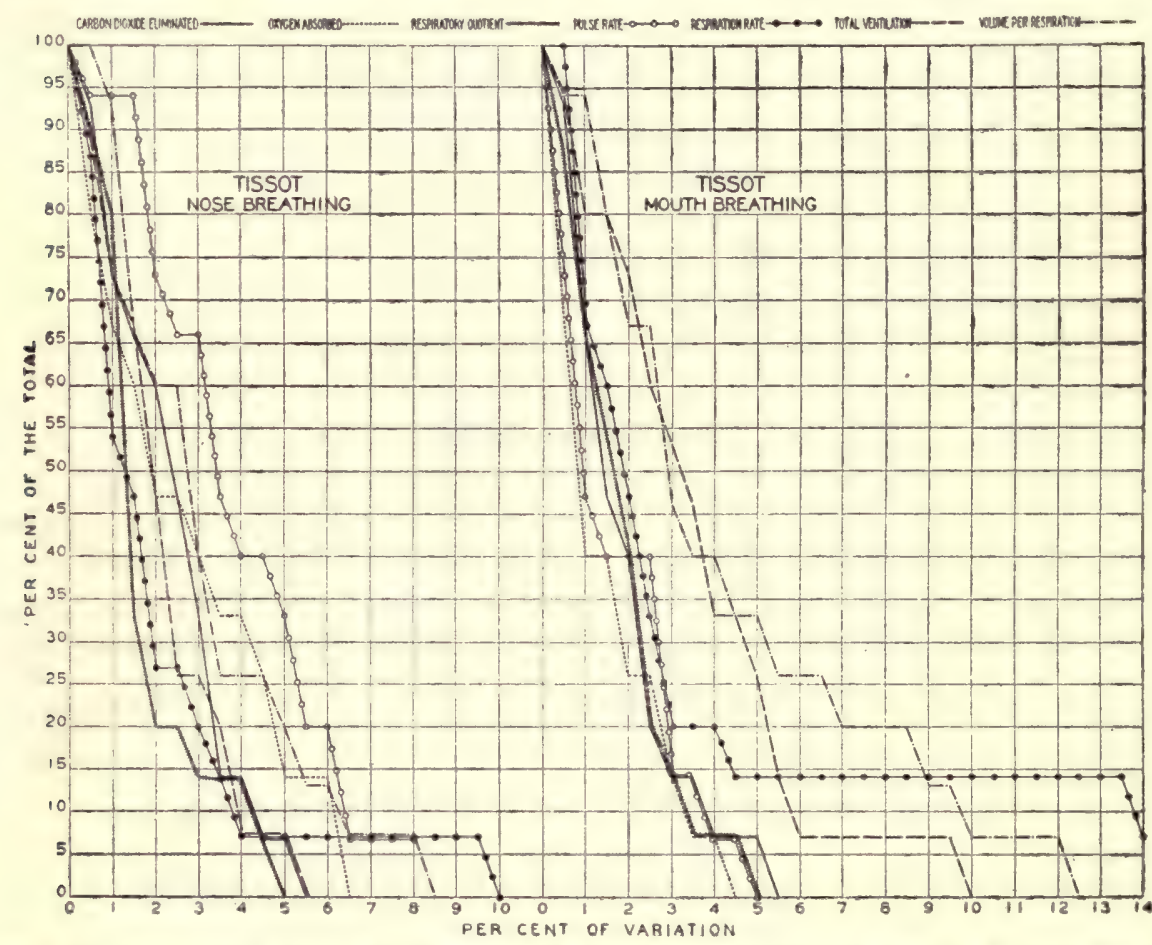

FIG. 49.-Probability curves for the series of comparison experiments with nose- and mouthbreathing (Tissot apparatus).

The ordinates indicate the percentage of the total number of periods and the abscissæ the percentage of variation from the average.

noticeably more uniform with the mouth-breathing. The respirationrate and the volume per respiration have about the same degree of uniformity with both types of breathing, while the total ventilation is slightly more uniform with the nose-breathing.

The results of this comparison substantiate in the main the results obtained with the two preceding comparisons with the Benedict respiration apparatus, $i . e$., that the differences in the respiratory exchange between mouth- and nose-breathing are not large enough to be of great significance in a study of the respiratory exchange. 


\section{MASK AND NOSEPIECES WITH THE BENEDICT RESPIRATION APPARATUS (SPIROMETER UNIT).}

In the earlier development of the Benedict respiration apparatus, several attempts were made to use a mask. This mask was ordinarily of rubber, conical in shape, and held against the face by means of strips of elastic tape bound around the head. About the edge of the mask was rubber tubing which could be inflated. The results obtained with this mask were not very satisfactory and its use was discontinued, mainly on account of the uncertainty as to the air-tight closure about the face.

As a mask is used in many laboratories in connection with respiration work, it was deemed advisable to make a number of experiments in which the respiratory exchange with the subject wearing a mask was compared with that when he breathed through nosepieces. In this series of comparisons, the spirometer unit was used to measure the respiratory exchange. The mask employed was constructed of sheet lead in the form of a cone, the small end of the cone being soldered to a piece of brass tubing of about $25 \mathrm{~mm}$. internal diameter. The cone was next shaped so as to fit as closely as possible to the face of the selected subject; the superfluous portions were then cut away. The edges of the mask were covered with plasticene, a material used by children in modeling. This mask was connected to the respiration apparatus by means of short pieces of rubber tubing. To make sure of the air-tight closure about the face, the edges of the mask were smeared with soapsuds and kept moist throughout the experimental period; the slightest leak could thus be readily detected.

The pulse-rate was obtained with the Bowles stethoscope. A graphic record of the respiration-rate was secured from the movements of the spirometer bell. A similar graphic record of the degree of muscular repose was obtained by means of the lever bed arrangement ${ }^{1}$ in all of the experiments except those with L. E. E. and M. J. S. With the exception of M. J. S., all of the subjects were accustomed to the apparatus. The statistics of the five experiments are given in the following pages.

\section{STATISTICS OF EXPERIMENTS.}

J. K. M., July 19, 1912.-A preliminary experiment to study the possibilities of the mask method. Subject had lunch at noon; experiment began at $3^{\mathrm{h}} 35^{\mathrm{m}}$ p. m. Mask, 1 period; pneumatic nosepieces, 1 period. No pulse records taken; respiration-rate very regular in both periods. Average barometric pressure, $759.3 \mathrm{~mm}$.; average temperature of air in apparatus, $20^{\circ} \mathrm{C}$.

J. K. M., November 19, 1912.-Subject had breakfast before experiment. Mask, 3 periods; nosepieces, 2 periods; preliminary period, 35 minutes; periods with mask and nosepieees in series. Subject asleep in first and third periods with mask. Said he preferred mask, as the nosepieces irritated the edge of the nostrils, but otherwise had no preference. Pulse-rate in first two periods varied considerably, with a range of 5 to 6 beats per minute; in the last three periods it was uniform. Respiration-rate previous to experiment, 19 per minute. During experiment respiration regular in depth and rapidity. Average barometric pressure, $762.3 \mathrm{~mm}$.; temperature of air in apparatus, $22.4^{\circ} \mathrm{C}$. 
M. J. S., July 20, 1912.-Mask, 4 periods; glass nosepieces, 3 periods; periods alternating. Both mask and nosepieces tested with soapsuds. Subject preferred mask, as nosepieces made edges of his nostrils sore and with the mask he felt that he had more freedom in breathing. He complained of soreness and pain on the left side of body. No pulse records taken. Respirationrate at beginning of periods uneven, but became more regular by the middle of the period. Average barometric pressure, $765.7 \mathrm{~mm}$; average temperature of air in apparatus, $24.4^{\circ} \mathrm{C}$.

M. J. S., July 22, 1912.- Subject had midday lunch previous to experiment; experiment began at $1^{\mathrm{h}} 53^{\mathrm{m}}$ p. $\mathrm{m}$. Sat in Morris chair instead of lying on couch. Mask, 3 periods; pneumatic nosepieces, 3 periods; periods alternating. Pulse-rate varied in periods with mask and in second period with nosepieces, the range being from 5 to 6 beats per minute; pulse-rate very regular in the other periods with nosepieces. Respiration-rate very regular in all periods. Average barometric pressure, $757.2 \mathrm{~mm}$.; average temperature of air in apparatus, $20.9^{\circ} \mathrm{C}$.

L. E. E., November 18, 1912.-Mask, 3 periods; pneumatic nosepieces, 2 periods; periods with mask and nosepieces in series; preliminary period, $1 \frac{1}{2}$ hours. Subject thought it would be an advantage to have a weight attached
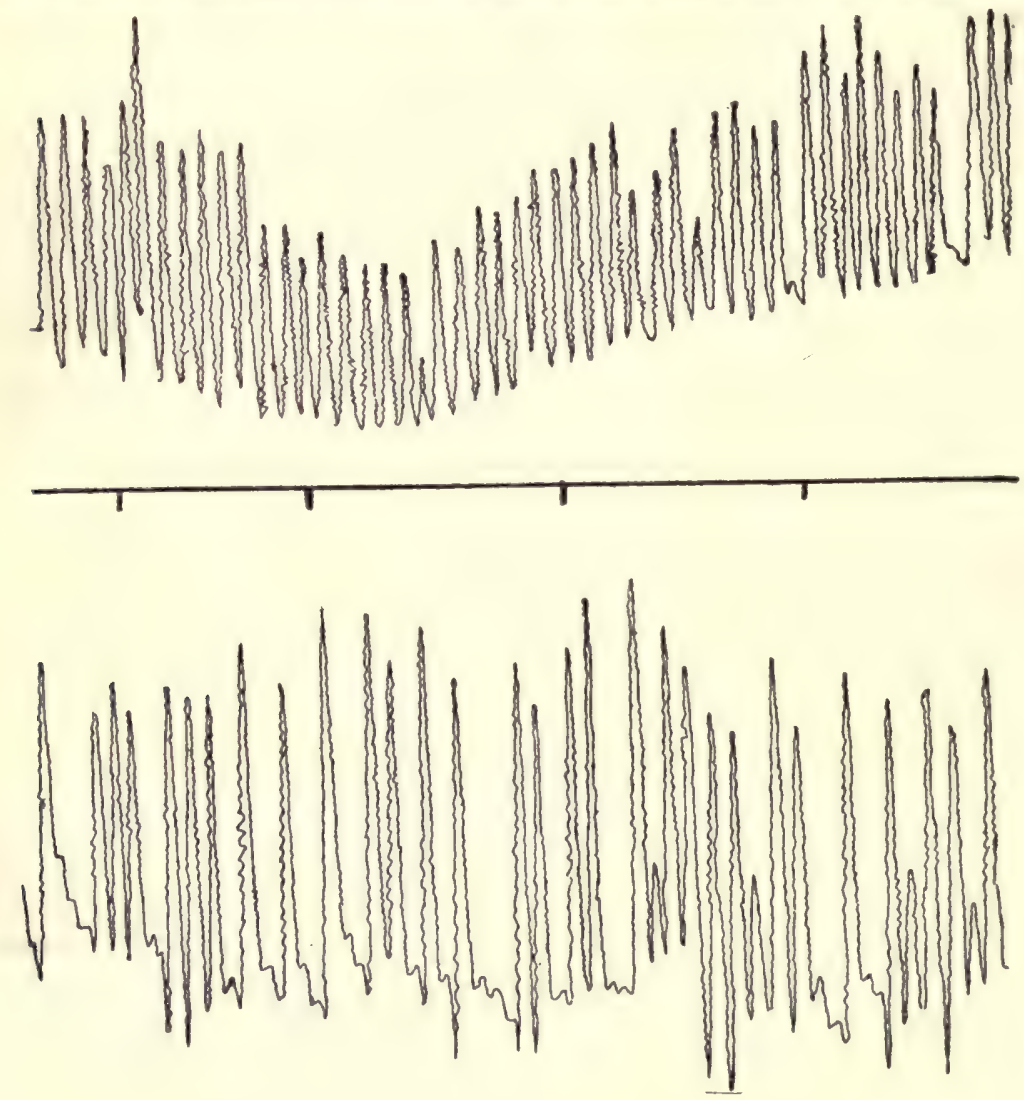

Fia. 50.-Types of respiration of subject L. E. E. as recorded from the spirometer bell in the second period on November 18, 1912.

Upper curve, beginning of period. Lower curve, end of period. Time line, minutes. 
to mask to press it more closely to the face. Pulse-rate very regular. Average respiration-rate previous to experiment, 17 per minute. ${ }^{1}$ During experiment, respiration somewhat irregular. In first period with mask, it was rapid and deep at first, but became slower and more shallow in the middle of the period; in second period with mask it was fairly regular at the beginning, but during the last half it was very irregular and there was considerable apncea. Portions of the records obtained are given in figure 50, showing the two types of respiration. In the last period with the mask, the respiration was very much like that in the preceding periods. In the periods with the nosepieces, the respiration was much more regular than in those with the mask. Average barometric pressure, $762.1 \mathrm{~mm}$. ; average temperature of the air in the apparatus, $21.0^{\circ} \mathrm{C}$.

\section{DISCUSSION OF RESULTS.}

The results of this series of comparisons are given in table 33 . The summary of the results shows that on the average there is practically no difference in the respiratory exchange with the two methods of breathing, only the total ventilation and the volume per respiration indicating any appreciable differences. The variations in the individual experiments are not in any case very large. With M. J. S. on July 20 , the carbon-dioxide elimination was 8 c.c. lower with the mask than with the nosepieces. With L. E. E. on November 18, the carbon-dioxide elimination was 10 c.c. and the respiratory quotient 0.045 lower with the mask than with the nosepieces, but in two of the periods with the mask there was irregular breathing and apnœa; consequently the carbon-dioxide values are not strictly normal. In practically all of the experiments the ventilation per minute was higher with the mask than with the nosepieces, but as the respiration-rate was not noticeably different, the increased volume per respiration must be due to the greater dead space with the mask. Assuming a dead space of 100 c.c. for the subject with both methods of breathing, we find by calculation that the dead space in the mask is about 40 to 70 c.c.

The probability curves for the different factors in this comparison have been plotted and are given in figure 51. The number of experiments is somewhat too small for obtaining good curves, but they show that in general the results with the mask are slightly more uniform than with the nose-breathing. This is especially noticeable in the curves for the oxygen consumption, the respiratory quotient, the total ventilation, and the volume per respiration.

All of the subjects were smooth-shaven, consequently no knowledge was obtained as to the applicability of the mask for men having a moustache or a beard. It is doubtful if the difficulties in making a mask air-tight under these circumstances can be overcome. As far as the measurement of the respiratory exchange is concerned, it is immaterial whether a mask or nosepieces are employed; but in using a mask, one must know the dead space in the mask in order to obtain the true ventilation during the experimental period.

\footnotetext{
${ }^{1}$ A new routine was established about this time in that records of the respiration-rate were taken in the preliminary rest period. In this way the normal value for the respiration-rate could be obtained for comparison with the values obtained during the experimental periods.
} 
TABLE 33.-Respiratory exchange in comparison experiments with mask and nosepiecesBenedict respiration apparatus (spirometer unit). (Without food.)

\begin{tabular}{|c|c|c|c|c|c|c|c|}
\hline $\begin{array}{l}\text { Subject, date, method, } \\
\text { and time. }\end{array}$ & $\begin{array}{l}\text { Carbon } \\
\text { dioxide } \\
\text { elimin- } \\
\text { ated per } \\
\text { minute. }\end{array}$ & $\begin{array}{c}\text { Oxygen } \\
\text { absorbed } \\
\text { per } \\
\text { minute. }\end{array}$ & $\begin{array}{c}\text { Respira- } \\
\text { tory } \\
\text { quotient. }\end{array}$ & $\begin{array}{c}\text { Average } \\
\text { pulse- } \\
\text { rate. }\end{array}$ & $\begin{array}{l}\text { Average } \\
\text { respira- } \\
\text { tion-rate. }\end{array}$ & $\begin{array}{l}\text { Ventila- } \\
\text { tion per } \\
\text { minute } \\
\text { (reduced). }\end{array}$ & $\begin{array}{l}\text { Volume } \\
\text { per } \\
\text { respira- } \\
\text { tion. }\end{array}$ \\
\hline \multicolumn{8}{|l|}{$\begin{aligned} J . K . M . \\
\text { July } 19,1912:\end{aligned}$} \\
\hline $\begin{array}{l}\text { Mask: } \\
3^{\mathrm{h}} 35^{\mathrm{m}} \text { p. } \mathrm{m}^{1} .\end{array}$ & $\begin{array}{l}c . c . \\
207\end{array}$ & $\begin{array}{l}c . c . \\
250\end{array}$ & 0.830 & & 16.2 & liters. & c.c. \\
\hline Nosepieces: & & & & & & 6.14 & 459 \\
\hline $4^{\mathrm{h}} 01^{\mathrm{m}}$ p. m. . . . & 216 & 252 & .855 & & 13.8 & 5.15 & 452 \\
\hline \multicolumn{8}{|l|}{$\begin{array}{l}\text { Nov. 19, 1912: } \\
\text { Mask: }\end{array}$} \\
\hline $8^{\mathrm{h}} 55^{\mathrm{m}}$ a. m. & 190 & 246 & .775 & 60.5 & 13.8 & 5.20 & 455 \\
\hline 919 a. $\mathrm{m}$. & 202 & 242 & .835 & 61.0 & 12.6 & 5.19 & 497 \\
\hline 950 a. $\mathrm{m}$. & 191 & 240 & .795 & 61.0 & 12.5 & 5.20 & 502 \\
\hline Average....... & 194 & 243 & .800 & 61.0 & 13.0 & 5.20 & 485 \\
\hline \multicolumn{8}{|l|}{ Nosepieces: } \\
\hline $10^{\mathrm{h}} 16^{\mathrm{m}}$ a. m. & 199 & 224 & .890 & 60.5 & 13.4 & 4.84 & 436 \\
\hline 1038 a. $\mathrm{m}$. & 186 & 227 & .820 & 59.5 & 13.0 & 4.51 & 418 \\
\hline Average....... & 193 & 226 & .855 & 60.0 & 13.2 & 4.68 & 427 \\
\hline \multicolumn{8}{|l|}{$\begin{array}{l}\text { M.J.S. } \\
\text { July 20, 1912: } \\
\text { Mask: }\end{array}$} \\
\hline $9^{h} 02^{m}$ a. m. & 195 & 249 & .785 & & 19.0 & 7.12 & 450 \\
\hline 955 a. m. & 186 & 246 & .760 & & 19.4 & 6.98 & 432 \\
\hline 1038 a. m. & 185 & 250 & .740 & & 20.4 & 7.25 & 427 \\
\hline 1137 a. m. & 191 & 247 & .775 & & 21.5 & 7.58 & 423 \\
\hline Average......... & 189 & 248 & .760 & & 20.1 & 7.23 & 493 \\
\hline \multicolumn{8}{|l|}{ Glass nosepieces: } \\
\hline $9^{h} 35^{m}$ a. m. & 197 & 240 & .820 & & 19.7 & 6.19 & 377 \\
\hline 1016 a. $\mathrm{m}$. & 189 & 248 & .760 & $\cdots$ & 17.4 & 5.85 & 404 \\
\hline 1114 a. $\mathrm{m} \ldots$ & 206 & 270 & .760 & ...... & 20.6 & 6.65 & 388 \\
\hline \multirow{3}{*}{\multicolumn{8}{|c|}{$\begin{array}{l}\text { July } 22,1912: \\
\text { Mask: }\end{array}$}} \\
\hline & & & & & & & \\
\hline & & & & & & & \\
\hline $\begin{array}{lll}1 & 53^{2} & \text { p. } \mathrm{m}^{2} \ldots \\
2 & 48 & \text { p. } \mathrm{m} . .\end{array}$ & $\begin{array}{l}216 \\
225\end{array}$ & $\begin{array}{l}239 \\
245\end{array}$ & $\begin{array}{l}.905 \\
.920\end{array}$ & $\begin{array}{l}66.0 \\
68.0\end{array}$ & 18.8 & $\begin{array}{l}7.04 \\
7.29\end{array}$ & $\begin{array}{l}455 \\
484\end{array}$ \\
\hline 408 p. m... & 217 & 240 & .900 & 65.5 & 16.5 & 6.92 & $\begin{array}{l}404 \\
509\end{array}$ \\
\hline Average............ & 219 & 241 & .910 & 66.5 & 17.9 & 7.08 & 483 \\
\hline \multicolumn{8}{|l|}{ Nosepieces: } \\
\hline $2^{\mathrm{h}} 24^{\mathrm{m}}$ p. m & 228 & 251 & .905 & 68.0 & 17.5 & 6.09 & 423 \\
\hline 344 p. m... & 225 & 245 & .920 & 66.0 & 16.7 & 6.02 & 438 \\
\hline 450 p. m.... & 203 & 245 & .830 & 67.0 & 17.9 & 5.93 & 402 \\
\hline Average.......... & 219 & 247 & .885 & 67.0 & 17.4 & 6.01 & 421 \\
\hline \multicolumn{8}{|l|}{$\begin{array}{l}\quad L . E . E . \\
\text { Nov. } 18,1912: \\
\text { Mask: }\end{array}$} \\
\hline $9^{\mathrm{h}} 20^{\mathrm{m}}$ a. m. . & 206 & 285 & .725 & 61.0 & 9.7 & 5.70 & 709 \\
\hline 940 a. $\mathrm{m}$. & 187 & 287 & .650 & 60.0 & 9.3 & 4.82 & 626 \\
\hline 1005 a. m.... & 188 & 278 & .675 & 59.5 & 8.8 & 4.91 & 673 \\
\hline Average........... & 194 & 283 & .685 & 60.0 & 9.3 & 5.14 & 669 \\
\hline \multicolumn{8}{|l|}{ Nosepieces: } \\
\hline $10^{\mathrm{h}} 31^{\mathrm{m}}$ a. m. & 202 & 270 & .750 & 64.0 & 8.9 & 4.55 & 617 \\
\hline 1156 a. $\mathrm{m} . .$. & 205 & 290 & .705 & 63.5 & 11.2 & 4.90 & 529 \\
\hline Average........... & 204 & 280 & .730 & 64.0 & 10.1 & 4.73 & 573 \\
\hline \multirow{3}{*}{$\begin{array}{l}\text { Arithmetical average of } \\
\text { all experiments with } \\
\text { mask................ } \\
\text { Arithmetical average of } \\
\text { all experiments with } \\
\text { nosepieces............ }\end{array}$} & 201 & 253 & .800 & 62.5 & 15.3 & 6.16 & 506 \\
\hline & & & & & & & \\
\hline & 206 & 252 & .815 & 63.5 & 14.7 & 5.36 & 453 \\
\hline
\end{tabular}




\section{GLASS AND PNEUMATIC NOSEPIECES WITH THE BENEDICT RESPIRATION APPARATUS (SPIROMETER UNIT).}

Two types of nosepieces have been used in the comparison experiments previously described: (1) the pneumatic nosepieces devised for use with the Benedict respiration apparatus and (2) the round glass nosepieces ordinarily used with the Tissot apparatus. The respiratory exchange with these two types of nosepieces was therefore compared in two experiments. The usual observations were made, the degree of muscular repose being recorded by means of the bed-lever arrangement. Both of the subjects were accustomed to the apparatus.

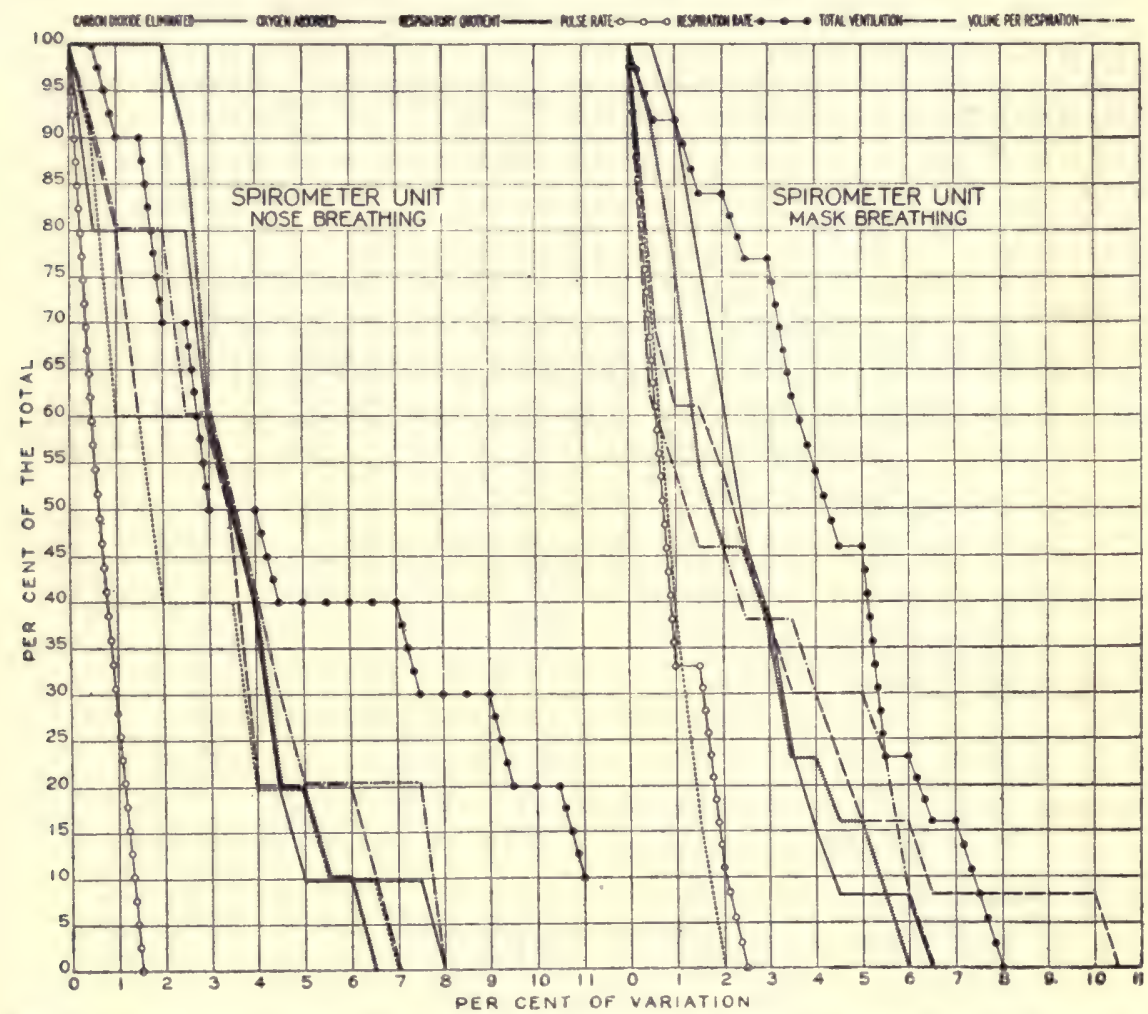

F16. 51.-Probability curves for the series of comparison experiments with nosepieces and mask (spirometer unit).

The ordinates indicate the percentage of the total number of periods and the abscisse represent the percentage of variation from the average.

\section{STATISTICS OF EXPERIMENTS.}

J. K. M., July 12, 1912.-Pneumatic nosepieces, 4 periods; glass nosepieces, 3 periods; preliminary period, 38 minutes; periods alternating after the first two periods. Subject drowsy the latter part of the experiment and stated that he preferred the glass nosepieces, as he could breathe more freely. Pulserate varied somewhat widely in all of the periods, the range being from 6 to 8 beats per minute. Respiration fairly regular. Average barometric pressure, $760.1 \mathrm{~mm}$.; average temperature of air in apparatus, $23.5^{\circ} \mathrm{C}$.

P. F. J., July 13, 1912.-Pneumatic nosepieces, 3 periods; glass nosepieces, 3 periods; periods alternating. Pulse-rate uniform, except in the first period 
with the glass nosepieces, when it varied from 61 to 69 beats per minute. Subject stated he was not asleep in this period. Respiration-rate varied, particularly in the first three periods. Average barometric pressure, 766.4 $\mathrm{mm}$. ; average temperature of air in the apparatus, $21^{\circ} \mathrm{C}$.

\section{DISCUSSION OF RESULTS.}

The results of the two experiments in this comparison are given in table 34. The average results show no marked difference in the respiratory exchange for the two types of nosepieces. These experiments were made in connection with other work and the number of compari-

TABLE 34.-Respiratory exchange in comparison experiments with glass and pmeumatic nosepieces-Benedict respiration apparatus (spirometer unit). (Without food.)

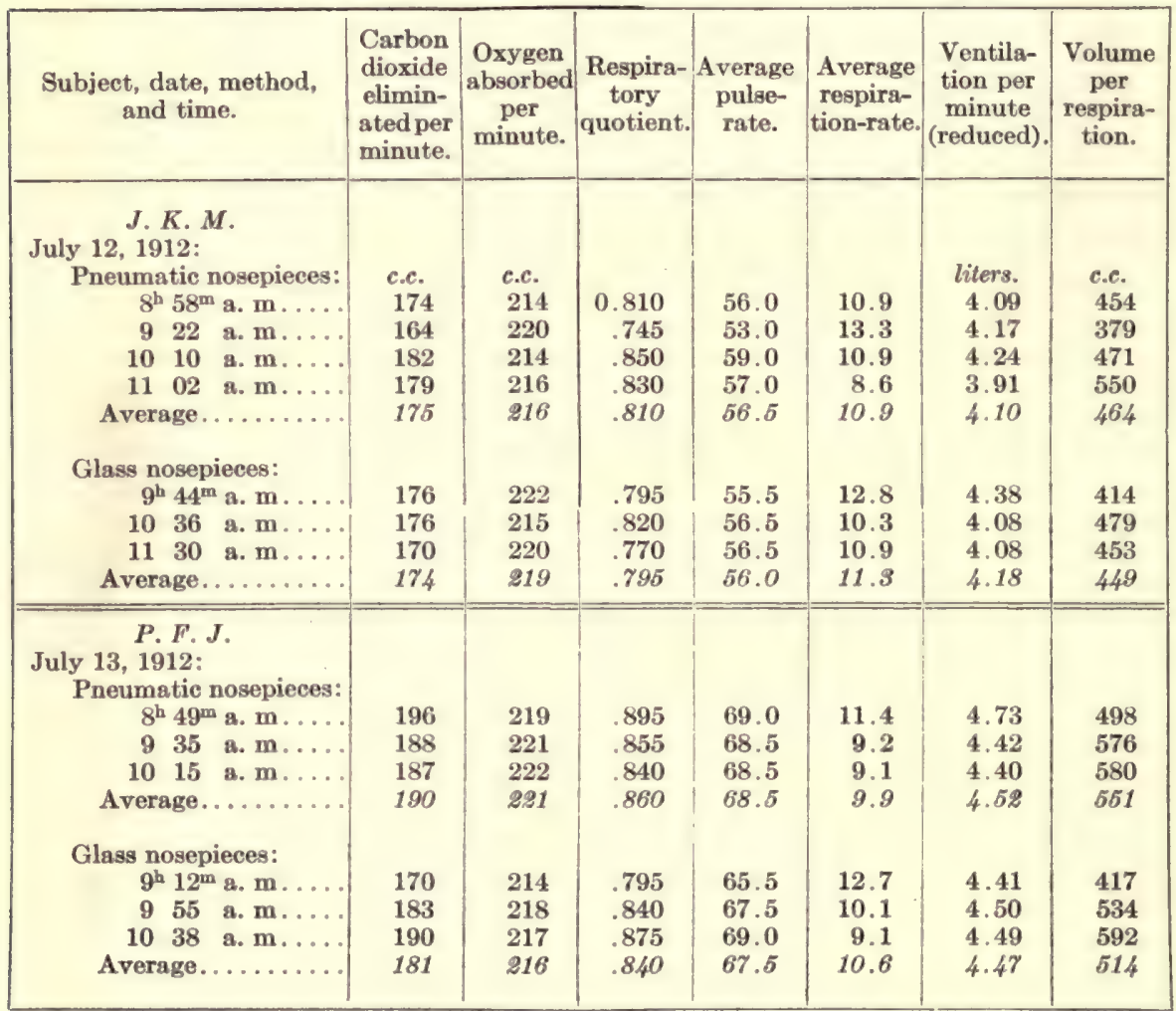

sons is so limited that no very definite conclusions can be drawn from them. A calculation of the uniformity in the results has been made, but the number of periods was too small to permit the plotting of curves. The most marked difference is shown in the total ventilation, which is more uniform with the glass nosepieces than with the pneumatic nosepieces. The other factors, carbon-dioxide elimination, oxygen consumption, etc., have practically the same degree of uniformity in both types of breathing. In one experiment the subject stated that it was easier breathing through glass nosepieces than through pneumatic nosepieces. The question as to which type of nosepieces is the more advisable to use is discussed in a later section. 
MUELLER VALVES AND TISSOT SPIROMETER AND THE BENEDICT RESPIRATION APPARATUS (SPIROMETER UNIT).

In view of the fact that the Mueller valves ${ }^{1}$ are still used in a number of laboratories for studying the respiratory exchange, it was considered desirable to make a series of experiments to test their efficiency. In these experiments the 200-liter Tissot spirometer was used with the Mueller valves to collect the expired air, and the results were compared with those obtained with the spirometer unit.

In the periods with the Mueller valves, the valves were supported by rods and wiring, so that with the subject lying on his back a valve hung on either side of him, just outside of his shoulders. Care was taken to have the valves hang perpendicularly in order that the water-level might always be at right angles to the sealed end of the tubing. The tee between the valves was so turned that the subject could breathe comfortably through them. From the exit valve a piece of rubber tubing led to the Tissot spirometer. The mouthpiece was used in all of the experiments, as both subjects preferred it.

Before sampling the air in the spirometer, a weight was placed on the spirometer bell and 5 to 10 liters of air forced out. A 300 c.c. gas-sampler was then connected with the tube at the bottom of the spirometer (see $A, B$, fig. 27, page 64 ) and when about 5 liters of air had been forced through the sampler the stopcocks were closed and the sampler disconnected. The air sample was then analyzed by means of the portable Haldane gas-analysis apparatus.

The pulse-rate was secured in this series of experiments with the Bowles stethoscope. In the periods with the Mueller valves, the record of the respiration was obtained by means of the chest pneumograph, but in the periods with the spirometer unit the respiration was recorded from the movements of the spirometer bell. No graphic record of the degree of muscular repose was obtained in this series, but both subjects were very quiet in all of the experiments. They were somewhat trained with the Benedict respiration apparatus and with the Tissot apparatus, but had not previously used the Mueller valves. The statistics of the five experiments are given in the following pages.

\section{STATISTICS OF EXPERIMENTS.}

W. J. T., March 18, 1913.- Spirometer unit, 3 periods; Mueller valves and Tissot spirometer, 3 periods; preliminary period, 1 hour 4 minutes. First period, spirometer unit; second and third periods, Mueller valves; periods with each method alternating thereafter. Subject drowsy in some of the periods. Pulse-rate for the most part uniform. Respiration-rate previous to experiment, 19 per minute. Respiration both in rate and character somewhat irregular during the experiment, particularly in the first period with the Mueller valves. Average barometric pressure, $780.5 \mathrm{~mm}$.; average temperature of the air in apparatus with Mueller valves, $18.1^{\circ} \mathrm{C}$; ; with spirometer unit, $20.5^{\circ} \mathrm{C}$.

W.J.T., March 29, 1913.-Mueller valves and Tissot spirometer, 4 periods; spirometer unit, 4 periods; preliminary period, 46 minutes; periods with each method in series. Pulse-rate very regular. Respiration-rate before experiment, 19 per minute; during experiment respiration uniform except in first 
period with the Mueller valves and second period with spirometer unit. Parts of the curves for these two periods are given in figures 52 and 53. Average barometric pressure, $773.8 \mathrm{~mm}$.; average temperature of air in apparatus with Mueller valves, $20.6^{\circ} \mathrm{C}$.; with spirometer unit, $21.1^{\circ} \mathrm{C}$.

J.J. G., March 19, 1913.-Mueller valves and Tissot spirometer, 3 periods; spirometer unit, 3 periods; preliminary period, 1 hour 5 minutes; periods with two methods alternated. Subject arose and urinated at $9^{\mathrm{h}} 25^{\mathrm{m}}$ a. m.; drowsy in second period with spirometer unit. The values for the carbon-dioxide production and oxygen consumption in the first period with Mueller valves are not included in averages, as there were indications that the sample of air was contaminated. Pulse-rate uniform in all periods. Respiration-rate before experiment, 21 per minute; during experiment very regular in type and rate. Average barometric pressure for Mueller valves, $772.5 \mathrm{~mm}$., and for spirometer unit, $771.8 \mathrm{~mm}$.; average temperature of air in apparatus with Mueller valves, $18.7^{\circ} \mathrm{C}$; with spirometer unit, $19.6^{\circ} \mathrm{C}$.

J. J. G., March 20, 1913. - Spirometer unit, 4 periods; Mueller valves and Tissot spirometer, 4 periods; preliminary period, 29 minutes; periods with two methods alternated. Pulse-rate regular throughout experiment. Respirationrate before experiment, 19 per minute; during experiment, fairly regular in depth. Average barometric pressure, $763.7 \mathrm{~mm}$.; average temperature of air in apparatus, $16.5^{\circ} \mathrm{C}$. with Mueller valves and $17.8^{\circ} \mathrm{C}$. with spirometer unit.
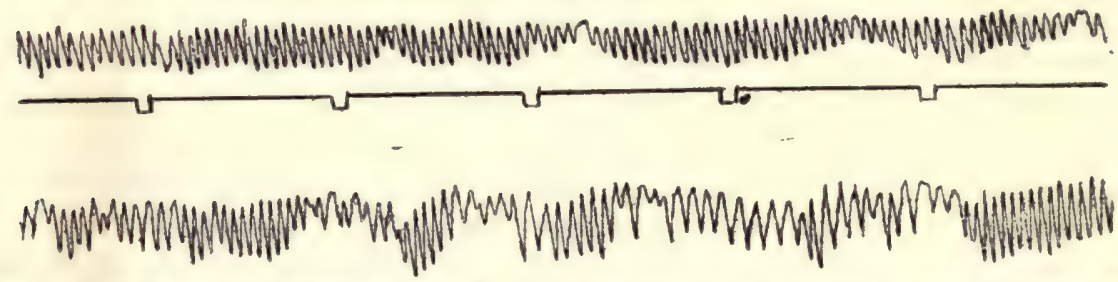

Frg. 52.-Types of respiration of subject W. J. T. as shown by the pneumograph in the first two periods with the Mueller valves and Tissot spirometer on March 29, 1913. Time line, minutes. Four-fifths original size.

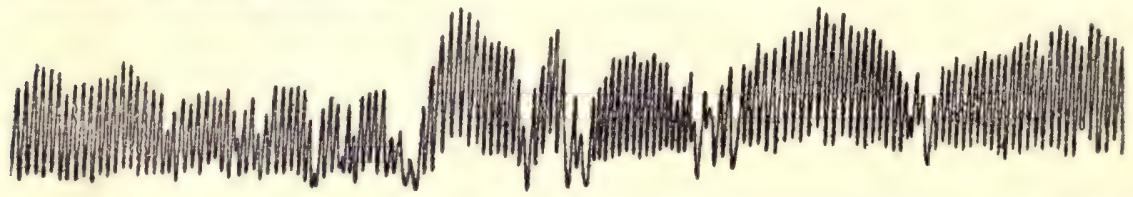

Fro. 53.-Type of respiration of subject W. J. T. as recorded from the spirometer bell in the second period with the spirometer unit on March 29, 1913. Time line, minutes. Threefourths original size.

J. J. G., April 2, 1913.-Spirometer unit, 4 periods; Mueller valves, 3 periods; preliminary period, 54 minutes; periods with each method in series. Pulse-rate fairly regular. Respiration-rate before experiment averaged 17 per minute; rate and type during experiment very regular. Average barometric pressure, $754.6 \mathrm{~mm}$.; temperature of air in apparatus with spirometer unit, $21.6^{\circ} \mathrm{C}$.; with Mueller valves, $19.5^{\circ} \mathrm{C}$.

\section{DISCUSSION OF RESULTS.}

The results of the individual experiments in this series are given in table 35 . The average of all of the experiments shows a slight difference between the results with the two methods, the carbon-dioxide elimination and oxygen consumption being higher with the Mueller valves than with the spirometer unit, although the average respiratory 
quotient is the same. The total ventilation and volume per respiration with the Mueller valves are also higher, the difference being due in part to the larger dead space with this method.

TABLE 35.-Respiratory exchange in comparison experiments with Benedict respiration apparatus (spirometer unit) and Mueller valves with Tissot spirometer. (Without food.)

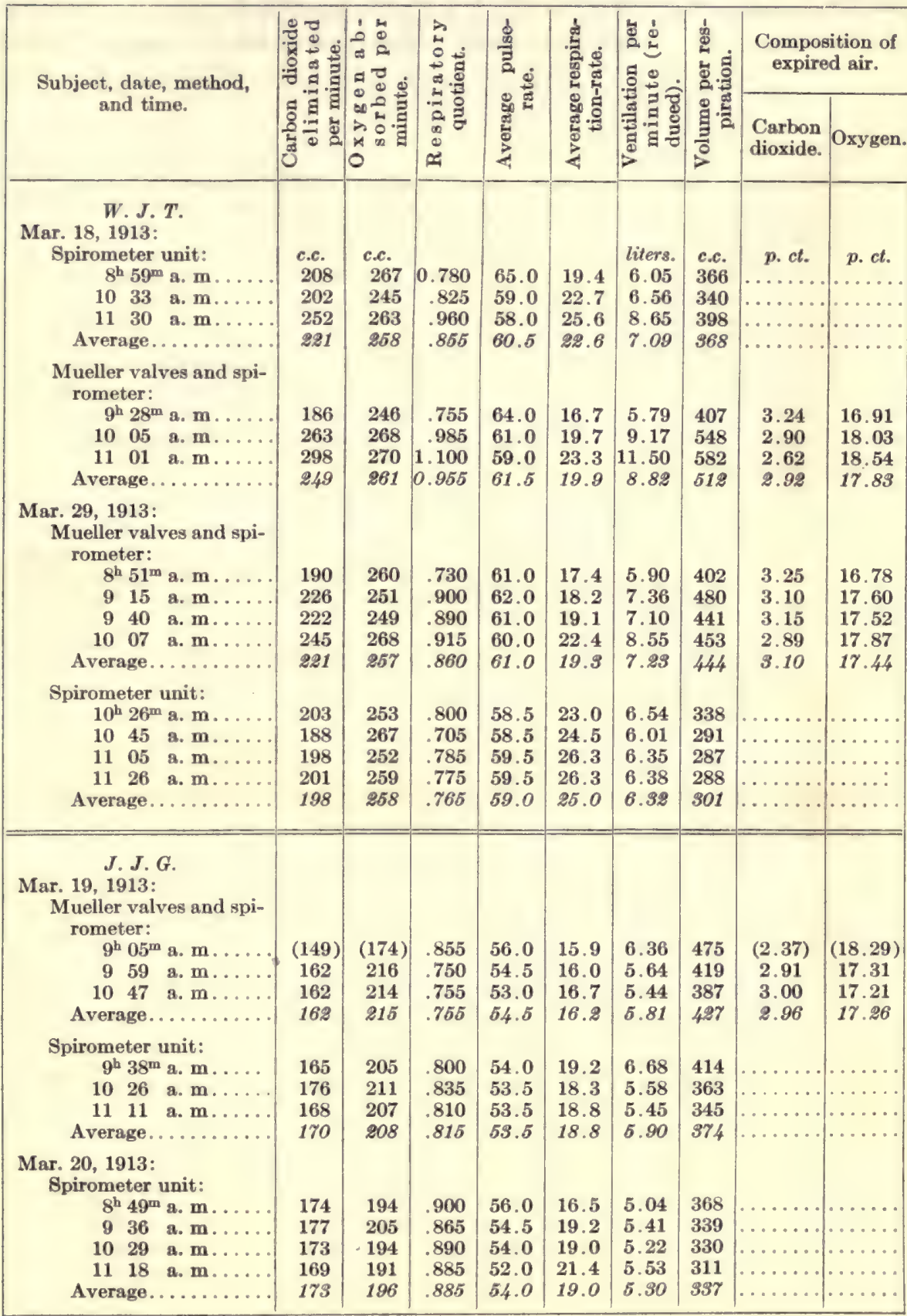


TABLE 35.-Respiratory exchange in comparison experiments with Benedict respiration apparatus (spirometer unit) and Mueller valves with Tissot spirometer. (Without food.)-(Continued.)

\begin{tabular}{|c|c|c|c|c|c|c|c|c|c|}
\hline \multirow{2}{*}{$\begin{array}{l}\text { Subject, date, method, } \\
\text { and time. }\end{array}$} & \multirow{2}{*}{ 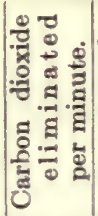 } & \multirow{2}{*}{ 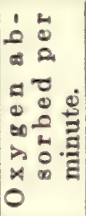 } & \multirow{2}{*}{ 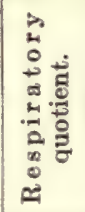 } & \multirow{2}{*}{ 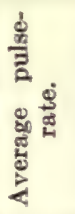 } & \multirow{2}{*}{ 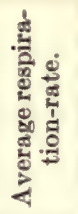 } & \multirow{2}{*}{ 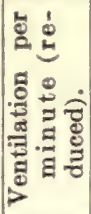 } & \multirow{2}{*}{ 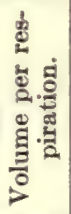 } & \multicolumn{2}{|c|}{$\begin{array}{l}\text { Composition of } \\
\text { expired air. }\end{array}$} \\
\hline & & & & & & & & $\begin{array}{l}\text { Carbon } \\
\text { dioxide. }\end{array}$ & Oxygen. \\
\hline $\begin{array}{l}\text { J. J. G. - Continued. } \\
\text { Mar. 20, 1913-Continued. } \\
\text { Mueller valves and spi- } \\
\text { rometer: } \\
9^{\mathrm{h}} 13^{\mathrm{m}} \text { a. m...... }\end{array}$ & c.c. & c.c. & & 56.5 & 15.4 & $\begin{array}{l}\text { liters. } \\
5.15\end{array}$ & $\begin{array}{l}\text { c.c. } \\
402\end{array}$ & p. ct. & p. ct. \\
\hline 958 a. m..... & 174 & 218 & 0.800 & 57.5 & 16.3 & 5.60 & 413 & 3.14 & 17.21 \\
\hline 1049 a. m..... & 168 & 221 & .760 & 55.5 & 16.8 & 5.84 & 419 & 2.91 & 17.34 \\
\hline 1140 a. $\mathrm{m} \ldots \ldots$ & 161 & 214 & .750 & 53.0 & 17.9 & 5.53 & 372 & 2.94 & 17.26 \\
\hline Average........... & 168 & 218 & $.7 \gamma 0$ & 55.5 & 16.6 & 5.63 & 402 & 3.00 & 17.27 \\
\hline $\begin{array}{l}\text { Apr. 2, 1913: } \\
\quad \text { Spirometer unit: } \\
\qquad 9^{\mathrm{h}} 09^{\mathrm{m}} \text { a. m. . . . . }\end{array}$ & 181 & 208 & .865 & 63.5 & 15.3 & 4.99 & 398 & & \\
\hline 925 a. m..... & 169 & 206 & .825 & 61.5 & 15.9 & 4.83 & 370 & & \\
\hline 945 s. m.... & 171 & 204 & .835 & 60.5 & 15.6 & 4.55 & 356 & & \\
\hline 1002 a. m..... & 169 & 208 & .810 & 59.5 & 16.7 & 4.72 & 345 & & \\
\hline Average............ & 178 & 207 & .835 & 61.5 & 15.9 & 4.77 & 367 & & \\
\hline $\begin{array}{l}\text { Mueller valves and spi- } \\
\text { rometer: }\end{array}$ & & & & & & & & & \\
\hline $10^{h} 23^{m}$ a. $m . .$. & 163 & 217 & .750 & 58.0 & 16.4 & 4.99 & 371 & 3.29 & 16.82 \\
\hline 1043 a. $\mathrm{m} \ldots .$. & 168 & 218 & .770 & 57.5 & 16.2 & 4.93 & 371 & 3.44 & 16.73 \\
\hline 1124 a. m..... & 172 & 214 & .805 & 56.5 & 16.0 & 5.08 & 387 & 3.42 & 16.90 \\
\hline Average...$\ldots \ldots \ldots$ & 168 & 216 & .780 & 57.5 & 16.2 & 5.00 & 976 & 3.98 & 16.82 \\
\hline $\begin{array}{l}\text { Arithmetical average of all } \\
\text { experiments with spi- } \\
\text { rometer unit. . . . . . . }\end{array}$ & 187 & 225 & .830 & 57.5 & 20.3 & 5.88 & 349 & & - \\
\hline $\begin{array}{l}\text { Arithmetical average of all } \\
\text { experiments with Muel- } \\
\text { ler valves and spirometer. }\end{array}$ & 194 & 233 & .83 & 58.0 & 17.6 & 6.48 & 432 & & \\
\hline
\end{tabular}

The differences shown in the individual experiments are given in table 36 , the experiments with the spirometer unit being used as a baseline. An examination of the figures in this table shows that the difference is not so uniform as would appear from the averages. For example, with W. J. T. the carbon-dioxide elimination is noticeably higher with the Mueller valves and the volume per respiration and ventilation per minute very much larger than with the spirometer unit. On the contrary, with J. J. G. the carbon-dioxide output is a little lower and the oxygen consumption slightly higher with the Mueller valves. The difference between the respiratory quotients in all of the experiments is very marked. The pulse-rate is in general higher with the Mueller valves than with the spirometer unit.

Many of the periods included in this comparison series would undoubtedly be excluded if only the normal figures were being considered. For example, the high carbon-dioxide elimination in the last period with the spirometer unit in the experiment with W. J. T. on March 18, 1913, 
is abnormal. This was probably due to over-ventilation, for if a calculation is made of the ventilation of the lungs other than that required to sweep out the normal dead space, it will be seen that there would be a greater volume of ventilation per unit of carbon dioxide in this period than in the other two periods with the spirometer unit. The 190 c.c. obtained in the experiment on March 29 for the carbon-dioxide elimination in the first period with the Mueller valves is also apparently

TABLE 36.-Variations of average results obtained with the Mueller valves and Tissot spirometer from those obtained with the spirometer unit.

\begin{tabular}{|c|c|c|c|c|c|c|c|c|}
\hline Subject. & Date. & $\begin{array}{l}\text { Carbon } \\
\text { dioxide } \\
\text { elimin- } \\
\text { ated per } \\
\text { minute. }\end{array}$ & $\begin{array}{c}\text { Oxygen } \\
\text { absorbed } \\
\text { per } \\
\text { minute. }\end{array}$ & $\begin{array}{c}\text { Respira- } \\
\text { tory } \\
\text { quotient. }\end{array}$ & $\begin{array}{c}\text { Average } \\
\text { pulse- } \\
\text { rate. }\end{array}$ & $\begin{array}{l}\text { Average } \\
\text { respira- } \\
\text { tion-rate. }\end{array}$ & $\begin{array}{l}\text { Ventila- } \\
\text { tion per } \\
\text { minute } \\
\text { (reduced). }\end{array}$ & $\begin{array}{c}\text { Volume } \\
\text { per } \\
\text { respira- } \\
\text { tion. }\end{array}$ \\
\hline $\begin{array}{l}\text { W. J. T... } \\
\text { J. J. G. . . }\end{array}$ & $\begin{array}{l}1913 \\
\text { Mar. } 18 \\
\text { Mar. } 29 \\
\text { Mar. } 19 \\
\text { Mar. 20 } \\
\text { Apr. } 2\end{array}$ & $\begin{array}{l}c . c . \\
+28 \\
+23 \\
-8 \\
-5 \\
-5\end{array}$ & $\begin{array}{l}\text { c.c. } \\
+3 \\
+1 \\
+7 \\
+22 \\
+9\end{array}$ & $\begin{array}{r}+0.100 \\
+\quad .095 \\
-\quad .060 \\
-\quad .115 \\
-\quad .055\end{array}$ & $\begin{array}{l}+1.0 \\
+2.0 \\
+1.0 \\
+1.5 \\
-4.0\end{array}$ & $\begin{array}{l}-2.7 \\
-5.7 \\
-2.6 \\
-2.4 \\
+\quad .3\end{array}$ & $\begin{array}{r}\text { liters. } \\
+1.73 \\
+\quad .91 \\
-\quad .09 \\
+\quad .23 \\
+\quad .23\end{array}$ & $\begin{array}{r}c . c . \\
+144 \\
+143 \\
+\quad 53 \\
+\quad 65 \\
+\quad 9\end{array}$ \\
\hline \multicolumn{2}{|c|}{ Average variation.... } & 14 & 8 & 0.085 & 2.0 & 2.7 & 0.64 & +83 \\
\hline
\end{tabular}

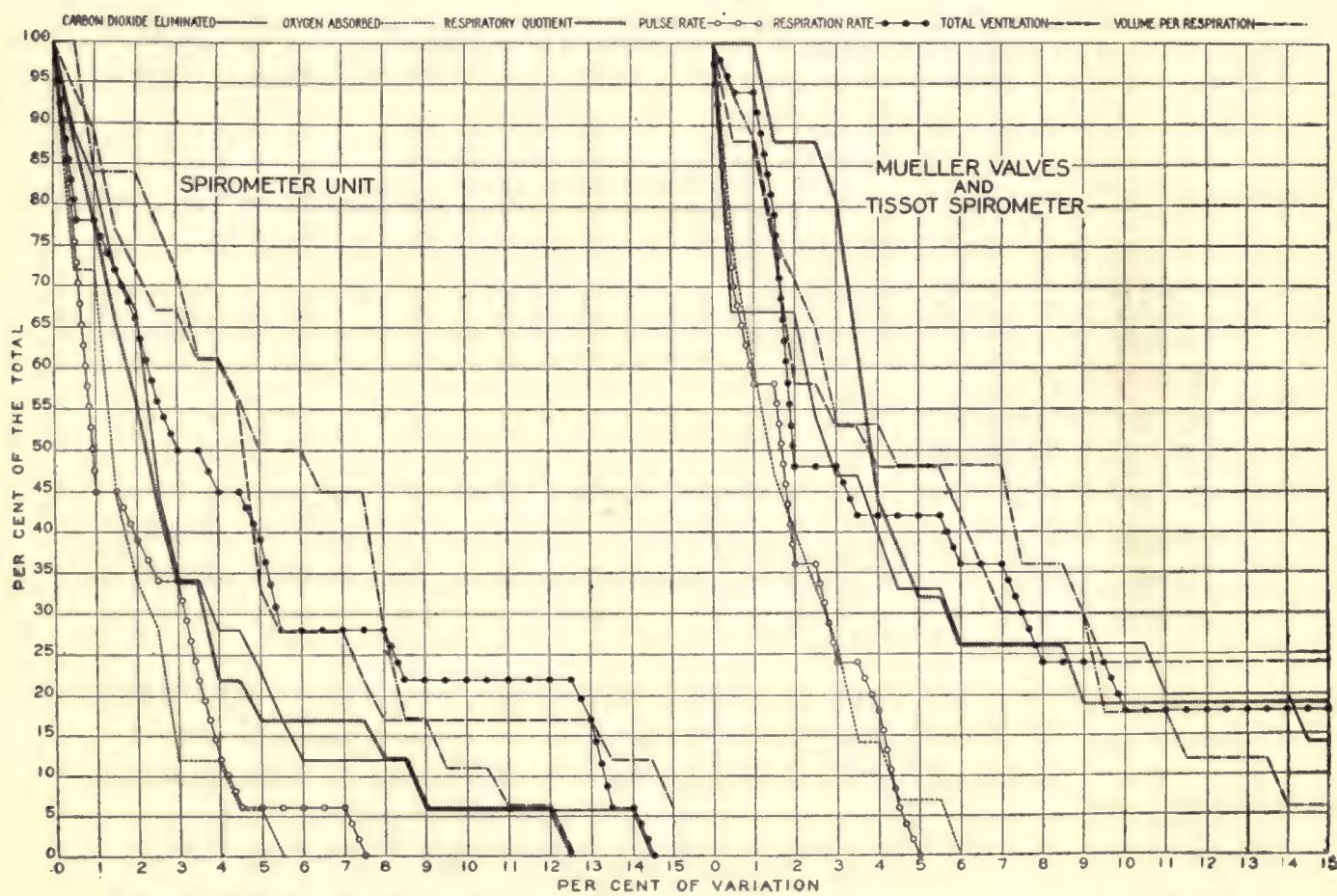

Fre. 54.-Probability curves for the series of comparison experiments with the spirometer unit and the Mueller valves.

The ordinates indicate the percentage of the total number of periods and the abscissæ indicate the percentage of variation from the average. 
abnormal. The carbon-dioxide elimination for the other two periods with this method does not compare well with the values obtained with the spirometer unit and if the last two values for the Mueller valves were considered abnormal, the value for the first period might be taken as normal. The average values in this experiment for the spirometer unit are influenced by the figures for the second period with this apparatus, as the values for the carbon-dioxide output and oxygen intake in this period are both abnormal; but there is no indication of error in the manipulation and the figures are accordingly included. If the values for the carbon-dioxide elimination and oxygen consumption for this period are excluded, the average respiratory quotient would be raised.

The values obtained with the subject J. J. G. present just the opposite picture to that for W. J. T., and it is difficult to state whether the value for the Mueller valves or that for the spirometer unit is correct. Both series of periods show good uniformity. The differences between the results with the two methods are not large, except that on March 20 the oxygen consumption is noticeably larger with the Mueller valves.

The degree of uniformity in the results shown by the curves in figure 54 is about the same with both apparatus, except that the respiratory quotient is more nearly uniform with the spirometer unit than with the Mueller valves.

Before a final conclusion is drawn regarding these two methods, the comparison experiments with the Mueller valves and the Tissot valves will be considered.

\section{MUELLER VALVES AND TISSOT VALVES.}

In addition to the preceding comparison, a series of experiments was carried out in which the Tissot valves and the Mueller valves were compared, the 200-liter Tissot spirometer being used to collect the expired air. In the first two of these experiments the regular routine for the use of the Tissot spirometer was not strictly followed. The bell of the spirometer is partly counterpoised by means of a weight suspended from a wheel ${ }^{1}$ and the increase in weight of the bell due to its rise when expired air is collected is automatically counterpoised by water running through a siphon from the spirometer tank to the counterpoise tube. In the first two experiments the siphon tube was not used, but the counterpoise tube was three-quarters full of water.

The methods of obtaining the various measurements were the same as in the previous comparison. The subjects used for the preceding series of experiments were subjects in this series, and one experiment was also made with a third subject. Thus two of the subjects were accustomed to both the Mueller and the Tissot valves. The third subject, J. H. H., had had no previous experience with the Mueller valves, but had been used in a number of experiments with the Tissot valves. The statisties of the seven experiments in this series follow. 


\section{STATISTICS OF EXPERIMENTS.}

W. J. T., A pril 5, 1913.-Mueller valves, 4 periods; Tissot valves, 3 periods; preliminary period, 43 minutes; periods with two types of valves in series. Counterpoise tube three-quarters full of water; no water running in siphon tube. Subject stated he noted no difference in inspiration and expiration with Mueller valves. Pulse-rate regular in all periods. Average respiration-rate in preliminary period, 18 to 19 per minute. Respiration-rate irregular in all periods, particularly in first period with Tissot valves. Average barometric pressure, $758.4 \mathrm{~mm}$.; average temperature of air in apparatus, $16.2^{\circ} \mathrm{C}$.

W.J.T., A pril 12, 1913.-Mueller valves, 3 periods; Tissot valves, 3 periods; preliminary period, 36 minutes; periods with two types of valves alternating. Counterpoise of spirometer two-thirds full of water; no water running in siphon tube. Subject stated that the breathing was easier with the Tissot valves than with the Mueller valves. He was drowsy at times. Pulse-rate fairly uniform. Average respiration-rate in preliminary period, 20 per minute; regular throughout each period; character of respiration can not be distinguished, as the pneumograph did not work properly. Average barometric pressure, $760.7 \mathrm{~mm}$.; average temperature of air in apparatus, $16.7^{\circ} \mathrm{C}$.

W. J. T., A pril 26, 1913.-Mueller valves, 2 periods; Tissot valves, 3 periods, preliminary period, 42 minutes; periods with two types of valves alternating. Subject stated that he found it easier to breathe through Mueller valves than through the Tissot valves. Pulse-rate fairly uniform. Normal respiration-rate before experiment, 20 per minute. Respirationrate during experiment regular in rate and character. Average barometric pressure, $762.9 \mathrm{~mm}$.; average temperature of air in apparatus, $19.4^{\circ} \mathrm{C}$.

J. J. G., A pril 8, 1913.-Tissot valves, 3 periods; Mueller valves, 2 periods; preliminary period, 1 hour 20 minutes; periods with two types of valves in series. Pulse-rate very uniform throughout experiment. Normal respirationrate before experiment, 17 per minute, records being taken for 1 hour previous to experimental period. Respiration in experiment uniform in rate; character could not be distinguished, as pneumograph did not work properly. Barometric pressure, $763.1 \mathrm{~mm}$.; average temperature of air in apparatus, $15.6^{\circ} \mathrm{C}$.

J. J. G., A pril 15, 1913.-Mueller valves, 3 periods; Tissot valves, 3 periods; preliminary period, 1 hour; periods with two types of valves alternating. Pulse-rate uniform in all of the periods. Average normal respiration-rate before experiment, 19 per minute; during experiment uniform in each period. Average barometric pressure, $760.5 \mathrm{~mm}$.; average temperature of air in apparatus, $17.0^{\circ} \mathrm{C}$.

J.J. G., A pril 22, 1918.-Mueller valves, 3 periods; Tissot valves, 3 periods; preliminary period, 30 minutes; periods with two types of valves alternating. Subject stated that he could see no difference in the two types of valves. Pulse-rate fairly uniform throughout experiment. Average normal respiration before experiment, 19 per minute; during experiment, fairly uniform in rate and character in each period. Average barometric pressure, $763.8 \mathrm{~mm}$.; average temperature of air in apparatus, $17.4^{\circ} \mathrm{C}$.

J. H. H., A pril 18, 1913.-Mueller valves, 3 periods; Tissot valves, 3 periods; preliminary period, 16 minutes; periods with two types of valves

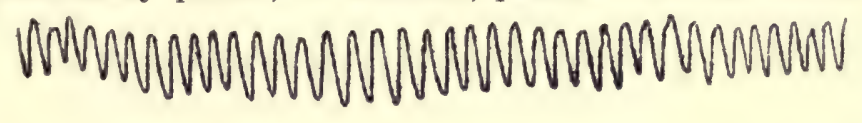

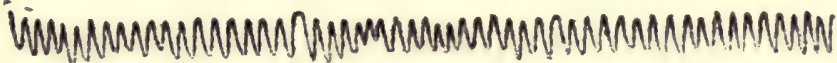

FrG. 55.-Type of respiration of subject J. H. H. in the fourth and fifth periods on April 18, 1913. Upper curve, Mueller valves; lower curve, Tissot valves. Original size. 
alternating. Subject found breathing with Mueller valves more difficult. Pulse-rate fairly uniform, except in first period with Tissot valves, when it varied from 54 to 61 . Average normal respiration-rate before experiment, 19 per minute. Rate during individual periods uniform, but differed with the two types of valves. The character of the respiration for the two methods of breathing is shown in figure 55, in which portions of the curves obtained for periods 4 and 5 are given. Average barometric pressure, $762.4 \mathrm{~mm}$.; average temperature of air in apparatus, $17.1^{\circ} \mathrm{C}$.

\section{DISCUSSION OF RESULTS.}

The results of the comparison experiments with the Mueller valves and the Tissot valves are given in table 37. The general averages show that the respiratory exchange is almost identical with the two types of valves. The volume per respiration is noticeably higher with the Mueller valves, this being accounted for in part by the lower respiration rate and the larger dead space with those valves.

TABLE 37.-Respiratory exchange in comparison experiments with Tissot valves and Mueller valves using Tissot spirometer. (Without food.)

\begin{tabular}{|c|c|c|c|c|c|c|c|c|c|}
\hline \multirow{2}{*}{$\begin{array}{c}\text { Subject, date, method, } \\
\text { and time. }\end{array}$} & \multirow{2}{*}{ 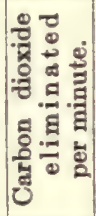 } & \multirow{2}{*}{ 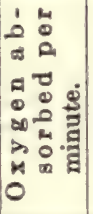 } & \multirow{2}{*}{ 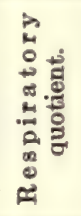 } & \multirow{2}{*}{ 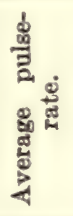 } & \multirow{2}{*}{ 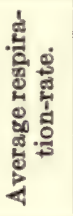 } & \multirow{2}{*}{ 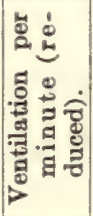 } & \multirow{2}{*}{ 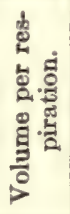 } & \multicolumn{2}{|c|}{$\begin{array}{l}\text { Composition of } \\
\text { expired air. }\end{array}$} \\
\hline & & & & & & & & $\begin{array}{l}\text { Carbon } \\
\text { dioxide. }\end{array}$ & Oxygen. \\
\hline $\begin{array}{c}\text { W.J.T. } \\
\text { Apr. } 5,1913:\end{array}$ & & & & & & & & & \\
\hline $\begin{array}{l}\text { Mueller valves: } \\
\qquad 8^{\mathrm{h}} 48^{\mathrm{m}} \mathrm{a} . \mathrm{m}\end{array}$ & $\begin{array}{l}\text { c.c. } \\
208\end{array}$ & $\begin{array}{l}\text { c.c. } \\
274\end{array}$ & 0.760 & 75.0 & 16.9 & $\begin{array}{l}\text { liters. } \\
6.20\end{array}$ & $\begin{array}{l}c . c . \\
445\end{array}$ & $\begin{array}{l}\text { p. ct. } \\
3.39\end{array}$ & $\begin{array}{l}\text { p. ct. } \\
16.74\end{array}$ \\
\hline $\begin{array}{lll}9 & 12 & 3 . \mathrm{m}\end{array}$ & 206 & 270 & .760 & 75.0 & 18.8 & 6.14 & 396 & 3.38 & 16.76 \\
\hline 937 a. m. & 212 & 272 & .775 & 71.5 & 18.6 & 6.32 & 412 & 3.38 & 16.83 \\
\hline 1002 a. m. & 219 & 277 & .790 & 69.5 & 20.3 & 6.76 & 404 & 3.27 & 17.02 \\
\hline Average........ & 211 & 273 & .775 & 73.0 & 18.7 & 6.36 & 414 & 3.36 & 16.84 \\
\hline $\begin{array}{l}\text { Tissot valves: } \\
10^{\mathrm{h}} 30^{\mathrm{m}} \text { a. } \mathrm{m} .\end{array}$ & 199 & 262 & .760 & 67.0 & 19.3 & 5.53 & 348 & 3.63 & 16.44 \\
\hline 1056 a.m. & 204 & 264 & 770 & 63.0 & 25.2 & $\begin{array}{l}0.03 \\
6.04\end{array}$ & $\begin{array}{l}290 \\
291\end{array}$ & $\begin{array}{l}3.03 \\
3.41\end{array}$ & 16.77 \\
\hline 1118 a.m. & 203 & 259 & .785 & 61.0 & 24.3 & 5.82 & 290 & 3.52 & 16.70 \\
\hline Average........ & 202 & 262 & .770 & 63.5 & 22.9 & 5.80 & 310 & 3.52 & 16.64 \\
\hline $\begin{array}{l}\text { Apr. 12, 1913: } \\
\text { Mueller valves: }\end{array}$ & & & & & & & & & \\
\hline 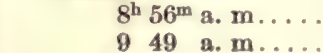 & $\begin{array}{l}203 \\
206\end{array}$ & $\begin{array}{l}261 \\
262\end{array}$ & $\begin{array}{l}.775 \\
.785\end{array}$ & $\begin{array}{l}68.0 \\
61.0\end{array}$ & $\begin{array}{l}18.6 \\
19.4\end{array}$ & $\begin{array}{l}6.19 \\
6.28\end{array}$ & $\begin{array}{l}402 \\
391\end{array}$ & $\begin{array}{l}3.32 \\
3.31\end{array}$ & $\begin{array}{l}16.91 \\
16.96\end{array}$ \\
\hline 1037 a. m.. & 218 & 271 & .805 & 59.0 & 19.0 & 6.66 & 424 & 3.31 & 17.03 \\
\hline Average......... & 209 & 265 & .790 & 62.5 & 19.0 & 6.38 & 406 & 3.31 & 16.97 \\
\hline $\begin{array}{l}\text { Tissot valves: } \\
9^{\mathrm{h}} 27^{\mathrm{m}} \text { a. } \mathrm{m} .\end{array}$ & 204 & 260 & .785 & 62.5 & 24.0 & 6.03 & 303 & 3.42 & 16.83 \\
\hline 1012 a. $\mathrm{m}$. & 198 & 255 & .775 & 60.0 & 24.2 & 6.09 & 304 & 3.29 & 16.95 \\
\hline 1102 a. m. & 211 & 256 & .825 & 61.5 & 26.4 & 6.88 & 315 & 3.10 & 17.36 \\
\hline Average........ & 204 & 257 & .795 & 61.5 & 24.9 & 6.33 & 307 & 3.27 & 17.05 \\
\hline $\begin{array}{l}\text { Apr. 26, 1913: } \\
\text { Mueller valves: }\end{array}$ & & & & & & & & & \\
\hline $\begin{array}{l}9 \\
9\end{array} 44$ a. $m$... & 204 & $\begin{array}{l}249 \\
249\end{array}$ & .835 & $\begin{array}{l}60.5 \\
56.5\end{array}$ & $\begin{array}{l}16.9 \\
16.4\end{array}$ & $\begin{array}{l}6.20 \\
6.02\end{array}$ & $\begin{array}{l}442 \\
442\end{array}$ & $\begin{array}{l}3.38 \\
3.41\end{array}$ & $\begin{array}{l}17.07 \\
16.97\end{array}$ \\
\hline Average......... & 206 & 249 & .825 & 58.0 & 16.7 & 6.11 & 448 & 3.40 & 17.08 \\
\hline 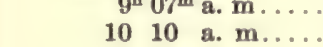 & $\begin{array}{l}212 \\
207\end{array}$ & 251 & .805 & $\begin{array}{l}57.0 \\
57.5\end{array}$ & $\begin{array}{l}18.0 \\
19.8\end{array}$ & $\begin{array}{l}5.80 \\
5.77\end{array}$ & 351 & $\begin{array}{l}3.08 \\
3.61\end{array}$ & $\begin{array}{l}16.81 \\
16.75\end{array}$ \\
\hline 1106 a. m. & (134) & (167) & .800 & 53.5 & 23.2 & 6.15 & 320 & $(2.21)$ & $(18.33)$ \\
\hline Average...... & 210 & 249 & .845 & 57.0 & 20.5 & 5.91 & 350 & 3.65 & 16.78 \\
\hline
\end{tabular}


TABLE 37.-Respiratory exchange in comparison experiments with Tissot valves and Mueller valves using Tissot spirometer. (Without food.) - Continued.

\begin{tabular}{|c|c|c|c|c|c|c|c|c|c|}
\hline \multirow{2}{*}{$\begin{array}{l}\text { Subject, date, method, } \\
\text { and time. }\end{array}$} & \multirow{2}{*}{ 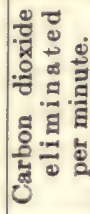 } & \multirow{2}{*}{ 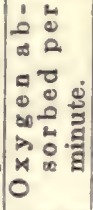 } & \multirow{2}{*}{ 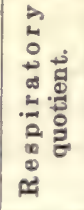 } & \multirow{2}{*}{ 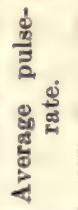 } & \multirow{2}{*}{ 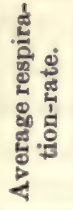 } & \multirow{2}{*}{ 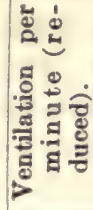 } & \multirow{2}{*}{ 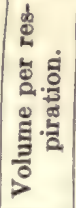 } & \multicolumn{2}{|c|}{$\begin{array}{l}\text { Composition of } \\
\text { expired air. }\end{array}$} \\
\hline & & & & & & & & $\begin{array}{l}\text { Carbon } \\
\text { dioxide. }\end{array}$ & Oxygen \\
\hline $\begin{array}{l}\text { J. J. G. } \\
\text { Apr. 8, 1913: }\end{array}$ & & & & & & & & & \\
\hline $\begin{array}{l}\text { Tissot valves: } \\
9^{\mathrm{h}} 50^{\mathrm{m}} \text { a. } \mathrm{m}\end{array}$ & $\begin{array}{l}\text { c.c. } \\
182\end{array}$ & $\begin{array}{l}\text { c.c. } \\
229\end{array}$ & 0.795 & 54.0 & 23.4 & $\begin{array}{l}\text { liters. } \\
6.03\end{array}$ & $\begin{array}{l}\text { c.c. } \\
310\end{array}$ & $\begin{array}{l}\text { p. ct. } \\
\text { 3.05 }\end{array}$ & $\begin{array}{l}\text { p. ct. } \\
17.31\end{array}$ \\
\hline 1013 a. m. & 165 & 202 & .820 & 53.5 & 18.6 & 4.84 & 313 & 3.44 & 16.93 \\
\hline 1045 a. $\mathrm{m}$ & 162 & 194 & .835 & 54.0 & 24.2 & 5.52 & 275 & 2.97 & 17.54 \\
\hline Average.... & 170 & 208 & .815 & 54.0 & 22.1 & 5.46 & 299 & 3.15 & 17.26 \\
\hline Mueller valves: & & & & & & & & & \\
\hline $11^{\mathrm{h}} 0$ & $\begin{array}{l}166 \\
163\end{array}$ & $\begin{array}{l}195 \\
194\end{array}$ & .850 & 54.0 & 15.9 & 5.04 & 382 & $\begin{array}{l}3.32 \\
3.31\end{array}$ & 17.19 \\
\hline $\begin{array}{r}1132 \text { a. } n \\
\text { Average..... }\end{array}$ & $\begin{array}{l}163 \\
165\end{array}$ & $\begin{array}{l}194 \\
195\end{array}$ & .845 & $\begin{array}{l}54.0 \\
54.0\end{array}$ & 15.5 & 4.98 & 387 & $\begin{array}{l}3.31 \\
3.39\end{array}$ & \\
\hline $\begin{array}{l}\text { Apr. 15, 1913: } \\
\text { Mueller valves: }\end{array}$ & & & & & & & & & \\
\hline $\begin{array}{c}g^{n} 20^{\mathrm{min}} \text { a. } n \\
10 \quad 10 \text { a. } n\end{array}$ & $\begin{array}{l}166 \\
171\end{array}$ & $\begin{array}{l}210 \\
214\end{array}$ & $\begin{array}{l}.790 \\
.800\end{array}$ & $\begin{array}{l}57.0 \\
59.5\end{array}$ & $\begin{array}{l}14.9 \\
13.7\end{array}$ & $\begin{array}{l}4.93 \\
4.91\end{array}$ & $\begin{array}{l}399 \\
433\end{array}$ & $\begin{array}{l}3.40 \\
3.51\end{array}$ & $\begin{array}{l}16.86 \\
16.77\end{array}$ \\
\hline 1100 a. m & 157 & 202 & .775 & 55.5 & 13.9 & 4.64 & 404 & 3.41 & 16.79 \\
\hline Average..... & 165 & 209 & .790 & 57.5 & 14.2 & 4.83 & 412 & 3.44 & 16.81 \\
\hline $\begin{array}{r}\text { Tissot valves: } \\
9^{\mathrm{h}} 44^{\mathrm{m}} \mathrm{a} .\end{array}$ & & & & & & & & & \\
\hline $\begin{array}{lll}10 & 37 & \text { a. } m\end{array}$ & $\begin{array}{l}172 \\
163\end{array}$ & $\begin{array}{l}206 \\
211\end{array}$ & .835 & $\begin{array}{l}57.0 \\
56.0\end{array}$ & 20.8 & $\begin{array}{l}5.42 \\
5.64\end{array}$ & $\begin{array}{l}315 \\
309\end{array}$ & & \\
\hline 1124 a. $\mathrm{m}$ & 175 & 215 & .815 & 56.0 & 19.1 & 5.25 & 333 & 3.3 & 17. \\
\hline Average...... & 170 & 211 & .805 & 56.5 & 20.7 & 5.44 & 319 & 3.17 & 17.22 \\
\hline $\begin{array}{l}\text { Apr. 22, 1913: } \\
\text { Mueller valves: }\end{array}$ & & & & & & & & & \\
\hline $9^{\mathrm{h}} 05^{\mathrm{m}} \mathrm{a}$ & 173 & 206 & .840 & 54.5 & 13.9 & 5.10 & 441 & 3.42 & 17.04 \\
\hline 1002 a. $n$ & 163 & 196 & .830 & 48.5 & 14.8 & 4.98 & 405 & & \\
\hline $\begin{array}{lll}10 & 57 \quad \text { a. }\end{array}$ & 182 & 215 & .845 & 55.0 & 14.0 & 5.26 & 453 & 3.4 & 16.98 \\
\hline Average ........ & 173 & 206 & .840 & 52.5 & 14.2 & 5.11 & 493 & 3.40 & 17.06 \\
\hline Tissot valves & & & & & & & & & \\
\hline & 165 & 196 & .840 & 54.0 & 15.4 & 4.68 & 366 & & 16.89 \\
\hline 1030 a. $n$ & 173 & 195 & .885 & 52.0 & 15.6 & 4.84 & 373 & 3.8 & 17. \\
\hline 1124 a. $\mathrm{m}$ & 189 & 211 & .895 & 53.5 & 20.7 & 6.22 & 362 & & \\
\hline Average.... . & 176 & 201 & .875 & 53.0 & 17.2 & 5.25 & 367 & 3.41 & 17.17 \\
\hline $\begin{array}{c}J . H . H . \\
\text { Apr. 18, 1913: } \\
\text { Mueller valves: } \\
8^{\mathrm{h}} 46^{\mathrm{m}} \text { a. } \mathrm{m}\end{array}$ & & & & & & & & & \\
\hline $\begin{array}{c}8^{n} 46^{\mathrm{m}} \mathrm{a} \text {. } \\
10 \mathrm{a} \text {. }\end{array}$ & $\begin{array}{l}223 \\
212\end{array}$ & $\begin{array}{l}236 \\
238\end{array}$ & $\begin{array}{l}.945 \\
.890\end{array}$ & $\begin{array}{l}58.0 \\
54.5\end{array}$ & $\begin{array}{r}9.4 \\
10.9\end{array}$ & $\begin{array}{l}6.81 \\
6.25\end{array}$ & $\begin{array}{l}873 \\
691\end{array}$ & & \\
\hline 1053 a. m. & 202 & 232 & .870 & 56.0 & 10.3 & 5.80 & 680 & & \\
\hline Average......... & 212 & 235 & .900 & 56.0 & 10.2 & 6.29 & 748 & 3.48 & 17.26 \\
\hline Tissot valves: & & & & & & & & & \\
\hline & 193 & 243 & .795 & 57.0 & 19.0 & 5.54 & 351 & 3.52 & \\
\hline 1028 a. 1 & 18 & 24 & .7 & 55.0 & & 4.95 & 347 & & \\
\hline 1121 a. $n$ & & 212 & .830 & & 17.7 & 5.36 & 366 & & \\
\hline Average...... & 184 & 232 & .795 & 56.5 & 18.0 & 5.28 & 355 & 3.53 & 16.73 \\
\hline $\begin{array}{l}\text { Arithmetical average of all } \\
\text { experiments with Muel- } \\
\text { ler valves............ }\end{array}$ & 192 & 233 & .825 & $\mathbf{5 9 . 0}$ & 15.5 & 5.73 & 463 & 3.38 & 17.02 \\
\hline $\begin{array}{l}\text { Anthmetical average of all } \\
\text { experiments with Tissot } \\
\text { valves....................... }\end{array}$ & 188 & 231 & .815 & 57.5 & 20.9 & 5.64 & 330 & 3.39 & 16.98 \\
\hline
\end{tabular}


The differences between the average results obtained in each experiment for the two types of valves are given in table 38 , the values for the Tissot valves being used as the basis of calculation. Considering the individual comparisons, it will be seen that the variations are not very large and are usually in one direction. For example, with W. J. T., two experiments show that the respiratory exchange with the Mueller valves is somewhat higher than with the Tissot valves, while the respiratory quotient is practically the same. The average respiration-rate, however, is lower with the Mueller valves; in two instances the respiration-rate with the Mueller valves is nearer the normal rate of 19 to 20 per minute than with the Tissot valves. The volume per respiration is noticeably higher with the Mueller valves. A peculiarity in the breathing of this subject was that the rate gradually increased during the morning and unfortunately, in the first comparison experiment with him, the periods with the two types of valves were not alternated. It is therefore somewhat difficult to decide whether the

TABLE 38.-Variations of average results obtained with Mueller valves from the average results obtained with Tissot valves.

\begin{tabular}{|c|c|c|c|c|c|c|c|c|}
\hline Subject. & Date. & $\begin{array}{l}\text { Carbon } \\
\text { dioxide } \\
\text { elimin- } \\
\text { ated per } \\
\text { minute. }\end{array}$ & $\begin{array}{c}\text { Oxygen } \\
\text { absorbed } \\
\text { per } \\
\text { minute. }\end{array}$ & $\begin{array}{l}\text { Respira- } \\
\text { tory } \\
\text { quotient. }\end{array}$ & $\begin{array}{c}\text { Average } \\
\text { pulse- } \\
\text { rate. }\end{array}$ & $\begin{array}{l}\text { Average } \\
\text { respira- } \\
\text { tion-rate. }\end{array}$ & $\begin{array}{l}\text { Ventila- } \\
\text { tion per } \\
\text { minute } \\
\text { (reduced). }\end{array}$ & $\begin{array}{l}\text { Volume } \\
\text { per } \\
\text { respira- } \\
\text { tion. }\end{array}$ \\
\hline $\begin{array}{l}\text { W. J. T.... } \\
\text { J. J. G.... }\end{array}$ & $\begin{array}{lr}2 & 1913 \\
\text { Apr. } & 5 \\
\text { Apr. } & 12 \\
\text { Apr. } & 26 \\
\text { Apr. } & 8 \\
\text { Apr. } & 15 \\
\text { Apr. } & 22 \\
\text { Apr. } & 18\end{array}$ & $\begin{array}{l}\text { c.c. } \\
+9 \\
+5 \\
-4 \\
-5 \\
-5 \\
-3 \\
+28\end{array}$ & $\begin{array}{r}c . c . \\
+11 \\
+\quad 8 \\
0 \\
-13 \\
-\quad 2 \\
+5 \\
+3\end{array}$ & $\begin{array}{l}+0.005 \\
-\quad .005 \\
-\quad .02 \\
+.03 \\
-.015 \\
-.035 \\
+.105\end{array}$ & $\begin{array}{c}+9.5 \\
+1.0 \\
+1.0 \\
0 \\
+1 \\
-0.5 \\
-0.5\end{array}$ & $\begin{array}{l}-4.2 \\
-5.9 \\
-3.8 \\
-6.4 \\
-6.5 \\
-3.0 \\
-7.8\end{array}$ & $\begin{array}{r}\text { liters. } \\
+0.56 \\
+\quad .05 \\
+\quad .20 \\
-\quad .45 \\
-\quad .61 \\
-\quad .14 \\
+1.01\end{array}$ & $\begin{array}{r}c . c . \\
+104 \\
+99 \\
+92 \\
+86 \\
+93 \\
+66 \\
+393\end{array}$ \\
\hline \multicolumn{2}{|c|}{ Average variation.... } & 8 & 6 & 0.03 & 2.0 & 5.3 & 0.43 & 133 \\
\hline
\end{tabular}

decreased metabolism shown by this subject with the Tissot valves is due to the valves themselves or to the fact that the subject became quieter as the experiment continued, with a consequent lowering of metabolism in the latter part of the morning. The first two comparison experiments indicate that the respiratory exchange was higher with the Mueller valves, while on April 26 the metabolism was practically the same with both types of valves.

On the other hand, the results of the experiments with J. J. G. indicate that the respiratory exchange is lower with the Mueller valves than with the Tissot valves, although the differences in the respiratory exchange with the two types of valves are not very large. In two cases the normal respiration-rate for this subject was approached by the respiration-rate with the Tissot valves. The comparison experiment with J. H. H. shows a decidedly different value in the amount of carbondioxide elimination, that with the Mueller valves being very much 
higher. This subject apparently did not breathe normally with the Mueller valves, the respiration-rate being only about 10 per minute, while the normal rate for J. H. H. on the same day was 19 per minute. This fact, together with the larger total ventilation, indicates that the effective ventilation of the lungs was greater with the Mueller valves than with the Tissot valves; consequently more carbon dioxide would be eliminated with the former valves.

The percentage variation of each individual period from the average of the experiment has been calculated for the values for each apparatus

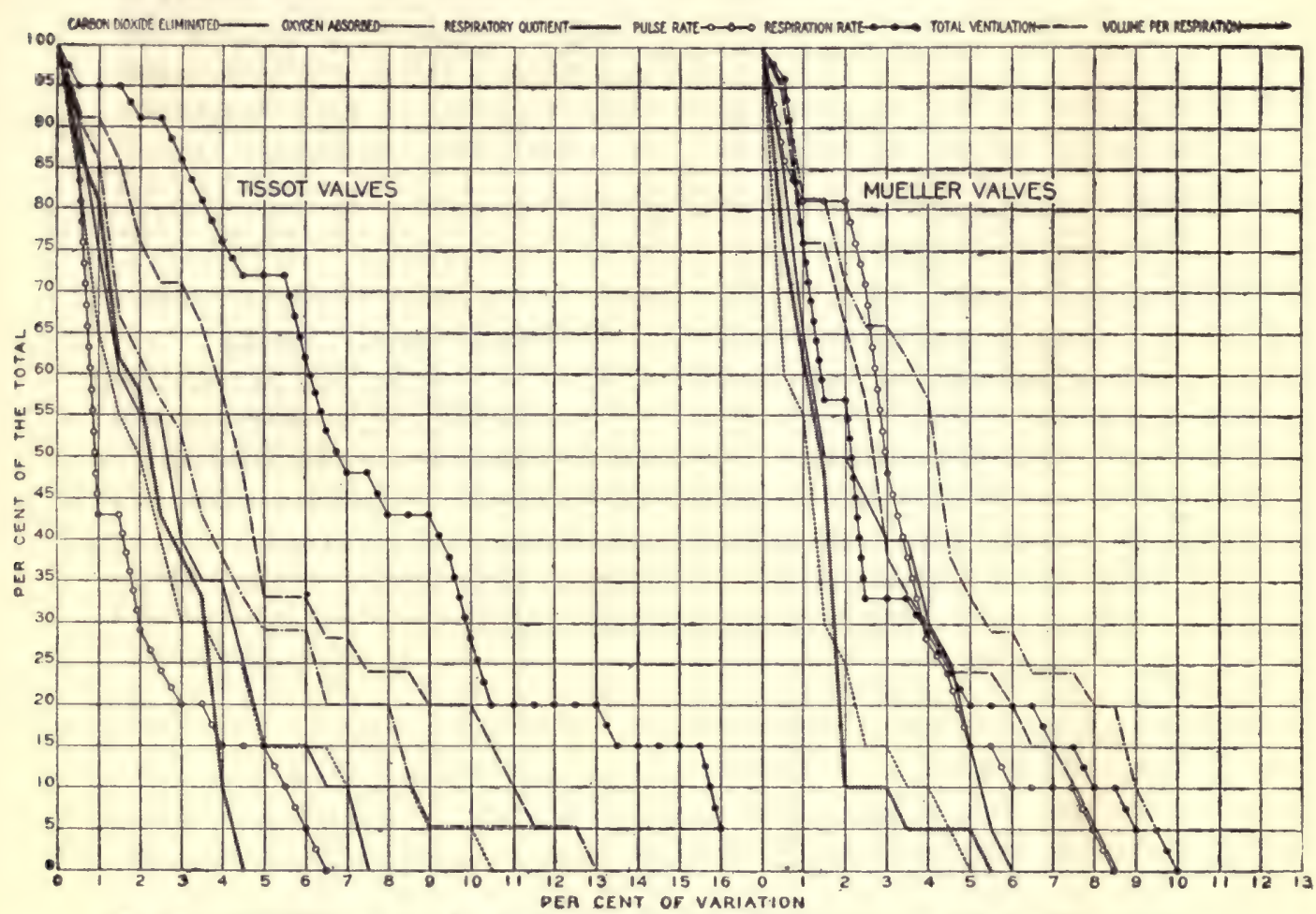

FIG. 56.-Probability curves for the series of comparison experiments with the Tissot valves and the Mueller valves.

The ordinates indicate the percentage of the total number of periods and the abscisser represent the percentage of variation from the average.

and the results given in the form of curves in figure 56. The carbondioxide elimination has about the same uniformity with both sets of valves, while the oxygen consumption, respiratory quotient, and respiration-rate are somewhat more uniform with the Mueller valves. The pulse-rate, however, is more uniform with the Tissot valves. There is not a very marked difference in the uniformity of results with either the total ventilation or the volume per respiration.

In general, it may be stated as a result of this series of comparisons and the one preceding, that it is possible to obtain entirely normal 
results with the Mueller valves. This is particularly true with subjects who have been trained in the use of the valves. This was shown by the fact that much more satisfactory results were obtained with W.J.T. and J. J. G. in the second series of experiments after they had become accustomed to the valves in the first series of experiments.

\section{BENEDICT RESPIRATION APPARATUS (SPIROMETER UNIT) WITH AND WITHOUT ADDITIONAL DEAD SPACE.}

In all apparatus employed for the determination of the respiratory exchange, when the subject is not inside a chamber, there is a volume of dead air which must be swept out at each respiration before fresh air can reach the respiratory tract of the subject. When inspiratory and expiratory valves are used, generally that part of the connecting tee-piece which is nearest the subject is filled with expired air at each expiration, and this must be replaced by fresh air. In a closed-circuit apparatus without valves there is likewise a dead space between the respiratory tract of the subject and the moving current of air inside the apparatus. The only exception to this rule is when a person inhales through the mouth and exhales through the nose, or vice versa.

In the construction and arrangement of all respiration apparatus, the attempt is always made to reduce the dead space as much as possible, for it has been assumed that marked increase in the dead space would result in such a disturbance in the respiratory exchange that the results would not represent the true values.

While in the construction of the Benedict universal respiration apparatus every effort was made to minimize the dead space between the subject and the moving current of air, in some of the experiments with $\mathrm{H}$. F. T. it became necessary to lengthen it in order that he might lie on his side.

The effect of thus varying the dead space between the subject and the moving current of air was accordingly studied with the spirometer unit in a considerable number of experiments. In this study the respiratory exchange with the normal dead space was compared with the results obtained when the dead space was arbitrarily increased by inserting a piece of rubber tubing of about $20 \mathrm{~mm}$. internal diameter between the three-way valve and the nosepieces or mouthpiece, varying the length of the rubber tubing as desired. The experiments were made in four series, the increase in the dead space being 45, 90, 135, and 224 c.c. respectively. There was usually a 5 -minute preliminary period of breathing through the nosepieces before the experimental period itself began.

The pulse-rate was recorded by means of the Bowles stethoscope; a graphic record of the respiration was obtained from the movements of the spirometer bell, and in many of the experiments an additional record was obtained with the chest pneumograph. In practically all of the experiments a record of the muscular activity was secured by a 
pneumograph fastened about the hips. The subjects were all members of the Laboratory force and, with the exception of W. F. O'H., were more or less trained subjects.

The statistics of the 13 experiments are given in the following pages. All of these experiments were made by Mr. P. F. Jones, whose assistance in this portion of the investigation I wish to acknowledge.

STATISTICS OF EXPERIMENTS WITH AN INCREASE IN DEAD SPACE OF 45 C.C.

J. K. M., September 20, 1912.-Without dead space, 3 periods; with dead space, 3 periods; first, second, and fourth periods without dead space, remaining periods with dead space. New form of glass nosepieces used (see page 62). Subject noted no difference between the periods, so far as ease of respiration was concerned, but did not like the glass nosepieces. Pulse-rate fairly regular. Respiration for the most part regular; slightly more regular in the periods with increased dead-air space than in those without. Sections of records obtained with each condition of experimenting are given in figures 57 and 58 . Average barometric pressure, $758.9 \mathrm{~mm}$. ; average temperature of air in apparatus, $22.6^{\circ} \mathrm{C}$.

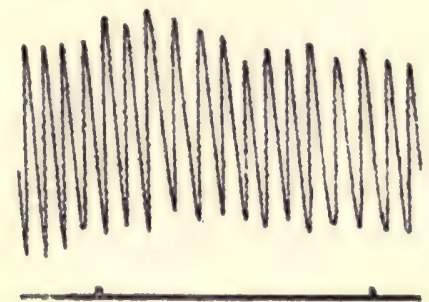

FIG. 57.

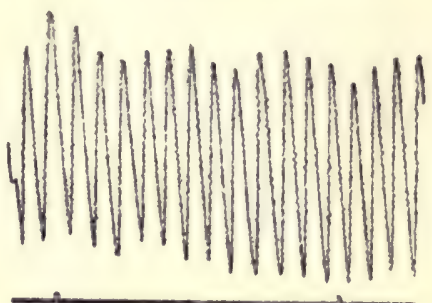

Fig. 58.

Fra. 57.-Type of respiration of subject J. K. M. without additional dead space on September 20, 1912. Original size.

Fro. 58.-Type of respiration of subject J. K. M. with 45 c.c. additional dead space on September 20, 1912. Original size.

J. B. T., September 23, 1912.-Without dead space, 4 periods; with dead space, 3 periods; first two periods without dead space, thereafter alternating. Subject reasonably quiet throughout experiment; increase in dead space did not seem to cause him any perceptible difficulty. Pulse-rate regular except in third and fourth periods without dead space. Respiration regular in rate and character in all of the periods. Average barometric pressure, $766.3 \mathrm{~mm}$.; average temperature of air in apparatus, $19.0^{\circ} \mathrm{C}$.

W.F. O'H., October 27, 1912.-Without dead space, 4 periods; with dead space, 3 periods; first two periods without dead space, thereafter alternating. Bandage used over subject's eyes. Subject stated that bandage made him somewhat more sleepy; when additional dead space was used he found it easier to breathe; with normal dead space he could inhale more easily, but there was some resistance in exhaling; he also stated that the vibration caused by the motor was less noticeable when the dead space was increased. Pulse-rate fairly regular in the individual periods. Respiration-rate in early part of experiment fairly regular, but in last three periods was irregular on account of subject's drowsiness; there were many periods of apnœea, and it was necessary for the observer to keep the subject awake. Sections of the records of respiration are given in figures 59 to 62 . The experiment was not particularly successful, owing to the wide variations in the degree of wakefulness of the subject. Average barometric pressure, $759.1 \mathrm{~mm}$; average temperature of air in apparatus, $20.0^{\circ} \mathrm{C}$. 


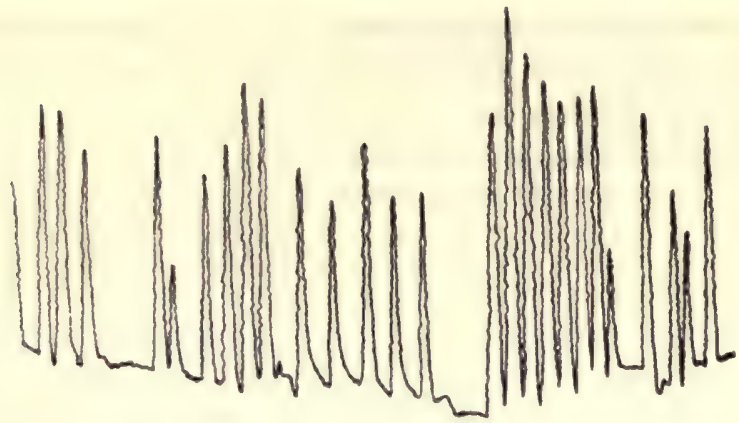

FIG. 59.-Type of respiration of subject W. F. O'H. in the third period with additional dead space on October 27, 1912. Original size.

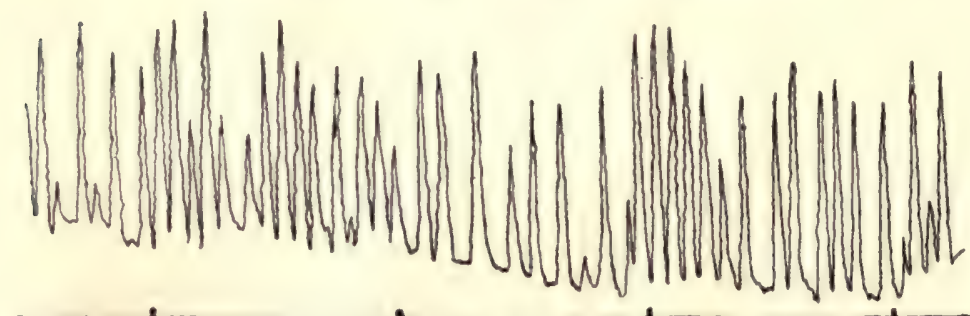

FIG. 60.-Type of respiration of subject W. F. O'H. at the end of the third period without additional dead space on Oetober 27,1912 . Original size.

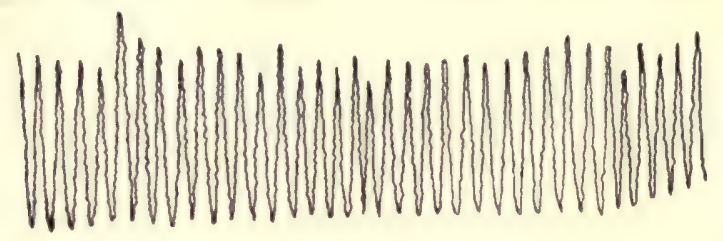

Fia. 61-Type of respiration of subject W. F. O'H. in the early part of the second period withou $t$ additional dead space on October 27, 1912. Original size.

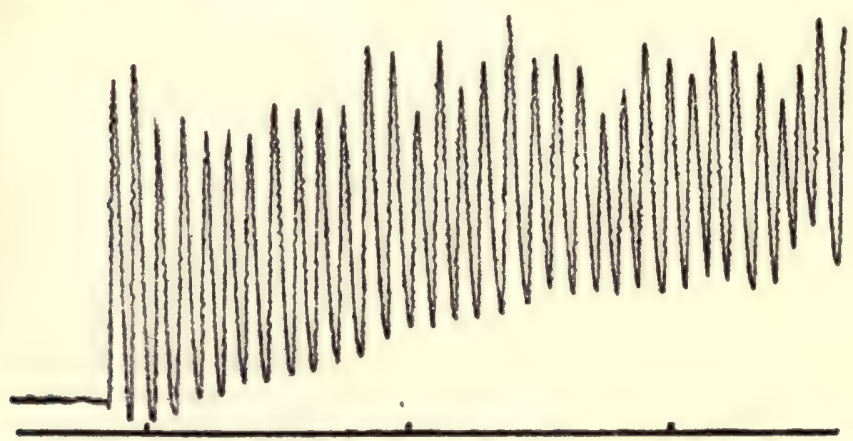

FrG. 62.-Type of respiration of subject W. F. O'H. at the beginning of the fourth period without additional dead space on October 27, 1912 . Original size. 
J. W. P., October 22, 1912.-Without dead space, 3 periods; with dead space, 3 periods; first, second, and fourth periods without dead space, remaining periods with dead space. Some difficulty was experienced in fitting the nosepieces closely to the nostrils of this subject; he was also somewhat active in second period with increased dead space. Pulse-rate fairly regular throughout experiment. Respiration irregular at times as to depth, although rate was regular; a portion of the respiration record for the second period with the dead space is given in figure 63. Average barometric pressure, $768.1 \mathrm{~mm}$.; average temperature of air in apparatus, $19.8^{\circ} \mathrm{C}$.

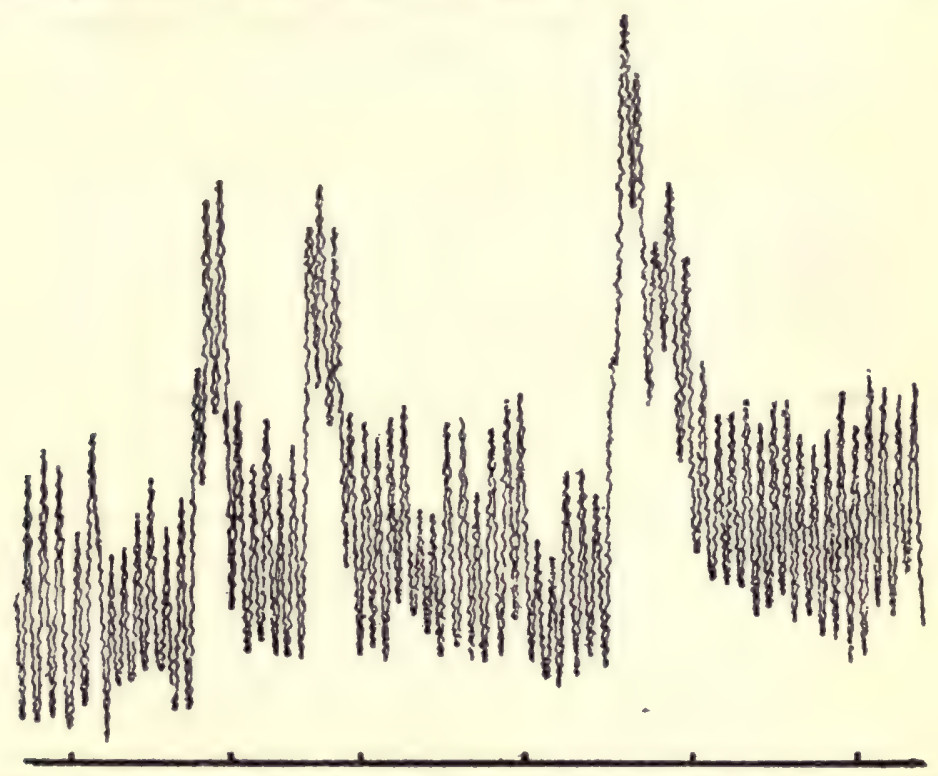

Fre. 63.-Type of respiration of subject J. W. P. in the second period with additional dead space on October 22, 1912. Original size.

STATISTICS OF EXPERIMENTS WITH AN INCREASE IN DEAD SPACE OF 90:C.C.

J. K. M., September 21, 1912.-Without dead space, 4 periods; with dead space, 3 periods; first two periods without dead space, thereafter alternating. Subject stated that he noted no difference in respiration with additional dead space; preferred pneumatic to glass nosepieces. Pulse-rate somewhat irregular in all periods, with wide variations in range. Respiration-rate regular in all periods, character being more regular in periods with dead space than in periods with normal dead space. Sections of the respiration record with each

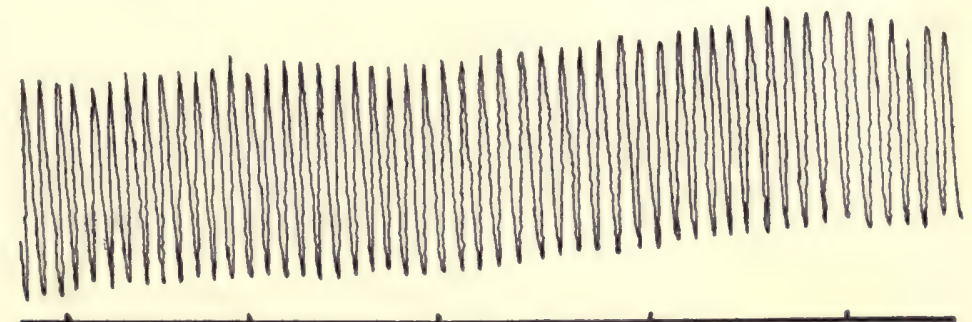

Fra. 64.-Type of respiration of subject J. K. M. with 90 c.c. additional dead space on September 21, 1912. Original size. 
type of respiration are given in figures 64 and 65 . Average barometric pressure, $766.8 \mathrm{~mm}$.; average temperature of air in apparatus, $19.4^{\circ} \mathrm{C}$.

J. K. M., October 31, 1912.-Without dead space, 3 periods; with dead space, 3 periods; first, second, and fourth periods without dead space, remaining periods with dead space. Subject noted no difference between the two conditions and was so unconscious of the change that he supposed one of the periods with an increased dead space to be a normal period. Pulse-rate varied somewhat in the different periods, the ranges varying from 6 to 15 beats per minute. The variations in pulse-rate were due to drowsiness and the necessity of waking subject occasionally. Respiration regular in all but fourth period. Average barometric pressure, $762.2 \mathrm{~mm}$.; average temperature of air in apparatus, $19.6^{\circ} \mathrm{C}$.

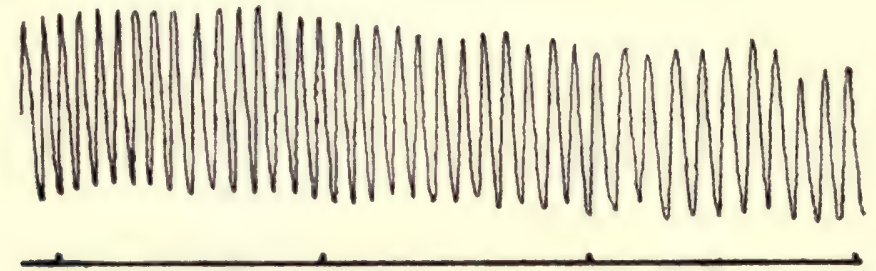

FIG. 65.-Type of respiration of subject J. K. M. without additional dead space on September 21, 1912. Original size.

J. B. T., October 26, 1912.-Without dead space, 3 periods; with dead space, 3 periods; first, second, and fourth periods without dead space, remaining periods with dead space. Subject somewhat active previous to third period with normal dead space, talking and moving about; this may account for the somewhat higher metabolism shown in that period. Respiration-rate regular, except in first and third normal periods; in the first period it decreased in rapidity toward the end; in the third normal period it was sometimes deep and slow, then rapid and shallow. Average barometric pressure, $753.8 \mathrm{~mm}$.; average temperature of air in apparatus, $18.9^{\circ} \mathrm{C}$.

J. B. T., November 1, 1912.- Without dead space, 3 periods; with dead space, 3 periods; first, second, and fourth periods without dead space, remaining periods with dead space. Subject noted no difference in breathing with the two conditions; he stated he was very comfortable throughout the experiment. Pulse-rate fairly uniform in all periods; respiration uniform in both character and rate in all periods. Average barometric pressure, $756.4 \mathrm{~mm}$.; average temperature of air in apparatus, $17.5^{\circ} \mathrm{C}$.

STATISTICS OF EXPERIMENTS WITH AN INCREASE IN DEAD SPACE OF 135 C.C.

T. M. C., November 8, 1912.-With dead space, 3 periods; without dead space, 3 periods; periods with the two methods alternating. New form of glass nosepieces used ${ }^{1}$ and tested for tightness with soapsuds. Pulse-rate in first period ranged from 68 to 76 ; in other periods it was uniform. Respiration both in depth and rate remarkably uniform in the individual periods. Subject said there was no difficulty in breathing, but the respiration was deeper than usual. Sections of the respiration records showing the two types of breathing are reproduced in figures 66 and 67 . Average barometric pressure, $747.8 \mathrm{~mm}$.; average temperature of air in apparatus, $20.3^{\circ} \mathrm{C}$.

P. F. J., November 7, 1912.-With dead space, 3 periods; without dead space, 3 periods; periods with and without additional dead space, alternating. 


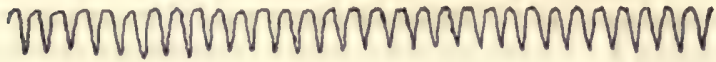

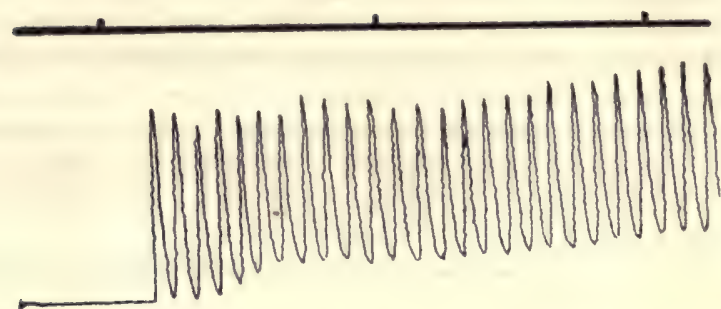

Frg. 66.-Type of respiration of subject T. M. C. without additional dead space on November

8, 1912. Upper curve, pneumograph record; lower curve, spirometer record. Original size.

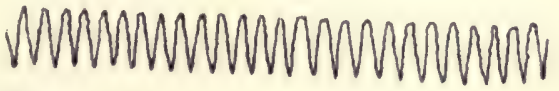

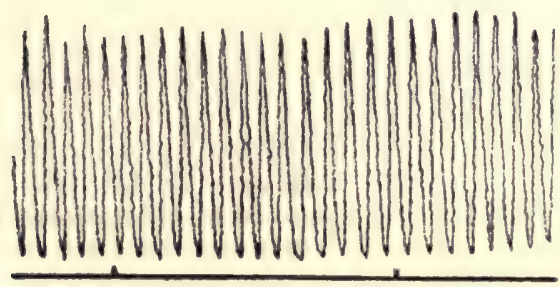

FIG. 67.-Type of respiration of subject T. M. C. with 135 c.c. additional dead space on November 8,1912 . Upper curve, pneumograph record; lower curve, spirometer record. Original size.
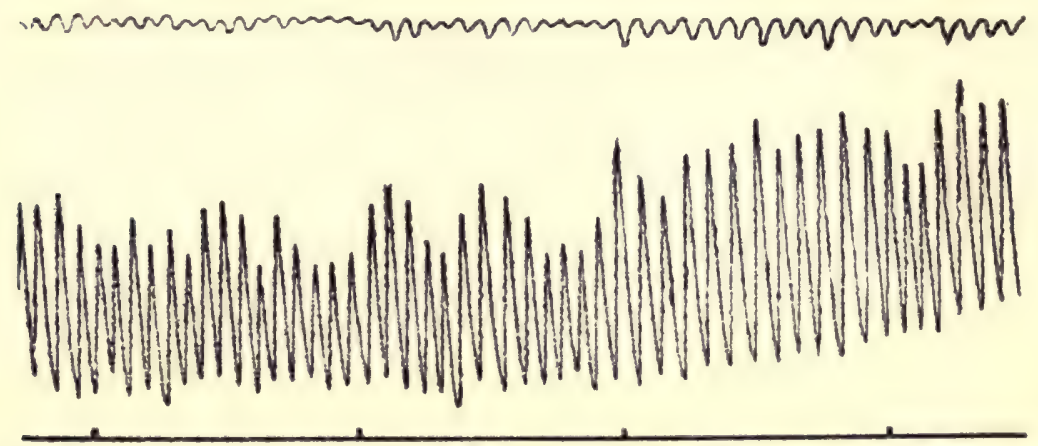

FrG. 68.-Type of respiration of subject P. F. J. without additional dead space on November 7. 1912. Upper curve, pneumograph record; lower curve, spirometer record. Original size. 
Subject drowsy in second and sixth periods; said he had some difficulty in breathing in first, fourth, and fifth periods, but in the others none at all. Was somewhat nervous, as he had not slept well the previous night. Pulserate uniform, except in fourth period, when the range was from 71 to $79^{\circ}$ Respiration in first period uniform, in second period uniform at first but later became more shallow; third period, uniform; fourth period, shallow in the middle of period; fifth period, uniform; sixth period, shallow at first, but deeper

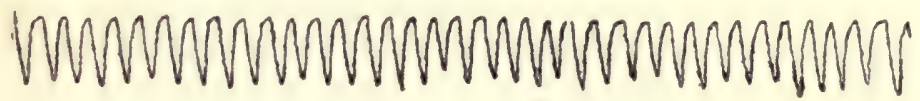

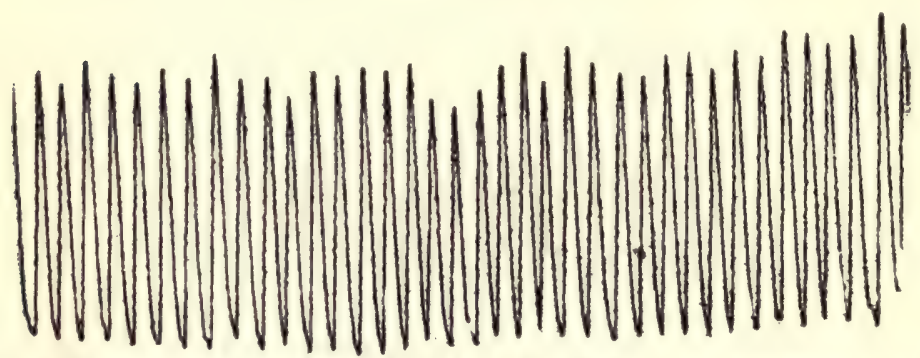

Fro. 69.-Type of respiration of subject P. F. J. with 135 c.c. additional dead space on November

7,1912 . Upper curve, pneumograph record; lower curve, spirometer record. Original size.

the last two-thirds of the period. Sections of the respiration curves obtained with the two methods are given in figures 68 and 69 . Average barometric pressure, $758.3 \mathrm{~mm}$.; average temperature of air in apparatus, $18.3^{\circ} \mathrm{C}$.

P. F. J., November 14, 1912.-Without dead space, 4 periods; with dead space, 4 periods; periods with and without additional dead space alternating. Subject stated that a desire to urinate during the last two or three periods

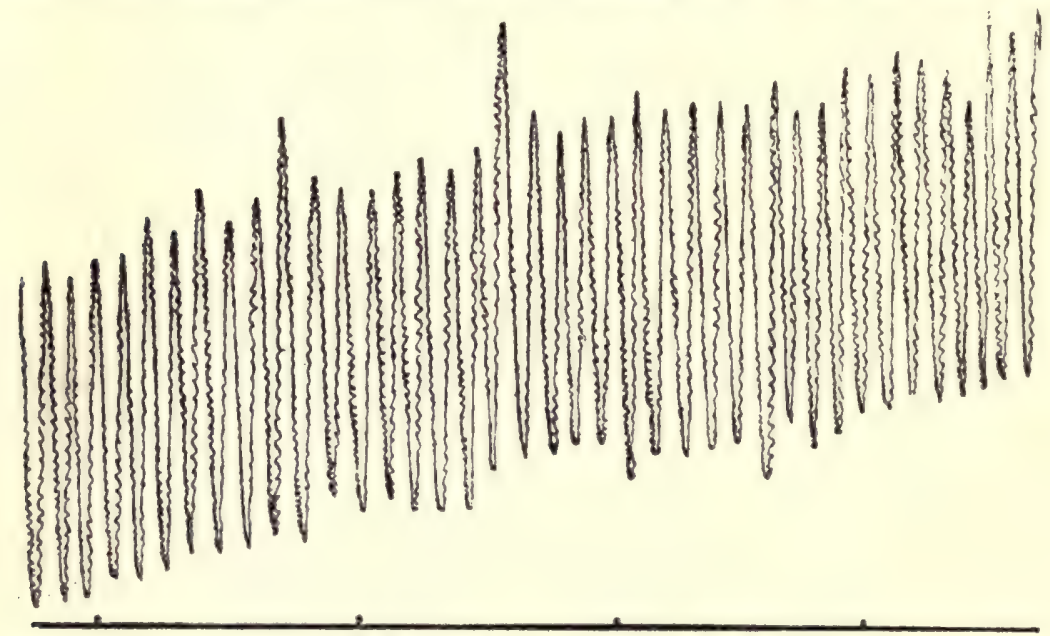

F1G. 70.-Type of respiration of subject J. B. T. with 224 c.c. additional dead space on December 7, 1912. Original size. 
distressed him, but otherwise he was perfectly comfortable. Pulse-rate very uniform in all of the periods. Respiration uniform in all periods, both as to rate and character. Average barometric pressure, $754.7 \mathrm{~mm}$.; average temperature of air in apparatus, $18.5^{\circ} \mathrm{C}$.

STATISTICS OF EXPERIMENTS WITH INCREASE IN DEAD SPACE OF 224 C.C.

J. K. M., December 3, 1912.-Without dead space, 3 periods; with dead space, 3 periods; periods with and without additional dead space alternating. New form of glass nosepieces used in normal periods; pneumatic nosepieces in periods with additional dead space. Subject very drowsy in normal periods. Pulse-rate somewhat variable in first four periods; uniform in last two periods. Respiration uniform in all periods, especially in the periods with the increased dead space. Average barometric pressure, $763.3 \mathrm{~mm}$.; average temperature of air in apparatus, $21.1^{\circ} \mathrm{C}$.

J. B. T., December 7, 1912.-Without dead space, 3 periods; with dead space, 3 periods; periods with and without additional dead space alternating. Pneumatic nosepieces used. Pulse-rate in first, second, third, and sixth periods uniform; in fourth period varied from 71 to 78 ; in fifth period, from 74 to 85 . Respiration very uniform in all periods, but subject said he had difficulty in breathing throughout the first period and again in the middle of the last period. Sections of the respiration curves are given in figures 70 and 71 . Average barometric pressure $759.7 \mathrm{~mm}$.; average temperature of air in apparatus $20.9^{\circ} \mathrm{C}$.

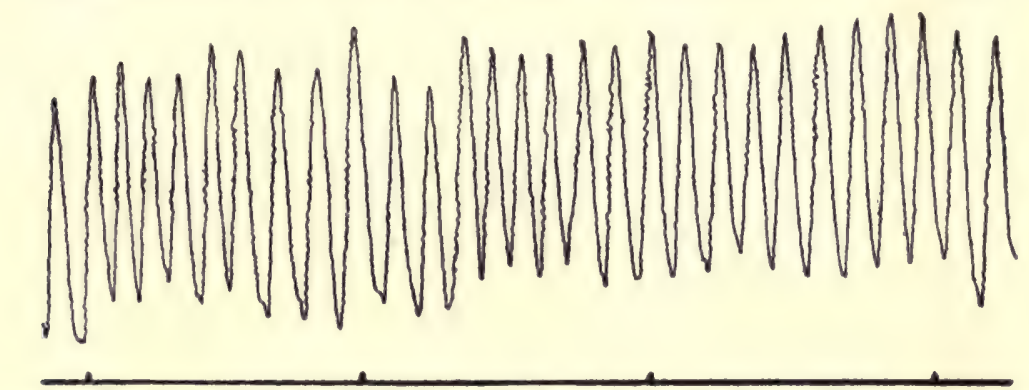

FrG. 71.-Type of respiration of subject J. B. T. without additional dead space on December 7, 1912. Original size.

DISCUSSION OF RESULTS.

The results of the comparison experiments with and without additional dead space in the spirometer unit are given in table 39. A study of these results shows but little, if any, difference in the two types of breathing with an additional dead space of not more than 224 c.c. 
TABLE 39.-Respiratory exchange in comparison experiments to study the effect of additional dead space. Benedict respiration apparatus (spirometer unit). (Without food.)

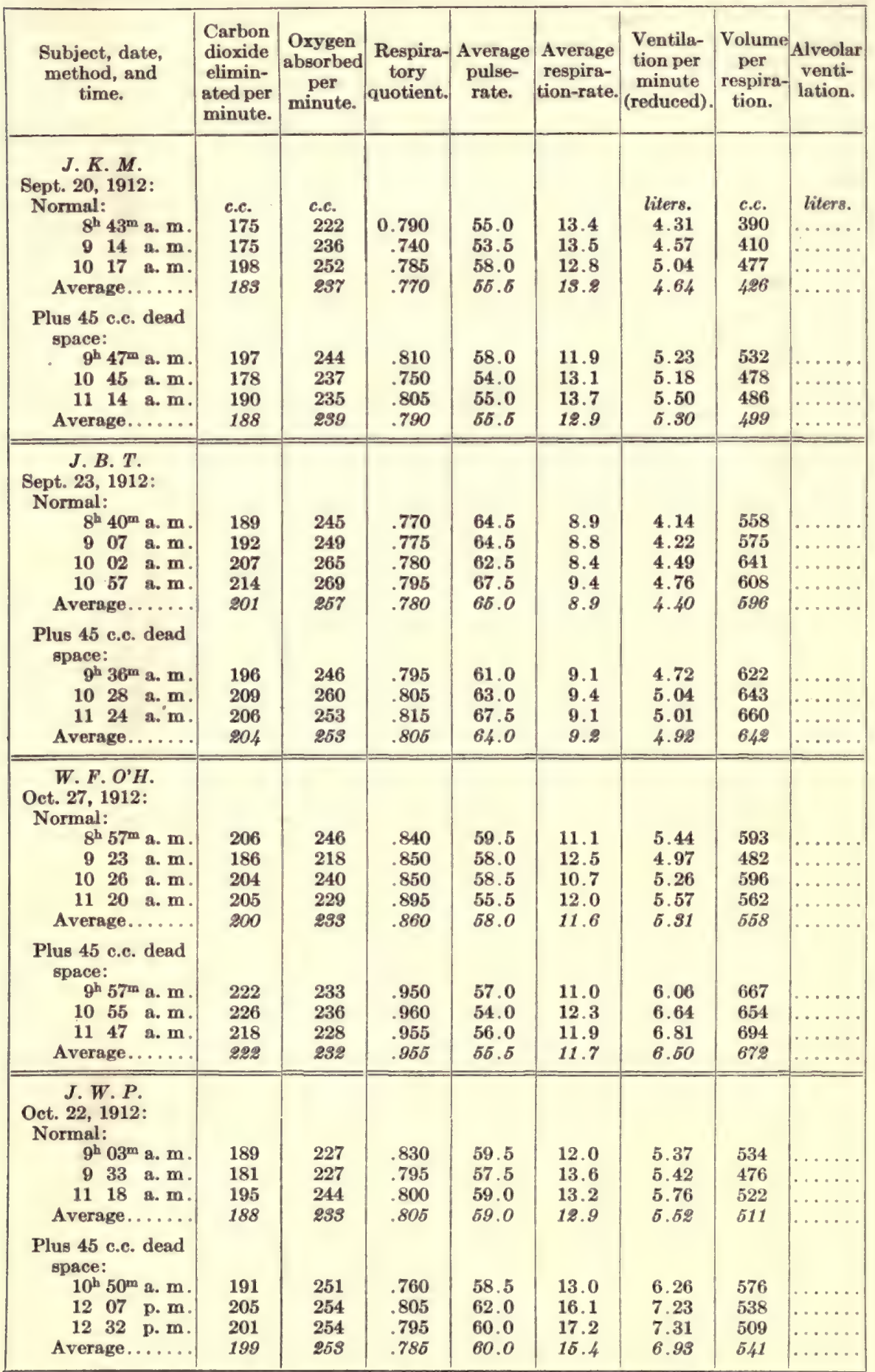


TABLE 39.-Respiratory exchange in comparison experiments to study the effect of additional dead space. Benedict respiration apparatus (spirometer unit). (Withoul food.)-Continued.

\begin{tabular}{|c|c|c|c|c|c|c|c|c|}
\hline $\begin{array}{l}\text { Subject, date, } \\
\text { method, and } \\
\text { time. }\end{array}$ & $\begin{array}{l}\text { Carbon } \\
\text { dioxide } \\
\text { elimain- } \\
\text { ated per } \\
\text { minute. }\end{array}$ & $\begin{array}{c}\text { Oxygen } \\
\text { absorbed } \\
\text { per } \\
\text { minute. }\end{array}$ & $\begin{array}{c}\text { Respira- } \\
\text { tory } \\
\text { quotient. }\end{array}$ & $\begin{array}{c}\text { Average } \\
\text { pulse- } \\
\text { rate. }\end{array}$ & $\begin{array}{c}\text { Average } \\
\text { respira- } \\
\text { tion-rate. }\end{array}$ & $\begin{array}{l}\text { Ventila- } \\
\text { tion per } \\
\text { minute } \\
\text { (reduced) }\end{array}$ & $\begin{array}{l}\text { Volume } \\
\text { per } \\
\text { respira- } \\
\text { tion. }\end{array}$ & $\begin{array}{l}\text { Alveolar } \\
\text { venti- } \\
\text { lation. }\end{array}$ \\
\hline \multirow{2}{*}{\multicolumn{9}{|c|}{$\begin{array}{l}\text { J. } K . M \\
\text { Sept. 21, 1912: } \\
\text { Normal: }\end{array}$}} \\
\hline & $\begin{array}{l}\text { c.c. } \\
177\end{array}$ & $\begin{array}{l}\text { c.c. } \\
233\end{array}$ & 0.760 & 51.0 & 13.8 & $\begin{array}{r}\text { liters. } \\
4.65\end{array}$ & $\begin{array}{l}\text { c.c. } \\
404\end{array}$ & $\begin{array}{r}\text { liters. } \\
\mathbf{3 . 5 5}\end{array}$ \\
\hline 915 a. $\mathrm{m}$ & 185 & 246 & .755 & 54.0 & 13.7 & 4.88 & 427 & 3.80 \\
\hline 1012 a. m. & 174 & 237 & .730 & 50.0 & 13.1 & 4.52 & 414 & 3.49 \\
\hline 1058 a. $\mathrm{m}$. & 174 & 235 & .740 & 52.0 & 12.8 & 4.50 & 421 & 3.50 \\
\hline Average....... & 178 & 238 & .745 & 52.0 & 13.4 & 4.64 & 417 & 3.57 \\
\hline \multirow{2}{*}{\multicolumn{9}{|c|}{$\begin{array}{l}\text { Plus } 90 \text { c.c. dead } \\
\text { space: } \\
\qquad 9^{\mathrm{h}} 46^{\mathrm{m}} \text { a. } \mathrm{m} .\end{array}$}} \\
\hline & 176 & 235 & .750 & 52.5 & 11.9 & 5.42 & 546 & 3.49 \\
\hline 1036 a. $\mathrm{m}$ & 179 & 232 & .770 & 56.0 & 13.8 & 5.94 & 516 & 3.69 \\
\hline 1121 a. $\mathrm{m}$. & 184 & 242 & .760 & 58.0 & 11.7 & 5.53 & 567 & 3.66 \\
\hline Average....... & 180 & 256 & .760 & 55.5 & 12.5 & 5.63 & 543 & 3.61 \\
\hline \multicolumn{9}{|l|}{$\begin{array}{l}\text { Oet. } 31,1912: \\
\text { Normal: }\end{array}$} \\
\hline $9^{\mathrm{h}} 04^{\mathrm{m}}$ a. m & 198 & 231 & .860 & 61.0 & 15.1 & 5.18 & 414 & 4.02 \\
\hline 936 a. m. & 184 & 217 & .845 & 52.0 & 14.2 & 4.47 & 379 & 3.35 \\
\hline 1045 a. $\mathrm{m}$. & 191 & 222 & .860 & 52.0 & 14.0 & 4.88 & 421 & 3.82 \\
\hline Average....... & 191 & 228 & .855 & 55.0 & 14.4 & 4.84 & 405 & 3.73 \\
\hline \multicolumn{9}{|l|}{$\begin{array}{l}\text { Plus } 90 \text { c.c. dead } \\
\text { space: }\end{array}$} \\
\hline $10^{\mathrm{h}} 10^{\mathrm{m}}$ a. m & 176 & 216 & .810 & 49.5 & 13.9 & 5.70 & 494 & 3.45 \\
\hline 1119 a. $\mathrm{m}$. & 202 & 223 & .905 & 59.0 & 14.8 & 6.40 & 522 & 4.03 \\
\hline 1150 a. $\mathrm{m}$. & 201 & 236 & .850 & 62.5 & 15.2 & 6.60 & 524 & 4.17 \\
\hline Average....... & 198 & 225 & .860 & 57.0 & 14.6 & 6.28 & 513 & 9.88 \\
\hline \multicolumn{9}{|l|}{$\begin{array}{l}\text { J. B. T. } \\
\text { Oct. } 26,1912 \text { : } \\
\text { Normal: }\end{array}$} \\
\hline $8^{\mathrm{h}} 58^{\mathrm{m}}$ a. $\mathrm{m}$ & 207 & 245 & .845 & 61.5 & 8.0 & 4.58 & 699 & 4.13 \\
\hline 948 a. $\mathrm{m}$. & 204 & 232 & .880 & 60.5 & 7.5 & 4.40 & 716 & 3.99 \\
\hline 1059 a. $\mathrm{m}$. & 236 & 263 & .895 & 62.0 & 12.0 & 5.67 & 577 & 4.92 \\
\hline Average....... & 216 & 247 & .875 & 61.5 & 9.2 & 4.88 & 664 & 4.35 \\
\hline \multicolumn{9}{|l|}{$\begin{array}{l}\text { Plus } 90 \text { c.c. dead } \\
\text { space: }\end{array}$} \\
\hline $10^{\mathrm{h}} 20^{\mathrm{m}}$ a. $\mathrm{m}$ & 234 & 246 & .950 & 65.5 & 8.0 & 5.82 & 888 & 4.76 \\
\hline 1132 a. $\mathrm{m}$. & 209 & 241 & .870 & 62.5 & 9.4 & 5.58 & 725 & 4.23 \\
\hline 1205 p. m. & 212 & 251 & .845 & 62.0 & 7.9 & 5.43 & 840 & 4.36 \\
\hline Average....... & 218 & 246 & .885 & 63.5 & 8.4 & 5.61 & 818 & 4.45 \\
\hline \multicolumn{9}{|l|}{$\begin{array}{l}\text { Nov. 1, 1912: } \\
\text { Normal: }\end{array}$} \\
\hline $9^{\mathrm{h}} 05^{\mathrm{m}}$ a. m. & 195 & 240 & .815 & 60.0 & 8.0 & 4.10 & 622 & 3.58 \\
\hline 934 a. m. & 200 & 248 & .805 & 58.5 & 8.7 & 4.35 & 607 & 3.77 \\
\hline 1038 a. $\mathrm{m}$. & 203 & 254 & .800 & 62.5 & 8.4 & 4.38 & 635 & 3.85 \\
\hline Average....... & 199 & 247 & .805 & 60.5 & 8.4 & 4.28 & 621 & 3.73 \\
\hline \multicolumn{9}{|l|}{$\begin{array}{l}\text { Plus } 90 \text { c.c. dead } \\
\text { space: }\end{array}$} \\
\hline $10^{\mathrm{h}} 07^{\mathrm{m}}$ a. m. & 200 & 246 & .815 & 61.0 & 8.5 & 5.05 & 722 & 3.77 \\
\hline 1118 a. m. & 197 & 252 & .780 & 63.0 & 8.2 & 4.55 & 676 & 3.31 \\
\hline 1149 a. $\mathrm{m}$. & 210 & 243 & .865 & 66.0 & 8.9 & 5.38 & 736 & 4.07 \\
\hline Average....... & 202 & 247 & .820 & 63.5 & 8.5 & 4.99 & 711 & 3.72 \\
\hline
\end{tabular}


TABIE 39.-Respiratory exchange in comparison experiments to study the effect of additional dead space. Benedict respiration apparatus (spirometer unit). (Without food.)-Continued.

\begin{tabular}{|c|c|c|c|c|c|c|c|c|}
\hline $\begin{array}{l}\text { Subject, date, } \\
\text { method, and } \\
\text { time. }\end{array}$ & $\begin{array}{l}\text { Carbon } \\
\text { dioxide } \\
\text { elimin- } \\
\text { ated per } \\
\text { minute. }\end{array}$ & $\begin{array}{c}\text { Oxygen } \\
\text { absorbed } \\
\text { per } \\
\text { minute. }\end{array}$ & $\begin{array}{c}\text { Respira- } \\
\text { tory } \\
\text { quotient. }\end{array}$ & $\begin{array}{l}\text { Average } \\
\text { pulse- } \\
\text { rate. }\end{array}$ & $\begin{array}{c}\text { Average } \\
\text { respira- } \\
\text { tion-rate. }\end{array}$ & $\begin{array}{c}\text { Ventila- } \\
\text { tion per } \\
\text { minute } \\
\text { (reduced). }\end{array}$ & $\begin{array}{c}\text { Volume } \\
\text { per } \\
\text { respira- } \\
\text { tion. }\end{array}$ & $\begin{array}{l}\text { Alveolar } \\
\text { venti- } \\
\text { lation. }\end{array}$ \\
\hline \multicolumn{9}{|l|}{$\begin{array}{c}T . M . C . \\
\text { Nov. } 8,1912: \\
\text { Plus } 135 \text { c.c. dead }\end{array}$} \\
\hline & c.c. & c.c. & & & & liters. & c.c. & liters. \\
\hline $8^{\mathrm{h}} 42^{\mathrm{m}}$ a. m. & 152 & 208 & 0.730 & 72.5 & 12.1 & 5.81 & 592 & 3.51 \\
\hline 953 a. $\mathrm{m}$ & 147 & & & 71.5 & 13.1 & 5.83 & 548 & 3.29 \\
\hline 1106 a. m. & 148 & 192 & .770 & 71.0 & 13.1 & 5.94 & 558 & 3.42 \\
\hline & 149 & 200 & .745 & 71.5 & 18.8 & 6.86 & 566 & 3.41 \\
\hline \multirow{2}{*}{\multicolumn{9}{|c|}{ Normal: }} \\
\hline & 147 & & & 73.0 & 11.7 & 3.99 & 420 & 3.18 \\
\hline 1029 a.m. & 149 & 199 & .750 & 69.0 & 12.2 & 4.13 & 417 & 3.29 \\
\hline 1135 a. m. & 151 & 206 & .730 & 66.5 & 12.1 & 4.06 & 413 & 3.24 \\
\hline Average....... & 149 & 205 & .735 & 69.5 & 18.0 & 4.06 & 417 & 3.84 \\
\hline \multirow{2}{*}{\multicolumn{9}{|c|}{$\begin{array}{c}P . F . J . \\
\text { Nov. } 7,1912: \\
\text { Plus } 135 \text { c.c. dead } \\
\text { space: } \\
g^{\mathrm{b}} 03^{\mathrm{m}} \text { a. m }\end{array}$}} \\
\hline & & & & & & & & \\
\hline $\begin{array}{l}9^{\mathrm{h}} 03^{\mathrm{m}} \text { a. } \mathrm{m} \\
1006 \text { a. } \mathrm{m}\end{array}$ & $\begin{array}{l}190 \\
193\end{array}$ & $\begin{array}{l}238 \\
251\end{array}$ & .800 & $\begin{array}{l}77.0 \\
79.5\end{array}$ & $\begin{array}{l}11.7 \\
12.8\end{array}$ & $\begin{array}{l}6.44 \\
6.70\end{array}$ & $\begin{array}{l}668 \\
635\end{array}$ & $\begin{array}{l}4.14 \\
4.17\end{array}$ \\
\hline $\begin{array}{lll}10 & 06 & \text { a. } \\
11 & 05 & \text { a. } \\
1\end{array}$ & $\begin{array}{l}193 \\
198\end{array}$ & 235 & .845 & 78.0 & 11.1 & 6.46 & 707 & 4.32 \\
\hline Average....... & 194 & 241 & .805 & 78.0 & 11.9 & 6.58 & 670 & 4.21 \\
\hline \multicolumn{9}{|l|}{ Normal: } \\
\hline $9^{h} 36^{m}$ a. m. & 188 & 237 & .790 & 79.0 & 12.9 & 4.88 & 458 & 3.92 \\
\hline 1034 a. $\mathrm{m}$. & 195 & & & 77.0 & 12.6 & 5.00 & 481 & 4.09 \\
\hline 1134 a. m. & 196 & 234 & .840 & 78.0 & 11.9 & 4.99 & 510 & 4.16 \\
\hline Average........ & 193 & 286 & .820 & 78.0 & 12.6 & 4.96 & 483 & 4.06 \\
\hline \multicolumn{9}{|l|}{$\begin{array}{l}\text { Nov. 14, 1912: } \\
\text { Normal: }\end{array}$} \\
\hline $8^{\mathrm{h}} 58^{\mathrm{m}}$ a. m. & 189 & 234 & .805 & 70.0 & 9.0 & 4.39 & 595 & 3.79 \\
\hline 942 a. $m$. & 181 & 233 & .790 & 67.5 & 11.2 & 4.71 & 512 & 3.93 \\
\hline 1025 a. $\mathrm{m}$. & 188 & 227 & .825 & 68.0 & 11.1 & 4.68 & 514 & 3.94 \\
\hline 1106 a. $\mathrm{m}$. & 186 & 236 & .790 & 68.5 & 12.1 & 4.80 & 484 & 3.97 \\
\hline Average........ & 186 & 283 & .800 & 68.6 & 10.8 & 4.65 & 526 & 3.91 \\
\hline \multirow{2}{*}{\multicolumn{9}{|c|}{$\begin{array}{l}\text { Plus } 135 \text { c.c. dead } \\
\text { space: } \\
9^{\mathrm{h}} 20^{\mathrm{m}} \text { a. m. }\end{array}$}} \\
\hline & 188 & 233 & .805 & 67.5 & 10.7 & 6.02 & 686 & 3.93 \\
\hline 1004 a. m. & 181 & 226 & .790 & 67.0 & 12.5 & 6.21 & 606 & 3.76 \\
\hline 1047 a. $\mathrm{m}$. & 181 & 229 & .790 & 67.5 & 11.1 & 6.16 & 677 & 4.05 \\
\hline 1129 a. $\mathrm{m}$. & 187 & 245 & $.765^{\circ}$ & 68.0 & 12.6 & 6.73 & 652 & 4.32 \\
\hline Average........ & 186 & 233 & .800 & 67.5 & 11.7 & 6.28 & 655 & 4.08 \\
\hline \multicolumn{9}{|l|}{$\begin{array}{l}J . K . M \\
\text { Dec. } 3,1912: \\
\text { Normal: }\end{array}$} \\
\hline $8^{\mathrm{h}} 50^{\mathrm{m}}$ a. m & 180 & 221 & .815 & 60.5 & 14.0 & 4.67 & 402 & 3.61 \\
\hline 947 a. m. & 186 & 229 & .810 & 61.5 & 15.1 & 4.19 & 334 & 2.98 \\
\hline 1046 a. $\mathrm{m}$. & 181 & 220 & .820 & 60.5 & 15.1 & 3.80 & 303 & 2.57 \\
\hline Average....... & 188 & 283 & .815 & 61.0 & 14.7 & 4.82 & 346 & 8.05 \\
\hline \multicolumn{9}{|l|}{$\begin{array}{l}\text { Plus } 224 \text { c.c. dead } \\
\text { space: }\end{array}$} \\
\hline $9^{\mathrm{h}} 21^{\mathrm{m}}$ a. m. & 191 & 218 & .875 & 63.0 & 14.0 & 7.02 & 604 & 2.99 \\
\hline 1016 a. $\mathrm{m}$. & 191 & 221 & .865 & 66.0 & 15.3 & 7.43 & 585 & 3.01 \\
\hline 1114 a. m. & 203 & 236 & .855 & 66.5 & 14.3 & 7.76 & 653 & 3.69 \\
\hline Average........ & 195 & 225 & .870 & 65.0 & 14.6 & 7.40 & 614 & $s .23$ \\
\hline
\end{tabular}


TABLE 39.-Respiratory exchange in comparison experiments to study the effect of additional dead space. Benedict respiration apparatus. (spirometer unit). (Without food). - Con.

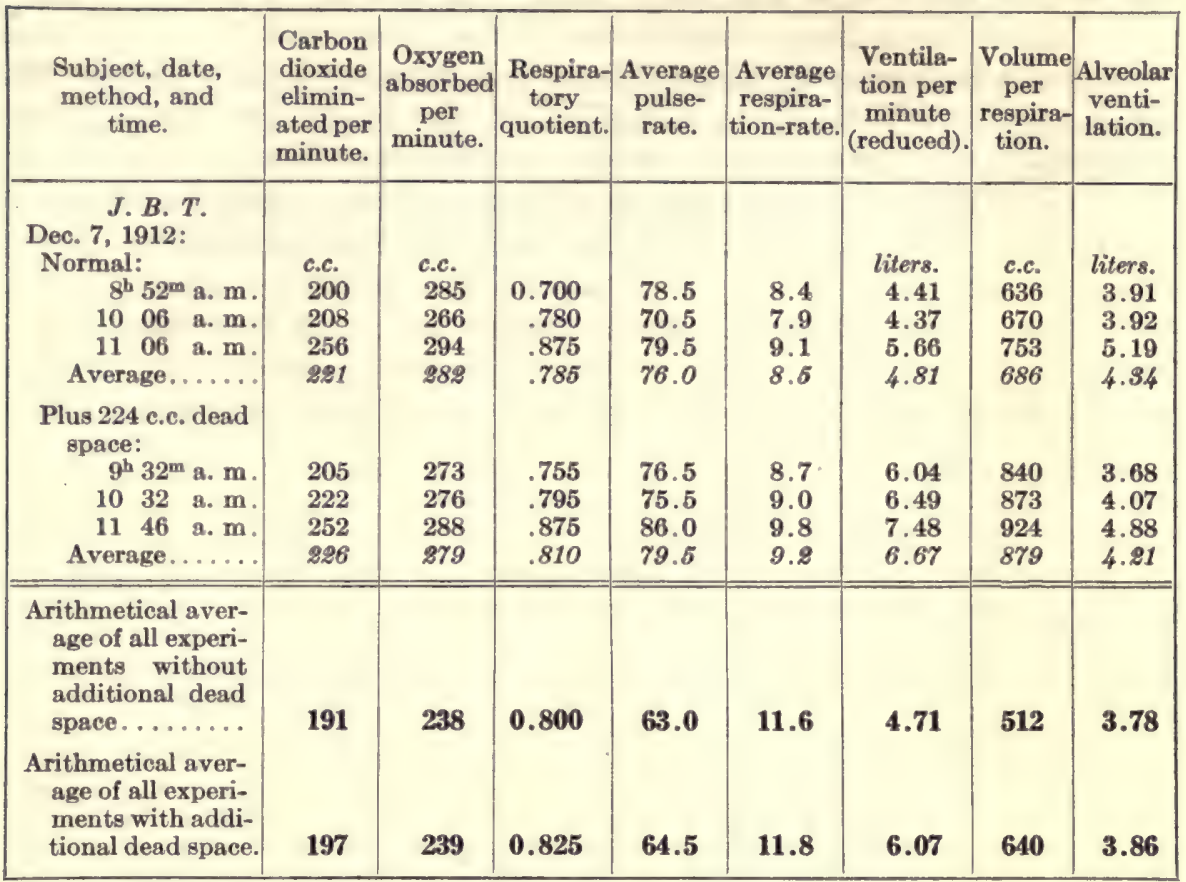

The variations of the individual comparisons are given in table 40 , using the normal values as a base-line. With a dead space of 45 c.c. the greatest variation in the respiratory exchange is shown in the experiment with W. F. O'H., in which the carbon-dioxide elimination was 22 c.c. higher with the additional dead space than with the apparatus as normally used. An inspection of the statistics for the individual periods shows, however, that this difference was due to the fact that in the periods normally carried out the subject was somewhat drowsy and there was considerable apnœea. With J. W. P. the respiratory exchange with the additional dead space was also slightly higher; but this was likewise due more to variations in the degree of muscular repose than to actual differences between the two methods. With the other two subjects the difference in the respiratory exchange with the two methods of breathing was insignificant. The four comparisons with 90 c.c. additional dead space show an even better agreement than with the 45 c.c. dead space, there being practically no difference in any of the factors measured except those for the ventilation of the lungs and the volume per respiration. With an additional dead space of 135 c.c. there is also a good agreement between the values for the respiratory exchange compared. With an additional dead space of 224 c.c. the carbon-dioxide output is slightly higher with the increased dead space than with the normal method, but this difference is not very great. 
A study of the results obtained for the ventilation of the lungs and the volume per respiration shows that in practically all of the experiments there was a larger ventilation of the lungs with the additional dead space than with the normal breathing; in other words, to have the same amount of effective ventilation of the lungs, the subject was obliged at each respiration to sweep out this increased dead space in addition to that of the normal dead space of the apparatus and the respiratory tract. The total ventilation of the lungs less that required to sweep out the natural dead space of the respiratory tract of the subject at each respiration may be designated as the alveolar ventilation. If an assumption is made that the natural dead space is 100 c.c. and the alveolar ventilation per minute is calculated by using this value,

TABLE 40.--Variations of average results obtained with dead space from those obtained witho $u$ dead space (spirometer unit).

\begin{tabular}{|c|c|c|c|c|c|c|c|c|c|}
\hline Subject. & Date. & 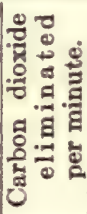 & 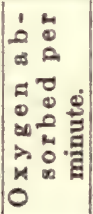 & 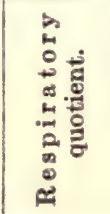 & 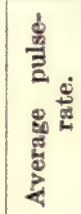 & 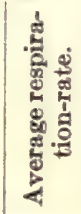 & 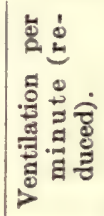 & 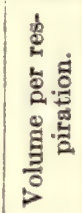 & 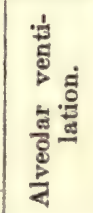 \\
\hline 45 c.c. & 191 & c.c. & c.c. & & & & liters. & & liters. \\
\hline & & +5 & +2 & +0.020 & 0 & -0.3 & +0.66 & +73 & \\
\hline J & ept. 2 & +3 & -4 & +.025 & -1 & +.3 & +.52 & +46 & \\
\hline & Oet. 2 & +22 & -1 & +.095 & -2.5 & +.1 & 1.19 & +114 & \\
\hline J. V & Oct. 22 & +11 & +20 & -.020 & +1.0 & +2.5 & 1.41 & +30 & \\
\hline $90 \mathrm{c.}$ & & & & & & & & & \\
\hline & Sept. 21 & +2 & -2 & +.015 & +3.5 & -.9 & +.99 & +126 & +0.04 \\
\hline & Oct. 31 & & +2 & +.005 & +2.0 & +.2 & +1 & +108 & +.15 \\
\hline & Oet. 26 & +2 & -1 & +.010 & +2.0 & -.8 & +.73 & +154 & +.10 \\
\hline & Nov. 1 & +3 & 0 & +.015 & +3.0 & +.1 & +0.71 & +90 & -.01 \\
\hline Ispace: & & & & & & & & & \\
\hline T. & $\begin{array}{l}\text { Nov. } 8 \\
\text { Nov. } 7\end{array}$ & $\begin{array}{r}0 \\
+\quad 1\end{array}$ & $\begin{array}{l}-3 \\
+5\end{array}$ & 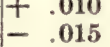 & $\begin{array}{c}+2.0 \\
0\end{array}$ & {$\left[\begin{array}{l}+ \\
-\quad .8 \\
-.6\end{array}\right.$} & $\begin{array}{l}+1.80 \\
+1.57\end{array}$ & $\begin{array}{l}+149 \\
+187\end{array}$ & $\begin{array}{l}+.17 \\
+\quad .15\end{array}$ \\
\hline & Nov. 14 & 0 & 0 & 0 & -1.0 & +.8 & +1.63 & +129 & +.11 \\
\hline 224 c.c & Dec. & +13 & +2 & $+\quad 055$ & +40 & -2 & +3 & +268 & $+\quad 18$ \\
\hline J. B. T & Dec. 7 & +5 & -3 & +.025 & +3.5 & +.7 & +1.86 & +193 & -.13 \\
\hline \multicolumn{2}{|l|}{ Average variation } & +5 & 4 & 0.025 & 2.0 & 0.6 & +1.36 & +128 & .08 \\
\hline
\end{tabular}

the total ventilation, and the respiration-rate per minute, it will be found that in the periods with the additional dead space the value for the alveolar ventilation is approximately equal to that in the normal periods plus the product of the respiration-rate and the additional dead space. These values have been calculated for all of the experiments except those with an additional dead space of 45 c.c.; in no case is the alveolar ventilation thus calculated more than 0.2 liter per minute higher in the periods with an additional dead space than in the normal periods. The assumed value of 100 c.c for the natural dead space is not accurate for all individuals, but it is immaterial what value is assumed, as in this case only differences are to be calculated. 
The uniformity in the results with the two methods of breathing has been calculated and the curves based upon these calculations are given in figure 72. These show that practically the same uniformity obtains with the additional dead space as in the experiments without this increase.

It will be seen, therefore, from the results obtained in this series of comparisons, that it is possible to increase the dead space of the Benedict respiration apparatus by attaching a long tube to the three-way valve without affecting the accuracy of the measurements of the respiratory exchange. This enables the experimenter to adapt the

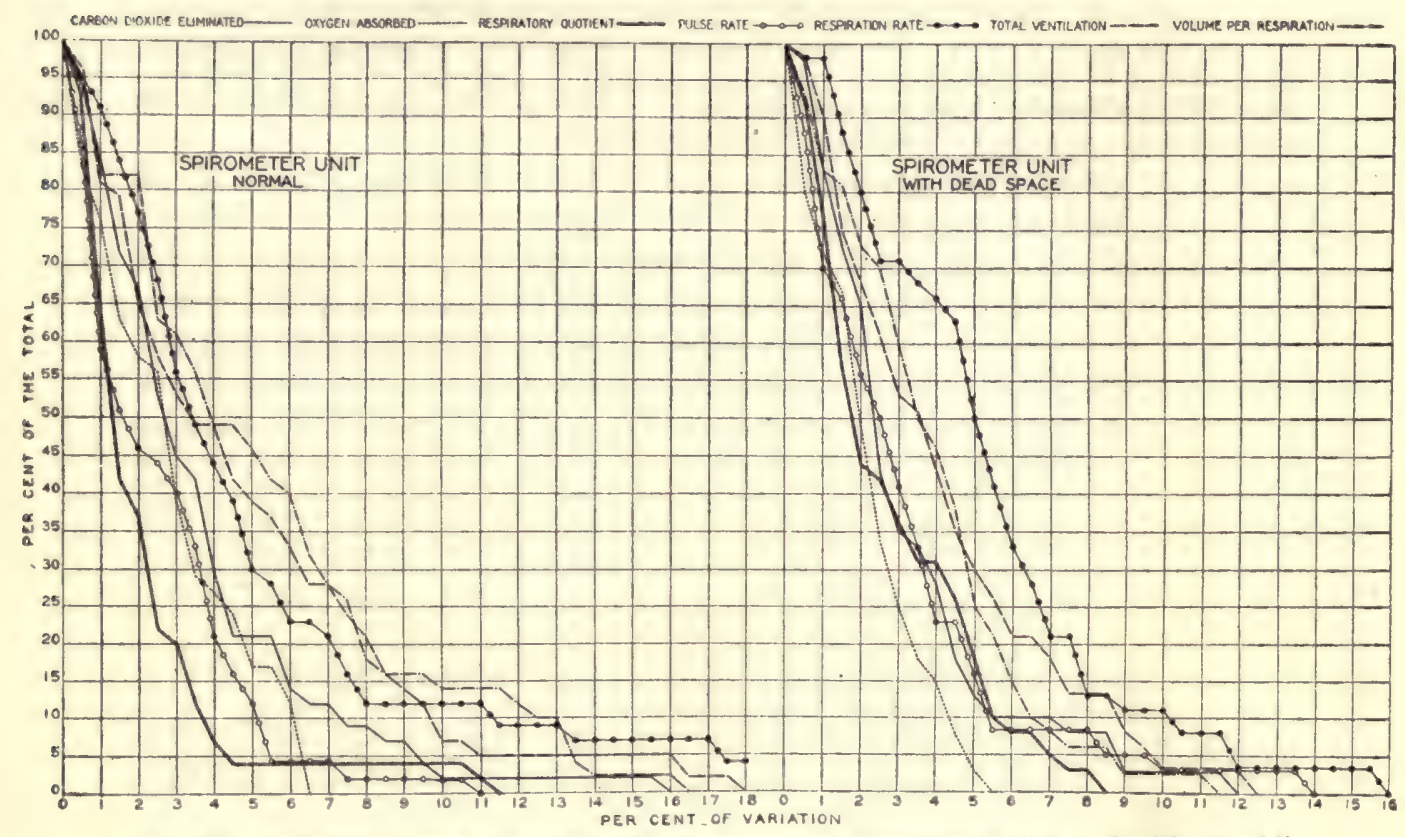

Fig. 72.-Probability curves for the series of comparison experiments with and without additional dead space (spirometer unit).

The ordinates indicate the percentage of the total number of periods and the abscissw represent the percentage of variation from the average.

apparatus more readily to different positions taken by the subject than would be practicable if it were necessary to keep the dead space as small as possible. While no series of experiments was carried out with other forms of apparatus, it would seem probable that the results could be applied to them as well as to the Benedict respiration apparatus.

TISSOT APPARATUS WITH AND WITHOUT AUTOMATIC COUNTERPOISE ON THE SPIROMETER BELL.

The Tissot spirometer is so arranged that each position of the bell is exactly counterpoised by means of a column of water in the counterpoise tube. ${ }^{1} \quad$ A siphon connects this column of water with the water in 
the tank, so that when changes in the position of the counterpoise tube take place the water in the tube is automatically brought to the same level as that of the water in the tank surrounding the bell. If it were not necessary to have the spirometer bell counterpoised exactly at each position the apparatus would be less complicated and there would be no necessity of making sure that the water flowed freely through the siphon between the two containers. A study was therefore made of the effect upon the respiratory exchange of discontinuing the siphon arrangement and partially, but not exactly, counterpoising the bell of the spirometer.

In some of the experiments the counterpoise tube was entirely empty, so that at the end of the period there was a slight pressure of air (less than $2 \mathrm{~mm}$. of water) inside the bell of the spirometer. This was due to the fact that the bell was heavier than the counterpoise tube. In other experiments the counterpoise tube was half full of water, so that at the beginning of the experiment there was a very slight diminished pressure inside the bell.

The Tissot valves were used in these experiments and either the pneumatic nosepieces or the mouthpiece, according to the desire of the subject. The pulse-rate was obtained with the Bowles stethoscope and the record of the respiration-rate by means of the chest pneumograph. The subjects, who were all more or less trained to the Tissot apparatus, were very quiet, except as noted in the statistics. The results of the seven experiments are given in the following pages.

\section{STATISTICS OF EXPERIMENTS.}

R. G., February 1, 1913.-With automatic counterpoise, 3 periods; without automatic counterpoise, 2 periods; periods with the two methods in series. During periods without counterpoise, the lead weight (see $R$, fig. 26) was removed; there was no water running in the siphon, and none in the counterpoise tube. Subject very quiet and awake all through experiment. Urinated at $9^{\mathrm{h}} 35^{\mathrm{m}}$ a. m. Pulse-rate uniform in all periods. Average preliminary respiration-rate 12 per minute. ${ }^{1}$ Respiration-rate during experiment uniform. Pneumograph so poorly adjusted that differences in character could not be seen. Subject noted no difference between two methods, but stated respiration was easy in all periods. Average barometric pressure, $752 \mathrm{~mm}$.; average temperature of air in apparatus, $19.1^{\circ} \mathrm{C}$.

R. G., February 4, 1913.-Without automatic counterpoise, 3 periods; with automatic counterpoise, 3 periods; preliminary period, 1 hour 2 minutes; periods with and without counterpoise in series. In periods without automatic counterpoise, counterpoise tube half full of water and no water running in siphon. Subject quiet and awake; urinated at $10^{\mathrm{h}} 40^{\mathrm{m}} \mathrm{a} . \mathrm{m}$. Pulse-rate very uniform. Average respiration-rate in preliminary period, 16 per minute; during experiment fairly uniform in rate. In first period, there was a tendency to apnœa; in the fourth period (the first with automatic counterpoise) there was the same tendency. Average barometric pressure, $757.0 \mathrm{~mm}$.; average temperature of air in apparatus, $19.1^{\circ} \mathrm{C}$.

W.J. T., March 1, 1913.-With automatic counterpoise, 3 periods; without automatic counterpoise, 2 periods; preliminary period 1 hour 25 minutes; 
periods with and without automatic counterpoise in series. In periods without automatic counterpoise, tube less than half full of water. Nosepieces used in first period; mouthpiece in other periods. Pulse-rate uniform in all series. Average respiration in preliminary period, $20 \mathrm{per}$ minute. In second period respiration wavy in character but normal in other periods. Sections of respiration curves showing the two types of respiration are given in figure 73. Subject stated that he preferred mouth-breathing to nose-breathing, but noted no difference between the two methods, except that he was more tired in the latter part of the morning. Average barometric pressure, $757.9 \mathrm{~mm}$.; average temperature of air in apparatus, $18.8^{\circ} \mathrm{C}$.

W.J.T., March 8, 1913.-Without automatic counterpoise, 4 periods; with automatic counterpoise, 3 periods; preliminary period, 49 minutes; periods with and without automatic counterpoise alternating for the most part. In periods without automatic counterpoise, no water in counterpoise and no water running in siphon. Mouthpiece used throughout experiment, but in third period no noseclip was used. Subject stated that he noted no difference in the two methods. The results obtained in the last period for the carbondioxide elimination and oxygen consumption are not included in the average, as the sample of air analyzed appears to have been contaminated with outside air. Pulse-rate fairly uniform in all periods. Average respiration-rate in pre-

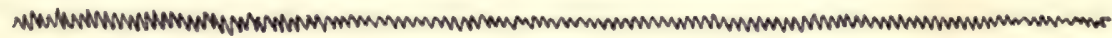

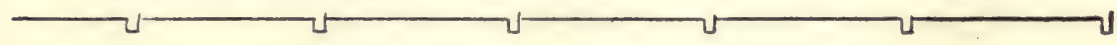

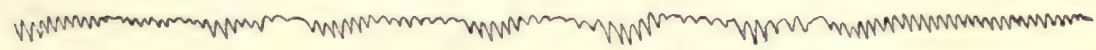

Fig. 73.-Types of respiration of subject W. J. T. in third and second periods on March $1,1913$. Note the irregular character of the respiration shown in the lower curve. Original size.

liminary period, 20 per minute. During experiment, respiration-rate irregular at times, particularly in fifth period. Average barometric pressure, 770.0 $\mathrm{mm}$.; average temperature of air in apparatus, $16.8^{\circ} \mathrm{C}$.

W.F.B., March 10,1913.-With automatic counterpoise, 3 periods; without automatic counterpoise, 2 periods; preliminary period, 46 minutes; periods with and without automatic counterpoise alternating. No water in counterpoise tube and none running in siphon. Subject quiet and awake throughout experiment. Pulse-rate uniform in all periods. Average respiration-rate in preliminary period, 11 per minute; between periods, average rate was 10, 9, 10,10 , and 9 respectively; during periods, regular in rate and even in character. Average barometric pressure, $765.1 \mathrm{~mm}$.; temperature of air in apparatus, $17.1^{\circ} \mathrm{C}$.

W.F. B., March 12, 1913.-Without automatic counterpoise, 3 periods; with automatic counterpoise, 3 periods; preliminary period, 42 minutes; periods with and without automatic counterpoise alternating. No water in counterpoise tube and no water running through siphon. Pulse-rate uniform throughout experiment. Average respiration-rate in preliminary period, 9 per minute; between periods, $6,9,8$, and 9 per minute, respectively; during experimental periods, respiration-rate very regular in character. Subject stated that during first period he found it difficult to breathe in and out; after third period he complained of a pain in left side of body which he thought was due to the pneumograph; the pneumograph was therefore loosened and moved to a lower point on the body, after which he said he felt more comfortable. 
Average barometric pressure, $769.6 \mathrm{~mm}$; average temperature of air in apparatus, $18.3^{\circ} \mathrm{C}$.

J. K. M., March 14, 1913.-With automatic counterpoise, 3 periods; without automatic counterpoise, 3 periods; preliminary period, 36 minutes; periods with and without counterpoise alternating. Counterpoise tube half full of water and no water running in siphon. The sample of air taken for the second period (without counterpoise) was not properly closed and it was contaminated with outside air; the results obtained for the carbon-dioxide production and oxygen consumption for this period are therefore too low. Pulse-rate uniform in all periods. Average respiration-rate in preliminary period, 20 per minute; between periods, 16, 17, 17, and 17 per minute, respectively. During the experimental periods the rate was uniform, but it was not possible to judge of the character of the respiration, owing to the fact that the pneumograph did not give a clear record. Subject stated that there was no resistance of any kind to the respiration. Average barometric pressure, 759.8 $\mathrm{mm}$. ; average temperature of air in apparatus, $16.5^{\circ} \mathrm{C}$.

\section{DISCUSSION OF RESULTS.}

The results of the experiments with the Tissot apparatus, in which the respiratory exchange with and without the counterpoise was compared, are given in table 41. The average values for all of the factors measured practically agree with the two methods.

The variations between the averages for each method in the individual experiments are shown in table 42 , the values for the experiments with the counterpoise being used for the base-line. These difference-

TABLE 41.-Respiratory exchange in comparison experiments with the Tissot apparatus with and without automatic counterpoising of the spirometer bell. (Without food.)

\begin{tabular}{|c|c|c|c|c|c|c|c|c|c|}
\hline \multirow{2}{*}{$\begin{array}{l}\text { Subject, date, method, } \\
\text { and time. }\end{array}$} & \multirow{2}{*}{ 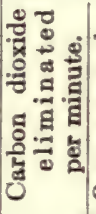 } & \multirow{2}{*}{ 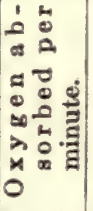 } & \multirow{2}{*}{ 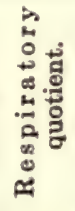 } & \multirow{2}{*}{ 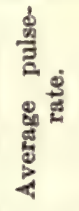 } & \multirow{2}{*}{ 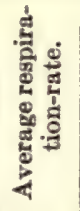 } & \multirow{2}{*}{ 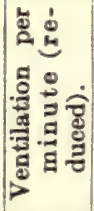 } & \multirow{2}{*}{ 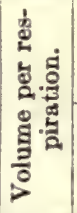 } & \multicolumn{2}{|c|}{$\begin{array}{l}\text { Composition of } \\
\text { expired air. }\end{array}$} \\
\hline & & & & & & & & $\begin{array}{l}\text { Carbon } \\
\text { dioxide. }\end{array}$ & Oxygen. \\
\hline \multicolumn{10}{|l|}{$\begin{array}{l}\qquad R . G . \\
\text { Feb. 1, 1913: } \\
\text { With automatic counter- }\end{array}$} \\
\hline $\begin{array}{l}\text { With automatic counter- } \\
\text { poise: } \\
9^{\mathrm{h}} 15^{\mathrm{m}} \text { a. m..... }\end{array}$ & $\begin{array}{l}c . c . \\
180\end{array}$ & $\begin{array}{l}\text { c.c. } \\
232\end{array}$ & 0.780 & 65.5 & 10.7 & $\begin{array}{c}\text { liters. } \\
4.36\end{array}$ & $\begin{array}{l}\text { c.c. } \\
499\end{array}$ & p. ct. & p. ct. \\
\hline 950 a. m..... & 196 & 229 & .855 & 64.5 & 11.1 & $\begin{array}{l}4.36 \\
4.68\end{array}$ & $\begin{array}{l}499 \\
516\end{array}$ & $\begin{array}{l}4.16 \\
4.22\end{array}$ & $\begin{array}{l}15.88 \\
16.19\end{array}$ \\
\hline 1019 a. m...... & 186 & 238 & .780 & 61.0 & 12.3 & 4.55 & 453 & 4.12 & 15.95 \\
\hline Average............ & 187 & 283 & .800 & 63.5 & 11.4 & 4.53 & 489 & 4.17 & 16.01 \\
\hline \multicolumn{10}{|l|}{$\begin{array}{l}\text { Without a utomatic } \\
\text { counterpoise: }\end{array}$} \\
\hline $11^{\mathrm{h}} 14^{\mathrm{m}}$ a. m......... & 164 & 207 & .790 & 62.5 & 12.1 & 4.13 & 418 & 3.99 & 16.16 \\
\hline 1139 a. m..... & 188 & 225 & .835 & 62.5 & 13.1 & 4.69 & 438 & 4.04 & 16.31 \\
\hline Average........... & 176 & 216 & .815 & 62.5 & 12.6 & $4 \cdot 41$ & 488 & 4.08 & 16.84 \\
\hline \multicolumn{10}{|l|}{$\begin{array}{l}\text { Feb. 4, 1913: } \\
\text { Without a u tom a tic } \\
\text { counterpoise: }\end{array}$} \\
\hline $9^{\mathrm{h}} 32^{\mathrm{m}}$ a. m....... & 181 & 228 & .795 & 62.0 & 12.5 & 4.65 & 452 & 3.92 & 16.25 \\
\hline 1000 a. $\mathrm{m} \ldots .$. & 207 & 244 & .850 & 62.0 & 13.3 & 5.23 & 478 & 3.99 & 16.42 \\
\hline 1023 a. m..... & 189 & 235 & .800 & 61.5 & 11.4 & 4.45 & 474 & 4.27 & 15.87 \\
\hline Average............ & 192 & 286 & .815 & 68.0 & 18.4 & 4.78 & 468 & 4.06 & 16.18 \\
\hline
\end{tabular}


TABLE 41.-Respiratory exchange in comparison experiments with the Tissol apparatus with and withont automatic counterpoising of the spirometer bell. (Without food.) - Continued.

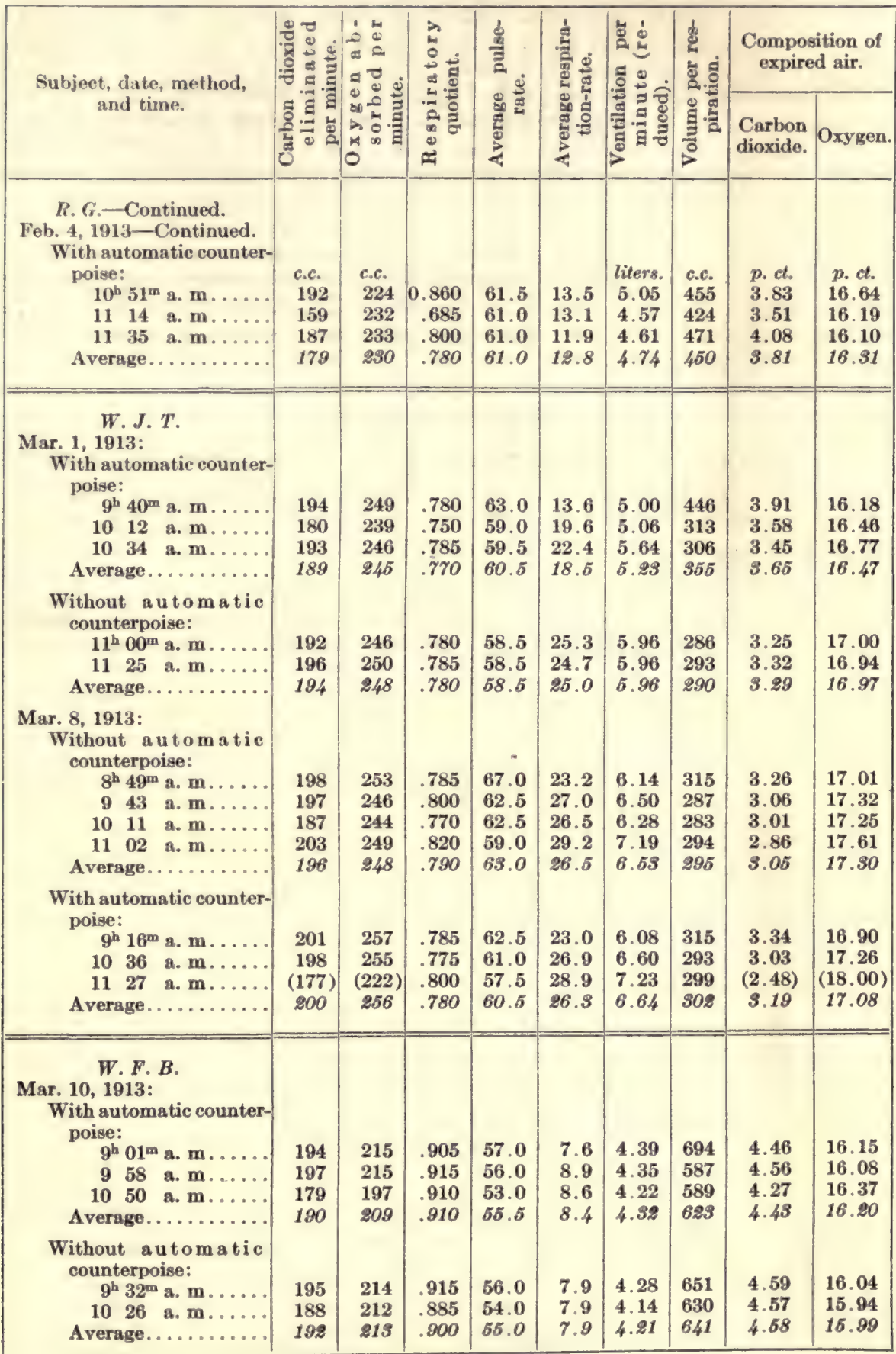


TABLE 41.-Respiratory exchange in comparison experiments with the Tissot apparatus with and without automatic counterpoising of the spirometer bell. (Without food.)-Cont.

\begin{tabular}{|c|c|c|c|c|c|c|c|c|c|}
\hline \multirow{2}{*}{$\begin{array}{l}\text { Subject, date, method, } \\
\text { and time. }\end{array}$} & \multirow{2}{*}{ 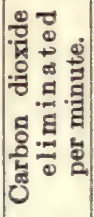 } & \multirow{2}{*}{ 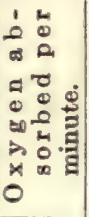 } & \multirow{2}{*}{ 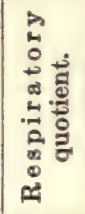 } & \multirow{2}{*}{ 总 } & \multirow{2}{*}{ 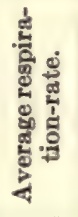 } & \multirow{2}{*}{ 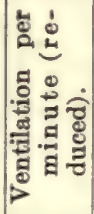 } & \multirow{2}{*}{ 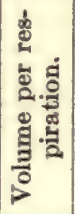 } & \multicolumn{2}{|c|}{$\begin{array}{l}\text { Composition of } \\
\text { expired air. }\end{array}$} \\
\hline & & & & & & & & $\begin{array}{l}\text { Carbon } \\
\text { dioxide. }\end{array}$ & Oxygen. \\
\hline 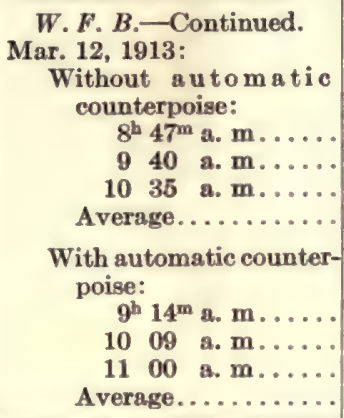 & $\begin{array}{l}\text { c.c. } \\
184 \\
191 \\
180 \\
185\end{array}$ & \begin{tabular}{l|}
$c . c$. \\
219 \\
220 \\
209 \\
816
\end{tabular} & $\begin{array}{r}0.840 \\
.865 \\
.860 \\
.855\end{array}$ & $\begin{array}{l}55.5 \\
53.5 \\
53.0 \\
54.0\end{array}$ & $\begin{array}{l}6.6 \\
6.5 \\
7.1 \\
6.7\end{array}$ & $\begin{array}{l}\text { liters. } \\
\mathbf{3 . 7 4} \\
\mathbf{3 . 9 1} \\
\mathbf{3 . 6 5} \\
\mathbf{3 . 7 7}\end{array}$ & $\begin{array}{l}\text { c.c. } \\
784 \\
778 \\
681 \\
748\end{array}$ & $\begin{array}{l}\text { p. } c t . \\
4.94 \\
4.90 \\
4.95 \\
4.99\end{array}$ & $\begin{array}{l}p . c t . \\
15.28 \\
15.48 \\
15.38 \\
15.38\end{array}$ \\
\hline 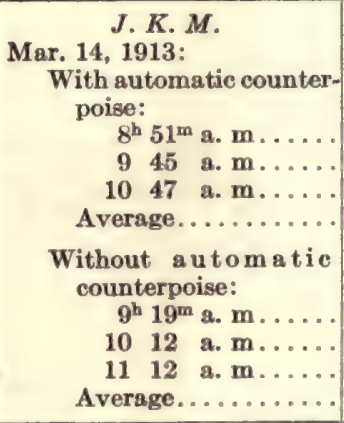 & $\begin{array}{c}(142) \\
185 \\
179 \\
188\end{array}$ & $\begin{array}{c}(176) \\
230 \\
223 \\
227\end{array}$ & $\begin{array}{l}.770 \\
.810 \\
.785 \\
.790\end{array}$ & $\begin{array}{l}62.5 \\
59.0 \\
58.0 \\
60.0\end{array}$ & $\begin{array}{l}13.9 \\
16.1 \\
12.2 \\
14.1\end{array}$ & $\begin{array}{l}4.45 \\
4.73 \\
4.16 \\
4.45\end{array}$ & $\begin{array}{l}425 \\
368 \\
343 \\
379\end{array}$ & $\begin{array}{c}(3.27) \\
3.96 \\
3.57 \\
3.77\end{array}$ & $\begin{array}{c}(17.10) \\
16.25 \\
16.71 \\
16.48\end{array}$ \\
\hline $\begin{array}{l}\text { Arithmetical average of all } \\
\text { experiments with auto- } \\
\text { matic counterpoise..... } \\
\text { Arithmetical average of all } \\
\text { experiments without } \\
\text { automatic counterpoise. }\end{array}$ & 187 & 231 & .810 & 59.5 & 14.0 & 4.83 & 471 & 4.02 & 16.23 \\
\hline
\end{tabular}

are not very large, varying from -11 c.c. to +13 c.c. for the carbondioxide elimination and from -17 c.c. to +8 c.c. for the oxygen consumption. The average respiratory quotient is practically identical for the two methods in all of the experiments. The pulse-rate is slightly lower in the periods with the counterpoise, but the other factors do not show large variations either way.

The percentage variations of the results of the individual periods from the averages of the experiments have also been calculated for this series and are plotted as curves in figure 74. On the whole the periods without the counterpoise show a slightly greater degree of uniformity than do those with the counterpoise, but the difference is not marked with any of the factors measured. 
From the results of these comparisons it may be seen that with the Tissot 200-liter spirometer, with a pressure on the air inside the bell varying from $+0.4 \mathrm{~mm}$. to $-0.4 \mathrm{~mm}$. of water, it is not necessary to counterpoise the spirometer bell exactly and automatically by the use of water in the counterpoise tube. These variations in pressure are too small to affect the respiratory exchange.

TABLE 42.-Variations of average results obtained without automatic counterpoise from those obtained with automatic counterpoise-Tissot apparatus.

\begin{tabular}{|c|c|c|c|c|c|c|c|c|}
\hline Subject. & Date. & $\begin{array}{l}\text { Carbon } \\
\text { dioxide } \\
\text { elimin- } \\
\text { ated per } \\
\text { minute. }\end{array}$ & $\begin{array}{c}\text { Oxygen } \\
\text { absorbed } \\
\text { per } \\
\text { minute. }\end{array}$ & $\begin{array}{l}\text { Respira- } \\
\text { tory } \\
\text { quotient. }\end{array}$ & $\begin{array}{l}\text { Average } \\
\text { pulse- } \\
\text { rate. }\end{array}$ & $\begin{array}{l}\text { Average } \\
\text { respira- } \\
\text { tion-rate }\end{array}$ & $\begin{array}{l}\text { Ventila- } \\
\text { tion per } \\
\text { minute } \\
\text { (reduced) }\end{array}$ & $\begin{array}{l}\text { Volume } \\
\text { per } \\
\text { respira- } \\
\text { tion. }\end{array}$ \\
\hline $\begin{array}{l}\text { R. G.... } \\
\text { W. J. T. } \\
\text { W. F. B.. } \\
\text { J. K. M.. }\end{array}$ & \begin{tabular}{ll}
\multicolumn{2}{c}{1913} \\
Feb. & 1 \\
Feb. & 4 \\
Mar. & 1 \\
Mar. & 8 \\
Mar. 10 \\
Mar. & 12 \\
Mar. & 14
\end{tabular} & $\begin{array}{l}c . c . \\
-11 \\
+13 \\
+5 \\
+4 \\
+2 \\
+0 \\
+2\end{array}$ & $\begin{array}{l}\text { c.c. } \\
-17 \\
+\quad 6 \\
+3 \\
+8 \\
+4 \\
0 \\
-1\end{array}$ & $\begin{array}{l}+0.015 \\
-.035 \\
+.01 \\
-.01 \\
-.01 \\
0.015 \\
+\quad .015\end{array}$ & $\begin{array}{l}-1.0 \\
+1.0 \\
-2.0 \\
-2.5 \\
-0.5 \\
+1.0 \\
-1.5\end{array}$ & $\begin{array}{l}+1.2 \\
-\quad .4 \\
+6.5 \\
+0.2 \\
-0.5 \\
-0.7 \\
+1.2\end{array}$ & $\begin{aligned} & \text { liter. } \\
&-0.12 \\
&+.04 \\
&+.73 \\
&-.11 \\
&-.11 \\
&+.12 \\
&+.27\end{aligned}$ & $\begin{array}{l}c . c . \\
-61 \\
+18 \\
-65 \\
-7 \\
+18 \\
+57 \\
-6\end{array}$ \\
\hline \multicolumn{2}{|c|}{ Average variation.... } & 5 & 6 & .015 & 1.5 & 1.5 & .21 & 33 \\
\hline
\end{tabular}

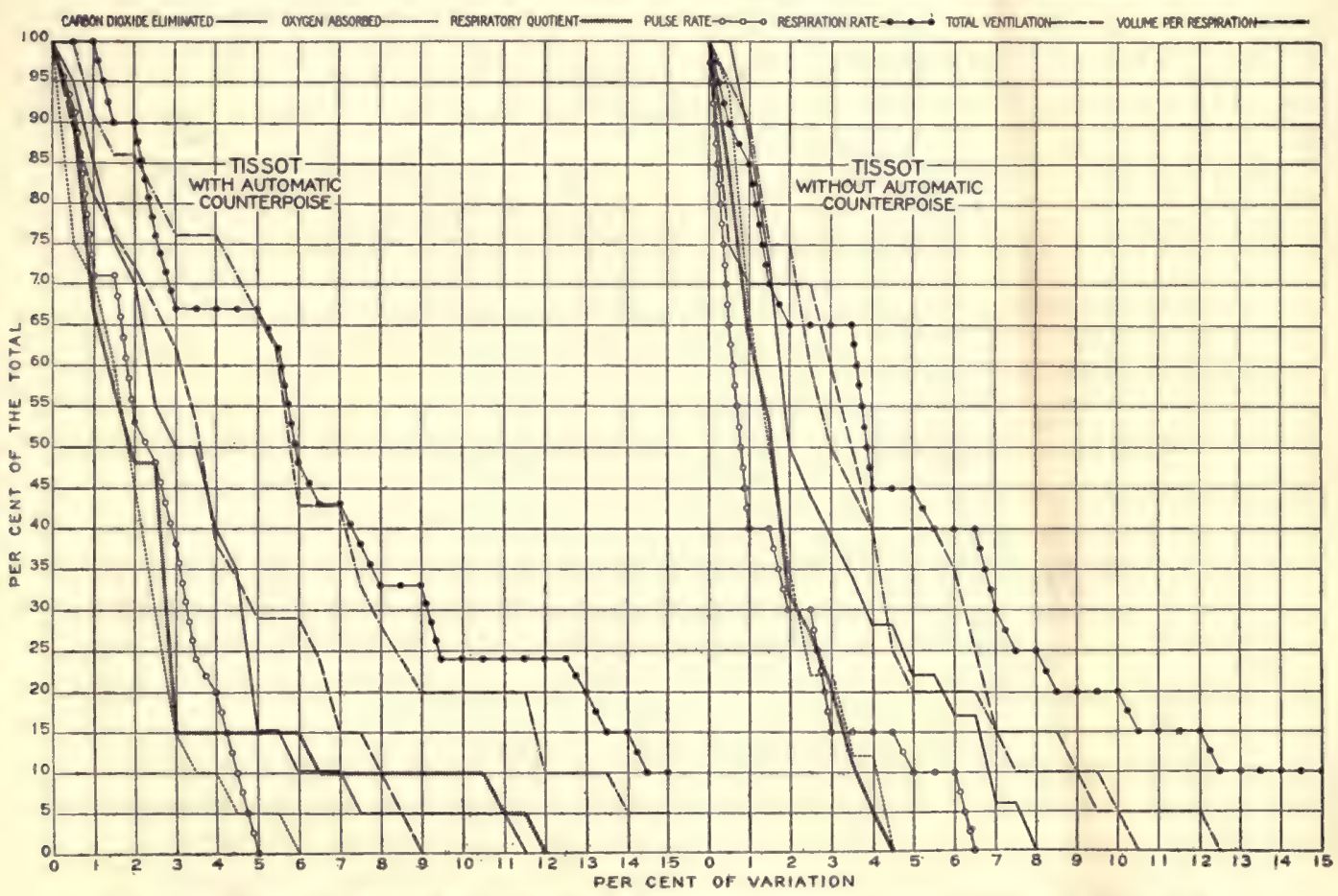

FrG. 74.-Probability curves for the series of comparison experiments with and without the counterpoise on the Tissot spirometer.

The ordinates indicate the percentage of the total number of periods and the abscissw represent the percentage of variation from the average. 



\section{PART III.}

\section{CRITICAL DISCUSSION OF RESPIRATION APPARATUS AND THEIR TECHNIQUE.}

In this investigation essentially two types of respiration apparatus have been employed. One is constructed on the Regnault-Reiset principle, sometimes designated as the "closed-circuit" or "direct" method; the other is on the "open-circuit" plan or the so-called "indirect" method, requiring the use of valves and apparatus to measure and analyze the expired air. The first type is represented by the two forms of the Benedict universal respiration apparatus (the tension-equalizer unit and the spirometer unit) and by Rolly's recent modification of the tension-equalizer unit. The indirect type is represented by the ZuntzGeppert, Tissot, and Douglas methods. As the experiments carried out in this investigation have shown that practically the same results can be obtained with all of these methods, it is of interest to consider the advantages and disadvantages of the different forms of respiration apparatus regularly employed in various laboratories and clinies. In this discussion, however, only those apparatus will be included which have been used in this research.

\section{BENEDICT UNIVERSAL RESPIRATION APPARATUS.}

The spirometer form of the Benedict universal respiration apparatus, which is coming more and more into use, has certain special advantages. One of these is the facility with which respiration experiments with periods of short duration may be made and the results calculated. The ease and rapidity with which this apparatus may be manipulated are especially appreciated by those who are required to make long experiments in which the later periods depend upon the results of those preceding. In many of the experiments in this laboratory, as, for instance, when the effect of a superimposed factor is being studied, it is necessary to know as soon as possible the results of the first two or three periods, so as to assure the experimenter that an accurate baseline has been established before the experiment is continued under the changed conditions. With this apparatus it is possible, with the help of one laboratory assistant, to make a series of three 15-minute periods and to calculate the results in the minimum time of an hour and a half. In fact, the results of the first two periods may be calculated while the succeeding periods are being carried out. In a long series of experiments made with a fasting man it was possible in every case to complete the calculations of the first two 15-minute periods by the end of the third period. This is possible with no other apparatus now in use for the determination of the respiratory exchange. 
A second advantage of this apparatus is the accurate picture of the respiration which may be obtained from the graphic record of the movements of the spirometer bell, by which any irregularity or abnormality is very accurately shown. For instance, these records indicate when the subject is drowsy; this is of special importance in comparing the results of respiration experiments, as the metabolism is greatly influenced by sleep. Information regarding any such irregularities is necessary in interpreting the respiratory quotients, as their value depends upon the normality of the breathing.

Still another advantage of the Benedict respiration apparatus is the fact that it dispenses with the use of gas-analysis apparatus and with the analysis of a large number of samples of expired air. Those who are accustomed to making these analyses know that such work is not only tedious but somewhat difficult, requiring special training to obtain accurate results.

Rolly ${ }^{1}$ has considered it necessary, in his experiments with a modified Benedict respiration apparatus, to make an analysis of the air in the apparatus at the beginning and end of the experimental period. $\mathrm{He}$ states that it is not possible to get exact values for the oxygen consumption without such gas analysis, as the composition of the air alters and the volume of the air also alters, owing to changes in barometric pressure and temperature. As was pointed out in the description ${ }^{2}$ of the original apparatus, theoretically corrections should be made for changes in barometric pressure and temperature and in the composition of the air of the apparatus, but practically it is not necessary. Grafe, ${ }^{3}$ in his discussion of the advantages and disadvantages of the Benedict apparatus and of Rolly's modification, points out that theoretically the results with Rolly's modification are more exact than with the original apparatus of Benedict, $i$. e., the tension-equalizer unit, but that the control experiments made with the latter are proof of its accuracy.

The slight difference between the two methods may be shown by comparing the respiratory quotients given by Rolly $^{4}$ with those obtained by computing them from Rolly's protocols. Rolly's respiratory quotients are $0.7991,0.7432$, and 0.773 . Those calculated by the Benedict method from Rolly's own figures for the carbon-dioxide production, oxygen consumption, and the nitrogen admitted with the oxygen are $0.7968,0.7398$, and 0.7845 respectively. In the last experiment, there was a change of $1 \mathrm{~mm}$. in the barometric pressure, which is unusually large. Even with this large variation in barometric pressure it is seen that the values for the respiratory quotient obtained by the two methods agree within 0.01 . Furthermore, an examination

1'Rolly and Rosiewiez, Deutsch. Archiv f. klin. Med., 1911, 103, p. 58.

${ }^{2}$ Benedict, Am. Journ. Physiol., 1909, 24, p. 345.

${ }^{3}$ Grafe, Abderhalden's Handbuch der biochemischen Methoden, Berlin and Vienna, 1913,

7, p. 472.

'Rolly and Rosiewicz, Deutsch. Archiv f. klin. Med., 1911, 103, p. 58, and Rolly, ibid., p. 117. 
of the results obtained by Rolly in several experiments in which the so-called "nitrogen balance" is given shows that even with the precautions he has taken regarding analysis, barometric pressure, and temperature there are wide variations in this balance, which are as high as 150 c.c. in some experiments. These may be due to variations in the content of the lungs between the beginning and end of the period, because of the difficulty in making a deep expiration. As his experimental period is about half an hour long, this would mean an error of 5 c.c. per minute. If the carbon-dioxide elimination were 200 c.c. per minute and the oxygen consumption 250 c.c. per minute, an error of 5 c.c. would change the respiratory quotient from 0.800 to 0.785 or to 0.815 ; this error is greater than would be expected with the precautions taken by Rolly.

Rolly's modification is larger than the original apparatus, having a volume of 18 liters as compared to 9 to 10 liters in the original. Thus changes in barometric pressure and temperature have a greater significance in his modification than with the original type; furthermore, his experimental periods are half an hour long while the experiments with the Benedict respiration apparatus are usually only 15 minutes long. These facts should be noted in considering the theoretical question of the influence of changes in temperature or pressure.

The analysis of the air at the beginning and end of an experimental period is of advantage as a test of the air-tight condition of that portion of the apparatus which is connected to the subject. The method of applying the correction for a leak as ascertained by analysis is, however, not so simple as it appears. In the first place, one must know the exact volume of the apparatus used. It is also necessary to have the composition of the air in the apparatus essentially the same as that in the man's lungs, otherwise the mixture of air in the apparatus and in the man's lungs will be different at the end from that at the beginning of the period. For example, if the percentage of nitrogen in the apparatus is 70 and in the man's lungs 80 , with the residual air 1,500 c.c. and the air-content of the respiration apparatus 20 liters, the percentage of nitrogen in the apparatus at the end of the period would be raised to 70.70. This would indicate an apparent absorption of oxygen amounting to 140 c.c. which did not actually take place. For such correction it is likewise necessary to have the volume of the apparatus at the beginning and end of the period precisely the same. One must also know very exactly the composition of the compressed oxygen used, and finally, one must have a very accurate gas-analysis apparatus. The aim of the operator with the Benedict apparatus should be to take every precaution to avoid leaks rather than to estimate such leaks as may occur. The question of this control and the use of various breathing appliances are subsequently discussed. ${ }^{1}$ In general, 
leaks in the apparatus and corrections for leaks may be taken as evidence of poor technique.

Rolly has also added another modification of the Benedict method which he considers necessary, $i$. $e$, , that of equalizing the pressure throughout the apparatus in order to know the true volume at the beginning and end of the experimental period. Tests made with the Benedict apparatus in this laboratory have shown that this is entirely unnecessary. If a reading is taken of the spirometer bell before the motor is started and the ventilating current is kept in motion for 15 minutes or longer, it will be found that the bell returns to the same position after the motor is stopped as that previous to the beginning of the test. Furthermore, if the movement of the drum is recorded during the 15 minutes that the ventilating current is in motion, after the drum has again settled into position it will be found that the record is a straight line and that there is no change in volume. The equalization of pressure in the apparatus is wholly unnecessary if the apparatus is constructed according to the design given by Benedict. If the method is employed of filling the spirometer bell to the same point while the apparatus is running, it is necessary to have an electric current which is very constant, otherwise there may be differences in compression in the apparatus due to slight differences in speed.

While the Benedict apparatus has many advantages, it also has certain disadvantages, previously pointed out by Benedict ${ }^{1}$, which require special care in the technique to overcome. As with all apparatus of the closed-circuit type, the slightest leak vitiates the results. Extremely small leaks may occur- - so small as to escape detection-and even a small leak may change the relation between the carbon-dioxide production and oxygen consumption from a probable figure to one which is wholly improbable; unless it is known absolutely that a leak has occurred, one is in grave doubt as to the necessity of rejecting the figure obtained. If, for example, the respiratory quotients for the successive periods of an experiment have been uniformly 0.85 , and the quotient drops in a subsequent period to 0.75 without an apparent cause, the logical inference would be that the change is due to a leak, and yet there may be no proof of it. This uncertainty regarding the occurrence of a leak makes it questionable to assume one. In a study made of the effect of a no-carbohydrate diet upon the respiratory exchange, it was found that in one period of an experiment there was a tendency for the respiratory quotient to be higher than that which would be expected. The pneumatic nosepieces were used in this experiment with a good subject, but upon inflating them to a somewhat greater distention, so that they fitted the nose still more closely, results were obtained which were more nearly in accord with earlier periods. 
The leaks in the various portions of the apparatus can be easily controlled; consequently the possibility of an error from this cause is not a serious disadvantage. If the apparatus has not been properly constructed, leaks occasionally occur in weak portions; these may be due to a defect in the rubber connections or to imperfections in the metal parts. They may, however, be detected before an experiment by setting the ventilating current in motion and noting the position of the pointer on the spirometer bell. If the apparatus is air-tight the pointer remains constant; if the apparatus leaks the position of the pointer changes one way or the other. Should such a change occur, it is only necessary to apply the usual tests to find what portion of the apparatus is defective.

The question of a leak in the connection between the subject and the apparatus, as, for example, in the mouthpiece or the nosepieces, is of much more significance and much more difficult to control, as it depends so largely on the cooperation of the subject. The kymograph records sometimes show when such a leak has actually taken place by a break in the regularity of the respiration and a change in the level of expiration. The leak may be so small, however, as to escape detection in this way, and, again, these irregularities in the respiration record may not be due to leaks at all, but to actual irregularities in the point to which the subject empties the lungs. The depth of expiration may be controlled by using a pneumograph around the chest and possibly another pneumograph around the abdomen. If these pneumographs are well adjusted and a sensitive tambour is used, it is nearly always found that changes in the regularity of the pneumograph record are accompanied by changes in the regularity of the respiration record.

In this connection a difficulty encountered in determining the oxygen consumption may be considered. It is always assumed in the determination of the oxygen consumption that the volume of the apparatus plus the volume of the respiratory tract of the subject is the same at the beginning of an experimental period as at the end. This means that when the valve is turned at the end of the period the subject has expired to exactly the same point as when the valve was turned at the beginning of the period. It can readily be seen that if there is a change in the actual volume of the lungs the value for the consumption of the oxygen will be seriously affected. Sometimes this change may be very gradual and at other times abrupt. When it is abrupt the spirometer record will show it definitely. It is, then, possible to calculate a correction for such change and apply it to the results.

Rolly has sought to overcome this difficulty by having the subject expire as completely as possible and turning the valve at the end of the forced expiration. This is objectionable for several reasons. In the first place, it calls the attention of the subject to his respiration and to 
the beginning and end of the experimental period, a practice which we have found very disadvantageous and liable to result in disturbing the normal respiration; it is desirable to conduct the experiment in such a manner that the subject has no knowledge as to the beginning and end of the periods. Furthermore, there is a question as to whether it is possible for the subject to expire voluntarily and forcibly to exactly the same point each time. Such a procedure would require considerable practice and the position in which the subject usually lies, $i . e$. , on his back, is not conducive to a perfect forced expiration. It is also necessary for the operator to turn the valve at exactly the moment when the subject has ended this forced expiration; this may make it necessary for him to hold this position until the valve is turned. In Rolly's modification there is no control on the accuracy of this valve movement; in the spirometer type of the Benedict respiration apparatus, an admirable control has been established on the turning of the valve at the exact end of the expiration.

This question of the volume of air in the lungs of the subject at the beginning and end of the experimental period is of the most vital importance in determining the oxygen consumption by the Benedict apparatus. After all other sources of error are eliminated, it remains the most important assumption bearing upon the fundamental principle of the determination of the oxygen consumption. In the earlier development of the apparatus this was apparently not a serious matter, as most of the subjects were more or less trained to breathing on respiration apparatus and accordingly breathed regularly and quietly, without an apparent variation in the volume of the lungs. When the apparatus was used with subjects who were unaccustomed to it this factor was somewhat more in evidence and, in many instances, it was apparent that the subjects were not breathing normally and regularly and that the volume of air in the lungs must be continually changing. In a study of the influence of a no-carbohydrate diet, it was found impossible to use a certain subject, owing to the fact that in several tests made with him, he apparently constantly increased the volume of air in the system in breathing instead of reducing it. "The fact that we were not obtaining trustworthy results agitated him and this caused even greater disturbances. Attempts were made at different times of the day to secure better results, but without marked success. There have also been other cases when it was very difficult to obtain uniform results. A comparison of the probability curves for the respiratory quotients obtained with the Benedict, Zuntz, and Tissot apparatus show that both with the Zuntz and with the Tissot apparatus the respiratory quotients are more uniform than with the Benedict apparatus. As the experiments in which these apparatus were compared were carried out under the same conditions, the lesser degree of uniformity with the Benedict apparatus is probably due to 
the oxygen determinations being affected by changes in the volume of the lungs. This disturbance, which does not play a noticeable part in the oxygen determination by the open-circuit methods, gradually disappears with most subjects as they become accustomed to the apparatus, so that practice plays a significant rôle.

When the motor is running and the air circulating, there is a slight mechanical vibration due to the movement of the blower and motor which varies with the apparatus used. This is at times noticeable, being referred to occasionally by subjects. It is an objectionable feature and constant attempts are being made to eliminate it.

The respiration is, as a rule, fairly normal with the Benedict respiration apparatus. The average subject breathes so regularly in quantity that the variations are not marked. Many subjects have stated that they were unable to tell whether they were breathing into the apparatus or into the open air. In fact, in one instance, a new subject was told that he would know when the valve was turned, as the air in the apparatus had a very slight odor. A few minutes after the valve had been turned he opened his mouth several times and, when asked why he did this, stated that he did not know that he was breathing into the apparatus.

The fact that subjects often fall asleep in experiments with this apparatus - much more frequently than in experiments with apparatus like the Zuntz-Geppert or the Douglas - gives evidence that the apparatus is certainly not unpleasant to breathe into and that the respiration is fairly normal. Coleman and Dubois ${ }^{1}$ have used the apparatus with a number of typhoid patients in Bellevue Hospital, New York. They state that, as a rule, patients are somewhat nervous the first time the apparatus is used, but soon become accustomed to the routine and seem to enjoy it, since they suffer no discomfort. They report difficulty in obtaining normal results with two individuals because of abnormal breathing, as they breathed too deeply or too rapidly. Rolly has also used his modification of this apparatus with many fever patients. The apparatus has likewise been employed with success by Professor H. M. Smith in his studies with athletes at Syracuse University. Dr. Paul Roth, of the Battle Creek Sanitarium, has, with this apparatus, studied the respiratory exchange of a large number of individuals, both normal and pathological, with very satisfactory results. More recently Dr. J. H. Means, of the Massachusetts General Hospital, and Dr. W. H. Boothby, of the Peter Bent Brigham Hospital, have used it for studies with patients in the hospitals mentioned.

Considerable time is necessary to acquire the technique of the apparatus, as it includes attention to many details outside of the usual routine in any series of respiration experiments, such as weighing the absorbers, making tests for leaks, adjusting properly the signal magnets, 
tambours, and kymographs, and similar matters. The manipulation of the apparatus requires the utmost concentration, but it can not be said to be really difficult and any investigator familiar with laboratory methods in physiology and chemistry should be able to carry out the routine without additional training.

Like any good physiological apparatus, this respiration apparatus necessitates a certain amount of constant care. If the apparatus is kept in good condition, our experience has been that after a month or two of disuse it can be employed for experiments with but little preliminary repair. Certain portions of the rubber connections deteriorate and must occasionally be renewed; if a superficial examination does not show such defects, a test for leaks immediately reveals any weakness of this character. The two three-way valves ${ }^{1}$ which provide for using either one of duplicate absorbing systems are sometimes found to leak and to require re-grinding. The other parts of the apparatus rarely need attention, provided they have been well constructed. The filling of the soda-lime bottles and sulphuric-acid containers does not need particular training. The soda-lime may be made, ${ }^{2}$ as has been the custom in this laboratory, or may be purchased ready for use in containers of suitable size.

In conclusion, it may be stated that the chief advantages of the Benedict respiration apparatus are the rapidity with which experiments of short duration may be carried out and the exact graphic record of the respiration which may be obtained with the spirometer type. The disadvantages are the difficulty in obtaining absolute freedom from leaks in the connections of the apparatus to the subject and the possibilities of unreliable determinations of the oxygen consumption due to irregularities in the volume of the lungs. With the majority of individuals, the breathing is normal and the results of the measurements of the respiratory exchange are accurate.

\section{ZUNTZ-GEPPERT APPARATUS.}

The Zuntz-Geppert apparatus is, in all probability, the most used respiration apparatus in existence. It has been very extensively employed in Europe, where an enormous amount of work has been done with it, and to a slight extent in American laboratories.

The apparatus has been criticized by Magnus-Levy ${ }^{3}$ in his description of it, also by Durig in his reports on the Monte Rosa expedition ${ }^{4}$ and on the effect of oxygen-rich atmospheres on the respiratory exchange. ${ }^{5}$ In all of these the discussion is mainly on the question of the gas analysis and on the probability of error and the limits of error

\footnotetext{
1See p. 41.

${ }^{2}$ Benedict and Talbot, Carnegie Inst. Wash. Pub. 201, 1914, p. 40; and Benedict, Deutsch. Archiv f. klin. Med., 1912, 107, p. 166.

${ }^{3}$ Magnus-Levy, Archiv f. d. ges. Physiol., 1894, 55, p. 1.

"Durig, Denkschriften der mathematisch-naturwissenschaftlichen Klasse der kaiserlichen Akademie der Wissenschaften, Vienna, 1909, 86, p. 116.

'Durig, Archiv f. Anatomie und Physiologie, Physiologische Abteilung, 1903, p. 209.
} 
allowable in exact research work. Loewy ${ }^{1}$ has made an investigation to find whether or not the respiration and the respiratory exchange are markedly affected by breathing through the Zuntz-Geppert respiration apparatus during muscular work. In this study he measured the respiratory exchange after a certain amount of muscular work had been done and also carried out experiments in which the respiratory exchange was measured both during the working period and in the period after the work had been completed. He found that the respiratory exchange in the period after work was not affected by breathing through the respiration apparatus during the period of work. Grafe ${ }^{2}$ has called attention to the criticisms of the Zuntz-Geppert apparatus and Rolly ${ }^{3}$ and Jaquet ${ }^{4}$ have also referred to the abnormal results which are sometimes obtained.

One of the criticisms brought against the Zuntz-Geppert method is that the sampling is not proportional; in other words, that the sample of air does not represent the true average. In experiments which were made in this laboratory a known amount of carbon dioxide was introduced into a current of air passing intermittently into the meter and it was found that the percentage of carbon dioxide recovered in the sample taken by the proportional method agreed well with the percentage calculated. The experiments were somewhat difficult to carry out, as it was not easy to arrange for the intermittent delivery of carbon dioxide into the air-current in such a manner that it would be readily calculated or determined. The air can be mixed to some extent before it reaches the sampling tube by inserting a large bottle or flask between the expiration valve and the entrance to the Elster meter. In experiments in which the expired air changed rapidly in composition such a procedure would be a disadvantage, as there would be a dead space through which the air would have to pass before a sample was taken. The mixing and sampling would thus lag behind the changes in the expired air. This is true even with the present arrangement, as the long tube between the man and the gas-meter is a dead space which must be swept out before the sample drawn through the tube will actually represent the composition of the air. This lag plays no great rôle unless the experimental periods are of extremely short duration and in periods of 15 or 20 minutes it is not of much importance. Geppert and Zuntz ${ }^{5}$ point out that the capacity of the tube from the valves to the sampling device should always be greater than the maximum expiration likely to occur during an experiment.

The Zuntz-Geppert method of proportional sampling was checked by Geppert $^{6}$ with a rabbit, all of the carbon dioxide produced being ab-

'Loewy, Archiv f. d. ges. Physiol., 1891, 49, p. 492.

2Grafe, Abderhalden's Handbuch der biochemischen Arbeitsmethoden, 1913, 7, p. 459.

${ }^{2}$ Rolly, Deutsch. Archiv f. klin. Med., 1908, 95, p. 75.

'Jaquet, Ergebnisse der Physiologie, 1902, 2, p. 457.

Geppert and Zuntz, Archiv f. d. ges. Physiol., 1888, 42, p. 189.

'Geppert, Archiv f. experimentelle Path. u. Pharm. 1886-87, 22, p. 373. 
sorbed by potassium-hydroxide solution and the amount found compared with the amount determined by an analysis of the proportional sample. The amounts of carbon dioxide obtained were 520.6 c.c. and 524.7 c.c., respectively. The whole method has been tested by Zuntz ${ }^{1}$ with burning candles, the experimental procedure being the same as in an ordinary respiration experiment. The average error found in the oxygen determination was -0.40 per cent and in the carbon-dioxide determination -2.15 per cent.

Schaternikoff ${ }^{2}$ states that the method of measuring the volume of air by means of a meter, when the air is being pushed through it intermittently, is liable to error because the inertia of the moving drum causes it to record more than the true volume.

Calibrations of the Elster meter were made in this investigation, in which air or oxygen was driven through it in exactly the same manner as in a respiration experiment. These calibrations gave factors for correction of 101.8 per cent and 100.8 per cent with continuous flow and 100.9 per cent with intermittent flow, these errors being no larger than would be expected in individual calibrations. In all probability, the measurement of the expired air by the Elster meter is accurate to within 1 or 2 per cent.

The level of the water in the Elster meter is of prime importance. To secure trustworthy results, it is essential that the meter should be level as any slight variation in the level of the water makes an appreciable difference in the results. In this laboratory we have installed on the outside of the meter a water-gage with a millimeter scale, by means of which the exact height of the water inside the meter can be noted at any time.

The Zuntz-Geppert gas-analysis apparatus can be made to give results which agree very well, particularly if that form is used in which the burettes are divided into 0.02 c.c. The manipulation of the Zuntz-Geppert gas-analysis apparatus is somewhat difficult, and it will be noted from the statisties ${ }^{3}$ of the experiments that in some of the comparisons with the Zuntz-Geppert method this gas-analysis apparatus was not used, but that the Haldane apparatus was substituted.

An advantage in using the Zuntz-Geppert gas-analysis apparatus is the fact that duplicate analyses may be carried out simultaneously, thus assuming identical conditions. This, however, insures only uniformity in the technique, but does not guarantee the accuracy of the technique or of the results. It has been my experience that it is more difficult to obtain duplicate results with any method when the determinations are made at different times than when they are all made at

\footnotetext{
${ }^{1}$ Zuntz and Hagemann, Landw. Jahrb., 1898, 27, Supplm. IIr, p. 10.

${ }^{2}$ Schaternikoff, A new method for determining the quantity of exhaled air in man and the quantity of carbon dioxide contained in it. (Russian.) Dissertation, 1899, Moscow.

${ }^{3}$ See p. 129.
} 
exactly the same time. It is therefore a better control of a method to make the duplicate determinations at different times and preferably on different days. I have always more confidence, for example, in the results of analyses of a sample of expired air when the second analysis is made on a different day than when the two analyses are made simultaneously or even immediately succeeding one another, provided the sample is stored in such manner as to prevent loss of carbon dioxide.

One serious objection to the Zuntz-Geppert gas-analysis apparatus is the fact that the gases are collected over water. Many experiments of various kinds in this laboratory have shown that the collection or storage over water of air containing carbon dioxide is a very bad practice, for even during the time of collection, i.e., 15 or 20 .minutes, there is a possibility of a slight disappearance of the carbon dioxide, which does not occur when the gas is collected over mercury. Magnus-Levy ${ }^{1}$ cites experiments which were carried out by Zuntz and Hagemann in which it was demonstrated that the analyses of expired air collected over mercury and over water gave, on the average, the same results; but the variations in the results obtained in analyzing carbon dioxide collected over water are considerable and it is questionable whether results varying so widely show that the method is a good one. While it may be possible that air collected in the burettes of the ZuntzGeppert gas-analysis apparatus and analyzed immediately suffers no significant loss of carbon dioxide, there is no question that air collected over water in glass samplers and analyzed later would lose a part of its carbon dioxide, and that such analyses would not give accurate results. This is particularly true if the samples are stored for 12 hours or more; the losses are then very large, and occur even when there is no water visible in the container. Even saturated air, collected in dry glass containers or over mercury, loses carbon dioxide if the samples are kept for several days, but the loss is not great enough to affect results in work on the respiratory exchange. The practice of collecting samples over water and then storing them for analysis must certainly be avoided in all respiration work in which the highest degree of accuracy is desired.

Durig $^{2}$ has pointed out a possibility of error in the determination of oxygen by the Zuntz-Geppert gas-analysis apparatus in that even when constant results are obtained after absorption by phosphorus it is possible that the oxygen is not all absorbed. He mentions particularly the fact that when time is limited there is a tendency for operators to neglect the last particle of absorption. The use of phosphorus in gas-analysis apparatus is by no means to be discouraged, however, in spite of this possibility. In our use of the Haldane appa-

${ }^{1}$ Magnuo-Levy, Archiv.f. d. ges. Physiol., 1894, 55, p. 20.

'Durig, Denkschriften der mathematisch-naturwiasenschaftlichen Klasse der kaiserlichen Akademie der Wissenschaften, Vienna, 1909, 86, p. 119. 
ratus, which has been adopted in this laboratory for gas analyses, phosphorus has been employed for the absorption of oxygen, and in one portable Haldane gas-analysis apparatus it was used for over 6 months without replacement. In its use, however, we take special care that, even after the readings are constant, the gas remains over the phosphorus for a sufficient length of time to insure complete absorption.

If the possibilities of the loss of carbon dioxide in the collection of the gas over water and of an incomplete absorption of oxygen by phosphorus are taken into consideration, it will be seen that these may be the apparent causes of the low respiratory quotients which are occasionally obtained with the Zuntz-Geppert gas-analysis apparatus. It is somewhat difficult, however, to understand why such quotients occur more frequently in fever than with normal people.

Hoppe-Seyler ${ }^{1}$ states that in spite of every attempt to have the valves, meter, and tubing free from resistance, breathing through the Zuntz-Geppert apparatus is not free breathing and that long-continued experiments can not be carried out with it. Katzenstein ${ }^{2}$ says that in spite of all care taken, the breathing through a mouthpiece and a meter must involve work and therefore a greater metabolism will result. The experiments carried out here, however, indicate that the respiration with the Zuntz-Geppert method is, on the whole, normal when the subjects have become accustomed to it. Even subjects who stated that they expected it to be hard to breathe through the valves have, after a few moments of breathing, found no great difficulty. After the respiration has become uniform it is apparently perfectly normal. The control upon the uniformity of the breathing can be obtained directly from readings of the meter, and if these are made every minute the results show whether or not there is a regularity in the ventilation of the lungs in the individual minutes. This is one of the advantages of the Zuntz-Geppert method and the readings thus obtained may be depended upon. The practice of reporting values to the single cubic centimeter or fraction thereof is not warranted by the general percentage accuracy of the apparatus, when the factors of the calibration, temperature, pressure, and the personal equation in reading the results are taken into consideration.

The manipulation of the Zuntz-Geppert apparatus is in part simple and in part somewhat complicated. Reading the individual ventilation figures requires constant attention. Furthermore, the adjustment of the valves is somewhat difficult, for in order to insure the most satisfactory results with this apparatus, it is necessary that the valves be so adjusted that resistance will be at a minimum and the valves will also close properly when there is suction or pressure. We have used both fish membrane and thin rubber membrane for the valves, but do

${ }^{1}$ Hoppe-Seyler, Zeitschr. f. physiol. Chemie, 1894, 19, p. 578.

${ }^{2}$ Katzenstein, Archiv f. d. ges. Physiol., 1891, 49, p. 380. 
not find that either one is better than the other. The manipulation of the gas-analysis apparatus is also difficult and, according to our experience, requires considerable training for its successful use.

To keep the apparatus in good condition does not require a great deal of attention, the parts needing most care being the valves and the gas-analysis apparatus. The valves should be so cared for that they will have the least resistance when used and be without leak. During experiments they should always be moist, as otherwise they do not functionate properly. The rubber connections in the gas-analysis apparatus are also liable to leak and to deteriorate. Setting up the apparatus requires skill in order to avoid breaking the different parts of the capillary tubing; the gas-analysis apparatus is especially large and cumbersome, with many parts to be connected. The caustic-potash solution in the carbon-dioxide pipette occasionally needs renewing. The phosphorus also needs attention occasionally, particularly if the apparatus has been used in a warm room, as in this case there is a tendency for the phosphorus to fuse together and to cause errors due to the occlusion of small bubbles of gas when the air is drawn from the phosphorus pipette. When in use, the apparatus should be frequently controlled by analyses of outdoor air, which is uniform in composition all over the world and in all kinds of weather. ${ }^{1}$

The apparatus used by Zuntz and his co-workers does not give a graphic record of the respiration, either in volume or in rate, and in order to have an accurate knowledge of the respiration-rate it is necessary to have some accessory apparatus.

The results obtained with the Zuntz-Geppert apparatus are reliable, provided the greatest care is taken in carrying out the experiments. When the breathing is normal this is particularly true as to the respiratory quotient, which represents the relation between the carbon dioxide and the oxygen. Even if there is a leak in some part of the measuring apparatus, the relationship expressed by the respiratory quotient would not be affected, but the total quantity of expired air would be less than the actual amount and consequently the total metabolism as measured would be too low. This would not be true if a certain portion of the expiration were lost, as, for example, the last portion or the beginning, as the ratio between the carbon dioxide and the oxygen is not the same in all portions of the expired air. In the manipulation of the apparatus there is practically no noise which would disturb the subject, and this quietness is conducive to good results.

When samples are being taken and stored continuously, as they may be over mercury, a series of experiments may be carried out with but very short intermissions, thus making the measurements practically without interruption; this is also true with the Benedict apparatus. The gas analyses necessitated by this method are, however, tedious and 
time-consuming and results can not be obtained so quickly as with the Benedict method. If the air is collected directly in the gas-analysis apparatus, the results may be obtained more quickly than if collected in a series of samples and analyzed later. In a series of experiments which can be definitely planned beforehand, this is not an objection.

In general, it can be stated that the Zuntz-Geppert method for the determination of the respiratory exchange in short periods is more difficult and complicated than the other methods used for this purpose. When the utmost precautions are taken to carry out experiments with this method in the way it should be used, the results of the measurements of the total gaseous exchange are reliable and comparable to those secured with the other methods considered in this research. The respiratory quotients are uniform and comparable to those obtained with other apparatus with which either nose- or mouth-breathing is employed.

\section{TISSOT APPARATUS.}

The general principle of the Tissot apparatus is that of an opencircuit apparatus, $i$. e., the inspired and expired air are separated and the expired air is collected, measured, and analyzed. From the results obtained, the respiratory exchange is calculated. The valves used in separating the inspired and expired air are very simple and of very light construction. The flap moves easily, offering practically no resistance to the passage of air. The valves need very little attention other than to see that they are dry and clean as there is no membrane to get out of order or to deteriorate. If properly taken care of, they should last indefinitely.

The valves have one disadvantage, however, in that the glass connecting the two metal parts is liable to become disconnected, especially if hot water is used for cleansing them; if accidentally dropped, the glass part is of course easily broken. The valves also have to be kept in a certain position in order to work efficiently. With the glass connection the position of the flap may be readily seen and the valves may be easily adjusted in the proper position on the tee-piece which connects them. The valves may be made less fragile by having the connection made of brass instead of glass, so that the whole valve will be of metal. This method of preventing breakage has the disadvantage that one can not see whether the valves are working properly, but if a notch is made in one end to indicate the proper position for use, and if care is taken to adjust the valves with the aid of this indicator, there is no reason why they should not work efficiently. Practically none of the subjects with whom we experimented complained that the valves did not move freely. Sometimes if the flap becomes clogged by the accumulation of material of any kind it will stick a little before opening. This can, however, be remedied by a thorough cleansing and polishing. If the valves are kept clean, the closure is perfect and 
when pressure is put on them in the reverse direction ordinarily no air will escape. In our use of them, they have given very satisfactory results, showing an efficiency when tested of 99 per cent in separating inspired and expired air. ${ }^{1}$ This is well within the limits of error in measurement of the total expired air.

When experimenting with subjects who are accustomed to breathing through the nose, it is somewhat better to use nosepieces than a mouthpiece. The nosepieces used with the Tissot apparatus are of special advantage because they permit very free breathing through the nose. They are not, however, so well-constructed mechanically as they should be, as they do not readily conform to the shape of the nose or to the openings of the nostrils. The glass nosepieces devised by Tissot are circular in cross-section, but should be elliptical, as this shape is more nearly that of the opening of the nostril. We have had nosepieces constructed on the elliptical principle which were found somewhat more comfortable than the round nosepieces. ${ }^{2}$

The respiration through these valves and nosepieces is very free and with the majority of the subjects in our experiments with this apparatus we have obtained very successful results. This was especially remarkable in the case of J. H. H. During one series of experiments with the Benedict respiration apparatus it was found practically impossible to obtain good results with him because of his inability to maintain a regular respiration, the volume of the air in the lungs varying so much that the determination of the oxygen absorbed could not be secured. A few experiments were made with him in which the Tissot apparatus was used with satisfactory results. Thereafter the Tissot apparatus was employed in experiments with this subject. The results of consecutive determinations with the two apparatus are given in table 43 . Later, a comparison of the two methods was made with the same subject and the respiratory quotients obtained with the Benedict apparatus did not show so great variations as in table 43 . The greatest range was from 0.795 to $0.845 .^{3}$

The differences shown in the results with the subject J. H. H. for the two methods, however, can not be due solely to the differences in the apparatus, for in all probability this subject became more or less trained as the experimenting progressed, and for that reason he would give more uniform results with either apparatus. The fact that we were able in the later experiments to obtain good results with both methods shows that practice and familiarity with the apparatus has a great influence upon the results. The principle involved in the Tissot method of determining the respiratory exchange is theoretically good for the determination of the respiratory quotient, because it depends upon the composition of the expired air and not on the measurement of volume. 
It was shown in the comparison between the Tissot and the Benedict apparatus that the respiratory quotients with the Tissot apparatus were more uniform than those secured with the Benedict method.

The Tissot spirometer used for the collection of expired air is easily manipulated in the way devised by the originator, as one has simply to adjust the counterpoise correctly for any position in which the drum stands, $i . e$., so that the weight of the counterpoise will keep the spirometer bell in exact equilibrium, the siphon device automatically maintaining the equilibrium thereafter. The siphon attachment operates without difficulty if there is sufficient water-pressure to forcethe air bubbles out of the siphon. When water-pressure is not available, use may

TABLE 43.-Results of consecutive experiments with Benedict and Tissot apparatus, showing difference in uniformity of respiratory quotients with subject $J . H$. H.

\begin{tabular}{|c|c|c|c|}
\hline \multicolumn{2}{|l|}{ Benedict apparatus. } & \multicolumn{2}{|l|}{ Tissot apparatus. } \\
\hline Date and time. & $\begin{array}{l}\text { Respira- } \\
\text { tory } \\
\text { quotient. }\end{array}$ & Date and time. & $\begin{array}{c}\text { Respira- } \\
\text { tory } \\
\text { quotient. }\end{array}$ \\
\hline 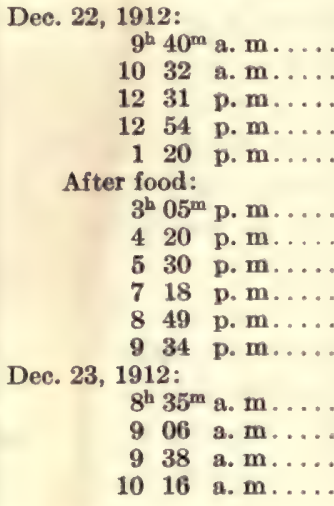 & $\begin{array}{r}1.12 \\
.66 \\
.84 \\
.71 \\
.86 \\
.82 \\
.85 \\
.85 \\
.85 \\
.74 \\
.75 \\
.76 \\
.76 \\
1.08 \\
1.02\end{array}$ & 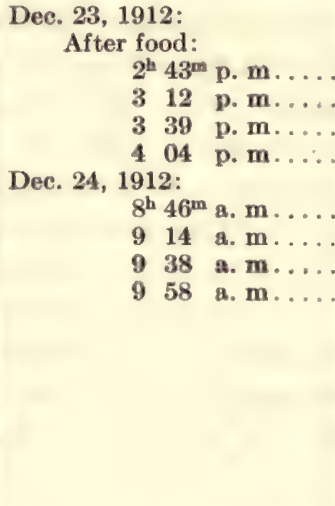 & $\begin{array}{r}0.73 \\
.73 \\
.74 \\
.73 \\
.75 \\
.73 \\
.72 \\
.75\end{array}$ \\
\hline
\end{tabular}

be made of a Mariotte flask, as described by Laulanie, ${ }^{1}$ or of a tank attached to the wall or some other support above the spirometer.

The spirometer is exceedingly sensitive to changes in the pressure of the air inside the bell, Tissot claiming it to be sensitive to $0.1 \mathrm{~mm}$. of water-pressure. While in our use of it we have not found so great a degree of sensitiveness, yet it is certainly sensitive to less than $1 \mathrm{~mm}$. of water-pressure. The series of comparison experiments in which a study was made of the effect of discarding the automatic device on the counterpoise showed that it is not necessary to have the spirometer so delicately counterpoised as Tissot has suggested, and, for all practical purposes, with normal subjects in measuring the respiratory

${ }^{1}$ Laulanie, fléments de physiologie, Paris, 1905, p. 344. 
exchange, it is sufficient to adjust the counterpoise to an equilibrium with the spirometer bell in a central position. This obviates the necessity of having a water-supply, only sufficient water being required to keep the level of the water inside the tank constant. Care must be taken, however, that the counterpoise is so adjusted that its weight does not exceed that of the drum by such an amount as would produce a decreased tension inside the spirometer which might be sufficient to open the valves and cause a movement of the drum independent of the movements due to the increase in the expired air. This would result in an error in the ventilation figures, although it would not affect the determination of the respiratory exchange.

The possible errors in the determination of the respiratory exchange by the Tissot method may be divided into two classes: one, those due to factors influencing the readings made in the measurement of the volume of the total air expired, the other due to factors influencing the sampling and the analysis of the expired air. The first two sources of error to be considered in the measurement of the total volume of expired air are those which affect the readings of the barometric pressure and the temperature of the air. The possible inaccuracy in the value for the barometric pressure is extremely small, for with any good barometer readings may be obtained to $0.1 \mathrm{~mm}$.; the error would thus be not more than $\pm 0.1 \mathrm{~mm}$., which is well within the limits of error in determining the respiratory exchange. For determining the temperature of the air in the spirometer, a thermometer may be placed in the opening provided at the top and readings made to $0.1^{\circ} \mathrm{C}$. It must be considered, however, whether a value thus obtained represents the true temperature of the air inside the spirometer. Errors may be avoided by having the water in the apparatus of the same temperature as the air in the room, so that air collected in the spirometer may be more nearly the temperature of the room than if extremely cold water were used.

To test the accuracy of the volume measurement, a series of experiments was made in which 50 liters of air were collected in a 50-liter spirometer, a 100-liter spirometer, and a 200-liter spirometer and allowed to remain for several days. The temperature was obtained each morning with the siphon automatic device actuating; the barometric pressure was also recorded. The volumes were then calculated to $0^{\circ} \mathrm{C}$. and $760 \mathrm{~mm}$. pressure. The variations obtained with the three spirometers were 0.7 liter for the 200 -liter spirometer, 0.2 liter for the 100-liter spirometer, and 0.5 liter for the 50-liter spirometer. The readings may be made more closely on the 50-liter spirometer than on the 200-liter spirometer, as the length of the scale is approximately the same with both apparatus; an increase in volume of 1 liter will therefore produce a greater rise with the 50-liter spirometer than with the 200 -liter spirometer. It is quite possible to read to 0.05 liter with 
the smaller spirometer, but only to 0.1 or 0.2 liter with the 200 -liter spirometer. This fact should be taken into consideration in experiments with the Tissot method. For example, in making experiments in which the periods are very short and the volume of air to be collected is not more than 50 liters, it is advisable to use the smaller spirometer, as more accurate readings may be obtained with it. In all but five of the comparison experiments in which the Tissot method was used the 200-liter spirometer was employed, the volume of air collected usually being 75 to 100 liters.

As the spirometer bell rises out of the water, some moisture adheres to the sides. This has a certain cooling effect, at least upon the outside, and may affect the volume of air inside the bell. To determine this possible influence, a 100-liter spirometer was filled as quickly as possible with room air and readings of the temperature of the air were taken every minute in the usual way, also readings of a thermometer hung as closely as possible to the water-level and to the side of the bell. The thermometer near the water-level showed a marked cooling effect after the bell had come to rest and the thermometer in the opening at the top of the spirometer bell indicated a simultaneous cooling effect upon the volume of air inside. By using the readings of the latter thermometer and calculating the volume of air in the spirometer to $0^{\circ} \mathrm{C}$. and 760 $\mathrm{mm}$. pressure, it was found that the variations in volume due to this cooling were less than 0.2 liter with a volume of 70 liters. Since this is less than 0.3 per cent, the possible error due to cooling must be very small, especially as the variation noted is also subject to possible errors in the reading of the volume of air and of the temperature.

The errors of the second class, $i . e$. , those affecting the sampling and analysis of the air collected in the spirometer, have occupied the attention of a great many observers. Durig ${ }^{1}$ has pointed out that there is a possibility of stratification in collecting expired air by this method and that such stratification may cause a considerable error when a large volume is sampled. To study this point and also to test the general accuracy of the Tissot method in the measurement of the carbondioxide content of expired air, a series of experiments was carried out in the following manner:

A pair of Tissot valves was attached to the hand spirometer ${ }^{2}$ by means of a glass tee. A small opening was made in the side of the glass tee and carbon dioxide was introduced from a cylinder of the compressed gas, the carbon dioxide passing through a 1-liter Bohr meter. The meter was immersed in a tank, as for the measurement of oxygen by the Benedict method. The outgoing valve was connected to a 200-liter Tissot spirometer. By raising and lowering the bell of the hand spirometer and drawing carbon dioxide intermittently through

'Durig, Archiv f. Anatomie und Physiologie. Physiologische Abteilung, 1903, p. 219.

'See p. 252. 
the side opening, it was possible to imitate the introduction of carbon dioxide into the large spirometer as in the ordinary respiration of a man. The spirometer was partly filled with air in this way, the ventilating being done more or less irregularly, so that the composition of the air might be as unequal as possible, although ordinarily nearly full respirations were simulated by the hand spirometer. The volume of carbon dioxide passed through the meter was then noted and the introduction of the gas stopped, the ventilation being continued for a few moments to sweep out all of the carbon dioxide in the tube leading to the spirometer. Readings of the volume and temperature of the air in the spirometer and the barometric pressure were next taken and a sample was drawn from the top of the spirometer in the usual manner, by means of a 300 c.c. sampler. The first and second samples were rejected and the gas was then put under pressure in the sampler. The air was analyzed with the Haldane gas-analysis apparatus.

The percentage content found in the first sample analyzed was 2.45 per cent; the amount calculated from the carbon dioxide introduced and that contained in the room air was 2.46 per cent. This sample was drawn at 10 a. m.; at $11^{\mathrm{h}} 15^{\mathrm{m}}$ a. m. another sample was drawn from the spirometer in the same way, the analysis showing 2.45 per cent of carbon dioxide present. At $2 \mathrm{p}$. m. still another sample was taken, which showed a carbon-dioxide content of 2.36 per cent. Another experiment of the same kind was carried out and a sample drawn at $3^{\mathrm{h}} 15^{\mathrm{m}} \mathrm{p}$. m. gave by analysis a carbon-dioxide content of 3.17 per cent, while the calculated percentage content was 3.15 per cent. Another sample was taken at $4^{\mathrm{h}} 10^{\mathrm{m}} \mathrm{p} . \mathrm{m}$., the average of the analyses giving a content of 3.15 per cent. Samples taken at $5 \mathrm{p}$. m. on this day and 8 a. $\mathrm{m}$. the following day gave a carbon-dioxide content of 2.93 per cent and 2.82 per cent respectively.

As this difference in the carbon-dioxide content shown in samples taken at different times might be due to stratification and a sample drawn from the top of the spirometer might contain less carbon dioxide than a sample of air taken from the lower part of the spirometer, it was desirable to determine the carbon-dioxide content of the air in other parts of the spirometer. Accordingly the spirometer bell was forced from a content of 94 liters down to a content of 12 liters and a sample was taken from the outlet at the bottom, where the expired air is usually introduced. An analysis of this sample gave 2.86 per cent of carbon dioxide, showing an actual loss of carbon dioxide due to absorption by the water. The following day another comparison was made in the same manner. The sample at $10 \mathrm{a}$. m. gave 3.16 per cent as compared with the calculated percentage of 3.31 per cent; at $11 \mathrm{a} . \mathrm{m}$., the analyses gave 3.14 per cent; at 12 noon, 3.04 per cent, and at $1^{\mathrm{h}} 45^{\mathrm{m}}$ p. m., a sample from the top of the spirometer gave 3.05 per cent, while one from the bottom of the spirometer gave 3.07 per cent. A determina- 
tion of the carbon-dioxide content on still another day gave an average result of 2.40 per cent as compared with the calculated content of 2.35 per cent.

The mixture of the air in the spirometer was also tested by another method. The 50-liter spirometer was used in this test and the samples were drawn through three copper tubes of very fine bore which were introduced into the spirometer bell through a rubber stopper in the side opening at the top. The shortest tube extended only just below the conical top of the spirometer; a second tube was so bent that it was carried half way down the inner wall of the spirometer bell in the space occupied by the bath; the third tube extended nearly to the bottom of the spirometer bell. Samples could thus be drawn from the air in the spirometer at three points, $i$. e., top, middle, and bottom. The spirometer was then filled with expired air, the subject at first breathing normally, then with forced expiration for several moments, and finally, near the end of the test, breathing quietly, so as to obtain varying composition of the expired air. Samples were drawn from the three points immediately after the experiment and the carbon dioxide was determined by means of the Haldane gas-analysis apparatus. Two tests were made in this manner, the results being as follows: On July 28,1911 , the percentage of carbon dioxide at the bottom of the spirometer was 3.43 per cent; at the middle, 3.42 per cent; and at the top, 3.43 per cent. On March 15, 1912, the percentage of carbon dioxide at the bottom of the spirometer was 3.59 per cent; at the middle, 3.57 per cent; and at the top, 3.59 per cent. The results of these two series of experiments indicate that the mixture of air in the spirometer approximated uniformity.

Loeffler ${ }^{1}$ studied the question of uniformity in the composition of the air throughout the Jaquet spirometer. He first introduced expired air into the spirometer and when half full the remaining space was filled with atmospheric air. He then drew samples of air from different portions of the spirometer and immediately analyzed them, finding that the composition of the air was identical in all parts of the spirometer.

As a final control upon the Tissot method, alcohol check tests were made in which the Tissot valves were used and the air collected in the spirometer and analyzed. The method of carrying out these tests was described in a previous section (see page 80 ). The successful completion of alcohol check tests with this apparatus presents many difficulties, for if the ventilation is too slow the lamp will go out; if it is too rapid the carbon-dioxide content will be too low. The results of the few tests which were made are given in table 44 . The air left in the spirometer after the third experiment was increased by the addition of outside air from 60 to 92.5 liters and an analysis was made, but 
the results did not agree with the calculated composition. Two more alcohol check tests were made and again the results were very unsatisfactory. The air in the spirometer was then forced out into a large Douglas bag, thoroughly mixed, and returned to the spirometer. The results of the subsequent analysis are given in the table as experiment 4 . Two additional tests were made in which the air was analyzed before and after mixing in a Douglas bag. The results are given in table 44 as experiments 5 and 6 . To find if the same conditions obtained during

TABLE 44.-Results of alcohol check tests with the Tissot apparatus.

\begin{tabular}{|c|c|c|c|c|c|c|}
\hline \multirow{2}{*}{$\begin{array}{c}\text { Experi- } \\
\text { ment } \\
\text { No. }\end{array}$} & \multirow{2}{*}{$\begin{array}{l}\text { Alcohol } \\
\text { burned. }\end{array}$} & \multicolumn{2}{|c|}{$\begin{array}{l}\text { Analysis of spi- } \\
\text { rometer air. }\end{array}$} & \multirow{2}{*}{$\begin{array}{l}\text { Respira- } \\
\text { tory } \\
\text { quotient. }\end{array}$} & \multicolumn{2}{|c|}{$\begin{array}{l}\text { Percentage of } \\
\text { theory found. }\end{array}$} \\
\hline & & $\begin{array}{l}\text { Carbon- } \\
\text { dioxide } \\
\text { increase. }\end{array}$ & $\begin{array}{l}\text { Oxygen } \\
\text { deficit. }\end{array}$ & & $\begin{array}{l}\text { Carbon- } \\
\text { dioxide } \\
\text { produced. }\end{array}$ & $\begin{array}{c}\text { Oxygen } \\
\text { con- } \\
\text { sumed. }\end{array}$ \\
\hline 1 & $\begin{array}{c}\text { c.c. } \\
2.50\end{array}$ & p. ct. & $\begin{array}{l}\text { p. ct. } \\
3.33\end{array}$ & 0.70 & $\begin{array}{l}\text { p. ct. } \\
102.7\end{array}$ & p.ct. \\
\hline 2 & 2.20 & $\begin{array}{l}2.00 \\
2.29\end{array}$ & $\begin{array}{l}0.00 \\
2.92\end{array}$ & $\ldots .$. & 105.6 & 90.1 \\
\hline 3 & 2.00 & 2.71 & 4.08 & .66 & 103.4 & 103.6 \\
\hline 4 & 2.44 & 12.48 & 3.71 & .67 & 100.7 & 100.1 \\
\hline \multirow[t]{2}{*}{5} & 3.00 & 2.35 & 3.67 & .64 & 96.4 & 100.3 \\
\hline & & 12.39 & 3.60 & .66 & 98.0 & 98.4 \\
\hline \multirow[t]{2}{*}{6} & 2.50 & 2.10 & 3. 12 & .67 & 101.5 & 100.5 \\
\hline & & ${ }^{12} .14$ & 3.05 & .70 & 103.4 & 98.2 \\
\hline
\end{tabular}

'Spirometer air mixed in a Douglas bag.

respiration experiments with man, expired air was collected in the spirometer and analyzed before and after mixing. The results are given in table 45 . In the first experiment the respiration was irregular; the ventilation per minute was as follows: $5,5,17,3,3,2,7,4$, and 4 liters. Two samples were taken, the first at $9^{\mathrm{h}} 30^{\mathrm{m}}$ a. $\mathrm{m}$. and the second at $3^{\mathrm{h}} 15^{\mathrm{m}} \mathrm{p} . \mathrm{m}$. Another experiment was carried out in which the respiration was quiet and regular, the ventilation per minute being $10,5,10,7,6.5,7,6.5,7$, and 6.5 liters. Similar experiments were

TABLE 45.-Effect upon the analyses of mixing spirometer air.

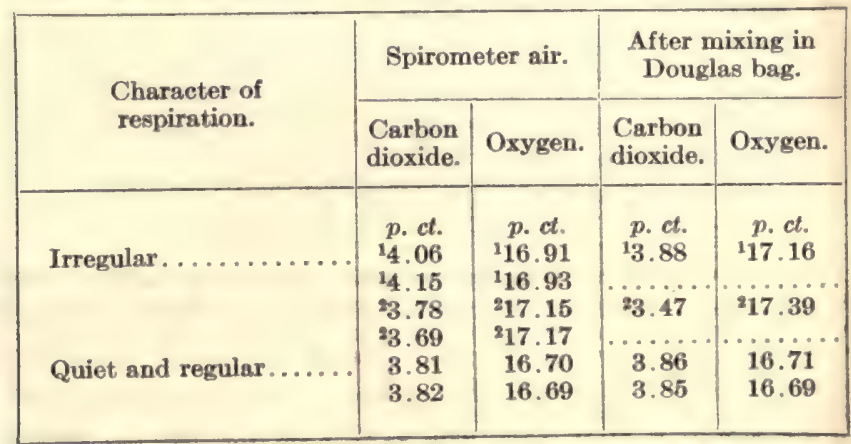

iSample taken at $9^{\mathrm{h}} 30^{\mathrm{m}}$ a. m. 2 Sample taken at $3^{\mathrm{h}} 15^{\mathrm{m}}$ p. $\mathrm{m}$. 
made by Mr. H. L. Higgins, which showed that with quiet, regular respiration there was practically no difference in composition.

From these experiments and the alcohol check tests it would appear that the uniformity in the composition of the air throughout the spirometer depends upon the character of the respiration. In the alcohol check tests the volume per respiration produced by the hand spirometer was very small, so that the movement was not sufficient to cause so complete a mixing of the air as in normal respiration. In the comparison experiments in this research in which the Tissot apparatus was used, the respiration was quiet and uniform and the good agreement of the results indicates that the composition of the expired air was uniform in all parts of the spirometer.

In conclusion, it may be stated that the manipulation of the Tissot apparatus is not difficult and that the results obtained with it are reliable and entirely comparable with those obtained with other respiration apparatus used for the determination of the respiratory exchange in short periods.

\section{DOUGLAS METHOD.}

The method of collecting expired air in a rubber bag has been employed by a number of investigators. ${ }^{1}$ Nearly every one of their investigations has been severely criticized because a rubber receptacle was used for collecting a mixture of gases containing an appreciable amount of carbon dioxide. It is a well-known fact that rubber has a tendency to absorb carbon dioxide and also to let it diffuse. Hufner ${ }^{2}$ found that a much larger amount of carbon dioxide was absorbed than of either oxygen or nitrogen. Kayser ${ }^{3}$ found that 1 c.c. of rubber at $0^{\circ}$ and $760 \mathrm{~mm}$. absorbed 1.3507 c.c. of carbon dioxide. Graham ${ }^{4}$ found that carbon dioxide passed through a rubber membrane much more rapidly than hydrogen or nitrogen. Atwater and Benedict, ${ }^{5}$ on the contrary, in using a rubber membrane in a sampling device found that there was no diffusion of carbon dioxide with a percentage of carbon dioxide in the air of not over 2 per cent, but there was an absorption and diffusion of water-vapor.

Douglas ${ }^{6}$ points out that care should be taken to obtain bags having a negligible amount of diffusion and the bags used in this investigation were both tested for this. A sample of air taken from the smaller bag on July 6,1912 , at $9^{\mathrm{h}} 45^{\mathrm{m}}$ a. m., gave 4.13 per cent of carbon dioxide; at $10^{\mathrm{h}} 45^{\mathrm{m}}$ a. m., 4.12 per cent; at $11^{\mathrm{h}} 45^{\mathrm{m}}$ a. m., 4.09 per cent;

\footnotetext{
${ }^{1}$ Regnard, Recherches expérimentales sur les variations pathologiques des combustions respiratoires, Paris, 1879, p. 286. Luciani, Das Hungern, Hamburg and Leipzig, 1890, p. 181. Marcet, A contribution to the history of the respiration of man. London, 1897, p. 11.

'Hufner, Wiedemann's Ann. d. Physik u. Chem., 1888, 34, p. 1.

${ }^{3}$ Kayser, Ann. d. Physik u. Chem., 1891, N. F., 43, p. 548.

${ }^{4}$ Graham, Proc. Royal Society, London, 1866, 15, p. 223.

${ }^{5}$ Atwater and Benedict, U. S. Dept. Agr., Office Expt. Stas. Bull. No. 136, 1903, p. 25.

'Douglas, Journ. Physiol., 1911, 42, Proc. Physiol. Soc., p. xvii.
} 
and at $1^{\mathrm{h}} 10^{\mathrm{m}}$ p. m., 4.03 per cent. There was some diffusion, but the rate was so slow that it played no rôle in experimental periods of 5 minutes' duration. Samples were also taken from the larger bag and gave the following results: $12^{\mathrm{h}} 10^{\mathrm{m}} \mathrm{p} . \mathrm{m} ., 3.53$ per cent of carbon dioxide; $1^{\mathrm{h}} 21^{\mathrm{m}}$ p. m., 3.46 per cent; $2^{\mathrm{h}} 30^{\mathrm{m}}$ p. m., 3.36 per cent; $3^{\mathrm{h}} 30^{\mathrm{m}}$ p. m., 3. 37 per cent. With the larger bag, the experimental periods were about 10 minutes in length and the sampling took place immediately after the period was over, so that this rate of diffusion, did not play a significant rôle.

Another possible source of error in the bag method is the difficulty of measuring the air in the bag accurately. It is practically impossible to empty the bag completely, and even when pressed flat and rolled, air still remains and additional air will be sucked back when the bag is again flattened. Douglas recommends using exactly the same procedure before and after the experiment, so as to have the amount of air driven from the bag during measurement the same as that which has actually been added to it. The accuracy of measurement is of special importance, as it is not possible to make long experiments with the bag method.

The agreement of duplicates in measuring volumes was tested with the larger bag by introducing a known weight of oxygen into the bag and then passing the gas through a 10-liter Bohr meter, noting the temperature, the barometric pressure, and the amount of gas registered by the meter. In one case 15.7 grams of oxygen were used and the meter reading showed that 99.5 per cent of the oxygen had passed through it; in a second case, 63.9 grams were used and the meter reading gave 100.2 per cent. In this instance, therefore, the duplicates were within 1 per cent; it should be noted that this included not only variations in the bag itself, but also in the weighing of the cylinder and in the reading of the meter.

As the air in the bag is thoroughly mixed by the kneading process, it is evident that a sample of air taken from the bag represents the average composition very exactly. In this regard the method is superior to all other open-circuit methods because of the possibility of thorough mixing.

One of the advantages of the Douglas apparatus is the fact that it is portable. Furthermore, by using several bags it is possible to carry out several experimental periods in quick succession. On the other hand, there is no control upon the regularity of respiration with this method, as only the total amount of expired air is known, but not the amount for individual portions of time. The periods must also be extremely short and should not be continued so long as to cause the subject to exhale against a noticeable pressure, for it is doubtful if normal respiratory exchange can be obtained under such circumstances.

The valves used by Douglas are the mica-flap valves of the SiebeGorman Company. We have found that these are sometimes unreli- 
able, and that when the respiration is quiet and free there is a liability toward back-leak. The more forcible part of the expiration passes through the expiration valve, but the end of the expiration, which is slower, may go back through the inspiration valve; consequently, if the portion lost has not the same ratio of carbon dioxide to oxygen as the portion collected, true respiratory quotients may not be obtained. A test ${ }^{1}$ of one of the valves showed a recovery of only 78 per cent of the air drawn through it. These valves may be safeguarded by attaching a long tube to them, so that the air which passes out through the expiration valve may be drawn in again with the next inhalation.

The Douglas method has recently been used by Carter $^{2}$ on tubercular patients in preference to the Zuntz-Geppert method. Henderson and Prince $^{3}$ have also employed it in some observations on "oxygen pulse and systolic discharge" and state that it is much simpler and easier to use, more accurate, and affords more nearly normal conditions as to the air breathed by the subject than any other device with which they are familiar.

In general, it is apparently more difficult to obtain reliable results with this method than with the other open-circuit methods. The bags used must be tested for diffusion and always handled in the same manner when emptying them before and after the experiment. Care must be taken not to have the periods long enough to cause the subject to exhale against pressure. The valves used should be of a reliable type or carefully safeguarded by a long tube on the ingoing valve. The apparatus is of advantage because of its portability.

\section{VALVES.}

In all methods for determining the respiratory exchange in which the inspired and expired air are separated, it is necessary to use some kind of valve for the separation. In this investigation several types of valves have been employed and their individual merits have been discussed in connection with the apparatus with which they were used. Those most easily and cheaply constructed are the Mueller valves, which can be made of materials found in almost any laboratory. The principal requirements are that they should have a wide opening through which the air passes; that the water seal should be so thin that it offers no resistance and yet at the same time sufficiently deep to prevent air from returning through the ingoing valve; and that they should be suspended or set in such a manner that they are perfectly level, so as to givean effective closure with a minimum amount of water.

The Zuntz valves, which are actually of the type devised by Speck, are effective in operation; the chief objections to them are their size

1See p. 252.

${ }^{2}$ Carter, Journ. Expt. Med., 1914, 20, p. 87.

${ }^{3}$ Henderson and Prince, Am. Journ. Physiol., 1914, 35, p. 109. 
and the necessity for an occasional renewal of the material which acts as a valve. It is also sometimes difficult so to adjust them that the resistance is not only absolutely minimum but their efficiency unimpaired. The membrane surrounding the valve also dries out readily on the inspiring valve and must be frequently moistened.

The Tissot valves give very satisfactory results. They are, however, quite fragile, the glass part between the two metal ends breaking easily and at times becoming loosened from the brass connections. Another disadvantage is that the valves must be perfectly level when used, so that the brass flap, which is very light and sensitive, will work properly. With suitable care the valves should not get out of order. They should be cleansed occasionally and the flap kept perfectly smooth to secure effective closure.

Both types of the Siebe-Gorman valves ${ }^{1}$ are inferior to the other valves mentioned and need more care when used in determining the respiratory exchange during rest.

To give efficient service, valves should offer a minimum amount of resistance, close perfectly, and be easy to care for and to keep in repair, so that they will be ready for use at any time. If the valves do not close perfectly and there is a back-leakage of air, an actual loss may result, with a consequent loss in the amount of air measured. When prevention of this loss of air is made by the use of a rubber tube on the intake side of the inspiratory valve, the measured volume of ventilation will tend to be greater than the true ventilation. It should be pointed out that in the interpretation of results the more information one has as to the character of the ventilation the more readily unusual results may be interpreted. It is always advisable to safeguard the inspiratory valve by attaching a tube to the intake.

A set of valves may be tested in two ways, for pressure and for efficiency, $i . e$., for absence of back-leak. If in the tee-piece which usually connects the ingoing and outgoing valves a side opening is made large enough to insert a rubber tube approximately 3 to $4 \mathrm{~mm}$. in diameter, and this rubber tube is connected with an ordinary water manometer, or a manometer with oil, the total variations in pressure may be determined during a respiration cycle. Some fluctuations in pressure are to be expected, for at the moment of inspiration there is a slight vacuum in the space between the two valves and at the moment of expiration there is a slight pressure. These variations, however, should not be very large. A set of valves in which the fluctuations in pressure exceed $\pm 5 \mathrm{~mm}$. of water is not desirable for use, as this pressure is greater than would be advisable in ordinary respiration. The variations in pressure may also be graphically recorded by connecting the pressure-tube to a tambour, with a pointer writing upon the 
smoked surface of a kymograph. Care must be taken, however, to make sure that there are no errors due to inertia and that the tambour is calibrated so that the pressure can be read directly.

Another test of the pressure may be made by noting the effect of using the valves with a spirometer, a meter, or a bag. If the pressure increases very largely under these conditions, it is doubtless too great; some means should therefore be taken to overcome it, either by weighting the counterpoise of the spirometer or by a device producing a slightly diminished pressure in the meter, such as that recommended by Magnus-Levy ${ }^{1}$ in connection with the sampling apparatus of the Zuntz-Geppert method.

The test for the efficiency of the valve, that is, for the quantitative separation of inspired and expired air, may be made by means of special apparatus. If a pair of valves is connected to the hand spirometer ${ }^{2}$ and a pointer writing upon a kymograph drum is attached to the bell of the spirometer, the natural respirations of a man breathing through the valves can be imitated and a direct record made of the total ventilation as determined by the hand spirometer. The air can then be collected in a large spirometer or passed through a meter and with proper precautions as to temperature and saturation a calculation may be made from the movements of the hand spirometer of the amount of air which has passed through the valves, and from the meter or large spirometer how much air has been received. The results of such a calculation will show the efficiency of the valves for separating the inspired and the expired air. Of course care must be taken that the movements of the hand spirometer are at approximately the rate and depth of normal breathing.

While no complete study of the efficiency of different valves has been made in this research, two series of experiments have been carried out by this method, one with the Tissot valves and the other with the Siebe-Gorman valves. In these experiments the Tissot valves gave results which were superior to those obtained with the Siebe-Gorman valves. The total efficiency of the Tissot valves was about 99 per cent, while that of the Siebe-Gorman valves may fall as low as 75 per cent, according to which valve is used for the inspiratory valve. It can be seen from these experiments that this method affords a good test of the efficiency of valves. Simultaneous with this efficiency test, a graphic record of the cycle of pressure may be obtained by means of a side tube in the manner previously described.

\section{BREATHING APPLIANCES.}

In the experiments here reported the different types of breathing appliances which have been used are the glass and pneumatic nosepieces, the rubber mouthpiece, and the mask. The last permits breathing either through the mouth or the nose, or both. 
PNEUMATIC NOSEPIECES.

The breathing apparatus used most frequently in this laboratory is the pneumatic nosepiece, ${ }^{1}$ which was devised in connection with the development of the Benedict respiration apparatus in an attempt to secure some breathing appliance which could be used by practically all subjects. Before this time a glass nosepiece, such as that used by Tissot, and a rubber mouthpiece of the Denayrouse form had been tried. Neither of these appliances gave markedly successful results, as it was found difficult to make them air-tight.

The deflated pneumatic nosepieces are inserted in the nose and air pressure applied until the rubber is inflated sufficiently to fit closely into all of the inequalities of the nostrils. These nosepieces have given very satisfactory results. They are easily made from materials which are readily obtained, such as rubber finger cots, rubber stoppers, glass tubing, and rubber tubing, but considerable time is required for their construction. The nosepieces are flexible and nearly every type of nostril can be fitted without great discomfort. This has been proved repeatedly in the Nutrition Laboratory by the fact that many of the subjects with whom they have been used have fallen asleep easily.

The nosepieces when inserted may be tested for leaks around the nostrils by a simple method. The subject draws air in through the nose, then closes with the hands the ends of the attachment to which the nosepieces are fastened and attempts to exhale through the nose; a leak will be detected by the air which passes out through the opening. The escaping air may be heard if the leak is large or felt against the skin if the hand is placed near the nosepieces. This method of testing is not, however, always absolutely reliable, for occasionally, when pressure is applied from within the nose, the nosepieces apparently fit closely but in use a slight loss of air occurs. This may be due to the fact that in normal breathing there is always a very slight dilation of the outer edge of the soft part of the nostril and this may be sufficient, when air is inhaled, to allow air to pass.

The best test for tightness is to apply soapsuds with a camel's hair brush, any leakage of air being shown by bubbles. In a series of experiments which are difficult and costly to repeat, the tightness of the nosepieces should be tested in this manner. The soapsuds should be continually applied throughout the experiment, or at least sufficiently often so that the space between the nosepieces and the nostrils will always be wet. Although Coleman and Dubois have employed this method in all of their experiments with typhoid-fever patients, it is annoying to some subjects, and if experiments are continued over a long period, as for example, for 12 hours daily, it may produce soreness which will make the subject distinctly uncomfortable. The use of 
soapsuds in this manner through the experiment also requires the constant attention of at least one assistant during the entire period. Unless the nosepieces are tested throughout the whole experiment, it is possible, after the experiment has begun, for a leak to oecur which may give inaccurate results with a closed-circuit apparatus, although the presence of the leak may not be positively known. As the pneumatic nosepieces deteriorate somewhat rapidly and require constant care to make sure that they are in perfect condition, they must be tested under water immediately before the beginning of each day's work, for it has at times been found that nosepieces which have been perfect on the night preceding the experiment may leak when used the next morning.

Occasionally a nosepiece slips out of place during a period. They also have a tendency to cause a mucous secretion in the nostril, which clogs the nose and interferes with the breathing. In several instances it has been necessary to use the mouthpiece instead of the nosepieces for this reason. When the nostril is exceedingly small, a smaller nosepiece has to be used and the opening may not be large enough for free respiration, so that an actual impediment to the breathing may result. Although these nosepieces are extremely flexible, they will not fit in every case, as the opening of the nostril varies markedly with different people. With some individuals it is practically impossible to make a circular-shaped nosepiece fit the nostril, as the opening of the nostril is not round, but long and narrow, with a point at each end. This makes it extremely difficult to find any kind of a nosepiece which will fit closely without leak.

In general the pneumatic nosepieces have found the widest application in this laboratory, because they are adaptable to most subjects and the most comfortable appliance to use. In our experimenting we have not found more than 10 subjects who were unable to use these nosepieces, and with only a small proportion of those who used them was soapsuds applied for the detection of possible leaks.

GLASS NOSEPIECES.

The glass nosepieces described by Tissot ${ }^{1}$ have been more or less employed in this research. They are always ready for use, practically indestructible with proper care, can be made in a large variety of sizes, and give a good opening for free breathing. On the other hand, as the round glass nosepieces when inserted are parallel to one another, the enlarged part of the glass presses against the cartilage between the nostrils and this pressure becomes exceedingly painful after a time. An attempt has been made to remedy this by making glass nosepieces with an oval instead of round cross-section, since this would conform more generally to the usual shape of the opening of the nostril; but the oval nosepieces have not proved so successful as had been expected. 
It is quite possible that the exact shape of the nosepieces has not yet been rightly determined.

These glass nosepieces can also be tested by the use of soapsuds. The use of pressure for testing is not, however, generally practicable, as the nosepieces are not dilatable and allow the air to escape between the glass and the nostril when pressure is put on the inside, thus practically enlarging the nostril without enlarging the nosepiece.

\section{MOUTHPIECE.}

While the mouthpiece ${ }^{1}$ has been more or less employed in this research, the pneumatic nosepieces have usually been preferred. Three objections are made to the use of a mouthpiece, $i$. $e$, , that the subjects do not like it, that constant care is necessary to prevent the escape of air, and that abnormal breathing may possibly result from its use.

The mouthpiece is not so agreeable as the nosepieces, for the thick piece of rubber used for the flange and held between the teeth and lips excites a flow of saliva in the mouth which is often extremely annoying to the subject. Furthermore, to prevent an escape of air, the subject must draw his lips up closely around the circular tube. There is a natural tendency to relax this firm closure of the lips and air may thus escape between the corners of the mouth and the rubber flange of the mouthpiece. The absence of leaks may be determined by using soapsuds, as with other breathing appliances. This was admirably demonstrated in a research on muscular work carried out by Benedict and Cathcart, ${ }^{2}$ in which the subject rode a bicycle and breathed through the mouthpiece into the respiration apparatus. In this series of experiments it was absolutely imperative that there should be no uncertainty regarding the measurement of the oxygen consumption. The only loss of air possible was about the mouthpiece, and soapsuds were constantly used over the mouth. That the loss of air was possible was proved by the fact that occasionally a small bubble formed in the soapsuds; when cautioned by the observer, however, the subject closed his mouth tightly and thus no leak occurred. With the mouthpiece it is easier to make sure that the closure is perfect, for if the subject keeps his lips drawn closely about the central tube there is very little, if any, probability of a leak.

When the mouthpiece is employed, the nose can easily be closed by means of a nose-clip. Most of the nose-clips used give great discomfort after they have been worn throughout the experimental period. The most comfortable nose-clip and the one commonly used at the present time is that made by Siebe, Gorman \& Co. This is provided with a thick felt pad and is so constructed that it fits closely to the outside of

1See description of type used on p. 54.

'Benedict and Catheart, Carnegie Inst. Wash. Pub. 187, 1913. 
the nostrils, the pressure against the nostril being regulated at will. This noseclip may be worn for a long time without discomfort.

That the breathing with the mouthpiece is not abnormal was shown in the comparison experiments carried out in this research with both the Benedict apparatus and the Tissot apparatus, in which mouthbreathing and nose-breathing were compared. The results obtained with the two methods of breathing were practically the same.

MASK.

The mask has been used in this research in a very few experiments, but only for the purpose of studying the effect on the respiratory exchange of this method of breathing. ${ }^{1}$ In the earlier use of the Benedict respiration apparatus, a rubber mask was employed which was held against the face by binding-strips of leather and tape. A pneumatic ring around the edge could be inflated when the mask was in position. In this research, however, a mask of lead and plasticene was used, similar to that employed by Bohr. ${ }^{2}$

With the mask the subject can breathe at will through the mouth or the nose and is not obliged to concentrate his mind upon keeping his mouth closed or taking care that the mouthpiece does not slip out of position. With this form of breathing appliance, however, it is much more difficult to prevent the escape of air than with either the mouthpiece or the nosepieces. The subject must hold his head practically rigid, as the slightest movement may cause a leak and a consequent loss of the whole experiment. The mask can not be used with a subject having a beard and a separate mask must be made for each individual. Furthermore, the air inside the mask acts as a dead space and increases the depth of the respiration. Some of the subjects have also complained that the air seems warm and stagnant inside the mask.

From our experience in this laboratory it does not seem advisable to use a mask and either the nosepiece or the mouthpiece is preferable from the standpoint of both the mechanical manipulation and the comfort of the subject. This is especially the case when many subjects are being used, particularly if they are not very much interested in the experiments. In experiments in which the investigators themselves are the subjects, it may be perfectly practicable to use a mask. In such experiments, however, the edges of the mask should be tested with soapsuds to make sure that no leaks occur. The mere fact that no leak is perceptible when pressure is used inside the mask is not an absolute proof of the absence of a leak, as the pressure inside the mask may tend to make the closure more perfect.

In general, it may be stated that the mask is the least preferable of the breathing appliances. The mouthpiece is the most reliable from 
the standpoint of an air-tight closure, but its use may be disagreeable to the subject. The glass nosepiece is not so practicable as the pneumatic nosepiece, which, with proper precautions, can be made to conform closely to the inequalities in the surface of the nostril and is the most comfortable for the average subject.

GAS ANALYSIS.

Practically all methods of determining the respiratory exchange require the use of gas-analysis apparatus in one form or another. Even determinations made with apparatus constructed on the RegnaultReiset principle may involve gas analysis, for Rolly, in his adaptation of the Benedict respiration apparatus, has considered it necessary to make air analyses to find whether or not the apparatus is air-tight. The difficulties experienced by many investigators with such apparatus led to the development of the Benedict respiration apparatus, for it is considered that the general construction and technique of this apparatus make gas analysis unnecessary in its use.

It is frequently claimed that gas analysis requires special technique, which many people are unable to acquire. It must be admitted that in going over the results of analyses obtained with various kinds of gas-analysis apparatus, it is not so easy to find duplicate results as would be expected. Another factor which must be taken into consideration is not only the ease or the difficulty in obtaining results, but also the amount of work involved. All analysts will agree that gas analysis is one of the most tedious operations connected with the determination of the respiratory exchange and becomes very monotonous when continued for any length of time. In fact, in this laboratory it has been found advisable to vary the work of the analysts, so that they may operate with the highest efficiency and with the least physical strain. At the same time it is perfectly logical to conclude that if an individual can not make gas analyses well enough to obtain accurate results, he should not be engaged in the study of the respiratory exchange, for it is probable that his results will be similarly inaccurate, as the technique of such investigations is somewhat difficult.

In this research two types of gas-analysis apparatus were used and the criticisms here set down will refer mainly to these two types. The Zuntz gas-analysis apparatus ${ }^{1}$ was employed in the first series of comparison experiments with the Zuntz-Geppert method, ${ }^{2}$ and very fair results were obtained with it. When each division of the burettes represents 0.02 c.c., it is quite possible to obtain duplicates to 0.02 per cent. The special advantage of this apparatus is the fact that the analysis may be made in duplicate in one operation rather than by drawing two samples and analyzing them successively. However, this simply means that one operation has been carried out twice in 
exactly the same manner, with no control upon the sampling. A sample which was incorrectly drawn may therefore be equally divided between the two burettes and yet duplicate results obtained. The apparatus is very large and cumbersome and has a great number of rubber connections which are liable to deteriorate, with consequent leaks. It also requires the use of large samples- 100 c.c. - so that if the analysis is not carried out immediately, a very large sample, at least 200 c.c. or more, must be collected in order to have sufficient air for flushing the connections when the samples are drawn. One of the chief objections to the Zuntz apparatus is the fact that the analysis is made over water. Practically all investigators are agreed that the collection and analysis of air samples over water is to be avoided if the carbon-dioxide content of a mixture of gases is to be determined to less than 0.05 per cent.

The major part of the analyses carried out in connection with this research were made with the two forms of the Haldane gas-analysis apparatus. ${ }^{1}$ In the earlier comparisons, the laboratory form of this apparatus was used exclusively. Phosphorus was successfully substituted for potassium pyrogallate as an absorbent, thus doing away with the necessity for repeated raising and lowering of the mercury reservoir and saving much time and labor in continuous work. The phosphorus also required less frequent renewal; but on the other hand it absorbed the oxygen more slowly than the potassium pyrogallate. In the later experimenting the portable form of the Haldane gas-analysis apparatus was used with very good success. Practically as good results were obtained with it as with the laboratory form and it was much more convenient to use. With both forms of the apparatus only a small sample is required, $i$.e., 20 c.c. for the larger apparatus and 10 c.c. for the portable apparatus. Smaller containers may therefore be used for collecting the samples, which is of advantage when space is limited and when large amounts of mercury are required. In all of the gas analyses with these two apparatus it has been the routine to collect the samples over mercury, so that both the collection and the analyses were made over mercury. .

It must be pointed out that while apparently many people have found it difficult to make gas analyses with sufficient accuracy for use in determining the respiratory exchange, yet in this laboratory a considerable number of individuals have been trained to use the Haldane gas-analysis apparatus with good success. For example, one young lady, who had had neither prior chemical training nor training in gas analysis, was instructed in the use of the Haldane apparatus and in two weeks was able to make satisfactory analyses of outdoor air and of expired air. This young lady was but one of several assistants who have been taught the technique in the same manner. The fact that 
we have had a wide experience in the use of various forms of gasanalysis apparatus may have been a factor in acquiring and teaching the technique of this apparatus.

To be able to place absolute reliance upon the results of the analyses, they must be controlled in some way. The best control of analyses of expired air is the analysis of samples of atmospheric air. Haldane ${ }^{1}$ points out that such analyses are sometimes used for calibrating his gas-analysis apparatus, as he assumes that the composition of outdoor air is constant, $i . e ., 20.93$ per cent for oxygen and 0.03 per cent for the carbon-dioxide content. Benedict, ${ }^{2}$ in studying the oxygen content of the atmospheric air, has found that both the carbon dioxide and the oxygen content are very constant at all seasons of the year and in all parts of the world where such investigations have been made. Wolff and $\mathrm{Heele}^{3}$ have recently based the accuracy of the gas-analysis apparatus used by them upon the constancy of the composition of outdoor air as reported by Benedict. Results of analyses of expired air can be properly taken as reliable when a series of analyses of outdoor air, made under the same conditions, show constancy.

In this laboratory it has been the practice to control our gas-analysis apparatus with frequent analyses of outdoor air, and when constant results could not be obtained with samples of outdoor air, the apparatus has been examined to find the cause of the discrepancies. In some cases it has been found that the burette was dirty; in other cases there has been a slight leak or the sample has been contaminated with outside air in transit. Unfortunately, we have no method of controlling the analyses of expired air; that is, we have no air that can be analyzed which is both similar in composition to expired air and constant in composition. While analyses of outdoor air may be made and accurate results obtained, it is barely possible that the sampling of the expired air may be imperfect and duplicate results still be obtained. Outdoor air has so nearly the composition of any air which may surround the apparatus that even if other air were admitted there would be no possible way of detecting it. Notwithstanding these facts, it is strongly recommended that all gas-analysis apparatus be controlled by analyses of outdoor air and that results be obtained in general within 0.02 per cent for either oxygen or carbon dioxide. The values for atmospheric air obtained by Benedict with the Haldane solution in a Sondén gas-analysis apparatus were for carbon dioxide 0.031 per cent, and for oxygen 20.952 per cent in carbon-dioxide-free air. ${ }^{2}$ Investigators do not, as a rule, publish the results of their analyses of atmospheric air, and when published, they frequently show large variations; these variations must certainly be taken as an indication that

${ }^{1}$ Haldane, Methods of Air Analysis, London, 1912, 44-45.

${ }^{2}$ Benedict, Carnegie Inst. Wash. Pub. 166, 1912, p. 114.

${ }^{3}$ Wolff and Heele, Journ. Physiol., 1914, 48, p. 430. 
similar, if not greater, errors also occur in their analyses of expired air. It is to be recommended that investigators publish their analyses of atmospheric air and thus indicate the general accuracy of their gas analyses.

In choosing a respiration apparatus, an investigator must consider whether or not he wishes to use gas-analysis apparatus. Those who do not should select some respiration apparatus which is constructed on the Regnault-Reiset principle, since, if properly manipulated, no gas analyses are necessary, the respiratory exchange being determined directly by either weight or volume. On the contrary, the acquirement of the technique of gas analysis is of great service, even in using an apparatus of the Regnault-Reiset type, as it may be desirable to determine the composition of various portions of the expired air, the residual air, or alveolar air in studies of this character. Furthermore, it is possible at the same time to study the ventilation and the effect upon the respiratory exchange of breathing atmospheres of varying composition. If, then, one has not acquired skill in gas analysis, the field of investigation is very much limited.

To sum up, therefore, gas analysis requires a great deal of time to carry out and is very tedious; an apparatus for determining the respiratory exchange which does not require such analysis is accordingly to be preferred. Furthermore, with a method in which the respiratory exchange may be determined directly, the results may be obtained more quickly than with a method involving gas analysis, for it is rarely possible to make such analyses as rapidly as the weighings and the computations can be made by the direct method, and at the same time obtain the necessary records of the pulse, respiration, and other factors included in a complete respiration experiment. The ability to use gas-analysis apparatus, however, extends widely the field of an investigator in respiration and respiratory exchange.

\section{ACCURACY AND INTERPRETATION OF RESULTS.}

In studying the respiratory exchange of man, some standard of accuracy is necessary in order that one may interpret the results and draw inferences from variations which may be found. If an experiment with three experimental periods is made with a man in a resting condition and without food for 12 hours or more, a certain constancy of results may be expected. The variations from this constancy are due to three things: Errors in the actual manipulation and the limits of accuracy, due to the apparatus itself; the accidental variations in the metabolism of man; and abnormalities in the respiration, such as dyspnœa, apnœa, and hyperpnœa.

The first source of variation must be eliminated so far as possible by the experimenter. To this end he must observe all the precautions 
which are prescribed in the manipulation of each type of apparatus. He must assure himself that the apparatus is in perfect condition and must control it frequently in order that he may depend upon his results. For example, if he is working with a closed-circuit apparatus, he must be perfectly sure that the apparatus is air-tight and will remain air-tight throughout the experimental period; also that the various absorption apparatus are functionating perfectly. If the method involves measurement with spirometers and gas analysis, these must be controlled so far as possible, the spirometers by calibration and the gas analyses by frequent comparisons with analyses of outdoor air.

As many controls as possible should also be used for the subject. Records of the pulse-rate, respiration-rate, and some graphic registration of the degree of repose should be obtained. In addition, data should be recorded as to his general condition, his previous condition, and any factors which may influence the respiration during the experiment, particularly those of a psychical nature.

Every precaution should be taken that the conditions under which the experiments are made are favorable to uniformity in results. For instance, the experiments should be made in a perfectly quiet room, where no interruptions will be likely to occur. It has been frequently observed in this laboratory that the unexpected and unnecessary entrance of a person into the room during an experiment has resulted in a very noticeable change in the pulse-rate and a consequent change in the metabolism. Sudden noises or sudden disturbances also result in variable values, particularly if the subjects are new and unaccustomed to the laboratory. Also, so far as possible, the manipulation of the apparatus should not be visible to the subject. With the Benedict apparatus it has been our custom to conceal the whole apparatus with a curtain in such a way that the subject can not see the spirometer moving, the valve turned, or any of the other operations connected with the progress of the experiment. In the use of the Tissot spirometer, it is desirable to place the spirometer behind the subject so that he can not see it rising as he exhales. Some subjects have had the idea that the object of the experiment was to fill the spirometer as rapidly as possible; obviously good results can not be obtained with these subjects.

If a subject is quiet, the pulse-rate is constant, and the apparatus is in good working condition, the values of the carbon dioxide and the oxygen obtained in three succeeding experimental periods should not vary more than 5 per cent. It has been the custom in this laboratory to expect results within 10 c.c. per minute for both the carbon-dioxide elimination and the oxygen consumption; even more closely agreeing results may be obtained.

It is rather difficult to state what the differences in the total metabolism of an individual may be from day to day. Magnus-Levy ${ }^{1}$ has cited 
possible differences as high as 15 per cent which are not apparently due to muscular movement, and says that no absolute predictions can be made as to the total metabolism of an individual. Benedict ${ }^{1}$ has recently made an extensive study of the variations in the daily resting metabolism of 35 normal individuals over periods varying from 5 days to 4 years and 5 months. He found that the total metabolism as measured by the oxygen intake may show variations from 3.5 per cent with an individual over a period of 12 days to 31.3 per cent with another individual over a period of 8 months. The average extent of variation was about 14 per cent.

The respiratory quotient should not vary to any great degree, certainly not more than 0.03 or 0.04 . From our experience with resting men in the post-absorptive condition, i. e., without food for 12 hours or more, it may be stated that the value for the respiratory quotient is fairly constant for a considerable length of time, certainly 2 or 3 hours, and consequently large variations in the respiratory quotient would not be expected during this period. For example, if a series of quotients were obtained of $0.77,0.70$, and 0.77 , the second quotient would be looked upon with suspicion, and a search would be made for the source of the possible error in the manipulation of the apparatus. The low quotient may be due to two causes: (1) too low a carbondioxide elimination, (2) an error in the measurement of the oxygen consumption, or possibly a combination of these errors.

The low carbon-dioxide elimination may be due to a perfectly natural cause, such as under-ventilation in apnœa. If a graphic record of the respiration has been obtained, either by means of a pneumograph or a spirometer, and this shows clearly that apnoea occurred, the cause of the low value for the carbon-dioxide elimination is known absolutely. If, then, the results are used, it will be with a clear understanding that the respiratory quotient 0.70 does not indicate the true character of the katabolism for that period.

Since the respiratory quotient is the relation between the volume of carbon dioxide produced and the volume of oxygen consumed, it may be calculated directly from the increase in the carbon dioxide and the deficit of the oxygen in the expired air. ${ }^{2}$ Analyses of expired air, such as are made with the open-circuit method, give the volumetric content of carbon dioxide and oxygen, and this ratio is in no way affected by variations in barometric pressure, temperature, or even slight muscular activity, but is dependent solely upon the character of the respiration and (if this is normal) upon the character of the katabolism taking place in the body.

\footnotetext{
${ }^{1}$ Benedict, Journ. Biol. Chem., 1915, 20, p. 291.

"Correction must be made, of course, for the carbon dioxide in inspired air and the change in percentage of the nitrogen in inspired and expired air.
} 
Durig $^{1}$ has pointed out that differences of small amounts in the oxygen consumption and the carbon-dioxide elimination per minute may result in large variations in the respiratory quotient if the differences are in opposite directions. There is, then, a double effect upon the respiratory quotient, and in that case the quotients are very variable. For example, in gas analysis, with a difference of 0.1 per cent, differences may be obtained of 0.04 to 0.05 in the respiratory quotient if the errors in the carbon-dioxide determination are in the opposite direction to those in the oxygen determination. Such variations, however, would be very large for gas analyses in which differences of not more than 0.02 to 0.04 per cent should be expected.

With many methods of gas analysis the errors tend to compensate one another, particularly if the gas analysis is made by means of a Haldane apparatus, when the low carbon-dioxide absorption will be compensated by a greater absorption in the potassium pyrogallate. The result in this case would be that the carbon-dioxide increase would be too small, while the oxygen percentage would be too high; the oxygen loss would then be too small, but unless the error due to incomplete absorption of carbon-dioxide by the potassium hydroxide was large, the ratio between the carbon dioxide increase and the oxygen deficit would not be markedly different from the actual ratio obtained by a correct analysis. With the Regnault-Reiset or closed-circuit method, on the contrary, the two determinations are made independently and there may be an error in one but no compensating error in the other. Consequently, wider variations may be found in the respiratory quotient by this method than with the open-circuit method. The determination of the respiratory quotient by the analysis of expired air is, therefore, the more logical method.

Respiratory quotients below 0.7 or above 1.00 , which are obtained with individuals without food and in a resting condition, must be looked upon with considerable suspicion. Thus far the accumulation of reliable evidence has not been sufficient to show that respiratory quotients much below 0.7 may be obtained, even with abnormal or pathological conditions. On the other hand, respiratory quotients over 1.00 can not be expected to occur unless there is some transformation of sugar into fat, but this is not likely to occur with a man who has not had food for 12 hours or more. Abnormal quotients such as these should be controlled by repeated observations in successive experiments in order to make certain of their accuracy. It must be pointed out that a very sharp distinction should be made between the probable accuracy of respiratory quotients obtained with an apparatus and the probable accuracy of the values obtained for the carbondioxide elimination and oxygen absorption. Accurate respiratory

\footnotetext{
${ }^{1}$ Durig, Denkschriften der mathematisch-naturwissenschaftichen Klasse der kaiserlichen Akademie der Wissenschaften, Vienna, 1909, 86, p. 118.
} 
quotients are much more difficult to obtain than accurate figures for the carbon-dioxide elimination and oxygen absorption.

The uniformity of results is also greatly dependent upon the amount of training which the subject has had. In general, one can not expect so good results from untrained subjects, particularly if they are pathological, as from trained subjects. This is generally true, regardless of the apparatus which is used. With no known respiration apparatus can an investigator be absolutely certain that the results obtained in a first experiment with a subject will be accurate. Magnus-Levy ${ }^{1}$ has stated that in one case it was necessary for him to make experiments with one subject daily for over 10 days before he was certain that there was not a slight diminished metabolism due to the lack of training.

In drawing conclusions, the results obtained must be very carefully examined and the different factors involved compared. For example, the values for the carbon-dioxide elimination should be compared with the values for the total ventilation and those for the total ventilation with the respiration-rate. Records of the pulse-rate and respirationrate are of great importance, and valuable evidence as to the character of the respiration may be secured from graphic records. An idea of the eharacter of the experiment may also be obtained from readings of the ventilation from minute to minute, which may be secured from the movements of the spirometer on the Benedict respiration apparatus or from the meter with the Zuntz-Geppert apparatus.

The condition of the subject at the time of the experiment must also be considered very carefully. For example, the results obtained in an experimental period which follows immediately after the subject has lain down upon the couch can not be expected to be comparable with those obtained in the experimental periods following or carried out some time later. A subject should rest quietly upon the couch for at least a half hour, preferably three-quarters of an hour, before the beginning of the experiment, unless a study is being made of the effect of the previous state upon the metabolism. In such a study, however, the same character of results would not be expected as would be obtained when experiments were being made for the purpose of establishing basal values for future work. In determining a base-line for later investigations, extreme care is necessary in the interpretation of results. Furthermore, as uniform results as possible should be secured, otherwise if a very small increase is superimposed upon a variable base-line there is no definite evidence that the increase is positive.

In general, when interpreting the results of experiments, one must distinguish between the variations due to the apparatus and variations due to the subject. The first can be eliminated within certain limits and these limits must be determined for each of the apparatus used.

${ }^{1}$ Magnus-Levy, Zeitschr. f. klin. Med., 1897, 33, p. 258. 
It is recommended that so far as possible all respiration apparatus be controlled by means of some method in which a known quantity of the gases is measured. For instance, candles, alcohol, ether, or other combustible materials may be burned, and, since their composition is definitely known, the oxidation products and oxygen requirement may be definitely measured and compared with the actual determinations made with the apparatus. It must be pointed out that such control tests only prove that the apparatus is theoretically accurate, but does not necessarily prove that all experiments made upon men with this apparatus will give accurate results. Too frequently an apparatus which has been proved to be theoretically correct has been used by investigators in a way in which it was not intended to be used or the experiments were not carried out under proper conditions or were not sufficiently controlled. Far-reaching conclusions and theoretical deductions have then been drawn from a very few experiments. The determination of the respiratory exchange of man in short periods and particularly of the respiratory quotient is a very difficult problem. Conservatism in the acceptance and interpretation of results is therefore strongly recommended because of the great number of variable factors involved in any respiration experiment and because of the great necessity of repeated observations before one can be absolutely certain of the results obtained.

I desire to express my thanks to Miss A. N. Darling for much assistance in the preparation and editing of the manuscript and to Professor Francis G. Benedict for advice and helpful criticism throughout this investigation.

Nutrition Laboratory OF THE

Carnegie Institution of Washington, Boston, Massachusetts, March 17, 1915. 




QP Carpenter, Thorne Martin

121 A comparison of methods

C3 for determining the respiratory exchange of man

Biological

\& Medieal

PLEASE DO NOT REMOVE CARDS OR SLIPS FROM THIS POCKET

UNIVERSITY OF TORONTO LIBRARY 
W.

1030.

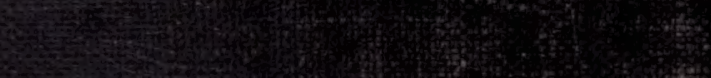

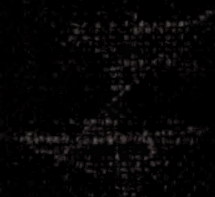

(3)

S.7.

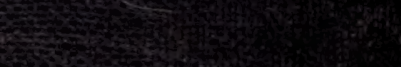

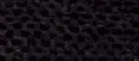

2.t.

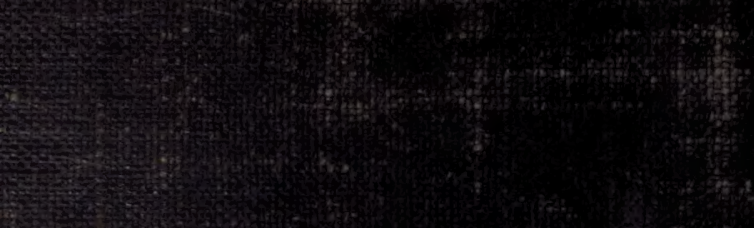

